EXPLORATORY INVESTIGATION OF LEGACY BRIDGE DATABASES IN VIRGINIA

\author{
A Thesis \\ Presented to \\ the faculty of the School of Engineering and Applied Science \\ University of Virginia \\ in partial fulfillment \\ of the requirements for the degree \\ Master of Science \\ by \\ James Taylor Johnston
}

May

2013 


\section{APPROVAL SHEET}

The thesis

is submitted in partial fulfillment of the requirements

for the degree of

Master of Science

James Taylor Johnston, Author

The thesis has been read and approved by the examining committee:

Dr. Steven Chase, Advisor

Dr. Brian Smith, Chair

Dr. Michael Brown

Dr. Jose Gomez

Accepted for the School of Engineering and Applied Science:

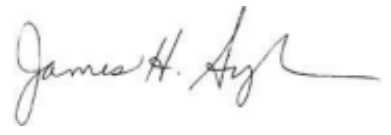

Dean, School of Engineering and Applied Science

May 


\title{
EXPLORATORY INVESTIGATION OF LEGACY BRIDGE DATABASES IN VIRGINIA
}

James Taylor Johnston

\begin{abstract}
This report is an investigation of data mining of legacy bridge databases, focusing on the Pontis and National Bridge Inventory databases maintained by the Virginia Department of Transportation (VDOT). This project is applied in nature as it was sponsored by the Virginia Center for Transportation Innovation and Research (VCTIR). Exploratory data analysis was performed using Microsoft Excel, and resulted in patterns and projections that were unavailable directly from the raw inspection report data.

Data mining techniques were applied to 6 sub-studies conducted in response to VDOT interests. First, district-based trends in various element condition states across Virginia were determined and the results were summarized using graphs created from the Pontis element data. Second, a state-wide identification of potential inspector data-entry errors was conducted. Third, as part of a wider VCTIR study, the effectiveness of zinc-based coating systems was investigated by looking at deterioration of steel girders using National Bridge Inventory (NBI) and Pontis data; deteriorated sections of girder elements on the same set of bridges were also identified. Fourth, joint closures occurring between 1995 and 2010 were discovered and categorized. Fifth, priority bridges were identified around Virginia, and trips to the nine VDOT district offices were taken to investigate which maintenance actions caused improvements to specific elements on those bridges. Lastly, linear least squares regression and Markov chain condition state transition modeling were performed on selected elements to predict deterioration.
\end{abstract}




\section{ACKNOWLEDGEMENTS}

I would first like to express my gratitude to Dr. Steven Chase who has been my primary advisor in this investigatory analysis. Without his guidance, the project would have been unstructured and meaningful conclusions would have been difficult to draw.

I also wish to thank the other members of the project advisory group: Mr. Adam Matteo, Mr. Jeffrey Milton, Mr. Rex Pearce, Dr. Michael Brown, and Mr. Prasad Nallapaneni. This group is part of the Bridge Information Systems Laboratory for Virginia that directed me towards the various side studies that were completed using the wealth of data in the Pontis database.

I wish to thank UVA for their financial support throughout this research endeavor that I have undertaken for them and the Virginia Center for Transportation Innovation and Research.

I would like to thank the VDOT district bridge engineers and their staff that assisted me in finding the requested inspection reports in the district office visit portion of this exploration. These people (and their associated district) were: Lance Click and Darryl Raines (Bristol), David Wright (Salem), Pettis Bond (Lynchburg), Thomas Lester and William Danzeisen (Richmond), Shannon Ternes and Andrew Werner (Hampton Roads), Leslie Danovich and Annette Adams (Fredericksburg), Christopher Williams and Jeremy McCray (Culpeper), Barton Boyd and Richard Burton (Staunton), and Ali Foroughi (Northern Virginia). Special thanks to Mr. McCray from the Culpeper district who took time out of multiple days to help locate folders both at the district office in the city of Culpeper and in the residency office in Charlottesville, as their inspection history database was in the process of reorganization between various locations.

Lastly, I wish to thank my mother, Althea Johnston, for her continued support and encouragement. 


\section{TABLE OF CONTENTS}

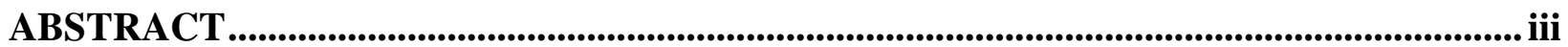

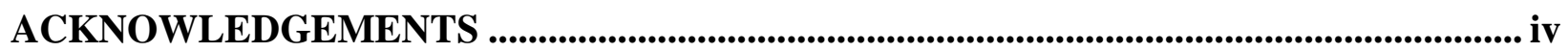

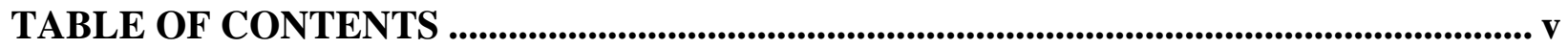

LIST OF TABLES .......................................................................................................... viii

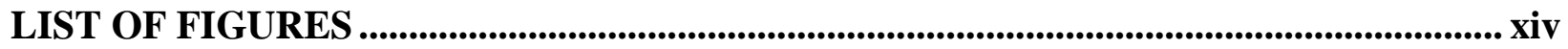

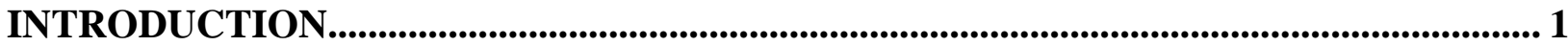

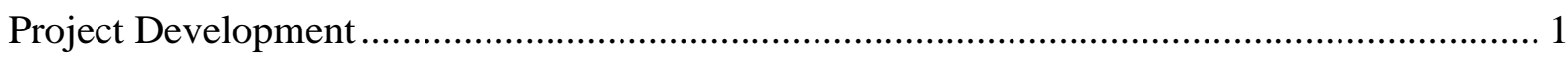

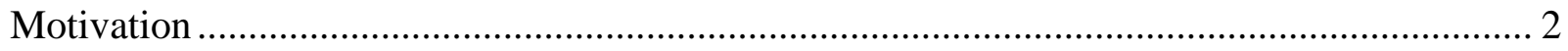

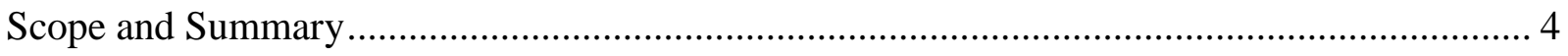

REVIEW OF THE LITERATURE ............................................................................................ 6

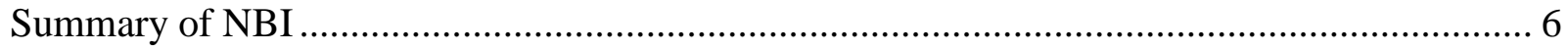

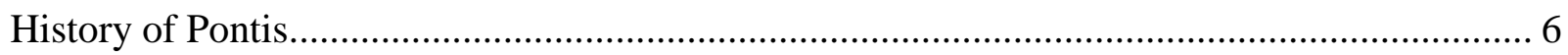

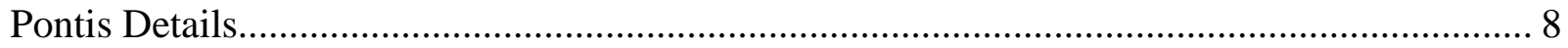

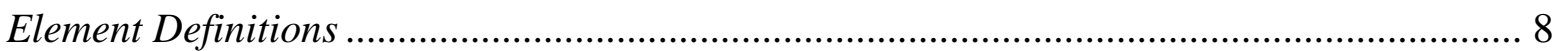

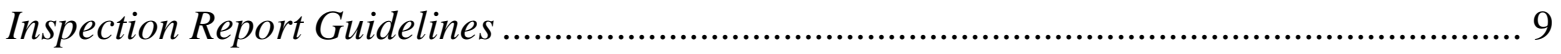

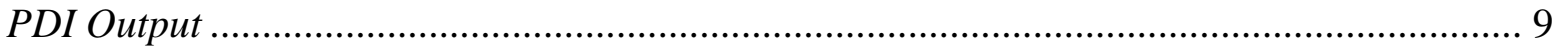

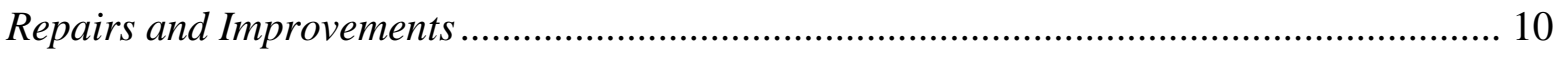

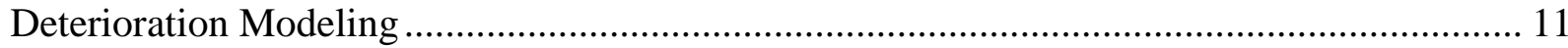

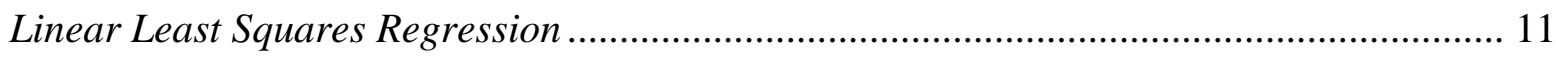

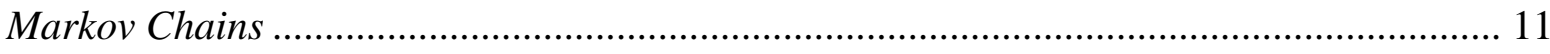

Knowledge Discovery in Databases and Data Mining............................................................ 12 


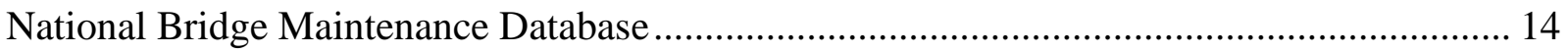

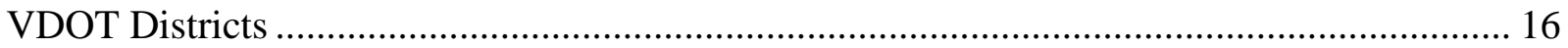

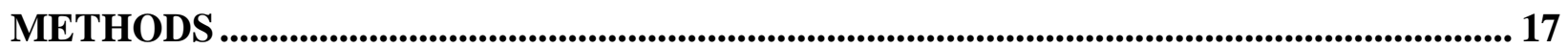

Original Pontis Analysis by District.................................................................................. 17

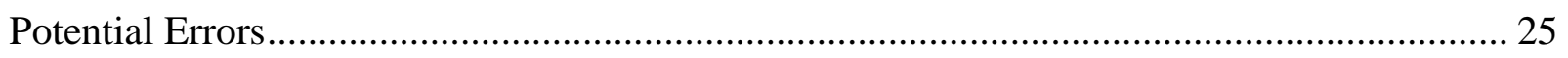

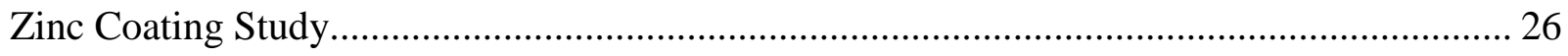

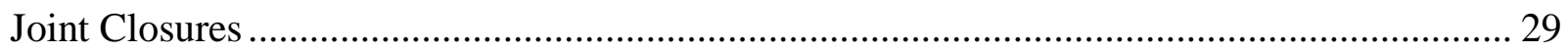

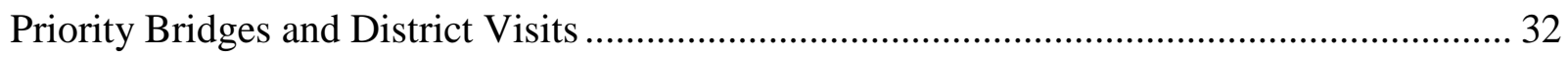

Linear Least Squares and Markov Modeling .......................................................................... 37

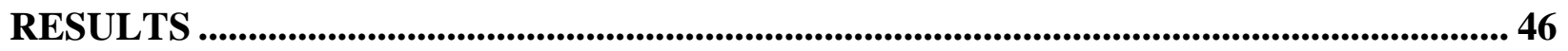

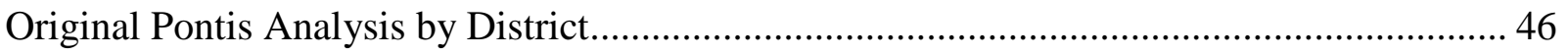

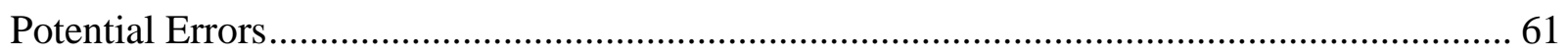

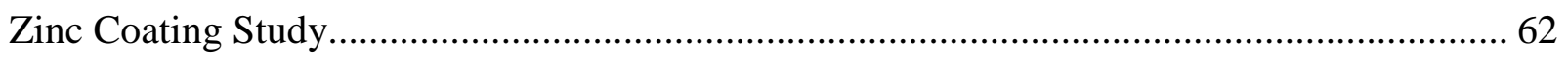

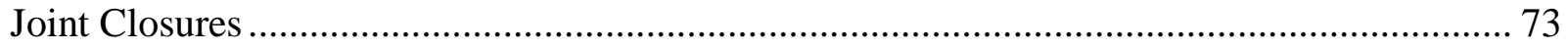

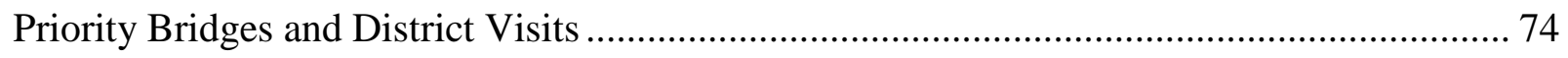

Linear Least Squares and Markov Modeling …………...................................................... 79

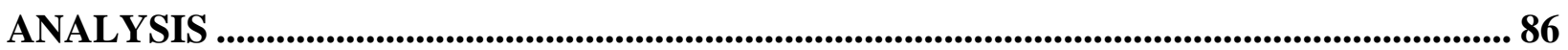

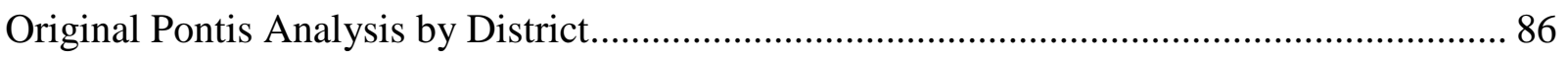

Potential Errors............................................................................................................... 91

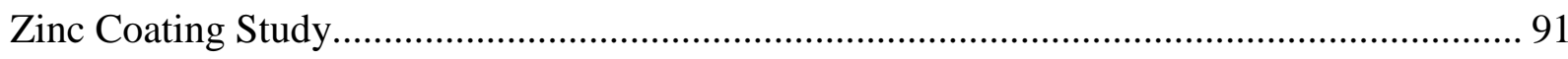

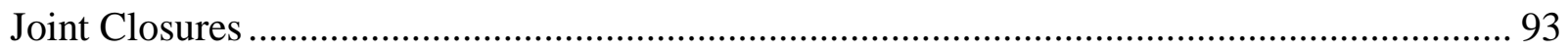

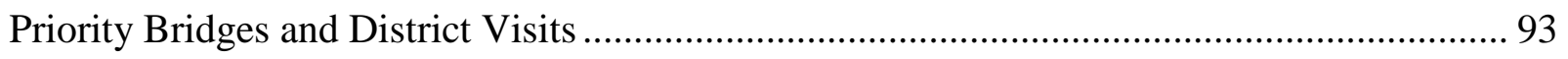


Linear Least Squares and Markov Chain Modeling.

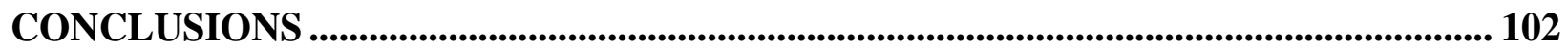

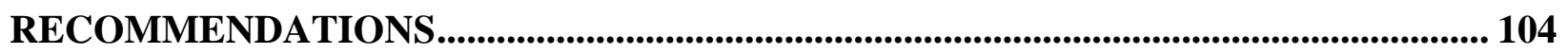

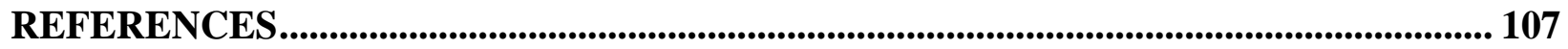

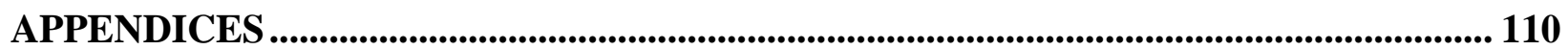

APPENDIX A - ELEMENT CODES AND DESCRIPTIONS ………………………......... 111

APPENDIX B - CONDITION STATE TRENDS ……………………………………....... 115

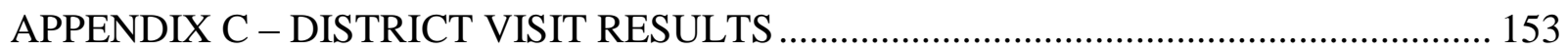

APPENDIX D - FULL LLS AND MARKOV CHAIN RESULTS ....................................... 167 


\section{LIST OF TABLES}

Table 1 - Example Selection of Raw District Analysis Data (District 1) .................................. 18

Table 2 - Example Selection of Counting Table for Element and Smart Flag Selection (District 1)

Table 3 - Example Selection for Unexpected Improvement (Element 334 - District 5).............. 20

Table 4 - Example Selection for Painting as Known Improvement (Element 108 - District 2).. 22

Table 5 - Example Selection for Summed Condition States (Element 334) …........................... 24

Table 6 - Example Selection for Age since Improvement (Element 334) ...................................... 24

Table 7 - Example Selection for Count of Reports by Improvement Year and Inspection Year

(Element 334)

Table 8 - Example Selection of Condition State Trend by Age since Improvement (Element 334)

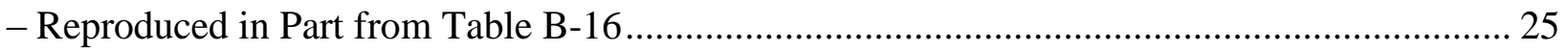

Table 9 - Example Error Finding Process - Selection from District 3 ........................................ 26

Table 10 - Example Selection from Zinc Study of Yearly Condition State by Quantity (Group 1)

Table 11 - Example Joint Elimination Processing (District 9) ……………………………........ 31

Table 12 - Example Selection of Priority Bridge Choices (District 2) .......................................... 34

Table 13 - Example Selection of Bridge Information for Report Finding (District 9) ................. 35

Table 14 - Example Selection of Table Taken to District Visit (District 2) .................................. 36

Table 15 - Example Selection of District Visit Complete Information (District 6)....................... 37

Table 16 - Example Selection of Linear Least Squares Appended Data - Element 215 - District

1

Table 17 - Example Selection for LLS Analysis Based on Age of Bridges - Element 107 ........ 39 
Table 18 - Example Selection for Number of Bridges Reporting by Age - Element 107 .......... 40

Table 19 - Example Selection of Table of Raw Data for Markov Processing (Element 107)...... 42

Table 20 - Example Selection of Summary Table of Raw Data Arranged by Age and Condition

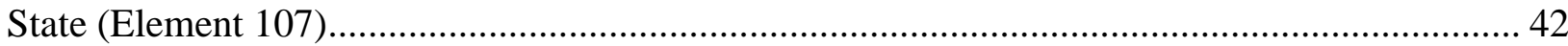

Table 21 - Example Selection of Age-Based Condition State Data (Element 107 - District 1) .. 43

Table 22 - Example Transition Probability Matrix - Element 107 - District 1 .......................... 44

Table 23 - Example Selection of Condition State Probability Prediction by Age - Element 107 -

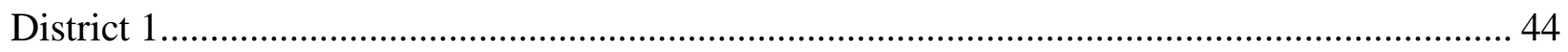

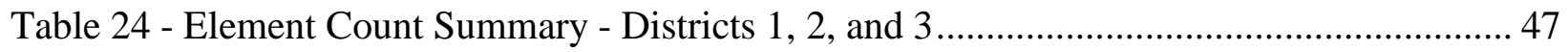

Table 25 - Element Count Summary - Districts 4, 5, and 6.............................................. 48

Table 26 - Element Count Summary - Districts 7, 8, and 9.............................................. 49

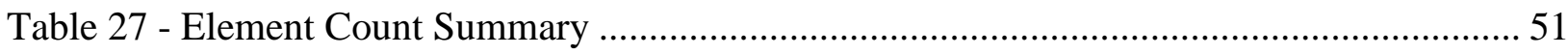

Table 28 - Smart Flag Count Summary .................................................................. 52

Table 29 - Smart Flag and Element Count Summary - Total Count ..................................... 53

Table 30 - Statewide Condition State Improvements by Year for Element 334 - Reproduced

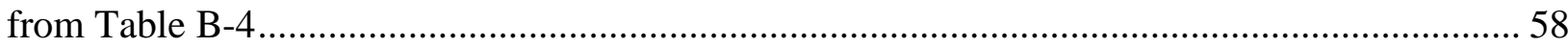

Table 31 - Statewide Condition State Improvements by Year for Smart Flag 358 - Reproduced

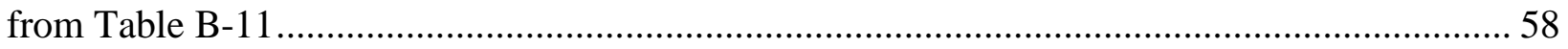

Table 32 - Statewide Condition State Trend by Age since Improvement for Element 108 -

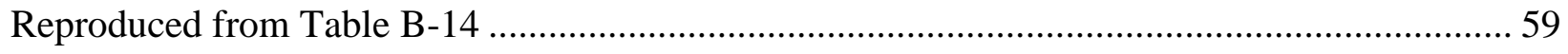

Table 33 - Statewide Condition State Trend by Age since Improvement for Element 706 -

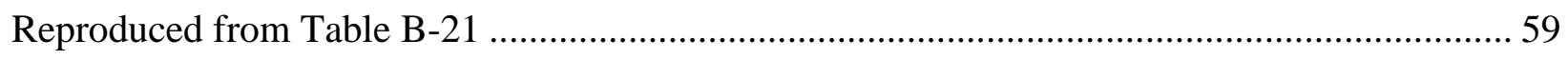

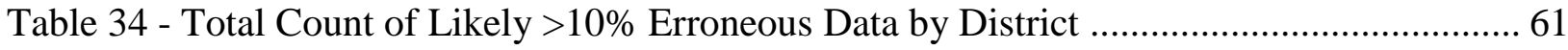


Table 35 - Average Year Built / Painted and Average Age of Bridge / Paint 72

Table 36 - Joint Closure Count Summary ….............................................................. 73

Table 37 - Number of Unexpected Improvements by District for Selected Element ................. 74

Table 38 - Maintenance Action Codes and Descriptions - Reproduced from Table C-25 …...... 75

Table 39 - District Visit Maintenance Summary - Element 107 - All Road Types - Reproduced

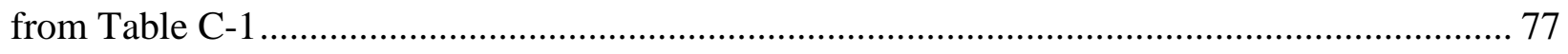

Table 40 - District Visit Maintenance Summary - Element 107 - Interstate Roads - Reproduced

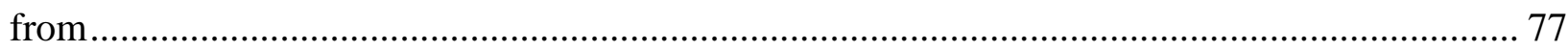

Table 41 - District Visit Maintenance Summary - Element 107 - Primary Roads - Reproduced

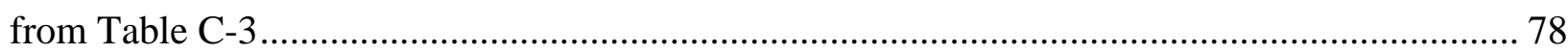

Table 42 - District Visit Maintenance Summary - Element 107 - Secondary Roads - Reproduced

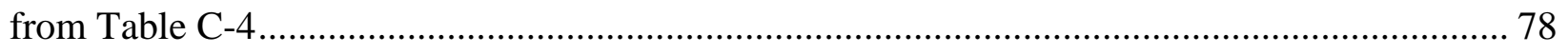

Table 43 - Linear Least Squares Summary for Top Elements by District ............................. 79

Table 44 - Markov Transition Probability Matrices - Element 107 - Districts 1 and 2 -

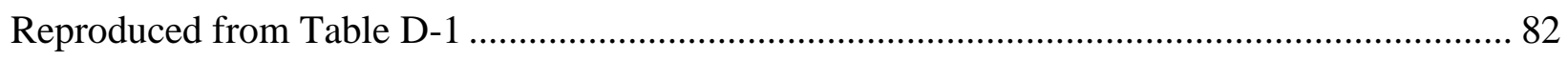

Table 45 - Markov Transition Probability Matrices - Element 107 - Districts 3 and 4 -

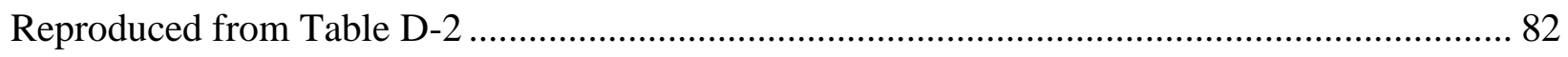

Table B-1 - Statewide Condition State Improvements by Year for Element 32 .................... 144

Table B-2 - Statewide Condition State Improvements by Year for Element 108 ................... 144

Table B-3 - Statewide Condition State Improvements by Year for Element 302 ................... 144

Table B-4 - Statewide Condition State Improvements by Year for Element 334 ................... 144

Table B-5 - Statewide Condition State Improvements by Year for Element 240 ................... 145

Table B-6 - Statewide Condition State Improvements by Year for Element 107 ................... 145 
Table B-7 - Statewide Condition State Improvements by Year for Smart Flag 359 ................ 145

Table B-8 - Statewide Condition State Improvements by Year for Smart Flag 702 ................ 145

Table B-9 - Statewide Condition State Improvements by Year for Smart Flag 706 ................ 146

Table B-10 - Statewide Condition State Improvements by Year for Smart Flag 363 .............. 146

Table B-11 - Statewide Condition State Improvements by Year for Smart Flag 358 .............. 146

Table B-12 - Statewide Condition State Improvements by Year for Smart Flag 704 .............. 146

Table B-13 - Statewide Condition State Trend by Age since Improvement for Element 32 ..... 147

Table B-14 - Statewide Condition State Trend by Age since Improvement for Element 108 ... 147

Table B-15 - Statewide Condition State Trend by Age since Improvement for Element 302 ... 147

Table B-16 - Statewide Condition State Trend by Age since Improvement for Element 334 ... 147

Table B-17 - Statewide Condition State Trend by Age since Improvement for Element 240 ... 148

Table B-18 - Statewide Condition State Trend by Age since Improvement for Element 107 ... 148

Table B-19 - Statewide Condition State Trend by Age since Improvement for Smart Flag 359148

Table B-20 - Statewide Condition State Trend by Age since Improvement for Smart Flag 702148

Table B-21 - Statewide Condition State Trend by Age since Improvement for Smart Flag 706149

Table B-22 - Statewide Condition State Trend by Age since Improvement for Smart Flag 363149

Table B-23 - Statewide Condition State Trend by Age since Improvement for Smart Flag 358149

Table B-24 - Statewide Condition State Trend by Age since Improvement for Smart Flag 704149

Table C-1 - District Visit Maintenance Summary - Element 107 - All Road Classifications .. 154

Table C-2 - District Visit Maintenance Summary - Element 107 - Interstate Roads .............. 154

Table C-3 - District Visit Maintenance Summary - Element 107 - Primary Roads ................ 155

Table C-4 - District Visit Maintenance Summary - Element 107 - Secondary Roads ............ 155

Table C-5 - District Visit Maintenance Summary - Element 108 - All Road Classifications .. 156 
Table C-6 - District Visit Maintenance Summary - Element 108 - Interstate Roads 156

Table C-7 - District Visit Maintenance Summary - Element 108 - Primary Roads 157

Table C-8 - District Visit Maintenance Summary - Element 108 - Secondary Roads 157

Table C-9 - District Visit Maintenance Summary - Element 301 - All Road Classifications .. 158

Table C-10 - District Visit Maintenance Summary - Element 301 - Interstate Roads 158

Table C-11 - District Visit Maintenance Summary - Element 301 - Primary Roads 159

Table C-12 - District Visit Maintenance Summary - Element 301 - Secondary Roads 159

Table C-13 - District Visit Maintenance Summary - Element 302 - All Road Classifications 160

Table C-14 - District Visit Maintenance Summary - Element 302 - Interstate Roads 160

Table C-15 - District Visit Maintenance Summary - Element 302 - Primary Roads 161

Table C-16 - District Visit Maintenance Summary - Element 302 - Secondary Roads 161

Table C-17 - District Visit Maintenance Summary - Element 311 - All Road Classifications 162

Table C-18 - District Visit Maintenance Summary - Element 311 - Interstate Roads 162

Table C-19 - District Visit Maintenance Summary - Element 311 - Primary Roads 163

Table C-20 - District Visit Maintenance Summary - Element 311 - Secondary Roads 163

Table C-21 - District Visit Maintenance Summary - Element 313 - All Road Classifications 164 Table C-22 - District Visit Maintenance Summary - Element 313 - Interstate Roads 164

Table C-23 - District Visit Maintenance Summary - Element 313 - Primary Roads 165

Table C-24 - District Visit Maintenance Summary - Element 313 - Secondary Roads 165

Table C-25 - Maintenance Action Codes and Descriptions 166

Table C-26 - Road Type Codes, Descriptions, and Classifications 166

Table D-1 - Markov Transition Probability Matrices - Element 107 - Districts 1 and 2.......... 188

Table D-2 - Markov Transition Probability Matrices - Element 107 - Districts 3 and 4. 188 
Table D-3 - Markov Transition Probability Matrices - Element 107 - Districts 5 and 6......... 188

Table D-4 - Markov Transition Probability Matrices - Element 107 - District 7 and 8 ........... 188

Table D-5 - Markov Transition Probability Matrices - Element 107 - District 9 and Total State 189

Table D-6 - Markov Transition Probability Matrices - Element 108 - Districts 1 and 2......... 189

Table D-7 - Markov Transition Probability Matrices - Element 108 - Districts 3 and 4......... 189

Table D-8 - Markov Transition Probability Matrices - Element 108 - Districts 5 and 6......... 189

Table D-9 - Markov Transition Probability Matrices - Element 108 - Districts 7 and 8......... 190

Table D-10 - Markov Transition Probability Matrices - Element 108 - District 9 and Total State 190

Table D-11 - Markov Transition Probability Matrices - Element 302 - Districts 1 and 2....... 190

Table D-12 - Markov Transition Probability Matrices - Element 302 - Districts 3 and 4....... 190

Table D-13 - Markov Transition Probability Matrices - Element 302 - Districts 5 and 6....... 191

Table D-14 - Markov Transition Probability Matrices - Element 302 - Districts 7 and 8 ....... 191

Table D-15 - Markov Transition Probability Matrices - Element 302 - District 9 and Total State

Table D-16 - Markov Transition Probability Matrices - Element 32 - Districts 1 and 2......... 191

Table D-17 - Markov Transition Probability Matrices - Element 32 - Districts 3 and 5......... 192

Table D-18 - Markov Transition Probability Matrices - Element 32 - Districts 7 and $8 \ldots \ldots . . . .192$

Table D-19 - Markov Transition Probability Matrix - Element 32 - Total State.................... 192 


\section{LIST OF FIGURES}

Figure 1 - Map of VDOT Districts (Courtesy of VDOT) ........................................................ 16

Figure 2 - Example Addition of Element 107 Data to 108 Table (District 8) ……....................... 21

Figure 3 - Element 32 (Timber Deck - with Asphaltic Concrete (AC) Overlay) Average

Condition State Trend - Improvement Noted - Reproduced from Figure B-1 55

Figure 4 - Element 32 Average Condition State Trend - Improvement Not Noted - Reproduced from Figure B-2

Figure 5 - Element 32 Normalized Condition State Trend - Improvement Noted - Reproduced from Figure B-3 56

Figure 6 - Element 32 Normalized Condition State Trend - Improvement Not Noted -

Reproduced from Figure B-4 56

Figure 7 - Element 32 Normalized Condition State Trend - Improvement Noted (Quantity

Analysis) - Reproduced from Figure B-5

Figure 8 - Element 32 Normalized Condition State Trend - Improvement Not Noted (Quantity

Analysis) - Reproduced from Figure B-6......

Figure 9 - Condition State Improvement Trend for Element 32 - Reproduced from Figure B-57

Figure 10 - Condition State Improvement Trend for Element 240 - Reproduced from Figure

B-58.

Figure 11 - Zinc Coating - Summary Graphs of Quantity in Each Condition State by Year -

Two-Year Grouping.

Figure 12 - Zinc Coating - Normalized Summary Graphs of Proportion of Inventory in Each Condition State Range by Year (Quantity Analysis) 
Figure 13 - Zinc Coating - Normalized Summary Graphs of Proportion of Inventory in Each Condition State Range by Year. 66

Figure 14 - Zinc Coating - Normalized Summary Graphs of Condition Rating Analysis by Year 67

Figure 15 - Zinc Coating - Summary Graphs of Quantity in Each Condition State by Age since

Painting 68

Figure 16 - Zinc Coating - Condition State Trends by Age since Painting 69

Figure 17 - Zinc Coating - Condition Rating Trends by Age since Painting .......................... 70

Figure 18 - Zinc Coating - Summary Trends for Condition State Deterioration ...................... 71

Figure 19 - Zinc Coating - Summary Trends for Condition Rating Deterioration.................... 71

Figure 20 - Condition State History and Linear Trendline by Age - Element 107 - Total State -

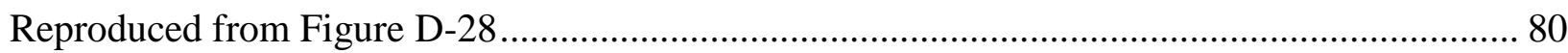

Figure 21 - Condition States Residuals (Prediction Minus Actual) by Age - Element 107 - Total

State - Reproduced from Figure D-29 81

Figure 22 - Number of Bridges Reporting by Age - Element 107 - Total State - Reproduced from Figure D-30 81

Figure 23 - Markov Chain Prediction Graphs - Element 107 - District 2 Prediction vs. Actual

Graphs - Reproduced from Figure D-62. 84

Figure B-1 - Element 32 (Timber Deck - with Asphaltic Concrete (AC) Overlay) Average

Condition State Trend - Improvement Noted 116

Figure B-2 - Element 32 Average Condition State Trend - Improvement Not Noted 116

Figure B-3 - Element 32 Normalized Condition State Trend - Improvement Noted. 117

Figure B-4 - Element 32 Normalized Condition State Trend - Improvement Not Noted 117 
Figure B-5 - Element 32 Normalized Condition State Trend - Improvement Noted (Quantity Analysis) 118

Figure B-6 - Element 32 Normalized Condition State Trend - Improvement Not Noted (Quantity Analysis) 118

Figure B-7 - Element 108 (Steel Open Girder with Timber Deck - Coated and Uncoated)

Average Condition State Trend - Improvement Noted 119

Figure B-8 - Element 108 Average Condition State Trend - Improvement Noted 119

Figure B-9 - Element 108 Normalized Condition State Trend - Improvement Noted 120

Figure B-10 - Element 108 Normalized Condition State Trend - Improvement Not Noted ..... 120 Figure B-11 - Element 108 Normalized Condition State Trend - Improvement Noted (Quantity Analysis) 121

Figure B-12 - Element 108 Normalized Condition State Trend - Improvement Not Noted

(Quantity Analysis)

Figure B-13 - Element 302 (Compression Joint Seal) Average Condition State Trend Improvement Noted 122

Figure B-14 - Element 302 Average Condition State Trend - Improvement Not Noted 122

Figure B-15 - Element 302 Normalized Condition State Trend - Improvement Noted 123

Figure B-16 - Element 302 Normalized Condition State Trend - Improvement Not Noted ..... 123

Figure B-17 - Element 302 Normalized Condition State Trend - Improvement Noted (Quantity Analysis) 124

Figure B-18 - Element 302 Normalized Condition State Trend - Improvement Not Noted (Quantity Analysis) 124 
Figure B-19 - Element 334 (Metal Bridge Railing - Coating) Average Condition State Trend -

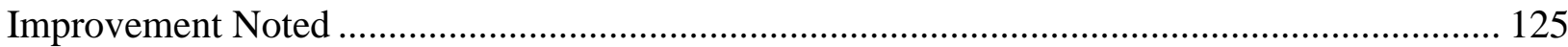

Figure B-20 - Element 334 Average Condition State Trend - Improvement Not Noted ......... 125

Figure B-21 - Element 334 Normalized Condition State Trend - Improvement Noted ........... 126

Figure B-22 - Element 334 Normalized Condition State Trend - Improvement Not Noted ..... 126

Figure B-23 - Element 240 (Metal Culvert) Average Condition State Trend - Improvement

Noted

Figure B-24 - Element 240 Average Condition State Trend - Improvement Not Noted

Figure B-25 - Element 240 Normalized Condition State Trend - Improvement Noted 128

Figure B-26 - Element 240 Normalized Condition State Trend - Improvement Not Noted ..... 128

Figure B-27 - Element 107 (Steel Open Girder - Coated) Average Condition State Trend Improvement Noted 129

Figure B-28 - Element 107 Average Condition State Trend - Improvement Not Noted 129

Figure B-29 - Element 107 Normalized Condition State Trend - Improvement Noted 130

Figure B-30 - Element 107 Normalized Condition State Trend - Improvement Not Noted ..... 130 Figure B-31 - Element 107 Normalized Condition State Trend - Improvement Noted (Quantity Analysis) 131

Figure B-32 - Element 107 Normalized Condition State Trend - Improvement Not Noted (Quantity Analysis) 131

Figure B-33 - Smart Flag 359 (Soffit of Concrete) Average Condition State Trend Improvement Noted 132

Figure B-34 - Smart Flag 359 Average Condition State Trend - Improvement Not Noted ...... 132 Figure B-35 - Smart Flag 359 Normalized Condition State Trend - Improvement Noted 133 
Figure B-36 - Smart Flag 359 Normalized Condition State Trend - Improvement Not Noted. 133

Figure B-37 - Smart Flag 702 (Drains) Average Condition State Trend - Improvement Noted134

Figure B-38 - Smart Flag 702 Average Condition State Trend - Improvement Not Noted ...... 134

Figure B-39 - Smart Flag 702 Normalized Condition State Trend - Improvement Noted........ 135

Figure B-40 - Smart Flag 702 Normalized Condition State Trend - Improvement Not Noted. 135

Figure B-41 - Smart Flag 706 (Soffit of Overhang of Concrete) Average Condition State Trend -

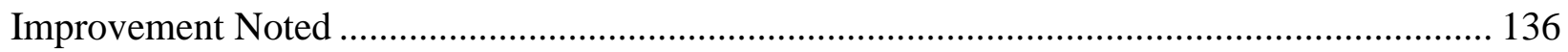

Figure B-42 - Smart Flag 706 () Average Condition State Trend - Improvement Not Noted .. 136

Figure B-43 - Smart Flag 706 Normalized Condition State Trend - Improvement Noted........ 137

Figure B-44 - Smart Flag 706 Normalized Condition State Trend - Improvement Not Noted. 137

Figure B-45 - Smart Flag 363 (Section Loss) Average Condition State Trend - Improvement

Noted 138

Figure B-46 - Smart Flag 363 Average Condition State Trend - Improvement Not Noted ...... 138

Figure B-47 - Smart Flag 363 Normalized Condition State Trend - Improvement Noted........ 139

Figure B-48 - Smart Flag 363 Normalized Condition State Trend - Improvement Not Noted. 139

Figure B-49 - Smart Flag 358 (Deck Cracking) Average Condition State Trend - Improvement

Noted 140

Figure B-50 - Smart Flag 358 Average Condition State Trend - Improvement Not Noted ...... 140

Figure B-51 - Smart Flag 358 Normalized Condition State Trend - Improvement Noted........ 141

Figure B-52 - Smart Flag 358 Normalized Condition State Trend - Improvement Not Noted. 141

Figure B-53 - Smart Flag 704 (Roadway Over Culverts) Average Condition State Trend -

Improvement Noted

Figure B-54 - Smart Flag 704 Average Condition State Trend - Improvement Not Noted ...... 142 
Figure B-55 - Smart Flag 704 Normalized Condition State Trend - Improvement Noted 143

Figure B-56 - Smart Flag 704 Normalized Condition State Trend - Improvement Not Noted . 143

Figure B-57 - Statewide Condition State Trend Lines by Age since Improvement for Elements

$32,108,302$, and 334

Figure B-58 - Statewide Condition State Trend Lines by Age since Improvement for Elements

240 and 107 and Smart Flags 359 and 702

Figure B-59 - Statewide Condition State Trend Lines by Age since Improvement for Smart Flags

$706,363,358$, and 704 152

Figure D-1 - Condition State History and Linear Trendline by Age - Element 107 - District 1

Figure D-2 - Condition States Residuals (Prediction Minus Actual) by Age - Element 107 -

District 1 168

Figure D-3 - Number of Bridges Reporting by Age - Element 107 - District 1 168

Figure D-4 - Condition State History and Linear Trendline by Age - Element 107 - District 2

Figure D-5 - Condition States Residuals (Prediction Minus Actual) by Age - Element 107 -

District 2 169

Figure D-6 - Number of Bridges Reporting by Age - Element 107 - District 2 169

Figure D-7 - Condition State History and Linear Trendline by Age - Element 107 - District 3

Figure D-8 - Condition States Residuals (Prediction Minus Actual) by Age - Element 107 -

District 3 170

Figure D-9 - Number of Bridges Reporting by Age - Element 107 - District 3 170 
Figure D-10 - Condition State History and Linear Trendline by Age - Element 107 - District 4

Figure D-11 - Condition States Residuals (Prediction Minus Actual) by Age - Element 107 -

District 4.

Figure D-12 - Number of Bridges Reporting by Age - Element 107 - District 4

Figure D-13 - Condition State History and Linear Trendline by Age - Element 107 - District 5

Figure D-14 - Condition States Residuals (Prediction Minus Actual) by Age - Element 107 -

District 5.

Figure D-15 - Number of Bridges Reporting by Age - Element 107 - District 5 .....

Figure D-16 - Condition State History and Linear Trendline by Age - Element 107 - District 6

Figure D-17 - Condition States Residuals (Prediction Minus Actual) by Age - Element 107 -

District 6. 173

Figure D-18 - Number of Bridges Reporting by Age - Element 107 - District 6 . 173

Figure D-19 - Condition State History and Linear Trendline by Age - Element 107 - District 7

Figure D-20 - Condition States Residuals (Prediction Minus Actual) by Age - Element 107 -

District 7.

Figure D-21 - Number of Bridges Reporting by Age - Element 107 - District 7. 174

Figure D-22 - Condition State History and Linear Trendline by Age - Element 107 - District 8 
Figure D-23 - Condition States Residuals (Prediction Minus Actual) by Age - Element 107 District 8 . 175

Figure D-24 - Number of Bridges Reporting by Age - Element 107 - District 8 175

Figure D-25 - Condition State History and Linear Trendline by Age - Element 107 - District 9

Figure D-26 - Condition States Residuals (Prediction Minus Actual) by Age - Element 107 -

District 9. 176

Figure D-27 - Number of Bridges Reporting by Age - Element 107 - District 9. 176

Figure D-28 - Condition State History and Linear Trendline by Age - Element 107 - Total State

Figure D-29 - Condition States Residuals (Prediction Minus Actual) by Age - Element 107 -

Total State 177

Figure D-30 - Number of Bridges Reporting by Age - Element 107 - Total State..... 177

Figure D-31 - Condition State History and Linear Trendline by Age - Element 108 - District 1

Figure D-32 - Condition States Residuals (Prediction Minus Actual) by Age - Element 108 -

District 1 178

Figure D-33 - Number of Bridges Reporting by Age - Element 108 - District 1. 178

Figure D-34 - Condition State History and Linear Trendline by Age - Element 108 - District 2

Figure D-35 - Condition States Residuals (Prediction Minus Actual) by Age - Element 108 -

District 2 . 179

Figure D-36 - Number of Bridges Reporting by Age - Element 108 - District 2. 179 
Figure D-37 - Condition State History and Linear Trendline by Age - Element 108 - District 3

Figure D-38 - Condition States Residuals (Prediction Minus Actual) by Age - Element 108 -

District 3. 180

Figure D-39 - Number of Bridges Reporting by Age - Element 108 - District 3. 180

Figure D-40 - Condition State History and Linear Trendline by Age - Element 108 - District 4

Figure D-41 - Condition States Residuals (Prediction Minus Actual) by Age - Element 108 -

District 4. 181

Figure D-42 - Number of Bridges Reporting by Age - Element 108 - District 4 181

Figure D-43 - Condition State History and Linear Trendline by Age - Element 108 - District 5

Figure D-44 - Condition States Residuals (Prediction Minus Actual) by Age - Element 108 -

District 5 . 182

Figure D-45 - Number of Bridges Reporting by Age - Element 108 - District 5. 182

Figure D-46 - Condition State History and Linear Trendline by Age - Element 108 - District 6

Figure D-47 - Condition States Residuals (Prediction Minus Actual) by Age - Element 108 -

District 6 . 183

Figure D-48 - Number of Bridges Reporting by Age - Element 108 - District 6 ..... 183

Figure D-49 - Condition State History and Linear Trendline by Age - Element 108 - District 7 
Figure D-50 - Condition States Residuals (Prediction Minus Actual) by Age - Element 108 District 7 184

Figure D-51 - Number of Bridges Reporting by Age - Element 108 - District 7 184

Figure D-52 - Condition State History and Linear Trendline by Age - Element 108 - District 8

Figure D-53 - Condition States Residuals (Prediction Minus Actual) by Age - Element 108 -

District 8 . 185

Figure D-54 - Number of Bridges Reporting by Age - Element 108 - District 8 . 185

Figure D-55 - Condition State History and Linear Trendline by Age - Element 108 - District 9 186

Figure D-56 - Condition States Residuals (Prediction Minus Actual) by Age - Element 108 -

District 9 186

Figure D-57 - Number of Bridges Reporting by Age - Element 108 - District 9. 186

Figure D-58 - Condition State History and Linear Trendline by Age - Element 108 - Total State 187

Figure D-59 - Condition States Residuals (Prediction Minus Actual) by Age - Element 108 -

Total State 187

Figure D-60 - Number of Bridges Reporting by Age - Element 108 - Total State..... 187

Figure D-61 - Markov Chain Prediction vs. Actual Graphs - Element 107 - Total State and

District 1 193

Figure D-62 - Markov Chain Prediction vs. Actual Graphs - Element 107 - Districts 2 and 3194

Figure D-63 - Markov Chain Prediction vs. Actual Graphs - Element 107 - Districts 4 and 5195

Figure D-64 - Markov Chain Prediction vs. Actual Graphs - Element 107 - Districts 6 and 7196 
Figure D-65 - Markov Chain Prediction vs. Actual Graphs - Element 107 - Districts 8 and 9197

Figure D-66 - Markov Chain Prediction vs. Actual Graphs - Element 108 - Total State and

District 1 198

Figure D-67 - Markov Chain Prediction vs. Actual Graphs - Element 108 - Districts 2 and 3199

Figure D-68 - Markov Chain Prediction vs. Actual Graphs - Element 108 - Districts 4 and 5200

Figure D-69 - Markov Chain Prediction vs. Actual Graphs - Element 108 - Districts 6 and 7201

Figure D-70 - Markov Chain Prediction vs. Actual Graphs - Element 108 - Districts 8 and 9202

Figure D-71 - Markov Chain Prediction vs. Actual Graphs - Element 302 - Total State and

District 1 203

Figure D-72 - Markov Chain Prediction vs. Actual Graphs - Element 302 - Districts 2 and 3204

Figure D-73 - Markov Chain Prediction vs. Actual Graphs - Element 302 - Districts 4 and 5205

Figure D-74 - Markov Chain Prediction vs. Actual Graphs - Element 302 - Districts 6 and 7206

Figure D-75 - Markov Chain Prediction vs. Actual Graphs - Element 302 - Districts 8 and 9207

Figure D-76 - Markov Chain Prediction vs. Actual Graphs - Element 32 - Total State and

District 1 208

Figure D-77 - Markov Chain Prediction vs. Actual Graphs - Element 32 - Districts 2 and 3.. 209

Figure D-78 - Markov Chain Prediction vs. Actual Graphs - Element 32 - Districts 5 and $7 . .210$

Figure D-79 - Markov Chain Prediction vs. Actual Graphs - Element 32 - District 8 211 


\section{INTRODUCTION}

This introduction is divided into three sections. The first section, Project Development, describes how this investigation came about and how it was guided by various internal VDOT forces. The second section, Motivation, presents the incentive behind the two main subtopics of the study and how other sub-studies emerged. The final section, Scope and Summary, outlines the format of the data mining process and its results.

\section{Project Development}

The National Bridge Inventory (NBI) and Pontis databases were created in 1972 and 1991, respectively, in response to developing congressional demands for stricter bridge inspection and management practices. These databases have provided the means for the Federal Highway Administration (FHWA) to determine funding priorities in bridge maintenance. The primary use of these databases has been by the Federal Highway Administration to manage the National Bridge Program. This project investigates data mining of these legacy databases and seeks to find previously unknown deterioration and improvement trends in Virginia bridges.

The NBI uses condition ratings to quantify the condition of each of 5 major components of the bridge: superstructure, substructure, deck, channels, and culverts (if present). A condition rating of 9 means that component is in pristine condition while a 0 means that component has failed. Pontis breaks these major components down further into elements such as compression joint seal (element 301) and steel open girder - painted (element 107); the full list of these elements and associated codes is available in Appendix A of this report. Pontis stores a condition state for each quantity (linear feet, square feet, each) of these elements on a bridge to 
quantify their condition, with 1 representing a pristine section of element, and a 3, 4, or 5 (depending on element) representing a severely deteriorated section of element.

The methods of Knowledge Discovery in Databases (KDD), specifically exploratory data mining, are predicated on the fact that the researcher does not know what the data can tell them when beginning the analysis. As Tukey pointed out, "exploratory data analysis can never be the whole story, but nothing else can serve as the foundation — as the first step" (Tukey, 1977). This project reduced the raw Pontis data to several usable tables and found unknown trends; additional studies of this data could produce additional patterns and conclusions.

The Virginia Center for Transportation Innovation and Research in Charlottesville, Virginia created a Bridge Information Systems Laboratory for Virginia to encompass the scope of this project. A project advisory group, comprised of VDOT bridge personnel, was also created to oversee and guide the work conducted by this Laboratory. The members of this group are Mr. Adam Matteo, Mr. Jeffrey Milton, Mr. Rex Pearce, Dr. Steven Chase, Dr. Michael Brown, and Mr. Prasad Nallapaneni. Monthly meetings were held to review ongoing work and generate ideas for the direction of the exploration to take in the month to follow. This active interaction and discussion was a key component of the project.

\section{Motivation}

Two main subtopics were identified for the initial explorations: an investigation on the effectiveness of maintenance actions and a study on the performance of zinc-based coating systems on girders. The objective of the maintenance study was to ascertain what specific actions were being undertaken to maintain various bridge elements, and to begin to analyze the effectiveness of these actions in the years since they were taken. This study was undertaken 
because there is no adequate and readily available bridge maintenance system in place for VDOT currently. Each of the 9 VDOT-maintained districts is in charge of its own system, and while some districts have elected to start an online repository of maintenance actions in recent years, others still use only paper reports for maintenance information.

The zinc performance study examined the coating performance of four groups of bridges. These were: simply supported bridges built after 1983 (group 1), continuous bridges built after 1983 (group 2), simply supported bridges built before 1984 and repainted after 1984 (group 3), and continuous bridges built before 1984 and repainted after 1984 (group 4). All bridges were VDOT-maintained, active, non-posted, with steel beams or girders, and concrete decks from across Virginia (district was ignored). The bridges were divided into these groups to investigate possible differences in performance between structures that had been shop-coated (groups 1 and 2) and structures that had been field-coated (groups 3 and 4). Also, it was postulated that there might be a difference in performance between simply supported structures and continuous structures due to the presence of additional bridge joints in the simply supported structures. This investigation was part of a larger VDOT project investigating the zinc-based coating that has been the primary paint system used on Virginia bridge girders since 1983. The task for this project was to identify Pontis condition state trends for these girders (element 107).

Due to the emergent nature of this project, several side studies arose. During the preparation of the raw data for analysis, condition state improvement and deterioration trends based on year of inspection were discovered and graphed. An investigation of condition state histories related to bridge age since improvement aimed to find more accurate element-based deterioration rates. Another side study was a state-wide identification of potential inspector dataentry errors. Another special analysis requested by VDOT was a classification of different types 
of joint closures that occurred between 1995 and 2010. Additionally, as an addendum to the zinc coating study, deteriorated sections (defined as quantity in condition state 4 and 5) were identified on element 107 (Steel Open Girder - Coated). Lastly, linear least squares regression and Markov chain condition state transition modeling were performed on selected elements to predict deterioration.

\section{Scope and Summary}

This project was applied in nature and focused more on refinement and application of previously developed methodologies than on developing new approaches. These methods were used to investigate legacy Pontis and NBI bridge condition data for VDOT. The project began with a review of exploratory data mining literature, followed by a selection and application of methods that were suitable for the problems posed by the project advisory group.

For the zinc study, various data filters were applied to the VDOT Pontis and NBI data to create data sets of bridges with zinc-based coatings. The data sets were analyzed and several descriptive statistics were produced. These were: the average element 107 condition state for each structure, the total quantity of element 107 and the percent in each condition state, the distribution of average condition states for element 107, the average superstructure condition rating, the distribution of superstructure condition ratings, the percentage of bridges with superstructure condition rating of 7 or higher, and the average years built and painted.

For the maintenance study, six elements were selected by perceived criticality, two each of beams, joints, and bearings. Improvements were identified in the Pontis data for these elements based on a condition state decrease of more than 1.00 from the maximum recorded condition state (between 1995 and 2010) to 2010 values. District visits were made to correlate 
likely maintained bridges (forty per district) with maintenance actions noted on inspection reports. These records are stored as physical written reports at each of the district offices. The results were further reported by classification of the road carried: interstate, primary, or secondary.

In the analysis of condition state trends, elements were first selected by count within each district. The raw Pontis data were reduced to condition state histories between 1995 and 2010, and trends were found for bridges that did and did not have a significant improvement noted. Bridges of varying sizes were given the same weight for most of the analysis. A study was performed using the total quantity of each element per district to investigate if there were any substantial differences in results, and no significant difference was discovered. Investigations of potential inspector errors and noted joint closures were also performed by applying filters to Pontis data. Summary tables and long lists of federal bridge ID numbers were produced and reported to VDOT.

The Linear Least Squares regression analysis required several custom macros that were applied to the Pontis data to generate deterioration trends. The Markov chain modeling produced transition probability matrices and associated deterioration prediction graphs. Both models were applied to element-level data at the state and district level.

Pontis element-level condition state data is sometimes converted to NBI condition ratings using a translator program to conform to established reporting procedures, but the translation results do not always correlate well to corresponding NBI data (Aldemir-Bektas \& Smadi, 2008). This project investigated unmodified NBI data in the zinc coating study, but the main focus was on mining the raw Pontis element-level condition state data. 


\section{REVIEW OF THE LITERATURE}

\section{Summary of NBI}

The National Bridge Inspection Standards (NBIS) were created in response to the 1967 failure of the Silver Bridge between West Virginia and Ohio that resulted in the death of 46 people. Implemented in the early 1970s by the Secretary of Transportation, under pressure from Congress, the NBIS set the specifications on the inspection of bridges on public roads. Information from these inspections is stored in the National Bridge Inventory (NBI) database, created in 1972. FHWA uses the NBI to allocate funds to the states for bridge replacement, rehabilitation and to some extent maintenance (Small, Philbin, Fraher, \& Romack, 1999).

\section{History of Pontis}

Pontis is a Bridge Management System (BMS) that has been adopted for use by 39 states / territories and 7 other agencies in the US, as well as 7 systems internationally. It was created and is maintained through the American Association of State Highway and Transportation (AASHTO)'s joint software development program, which allows agencies to create a unified management system through pooled resources. Pontis has thus been cheaper (to each agency) to both implement and maintain, plus it creates an industry standard of best practice to help standardize bridge management at the national level (Robert, Marshall, Shepard, \& Aldayuz, 2003).

Unlike the NBI database, which stores all information in one massive file, Pontis is based upon a Relational Database Management System. This means that the information is stored in tables that are interconnected, in order to more efficiently reference data in related tables. These tables store records in separate rows and data fields in separate columns. The tables are related 
to each other by key data fields in other tables (unit prices, probabilities, etc.). These systems provide methods to efficiently enter, store, and generate reports from data (Chase, 2011).

Pontis was created in 1991 in response to the Intermodal Surface Transportation Efficiency Act (ISTEA) from Congress requiring each state Department of Transportation (DOT) to implement a more functional / detailed BMS. Funding came from the federal level via contract to industry. A previous system, the NBIS, provided overall condition ratings for each bridge at the deck, superstructure, substructure, channel, and culvert component levels. That was determined to be too subjective (based too heavily on the experience of the bridge inspector), with funding ultimately believed to be going to the wrong bridges (Gutkowski \& Arenella, 1998).

In response to this, Pontis was developed and is a more quantitative BMS that looks at structures at the element level. These elements are well-defined subdivisions of bridge systems such as girders, joints, decks, and railings, each of which is further broken down by material type. Thus, each component of the NBIS (such as superstructure) is broken down into many more detailed elements. Being able to know which specific elements contribute most to the deteriorated state of a bridge allows funding to more effectively be used on maintenance. Additionally, Pontis supports the entire bridge management life cycle, providing methods for inventorying, inspecting, performing needs assessment, strategy development, and project / program growth (AASHTO, Pontis User Manual, 2005).

The NBI database stores condition information on five aggregate structural units (deck, superstructure, substructure, channel, and culvert) by assigning a condition rating (occasionally abbreviated CR in tables / graphs) to each of these components of a bridge on a scale from 9 (perfect) to 1 (severe deterioration / failure). Pontis, on the other hand, assigns each defined 
element a condition state (occasionally abbreviated CS in tables / graphs) on a scale from 1 (perfect) to 3, 4, or 5 (severe deterioration / failure), depending on the element. Inspectors using the NBIS would apply an average condition rating to each component of the bridge while those using Pontis break down the condition assessment into the units each element is assigned.

Elements can also be quantitative. For example, girders are assigned linear footage while elements such as bearings are assigned "each", thereby quantifying the total number of bearings on a given bridge. Pontis is thus a more descriptive inspection tool enabling the determination of how much of a certain element of the bridge is in a truly deteriorated condition. Pontis also contains "smart flag" elements that track types of deterioration different from those listed in the structural element condition state definitions. Smart flags, such as scour and traffic impact damage, are used to record conditions on the bridge that "do not exhibit a logical pattern of deterioration" (VDOT, 2007). This thesis used data from both databases but focused on Pontis data because it was more detailed.

\section{Pontis Details}

\section{Element Definitions}

The Virginia Pontis Element Data Collection Manual defines 111 elements and associated condition states that can be tracked on bridges in the state of Virginia. One hundred of these are known as Commonly Recognized (CoRe) elements. These CoRe elements have identical definitions between agencies in order to facilitate more uniform data collection and analysis nationally. The Pontis guidelines allow users to add their own additional elements to track the condition of further components states wish to track, and the other eleven elements were uniquely defined by the Virginia DOT. These 111 elements define common bridge 
components in terms of component function and material, such as 'Steel Open Girder - Coated', 'Timber Bridge Railing' and 'Elastomeric Bearing'. Additionally, in Virginia, there are nineteen smart flags recorded, eight of which are CoRe and the remaining eleven are uniquely defined by the Virginia DOT (VDOT, 2007). The full list of the 111 elements and nineteen smart flags is presented in Appendix A.

\section{Inspection Report Guidelines}

The National Bridge Inspection Standards set forth the requirements and the general guidelines for responsibility of inspection of state and federal bridges. These include the qualifications for different levels of inspection personnel, different types of inspections and suggested associated frequencies, general inspection procedures, and fields in common data collection tables (Chase, 2010). The specific procedures for inspection and reporting are outlined in the AASHTO Manual for Bridge Evaluation (AASHTO, 2011), the Bridge Inspector's Reference Manual (Ryan, Hartle, Mann, \& Danovich, 2006), the Recording and Coding Guide (FHWA, 1995), and the AASHTO Maintenance Manual for Roadways and Bridges (AASHTO, 2007). These documents explain in detail the different bridge members, explain common defects, and define the associated condition ratings for the superstructure, substructure and deck. The Pontis Element Data Collection Manual defines the condition state guidelines for the Pontis element-level inspection reporting (VDOT, 2007).

\section{PDI Output}

Pontis Data Interchange (PDI) files are text files recognizable by Pontis either as imports from another program or as exports in the form of reports. The PDI files contain the data for all 
bridges in the database and formatting information such as Metric / English units, date format, left / right justification, and other rules that are either column-specific or table-wide. The columns and tables used by Pontis in storing bridge / inspection data are thereby converted into PDI files which can be imported into Microsoft Excel as Comma Separated Values Files.

Five of these PDI-based Excel files were used over the course of this project. The Bridge table contains physical, administrative, and operation characteristics of structures. The ElemInsp table contains the Pontis element-level inspection reports including quantity of each element in each condition state for a bridge per inspection cycle. The InspEvnt table contains one entry per inspection, reporting specifics such as inspection type, inspector identification, and structurelevel results. The Roadway table contains information about all roadways on and under each structure, with fields such as route number, truck traffic, detour length, and number of lanes. The UserBrdg table is defined by the agency and contains additional information about bridges; VDOT uses fields such as approach pier type, utilities present, year repainted, and drain dimensions (AASHTO, Pontis Technical Manual, 2005).

\section{Repairs and Improvements}

Pontis makes a distinction between repairs and improvements. The former comprises routine maintenance (girder painting, deck overlays, patching, etc.) whereas improvements aim to fix functional deficiencies such as vertical clearance, bridge width, or strength capacity. Maintenance is considered a dynamic and ongoing process, while improvement is dealt with as a one-time solution to a deficiency and is considered static (Golabi \& Shepard, 1997). An "improvement" (decrease) in element condition state would likely be achieved by either of the 
above types of work. The associated improvement for a bridge in the NBI database would be a condition rating increase.

\section{Deterioration Modeling}

Two main types of modeling available for deterioration prediction are deterministic and stochastic. Deterministic models include regression analyses that can create trends that follow data linearly or in a quadratic / cubic / higher power manner. The stochastic model is more probabilistic and attempts to account for more of the perceived randomness associated with deterioration of a bridge element such as the paint system (Zayed, Chang, \& Fricker, 2002).

\section{Linear Least Squares Regression}

The most commonly used deterministic model is linear least squares regression in which a line is fit to a set of data. The form of the solution is $y=f(x ; \beta)=\beta_{0}+B_{1} x_{1}+\beta_{2} x_{2}+\cdots$. This method does not always result in a straight line product; the "linear" merely refers to a oneto-one mapping between the known coefficients and unknown parameters (NIST/SEMATECH, 2012). The equation of the solution is calculated by minimizing the sum of the squared differences between the y values of the data set with the y values of the model.

\section{Markov Chains}

A stochastic model that lends itself well to the finite condition state description associated with Pontis data is Markov chains. In this type of model, a probability is assigned to each possible transition, which in this case correlates to changes from condition state 1 to 2,2 to 
3, etc. in a given time period (one year in this case). These transition probabilities can be determined based on averages from historical deterioration for each element or from expert judgment from experienced bridge engineers. The single table of deterioration probabilities created from historical averages does not take into account the history of each specific element; it is limited to the average of the entire population and / or experts' experiences (Morcous, 2006).

\section{Knowledge Discovery in Databases and Data Mining}

As computational power increases in the digital age, our ability to store vast quantities of data has greatly increased. The flood of information that is generated by our advanced data collection methods can be difficult to make sense of, generating a need for advanced techniques for application of statistical techniques (Frawley, Piatesky-Shapiro, \& Matheus, 1992). The field of Knowledge Discovery in Databases (KDD) aims to develop more efficient tools for exploring large volumes of data with the end goal being a more thorough understanding of the results. As Fayyad, Piatetsky-Shapiro, \& Smyth stated in 1996, the value of data storage is determined by “our ability to extract useful reports, spot interesting events and trends, support decisions and policy based on statistical analysis and inference, and exploit the data to achieve business, operational, or scientific goals” (Fayyad, Piatetsky-Shapiro, \& Smyth, 1996, p. 27).

A distinction can be made between KDD and "data mining", where KDD is the entire procedure of drawing meaningful conclusions from patterns found in raw data while data mining is specifically the extraction of results from reduced data sets (Fayyad \& Stolorz, 1997).

Reducing the data to usable form, mining that information, and analyzing the types of results obtained are all necessary steps to the KDD process, often taken in an iterative manner. The application of these methods was explored in this project. Pre-defined report-generating 
processes often do not work well for new types of analysis as it may be difficult to know what results to expect, so new methods must often be developed over the course of the exploration.

The ultimate goal of KDD, as implied by its name, is an increased knowledge of the data. This is accomplished through identification of valid, useful, novel, and understandable patterns. Validity can be verified through certainty measures such as accuracy of predictions made. Usefulness can be quantified by a (predicted or actual) monetary gain or savings in time due to modifying a process. Novelty here means the results are new to the system being analyzed, if not also the user specifically, and is somewhat subjective. Understandability, to the researcher and the audience of the findings, is also rather subjective and can be partially represented by the simplicity of the results (Fayyad \& Stolorz, 1997).

Effective investigation of data requires both the tools and the understanding to direct the analysis. The tools encompass different analytical techniques (as well as the computers themselves), while understanding the nature of the data and the expected results helps guide which types of studies to pursue. Exploratory data analysis is a useful detective method to determine trends on which to perform more judicial confirmatory data analysis. Providing summary statistics, such as averages and extrema, can be useful tools in dealing with large sets of data, but they necessarily reduce the full value of the details stored in each piece of data (Tukey, 1977).

In exploratory data analysis, the precise types of results to be created are often not known at the beginning of the exploration. Researchers must therefore "examine the data, in search of structures that may indicate deeper relationships between cases or variables" (Hand, Mannila, \& Smyth, 2001, p. 53). These deeper relationships provide statistics to more easily infer significant conclusions and suggest meaningful recommendations. Visualization was frequently utilized; 
this method uses the pattern-finding ability of the human brain to detect trends when data is presented in certain ways, such as different types of graphs (Hand, Mannila, \& Smyth, 2001).

\section{National Bridge Maintenance Database}

One of the primary motivations for this project was that a comprehensive database for bridge maintenance information collection and storage does not exist. Hearn, Thompson, Mystkowski, and Hyman (2010) describe a suggested framework for such a resource, the National Bridge Maintenance Database (NBMD). This proposed database aims to create a uniform format to collect, report, and store information on bridge maintenance actions while allowing the various DOTs to continue their current practices for in-the-field data recording. As such, it would be a recipient of data, and the condition and inventory data would conform to the existing U.S. NBI and Pontis systems. As in any database, the NBMD would become more useful as more data is collected from around a given state, in terms of years of condition history and number of bridges reporting.

The NBMD report contains an implementation plan outlining general steps to follow to guide this database into effective widespread use. First, funding would be secured through a supporting agency, which could be a research organization, a federal group, or a pool of state agencies. Next, a technical working group of DOTs would sign on for a certain number of years, committing to annual data upload of maintenance, inventory, and condition values. They would also evaluate the NBMD outputs to assess the effectiveness and recommend new applications as they see fit. A contractor would be brought on board to perform system maintenance, develop the specific data processors, provide technical support, maintain table standards, and in general execute regular upkeep. Next is smoothing the integration with Pontis so that NBMD element- 
level maintenance costs can effectively be used to update the Pontis preservation model. Lastly, methods would be developed to integrate NBMD with DOT enterprise software. (Hearn, Thompson, Mystkowski, \& Hyman, 2010).

The NBMD is a maintenance event archive, storing data on the structure, the maintenance operation / date, the resources used, and the outcome. Fourteen bridge "components" are used as objects of maintenance actions, including the 5 from the NBIS (Deck, Superstructure, etc.) and 7 more that many DOTS use (such as Joints and Bearings), as well as overall Bridge and non-Bridge repairs. Eight standard maintenance operations are defined, including cleaning / clearing, coating, partial replacement, and emergency response work. Each of these in turn has several activities associated with it (e.g. spot painting and chemical treatments for "coating") as well as frequent uses of these activities in practice. For example, chemical treatments are used as preservatives for timber decks / superstructures / etc. while herbicides and pesticides are used on earth slopes and banks.

Overall, the report presents a framework for a database that is designed to assist DOTs with maintenance data storage and assessment at minimal levels of additional cost and effort per result, especially as more states would adopt it into use. An important aspect of the NBMD is that the methods of evaluation (e.g. discount / inflation rates and cost data) come from users, so its results are more customizable to each DOT's specific conditions (Hearn, Thompson, Mystkowski, \& Hyman, 2010).

The project advisory group was curious if there was any correlation between the general geographic environments bridges were in around Virginia and their element deterioration, as well as the maintenance actions performed on them. This resulted in many of the results of the project being subdivided into the different VDOT districts. 


\section{VDOT Districts}

There are nine districts in VDOT that maintain roads in different regions of Virginia.

The regional boundaries are shown in Figure 1. The VDOT-assigned district numbers (1-9) were used throughout this project for reference and are:

$1-$ Bristol

2 - Salem

3 - Lynchburg

4 - Richmond

5 - Hampton Roads

6 - Fredericksburg

7 - Culpeper

8 - Staunton

9 - Northern Virginia

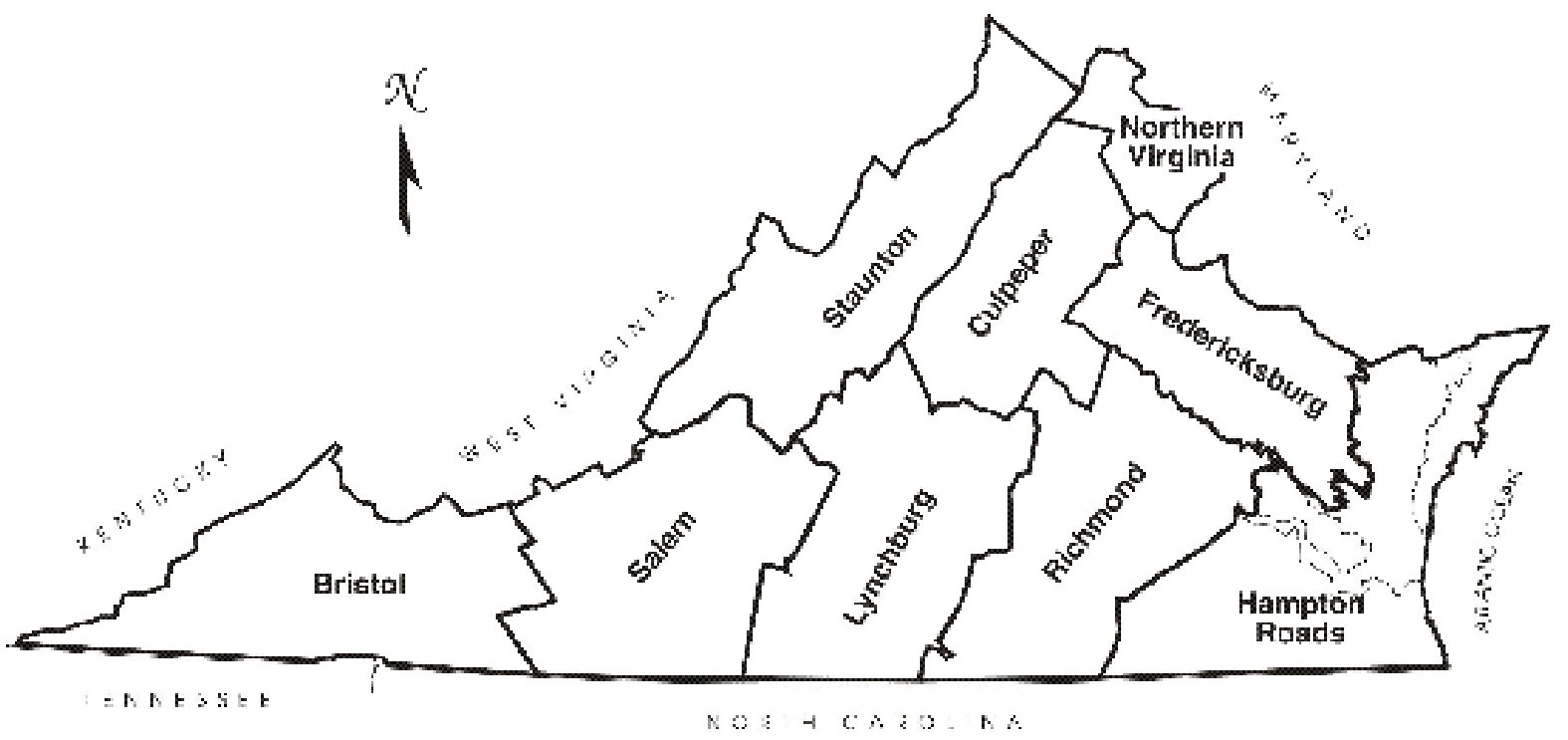

Figure 1 - Map of VDOT Districts (Courtesy of VDOT) 


\section{METHODS}

This study included several different investigations suggested by the members of the project advisory group. This section is organized into those subtopics as they were investigated for this project, employing different data mining methods and techniques. The subsections are: Original Pontis Analysis by District, Potential Errors, Zinc Coating Study, Joint Closures, Priority Bridges and District Visits, and Linear Least Squares and Markov Modeling.

\section{Original Pontis Analysis by District}

The data analysis portion of this work began with a large PDI file containing text strings of information from the Pontis database for all of the bridges in Virginia over the period 1995 2010. Visual Basic for Applications (VBA) code was used to separate the needed information by district into 9 different Excel files. The tables created contained each inspection report in a separate row and each unique extracted piece of information in a separate column. Several userdefined columns were created to be used in the analysis of the inspection report data, including numerous cells converting digits contained within quotation marks (as text strings) into Excelrecognized numbers. Also included was a column calculating the average condition state (marked AVG CS) for the given element and bridge. This data provided the basis for the analyses in this section of the report. An example of some of the extracted data is shown in Table 1. The "PCT\#" columns represent the percent of the given element in each condition state for each inspection report, and the QTY columns represent the quantity of the given element in each condition state (in linear feet, square feet, or each, depending on the element). 


\begin{tabular}{|r|l|l|r|r|r|r|r|r|r|r|r|}
\hline ElemKey & YR Built & Inspection Date & PCT1 & PCT2 & PCT3 & PCT4 & PCT5 & AVG CS & QTY1 & QTY2 \\
\hline 241 & 1969 & $1998 / 02 / 06$ & 89.000 & 11.000 & 0.000 & 0.000 & 0.000 & 1.110 & 74.000 & 9.000 \\
\hline 241 & 1969 & $1996 / 03 / 04$ & 92.600 & 7.400 & 0.000 & 0.000 & 0.000 & 1.074 & 77.000 & 6.000 \\
\hline 241 & 1969 & $2000 / 02 / 03$ & 89.000 & 11.000 & 0.000 & 0.000 & 0.000 & 1.110 & 74.000 & 9.000 \\
\hline 241 & 1969 & $2002 / 02 / 05$ & 83.100 & 15.800 & 1.100 & 0.000 & 0.000 & 1.180 & 69.000 & 13.000 \\
\hline 241 & 1969 & $2004 / 03 / 12$ & 83.100 & 15.800 & 1.100 & 0.000 & 0.000 & 1.180 & 69.000 & 13.000 \\
\hline 241 & 1969 & $2006 / 02 / 17$ & 83.100 & 15.800 & 1.100 & 0.000 & 0.000 & 1.180 & 69.000 & 13.000 \\
\hline 241 & 1969 & $2008 / 02 / 22$ & 83.100 & 15.800 & 1.100 & 0.000 & 0.000 & 1.180 & 69.000 & 13.000 \\
\hline 241 & 1969 & $2010 / 02 / 09$ & 83.100 & 15.800 & 1.100 & 0.000 & 0.000 & 1.180 & 69.000 & 13.000 \\
\hline 298 & 1969 & $2010 / 02 / 09$ & 100.000 & 0.000 & 0.000 & 0.000 & 0.000 & 1.000 & 2.000 & 0.000 \\
\hline 299 & 1969 & $2010 / 02 / 09$ & 100.000 & 0.000 & 0.000 & 0.000 & 0.000 & 1.000 & 4.000 & 0.000 \\
\hline 361 & 1969 & $2008 / 02 / 22$ & 100.000 & 0.000 & 0.000 & 0.000 & 0.000 & 1.000 & 1.000 & 0.000 \\
\hline
\end{tabular}

Table 1 - Example Selection of Raw District Analysis Data (District 1)

There were too many element types that are routinely inspected to reasonably analyze all of them for each district, so a selection process was implemented to choose the most numerous elements. In a new worksheet, a table was created with each of the bridges in a given district in a separate row and all of the elements present on each bridge in that district in separate columns. A count-if function was performed on each cell (bridge / element intersection), which resulted in the number of inspection reports from 1995-2010 for that element and bridge. Table 2 shows an example portion of this table for District 1; for example, bridge 16747 had 7 recorded inspection reports containing element 12 . The number of reports was totaled and ordered from highest to lowest along with the top 10 smart flags and top 20 elements selected from each district for analysis. A summary of the top elements and smart flags for each district is presented in Table 24 through Table 26. Throughout this project, elements and smart flags were tracked of using their 3-digit numeric designation; if the corresponding text was desired (i.e. Element 234 is 'Reinforced concrete pier cap') a link was created to that element in a separate reference sheet in Excel. This worksheet served as a lookup table for element and smart flag numbers to corresponding description. This was as documented in the Pontis element definitions, and is reproduced in this report as Appendix A. 


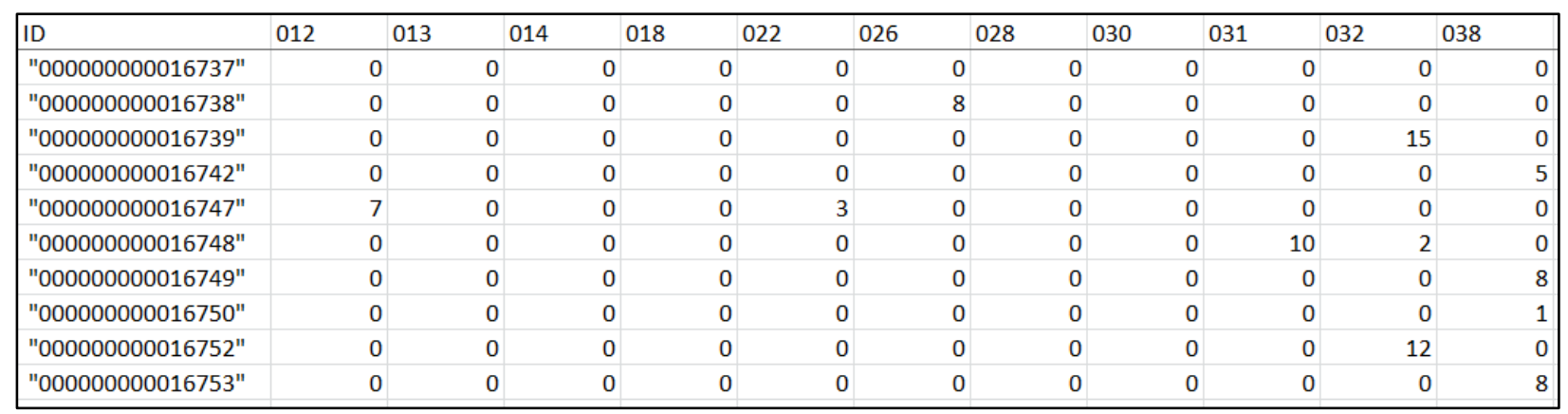

Table 2 - Example Selection of Counting Table for Element and Smart Flag Selection (District 1)

For the elements that were selected for analysis, the corresponding BridgeIDs, years built, inspection dates, and average condition states were copied into separate worksheets titled with the respective element numbers. A subsequent table was constructed with unique bridge numbers in each row and the years 1995-2010 in separate columns. For each cell in the table, a formula was used that averaged any condition states for that element from reports matching the given bridge and inspection year. Cells were color-coded to be grey when there was no inspection report (marked as condition state 0.00 ), green when the average condition state was between 1.00 and 1.99 , yellow for 2.00 to 2.99 , orange for 3.00 to 3.99 , and red for 4.00 to 5.00 . Several additional columns were created; one for the most recent condition state as of 2010, one for the age of the bridge in 2010, and lastly a column for whether there was an "Unexpected Improvement". This final column reported TRUE or FALSE. If the maximum condition state of the given bridge over the years 1995 - 2010 was more than 1.00 condition state points higher than the most recent condition state it was marked as 'true' and if not it was marked 'false'. This threshold was chosen to only identify bridges with significant improvements likely to be caused by major repairs. Table 3 shows a selection from this table for element 334 in District 5. Note that the bridge at the bottom displays a condition state decrease of 2.00 , so it is noted as 
having an unexpected improvement. The decrease from a max of 3.00 to the current condition state was assumed to correspond to a significant repair. The amount of decrease associated with different interventions depends on the element, but for this project the $>1.00$ condition state decrease was used for all elements except joints. A separate, more lenient, improvement criterion was used for joint elements; this is described under the 'Priority Bridges and District Visits' heading later in this section.

\begin{tabular}{|c|c|c|c|c|c|c|c|c|c|}
\hline 2004 & 2005 & 2006 & 2007 & 2008 & 2009 & 2010 & $\mathrm{CR}$ & Age & Unexpected Improvement \\
\hline 0.00 & 0.00 & 0.00 & 0.00 & 1.36 & 0.00 & 0.00 & 1.36 & 44 & FALSE \\
\hline 1.00 & 0.00 & 1.00 & 0.00 & 1.00 & 0.00 & 1.00 & 1.00 & 33 & FALSE \\
\hline 1.00 & 0.00 & 1.00 & 1.00 & 1.00 & 1.00 & 1.00 & 1.00 & 59 & FALSE \\
\hline 1.00 & 1.00 & 1.00 & 1.00 & 1.00 & 0.00 & 1.00 & 1.00 & 81 & FALSE \\
\hline 0.00 & 1.44 & 0.00 & 1.00 & 1.00 & 1.00 & 1.00 & 1.00 & 40 & FALSE \\
\hline 0.00 & 1.77 & 0.00 & 1.52 & 1.52 & 1.52 & 0.00 & 1.52 & 49 & FALSE \\
\hline 0.00 & 0.00 & 0.00 & 0.00 & 0.00 & 0.00 & 2.49 & 2.49 & 36 & FALSE \\
\hline 1.00 & 0.00 & 1.19 & 0.00 & 1.19 & 1.23 & 1.00 & 1.00 & 36 & FALSE \\
\hline 1.05 & 1.10 & 1.10 & 1.10 & 1.10 & 1.10 & 1.10 & 1.10 & 78 & FALSE \\
\hline 1.00 & 0.00 & 1.00 & 0.00 & 1.00 & 0.00 & 1.00 & 1.00 & 49 & FALSE \\
\hline 3.00 & 3.00 & 1.00 & 1.00 & 1.00 & 1.00 & 1.00 & 1.00 & 30 & TRUE \\
\hline
\end{tabular}

Table 3 - Example Selection for Unexpected Improvement (Element 334 - District 5)

After preliminary analysis of the elements, the highest number of unexpected improvements occurred to element 107 (steel open girder - coated). After initial discussion with the project advisory group about potential inconsistencies in the analysis, it was revealed that there could be some irregularities with the steel open girder elements (107 and 108). The element 108 classification, as distinct from element 107, was created in 2006 to signify only the steel open girders under timber decks (these would likely have different deterioration rates and maintenance plans). This required additional work to segregate these bridges. The bridges listed as containing 108 were copied in Excel next to those originally containing 107 and a filter was created to identify those bridges which appeared in both lists. The data from the bridges that 
were reclassified by VDOT as having element 108, that had previously been marked 107, were appended to the existing 2006-2010 element 108 data. These bridges were analyzed separately from those that remained as 107 past 2005, and the final improvement count for each element was based upon the revised bridge lists. Figure 2 below shows the condition state histories for the same bridges in District 8 before and after this coding transition was accounted for. The top table shows just the inspection reports recorded after element 108 was coded while the bottom table has the elements previously coded as element 107 (that were ultimately 108) appended. As the new classification went into effect in the middle of 2006, some bridges had data from element 108 from that year and other had to be appended from the previous element 107 data.

\begin{tabular}{|c|c|c|c|c|c|c|c|c|c|c|c|c|c|c|c|c|c|}
\hline Unique brkey & Unique YR Built & 1995 & 1996 & 1997 & 1998 & 1999 & 2000 & 2001 & 2002 & 2003 & 2004 & 2005 & 2006 & 2007 & 2008 & 2009 & 2010 \\
\hline "000000000000901" & 1992 & 0.00 & 0.00 & 0.00 & 0.00 & 0.00 & 0.00 & 0.00 & 0.00 & 0.00 & 0.00 & 0.00 & 0.00 & 0.00 & 1.13 & 0.00 & 1.13 \\
\hline "000000000001068" & 1932 & 0.00 & 0.00 & 0.00 & 0.00 & 0.00 & 0.00 & 0.00 & 0.00 & 0.00 & 0.00 & 0.00 & 2.50 & 0.00 & 2.50 & 0.00 & 2.50 \\
\hline "000000000001069" & 1932 & 0.00 & 0.00 & 0.00 & 0.00 & 0.00 & 0.00 & 0.00 & 0.00 & 0.00 & 0.00 & 0.00 & 2.63 & 0.00 & 2.63 & 0.00 & 2.63 \\
\hline "000000000001072" & 1976 & 0.00 & 0.00 & 0.00 & 0.00 & 0.00 & 0.00 & 0.00 & 0.00 & 0.00 & 0.00 & 0.00 & 1.64 & 0.00 & 1.64 & 0.00 & 1.64 \\
\hline "000000000001073" & 1932 & 0.00 & 0.00 & 0.00 & 0.00 & 0.00 & 0.00 & 0.00 & 0.00 & 0.00 & 0.00 & 0.00 & 2.15 & 0.00 & 2.15 & 0.00 & 2.15 \\
\hline "000000000001086" & 1932 & 0.00 & 0.00 & 0.00 & 0.00 & 0.00 & 0.00 & 0.00 & 0.00 & 0.00 & 0.00 & 0.00 & 2.38 & 0.00 & 2.38 & 0.00 & 2.38 \\
\hline "000000000001093" & 1932 & 0.00 & 0.00 & 0.00 & 0.00 & 0.00 & 0.00 & 0.00 & 0.00 & 0.00 & 0.00 & 0.00 & 0.00 & 0.00 & 1.00 & 0.00 & 1.00 \\
\hline "000000000001094" & 1932 & 0.00 & 0.00 & 0.00 & 0.00 & 0.00 & 0.00 & 0.00 & 0.00 & 0.00 & 0.00 & 0.00 & 0.00 & 0.00 & 1.20 & 0.00 & 1.20 \\
\hline Unique brkey & Unique YR Built & 1995 & 1996 & 1997 & 1998 & 1999 & 2000 & 2001 & 2002 & 2003 & 2004 & 2005 & 2006 & 2007 & 2008 & 2009 & 2010 \\
\hline "000000000000901" & 1992 & 0.00 & 1.00 & 0.00 & 0.00 & 0.00 & 1.00 & 0.00 & 1.13 & 0.00 & 1.13 & 0.00 & 1.13 & 0.00 & 1.13 & 0.00 & 1.13 \\
\hline "000000000001068" " & 1932 & 0.00 & 0.00 & 0.00 & 0.00 & 0.00 & 2.00 & 0.00 & 2.50 & 0.00 & 2.50 & 0.00 & 2.50 & 0.00 & 2.50 & 0.00 & 2.50 \\
\hline "000000000001069" & 1932 & 0.00 & 0.00 & 0.00 & 1.10 & 0.00 & 2.00 & 0.00 & 2.63 & 0.00 & 2.63 & 0.00 & 2.63 & 0.00 & 2.63 & 0.00 & 2.63 \\
\hline "000000000001072" & 1976 & 0.00 & 1.00 & 0.00 & 0.00 & 0.00 & 1.00 & 0.00 & 1.64 & 0.00 & 1.64 & 0.00 & 1.64 & 0.00 & 1.64 & 0.00 & 1.64 \\
\hline "000000000001073" & 1932 & 0.00 & 0.00 & 0.00 & 0.00 & 0.00 & 2.00 & 0.00 & 2.15 & 0.00 & 2.15 & 0.00 & 2.15 & 0.00 & 2.15 & 0.00 & 2.15 \\
\hline "000000000001086" & 1932 & 0.00 & 2.25 & 0.00 & 0.00 & 0.00 & 2.25 & 0.00 & 2.38 & 0.00 & 2.38 & 0.00 & 2.38 & 0.00 & 2.38 & 0.00 & 2.38 \\
\hline "000000000001093" & 1932 & 0.00 & 0.00 & 0.00 & 0.00 & 0.00 & 1.00 & 0.00 & 1.00 & 0.00 & 1.00 & 0.00 & 1.00 & 0.00 & 1.00 & 0.00 & 1.00 \\
\hline "000000000001094" & 1932 & 0.00 & 0.00 & 0.00 & 1.00 & 0.00 & 1.00 & 0.00 & 1.20 & 0.00 & 1.20 & 0.00 & 1.20 & 0.00 & 1.20 & 0.00 & 1.20 \\
\hline
\end{tabular}

Figure 2 - Example Addition of Element 107 Data to 108 Table (District 8)

It was noted that known painting that occurred on elements 107 and 108 could show up as "unexpected improvements". By comparing the year of the element improvements to the most recent year the bridges were painted (from the UserBrdg PDI file), only the improvements that were likely to be significant non-painting maintenance were counted in the final summary. Table 4 shows a selection from District 2 of element 108 where there were 4 bridges that fit the criteria for unexpected improvement, but 2 of those bridges (Paint Yr. 2009 and 2004) had the 
condition state decrease noted as the result of painting. The rightmost column is labeled "For Q. An." as this refined true / false distinction was also used in the quantity analysis that is discussed later in this section; it was also used in all further analysis containing 107 / 108 data.

\begin{tabular}{|c|c|c|c|c|c|c|c|c|c|c|c|c|}
\hline 2004 & 2005 & 2006 & 2007 & 2008 & 2009 & $2010 \mathrm{CR}$ & & Age & Unexpected Improvement & Paint Yr (Impr.) & Paint Improvement? & For Q An. \\
\hline 0.00 & 1.00 & 0.00 & 1.00 & 0.00 & 1.00 & 0.00 & 1.00 & 78 & FALSE & & & FALSE \\
\hline 2.40 & 0.00 & 2.40 & 0.00 & 3.39 & 2.38 & 0.00 & 2.38 & 78 & TRUE & "2009" & TRUE & FALSE \\
\hline 2.48 & 0.00 & 1.00 & 0.00 & 1.00 & 0.00 & 1.00 & 1.00 & 78 & TRUE & "2004" & TRUE & FALSE \\
\hline 4.57 & 0.00 & 4.57 & 0.00 & 2.79 & 0.00 & 1.00 & 1.00 & 78 & TRUE & "1980" & FALSE & TRUE \\
\hline 0.00 & 1.00 & 0.00 & 1.00 & 0.00 & 1.00 & 0.00 & 1.00 & 78 & FALSE & & & FALSE \\
\hline 0.00 & 3.22 & 0.00 & 4.44 & 2.72 & 0.00 & 1.00 & 1.00 & 78 & TRUE & "1976" & FALSE & TRUE \\
\hline
\end{tabular}

Table 4 - Example Selection for Painting as Known Improvement (Element 108 - District 2)

A separate workbook was created to perform additional analysis on the most numerous elements across the state. The elements chosen for further analysis of condition state trends between and within districts were selected by ranking the most numerous elements both in terms of bridges listing inspection reports for that element and by the number of 'unexpected improvements' that occurred (as shown in Table 27, Table 28, and Table 29 in the Results section of this report). For each of these elements, the yearly data from the separate district files were copied into a new worksheet, separated by those with unexpected improvements and those without, and then further separated by district. From here, the average condition state per year per district could be calculated in addition to the number of bridges in different ranges of condition states. The average condition state data were used to create line graphs over time, such as Figure 4, showing condition state trends across districts and the entire state.

“100\% stacked column” plots, such as Figure 5, were created to show the percent of bridges in the given district or across the state in various condition state ranges by year for the given element. These ranges were 1.00 to 1.99 (marked as a green bar), 2.00 to 2.99 (yellow), 3.00 to 3.99 (orange) and 4.00 to 5.00 (red). A separate analysis was run on the quantity (linear 
feet, square feet, each, etc.) of the same elements across the state, with the intent of providing more weight to the condition states of elements in larger bridges (as compared to the original analysis which placed equal value on bridge of all sizes). $100 \%$ stacked columns were created from this quantity analysis as well, such as Figure 7, for comparison to those from the original analysis. Each graph type was created for the bridges that were found to have had an unexpected improvement, and for those that had not. Similar analyses were performed on 6 smart flags and 6 elements to investigate the condition state improvement based on the year the improvement occurred in, referred to as the year of improvement analysis, and deterioration based on the age in years of each bridge since improvement, referred to as the age since improvement analysis. These investigations were performed to explore the trends in improvement of different elements and when deterioration began after these improvements occurred.

For the year of improvement analysis, additional columns were created for each inspection report denoting the year of improvement, and the condition states before and after improvement. This was accomplished using Visual Basic code that found the year in which a condition state decrease of greater than 1.00 occurred and reported the lower number as the condition state after, and the higher value from the previous report (regardless of how many years earlier it was) as the condition state before. The data from these last two columns were then put into a new table using an averageif command, averaging the associated before and after improvement condition states for each year of improvement. This information is presented in tables in Appendix B.

For the age since improvement analysis, a table was created with the year the improvement occurred heading the columns and the year inspected heading the rows. The cells in this table were filled in with the total summed condition states for all inspection reports 
matching both criteria for the given element. A selection from this table used in the analysis of element 334 is shown in Table 5. Additional tables with the same headings were created to denote the age of each cell (as in Table 6), and the total count of reports for each cell (as in Table 7). The table showing age displays "- 1 " if the inspection year of a cell is before the associated construction year, in order to not show up in the summary table.

\begin{tabular}{|r|r|r|r|r|r|r|r|r|r|r|}
\hline & 1995 & 1996 & 1997 & 1998 & 1999 & 2000 & 2001 & 2002 & 2003 & 2004 \\
\hline 1995 & 0 & 0 & 0 & 1 & 0 & 0 & 0 & 0 & 7.5 & 0 \\
\hline 1996 & 0 & 0 & 0 & 8.5 & 0 & 6.072 & 1 & 12.694 & 11.99 & 4.912 \\
\hline 1997 & 0 & 0 & 0 & 6.515 & 10 & 17.54 & 12 & 19.11 & 15.47 & 7.11 \\
\hline 1998 & 0 & 0 & 0 & 6.633 & 2.5 & 9 & 11.976 & 28.9 & 14.48 & 13.978 \\
\hline 1999 & 0 & 0 & 0 & 4 & 4 & 16.521 & 6.401 & 32.472 & 27.97 & 10.707 \\
\hline 2000 & 0 & 0 & 0 & 8.633 & 1 & 12.947 & 20.949 & 34.51 & 16.98 & 25.023 \\
\hline 2001 & 0 & 0 & 0 & 3 & 3 & 5.6 & 9.5 & 33.061 & 29.97 & 19.015 \\
\hline 2002 & 0 & 0 & 0 & 6.633 & 1 & 14.6 & 9.5 & 16.744 & 17.48 & 28.632 \\
\hline
\end{tabular}

Table 5 - Example Selection for Summed Condition States (Element 334)

\begin{tabular}{|r|r|r|r|r|r|r|r|r|r|r|}
\hline & 1995 & 1996 & 1997 & 1998 & 1999 & 2000 & 2001 & 2002 & 2003 & 2004 \\
\hline 1995 & 0 & -1 & -1 & -1 & -1 & -1 & -1 & -1 & -1 & -1 \\
\hline 1996 & 1 & 0 & -1 & -1 & -1 & -1 & -1 & -1 & -1 & -1 \\
\hline 1997 & 2 & 1 & 0 & -1 & -1 & -1 & -1 & -1 & -1 & -1 \\
\hline 1998 & 3 & 2 & 1 & 0 & -1 & -1 & -1 & -1 & -1 & -1 \\
\hline 1999 & 4 & 3 & 2 & 1 & 0 & -1 & -1 & -1 & -1 & -1 \\
\hline 2000 & 5 & 4 & 3 & 2 & 1 & 0 & -1 & -1 & -1 & -1 \\
\hline 2001 & 6 & 5 & 4 & 3 & 2 & 1 & 0 & -1 & -1 & -1 \\
\hline 2002 & 7 & 6 & 5 & 4 & 3 & 2 & 1 & 0 & -1 & -1 \\
\hline
\end{tabular}

Table 6 - Example Selection for Age since Improvement (Element 334) 


\begin{tabular}{|r|r|r|r|r|r|r|r|r|r|r|}
\hline & 1995 & 1996 & 1997 & 1998 & 1999 & 2000 & 2001 & 2002 & 2003 & 2004 \\
\hline 1995 & 0 & 0 & 0 & 1 & 0 & 0 & 0 & 0 & 3 & 0 \\
\hline 1996 & 0 & 0 & 0 & 3 & 0 & 4 & 1 & 6 & 5 & 2 \\
\hline 1997 & 0 & 0 & 0 & 2 & 3 & 6 & 4 & 7 & 6 & 3 \\
\hline 1998 & 0 & 0 & 0 & 5 & 1 & 2 & 6 & 11 & 5 & 6 \\
\hline 1999 & 0 & 0 & 0 & 2 & 4 & 5 & 3 & 12 & 10 & 4 \\
\hline 2000 & 0 & 0 & 0 & 5 & 1 & 8 & 6 & 13 & 6 & 12 \\
\hline 2001 & 0 & 0 & 0 & 1 & 3 & 3 & 8 & 11 & 10 & 8 \\
\hline 2002 & 0 & 0 & 0 & 4 & 1 & 8 & 5 & 15 & 6 & 10 \\
\hline
\end{tabular}

Table 7 - Example Selection for Count of Reports by Improvement Year and Inspection Year (Element 334)

The total summed condition states and total inspection report counts per year since improvement were then calculated using "sumifs" Excel commands. The inputs for this command summed over the desired age in the "Age since Improvement" table using the associated cells in the "Summed Condition State" and "Report Count" tables. The first 8 years of this table are shown for element 334 as Table 8, and is reproduced in full in Appendix B as Table B-16.

\begin{tabular}{|l|r|r|r|r|r|r|r|r|r|}
\hline Age & 0 & 1 & 2 & 3 & 4 & 5 & 6 & 7 & 8 \\
\hline Total & 145.49 & 51.65 & 121.14 & 45.58 & 103.83 & 38.77 & 66.33 & 28.95 & 37.84 \\
\hline Count & 125 & 40 & 101 & 38 & 86 & 31 & 55 & 23 & 33 \\
\hline Average & 1.16 & 1.29 & 1.20 & 1.20 & 1.21 & 1.25 & 1.21 & 1.26 & 1.15 \\
\hline
\end{tabular}

Table 8 - Example Selection of Condition State Trend by Age since Improvement (Element 334) - Reproduced in Part from Table B-16

\section{Potential Errors}

Upon closer analysis of specific inspection report data, it became apparent that several of the data entries contained conflicting pieces of information. The most important data for this analysis were the condition states, so it was important to ensure correctness of the information in 
those cells. An analysis was performed to identify those inspection reports which might contain errors.

To accomplish this, a new column was created in the original district workbooks to note if, for any condition state, the amount listed in the "quantity" cell was more than $10 \%$ different from the quantity calculated from the percent in the corresponding condition state multiplied by the total quantity. This threshold was chosen to select only those differences that were significant enough to not likely be attributed to rounding errors in quantity calculation. A workbook was created containing all data (for all elements) for bridges with inspection reports with likely errors based on this criterion. This list could be used for checking the accuracy of the reports for those bridges and a potential investigation into correcting the inaccuracies in these Pontis data. Table 9 below shows several reports that were identified as having this type of error; notice how the reported quantities (“QTY\#”) do not show agreement with the associated quantities calculated from the reported percent fields (“QP\#”).

\begin{tabular}{|l|r|r|r|r|r|r|r|r|r|r|r|r|}
\hline brkey & QTY1 & QTY2 & QTY3 & QTY4 & QTY5 & TotQty & QP1 & QP2 & QP3 & QP4 & QP5 \\
\hline "000000000001322" & 0.000 & 0.000 & 1242.000 & 0.000 & 0.000 & 1242.000 & 1242.000 & 0.000 & 0.000 & 0.000 & 0.000 \\
\hline "000000000001322" & 0.000 & 0.000 & 1242.000 & 0.000 & 0.000 & 1242.000 & 1242.000 & 0.000 & 0.000 & 0.000 & 0.000 \\
\hline "000000000001339" & 0.000 & 1735.000 & 0.000 & 0.000 & 0.000 & 1735.000 & 0.000 & 0.000 & 1735.000 & 0.000 & 0.000 \\
\hline "000000000001340" & 0.000 & 2497.000 & 0.000 & 0.000 & 0.000 & 2497.000 & 0.000 & 0.000 & 2497.000 & 0.000 & 0.000 \\
\hline "000000000001340" & 0.000 & 2497.000 & 0.000 & 0.000 & 0.000 & 2497.000 & 2497.000 & 0.000 & 0.000 & 0.000 & 0.000 \\
\hline "000000000001365" & 4.000 & 0.000 & 0.000 & 0.000 & 0.000 & 4.000 & 0.000 & 0.000 & 0.000 & 0.000 & 0.000 \\
\hline "000000000001382" & 0.000 & 0.000 & 349.000 & 0.000 & 0.000 & 349.000 & 0.000 & 349.000 & 0.000 & 0.000 & 0.000 \\
\hline
\end{tabular}

\section{Zinc Coating Study}

The zinc coating sub-study was originally performed on data current to the end of 2010 and then later updated with data current as of September 17, 2012. In order to streamline this 
update process, the same bridges were considered between the two dates, as opposed to redefining the structure list with the few new bridges built in the intervening twenty months. PDI Export files were obtained from VDOT for the 2010 and then the 2012 Pontis databases. Visual Basic code was written that extracted data from tables in the PDI export file, namely the Bridge, Element Inspection, Roadway, User Bridge, and Inspection Event Tables. Four separate worksheets were created, one for each of the four prescribed groups.

A quantity analysis was performed for each group, for each of the years 2000-2012. The early years (1995-1999) often had inconsistent or incomplete data and were thus ignored for this analysis. The quantity in each condition state and total quantity were summed for each group, and the percentage in each condition state was calculated. A table was created for each group with the inspection years as column headings and condition states as row headings; an example of the first 7 years of this is shown in Table 10 for Group 1 bridges. Bar graphs were produced from these tables and are shown in Figure 11, in the Results section of this report.

\begin{tabular}{|c|c|c|c|c|c|c|c|}
\hline & 2000 & 2001 & 2002 & 2003 & 2004 & 2005 & 2006 \\
\hline Total & 56315.0 & 51695.0 & 55094.0 & 47326.5 & 63517.0 & 53080.3 & 56858.0 \\
\hline CS 1 & 54784.1 & 51152.5 & 53576.7 & 46434.5 & 61649.8 & 51970.3 & 54627.2 \\
\hline CS 2 & 1510.7 & 542.5 & 1490.8 & 888.8 & 1823.5 & 1104.5 & 2174.8 \\
\hline CS 3 & 20.3 & 0.0 & 20.3 & 3.1 & 35.5 & 4.3 & 49.2 \\
\hline CS 4 & 0.0 & 0.0 & 6.2 & 0.0 & 6.2 & 0.0 & 6.2 \\
\hline CS 5 & 0.0 & 0.0 & 0.0 & 0.0 & 0.0 & 0.0 & 0.0 \\
\hline
\end{tabular}

Table 10 - Example Selection from Zinc Study of Yearly Condition State by Quantity (Group 1)

Another set of tables was created based on percentages of bridges in various condition states, normalizing the quantity of element 107 per bridge. For each group, a table was created, with bridge IDs in separate rows and years 2000 thru 2012 in separate columns. The cells at the intersection of these headings summed up the total condition states for all inspection reports that 
occurred in the given year for the given bridge ID. A similar table was created that summed the number of inspection reports per bridge per year. These two tables were used to produce a third table which divided the condition state summation by the number of inspections per year to get the average condition state per bridge per inspection year. Thus the number of bridges in each condition state range $(1.00-1.99,2.00-2.99,3.00-3.99$, and $4.00-5.00)$ was determined. Normalizing these values by the number of bridges inspected each year produced the graphs shown in Figure 13.

Summary statistics for the NBI superstructure condition ratings were also requested. Tables similar to the condition state analysis were created, referencing a column of superstructure condition ratings instead of the element 107 condition states. These tables were graphed similarly to the previous analysis (but on the 9 to 1 NBI scale as opposed to the 1 to 5 Pontis scale), and are shown in Figure 14. Because the primary focus of this special project was to examine the performance of VDOT's zinc-based coating system, an additional analysis of the Pontis data was conducted which determined the age of the coating system of each bridge in each group at the time of each inspection. The results are summarized in Figure 15. The results were also used to calculate the average condition state for Element 107 for different coating ages. Graphs of the average condition states of Element 107 by age of coating and average superstructure condition ratings by age of coating are presented as Figure 16 and Figure 17. Six year moving average trendlines were added to the graphs to show the gradual change in condition with coating age. The six year moving average trendlines for the four groups were graphed for the element 107 condition states and superstructure condition ratings as Figure 18 and Figure 19. 
Following the coating study, a list of bridges was created with quantities of element 107 (steel open girder - coated) in their most deteriorated form. All of the bridges that met the criteria for the coating study were further filtered for any non-zero quantity of condition states 4 and/or 5. These were compiled in a new spreadsheet along with another column which showed which group from the coating study each of these deteriorated state bridges fell in.

\section{Joint Closures}

The project advisory group requested a study on joint closures to complement the zincbased girder coating analysis. The requested information was a list, separated by district, of every bridge in the state that had had all or part of any of the joint elements eliminated over the past 15 years. To implement the desired filter, all the inspection report rows were copied into each district's worksheet from the earlier Pontis-extracted Excel data, including the corresponding bridge IDs, the element number, the inspection date, and the total quantity of the element in each row. The inspection reports for all joint elements were included because if the joint was entirely removed, that element would not show up in the next report. The only way to know would be to compare an earlier report that did have the element to a later report containing only other elements. Bridges where a joint element was replaced with a different joint element, or the quantity was reduced (but not entirely eliminated), were also found using the method described below.

A new column extracted the year from the inspection date, and then the entire workbooks were sorted on bridge ID and then inspection year, to chronologically order the data by ascending bridge ID. Separate columns were created for each of the joint elements (300, 301, 302,303 , and 304), and these columns were populated with the corresponding quantity of each 
element if that row contained that element, or 0 if it did not. Another column was created for each joint element that found the maximum value of the given inspection year and bridge; this would populate with the quantity of the given joint element if it was inspected in a certain year, or 0 if it was not. The value of next column was determined by an IF statement, marking TRUE for any entry that had a $>10 \%$ decrease in quantity in any of the 5 elements from the row directly above it, as long as the bridge ID was the same. $10 \%$ was chosen as a threshold for when the decrease was likely due to actual closure and not just due to a rounding error or a different calculation of the linear footage of the given joint element on the bridge. Therefore, the bridges marked as TRUE had a reduction in any of the 5 joint element quantities between two consecutive inspection reports, from a non-zero number to 0 or at least a decrease of $>0.1$ times the previous quantity. The next column, "Date of Change", displays the year the quantity decreased or "N/A" if there is none. The total number of $>10 \%$ quantity reductions for any joint elements in that district were also calculated.

Table 11 is a selection from the District 9 joint elimination Excel file. Note there are 27 linear feet of element 300 in 2003, so the corresponding cell in "300 FULL" has 27 in it and the "300 MAX" column is filled in with 27 for all rows matching the bridge ID and inspection year. In 2005, there is no record of element 300 from this data, so 0 is filled in for those columns and the "ELIMINATION" column shows TRUE, with 2005 as the "Date of Change". A final column was added to denote if the change resulted in a complete elimination (as seen in Table 11), a decrease in quantity (between the $10 \%$ minimum criteria and a $99 \%$ decrease), or just a one-to-one change in joint type (for example, pourable joints replaced with compression joints). 


\begin{tabular}{|c|c|c|c|c|c|c|c|c|c|c|c|c|c|c|c|c|c|}
\hline brkey & ElemKey & Inspection Date & TotQty & Insp Year & 300 FULL & 301 FULL & 302 FULL & 303 FULL & 304 FULL & 300 MAX & 301 MAX & 302 MAX & 303 MAX & 304 MAX & ELIMINATION & Date of Change & TYPE \\
\hline 6185 & 12 & $2003 / 02 / 03$ & 620.000 & 2003 & 0 & 0 & 0 & 0 & 0 & 27 & 0 & 0 & 0 & 0 & FALSE & $\mathrm{N} / \mathrm{A}$ & \\
\hline 6185 & 106 & $2003 / 02 / 03$ & 271.000 & 2003 & 0 & 0 & 0 & 0 & 0 & 27 & 0 & 0 & 0 & 0 & FALSE & N/A & \\
\hline 6185 & 205 & $2003 / 02 / 03$ & 6.000 & 2003 & 0 & 0 & 0 & 0 & 0 & 27 & 0 & 0 & 0 & 0 & FALSE & N/A & \\
\hline 6185 & 215 & $2003 / 02 / 03$ & 28.000 & 2003 & 0 & 0 & 0 & 0 & 0 & 27 & 0 & 0 & 0 & 0 & FALSE & N/A & \\
\hline 6185 & 234 & $2003 / 02 / 03$ & 27.000 & 2003 & 0 & 0 & 0 & 0 & 0 & 27 & 0 & 0 & 0 & 0 & FALSE & $\mathrm{N} / \mathrm{A}$ & \\
\hline 6185 & 286 & $2003 / 02 / 03$ & 2.000 & 2003 & 0 & 0 & 0 & 0 & 0 & 27 & 0 & 0 & 0 & 0 & FALSE & $\mathrm{N} / \mathrm{A}$ & \\
\hline 6185 & 300 & $2003 / 02 / 03$ & 27.000 & 2003 & 27 & 0 & 0 & 0 & 0 & 27 & 0 & 0 & 0 & 0 & FALSE & N/A & \\
\hline 6185 & 313 & $2003 / 02 / 03$ & 12.000 & 2003 & 0 & 0 & 0 & 0 & 0 & 27 & 0 & 0 & 0 & 0 & FALSE & $\mathrm{N} / \mathrm{A}$ & \\
\hline 6185 & 321 & $2003 / 02 / 03$ & 2.000 & 2003 & 0 & 0 & 0 & 0 & 0 & 27 & 0 & 0 & 0 & 0 & FALSE & $\mathrm{N} / \mathrm{A}$ & \\
\hline 6185 & 331 & $2003 / 02 / 03$ & 91.000 & 2003 & 0 & 0 & 0 & 0 & 0 & 27 & 0 & 0 & 0 & 0 & FALSE & N/A & \\
\hline 6185 & 701 & $2003 / 02 / 03$ & 1.000 & 2003 & 0 & 0 & 0 & 0 & 0 & 27 & 0 & 0 & 0 & 0 & FALSE & N/A & \\
\hline 6185 & 12 & $2005 / 01 / 25$ & 610.373 & 2005 & 0 & 0 & 0 & 0 & 0 & 0 & 0 & 0 & 0 & 0 & TRUE & 2005 & ELIM \\
\hline 6185 & 106 & $2005 / 01 / 25$ & 271.000 & 2005 & 0 & 0 & 0 & 0 & 0 & 0 & 0 & 0 & 0 & 0 & FALSE & N/A & \\
\hline 6185 & 205 & $2005 / 01 / 25$ & 6.000 & 2005 & 0 & 0 & 0 & 0 & 0 & 0 & 0 & 0 & 0 & 0 & FALSE & N/A & \\
\hline 6185 & 215 & $2005 / 01 / 25$ & 28.000 & 2005 & 0 & 0 & 0 & 0 & 0 & 0 & 0 & 0 & 0 & 0 & FALSE & N/A & \\
\hline 6185 & 234 & $2005 / 01 / 25$ & 27.000 & 2005 & 0 & 0 & 0 & 0 & 0 & 0 & 0 & 0 & 0 & 0 & FALSE & $\mathrm{N} / \mathrm{A}$ & \\
\hline 6185 & 286 & $2005 / 01 / 25$ & 2.000 & 2005 & 0 & 0 & 0 & 0 & 0 & 0 & 0 & 0 & 0 & 0 & FALSE & N/A & \\
\hline 6185 & 313 & $2005 / 01 / 25$ & 12.000 & 2005 & 0 & 0 & 0 & 0 & 0 & 0 & 0 & 0 & 0 & 0 & FALSE & N/A & \\
\hline 6185 & 321 & $2005 / 01 / 25$ & 2.000 & 2005 & 0 & 0 & 0 & 0 & 0 & 0 & 0 & 0 & 0 & 0 & FALSE & $\mathrm{N} / \mathrm{A}$ & \\
\hline 6185 & 331 & $2005 / 01 / 25$ & 91.000 & 2005 & 0 & 0 & 0 & 0 & 0 & 0 & 0 & 0 & 0 & 0 & FALSE & $\mathrm{N} / \mathrm{A}$ & \\
\hline 6185 & 701 & $2005 / 01 / 25$ & 1.000 & 2005 & 0 & 0 & 0 & 0 & 0 & 0 & 0 & 0 & 0 & 0 & FALSE & N/A & \\
\hline 6185 & 12 & $2007 / 02 / 08$ & 610.373 & 2007 & 0 & 0 & 0 & 0 & 0 & 0 & 0 & 0 & 0 & 0 & FALSE & $\mathrm{N} / \mathrm{A}$ & \\
\hline 6185 & 106 & $2007 / 02 / 08$ & 271.000 & 2007 & 0 & 0 & 0 & 0 & 0 & 0 & 0 & 0 & 0 & 0 & FALSE & $\mathrm{N} / \mathrm{A}$ & \\
\hline 6185 & 205 & $2007 / 02 / 08$ & 6.000 & 2007 & 0 & 0 & 0 & 0 & 0 & 0 & 0 & 0 & 0 & 0 & FALSE & $\mathrm{N} / \mathrm{A}$ & \\
\hline 6185 & 215 & $2007 / 02 / 08$ & 35.662 & 2007 & 0 & 0 & 0 & 0 & 0 & 0 & 0 & 0 & 0 & 0 & FALSE & N/A & \\
\hline 6185 & 234 & $2007 / 02 / 08$ & 27.000 & 2007 & 0 & 0 & 0 & 0 & 0 & 0 & 0 & 0 & 0 & 0 & FALSE & N/A & \\
\hline
\end{tabular}

Table 11 - Example Joint Elimination Processing (District 9) 


\section{Priority Bridges and District Visits}

A separate workbook on priority bridges was created to store the data for bridges containing the most numerous elements that were deemed important based on the experience of the Principal Investigator of the project, Dr. Steven Chase. The three main categories were deck / girder systems, joints, and bearings, each of which contained two elements that had been analyzed previously. The resulting list of six elements was: 107 (steel open girder - coated), 108 (steel open girder with timber deck - coated and uncoated), 301 (pourable joint seal), 302 (compression joint seal), 311 (moveable bearing), and 313 (fixed bearing). The previously calculated color-coded yearly condition state data and year built were copied for each of the chosen elements into a corresponding worksheet and kept in order by district.

The number of unexpected improvements for the joints (elements 301 / 302) was lower than expected by the project advisory group, which was attributed to the $>1.00$ condition state decrease criteria being too large for most joint improvements. A much more lenient 0.05 condition state decrease at any point criteria was then implemented in order to include many more subtle improvements to the joints. This revised criteria increased the number of bridges with improvements noted for those elements anywhere from about 2 to 11 times depending on the district (compare the "301>1" and "302>1" row totals to the more lenient "301" and "302" row totals in Table 37).

For each of the 9 VDOT districts, 40 bridges were chosen that contained unexpected improvements to the six elements listed above. Bridges containing the more lenient 0.05 condition state decrease criteria for joint elements (301 \& 302) were included, but bridges where the improvement to the girder elements $(107 \& 108)$ had been noted in Pontis as repainting (as discussed above) were omitted, as the type of maintenance was known. Selection was made 
from the available bridges by element, attempting to keep the number of bridges containing each of the 6 elements approximately equal. There were few significant improvements made to the bearing elements ( $311 \& 313)$, so almost all of them were included in the respective districts while the large number of bridges with improvements to the joint elements (301 \& 302) meant a random selection of about 10 per district was made. The summary table of the number of improvements per element per district can be found as Table 37.

Table 12 shows the first twenty rows of the priority bridge selection worksheet for District 2. Each column represents the federal IDs for bridges that were shown to have an improvement to the given element. The modified criteria of no noted paint maintenance is applied to elements 107 and 108, while "301 new" and "302 new" denote the more lenient 0.05 condition state decrease improvement criteria for those joint elements. You can see that there are far fewer bridges with the bearing elements (311 and 313) than the other elements for this district. The total available count per element for District 2 is: element $107-21$ bridges, $108-$ $64,301-88,302-139,311-5$, and element $313-1$ bridge. The last column, "Multiple", is a listing of all bridges that contained improvements to multiple elements; these bridges were automatically selected to maximize the inspection results obtained from 40 reports. Bridge 3450 is bolded because it has 3 elements $(107,301, \& 302)$ that were improved. 


\begin{tabular}{|c|c|c|c|c|c|c|}
\hline $\begin{array}{c}107-\text { No } \\
\text { Paint }\end{array}$ & $\begin{array}{c}108-\text { No } \\
\text { Paint }\end{array}$ & 301 New & 302 New & 311 & 313 & Multiple \\
\hline 3183 & 2673 & 2576 & 2587 & 4569 & 12120 & 3183 \\
\hline 3230 & 2676 & 2592 & 2651 & 8372 & & 3450 \\
\hline 3232 & 2694 & 2600 & 2652 & 10166 & & 4569 \\
\hline 3450 & 2719 & 2709 & 2717 & 12120 & & 8372 \\
\hline 4569 & 2725 & 2903 & 2865 & 22513 & & 8374 \\
\hline 4571 & 2739 & 3183 & 2882 & & & 8375 \\
\hline 4750 & 2890 & 3196 & 2901 & & & 12120 \\
\hline 8335 & 2914 & 3198 & 3220 & & & 12290 \\
\hline 8336 & 3357 & 3200 & 3222 & & & 12357 \\
\hline 8374 & 4624 & 3202 & 3229 & & & 14571 \\
\hline 8375 & 4648 & 3218 & 3234 & & & 14863 \\
\hline 10126 & 4663 & 3219 & 3235 & & & 22510 \\
\hline 12290 & 4681 & 3345 & 3236 & & & \\
\hline 12357 & 4691 & 3366 & 3276 & & & \\
\hline 14571 & 4752 & 3438 & 3277 & & & \\
\hline 14863 & 5496 & 3450 & 3353 & & & \\
\hline 14899 & 5497 & 3528 & 3450 & & & \\
\hline 14901 & 5527 & 3533 & 3527 & & & \\
\hline 21744 & 5544 & 4554 & 4542 & & & \\
\hline 22416 & 5555 & 4632 & 4543 & & & \\
\hline & & & & & & \\
\hline
\end{tabular}

Table 12 - Example Selection of Priority Bridge Choices (District 2)

Once the 40 bridges per districts were selected, arrangements were made to investigate the paper inspection reports in person at the bridge inspection divisions at the 9 VDOT district offices. For each district, the list of 40 bridges had additional columns added to cross reference the county the bridge was in, the route number carried, and the Virginia structure number in addition to the federal ID; an example of some of the bridges for District 9 is shown in Table 13. These columns used the "lookup" Excel command, searching in the following PDI output tables (for the desired information): Bridge (for "County"), Userbrdg (for "VA Structure \#"), and Roadway (for "Route \#"). These lists were sent ahead to the districts to have the reports ready upon arrival, and dates selected to extract the desired information. 


\begin{tabular}{|c|c|c|c|}
\hline $\begin{array}{c}\text { Federal } \\
\text { ID }\end{array}$ & County & $\begin{array}{c}\text { VA Structure } \\
\#\end{array}$ & Route \# \\
\hline 6189 & "029" & "2191" & "00674" \\
\hline 6200 & $" 029 "$ & $" 5002 "$ & $" 00495 "$ \\
\hline 6297 & $" 029 "$ & $" 1120 "$ & $" 00066 "$ \\
\hline 6301 & $" 029 "$ & $" 1122 "$ & "00050" \\
\hline 6370 & "029" & "2218" & "00495" \\
\hline 6656 & "029" & "6097" & "00603" \\
\hline
\end{tabular}

Table 13 - Example Selection of Bridge Information for Report Finding (District 9)

Site visits were then made to the districts to look at the inspection reports from the 360 total selected bridges in order to determine what maintenance actions were recorded as having been taken to cause the condition states to improve. Before each visit, an Excel table was created with each expected improvement for the chosen 40 bridges in that district as well as the year that the extracted Excel data showed an improvement for each element and bridge. Another set of columns was filled in ahead of time for the condition state of the selected element before and after the improvement occurred. An example of the table taken to the different districts is shown in Table 14 for the first several bridges in District 2. Because the criteria was a greater than 1.00 decrease from maximum condition state to current condition state, there was not necessarily a two report period for each improvement, as occasionally the decrease would be split between multiple years. The element 107 condition state for Bridge 12290 in Table 14, for example, decreases by exactly 1.00 from 2006 to 2008, which wouldn't show up under the improvement criteria except it further decreases in the 2010 report. Bridges that had improvements to multiple elements had a different entry (row) for each improvement noted. 


\begin{tabular}{|c|c|c|c|c|c|c|}
\hline Element & $\begin{array}{c}\text { Structure } \\
\text { ID }\end{array}$ & $\begin{array}{c}\text { Years } \\
\text { Expected }\end{array}$ & $\begin{array}{c}\text { Year } \\
\text { Performed }\end{array}$ & $\begin{array}{c}\text { Work } \\
\text { Performed }\end{array}$ & $\begin{array}{c}\text { Condition } \\
\text { State Before }\end{array}$ & $\begin{array}{c}\text { Condition } \\
\text { State After }\end{array}$ \\
\hline 107 & 3183 & 20082010 & & & 2.13 & 1.10 \\
\hline 107 & 3450 & 20062008 & & & 3.22 & 1.00 \\
\hline 107 & 4569 & 20052007 & & & 3.12 & 1.00 \\
\hline 107 & 8336 & 20052007 & & & 3.50 & 1.51 \\
\hline 107 & 8374 & 20052007 & & & 2.88 & 1.00 \\
\hline 107 & 8375 & 20072009 & & & 2.40 & 1.00 \\
\hline 107 & 12290 & 20062008 & & & 2.12 & 1.12 \\
\hline 107 & 12357 & 20012003 & & & 3.28 & 1.05 \\
\hline 107 & 14571 & 20072009 & & & 2.67 & 1.22 \\
\hline 107 & 14863 & 20072009 & & & 2.64 & 1.00 \\
\hline 107 & 21744 & 20062007 & & & 2.64 & 1.00 \\
\hline 107 & 22510 & 20002002 & & & 3.00 & 1.00 \\
\hline 108 & 2694 & 20082009 & & & 3.35 & 2.30 \\
\hline
\end{tabular}

Table 14 - Example Selection of Table Taken to District Visit (District 2)

A column was filled in on-site with the type of work performed (or lack thereof) and the year in which this work was done (or the year in which improvement with no maintenance was noted); these codes are explained in Appendix C in Table C-25. Table 15 shows a selection from the completed table of data from the visit to District 6 (Fredericksburg), displaying the information prepared before the visit and the data added in the field. An additional column was added after the visits to include the quantity of the element in each row, and another for the road type. These columns were filled in by using a lookup command to reference the corresponding quantity and road type from the Quantity field in the InspEvnt PDI table and the Functional Class field in the Roadway PDI table, respectively. The Road Type numbers refer to the detailed descriptions in Appendix C as Table C-26, which were reduced to Primary, Secondary, and Interstate roads for the road classification distinction used in the analysis. 


\begin{tabular}{|c|c|c|c|c|c|c|c|c|c|}
\hline Element & Structure ID & Years Expected & Year Performed & Work Performed & CS Before & CS After & QTY & Road Type & Road Classification \\
\hline 107 & 17926 & 20042006 & 2006 & CP & 3.12 & 2.12 & 633.039 & 14 & Primary \\
\hline 107 & 17926 & 20062008 & 2008 & NM & 2.12 & 1.01 & 633.039 & 14 & Primary \\
\hline 107 & 17991 & 20002002 & 2002 & CP & 2.10 & 1.00 & 312.861 & 17 & Secondary \\
\hline 108 & 10571 & 19982000 & 1998 & TE & 3.68 & 1.13 & 57.000 & 9 & Secondary \\
\hline 108 & 18035 & 19951996 & 1995 & TE & 4.00 & 3.01 & 67.000 & 9 & Secondary \\
\hline 108 & 18035 & 20012002 & 2002 & NM & 3.01 & 1.85 & 67.000 & 9 & Secondary \\
\hline 301 & 4398 & 20082009 & 2009 & NM & 2.73 & 2.00 & 10.029 & 6 & Primary \\
\hline \hline
\end{tabular}

Table 15 - Example Selection of District Visit Complete Information (District 6)

\section{Linear Least Squares and Markov Modeling}

A macro was written to perform linear least squares (LLS) regression for each bridge to forecast the average condition states over the next several years. The number of years after 2010 to predict the average condition state is user-defined in the code. The macro calculated the average condition states, by inspection year, of all the bridges (for a certain element) in a certain district that were not marked as having an unexpected improvement. LLS regression was then performed on these average values in order to calculate an average slope for the bridges that deteriorated (or improved less than the 1.00 max-to-current condition state criteria). Finally, the macro applied this slope to the most recent condition state of each bridge in order to forecast the condition states and select which bridges might have important elements reaching advanced deterioration levels. The y-intercept of this prediction line is back-calculated from the slope and most recent condition state. Another option that was coded was to perform LLS on each bridge separately, projecting the calculated slope out from the most recent condition state for that bridge. This required creating certain rules if there was 0 or negative slope, or if there was only one data point for that bridge; namely, at that point using the average slope for that district and element and projecting that from the most recent condition state. 
Table 16 shows the first several additional columns that the individual LLS regression macro added to the element 215 information for District 1. Everything left of the "Slope" column was created for the earlier condition state trend analysis (such as Table 3). The columns headed with years are the condition state predictions from the given slope and intercept, and they continue the user-defined number of years after 2010. For the individual LLS macro results shown below, the slope and intercept information are unique to the condition state history of each bridge analyzed. Below all the individual bridge rows, the LLS regression for the average condition state history for the selected element on all bridges in the given district is performed.

\begin{tabular}{|c|c|c|c|c|c|c|c|}
\hline 2010 & CR & Age & Unexpected Improvement & Slope & Intercept & 2011 & 2012 \\
\hline 1.15 & 1.15 & 69 & FALSE & 0.001 & -1.535 & 1.157 & 1.158 \\
\hline 0.00 & 1.21 & 61 & FALSE & 0.013 & -24.939 & 1.276 & 1.289 \\
\hline 0.00 & 1.17 & 69 & FALSE & 0.004 & -7.403 & 1.167 & 1.167 \\
\hline 1.00 & 1.00 & 63 & FALSE & 0.000 & 1.000 & 1.000 & 1.000 \\
\hline 1.04 & 1.04 & 60 & FALSE & 0.001 & -0.834 & 1.042 & 1.042 \\
\hline 1.00 & 1.00 & 60 & FALSE & 0.000 & 1.000 & 1.000 & 1.000 \\
\hline 1.11 & 1.11 & 60 & FALSE & 0.005 & -8.510 & 1.115 & 1.115 \\
\hline 1.12 & 1.12 & 60 & FALSE & 0.001 & -0.702 & 1.122 & 1.122 \\
\hline 1.09 & 1.09 & 45 & FALSE & 0.004 & -6.680 & 1.112 & 1.116 \\
\hline 1.28 & 1.28 & 42 & FALSE & 0.008 & -15.173 & 1.283 & 1.287 \\
\hline 1.47 & 1.47 & 38 & FALSE & 0.020 & -38.364 & 1.472 & 1.472 \\
\hline
\end{tabular}

Table 16 - Example Selection of Linear Least Squares Appended Data - Element 215 - District 1

Linear least squares regression was also performed based on age of bridge on the same set of structures (all bridges without the desired elements undergoing "unexpected improvements") for the beam elements, 107 and 108. This analysis began by calculating the average condition states for each bridge, based on quantity in each condition state. The averageif command was then used, matching the number of years that elapsed between bridge construction and the inspection report with the age in the corresponding column in order to quantify the 
average deterioration of the given element as the bridges age in that district. 10 years of these averages are shown for element 107 in Table 17 below, each column representing a different age of bridge while each row represents a different district or total state. These averages were then graphed over the 100 year timeline for all cells with bridges representing that age in the given district (or total state). Trendlines (and the associated best fit equations) were added using Excel's linear least squares trendline function; this process produced graphs such as Figure 20 in the Results section.

\begin{tabular}{|c|r|r|r|r|r|r|r|r|r|r|r|}
\hline CS History> & 0 & 1 & 2 & 3 & 4 & 5 & 6 & 7 & 8 & 9 & 10 \\
\hline D1 & 1 & 1 & 1.001741 & 1 & 1.002964 & 1.000045 & 1.006963 & 1.000675 & 1.00524 & 1.008593 & 1.012153 \\
\hline D2 & 1 & 1 & 1 & 1 & 1.138458 & 1 & 1.000282 & 1 & 1.157983 & 1 & 1.133187 \\
\hline D3 & 1 & 1 & 1 & 1 & 1.000165 & 1 & 1.006977 & 1.000216 & 1.009315 & 1.000176 & 1.002346 \\
\hline D4 & 1 & 1 & 1.000216 & 1 & 1.001473 & 1 & 1.001411 & 1 & 1.013046 & 1.000847 & 1.026772 \\
\hline D5 & 1 & 1 & 1 & 1 & 1 & 1 & 1.006799 & 1.004338 & 1.002548 & 1.013529 & 1.013078 \\
\hline D6 & 1 & 1 & 1 & 1 & 1 & 1 & 1 & 1 & 1.000302 & 1 & 1.224734 \\
\hline D7 & 1 & 1 & 1 & & 1 & 1 & 1.002261 & 1 & 1.002447 & 1 & 1.011917 \\
\hline D8 & 1 & 1 & & 1 & 1 & 1.001236 & 1 & 1.076451 & 1.492331 & 1.062262 & 1.302296 \\
\hline D9 & 1 & 1.001274 & 1 & 1.000704 & 1.001693 & 1 & 1.009456 & 1.004671 & 1.020688 & 1.00434 & 1.013046 \\
\hline Total & 1 & 1.000498 & 1.000247 & 1.000391 & 1.011182 & 1.000036 & 1.00555 & 1.005785 & 1.028075 & 1.008529 & 1.03205 \\
\hline
\end{tabular}

Table 17 - Example Selection for LLS Analysis Based on Age of Bridges - Element 107

The yearly values from the linear trendlines were calculated using the slope and intercept from the best fit equations Excel produced. Residuals were calculated for each year by subtracting the actual condition state from the modeled condition state. These data sets were graphed against bridge age, such as Figure 21 in the Results section. The number of bridges reporting for each age was also kept track of in order to understand when there is a significant amount of data for a condition state data point and when the results are more easily skewed by a few bridges. This statistic is represented in tables like Table 18 below (columns representing age of bridges reporting) and also graphed by each district such as in Figure 22 in the Results section. 


\begin{tabular}{|c|r|r|r|r|r|r|r|}
\hline Count by Age & 0 & 1 & 2 & 3 & 4 & 5 & 6 \\
\hline 1 & 32 & 6 & 37 & 10 & 40 & 15 & 54 \\
\hline 2 & 30 & 11 & 38 & 4 & 43 & 7 & 48 \\
\hline 3 & 19 & 2 & 19 & 7 & 27 & 7 & 41 \\
\hline 4 & 33 & 16 & 40 & 20 & 40 & 20 & 70 \\
\hline 5 & 21 & 13 & 26 & 10 & 37 & 18 & 38 \\
\hline 6 & 6 & 4 & 9 & 5 & 8 & 5 & 9 \\
\hline 7 & 12 & 1 & 8 & 0 & 15 & 1 & 16 \\
\hline 8 & 2 & 1 & 0 & 3 & 5 & 5 & 8 \\
\hline 9 & 45 & 24 & 67 & 53 & 84 & 52 & 84 \\
\hline Total & 200 & 78 & 244 & 112 & 299 & 130 & 368 \\
\hline
\end{tabular}

Table 18 - Example Selection for Number of Bridges Reporting by Age - Element 107

The form of the solution of the linear least squares regression based on inspection year is:

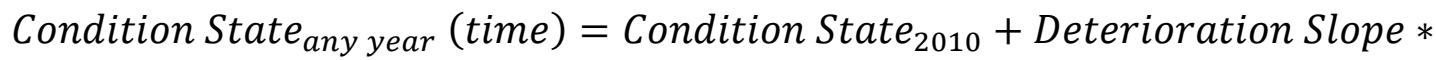
(\# of years since 2010). The form of the solution for the regression based on age of bridge is

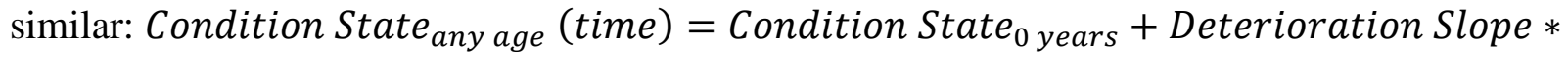
(\# of years since construction). The independent variable in both cases is time (in years), and the dependent variable is the resulting condition state.

The second type of deterioration modeling performed on the Pontis condition state data for this project was Markov chain creation. Deterioration trends were predicted for 4 elements in this exploration: 107 (Steel Open Girder - Coated), 108 (Steel Open Girder with Timber Deck Coated and Uncoated), 302 (Compression Joint Seal) and 32 (Timber Deck - with asphaltic concrete (AC) Overlay). The quantity of these elements in each condition state was used for this modeling, as compared to using the average condition state of an element on each bridge. This data was compiled in a large table comprised of the following column headings: bridge IDs, the year they were built, the quantity of the given element for each bridge in condition state 1, 2, 3 , 4, and 5 for each of the inspection years 1995 - 2010, and finally the district the bridges are in. An example of this is shown in Table 19 below, representing the left 14 (out of 83 total) columns 
and top 17 (out of 3293 total) rows of the raw data table for element 107. As an example, the quantity of element 107 on bridge number 2994 from the inspection report in 1995 is 304.82 feet in condition state 1 and 152.18 feet in condition state 2 .

To the right of this is a table of equal size, showing the age of each data point in the raw data table, calculated by subtracting the year each bridge was built from the inspection year corresponding to the current column; for example a bridge built in 1985 would show 10101010 10111111 etc. To the right of this is another table of equal size, showing the condition state each data point in the raw data table represents; A summary table is created that performs a sumifs function on the raw data to find the total quantity of each element in each condition state at each year of age since construction. An example of this is shown in Table 20 below, representing the left 13 (out of 506 total) columns of this summary data table for element 107, separated by district as D1, D2, etc. and total combined across the state. For example, the total quantity of element 107 in condition state 1 at 0 years after the respective construction of all bridges in District 1 is 12197.37 feet. 


\begin{tabular}{|c|c|c|c|c|c|c|c|c|c|c|c|c|c|}
\hline \multirow[t]{2}{*}{ Unique brkey } & Unique YR Built & 1995 & 1995 & 1995 & 1995 & 1995 & 1996 & 1996 & 1996 & 1996 & 1996 & 1997 & 1997 \\
\hline & TOTAL & 1 & 2 & 3 & 4 & 5 & 1 & 2 & 3 & 4 & 5 & 1 & 2 \\
\hline "000000000002960" & 1927 & 324.00 & 0.00 & 0.00 & 0.00 & 0.00 & 324.00 & 0.00 & 0.00 & 0.00 & 0.00 & 0.00 & 0.00 \\
\hline "000000000002961" & 1927 & 262.00 & 0.00 & 0.00 & 0.00 & 0.00 & 262.00 & 0.00 & 0.00 & 0.00 & 0.00 & 0.00 & 0.00 \\
\hline "000000000002963" & 2008 & 0.00 & 0.00 & 0.00 & 0.00 & 0.00 & 0.00 & 0.00 & 0.00 & 0.00 & 0.00 & 0.00 & 0.00 \\
\hline "000000000002966" & 1974 & 0.00 & 0.00 & 0.00 & 0.00 & 0.00 & 121.58 & 16.42 & 0.00 & 0.00 & 0.00 & 0.00 & 0.00 \\
\hline "000000000002978" & 1987 & 274.00 & 0.00 & 0.00 & 0.00 & 0.00 & 0.00 & 0.00 & 0.00 & 0.00 & 0.00 & 0.00 & 0.00 \\
\hline "000000000002992" & 1974 & 366.00 & 0.00 & 0.00 & 0.00 & 0.00 & 0.00 & 0.00 & 0.00 & 0.00 & 0.00 & 175.68 & 172.02 \\
\hline "000000000002993" & 1974 & 366.00 & 0.00 & 0.00 & 0.00 & 0.00 & 0.00 & 0.00 & 0.00 & 0.00 & 0.00 & 0.00 & 173.85 \\
\hline "000000000002994" & 1974 & 304.82 & 152.18 & 0.00 & 0.00 & 0.00 & 0.00 & 0.00 & 0.00 & 0.00 & 0.00 & 0.00 & 380.68 \\
\hline "000000000002996" & 1974 & 0.00 & 0.00 & 0.00 & 0.00 & 0.00 & 0.00 & 0.00 & 0.00 & 0.00 & 0.00 & 0.00 & 0.00 \\
\hline "000000000002998" & 1974 & 0.00 & 0.00 & 0.00 & 0.00 & 0.00 & 0.00 & 0.00 & 0.00 & 0.00 & 0.00 & 685.00 & 0.00 \\
\hline "000000000003000" & 1974 & 0.00 & 0.00 & 0.00 & 0.00 & 0.00 & 683.00 & 0.00 & 0.00 & 0.00 & 0.00 & 0.00 & 0.00 \\
\hline "000000000003016" & 1974 & 0.00 & 0.00 & 0.00 & 0.00 & 0.00 & 212.30 & 0.00 & 10.70 & 0.00 & 0.00 & 0.00 & 0.00 \\
\hline "000000000003019" & 1974 & 0.00 & 0.00 & 0.00 & 0.00 & 0.00 & 0.00 & 0.00 & 0.00 & 0.00 & 0.00 & 0.00 & 325.19 \\
\hline "000000000003021" & 1974 & 0.00 & 0.00 & 0.00 & 0.00 & 0.00 & 0.00 & 0.00 & 0.00 & 0.00 & 0.00 & 0.00 & 540.55 \\
\hline "000000000003022" & 1974 & 0.00 & 0.00 & 0.00 & 0.00 & 0.00 & 0.00 & 0.00 & 0.00 & 0.00 & 0.00 & 0.00 & 152.50 \\
\hline
\end{tabular}

Table 19 - Example Selection of Table of Raw Data for Markov Processing (Element 107)

\begin{tabular}{|c|c|c|c|c|c|c|c|c|c|c|c|c|}
\hline Age & 0 & 0 & 0 & 0 & 0 & 1 & 1 & 1 & 1 & 1 & 2 & 2 \\
\hline $\mathrm{CS}$ & 1 & 2 & 3 & 4 & 5 & 1 & 2 & 3 & 4 & 5 & 1 & 2 \\
\hline D1 & 12197.37 & 0.00 & 0.00 & 0.00 & 0.00 & 943.35 & 0.00 & 0.00 & 0.00 & 0.00 & 17037.94 & 29.72 \\
\hline D2 & 12838.00 & 0.00 & 0.00 & 0.00 & 0.00 & 5037.38 & 0.00 & 0.00 & 0.00 & 0.00 & 16495.88 & 0.00 \\
\hline D3 & 3548.57 & 0.00 & 0.00 & 0.00 & 0.00 & 1638.70 & 0.00 & 0.00 & 0.00 & 0.00 & 4510.16 & 0.00 \\
\hline D4 & 21427.20 & 0.00 & 0.00 & 0.00 & 0.00 & 7505.43 & 0.00 & 0.00 & 0.00 & 0.00 & 21181.09 & 4.58 \\
\hline D5 & 29709.70 & 0.00 & 0.00 & 0.00 & 0.00 & 8564.33 & 0.00 & 0.00 & 0.00 & 0.00 & 38291.55 & 0.00 \\
\hline D6 & 1598.83 & 0.00 & 0.00 & 0.00 & 0.00 & 627.73 & 0.00 & 0.00 & 0.00 & 0.00 & 2332.85 & 0.00 \\
\hline D7 & 4550.34 & 0.00 & 0.00 & 0.00 & 0.00 & 280.00 & 0.00 & 0.00 & 0.00 & 0.00 & 3170.34 & 0.00 \\
\hline D8 & 1299.00 & 0.00 & 0.00 & 0.00 & 0.00 & 59.00 & 0.00 & 0.00 & 0.00 & 0.00 & 0.00 & 0.00 \\
\hline D9 & 24810.32 & 0.00 & 0.00 & 0.00 & 0.00 & 15780.24 & 20.13 & 0.00 & 0.00 & 0.00 & 35911.76 & 0.00 \\
\hline Total & 111979.32 & 0.00 & 0.00 & 0.00 & 0.00 & 40436.16 & 20.13 & 0.00 & 0.00 & 0.00 & 138931.57 & 34.30 \\
\hline
\end{tabular}

Table 20 - Example Selection of Summary Table of Raw Data Arranged by Age and Condition State (Element 107) 
Separate workbooks were then created, for each district and the entire state, containing several tables to facilitate the Markov transition probability matrix creation. The first of these tables was a restructuring of the age and condition state data with the years 0 to 100 heading the rows and condition states 1 through 5 heading the columns, as well as a column for the total quantity at that age. The result is a table such as Table 21 for the data from the D1 row of Table 20 (from the raw data worksheet).

\begin{tabular}{|c|r|r|r|r|r|r|}
\hline Age & 1 & 2 & 3 & 4 & 5 & Sum \\
\hline 0 & 12197.37 & 0 & 0 & 0 & 0 & 12197.37 \\
\hline 1 & 943.35 & 0 & 0 & 0 & 0 & 943.35 \\
\hline 2 & 17037.94 & 29.72 & 0 & 0 & 0 & 17067.66 \\
\hline 3 & 4840.491 & 0 & 0 & 0 & 0 & 4840.491 \\
\hline 4 & 17522.9 & 52.09248 & 0 & 0 & 0 & 17574.99 \\
\hline 5 & 6830.186 & 0.305483 & 0 & 0 & 0 & 6830.491 \\
\hline 6 & 21873.89 & 153.3767 & 0 & 0 & 0 & 22027.27 \\
\hline 7 & 6762.196 & 4.57 & 0 & 0 & 0 & 6766.766 \\
\hline 8 & 18427.81 & 97.07748 & 0 & 0 & 0 & 18524.88 \\
\hline 9 & 8119.712 & 70.38 & 0 & 0 & 0 & 8190.092 \\
\hline 10 & 21170.08 & 260.4405 & 0 & 0 & 0 & 21430.52 \\
\hline 11 & 9462.404 & 209.5981 & 28.8 & 0 & 0 & 9700.802 \\
\hline 12 & 18052.68 & 255.3376 & 4.26629 & 0 & 0 & 18312.28 \\
\hline
\end{tabular}

Table 21 - Example Selection of Age-Based Condition State Data (Element 107 - District 1)

An equivalently sized table was then created with the quantity in each condition state per age divided into the sum of the quantity per age in order to get the percent of the bridges that were in each condition state at each year of age since construction. The next table is the estimated transition probability matrix such as Table 22 (similar to Table D-1 in Appendix D with the additional first column), with the column headings representing the condition state after transition. The first column represents the probability that bridges in each condition state will remain in that condition state over the next year, and is originally a guess that is later refined. 
The likelihood, therefore, of any quantity falling into the next worse condition state is 1 minus the first-column-value. 0 s fill in all other transition probabilities, resulting in an upper bidiagonal matrix. This approach limited the model to only reflect deterioration because the probability of any quantity of an element improving in condition was made to be 0 .

\begin{tabular}{|c|c|c|c|c|c|c|}
\hline & D1 & 1 & 2 & 3 & 4 & 5 \\
\hline 0.990 & 1 & 0.990 & 0.010 & 0.000 & 0.000 & 0.000 \\
\hline 0.972 & 2 & 0.000 & 0.972 & 0.028 & 0.000 & 0.000 \\
\hline 0.976 & 3 & 0.000 & 0.000 & 0.976 & 0.024 & 0.000 \\
\hline 0.961 & 4 & 0.000 & 0.000 & 0.000 & 0.961 & 0.039 \\
\hline 1.000 & 5 & 0.000 & 0.000 & 0.000 & 0.000 & 1.000 \\
\hline
\end{tabular}

Table 22 - Example Transition Probability Matrix - Element 107 - District 1

From this matrix, a table was created similar to the earlier table showing the percent in each condition state by age since construction. This new table, such as Table 23 , is based on the theoretical values from the transition probability matrix multiplied by itself for each year up to 100. Seven transitions are illustrated in Table 22. The rows represent the age of the elements, and the columns represent the 5 condition states and the average condition state (calculated from the proportion in condition state $1 * 1+$ the proportion in condition state $2 * 2$, etc.).

\begin{tabular}{|l|l|l|l|l|l|l|}
\hline & 1 & 2 & 3 & 4 & 5 & Average \\
\hline 0 & 1 & 0 & 0 & 0 & 0 & 1 \\
\hline 1 & 0.989967 & 0.010033 & 0 & 0 & 0 & 1.010033 \\
\hline 2 & 0.980036 & 0.019686 & 0.000279 & 0 & 0 & 1.020243 \\
\hline 3 & 0.970203 & 0.028971 & 0.000819 & $6.8 \mathrm{E}-06$ & 0 & 1.030629 \\
\hline 4 & 0.96047 & 0.037899 & 0.001604 & $2.65 \mathrm{E}-05$ & $2.62 \mathrm{E}-07$ & 1.041188 \\
\hline 5 & 0.950834 & 0.046482 & 0.002618 & $6.46 \mathrm{E}-05$ & $1.29 \mathrm{E}-06$ & 1.051918 \\
\hline 6 & 0.941295 & 0.054729 & 0.003846 & 0.000126 & $3.78 \mathrm{E}-06$ & 1.062815 \\
\hline 7 & 0.931851 & 0.062652 & 0.005274 & 0.000215 & $8.64 \mathrm{E}-06$ & 1.073879 \\
\hline
\end{tabular}

Table 23 - Example Selection of Condition State Probability Prediction by Age - Element 107 - District 1 
Finally, a table is created displaying the squared difference between each actual proportion (from the inspection reports) and theoretical proportion (from the transition probability matrix); the resulting table is again of equivalent size. All of these squared errors were then added together in a cell below the error table. The Excel Solver analysis tool was used to minimize this total error by changing the modeled transition probabilities (the first 4 values in the left column in Table 22) while keeping them between 0.00 and 1.00. This process is repeated iteratively to reach a smaller than $1.0 * 10^{-6}$ change in total error between successive attempts. Excel displays the transition probability matrix that produced the minimal total squared error when its results are compared to the actual data. The result is a deterioration model for that district and element. The process is repeated for each element and district desired; in this report, Markov chains were created for elements 107, 108, 302, and 32 in the different districts and total across the state. In this analysis, the state total is from the summed bridges with no regard to district, it is therefore weighted toward districts with more linear feet of the desired element.

These transition probability matrices are also graphically represented by creating $100 \%$ stacked area graphs over 100 years from the theoretical predicted data in tables such as Table 23, color-coded by condition state resulting in graphs such as the left, smooth graphs in Figure D-61. These predicted probability graphs are accompanied by their corresponding probability graphs produced from the actual Pontis data, such as the right, more jagged graphs in the same figure. 


\section{RESULTS}

\section{Original Pontis Analysis by District}

The top 10 smart flags and the top 20 elements are summarized in Table 24, Table 25, and Table 26, as selected by the highest total number of bridges in the given district containing

them. The tables are separated by district, with districts 1, 2, and 3 in Table 24, districts 4, 5, and 6 in Table 25, and districts 7, 8 and 9 in Table 26. For each district sub-table, the first column presents the number of improvements noted for the given smart flag or element, the second column displays the three digit element key, and the third column lists the number of bridges in the given district that that smart flag or element is present on. In the first column, red numbers represent smart flags while black numbers signify elements. 


\begin{tabular}{|c|c|c|}
\hline \multicolumn{3}{|c|}{ District 1} \\
\hline \# Improv. & Ekey & Count \\
\hline 1 & 295 & 2424 \\
\hline 16 & 215 & 1968 \\
\hline 20 & 706 & 1645 \\
\hline 53 & 359 & 1514 \\
\hline 1 & 708 & 1366 \\
\hline 19 & 331 & 1309 \\
\hline 3 & 361 & 1120 \\
\hline 4 & 704 & 953 \\
\hline 44 & 107 & 947 \\
\hline 12 & 358 & 874 \\
\hline 67 & 334 & 686 \\
\hline 16 & 363 & 635 \\
\hline 0 & 298 & 621 \\
\hline 28 & 234 & 604 \\
\hline 2 & 241 & 597 \\
\hline 0 & 299 & 594 \\
\hline 81 & 108 & 545 \\
\hline 4 & 210 & 521 \\
\hline 5 & 313 & 487 \\
\hline 41 & 032 & 478 \\
\hline 0 & 333 & 478 \\
\hline 20 & 702 & 465 \\
\hline 28 & 302 & 460 \\
\hline 6 & 297 & 435 \\
\hline 8 & 039 & 425 \\
\hline 7 & 311 & 417 \\
\hline 3 & 217 & 402 \\
\hline 0 & 240 & 367 \\
\hline 0 & 285 & 361 \\
\hline 23 & 205 & 335 \\
\hline
\end{tabular}

\begin{tabular}{|c|c|c|}
\hline \multicolumn{3}{|c|}{ District 2} \\
\hline \# Improv. & Ekey & Count \\
\hline 47 & 702 & 1771 \\
\hline 17 & 215 & 1770 \\
\hline 3 & 295 & 1523 \\
\hline 21 & 107 & 1227 \\
\hline 1 & 361 & 1162 \\
\hline 6 & 704 & 996 \\
\hline 39 & 331 & 932 \\
\hline 38 & 359 & 852 \\
\hline 64 & 108 & 809 \\
\hline 119 & 032 & 734 \\
\hline 37 & 706 & 675 \\
\hline 15 & 363 & 666 \\
\hline 16 & 334 & 665 \\
\hline 0 & 241 & 644 \\
\hline 0 & 298 & 576 \\
\hline 22 & 234 & 574 \\
\hline 13 & 285 & 523 \\
\hline 20 & 358 & 520 \\
\hline 1 & 313 & 515 \\
\hline 33 & 302 & 473 \\
\hline 0 & 299 & 441 \\
\hline 0 & 708 & 404 \\
\hline 9 & 210 & 394 \\
\hline 11 & 205 & 385 \\
\hline 5 & 311 & 381 \\
\hline 21 & 240 & 351 \\
\hline 7 & 330 & 349 \\
\hline 2 & 286 & 314 \\
\hline 20 & 332 & 293 \\
\hline 0 & 310 & 287 \\
\hline
\end{tabular}

\begin{tabular}{|c|c|c|}
\hline \multicolumn{3}{|c|}{ District 3} \\
\hline \# Improv. & Ekey & Count \\
\hline 3 & 295 & 1170 \\
\hline 6 & 215 & 908 \\
\hline 1 & 704 & 887 \\
\hline 4 & 361 & 871 \\
\hline 4 & 702 & 659 \\
\hline 4 & 107 & 647 \\
\hline 3 & 331 & 595 \\
\hline 10 & 359 & 531 \\
\hline 4 & 241 & 526 \\
\hline 6 & 285 & 510 \\
\hline 6 & 286 & 490 \\
\hline 8 & 706 & 475 \\
\hline 14 & 234 & 458 \\
\hline 0 & 298 & 445 \\
\hline 60 & 302 & 422 \\
\hline 1 & 313 & 405 \\
\hline 36 & 240 & 380 \\
\hline 17 & 108 & 379 \\
\hline 3 & 358 & 377 \\
\hline 35 & 032 & 370 \\
\hline 0 & 299 & 370 \\
\hline 6 & 205 & 341 \\
\hline 14 & 334 & 318 \\
\hline 1 & 311 & 289 \\
\hline 0 & 363 & 268 \\
\hline 1 & 210 & 246 \\
\hline 0 & 708 & 231 \\
\hline 12 & 332 & 206 \\
\hline 4 & 012 & 196 \\
\hline 0 & 310 & 194 \\
\hline
\end{tabular}

Table 24 - Element Count Summary - Districts 1, 2, and 3 


\begin{tabular}{|c|c|c|}
\hline \multicolumn{3}{|c|}{ District 4 } \\
\hline \# Improv. & Ekey & Count \\
\hline 1 & 295 & 1384 \\
\hline 28 & 215 & 1376 \\
\hline 26 & 331 & 1099 \\
\hline 2 & 704 & 977 \\
\hline 24 & 107 & 957 \\
\hline 36 & 234 & 868 \\
\hline 15 & 313 & 861 \\
\hline 11 & 285 & 851 \\
\hline 21 & 706 & 770 \\
\hline 6 & 358 & 759 \\
\hline 23 & 702 & 744 \\
\hline 17 & 205 & 740 \\
\hline 2 & 241 & 684 \\
\hline 51 & 359 & 683 \\
\hline 131 & 301 & 681 \\
\hline 12 & 311 & 665 \\
\hline 3 & 361 & 613 \\
\hline 7 & 321 & 591 \\
\hline 1 & 298 & 561 \\
\hline 2 & 299 & 521 \\
\hline 4 & 707 & 464 \\
\hline 9 & 286 & 438 \\
\hline 0 & 026 & 414 \\
\hline 21 & 012 & 413 \\
\hline 2 & 330 & 413 \\
\hline 27 & 302 & 408 \\
\hline 0 & 310 & 357 \\
\hline 15 & 240 & 356 \\
\hline 4 & 363 & 315 \\
\hline 15 & 108 & 247 \\
\hline & & \\
\hline 13 &
\end{tabular}

\begin{tabular}{|c|c|c|}
\hline \multicolumn{3}{|c|}{ District 5} \\
\hline \# Improv. & Ekey & Count \\
\hline 5 & 215 & 865 \\
\hline 2 & 331 & 758 \\
\hline 1 & 295 & 643 \\
\hline 0 & 234 & 630 \\
\hline 4 & 107 & 570 \\
\hline 8 & 285 & 565 \\
\hline 0 & 313 & 494 \\
\hline 0 & 311 & 484 \\
\hline 1 & 205 & 483 \\
\hline 1 & 706 & 481 \\
\hline 0 & 704 & 429 \\
\hline 3 & 361 & 424 \\
\hline 0 & 321 & 409 \\
\hline 14 & 302 & 405 \\
\hline 6 & 359 & 400 \\
\hline 16 & 301 & 369 \\
\hline 0 & 707 & 353 \\
\hline 4 & 358 & 340 \\
\hline 0 & 241 & 310 \\
\hline 0 & 298 & 280 \\
\hline 2 & 701 & 269 \\
\hline 6 & 018 & 261 \\
\hline 3 & 026 & 248 \\
\hline 8 & 702 & 243 \\
\hline 0 & 299 & 227 \\
\hline 3 & 334 & 204 \\
\hline 2 & 109 & 182 \\
\hline 10 & 108 & 159 \\
\hline 0 & 310 & 158 \\
\hline 10 & 032 & 155 \\
\hline
\end{tabular}

\begin{tabular}{|c|c|c|}
\hline \multicolumn{3}{|c|}{ District 6} \\
\hline \# Improv. & Ekey & Count \\
\hline 0 & 295 & 584 \\
\hline 4 & 361 & 430 \\
\hline 7 & 704 & 426 \\
\hline 0 & 298 & 372 \\
\hline 1 & 241 & 337 \\
\hline 0 & 215 & 317 \\
\hline 0 & 299 & 310 \\
\hline 2 & 331 & 263 \\
\hline 0 & 708 & 247 \\
\hline 2 & 359 & 213 \\
\hline 2 & 706 & 193 \\
\hline 2 & 107 & 191 \\
\hline 5 & 234 & 189 \\
\hline 2 & 358 & 183 \\
\hline 0 & 313 & 147 \\
\hline 0 & 363 & 142 \\
\hline 3 & 285 & 137 \\
\hline 0 & 360 & 137 \\
\hline 3 & 205 & 119 \\
\hline 2 & 334 & 119 \\
\hline 0 & 333 & 116 \\
\hline 1 & 311 & 115 \\
\hline 3 & 012 & 108 \\
\hline 3 & 286 & 104 \\
\hline 4 & 302 & 102 \\
\hline 0 & 321 & 96 \\
\hline 0 & 310 & 95 \\
\hline 10 & 301 & 94 \\
\hline 1 & 240 & 92 \\
\hline 2 & 108 & 78 \\
\hline
\end{tabular}

Table 25 - Element Count Summary - Districts 4, 5, and 6 


\begin{tabular}{|c|c|c|}
\hline \multicolumn{3}{|c|}{ District 7} \\
\hline \# Improv. & Ekey & Count \\
\hline 1 & 295 & 966 \\
\hline 6 & 215 & 913 \\
\hline 1 & 361 & 871 \\
\hline 1 & 704 & 673 \\
\hline 13 & 359 & 625 \\
\hline 5 & 331 & 545 \\
\hline 19 & 107 & 484 \\
\hline 2 & 241 & 468 \\
\hline 0 & 298 & 353 \\
\hline 0 & 299 & 331 \\
\hline 18 & 334 & 327 \\
\hline 4 & 363 & 289 \\
\hline 4 & 234 & 284 \\
\hline 9 & 108 & 246 \\
\hline 0 & 706 & 232 \\
\hline 35 & 240 & 223 \\
\hline 0 & 313 & 214 \\
\hline 2 & 210 & 198 \\
\hline 0 & 333 & 197 \\
\hline 4 & 039 & 192 \\
\hline 4 & 285 & 192 \\
\hline 3 & 205 & 191 \\
\hline 9 & 302 & 172 \\
\hline 0 & 311 & 165 \\
\hline 7 & 032 & 164 \\
\hline 6 & 018 & 159 \\
\hline 1 & 330 & 156 \\
\hline 5 & 358 & 128 \\
\hline 0 & 708 & 89 \\
\hline 1 & 360 & 79 \\
\hline
\end{tabular}

\begin{tabular}{|c|c|c|}
\hline \multicolumn{3}{|c|}{ District 8} \\
\hline \# Improv. & Ekey & Count \\
\hline 1 & 295 & 2067 \\
\hline 14 & 215 & 1968 \\
\hline 19 & 359 & 1341 \\
\hline 8 & 331 & 1180 \\
\hline 2 & 704 & 1174 \\
\hline 5 & 241 & 1056 \\
\hline 3 & 361 & 815 \\
\hline 21 & 107 & 804 \\
\hline 17 & 234 & 632 \\
\hline 4 & 706 & 609 \\
\hline 41 & 302 & 568 \\
\hline 2 & 299 & 566 \\
\hline 20 & 334 & 556 \\
\hline 1 & 313 & 544 \\
\hline 6 & 210 & 515 \\
\hline 1 & 298 & 506 \\
\hline 1 & 330 & 441 \\
\hline 6 & 205 & 437 \\
\hline 22 & 032 & 435 \\
\hline 19 & 108 & 427 \\
\hline 3 & 285 & 420 \\
\hline 0 & 039 & 409 \\
\hline 2 & 311 & 401 \\
\hline 14 & 702 & 382 \\
\hline 4 & 363 & 354 \\
\hline 3 & 358 & 351 \\
\hline 1 & 360 & 251 \\
\hline 12 & 240 & 204 \\
\hline 8 & 012 & 193 \\
\hline 3 & 013 & 169 \\
\hline & & \\
\hline
\end{tabular}

\begin{tabular}{|c|c|c|}
\hline \multicolumn{3}{|c|}{ District 9} \\
\hline \# Improv. & Ekey & Count \\
\hline 4 & 295 & 1386 \\
\hline 3 & 704 & 951 \\
\hline 3 & 215 & 865 \\
\hline 5 & 241 & 792 \\
\hline 18 & 331 & 713 \\
\hline 0 & 298 & 691 \\
\hline 4 & 361 & 683 \\
\hline 0 & 299 & 659 \\
\hline 5 & 706 & 646 \\
\hline 1 & 313 & 540 \\
\hline 3 & 107 & 505 \\
\hline 0 & 358 & 498 \\
\hline 7 & 234 & 492 \\
\hline 0 & 707 & 478 \\
\hline 2 & 321 & 471 \\
\hline 3 & 285 & 432 \\
\hline 2 & 311 & 431 \\
\hline 11 & 359 & 403 \\
\hline 0 & 205 & 402 \\
\hline 0 & 026 & 391 \\
\hline 9 & 302 & 389 \\
\hline 0 & 708 & 342 \\
\hline 5 & 334 & 315 \\
\hline 7 & 701 & 288 \\
\hline 2 & 310 & 237 \\
\hline 3 & 210 & 216 \\
\hline 2 & 286 & 213 \\
\hline 1 & 092 & 210 \\
\hline 17 & 301 & 201 \\
\hline 4 & 300 & 172 \\
\hline 13 & 108 & 63 \\
\hline
\end{tabular}

Table 26 - Element Count Summary - Districts 7, 8, and 9 
The most often improved smart flags and elements, as ordered by the average number of improvements per district, are displayed in Table 27 and Table 28. Not all districts had the same top smart flags and elements by improvement, so not every smart flag and element was present in all 9 districts. A cut-off of 6 out of 9 districts reporting was chosen to encompass smart flags and elements that were adequately represented across the state in this analysis.

In Table 27, elements are shown, with the top 9 well-represented elements highlighted in yellow, denoting those with an average of more than 10 improvements per district. In Table 28, the smart flags are shown, with the top 7 well-represented smart flags highlighted in red, denoting those with more than 2 improvements per district.

Table 29 shows the top smart flags and elements organized by the average number of bridges containing them per district, with the top 8 elements highlighted in orange and the top 9 smart flags highlighted in blue; the cut-off was 400 bridges with the given smart flag or element per district. The purpose of highlighting above certain thresholds was to denote those smart flags and elements that were relatively prolific (by total count or by improvement count) among similar categories. For this project, only the top elements by improvement were analyzed; element 295 (reinforced concrete wingwalls), for example, was present on the most bridges but rarely received a significant improvement in condition state; therefore, it was not analyzed.

The improvement thresholds (in Table 27 and Table 28) are different for smart flags and elements because of a much lower number of improvements showing up in this analysis for smart flags. In Table 27, Table 28, and Table 29, the first column is the 3 digit element key, the second column is the number of districts represented for that smart flag or element, the third column is the total number of improvements or bridge count per smart flag or element, the fourth 
column is the number of improvements or bridge count per district per smart flag or element, and the fifth column is the textual description of the given smart flag or element.

\begin{tabular}{|c|c|c|c|c|}
\hline Element & Districts & $\begin{array}{c}\text { Total } \\
\text { Improvements }\end{array}$ & $\begin{array}{c}\text { Divided } \\
\text { Improvements } \\
\end{array}$ & Description \\
\hline 301 & 4 & 174 & 43.50 & Pourable Joint Seal \\
\hline 032 & 6 & 234 & 39.00 & Timber Deck - with asphaltic concrete (AC) Overlay \\
\hline 108 & 9 & 230 & 25.56 & V Steel Open Girder with Timber Deck - Coated and Uncoated \\
\hline 302 & 9 & 225 & 25.00 & Compression Joint Seal \\
\hline 334 & 8 & 145 & 18.13 & Metal Bridge Railing - Coated \\
\hline 240 & 7 & 120 & 17.14 & Metal Culvert \\
\hline 332 & 2 & 32 & 16.00 & Timber Bridge Railing \\
\hline 107 & 9 & 142 & 15.78 & Steel Open Girder - Coated \\
\hline 234 & 9 & 133 & 14.78 & Reinforced Concrete Pier Cap \\
\hline 331 & 9 & 122 & 13.56 & Reinforced Concrete Bridge Railing \\
\hline 215 & 9 & 95 & 10.56 & Reinforced Concrete Abutment \\
\hline 012 & 4 & 36 & 9.00 & Concrete Deck - Bare - with Uncoated Reinforcement \\
\hline 205 & 9 & 70 & 7.78 & Reinforced Concrete Column or Pile Extension \\
\hline 018 & 2 & 12 & 6.00 & Concrete Deck - Thin Overlay (less than $1^{\prime \prime}$ ) - no AC Overlay \\
\hline 297 & 1 & 6 & 6.00 & V Other Material Wingwalls \\
\hline 285 & 9 & 51 & 5.67 & V Slope - Protected \\
\hline 286 & 5 & 22 & 4.40 & V Slope - Unprotected \\
\hline 210 & 6 & 25 & 4.17 & Reinforced Concrete Pier Wall \\
\hline 039 & 3 & 12 & 4.00 & Concrete Slab - with AC Overlay - without Membrane \\
\hline 300 & 1 & 4 & 4.00 & Strip Seal Expansion Joint \\
\hline 311 & 9 & 30 & 3.33 & Moveable Bearing (Roller, sliding, etc.) \\
\hline 013 & 1 & 3 & 3.00 & Concrete Deck - with AC Overlay - without Membrane \\
\hline 217 & 1 & 3 & 3.00 & Other Material Abutment \\
\hline 330 & 4 & 11 & 2.75 & Metal Bridge Railing - Uncoated \\
\hline 313 & 9 & 24 & 2.67 & Fixed Bearing \\
\hline 241 & 9 & 21 & 2.33 & Concrete Culvert \\
\hline 321 & 4 & 9 & 2.25 & Reinforced Concrete Approach Slab \\
\hline 109 & 1 & 2 & 2.00 & P/S Concrete Open Girder \\
\hline 295 & 9 & 15 & 1.67 & V Reinforced Concrete Wingwalls \\
\hline 026 & 3 & 3 & 1.00 & Concrete Deck - Bare - with Coated Reinforcement \\
\hline 092 & 1 & 1 & 1.00 & V Reinforced Concrete Sidewalk \\
\hline 310 & 6 & 2 & 0.33 & Elastomeric Bearing \\
\hline 333 & 3 & 0 & 0.00 & Timber Bridge Railing \\
\hline
\end{tabular}

Table 27 - Element Count Summary 


\begin{tabular}{|c|c|c|c|c|}
\hline $\begin{array}{c}\text { Smart } \\
\text { Flag }\end{array}$ & Districts & $\begin{array}{c}\text { Total } \\
\text { Improvements }\end{array}$ & $\begin{array}{c}\text { Divided } \\
\text { Improvements }\end{array}$ & Description \\
\hline 359 & 9 & 203 & 22.56 & Smart Flag - Soffit of Conc \\
\hline 702 & 6 & 116 & 19.33 & V Smart Flag - Drains \\
\hline 706 & 9 & 98 & 10.89 & V Smart Flag - Soffit of Overhang of Conc \\
\hline 363 & 7 & 43 & 6.14 & Smart Flag - Section Loss \\
\hline 358 & 9 & 55 & 6.11 & Smart Flag - Deck Cracking \\
\hline 701 & 2 & 9 & 4.50 & V Smart Flag - Utilities \\
\hline 361 & 9 & 26 & 2.89 & Smart Flag - Scour - \\
\hline 704 & 9 & 26 & 2.89 & V Smart Flag - Roadway Over Culverts \\
\hline 707 & 3 & 4 & 1.33 & V Smart Flag - Soffit of Conc \\
\hline 360 & 3 & 2 & 0.67 & Smart Flag - Settlement \\
\hline 299 & 9 & 4 & 0.44 & V Smart Flag - Culvert Wingwall \\
\hline 298 & 9 & 2 & 0.22 & V Smart Flag - Culvert Endwall/Headwall \\
\hline 708 & 6 & 1 & 0.17 & V Smart Flag - Debris in Channel - \\
\hline
\end{tabular}

Table 28 - Smart Flag Count Summary

\begin{tabular}{|c|c|c|c|c|}
\hline Element \# & Districts & Total & Divided & Description \\
\hline 295 & 9 & 12147 & 1349.67 & V Reinforced Concrete Wingwalls \\
\hline 215 & 9 & 10950 & 1216.67 & Reinforced Concrete Abutment \\
\hline 704 & 9 & 7466 & 829.56 & V Smart Flag - Roadway Over Culverts \\
\hline 331 & 9 & 7394 & 821.56 & Reinforced Concrete Bridge Railing \\
\hline 361 & 9 & 6989 & 776.56 & Smart Flag - Scour - \\
\hline 359 & 9 & 6562 & 729.11 & Smart Flag - Soffit of Conc \\
\hline 702 & 6 & 4264 & 710.67 & V Smart Flag - Drains \\
\hline 107 & 9 & 6332 & 703.56 & Steel Open Girder - Coated \\
\hline 706 & 9 & 5726 & 636.22 & V Smart Flag - Soffit of Overhang of Conc \\
\hline 241 & 9 & 5414 & 601.56 & Reinforced Concrete Pier Cap \\
\hline 234 & 9 & 4731 & 525.67 & V Smart Flag - Culvert Endwall/Headwall \\
\hline 298 & 9 & 4405 & 489.44 & Fixed Bearing \\
\hline 313 & 9 & 4207 & 467.44 & Smart Flag - Deck Cracking \\
\hline 358 & 9 & 4030 & 447.78 & V Smart Flag - Culvert Wingwall \\
\hline 299 & 9 & 4019 & 446.56 & V Smart Flag - Debris in Channel - \\
\hline 708 & 6 & 2679 & 446.50 & V Slope - Protected \\
\hline 285 & 9 & 3991 & 443.44 & V Other Material Wingwalls \\
\hline 297 & 1 & 435 & 435.00 & V Smart Flag - Soffit of Conc \\
\hline 707 & 3 & 1295 & 431.67 & Other Material Abutment \\
\hline 217 & 1 & 402 & 402.00 & \\
\hline & & & & \\
\hline
\end{tabular}




\begin{tabular}{|c|c|c|c|c|}
\hline 334 & 8 & 3190 & 398.75 & Metal Bridge Railing - Coated \\
\hline 321 & 4 & 1567 & 391.75 & Reinforced Concrete Approach Slab \\
\hline 032 & 6 & 2336 & 389.33 & Timber Deck - with asphaltic concrete (AC) Overlay \\
\hline 205 & 9 & 3433 & 381.44 & Reinforced Concrete Column or Pile Extension \\
\hline 302 & 9 & 3399 & 377.67 & Compression Joint Seal \\
\hline 311 & 9 & 3348 & 372.00 & Moveable Bearing (Roller, sliding, etc \\
\hline 026 & 3 & 1053 & 351.00 & Concrete Deck - Bare - with Coated Reinforcement \\
\hline 210 & 6 & 2090 & 348.33 & Reinforced Concrete Pier Wall \\
\hline 039 & 3 & 1026 & 342.00 & Concrete Slab - with AC Overlay - without Membrane \\
\hline 330 & 4 & 1359 & 339.75 & Metal Bridge Railing - Uncoated \\
\hline 301 & 4 & 1345 & 336.25 & Pourable Joint Seal \\
\hline 108 & 9 & 2953 & 328.11 & V Steel Open Girder with Timber Deck - Coated and Uncoated \\
\hline 286 & 5 & 1559 & 311.80 & V Slope - Unprotected \\
\hline 363 & 9 & 2669 & 296.56 & Smart Flag - Section Loss \\
\hline 240 & 7 & 1973 & 281.86 & Metal Culvert \\
\hline 701 & 2 & 557 & 278.50 & V Smart Flag - Utilities \\
\hline 333 & 3 & 791 & 263.67 & Timber Bridge Railing \\
\hline 332 & 2 & 499 & 249.50 & Timber Bridge Railing \\
\hline 012 & 4 & 910 & 227.50 & Concrete Deck - Bare - with Uncoated Reinforcement \\
\hline 310 & 6 & 1328 & 221.33 & Elastomeric Bearing \\
\hline 018 & 2 & 420 & 210.00 & Concrete Deck - Thin Overlay (less than 1") - no AC Overlay \\
\hline 092 & 1 & 210 & 210.00 & V Reinforced Concrete Sidewalk \\
\hline 109 & 1 & 182 & 182.00 & P/S Concrete Open Girder \\
\hline 300 & 1 & 172 & 172.00 & Strip Seal Expansion Joint \\
\hline 013 & 1 & 169 & 169.00 & Concrete Deck - with AC Overlay - without Membrane \\
\hline 360 & 3 & 467 & 155.67 & Smart Flag - Settlement \\
\hline 013 & 2 & 169 & 84.50 & Concrete Deck - with AC Overlay - without Membrane \\
\hline
\end{tabular}

Table 29 - Smart Flag and Element Count Summary - Total Count

The condition state trends from 1995 to 2010 for the top 6 elements and the top 6 smart flags, based on number of improvements, are shown in Appendix B from Figure B-1 to Figure B-56, only including smart flags and elements represented in at least 6 districts. The graphs for element 32 have been reproduced as Figure 3 through Figure 8 as examples for the results and analysis sections. The 32 graphs from the elements were also created separately for the 9 VDOT districts (containing just the data for bridges within each district), and these 288 graphs are available in a separate Excel file through VDOT. 
The first type of graph, exemplified by Figure 3, is a line graph of the average condition state of the specified element for all bridges that were marked as having an unexpected improvement to the given element from 1995 to 2010. The second type of graph, such as Figure 5, contains $100 \%$ stacked column plots, which represent the number of bridges with the specified element in each condition state, normalized so each column is the same total height showing only the bridges that were marked as having an unexpected improvement to the given element. Green signifies condition states 1.00 to 1.99 , yellow signifies 2.00 to 2.99 , orange signifies 3.00 to 3.99, and red signifies 4.00 to 5.00. The third type of graph, such as Figure 7, is also normalized stacked column plots, but based on the total quantity of the specified elements across the state in each condition state. Each of these types has corresponding graphs for bridges that did not show an unexpected improvement for the given element, such as Figure 4, Figure 6, and Figure 8. Only elements chosen for quantity analysis (elements 32, 108, 302, and 107) have the quantity graphs (such as Figure 7 and Figure 8). After 4 elements were examined by quantity, it was decided to stop that analysis as the results were very similar to the previous analysis. 


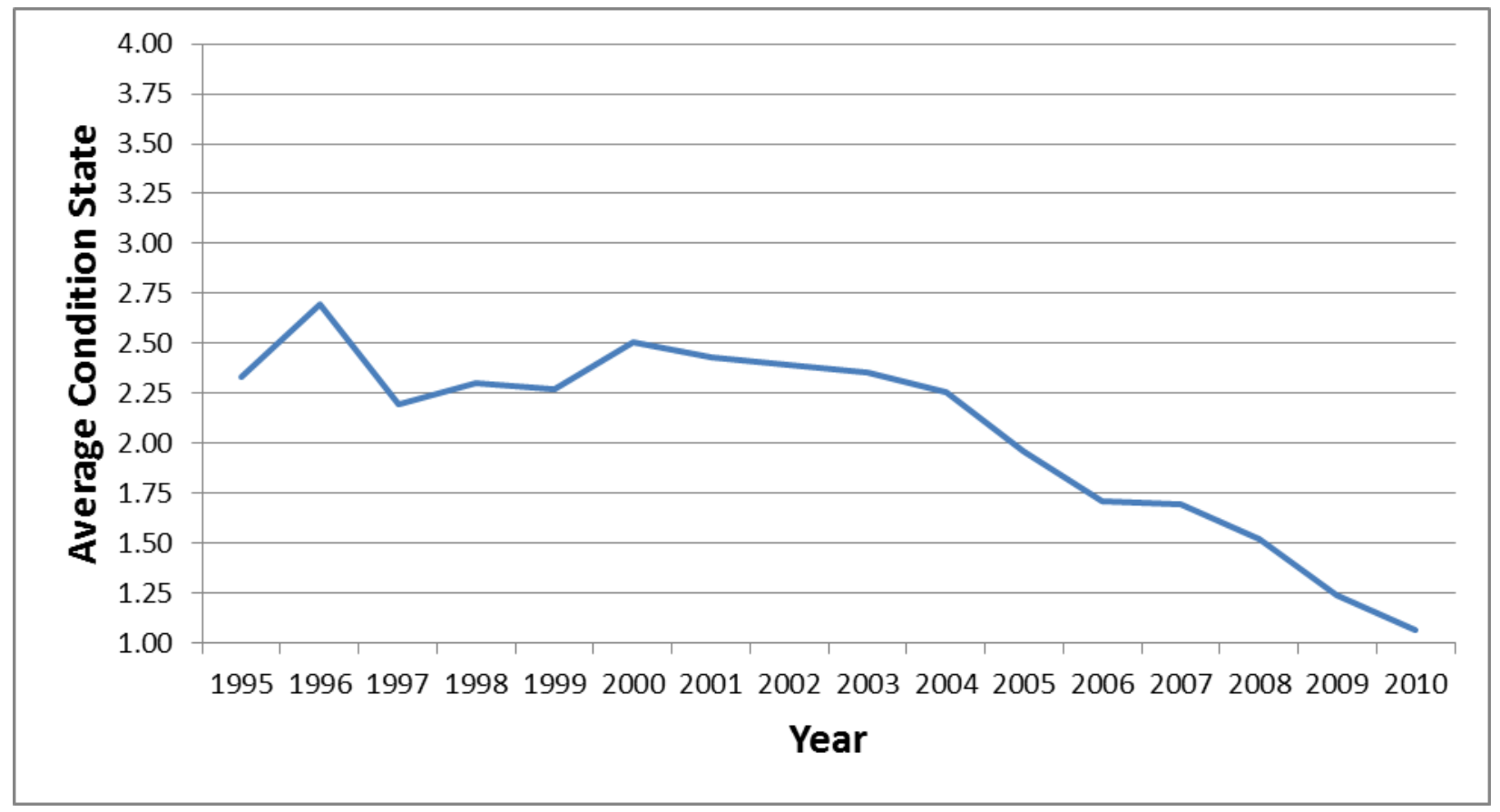

Figure 3 - Element 32 (Timber Deck - with Asphaltic Concrete (AC) Overlay) Average Condition State Trend Improvement Noted - Reproduced from Figure B-1

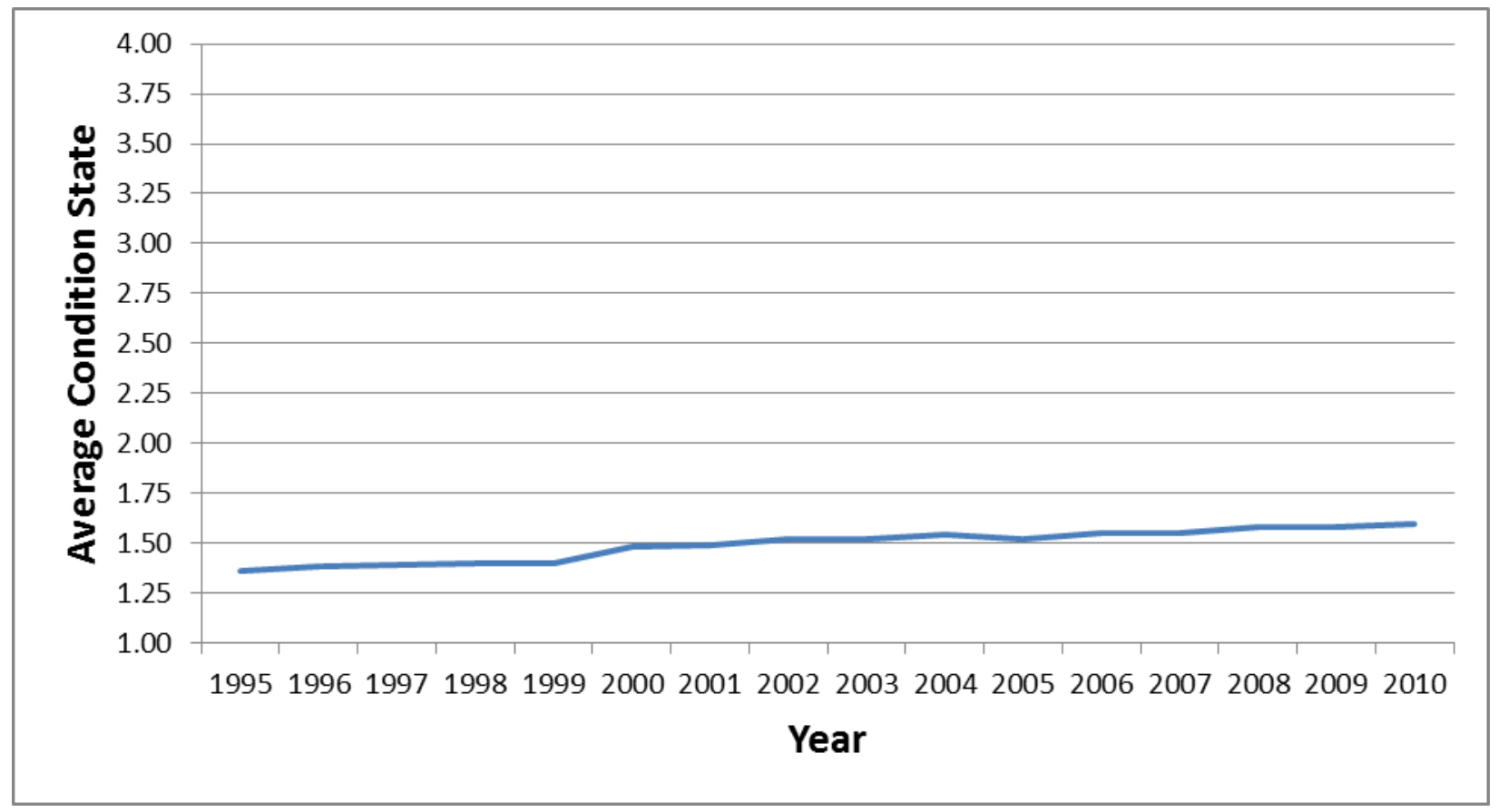

Figure 4 - Element 32 Average Condition State Trend - Improvement Not Noted - Reproduced from Figure B-2 


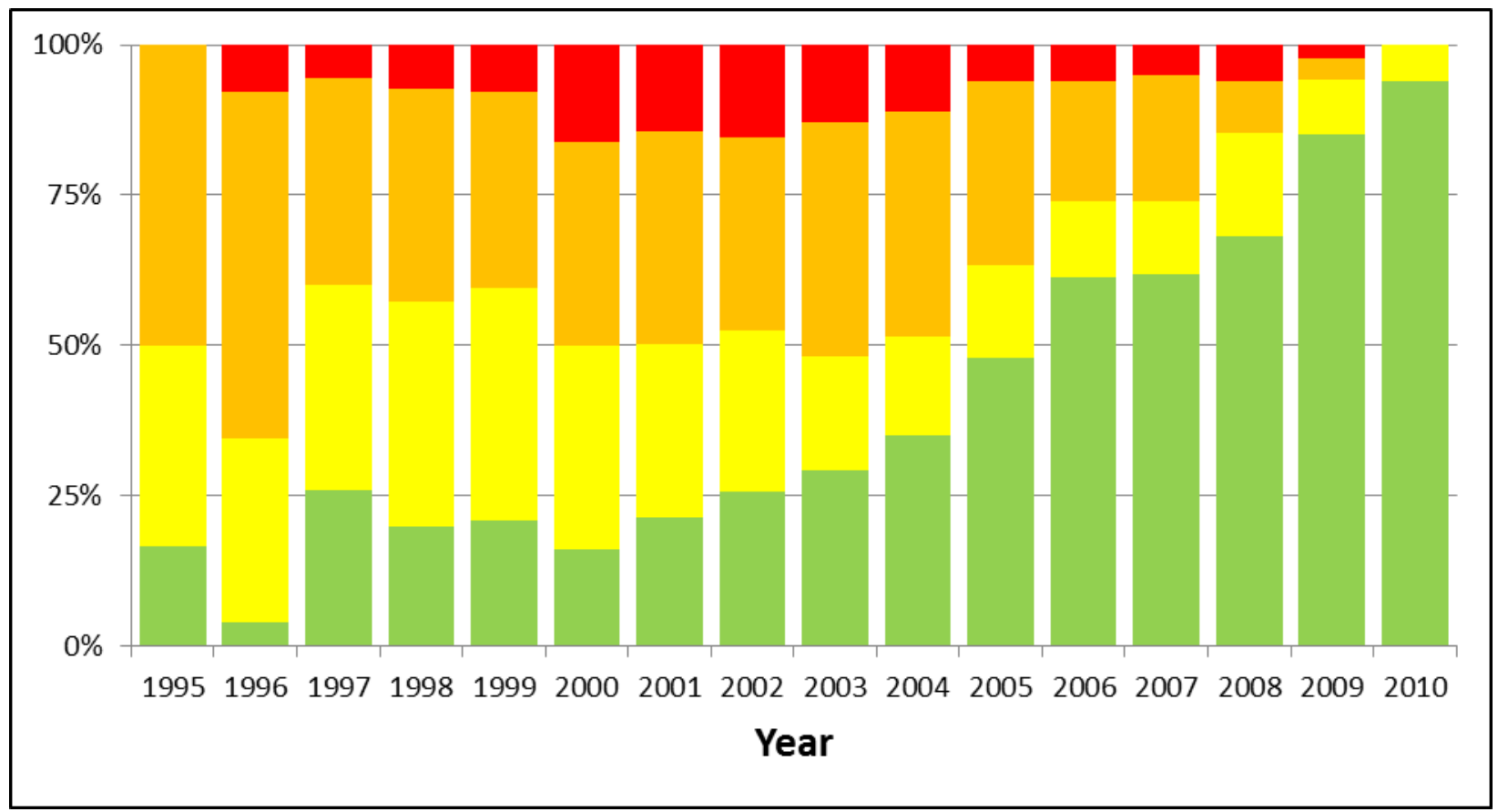

Figure 5 - Element 32 Normalized Condition State Trend - Improvement Noted - Reproduced from Figure B-3

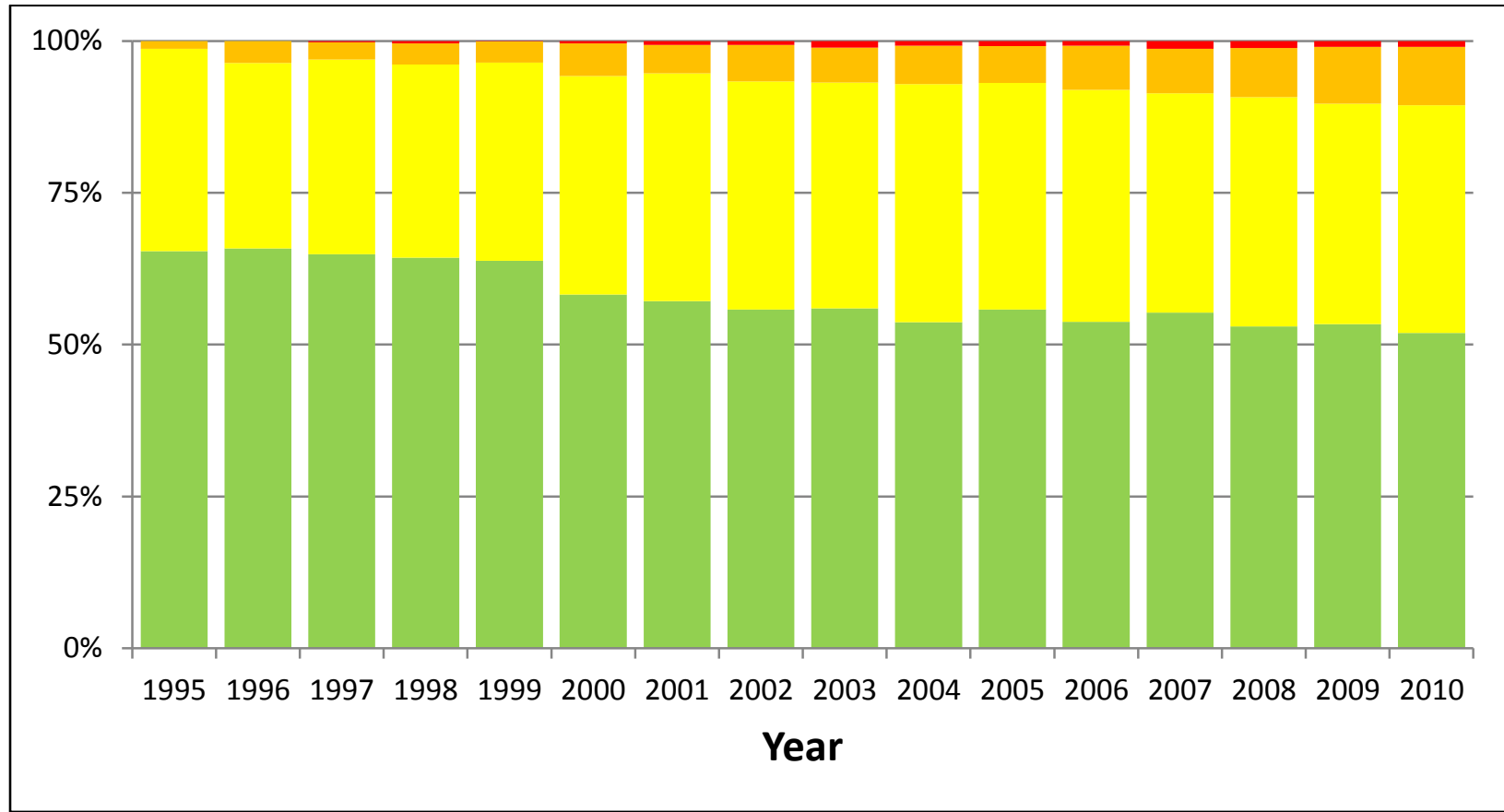

Figure 6 - Element 32 Normalized Condition State Trend - Improvement Not Noted - Reproduced from Figure B-4 


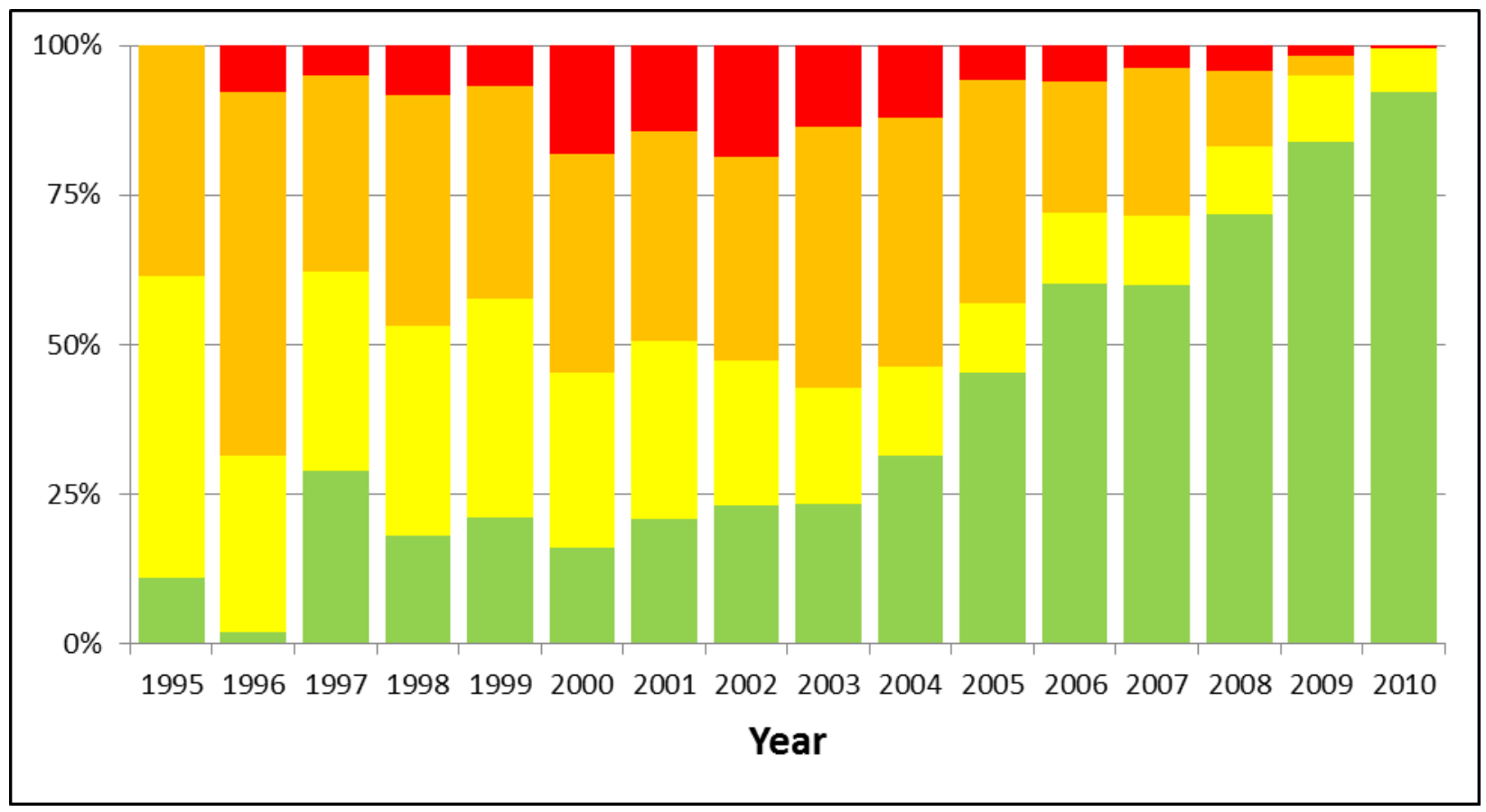

Figure 7 - Element 32 Normalized Condition State Trend - Improvement Noted (Quantity Analysis) - Reproduced from Figure B-5

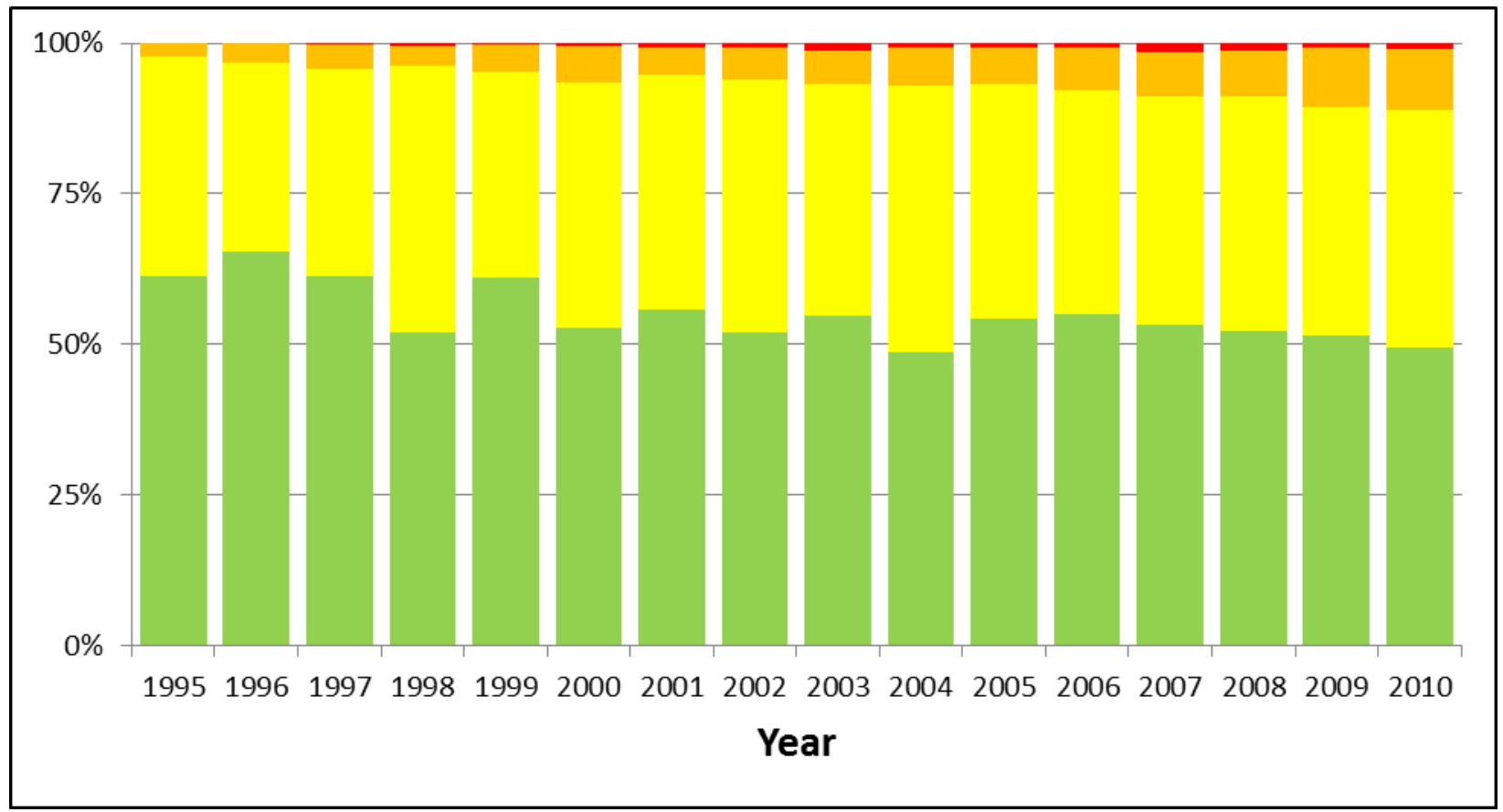

Figure 8 - Element 32 Normalized Condition State Trend - Improvement Not Noted (Quantity Analysis) Reproduced from Figure B-6 
The results of the year of improvement analysis are presented in tables such as Table 30 and Table 31 below and in their entirety in Appendix B as Table B-1 through Table B-12. The first row is the year of improvement, the second row is the number of bridges showing improvement in that year, and the last two rows are the average condition states recorded directly before and after that improvement occurred. Years with no improvements (often the early years, close to 1995) show 0.00 for before and after condition states. The full collection of tables in the appendix shows that some smart flags' and elements' condition states drop closer to 1 after improvement than others. Element 108 (Steel Open Girder with Timber Deck - Coated and Uncoated) and Smart Flag 359 (Soffit of Concrete) average around 1.55 after improvement compared to Element 302 (Compression Joint Seal) and Smart Flag 358 (Deck Cracking) which reached around 1.05 after improvement.

\begin{tabular}{|l|l|l|l|l|l|l|l|l|l|l|l|l|l|l|l|l|}
\hline Year & 1995 & 1996 & 1997 & 1998 & 1999 & 2000 & 2001 & 2002 & 2003 & 2004 & 2005 & 2006 & 2007 & 2008 & 2009 & 2010 \\
\hline Count & 0 & 0 & 0 & 5 & 4 & 8 & 8 & 15 & 12 & 13 & 16 & 11 & 11 & 5 & 8 & 9 \\
\hline Before & 0.00 & 0.00 & 0.00 & 3.00 & 3.13 & 3.63 & 3.49 & 2.94 & 3.00 & 2.97 & 2.89 & 2.85 & 2.60 & 2.85 & 2.79 & 3.08 \\
\hline After & 0.00 & 0.00 & 0.00 & 1.33 & 1.00 & 1.62 & 1.19 & 1.12 & 1.11 & 1.21 & 1.20 & 1.12 & 1.00 & 1.00 & 1.15 & 1.09 \\
\hline
\end{tabular}

Table 30 - Statewide Condition State Improvements by Year for Element 334 - Reproduced from Table B-4

\begin{tabular}{|l|l|l|l|l|l|l|l|l|l|l|l|l|l|l|l|l|}
\hline Year & 1995 & 1996 & 1997 & 1998 & 1999 & 2000 & 2001 & 2002 & 2003 & 2004 & 2005 & 2006 & 2007 & 2008 & 2009 & 2010 \\
\hline Count & 0 & 0 & 0 & 1 & 5 & 2 & 3 & 3 & 1 & 2 & 0 & 3 & 2 & 8 & 9 & 11 \\
\hline Before & 0.00 & 0.00 & 0.00 & 4.00 & 3.20 & 3.00 & 3.00 & 3.00 & 3.00 & 3.00 & 0.00 & 3.33 & 3.00 & 3.25 & 3.11 & 3.36 \\
\hline After & 0.00 & 0.00 & 0.00 & 1.00 & 1.00 & 1.00 & 1.00 & 1.00 & 1.00 & 1.00 & 0.00 & 1.00 & 1.00 & 1.13 & 1.00 & 1.23 \\
\hline
\end{tabular}

Table 31 - Statewide Condition State Improvements by Year for Smart Flag 358 - Reproduced from Table B-11 
The results from the age since improvement analysis are presented in tables such as Table 32 and Table 33 below and in their entirety in Appendix B as Table B-13 through Table B-24. The first row is the age in years of the bridges containing the element being analyzed that underwent a condition state improvement of greater than 1.00 . The second row is the summation of the condition states at the age shown at the top of that column. Similarly, the third row is the total count of all the bridges with inspection reports denoting a condition state improvement of greater than 1.00, at the age shown in the corresponding cell in the first row. The last row is the average condition state by year, calculated by dividing the total by the count for each age in which the count was non-zero (in order to avoid a divide-by-zero error).

\begin{tabular}{|c|c|c|c|c|c|c|c|c|c|c|c|c|c|c|c|c|}
\hline Age & 0 & 1 & 2 & 3 & 4 & 5 & 6 & 7 & 8 & 9 & 10 & 11 & 12 & 13 & 14 & 15 \\
\hline Total & 333.9 & 143.7 & 283.7 & 124.9 & 174.2 & 103.6 & 116.5 & 61.5 & 68.8 & 35.6 & 41.6 & 17.0 & 8.9 & 5.0 & 0.0 & 0.0 \\
\hline Count & 213 & 72 & 164 & 56 & 101 & 49 & 71 & 34 & 40 & 23 & 24 & 12 & 7 & 3 & 0 & 0 \\
\hline Average & 1.57 & 2.00 & 1.73 & 2.23 & 1.72 & 2.11 & 1.64 & 1.81 & 1.72 & 1.55 & 1.73 & 1.41 & 1.28 & 1.68 & & \\
\hline
\end{tabular}

Table 32 - Statewide Condition State Trend by Age since Improvement for Element 108 - Reproduced from Table B-14

\begin{tabular}{|c|c|c|c|c|c|c|c|c|c|c|c|c|c|c|c|c|}
\hline Age & 0 & 1 & 2 & 3 & 4 & 5 & 6 & 7 & 8 & 9 & 10 & 11 & 12 & 13 & 14 & 15 \\
\hline Total & 134.50 & 21.00 & 74.00 & 18.00 & 45.00 & 9.00 & 39.00 & 10.00 & 21.00 & 5.00 & 7.00 & 0.00 & 0.00 & 0.00 & 0.00 & 0.00 \\
\hline Count & 96 & 15 & 59 & 13 & 36 & 8 & 30 & 8 & 15 & 3 & 5 & 0 & 0 & 0 & 0 & 0 \\
\hline Average & 1.40 & 1.40 & 1.25 & 1.38 & 1.25 & 1.13 & 1.30 & 1.25 & 1.40 & 1.67 & 1.40 & & & & & \\
\hline
\end{tabular}

Table 33 - Statewide Condition State Trend by Age since Improvement for Element 706 - Reproduced from Table B-21 
The results of the age since improvement analysis are also presented in graphs such as Figure 9 and Figure 10 below and in their entirety in Appendix B as Figure B-57 through Figure B-59. These figures are graphical depictions of the Average Condition State vs. Age data from the associated tables (such as Table 32). The vertical axes were all forced to 1.00 to 3.00 (condition state value) and the horizontal axes forced to 0 to 12 (years) in order to keep the scales the same on all graphs in the set. The graphs created are straight lines between data points (with no smoothing), so elements with particularly up-and-down data show quite jagged results, such as element 240 in Figure 10.

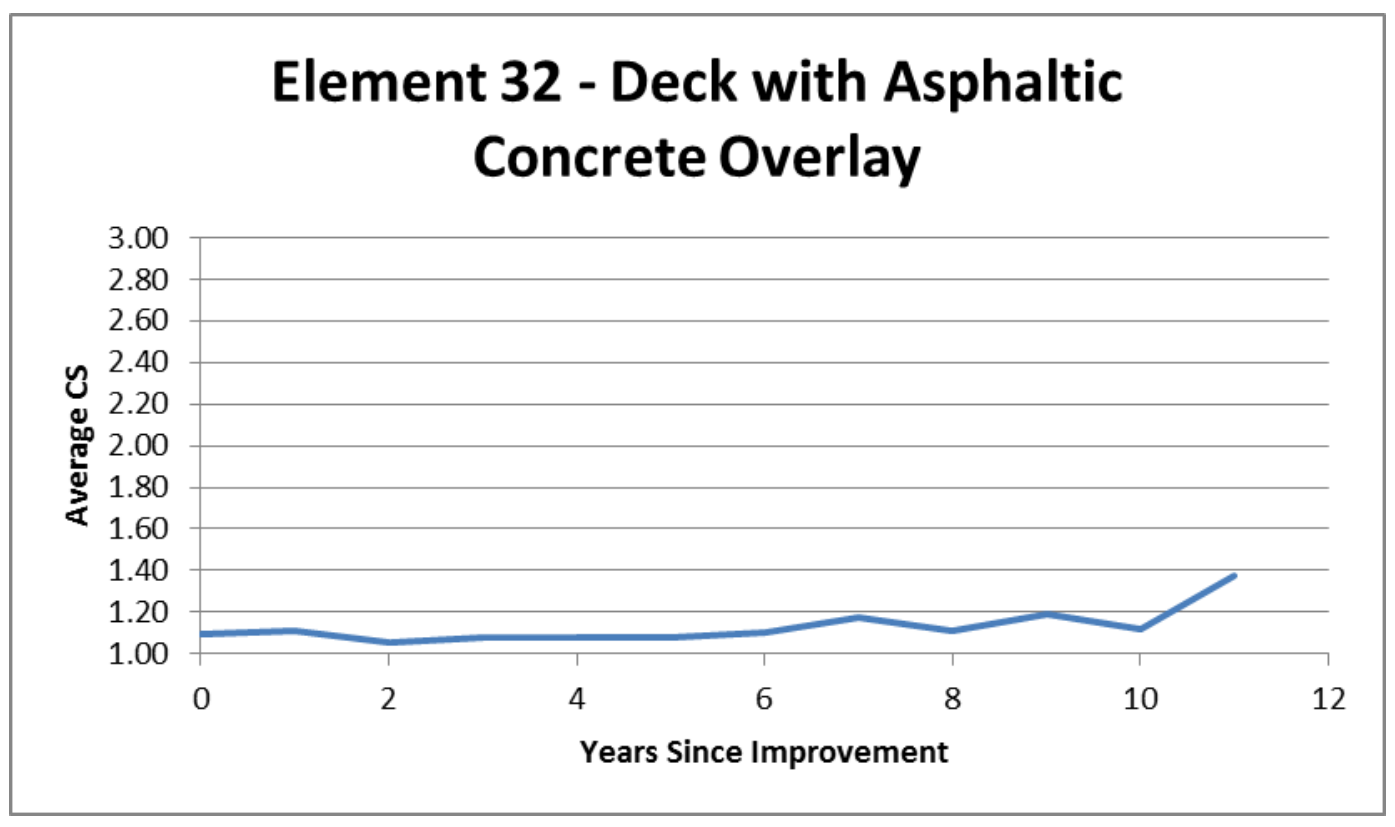

Figure 9 - Condition State Improvement Trend for Element 32 - Reproduced from Figure B-57 


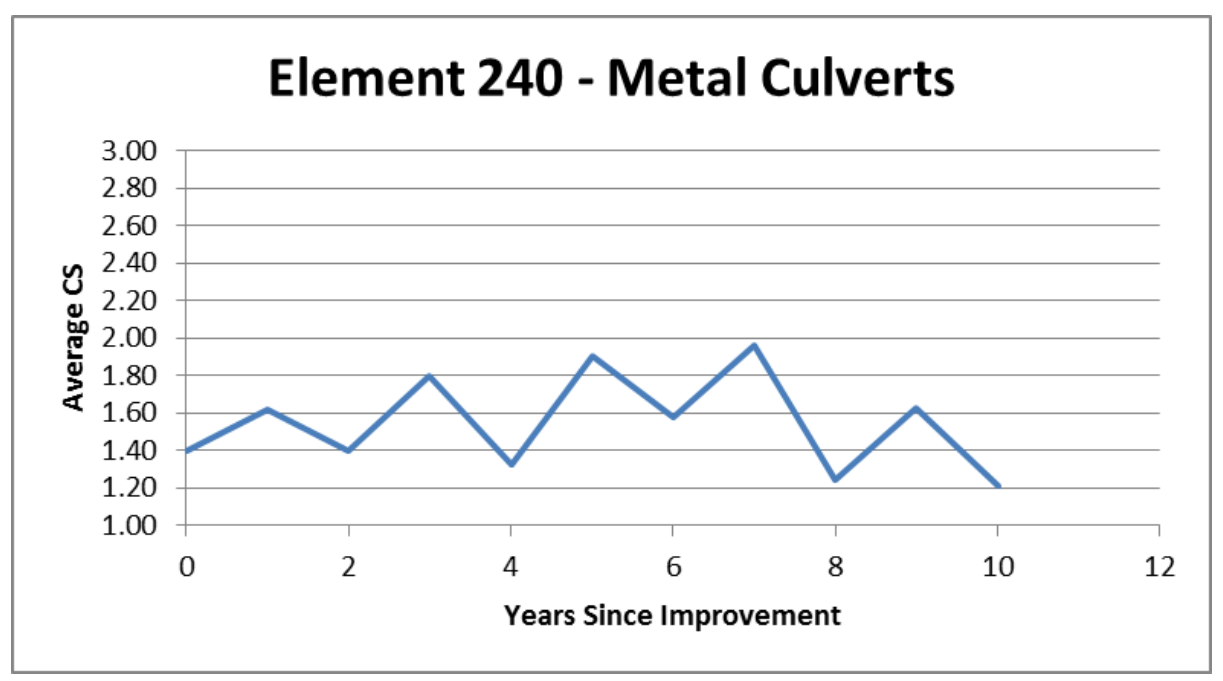

Figure 10 - Condition State Improvement Trend for Element 240 - Reproduced from Figure B-58

\section{Potential Errors}

Table 34 below shows the summary numbers for the side investigation on potential errors using the threshold of a 10\%+ difference between reported quantity and reported percent * total quantity. The middle column displays the number of inspection reports in each district and total across the state that met this criterion. The right column shows the number of bridges that met this criterion to see how many of the errors occurred on the same bridges and which were unique.

\begin{tabular}{|c|c|c|}
\hline District & \# of Reports with Errors & \# of Bridges with Errors \\
\hline 1 & 537 & 189 \\
\hline 2 & 418 & 215 \\
\hline 3 & 338 & 190 \\
\hline 4 & 309 & 210 \\
\hline 5 & 140 & 89 \\
\hline 6 & 290 & 69 \\
\hline 7 & 190 & 77 \\
\hline 8 & 306 & 173 \\
\hline 9 & 257 & 141 \\
\hline Total & 2785 & 1353 \\
\hline
\end{tabular}

Table 34 - Total Count of Likely $>10 \%$ Erroneous Data by District 
The full list of reports / bridges showing 10\%+ difference (between quantity and total quantity * percent for any condition state) is provided in a separate Excel file through VDOT.

\section{Zinc Coating Study}

The results of the zinc coating study are displayed graphically in Figure 11 through Figure 19. For the quantity analysis graphs (Figure 11, Figure 12, and Figure 15), only whole number condition state values are present because there is no averaging between bridges. Note the different horizontal scales in Figure 11 and Figure 15. In Figure 11, data was grouped by two-year inspection cycles to eliminate the biannual cyclic variation in this particular analysis.

For all condition state bar graphs, green represents condition state 1.00 to 1.99 , yellow is condition state 2.00 to 2.99 , orange is 3.00 to 3.99 , and red is 4.00 to 4.99 . For the condition rating bar graphs (Figure 14), condition rating 9 is shown in purple, down through blue, green, yellow, and red to condition rating 1 as maroon, as displayed in the legend. The graphs also display the approximate percentages of bridges in 2012 that were in condition rating 7 or higher, using double-sided black arrows. This proportion in other years can be found by reading the percentage covered by the combined purple, blue, and dark green bars.

The element 107 condition state deterioration trends (data points and 6-year moving average) by age since coating for the 4 groups are shown in Figure 16. Both the yearly data points and the calculated 6-year moving averages (with each average value represented as a data point in the sixth year) are plotted. The deterioration of the superstructure condition rating is shown in similar graphs in Figure 17. Note the inversion of the y-axis, as deterioration is a decrease in condition rating but an increase in condition state. The condition state axis is held to 1.0 to 2.2 and the condition rating axis is held to 9.0 to 4.0 for consistency. The 6 -year moving 
averages of the 4 groups are also graphed on the same plot for the condition state and condition rating analyses as Figure 18 and Figure 19, respectively, for easier comparison. 


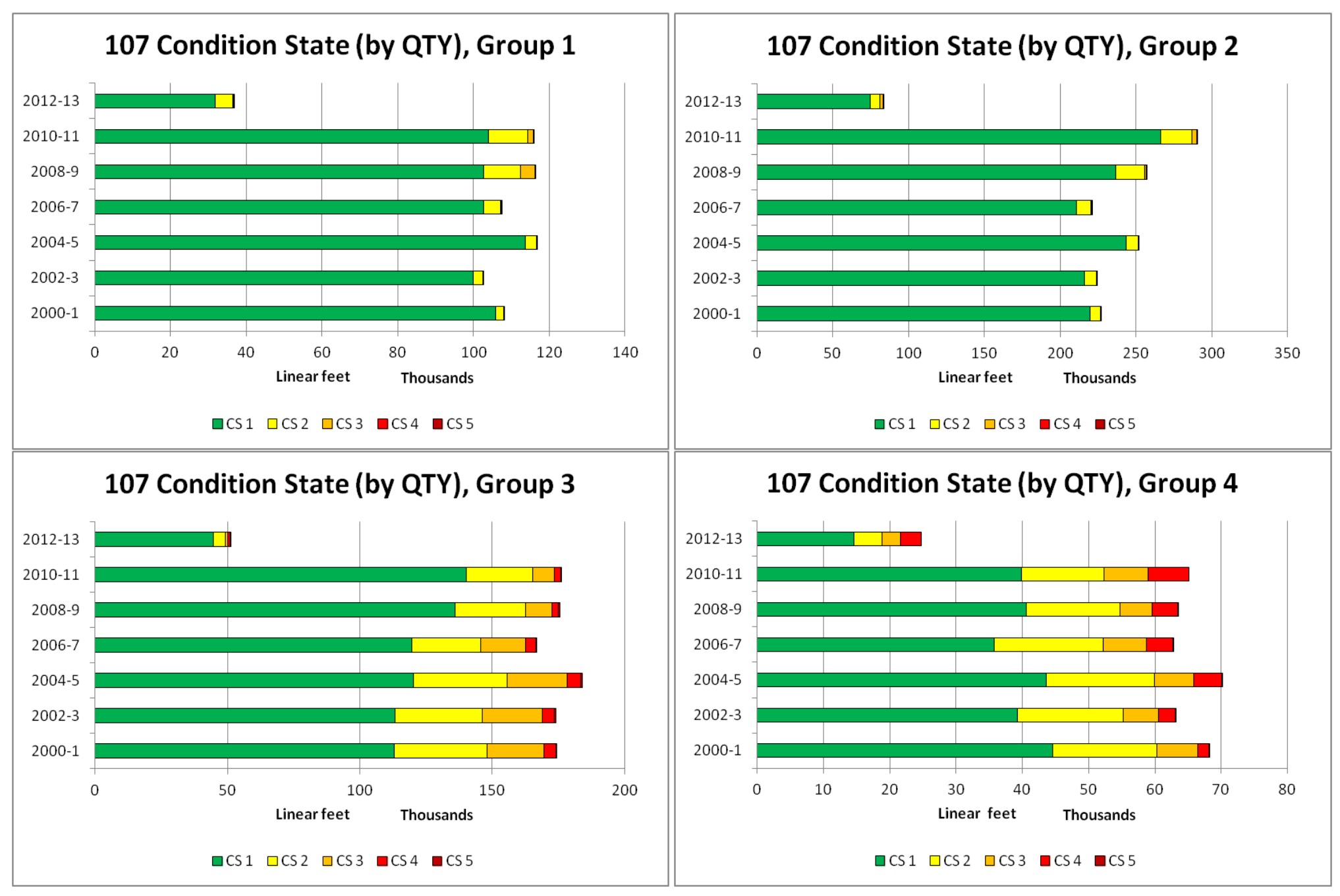

Figure 11 - Zinc Coating - Summary Graphs of Quantity in Each Condition State by Year - Two-Year Grouping 


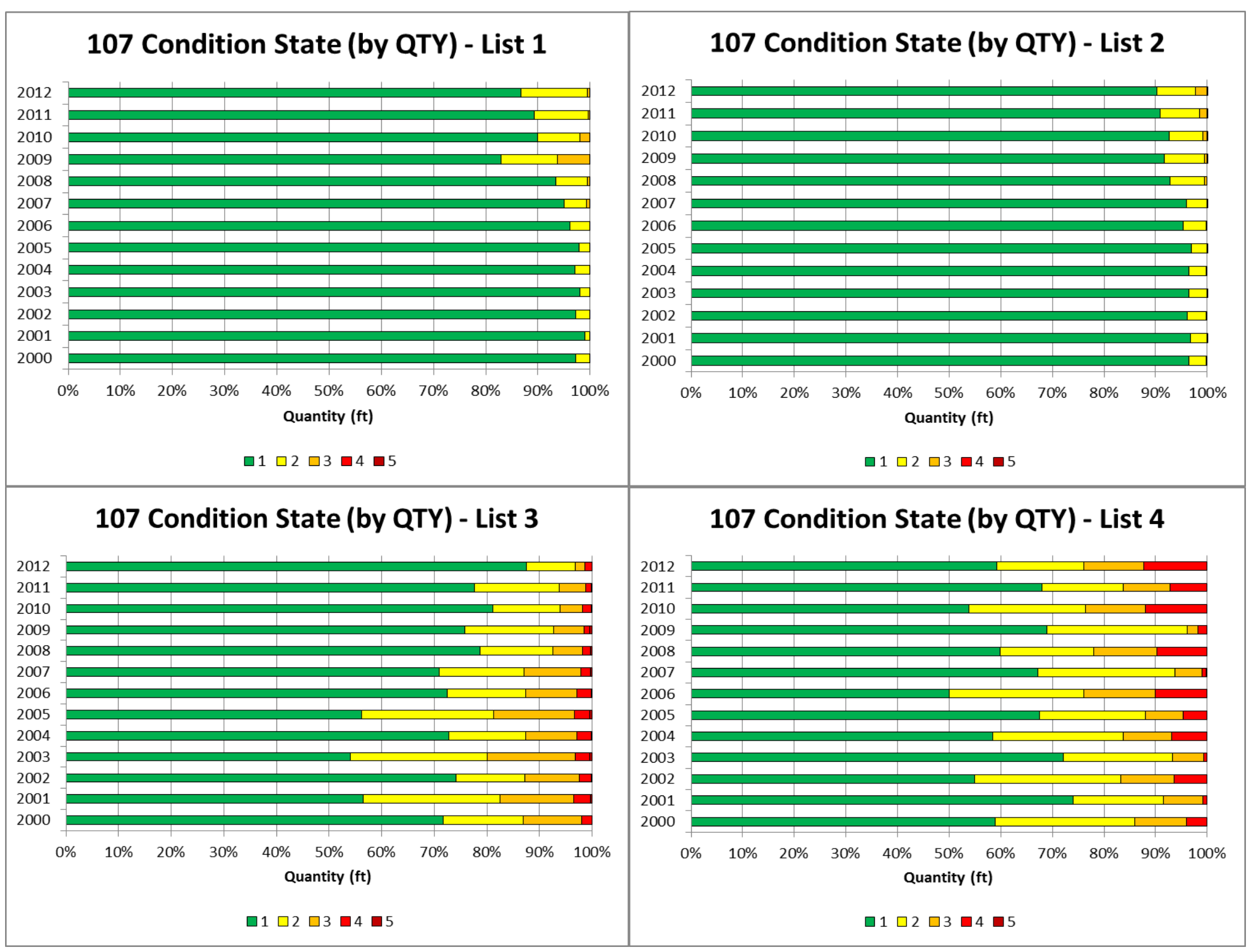

Figure 12 - Zinc Coating - Normalized Summary Graphs of Proportion of Inventory in Each Condition State Range by Year (Quantity Analysis) 


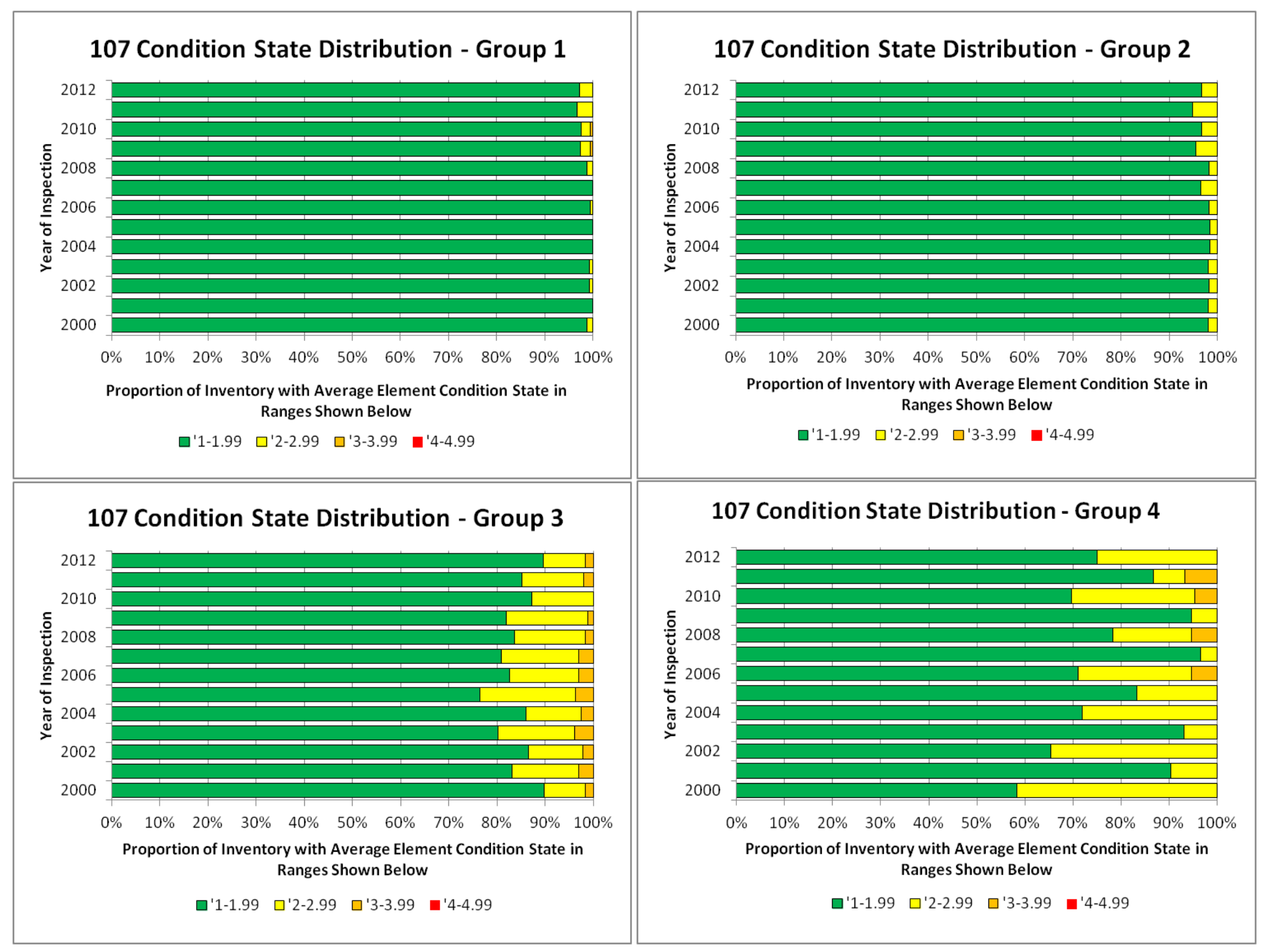

Figure 13 - Zinc Coating - Normalized Summary Graphs of Proportion of Inventory in Each Condition State Range by Year 


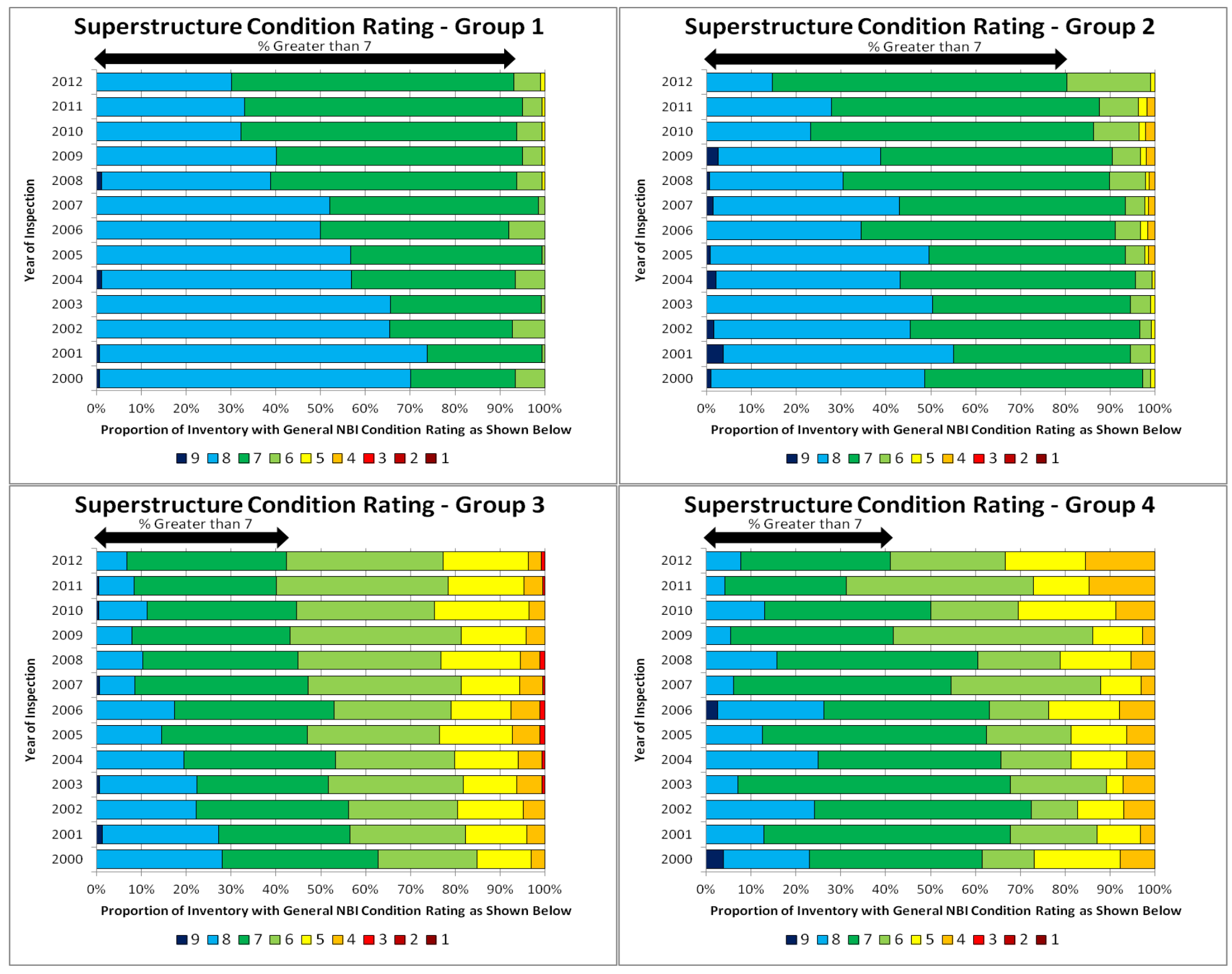

Figure 14 - Zinc Coating - Normalized Summary Graphs of Condition Rating Analysis by Year 


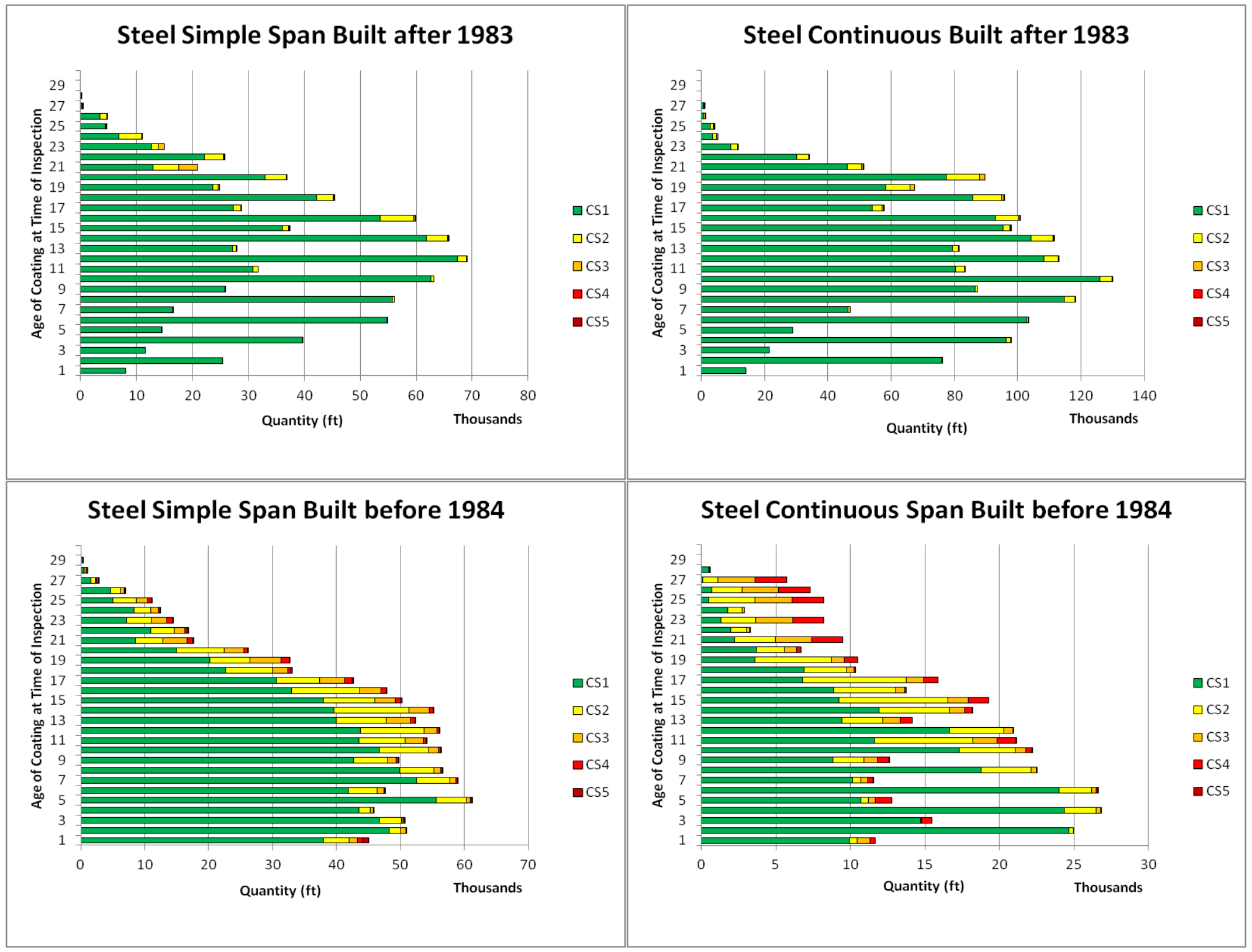

Figure 15 - Zinc Coating - Summary Graphs of Quantity in Each Condition State by Age since Painting 


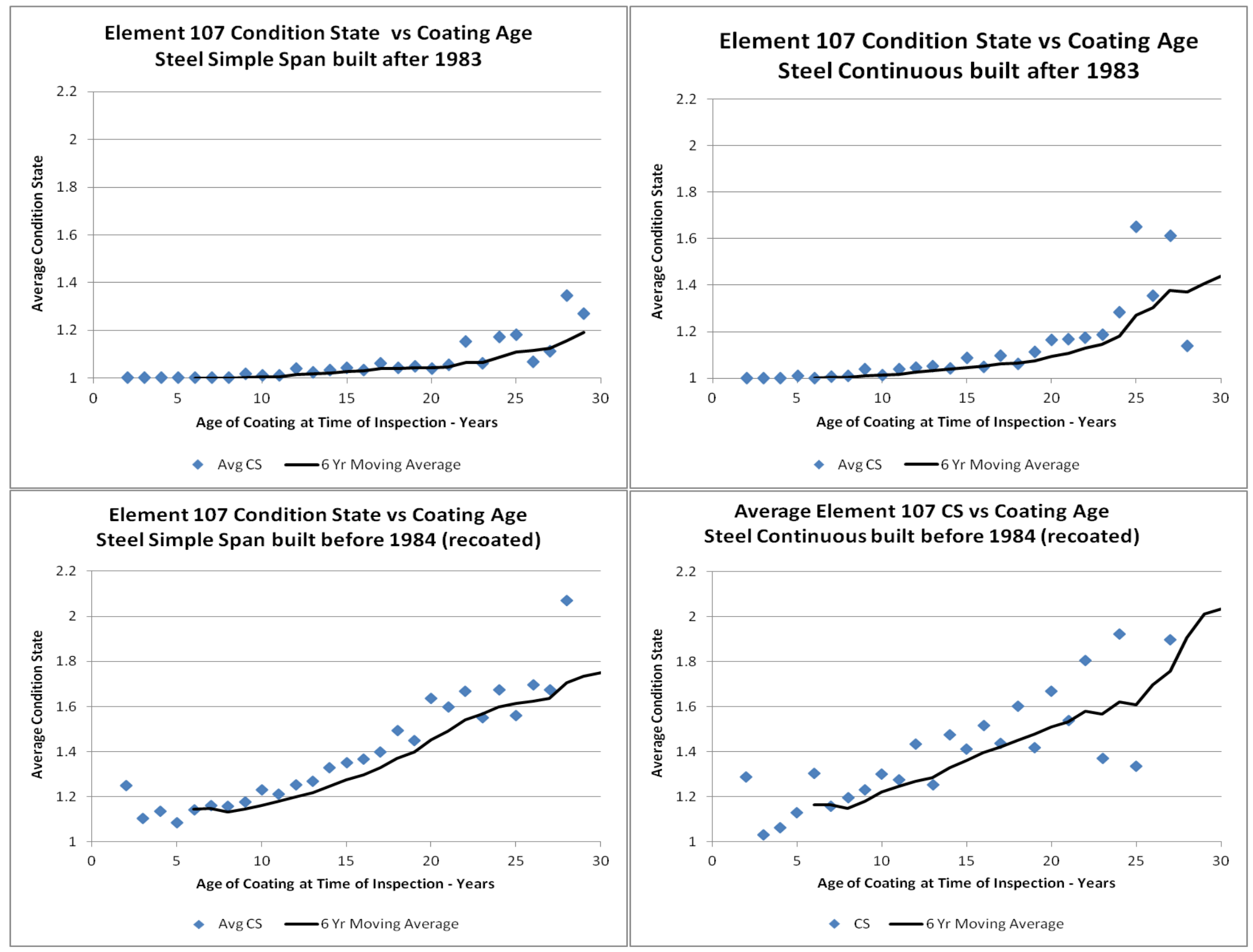

Figure 16 - Zinc Coating - Condition State Trends by Age since Painting 


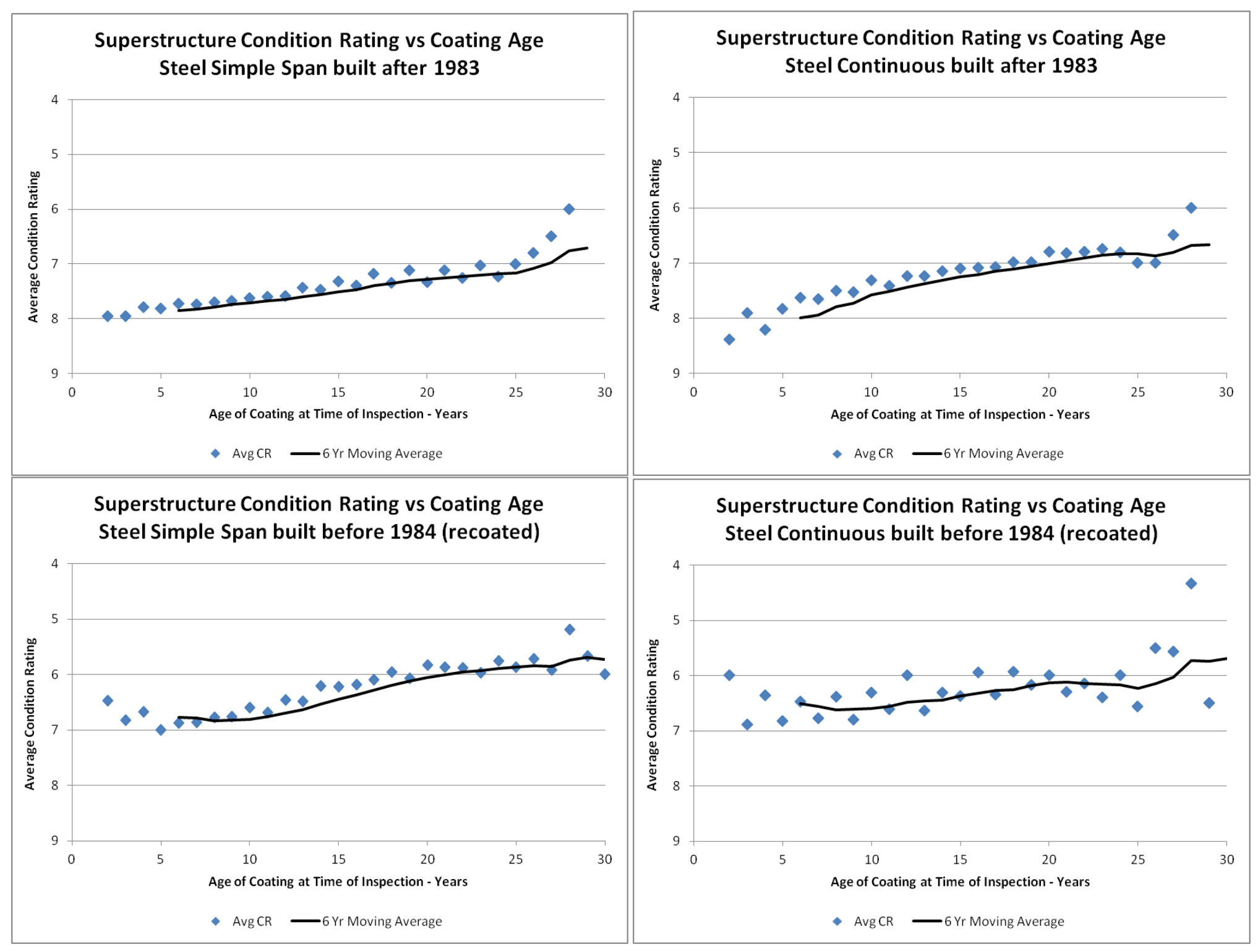

Figure 17 - Zinc Coating - Condition Rating Trends by Age since Painting 


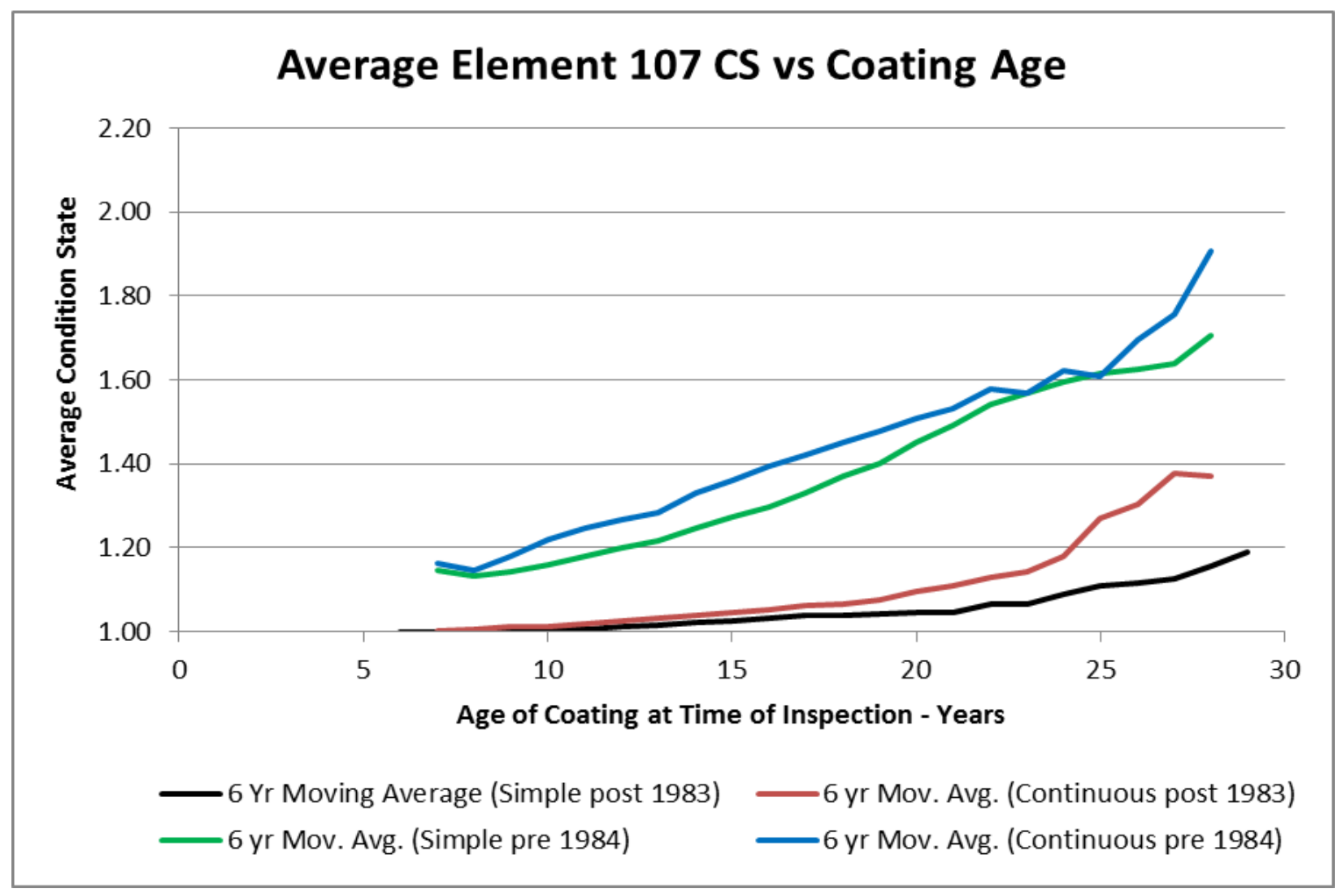

Figure 18 - Zinc Coating - Summary Trends for Condition State Deterioration

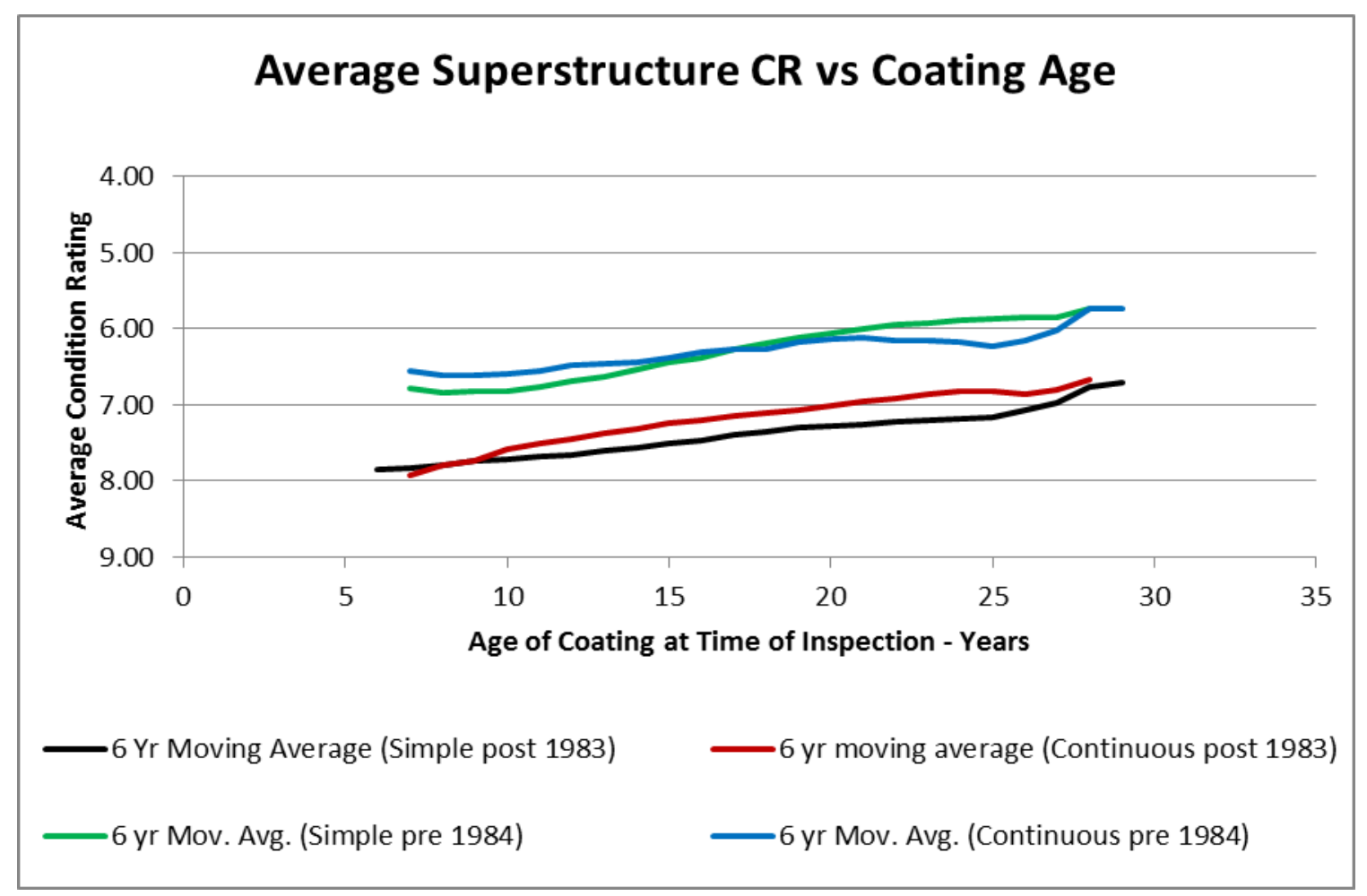

Figure 19 - Zinc Coating - Summary Trends for Condition Rating Deterioration 
Table 35 shows the summary statistics for the average year the bridges in the zinc analysis were built and painted and the corresponding age of the bridge and the paint systems as of 2012 . The different fields are divided by row into the bridges in each of the 4 groups.

\begin{tabular}{|c|c|c|c|c|}
\hline & \multicolumn{2}{|c|}{ Average Year Bridge Was: } & \multicolumn{2}{c|}{ Average Age (in Years) of: } \\
\hline Group & Built & Painted & Bridge & Paint \\
\hline 1 & 1993 & 1993 & 19 & 19 \\
\hline 2 & 1995 & 1995 & 17 & 17 \\
\hline 3 & 1961 & 1996 & 51 & 16 \\
\hline 4 & 1962 & 1997 & 50 & 15 \\
\hline
\end{tabular}

Table 35 - Average Year Built / Painted and Average Age of Bridge / Paint

The numbers of bridges in each group were: group 1 (326 bridges), group 2 (284 bridges), group 3 (381 bridges) and group 4 ( 82 bridges). Group 4 has significantly fewer bridges than the other groups and this is reflected in higher variation in the descriptive statistics. It is also noted that the number of bridges and the quantity of Element 107 decreases significantly for coating age greater than 20 years. This also is reflected in higher variability in the average condition state.

The deteriorated quantity analysis selected bridges with a non-zero quantity of element 107 in condition states 4 and / or 5 in their most recent inspection report as of September 17, 2012. This set of bridges was already filtered for the zinc study, so they are all VDOTmaintained, active, non-posted, steel beam/girder, concrete deck bridges. The number of deteriorated quantity bridges appearing in each group is as follows: 8 in group 1, 4 in group 2, 90 in group 3, and 19 in group 4, for a total of 121 bridges. The full list of these bridges is available in a separate Excel file through VDOT. 


\section{Joint Closures}

The summary count data from the joint elimination study are shown in Table 36 below. The bridges that had joint quantities entirely eliminated are represented by the "ELIM" row heading. The count of bridges that had joint quantities decrease between $10 \%$ and $99 \%$ are shown in the "DECR" row. The bridges with quantity of one joint element being replaced by an equivalent quantity of another joint element are displayed as "DIFF".

\begin{tabular}{|c|c|c|c|c|c|c|c|c|c|c|}
\hline Joints & \multicolumn{10}{|c|}{ District } \\
\hline Summary & 1 & 2 & 3 & 4 & 5 & 6 & 7 & 8 & 9 & Total \\
\hline ELIM & 98 & 82 & 43 & 154 & 129 & 22 & 32 & 77 & 60 & 697 \\
\hline DECR & 28 & 31 & 10 & 105 & 61 & 8 & 22 & 56 & 49 & 370 \\
\hline DIFF & 55 & 61 & 30 & 105 & 83 & 7 & 27 & 49 & 39 & 456 \\
\hline Total & 181 & 174 & 83 & 364 & 273 & 37 & 81 & 182 & 148 & 1523 \\
\hline
\end{tabular}

Table 36 - Joint Closure Count Summary

The full list of bridges showing joint deterioration is provided in a separate Excel file through VDOT. Each row contains the federal bridge ID, the year the quantity decreased, and which of the three elimination options occurred on each bridge. 


\section{Priority Bridges and District Visits}

The summary of the priority bridges is shown in Table 37 below. The top section of the table shows the number of bridges containing unexpected improvements to the element listed at left within the district listed at the top. These are summed both by district and by element, with the element descriptions at right. The bottom portion of the table is arranged similarly, showing bridge counts for alternate, unused classifications of four of the selected elements. Elements 107 and 108 have the bridges added back in where, based on the inspection report data, painting is the reported (and therefore "known") improvement. Elements 301 and 302 in the bottom section use the stricter $>1.00$ condition state decrease criteria that the other elements use (while elements 301 and 302 in the top section use the $>0.05$ condition state decrease criteria).

\begin{tabular}{|c|c|c|c|c|c|c|c|c|c|c|l|}
\hline & \multicolumn{7}{|c|}{ District Number } & & \\
\hline Element Number & 1 & 2 & 3 & 4 & 5 & 6 & 7 & 8 & 9 & Total & Element Description \\
\hline 107 & 44 & 21 & 4 & 24 & 4 & 2 & 19 & 21 & 3 & 142 & Steel Open Girder - Coated \\
\hline 108 & 81 & 64 & 17 & 15 & 10 & 2 & 9 & 19 & 13 & 230 & V Steel Open Girder with Timber Deck - Coated and Uncoated \\
\hline 311 & 7 & 5 & 1 & 12 & 0 & 1 & 0 & 2 & 2 & 30 & Moveable Bearing (Roller, sliding, etc.) \\
\hline 313 & 5 & 1 & 1 & 15 & 0 & 0 & 0 & 1 & 1 & 24 & Fixed Bearing \\
\hline 301 & 55 & 88 & 3 & 349 & 88 & 30 & 41 & 13 & 63 & 730 & Pourable Joint Seal \\
\hline 302 & 97 & 139 & 139 & 116 & 105 & 22 & 40 & 157 & 110 & 925 & Compression Joint Seal \\
\hline Total to Select From & 289 & 318 & 165 & 531 & 207 & 57 & 109 & 213 & 192 & 2081 & \\
\hline 107 w/ Paint & 44 & 69 & 17 & 47 & 7 & 10 & 41 & 96 & 6 & 337 & Steel Open Girder - Coated \\
\hline 108 w/ Paint & 127 & 189 & 34 & 23 & 21 & 3 & 33 & 38 & 16 & 484 & V Steel Open Girder with Timber Deck - Coated and Uncoated \\
\hline $301>1$ & 23 & 43 & 2 & 131 & 16 & 10 & 8 & 5 & 17 & 255 & Pourable Joint Seal \\
\hline $302>1$ & 28 & 33 & 60 & 27 & 14 & 4 & 9 & 41 & 9 & 225 & Compression Joint Seal \\
\hline
\end{tabular}

Table 37 - Number of Unexpected Improvements by District for Selected Element 
The final list of maintenance actions (and acronyms used) was developed over the course of the study as data became available and is shown in Appendix C as Table C-25, reproduced below as Table 38. "No Maintenance Noted" refers to situations where there is no recorded work done over a period where the paper reports agree with the Pontis data showing an improvement for the given bridge and element. If there is no agreement (the paper report does not show the expected improvement), that entry is marked as "Typographical Error", which can then likely be attributed to a mistake at some stage of inspection report data entry or Excel analysis. The remaining actions refer to different types of work done (often specific to an element or category of elements) that were noted in a given inspection report as having occurred since the previous inspection report.

\begin{tabular}{|c|c|}
\hline Code & Corresponding Maintenance Action \\
\hline CP & Cleaned / Painted Structural Steel \\
\hline SR & Superstructure Replaced \\
\hline RB & Replaced Some Beams / Girders \\
\hline DJ & Deck Joints Resealed \\
\hline BR & Bearings Replaced \\
\hline BP & Bearings Painted \\
\hline AQ & Added Quantity (in CS 1) \\
\hline RR & Road Repaved \\
\hline NM & No Maintenance Noted \\
\hline TE & Typographical Error \\
\hline ND & No Data \\
\hline OT & Other \\
\hline
\end{tabular}

Table 38 - Maintenance Action Codes and Descriptions - Reproduced from Table C-25

The final products of the district visits are shown in summary tables by element and road classification, displaying the relative percentage of number of times each maintenance action is taken on each set of bridges. These tables are presented in their entirety in Appendix $\mathrm{C}$ as Table C-1 through Table C-24, and a subset for element 107 is shown as Table 39 through Table 42. 
Each table is divided into the 9 districts and the entire state by column. The top data row displays the count of bridges containing the desired element on the specified road classification, followed by the average and total quantity of the given element per bridge within each district. The remaining rows display the percent, in each district, of the selected bridges where the cause of improvement was due to the maintenance action coded in the leftmost column (defined in Table 38). The "Total" column shows the combined maintenance values divided into the state total count, as opposed to simply averaging the district values.

The completed inspection tables from the district visits, such as Table 15, are available in a separate Excel file through VDOT. 


\begin{tabular}{|c|c|c|c|c|c|c|c|c|c|c|}
\hline Total & \multicolumn{10}{|c|}{ District } \\
\hline Element 107 & 1 & 2 & 3 & 4 & 5 & 6 & 7 & 8 & 9 & Total \\
\hline Count & 6 & 12 & 4 & 15 & 5 & 3 & 8 & 14 & 3 & 70 \\
\hline Avg. Quant. & 338.26 & 407.83 & 245.43 & 912.99 & 624.76 & 526.31 & 497.64 & 339.62 & 297.33 & 513.29 \\
\hline Tot. Quant. & 2029.56 & 4893.94 & 981.73 & 13694.82 & 3123.80 & 1578.94 & 3981.11 & 4754.63 & 892.00 & 35930.52 \\
\hline$C P$ & $50.0 \%$ & $66.7 \%$ & $0.0 \%$ & $40.0 \%$ & $20.0 \%$ & $66.7 \%$ & $75.0 \%$ & $71.4 \%$ & $0.0 \%$ & $51.4 \%$ \\
\hline$S R$ & $50.0 \%$ & $0.0 \%$ & $25.0 \%$ & $20.0 \%$ & $0.0 \%$ & $0.0 \%$ & $0.0 \%$ & $0.0 \%$ & $0.0 \%$ & $10.0 \%$ \\
\hline$R B$ & $0.0 \%$ & $0.0 \%$ & $0.0 \%$ & $6.7 \%$ & $0.0 \%$ & $0.0 \%$ & $0.0 \%$ & $0.0 \%$ & $0.0 \%$ & $1.4 \%$ \\
\hline$A Q$ & $0.0 \%$ & $0.0 \%$ & $0.0 \%$ & $0.0 \%$ & $0.0 \%$ & $0.0 \%$ & $0.0 \%$ & $0.0 \%$ & $0.0 \%$ & $0.0 \%$ \\
\hline NM & $0.0 \%$ & $16.7 \%$ & $75.0 \%$ & $33.3 \%$ & $80.0 \%$ & $33.3 \%$ & $25.0 \%$ & $28.6 \%$ & $100.0 \%$ & $34.3 \%$ \\
\hline$T E$ & $0.0 \%$ & $16.7 \%$ & $0.0 \%$ & $0.0 \%$ & $0.0 \%$ & $0.0 \%$ & $0.0 \%$ & $0.0 \%$ & $0.0 \%$ & $2.9 \%$ \\
\hline$O T$ & $0.0 \%$ & $0.0 \%$ & $0.0 \%$ & $0.0 \%$ & $0.0 \%$ & $0.0 \%$ & $0.0 \%$ & $0.0 \%$ & $0.0 \%$ & $0.0 \%$ \\
\hline
\end{tabular}

Table 39 - District Visit Maintenance Summary - Element 107 - All Road Types - Reproduced from Table C-1

\begin{tabular}{|c|c|c|c|c|c|c|c|c|c|c|}
\hline Interstate & \multicolumn{10}{|c|}{ District } \\
\hline Element 107 & 1 & 2 & 3 & 4 & 5 & 6 & 7 & 8 & 9 & Total \\
\hline Count & 1 & 3 & 0 & 4 & 3 & 0 & 5 & 7 & 0 & 23 \\
\hline Avg. Quant. & 506.94 & 443.98 & N/A & 974.14 & 305.93 & N/A & 483.86 & 291.27 & N/A & 483.10 \\
\hline Tot. Quant. & 506.94 & 1331.93 & 0.00 & 3896.55 & 917.80 & 0.00 & 2419.31 & 2038.89 & 0.00 & 11111.41 \\
\hline$C P$ & $100.0 \%$ & $100.0 \%$ & N/A & $25.0 \%$ & $0.0 \%$ & N/A & $80.0 \%$ & $57.1 \%$ & N/A & $56.5 \%$ \\
\hline$S R$ & $0.0 \%$ & $0.0 \%$ & N/A & $50.0 \%$ & $0.0 \%$ & N/A & $0.0 \%$ & $0.0 \%$ & N/A & $8.7 \%$ \\
\hline$R B$ & $0.0 \%$ & $0.0 \%$ & N/A & $0.0 \%$ & $0.0 \%$ & N/A & $0.0 \%$ & $0.0 \%$ & N/A & $0.0 \%$ \\
\hline$A Q$ & $0.0 \%$ & $0.0 \%$ & N/A & $0.0 \%$ & $0.0 \%$ & N/A & $0.0 \%$ & $0.0 \%$ & N/A & $0.0 \%$ \\
\hline NM & $0.0 \%$ & $0.0 \%$ & N/A & $25.0 \%$ & $100.0 \%$ & N/A & $20.0 \%$ & $42.9 \%$ & N/A & $34.8 \%$ \\
\hline$T E$ & $0.0 \%$ & $0.0 \%$ & N/A & $0.0 \%$ & $0.0 \%$ & N/A & $0.0 \%$ & $0.0 \%$ & N/A & $0.0 \%$ \\
\hline$O T$ & $0.0 \%$ & $0.0 \%$ & N/A & $0.0 \%$ & $0.0 \%$ & N/A & $0.0 \%$ & $0.0 \%$ & N/A & $0.0 \%$ \\
\hline
\end{tabular}

Table 40 - District Visit Maintenance Summary - Element 107 - Interstate Roads - Reproduced from Table C-2 


\begin{tabular}{|c|c|c|c|c|c|c|c|c|c|c|}
\hline Primary & \multicolumn{10}{|c|}{ District } \\
\hline Element 107 & 1 & 2 & 3 & 4 & 5 & 6 & 7 & 8 & 9 & Total \\
\hline Count & 3 & 2 & 1 & 10 & 2 & 2 & 0 & 1 & 3 & 24 \\
\hline Avg. Quant. & 274.38 & 415.21 & 332.00 & 947.72 & 1103.00 & 633.04 & $\mathrm{~N} / \mathrm{A}$ & 495.95 & 297.33 & 680.12 \\
\hline Tot. Quant. & 823.14 & 830.42 & 332.00 & 9477.19 & 2206.00 & 1266.08 & 0.00 & 495.95 & 892.00 & 16322.77 \\
\hline$C P$ & $66.7 \%$ & $100.0 \%$ & $0.0 \%$ & $50.0 \%$ & $50.0 \%$ & $50.0 \%$ & $\mathrm{~N} / \mathrm{A}$ & $0.0 \%$ & $0.0 \%$ & $45.8 \%$ \\
\hline$S R$ & $33.3 \%$ & $0.0 \%$ & $0.0 \%$ & $10.0 \%$ & $0.0 \%$ & $0.0 \%$ & $\mathrm{~N} / \mathrm{A}$ & $0.0 \%$ & $0.0 \%$ & $8.3 \%$ \\
\hline$R B$ & $0.0 \%$ & $0.0 \%$ & $0.0 \%$ & $0.0 \%$ & $0.0 \%$ & $0.0 \%$ & $\mathrm{~N} / \mathrm{A}$ & $0.0 \%$ & $0.0 \%$ & $0.0 \%$ \\
\hline$A Q$ & $0.0 \%$ & $0.0 \%$ & $0.0 \%$ & $0.0 \%$ & $0.0 \%$ & $0.0 \%$ & $\mathrm{~N} / \mathrm{A}$ & $0.0 \%$ & $0.0 \%$ & $0.0 \%$ \\
\hline$N M$ & $0.0 \%$ & $0.0 \%$ & $100.0 \%$ & $40.0 \%$ & $50.0 \%$ & $50.0 \%$ & $\mathrm{~N} / \mathrm{A}$ & $100.0 \%$ & $100.0 \%$ & $45.8 \%$ \\
\hline$T E$ & $0.0 \%$ & $0.0 \%$ & $0.0 \%$ & $0.0 \%$ & $0.0 \%$ & $0.0 \%$ & $\mathrm{~N} / \mathrm{A}$ & $0.0 \%$ & $0.0 \%$ & $0.0 \%$ \\
\hline$O T$ & $0.0 \%$ & $0.0 \%$ & $0.0 \%$ & $0.0 \%$ & $0.0 \%$ & $0.0 \%$ & $\mathrm{~N} / \mathrm{A}$ & $0.0 \%$ & $0.0 \%$ & $0.0 \%$ \\
\hline
\end{tabular}

Table 41 - District Visit Maintenance Summary - Element 107 - Primary Roads - Reproduced from Table C-3

\begin{tabular}{|c|c|c|c|c|c|c|c|c|c|c|}
\hline Secondary & \multicolumn{10}{|c|}{ District } \\
\hline Element 107 & 1 & 2 & 3 & 4 & 5 & 6 & 7 & 8 & 9 & Total \\
\hline Count & 2 & 7 & 3 & 1 & 0 & 1 & 3 & 6 & 0 & 23 \\
\hline Avg. Quant. & 349.74 & 390.23 & 216.58 & 321.09 & N/A & 312.86 & 520.60 & 369.96 & N/A & 369.41 \\
\hline Tot. Quant. & 699.48 & 2731.60 & 649.73 & 321.09 & 0.00 & 312.86 & 1561.80 & 2219.79 & 0.00 & 8496.34 \\
\hline$C P$ & $0.0 \%$ & $42.9 \%$ & $0.0 \%$ & $0.0 \%$ & N/A & $100.0 \%$ & $66.7 \%$ & $100.0 \%$ & N/A & $52.2 \%$ \\
\hline$S R$ & $100.0 \%$ & $0.0 \%$ & $33.3 \%$ & $0.0 \%$ & N/A & $0.0 \%$ & $0.0 \%$ & $0.0 \%$ & N/A & $13.0 \%$ \\
\hline$R B$ & $0.0 \%$ & $0.0 \%$ & $0.0 \%$ & $100.0 \%$ & N/A & $0.0 \%$ & $0.0 \%$ & $0.0 \%$ & N/A & $4.3 \%$ \\
\hline$A Q$ & $0.0 \%$ & $0.0 \%$ & $0.0 \%$ & $0.0 \%$ & N/A & $0.0 \%$ & $0.0 \%$ & $0.0 \%$ & N/A & $0.0 \%$ \\
\hline$N M$ & $0.0 \%$ & $28.6 \%$ & $66.7 \%$ & $0.0 \%$ & N/A & $0.0 \%$ & $33.3 \%$ & $0.0 \%$ & N/A & $21.7 \%$ \\
\hline$T E$ & $0.0 \%$ & $28.6 \%$ & $0.0 \%$ & $0.0 \%$ & N/A & $0.0 \%$ & $0.0 \%$ & $0.0 \%$ & N/A & $8.7 \%$ \\
\hline$O T$ & $0.0 \%$ & $0.0 \%$ & $0.0 \%$ & $0.0 \%$ & N/A & $0.0 \%$ & $0.0 \%$ & $0.0 \%$ & N/A & $0.0 \%$ \\
\hline
\end{tabular}

Table 42 - District Visit Maintenance Summary - Element 107 - Secondary Roads - Reproduced from Table C-4 


\section{Linear Least Squares and Markov Modeling}

The parameters for the LLS deterioration models are displayed in Table 43 for each of the 6 top elements in each of the 9 districts and then averaged across the state. Each line is completely described using its slope and the condition state of the bridge in 2010, which is more meaningful than the y-intercept. This intercept would have been the theoretical condition state at year 0 AD.

The averages are equally weighted means of the 9 districts' values (the number of bridges per district is not taken into account here).

\begin{tabular}{|c|c|c|c|c|c|c|c|c|c|c|c|}
\hline & & \multicolumn{10}{|c|}{ District } \\
\hline Element & & 1 & 2 & 3 & 4 & 5 & 6 & 7 & 8 & 9 & Average \\
\hline \multirow[b]{2}{*}{107} & Slope & 0.0048 & 0.0049 & 0.0033 & 0.0044 & 0.0037 & 0.0045 & 0.0041 & 0.0042 & 0.0032 & 0.0041 \\
\hline & 2010 CS & 1.6841 & 1.8459 & 1.2790 & 1.8292 & 1.4903 & 1.7414 & 1.7100 & 1.7602 & 1.2716 & 1.6235 \\
\hline \multirow[b]{2}{*}{108} & Slope & 0.0092 & 0.0079 & 0.0060 & 0.0059 & 0.0039 & 0.0085 & 0.0061 & 0.0074 & 0.0065 & 0.0068 \\
\hline & 2010 CS & 3.1021 & 3.0124 & 2.2572 & 2.4014 & 1.5889 & 3.0651 & 2.5003 & 2.6439 & 2.7920 & 2.5959 \\
\hline \multirow[b]{2}{*}{301} & Slope & -0.0046 & 0.0161 & -0.0299 & 0.0227 & 0.0130 & 0.0073 & 0.0244 & 0.0179 & 0.0267 & 0.0104 \\
\hline & 2010 CS & 1.8203 & 1.7749 & 1.5566 & 1.8794 & 1.2918 & 2.0160 & 1.8706 & 1.8585 & 1.6366 & 1.7450 \\
\hline \multirow[b]{2}{*}{302} & Slope & 0.0169 & 0.0157 & 0.0104 & 0.0273 & 0.0232 & 0.0220 & 0.0348 & 0.0146 & 0.0201 & 0.0206 \\
\hline & 2010 CS & 1.4129 & 1.4857 & 1.4333 & 1.5438 & 1.5645 & 1.8874 & 1.8271 & 1.4289 & 1.3998 & 1.5537 \\
\hline \multirow[b]{2}{*}{311} & Slope & 0.0101 & 0.0048 & 0.0033 & 0.0239 & 0.0153 & 0.0085 & 0.0131 & 0.0038 & 0.0213 & 0.0116 \\
\hline & 2010 CS & 1.5250 & 1.5890 & 1.4321 & 1.6586 & 1.5773 & 1.6789 & 1.5986 & 1.4444 & 1.3938 & 1.5442 \\
\hline \multirow[b]{2}{*}{313} & Slope & 0.0032 & 0.0000 & 0.0064 & 0.0127 & 0.0126 & 0.0020 & 0.0061 & 0.0011 & 0.0117 & 0.0062 \\
\hline & 2010 CS & 1.3929 & 1.3945 & 1.2100 & 1.4923 & 1.5204 & 1.5018 & 1.4747 & 1.3827 & 1.2114 & 1.3978 \\
\hline
\end{tabular}

Table 43 - Linear Least Squares Summary for Top Elements by District 
The condition state history by age linear regression analysis produced graphs such as Figure 20 below. The data points are shown in blue for each of the years 0 to 100 where there was at least on bridge reporting a condition state. The sloping black line through the data is the trendline produced by Excel's linear least squares best fit calculations. Near that line is the equation in $\mathrm{y}=\mathrm{mx}+\mathrm{b}$ form, where " $\mathrm{m}$ " represents the slope of average deterioration and " $\mathrm{b}$ " represents the modeled condition state at 0 years of age. The complete set of these graphs can be found at the beginning of Appendix D in this report.

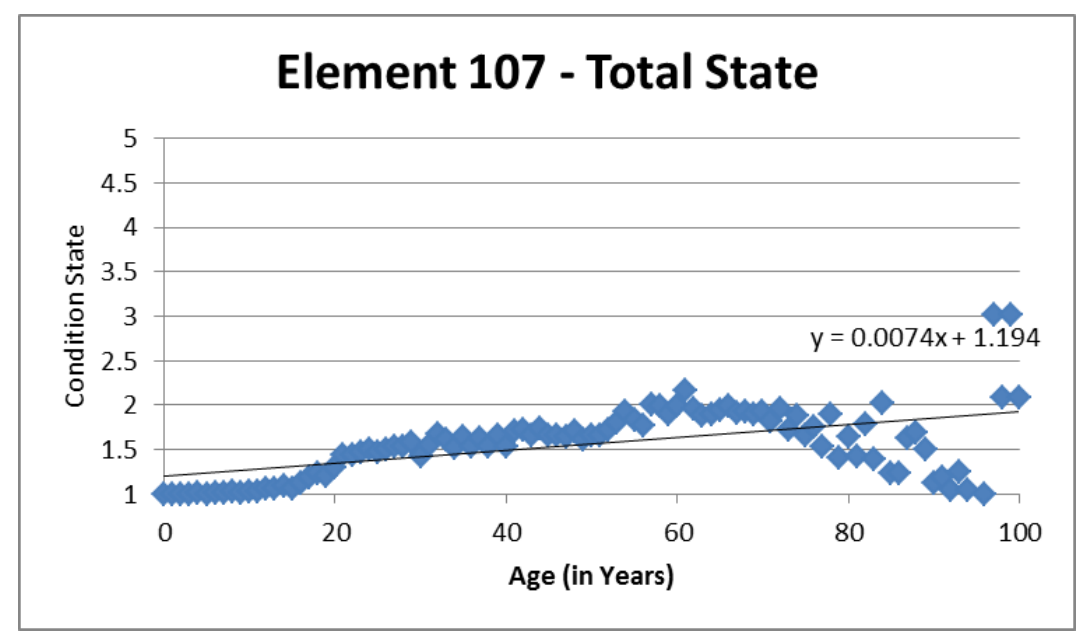

Figure 20 - Condition State History and Linear Trendline by Age - Element 107 - Total State - Reproduced from Figure D-28

The second set of graphs from the linear least squares regression analysis based on the age of bridges displays the residuals from subtracting the actual reported condition state from the condition state calculated from the linear trendline equation for the same age. These values are shown over each year of the 100 year time frame that at least one bridge reported condition state data for the given element in the given district / total state. Figure 21 below shows this set of data for the total state for element 107; the district specific graphs and those for element 108 are presented at the beginning of Appendix D in this report. 


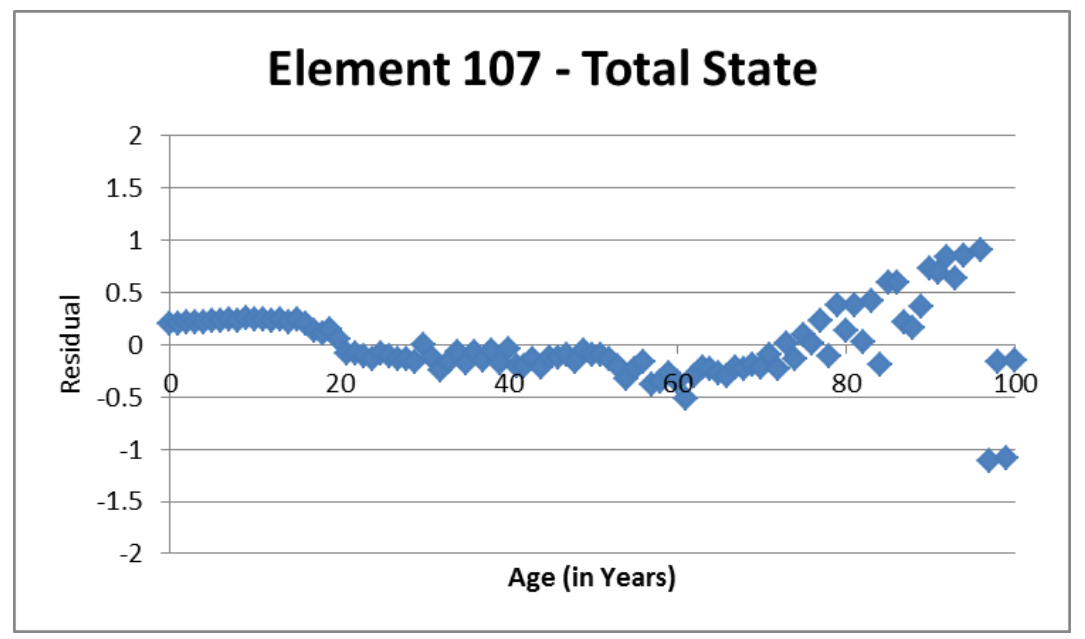

Figure 21 - Condition States Residuals (Prediction Minus Actual) by Age - Element 107 - Total State Reproduced from Figure D-29

The numbers of bridges contributing condition state data for each district (and total state) for each age are represented in graphs such as Figure 22 below. The complete set of these graphs for element 107 and element 108 in each district are presented at the beginning of Appendix D of this report. Note that the vertical scale for each element is consistent between districts in order to better appreciate the relative number of bridges reporting for each element from different regions around the state; the "total state" graphs have necessarily higher scales.

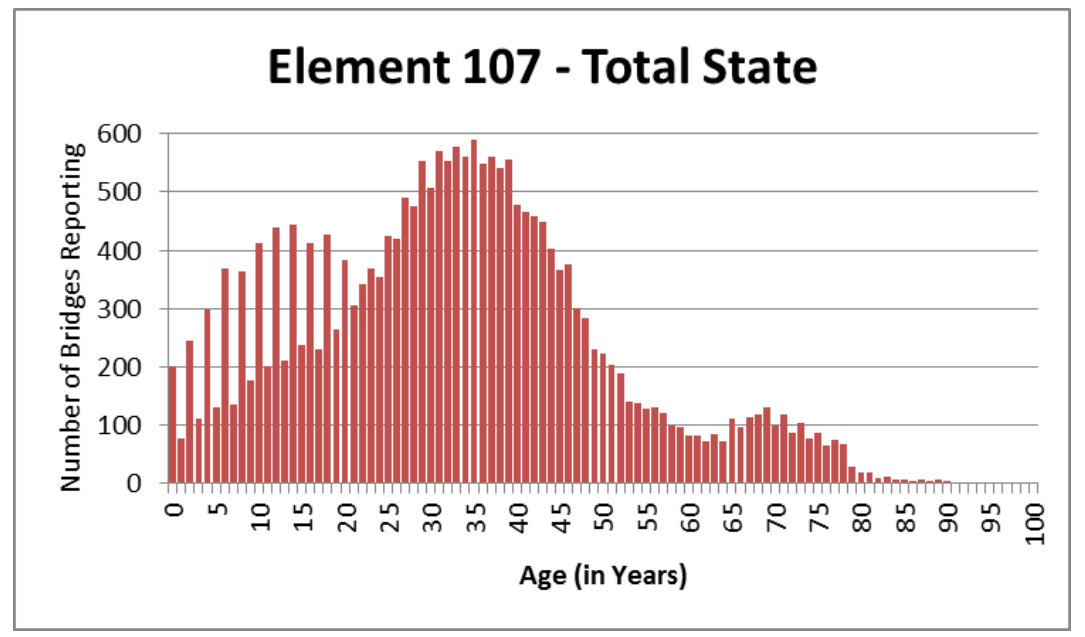

Figure 22 - Number of Bridges Reporting by Age - Element 107 - Total State - Reproduced from Figure D-30 
The transition probability matrices from the Markov chain modeling are presented in their entirety in Appendix D as Table D-1 through Table D-19, 4 of which are reproduced below as Table 44 and Table 45, for element 107 in Districts 1 through 4. The left column represents the condition state the quantity of the given element is in at the beginning of the time step (a year in this report), and each column heading represents the potential condition states that element can end the year in. For this project, an element only had the option of remaining in its current condition state or deteriorating to the next worst condition state.

\begin{tabular}{|c|c|c|c|c|c|}
\hline D1 & 1 & 2 & 3 & 4 & 5 \\
\hline 1 & 0.990 & 0.010 & 0.000 & 0.000 & 0.000 \\
\hline 2 & 0.000 & 0.972 & 0.028 & 0.000 & 0.000 \\
\hline 3 & 0.000 & 0.000 & 0.976 & 0.024 & 0.000 \\
\hline 4 & 0.000 & 0.000 & 0.000 & 0.961 & 0.039 \\
\hline 5 & 0.000 & 0.000 & 0.000 & 0.000 & 1.000 \\
\hline
\end{tabular}

\begin{tabular}{|c|c|c|c|c|c|}
\hline D2 & 1 & 2 & 3 & 4 & 5 \\
\hline 1 & 0.982 & 0.018 & 0.000 & 0.000 & 0.000 \\
\hline 2 & 0.000 & 0.975 & 0.025 & 0.000 & 0.000 \\
\hline 3 & 0.000 & 0.000 & 0.993 & 0.007 & 0.000 \\
\hline 4 & 0.000 & 0.000 & 0.000 & 0.993 & 0.007 \\
\hline 5 & 0.000 & 0.000 & 0.000 & 0.000 & 1.000 \\
\hline
\end{tabular}

Table 44 - Markov Transition Probability Matrices - Element 107 - Districts 1 and 2 - Reproduced from Table D-1

\begin{tabular}{|c|c|c|c|c|c|}
\hline D3 & 1 & 2 & 3 & 4 & 5 \\
\hline 1 & 0.994 & 0.006 & 0.000 & 0.000 & 0.000 \\
\hline 2 & 0.000 & 0.977 & 0.023 & 0.000 & 0.000 \\
\hline 3 & 0.000 & 0.000 & 0.979 & 0.021 & 0.000 \\
\hline 4 & 0.000 & 0.000 & 0.000 & 0.992 & 0.008 \\
\hline 5 & 0.000 & 0.000 & 0.000 & 0.000 & 1.000 \\
\hline
\end{tabular}

\begin{tabular}{|c|c|c|c|c|c|}
\hline D4 & 1 & 2 & 3 & 4 & 5 \\
\hline 1 & 0.987 & 0.013 & 0.000 & 0.000 & 0.000 \\
\hline 2 & 0.000 & 0.972 & 0.028 & 0.000 & 0.000 \\
\hline 3 & 0.000 & 0.000 & 0.977 & 0.023 & 0.000 \\
\hline 4 & 0.000 & 0.000 & 0.000 & 0.985 & 0.015 \\
\hline 5 & 0.000 & 0.000 & 0.000 & 0.000 & 1.000 \\
\hline
\end{tabular}

Table 45 - Markov Transition Probability Matrices - Element 107 - Districts 3 and 4 - Reproduced from Table D-2

These modeled deterioration tables and corresponding actual data deterioration tables are also shown in graphical form in their entirety in Appendix D as Figure D-61 through Figure D-79, 2 of which are reproduced as Figure 23. The graphs are color coded such that, for elements 107 and 108, green represents the percentage (by quantity) of the given element in the 
selected district that has a condition state of 1 , yellow represents 2 , orange represents 3 , bright red represents 4 , and dark red represents the ultimate deterioration of condition state 5 . For element 32 , which only has 4 coded condition states, green is 1 , yellow is 2 , orange is 3 , and dark red is 4 . For element 32 , which only has 3 coded condition states, green is 1 , orange is 2 , and dark red is 3. These slightly varying color selections are in an attempt to display similar levels of deterioration (on different elements) in the same colors. 


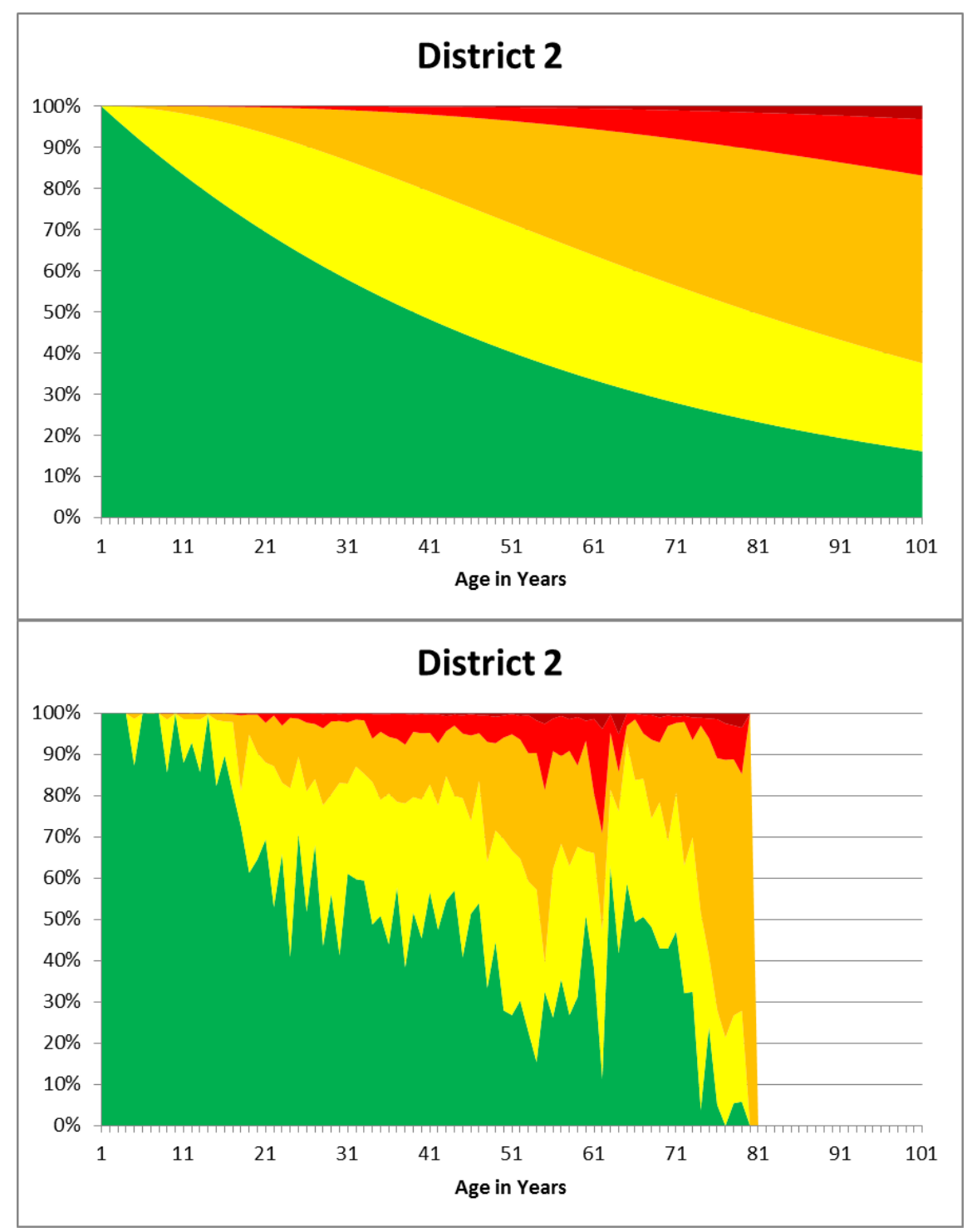

Figure 23 - Markov Chain Prediction Graphs - Element 107 - District 2 Prediction vs. Actual Graphs Reproduced from Figure D-62

Element 108, which is a girder under a timber deck, displays a noticeably more rapid deterioration rate than element 107, which is a girder under a concrete deck. From Table D-5 and Table D-10, we can see that for the total state, element 108's chances at staying in condition states $1,2,3$, and 4 between consecutive years are $0.990,0.980,0.977$, and 0.986 , respectively, whereas the associated element 107 values are $0.975,0.964,0.980$, and 0.983 . The result is a 
fairly large difference in projected deterioration over 100 years, due almost entirely to the first two transitions, as seen in the Total State graphs in Figure D-61 and Figure D-66.

It can be seen that certain elements in certain districts show particularly high levels of deterioration, such as element 302 - District 6 (in Figure D-74). For elements 107 and 108, districts 3 and 5 have slightly lower deterioration rates than the other districts and District 1 has a somewhat greater chance of deteriorating to condition state 5 over time. In general, however, consistent district-specific trends in this section of the analysis between elements were not noted.

Element 32 is shown to be less likely to deteriorate past condition state 2 once it reaches it than the other elements are. This is presented by transition probability matrices with a larger probability in the condition state $2-2$ cell (such as in Table D-17) and with increased thickness of the yellow bands of the graphs (such as District 3 in Figure D-77). 


\begin{abstract}
ANALYSIS
Original Pontis Analysis by District

The data from the first several years of the Pontis database's existence are more likely prone to errors in reporting, inspection, or recording as the system had not become standardized yet and inspectors were becoming familiar with the element-level inspection techniques. Many of the graphs produced using data from the first three years (1995-1997) show seemingly random fluctuations that are inconsistent with the remaining data. These deviations can perhaps be explained by the variance in the styles of early Pontis data collection and reporting before they became more standardized by the turn of the century. Additionally, in the early years of Pontis inspection reporting, only a small percentage of the total bridge population reported data at the element level; this could also contribute to the variation of data in the early years.

As there were too many elements to analyze each one for every district, the selection process based on total number of inspection reports for each element was used to narrow down the field. After the next stage of analysis, the district summary tables by count and improvement could be created (Table 24 through Table 26), showing that the amount of unexpected improvements often did not line up with those elements that simply had more inspection data. It is thus possible that elements that were less numerous and therefore not analyzed in this report could have had many improvements and be worth additional investigation.

Due to the timing of maintenance and inspection in a given year, sometimes condition state decreases occurred in the year the maintenance action was noted, whereas sometimes they do not show up until the next inspection report. The only effect this should have on the data is analysis based on the year of improvement. For example, if a bridge was repaired in 2002 but
\end{abstract}


the next inspection and associated condition state decrease wasn't until 2004, this analysis would show the improvement occurring in 2004.

However, sometimes a bridge was inspected, repaired, and then re-inspected in the same year. While the methods of this investigation take this possibility into account and display the median condition state for that year, the result is a condition state decrease split between the true before and after values. As an example of how this might alter the apparent data for a bridge, say an element was in condition state 2.50 in February 2002 and was repaired to condition state 1.00 in June 2002, and was reported as such in September 2002. The resulting condition state cell for 2002 would display 1.75 and not decrease to 1.00 until the following inspection, say 2004. This bridge would thus not show up as having an "unexpected improvement" given the $>1.00$ criteria, even though the repair ultimately caused a 1.5 condition state decrease. This example illustrates a rare occurrence, but demonstrates how the analysis process could contain specific loopholes.

All of the line and normalized column graphs show the expected general deterioration occurring in the bridges that did not show a condition state improvement of greater than 1 . The line graphs (such as Figure 4) have a positive slope, denoting the condition increasing. If the criterion for improvement was made more lenient, such as a $>0.5$ condition state decrease, it is likely that the bridges without improvement would show more dramatic worsening. This is because as the criteria is brought closer to any non-zero condition state decrease it becomes increasingly likely that the bridges remaining in the "no improvement noted" category would be comprised entirely of bridges that only deteriorate. Any change to make the criterion closer to 0 increases the chances of inspector variance showing up (as a form of "no maintenance noted" in 
this report). It is also much more likely for two inspectors to report the same condition state with a difference of 0.1 condition state than 1.0 condition state.

Similarly, the line and normalized column graphs representing the bridges that did show an improvement (such as Figure 3) based on the criteria in this report depict a general decrease in condition state. These graphs show data from all of these bridges for the entire 15 year time frame, meaning bridges that were improved in 2008 still had their deteriorated condition state mixed into the 2006 average. This ultimately means these graphs show that bridges whose average condition states were shown to dramatically decrease show a large improvement in general over the entire time frame. The analysis tracking condition state change as a function of age since improvement was undertaken to attempt to account for the noise in these graphs and provide more meaningful trends.

The quantity analysis of the condition state trend portion of this report yielded graphs very similar to the analysis that weighted each bridge equally (compare Figure 6 and Figure 8). Slight differences occur due to bridges having different quantities of elements across the state, but these differences were not significant enough across even a single district to merit quantity analysis for more than 4 elements. The first few years were more likely to have variation between the two analyses because there were fewer bridges reporting, so chances were higher that the standard deviation of bridge size would be large enough to produce a visible graphical difference.

The deck and slab elements, of which element 32 was chosen for the quantity analysis, have quantity units in the Pontis Element Definition Manual listed as "EACH". However, the total quantity listed is still the square footage of the bridge, so the only difference the "EACH" makes is that the inspector has to pick a single condition state to represent the entire bridge deck 
(it becomes more similar to the NBI inspections in this way). As the process of analyzing the quantity in this report multiplies the total quantity by the percent in each condition state, the resulting quantity analysis graphs yield similar results for both the deck and non-deck elements. Similarly, as the Markov transition analysis was performed using the probability that a certain quantity of each element would deteriorate in a given year, the results for element 32 are only affected by the "EACH" unit in that the Pontis data shows that bridges stay in condition state 1 for several years and then suddenly become entirely in condition state 2 .

The condition state trends by age since improvement tables (Table B-13 through Table B-24) show that there are many more data points at lower ages than higher ages (years 1 and 2 compared to, say, 10 year old data). This is because consistent data, from which element improvement was derived, only starts around year 1998, meaning most improvements aren't noted until the 2000s. Each combination of consecutive improvement - inspection years will yield 1 year old data, while 10 year old data, for example, only comes from the case of a 2000 improvement with a 2010 inspection (or a rare 1999-2009, 1998-2008, 1997-2007 pairing).

The algorithm to calculate the year of improvement (for the age since improvement analysis) has several assumptions that have important bearing on the results obtained. In instances in which there was no pair of consecutive years with a condition state decrease of greater than 1.00, the bridge was ignored for this analysis (these bridges still showed up under the previous criteria of a $>1.00$ condition state decrease from the maximum condition state to the 2010 condition state). In instances in which there were multiple years between the previous inspection and the one in which the lower condition state was noted (such as with a two year inspection cycle), the improvement was recorded as occurring in the first year the lower condition state was reported. In rare instances where multiple condition state drops greater than 
1.00 were recorded, the algorithm reports the improvement year as the first (earliest) of such decreases.

The graphs following the average condition state of the improved bridges based on the number of years since improvement did not often show the desired trend. Figure 9 shows that element 32 deteriorated as expected as the bridges containing that element aged since the occurrence of the improvement noted in this analysis. Most of the elements produced graphs such as element 323 in Figure 10, however, where the condition state does not show any discernible trend at all, jumping up and down equally as the age increases. It would be expected that after the single year of condition state decrease corresponding to the improvement noted the bridges would then deteriorate normally until the next intervention.

A potential source of error was that there was no check if there was another improvement (of any condition state decrease) after the initial decrease. This could cause slight variations in the data, but averaged across the state it should not have stopped the bridges from showing increased deterioration with age. Also with these graphs, only years in which there were 5 or more bridges reporting were displayed, which mainly affected the number of years to display per element in Figure B-57 through Figure B-59.

Bridges are often inspected every other year, so data from those bridges follow a biannual trend. This is especially true after important structural elements, such as the girders, are improved because the bridge is no longer in an advanced deterioration state and it can be monitored less frequently. This manifests itself in the data if the set of bridges inspected on even years happens to have a different average condition state than those inspected on odd years. The biannual trend shows up in several parts of this analysis, such as the yearly normalized column graphs (as in Figure B-17), the average condition state trends by age since improvement (as in 
Figure B-58), the zinc coating analysis (see group 4 in Figure 13 and Figure 14) and in the Markov modeling process (much higher quantity in even years in Table 21, especially right after improvement).

\section{Potential Errors}

As can be seen from Table 34, the number of potential errors varies significantly by district, following the general trends of the number of bridges in each district. Out of 22581 total bridges in the database, 1353 were noted to have potential quantity recording errors at some point in their Pontis inspection history; this is $6.0 \%$ of the total bridges. It can be seen that about half of the potential errors occur on additional reports from the same set of bridges (1353 / 2785 $=48.6 \%$.

\section{Zinc Coating Study}

The graphs from the zinc coating study (Figure 11 through Figure 19) show trends for element 107 (or the superstructure) on all bridges meeting the zinc coating criteria, regardless of whether significant improvements were shown or not. This means that the deterioration trends shown should be indicative of the actual average worsening of condition of the beams from 1995 to 2010 .

Figure 15 shows the relative amount of data (by quantity) being graphed in each year of age since painting. The biannual trend is quite visible and prompted every two years of data to be graphed together in the similar Figure 11. The data trails off after about 20 years because the zinc coating system began to be used in 1983 (age 29 years as of the analysis), and took several years to catch on. There is also not as much data for the lower years for several of the groups 
due to Pontis data collection starting in 1995. For example, bridges painted in 1993 can only have data starting at 2 years; those painted in 1992 can only have data starting at 3 years, etc. The result is a bell shape, best seen in the bridges built after 1983 (groups 1 and 2 of Figure 15).

The condition state analysis graphs by quantity and by bridge, as seen in Figure 12 and Figure 13, respectively, follow the same general trends, but the quantity analysis contains a slightly higher percentage of the bar graphs denoting worse condition states. An example helps to illustrate the difference. If a bridge has 100 linear feet of element 107, and 25 feet are in condition state 2 while 75 feet are in condition state 1, the quantity bar graph for that bridge will be $25 \%$ yellow (and $75 \%$ green), but the overall bridge will average to 1.25 and stay $100 \%$ green for the by-bridge bar graph. This difference was consistent between the vast majority of the quantity graphs and the by-bridge graphs. For this reason, the quantity analysis was not included in the after-paint analysis.

The average condition states versus age of paint graphs (by group in Figure 16 and summarized in Figure 18) yield consistent results between similar bridges (groups 1 and 2, and groups 3 and 4). The initial condition state was close to 1 for groups 1 and 2, with a gradual increase in condition state (deterioration) over time. It appears that the condition state remains close to 1 for approximately 10 years, and then begins to show a gradual worsening of condition for the next 10 years. The rate of deterioration seems to accelerate after 20 years, with this trend slightly more significant in the continuous bridges. For groups 3 and 4 (the recoated bridges), the average condition state after recoating is about 1.2 and there is a gradual and immediate deterioration trend which remains fairly constant over the next 25 years.

The trends for superstructure condition rating for these bridges are presented by group in Figure 17 and are summarized in Figure 19. These trends are similar to those described for the 
condition state analysis but with less variability. This is likely because the condition rating is determined by the condition of many elements other than just the girders. These other elements may have been improved separately from the girders or merely display different deterioration rates. The condition rating trends for simple and continuous span bridges show no significant difference between the two, and recoated bridges have generally worse condition ratings than shop coated bridges for the same age of coating.

\section{Joint Closures}

The joint closure summary in Table 36 shows that the proportion of joint eliminations where the action is total elimination is $45.8 \%$, the proportion where it is a decrease in quantity is $24.3 \%$, and the proportion where one joint element is replaced with equivalent quantity of a different joint element is $29.9 \%$. The table illustrates that there was a wide variation by district in the number of joint modifications this investigation discovered, from 81 in District 7 to 364 in District 4.

\section{Priority Bridges and District Visits}

The criteria for what an "unexpected improvement" was had great bearing on the population of bridges chosen for analysis with or without improvement. Bridges that had a $>1.00$ condition state decrease between two consecutive years but then deteriorated back up to a total of less than 1.00 condition state of the max did not get selected. On the other hand, bridges that, for whatever reason, showed multiple smaller improvements (none of which were singly more than 1.00) that totaled an improvement of more than 1.00 did get selected. The $>0.05$ condition state decrease as a more lenient criterion for the two joint elements (301 and 302) 
likely increased their sensitivity to inspector variance (as a form of "no maintenance noted" in this report) over the other elements. That modified criterion was only used in the selection of priority bridges for the district visits so it only affected the population of bridges in that substudy.

The list of which bridges have a $>1.00$ decrease from max to current condition state was produced by the computer, but the manual process of selecting the years of improvement from those bridges sometimes revealed multiple condition state drops. When there were multiple significant improvements (more than a 0.50 condition state decrease between two consecutive inspection reports) on the same bridge, each improvement was listed as a separate row in this analysis in order to maximize the data from the same population of inspection reports. In instances where the quantity of the element changed on a given bridge, the most recently entered quantity was used in the final tables in Appendix C.

The process for selecting priority bridges to investigate for the district visit sub-study meant the results are representative, and not complete. Forty bridges was the cutoff chosen to keep the workload reasonable for the inspection personnel finding and returning the reports, and allowing the trips to take place in a single work day even in the farther away districts. When there were 10 or fewer bridges for an element in a district, all of those bridges were able to be selected, while a random number generator was used in cases with more than that. The result is that almost all of the bearing elements (311 and 313) were included, a moderate portion of the beam elements (107 and 108) was included, and only a small, but hopefully characteristic, selection of the joint elements (301 and 302) was able to be included. Using the cutoff criteria from this report, the statewide selected / available bridge ratios for the three element groups are 46 / $54(85.2 \%)$ for bearings, 165 / 372 (44.4\%) for beams, and 218 / 1655 (13.2\%) for joints. 
Part of the preparation process for the beam elements (107 and 108) was to remove those bridges where the unexpected improvement was shown to correlate to repainting noted in a separate "year repainted" field in the inspection reports (referenced from the InspEvnt PDI table). However, it can be seen from Table C-1 and Table C-5 that $51.4 \%$ and $10.5 \%$ of the bridges with elements 107 and 108, respectively, still had painting as the maintenance causing improvement as discovered from the "work done" section in the inspection reports. This means that the "year repainted" field was likely overlooked in the inspection process of these bridges.

"No maintenance noted" showed up consistently as around a third of the improvements in the different elements, between $24.2 \%$ for element 108 and $42.1 \%$ for element 313 . A main possibility for why these show up so frequently is merely inspector variation, where two inspection reports show similar or identical notes (similar level of deterioration), but one will give a condition state of 3.6 and the next will give a condition state of 2.4 , for example. These differences were often noted between a private consultant and a state inspector but occasionally the difference would even be between two inspection reports from the same inspector. When the notes are significantly different, it is likely that some maintenance action occurred but was not marked in the "work done" section (or anywhere else on the report), and the type of work could therefore not be classified for this report.

From Appendix C, it can be seen that District 4, the Richmond district, has a much higher quantity of elements 107 and 108 (beam elements) per bridge than the other districts. This indicates there were longer / wider bridges in the Richmond district, or that they had a denser arrangement of beams per bridge.

The tables in Appendix $\mathrm{C}$ created by separating the different roadway classifications show information both on the relative proportion of each element improved on each type of road 
and the maintenance actions taken on each. Element 107 is evenly split between the three classifications, while element 108 only appears on bridges carrying secondary roads (and a single primary road); this confirms that timber decks are rarely used on major roads. Element 302 is more or less evenly split between the three classifications, while element 301 has more than $60 \%$ on secondary roads, with similar overall counts; this leads to the deduction that the pourable joint seals are improved (and used) less on major roads than the compression joint seals are. For the bearing elements (311 and 313), the highest levels of superstructure replacement came on secondary roads.

\section{Linear Least Squares and Markov Chain Modeling}

The results of the LLS regression analysis (presented in Table 43) show general deterioration trends of 6 elements in the form of condition state slope predictions and the average condition states of those elements in 2010, separated by district. Of the 3 element groups, averaged across the state, the bearing elements (311 and 313) have the lowest 2010 condition state at around 1.42. This was followed by the joint elements (301 and 302) next with a 2010 condition state around 1.65. The beam elements' 2010 condition state averaged around 2.10, with element 108 (beam under timber deck) almost a full condition state worse than element 107 (beam under concrete deck) at 2.60 vs. 1.62, respectively. Additionally, element 108 deteriorated 1.66 times as fast as element 107. Both beam statistics indicate that the timber decks do not protect the beams as well as concrete decks do.

A reason why it may be difficult to apply linear regression to condition state deterioration is that the language of the condition state definitions do not yield even spacing. For example, the physical difference between elements in condition states 1 and 2 will not be the same as between 
condition states 2 and 3 and they may take a different amount of time to deteriorate, even though both are a decrease of 1.00. Also, the physical deterioration itself is likely nonlinear, with more advanced deterioration (pitting, corrosion, etc.) occurring only after the coating / paint systems weaken.

The LLS condition state deterioration slopes are fairly low on average, as 0.01 means that it takes 100 years with no improvements for the element to deteriorate 1 condition state. A possible explanation for why this is so low is that bridges built after 1995 only have data beginning at their year of construction, usually at pristine condition states, so the data could be skewed to give a set containing these bridges an unrepresentatively low deterioration average. The degree of this skew would be based on the proportion of bridges in a certain district (for a given element) that were recently built; more new bridges likely means more skew. This would result in slopes that are lower than the true deterioration level, in a few extreme cases even looking as though the bridge average condition states are improving (see element 301 in districts 1 and 3). Bridges improving less than the 1.00 "unexpected improvement" criteria were included in the data for average regression, so they could also lessen the slope from the true deterioration.

For the linear least square condition state deterioration trends based on age of bridge for element 107 (such as Figure D-28), it can be seen that bridges often wait 15-20 years before element 107 deterioration begins. At that time, the condition state decreases at a moderate rate until it reaches about 2.00 around 75 years of age, at which point the data generally stops following any discernible trends. The residual graphs (such as Figure D-29) show the same trends as functions of how far below the modeled trendlines the actual data are (by subtracting them at each year). As the trendlines are forced to be linear while the actual deterioration 
follows the above trends, the residuals are generally positive to start, then go slightly negative, then become more variable (positively and negatively) at older ages.

The graphs showing the number of bridges reporting element 107 by age (such as Figure D-30) show that there is a steep drop-off of bridges reporting after about 45 years, and again around 75 years, so the condition states from the upper years are more prone to variation. Additionally, as the Pontis data was not collected until 1995 (up until 2010 for this report), we do not know the condition state histories of bridges outside of that timeframe, we merely have a window of information. For example, a bridge built in 1998 gives us 1-12 year data while a bridge built in 1950 gives us 45-60 year data. This means we do not have more than 15 years of data for any bridge, and there me be some error introduced by following different bridges' condition states as age increases.

Element 108 (a beam under a timber deck) shows a somewhat different trend. Deterioration appears to occur more immediately (as seen in Figure D-58), with the condition state increasing to 2.5 over the first twenty years and then staying there until the data becomes sporadic after about 80 years, when there were fewer bridges reporting. On that note, it is interesting that in the graphs of the number of bridges reporting element 108, such as Figure D-60, there was a giant spike in the population of bridges reporting element 108 in the seventy to eighty year range, indicating a large number of new timber deck bridges being constructed in the early- to mid-1930s. This is possibly because this was the era of President Franklin Delano Roosevelt's New Deal, which created many construction projects; several of these were bridges in rural Virginia where they would be likely to have been built with timber decks. Additionally, VDOT may have picked up existing bridges into their NBI database at this time; if they did not 
have information on bridge construction they may have entered the year they acquired each bridge as the year of its construction.

Due to the transition constraints the Markov modeling was performed under, all Markov graphs (Figure D-61 through Figure D-79) show steady deterioration between condition states, with each condition state boundary having a distinct deterioration percent depending on the given district and element. Due to the nature of the transition probability matrix multiplication, the boundary from condition state 1 to 2 (green to yellow) must start at its steepest and decrease asymptotically while the other boundaries will change from increasing to decreasing slope at some age. For example, in the modeled plot for element 108 in District 7 (bottom left graph in Figure D-69), the inflection point for condition state 2 to 3 (yellow to orange) occurs at around 30 years and the inflection point for condition state 3 to 4 (orange to bright red) occurs at around 60 years.

It should be noted that element 32 (Table D-16 through Table D-19 and Figure D-76 through Figure D-79) never deteriorates past condition state 3 and element 302 (Table D-11 through Table D-15 and Figure D-71 through Figure D-75) never deteriorates past condition state 4. On the other hand, elements 107 (Table D-1 through Table D-5 and Figure D-61 through Figure D-65) and 108 (Table D-6 through Table D-10 and Figure D-66 through Figure D-70) deteriorate to condition state 5. This is because in the element definitions in the Pontis Element Data Collection Manual, only 3 condition states are coded for element 32 and 4 condition states coded for element 302 (VDOT, 2007). Thus, it is important to keep in mind that the lowest coded condition state per element represents the maximum state of deterioration: condition state 3 in element 32 is analogous to condition state 4 in element 302 and condition state 5 in elements 107 or 108; these are all colored dark red to indicate similarly advanced levels of deterioration. 
Element 32 (Timber deck - with asphaltic concrete (AC) overlay) only has 6 districts reporting for the Markov section of the analysis. This is because in the original element selection by count (see Table 24 through Table 26), element 32 did not appear in the top 20 elements for districts 4, 6, and 9 and did not undergo quantity analysis in those districts.

The first step of the Markov modeling in this investigation was to limit the data selection to those bridges with the desired elements showing an unexpected improvement as per the criterion of a $>1.00$ condition state decrease from max to current. Similarly, the first step of the LLS regression across an entire district was to create an average deterioration slope / intercept based on those bridges without an "unexpected improvement". As in many of the previous sections of this report, the results are affected by the bridges that do or do not meet this criterion. If the threshold for improvement is decreased, more bridges with maintenance actions (that are unrepresentative of the desired natural deterioration) would likely be omitted. However, there would also be an increased chance of "improvements" being inspector variance (as a form of "no maintenance noted" in this report) as the criterion is brought closer to any condition state decrease at all.

One of the main limitations of the Markov chain modeling in this report (due to available data) is the inherent assumption that bridges were not improved from the time of construction until 1995. Due to the lack of Pontis inspection report data before 1995, it would be impossible to know if a bridge had deteriorated naturally over the course of its life until 1995, or had just been recently repaired in 1994 at age 50 years, say. The result of this is an unknown proportion of the data that could cause the transition probability matrices to have skewed deterioration rates. As the matrices created from this analysis could include bridges that were improved but not noted, the true natural deterioration rates are likely slightly greater than those presented in this 
report. The relationships between the deteriorations in various elements shown in this report may or may not stay the same if these unknown improvements were omitted, depending on which bridges they occurred on and when.

It is also useful to remember the Markov modeling in this report used the quantity data for the desired elements as opposed to averaging the deterioration equally for each bridge. This will provide greater weight to the deterioration of elements on bridges with a larger quantity of the elements selected. As discussed earlier in the Analysis section, element 32 which has a recorded quantity unit of "each" still provides square footage data, and as this is used in the analysis, more weight is given to larger bridges for that element as well.

From the 100 year graphs showing the Markov model vs. the actual condition state data (such as Figure D-61), one can compare the relative accuracy of the models between districts and elements. Each graph set has some disparity due to the fact that 100 years of real world variation has been modeled using a single transition matrix (multiplied out over 100 years). This means that those graphs of real world data that more closely follow steady condition state deterioration will be more closely modeled by the Markov chains that Excel optimized. 


\section{CONCLUSIONS}

Through numerous data mining investigations of Pontis and NBI data, exploratory

analysis was shown to be useful in providing information that was not obvious before this project.

The first study, characterizing the performance of coatings on girders under Virginia bridges, was successful. Deterioration trends were found by analyzing the Pontis and NBI data that were not observable from the raw data. Overall, the zinc-based coating system seems to be performing well. The condition states and ratings for coatings on older bridges, which have been repainted, deteriorate more quickly than those on newer bridges with shop coating. A significant difference was not observed in the performance of simple span and continuous span bridges.

The second study of quantitatively looking at which VDOT maintenance practices are being undertaken and how they are recorded through investigating the district reports was partially successful. The current information regarding maintenance actions appears to be insufficient for the ultimate questions VDOT is asking relating to maintenance cost effectiveness. Still, much has been and can be learned from analysis of the actions that have been recorded.

Several side studies arose over the course of this project and they also provided useful statistics. Condition state trends were plotted for bridges that did and did not undergo a significant improvement. Summary tables and complete ID lists were created for bridges with potential reporting errors, bridges with joint elements that were eliminated, and those with beam sections in advanced deterioration condition were also produced. Several linear least squares algorithms (macros) were created in Visual Basic for Applications to predict deterioration of 6 
selected elements. Markov transition probability matrices and associated deterioration graphs for a 100-year horizon were created for 4 elements.

Significant variations in results and perceived conclusions can arise from slight variations in the raw data. As such, if more studies are conducted with the intent of extracting meaningful information, stricter inspector standards should be implemented. 


\section{RECOMMENDATIONS}

The $>1.00$ decrease from max to current condition state criterion for "unexpected improvement" affected the cut-offs for much of the analysis in this project, deciding which set different bridge condition state trends were graphed in, which priority bridges were selected for district visits, and excluding "improved" bridges from both modeling methods. Future research could modify this criterion, by element if desired, to include a different population of bridges as those having an improvement (similar to how the joint improvement criterion was decreased for the district visit study in this project).

Selection of elements for analysis in this report was based on the number of bridges they appeared on and the number of improvements noted. Various parts of the project could be performed on additional elements of interest based on criticality to bridge performance, anticipated maintenance / deterioration, or other criteria by a future researcher.

The Pontis analysis methods used in this report on VDOT data could be modified and used in other states that use Pontis depending on what their specific areas of maintenance interest are. For additional states, many of the investigations from this report would have corollaries in their NBI data if similar trends were desired. The methods from the NBI part of the zinc coating study in this project could also be directly applied to the NBI data in any state.

If stricter inspector criteria for the rating of condition states were implemented, inspector variance (as a form of "no maintenance noted" in the district visit section of this report) would likely be reduced. This project could merely conclude that if the quantities and notes were the same but the condition state was different, there would likely be a difference of inspector opinion as the reason for disparity. It may be useful, therefore, to conduct an additional investigation on inspector variance. A supplementary study could, through closer analysis of inspection report 
data and interviews with inspectors, explore the extent of the variability, the causes of differences in opinions, and propose ways to mitigate that error. Relatedly, it would be useful to ensure that painting gets noted in the "year repainted" field on the inspector reports. That way, the field could be more meaningfully felt to represent the entire population of bridges that were repainted.

It would be useful to institute a uniform state-wide database for storing information on maintenance actions in Virginia. This way, it would be much easier to perform investigations on the effectiveness of various actions, and computer resources could more readily be used in the analysis of maintenance on many additional elements. This system would likely by similar to the proposed National Bridge Maintenance Database (Hearn, Thompson, Mystkowski, \& Hyman, 2010) described in the Review of the Literature earlier in this report.

Following this report, it is suggested that more streamlined methods be developed to run the analyses that are deemed most useful to VDOT. This way, as more data is produced and becomes available to researchers, the tables and graphs can be updated so analysis can continue with minimal additional human interaction. More sophisticated statistical methods, perhaps using more advanced software packages than Microsoft Excel, can also be applied to this data as additional desired results are precisely defined in projects continuing this work.

The project advisory group expressed interest in exploring the feasibility of defining subelements for girder ends below joints as a way of better capturing coating performance for VDOT's steel painted bridges. This arose from a discussion of their personal experiences investigating beam deterioration.

Trends of element 107 beam deterioration under various cases of joint elimination could be found by filtering the bridges from the zinc coating study by the results of the joint 
elimination study. Other similar studies for potential correlation could be completed by analyzing modifications (quantity reduction, replacement, etc.) of other elements, or performing lookups on various bridge traffic or environmental categories, and filtering the zinc coating element 107 condition state and rating data by the resulting bridge IDs.

As with any computation-based research, the analysis will become quicker as computer hardware and software resources improve. This progress allows for more complex data manipulation and expanded interconnectivity of Excel cells to streamline any raw data addition / modification. Additionally, as time progresses and more data is collected in the Pontis system, the methods presented in this report will return more representative data with more years of recorded condition state trends on elements with and without improvements noted. This will also refine the accuracy of the LLS and Markov prediction models this project developed. Average element value Linear Least Squares regression analysis by district could be performed on additional elements by running the macro on the data in the desired elements' worksheets in the district-specific Excel files available through VDOT. District 1 contains the macros: "LLS_Predict_Lite" for most elements and "LLS_Predict_Lite_107_or_108" for elements 107 and 108. For the same set of files, running the individual bridge LLS macro (“LLS_Predict") would result in a separate regression analysis being performed on each bridge's unique condition state history. Lastly, the LLS analysis by age of bridge could be run on elements other than 107 and 108.

The comparison of the element-level deterioration (LLS and Markov chain) models developed in this project to existing regression analyses and Markov chain transition probability matrices is left to another investigation. 


\section{REFERENCES}

AASHTO. (2005). Pontis Technical Manual.

AASHTO. (2005). Pontis User Manual.

AASHTO. (2007). Maintenance Manual for Roadways and Bridges.

AASHTO. (2011). Manual For Bridge Evaluation.

Aldemir-Bektas, B., \& Smadi, O. G. (2008). A Discussion on the Efficiency of NBI Translator Algorithm. Proceedings of the Tenth International Conference on Bridge and Structure Management, October 20-22, 2008, Transportation Research E-Circular.

Chase, S. B. (2010). Slides from Bridge Inspection Short Course. Charlottesville, Virginia. Retrieved from UVA Collab.

Chase, S. B. (2011). Slides from Infrastructure Management Class. Lecture 15. Charlottesville, Virginia. Retrieved from UVA Collab.

Fayyad, U., \& Stolorz, P. (1997). Data mining and KDD: Promises and challenges. Future Generation Computer Systems, 13(2), 99-115.

Fayyad, U., Piatetsky-Shapiro, G., \& Smyth, P. (1996). The KDD process for extracting useful knowledge from volumes of data. Communications of the ACM, 39(11), 27-34.

FHWA. (1995). Recording and Coding Guide for the Structure Inventory and Appraisal of the Nation's Bridges.

Frawley, W. J., Piatesky-Shapiro, G., \& Matheus, C. J. (1992). Knowledge discovery in databases: An overview. AI magazine, 13(3), 57-70. 
Golabi, K., \& Shepard, R. (1997). Pontis: A system for maintenance optimization and improvement of US bridge networks. Interfaces, 27(1), 71-88.

Gutkowski, R. M., \& Arenella, N. D. (1998). Investigation of PONTIS, a Bridge Management Software. Mountain-Plains Consortium, 98-95.

Hand, D., Mannila, H., \& Smyth, P. (2001). Principles of Data Mining.

Hearn, G., Thompson, P. D., Mystkowski, W., \& Hyman, W. (2010). Framework for a National Database for Maintenance Actions on Highway Bridges. Washington, D.C.: National Cooperative Highway Research Program - Report 668.

Morcous, G. (2006). Performance prediction of bridge deck systems using Markov chains. Journal of Performance Constructed Facilities, 20(2), 146-155.

NIST/SEMATECH. (2012). Section 4.1.4.1. Linear Least Squares Regression. Retrieved from eHandbook of Statistical Methods: http://www.itl.nist.gov/div898/handbook/pmd/section1/pmd141.htm

Robert, W. E., Marshall, A. R., Shepard, R., \& Aldayuz, J. (2003). The Pontis bridge management system: State-of-the-practice in implementation and development. Proceedings of the 9th International Bridge Management Conference, (pp. 49-60).

Ryan, T. W., Hartle, R. A., Mann, J. E., \& Danovich, L. J. (2006). Bridge Inspector's Reference Manual.

Small, E. P., Philbin, T., Fraher, M., \& Romack, G. P. (1999). Current status of bridge management system implementation in the United States. 8th Internation Bridge Management Conference, (pp. A-1/1-16). 
Tukey, J. W. (1977). Exploratory Data Analysis. Addison-Wesley.

VDOT. (2007). Pontis Element Data Collection Manual.

Zayed, T. M., Chang, L., \& Fricker, J. D. (2002). Statewide performance function for steel bridge protection systems. Journal of Performance of Constructed Facilities, 16(2), 4654. 
APPENDICES 
APPENDIX A - ELEMENT CODES AND DESCRIPTIONS

Derived from the Pontis Element Data Collection Manual (VDOT, 2007) 


\begin{tabular}{|c|c|}
\hline Code & Element Description \\
\hline 012 & Concrete Deck - Bare - with Uncoated Reinforcement \\
\hline 013 & Concrete Deck - with AC Overlay - without Membrane \\
\hline 014 & Concrete Deck - with AC Overlay - with Membrane \\
\hline 018 & Concrete Deck - Thin Overlay (less than 1") - no AC Overlay \\
\hline 022 & Concrete Deck - Rigid Overlay (greater than 1") - no AC Overlay \\
\hline 026 & Concrete Deck - Bare - with Coated Reinforcement \\
\hline 027 & Concrete Deck - with Cathodic Protection \\
\hline 028 & Steel Deck - Open Grid \\
\hline 029 & Steel Deck - Concrete Filled Grid \\
\hline 030 & Metal Deck - Corrugated/Orthotropic, Etc \\
\hline 031 & Timber Deck \\
\hline 032 & Timber Deck - with asphaltic concrete (AC) Overlay \\
\hline 038 & Concrete Slab - Bare - with Uncoated Reinforcement \\
\hline 039 & Concrete Slab - with AC Overlay - without Membrane \\
\hline 040 & Concrete Slab - with AC Overlay - with Membrane \\
\hline 044 & Concrete Slab - Thin Overlay (less than 1") - no AC Overlay \\
\hline 048 & Concrete Slab - Rigid Overlay (greater than 1") - no AC Overlay \\
\hline 052 & Concrete Slab - Bare - with Coated Reinforcement \\
\hline 053 & Concrete Slab - with Cathodic Protection \\
\hline 054 & Timber Slab \\
\hline 055 & Timber Slab - with asphaltic concrete (AC) Overlay \\
\hline 092 & V Reinforced Concrete Sidewalk \\
\hline 094 & V Timber Sidewalk \\
\hline 098 & V Steel Sidewalk, Open Grid - Coated \\
\hline 101 & Steel Closed Web/Box Girder - Uncoated \\
\hline 102 & Steel Closed Web/Box Girder - Coated \\
\hline 104 & P/S Concrete Voided and Unvoided Closed Web/Box Girder \\
\hline 105 & Reinforced Concrete Voided and Unvoided Closed Web/Box Girder \\
\hline 106 & Steel Open Girder - Uncoated \\
\hline 107 & Steel Open Girder - Coated \\
\hline 108 & V Steel Open Girder with Timber Deck - Coated and Uncoated \\
\hline 109 & P/S Concrete Open Girder \\
\hline 110 & Reinforced Concrete Open Girder \\
\hline 111 & Timber Open Girder \\
\hline 112 & Steel Stringer - Uncoated \\
\hline 113 & Steel Stringer - Coated \\
\hline 115 & $\mathrm{P} / \mathrm{S}$ Concrete Stringer \\
\hline 116 & Reinforced Concrete Stringer \\
\hline 117 & Timber Stringer \\
\hline 120 & Steel Bottom Chord of Through Truss - Uncoated \\
\hline 121 & Steel Bottom Chord of Through Truss - Coated \\
\hline 125 & Steel Through Truss excluding bottom chord - Uncoated \\
\hline
\end{tabular}




\begin{tabular}{|c|c|}
\hline 126 & Steel Through Truss excluding bottom chord - Coated \\
\hline 130 & Steel Deck Truss - Uncoated \\
\hline 131 & Steel Deck Truss - Coated \\
\hline 135 & Timber Truss or Arch \\
\hline 140 & Steel Arch - Uncoated \\
\hline 141 & Steel Arch - Coated \\
\hline 143 & P/S Concrete Arch \\
\hline 144 & Reinforced Concrete Arch \\
\hline 145 & Other Material Arch \\
\hline 146 & Steel Cable - Uncoated (not embedded in concrete) \\
\hline 147 & Steel Cable (not embedded in concrete) - Coated \\
\hline 151 & Steel Floor Beam - Uncoated \\
\hline 152 & Steel Floor Beam - Coated \\
\hline 154 & P/S Concrete Floor Beam \\
\hline 155 & Reinforced Concrete Floor Beam \\
\hline 156 & Timber Floor Beam \\
\hline 160 & Steel Pin and/or Pin \& Hanger Assembly - Uncoated \\
\hline 161 & Steel Pin and/or Pin \& Hanger Assembly - Coated \\
\hline 201 & Steel Column or Pile Extension - Uncoated \\
\hline 202 & Steel Column or Pile Extension - Coated \\
\hline 204 & P/S Concrete Column or Pile Extension \\
\hline 205 & Reinforced Concrete Column or Pile Extension \\
\hline 206 & Timber Column or Pile Extension \\
\hline 210 & Reinforced Concrete Pier Wall \\
\hline 211 & Other Material Pier Wall \\
\hline 215 & Reinforced Concrete Abutment \\
\hline 216 & Timber Abutment \\
\hline 217 & Other Material Abutment \\
\hline 220 & Reinforced Concrete Submerged Pile Cap/Footing \\
\hline 225 & Steel Submerged Pile \\
\hline 226 & P/S Concrete Submerged Pile \\
\hline 227 & Reinforced Concrete Submerged Pile \\
\hline 228 & Timber Submerged Pile \\
\hline 230 & Steel Pier Cap - Uncoated \\
\hline 231 & Steel Pier Cap - Coated \\
\hline 233 & P/S Concrete Pier Cap \\
\hline 234 & Reinforced Concrete Pier Cap \\
\hline 235 & Timber Pier Cap \\
\hline 240 & Metal Culvert \\
\hline 241 & Concrete Culvert \\
\hline 242 & Timber Culvert \\
\hline 243 & Other Culvert \\
\hline 285 & V Slope - Protected \\
\hline
\end{tabular}




\begin{tabular}{|c|c|}
\hline 286 & V Slope - Unprotected \\
\hline 295 & V Reinforced Concrete Wingwalls \\
\hline 296 & V Timber Wingwalls \\
\hline 297 & V Other Material Wingwalls \\
\hline 298 & Smart Flag - Culvert Endwall/Headwall \\
\hline 298 & V Smart Flag - Culvert Endwall/Headwall \\
\hline 299 & V Smart Flag - Culvert Wingwall \\
\hline 300 & Strip Seal Expansion Joint \\
\hline 301 & Pourable Joint Seal \\
\hline 302 & Compression Joint Seal \\
\hline 303 & Assembly Joint/Seal \\
\hline 304 & Open Expansion Joint \\
\hline 310 & Elastomeric Bearing \\
\hline 311 & Moveable Bearing (Roller, sliding, etc.) \\
\hline 312 & Enclosed/Concealed Bearing or Bearing System \\
\hline 313 & Fixed Bearing \\
\hline 314 & Pot Bearing \\
\hline 315 & Disk Bearing \\
\hline 320 & Prestressed Concrete Approach Slab \\
\hline 321 & Reinforced Concrete Approach Slab \\
\hline 330 & Metal Bridge Railing - Uncoated \\
\hline 331 & Reinforced Concrete Bridge Railing \\
\hline 332 & Timber Bridge Railing \\
\hline 334 & Metal Bridge Railing - Coated \\
\hline 356 & Smart Flag - Steel Fatigue \\
\hline 357 & Smart Flag - Pack Rust \\
\hline 358 & Smart Flag - Deck Cracking \\
\hline 359 & Smart Flag - Soffit of Conc \\
\hline 360 & Smart Flag - Settlement \\
\hline 361 & Smart Flag - Scour - \\
\hline 362 & Smart Flag - Traffic Impact Damage \\
\hline 363 & Smart Flag - Section Loss \\
\hline 444 & V Mechanically Stabilized Earth - Abutment \\
\hline 701 & V Smart Flag - Utilities \\
\hline 702 & V Smart Flag - Drains \\
\hline 703 & V Smart Flag - Lighting \\
\hline 704 & V Smart Flag - Roadway Over Culverts \\
\hline 706 & V Smart Flag - Soffit of Overhang of Conc \\
\hline 707 & V Smart Flag - Soffit of Conc \\
\hline 708 & V Smart Flag - Debris in Channel - \\
\hline 709 & V Smart Flag - Replacement - \\
\hline 710 & V Smart Flag-Deck Replacement \\
\hline 738 & Concrete Slab - Covered with Fill \\
\hline
\end{tabular}


APPENDIX B - CONDITION STATE TRENDS 


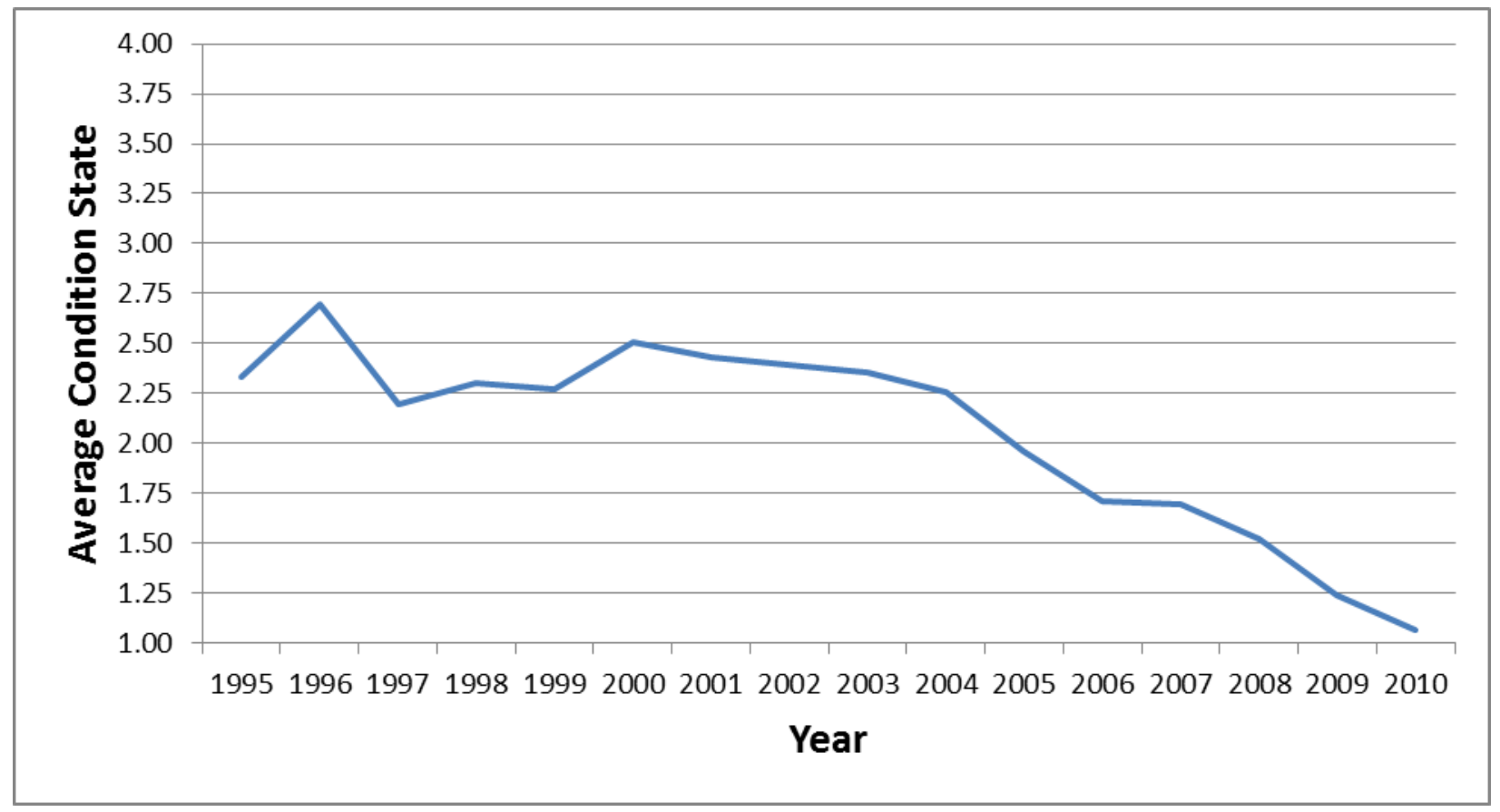

Figure B-1 - Element 32 (Timber Deck - with Asphaltic Concrete (AC) Overlay) Average Condition State Trend Improvement Noted

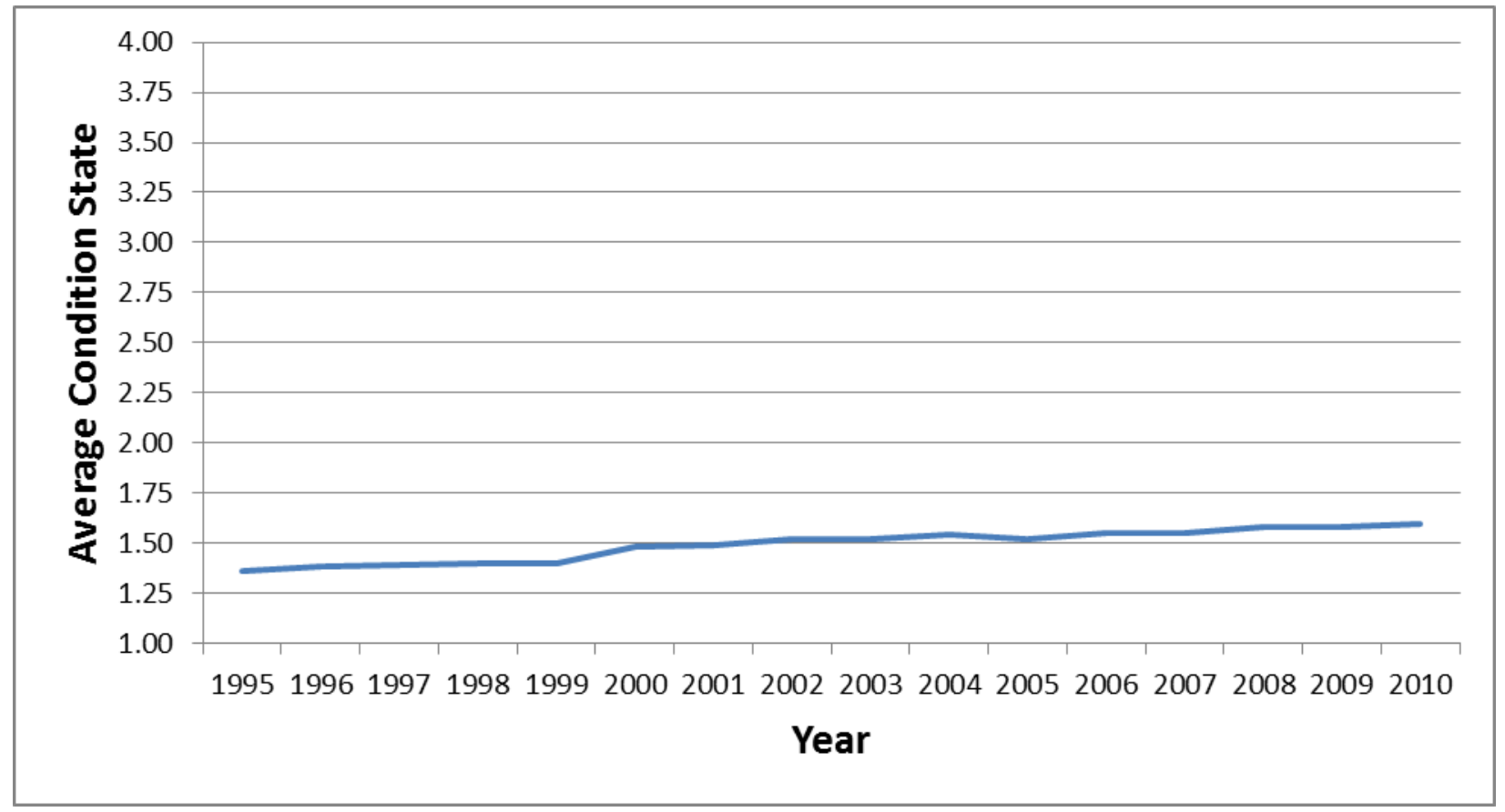

Figure B-2 - Element 32 Average Condition State Trend - Improvement Not Noted 


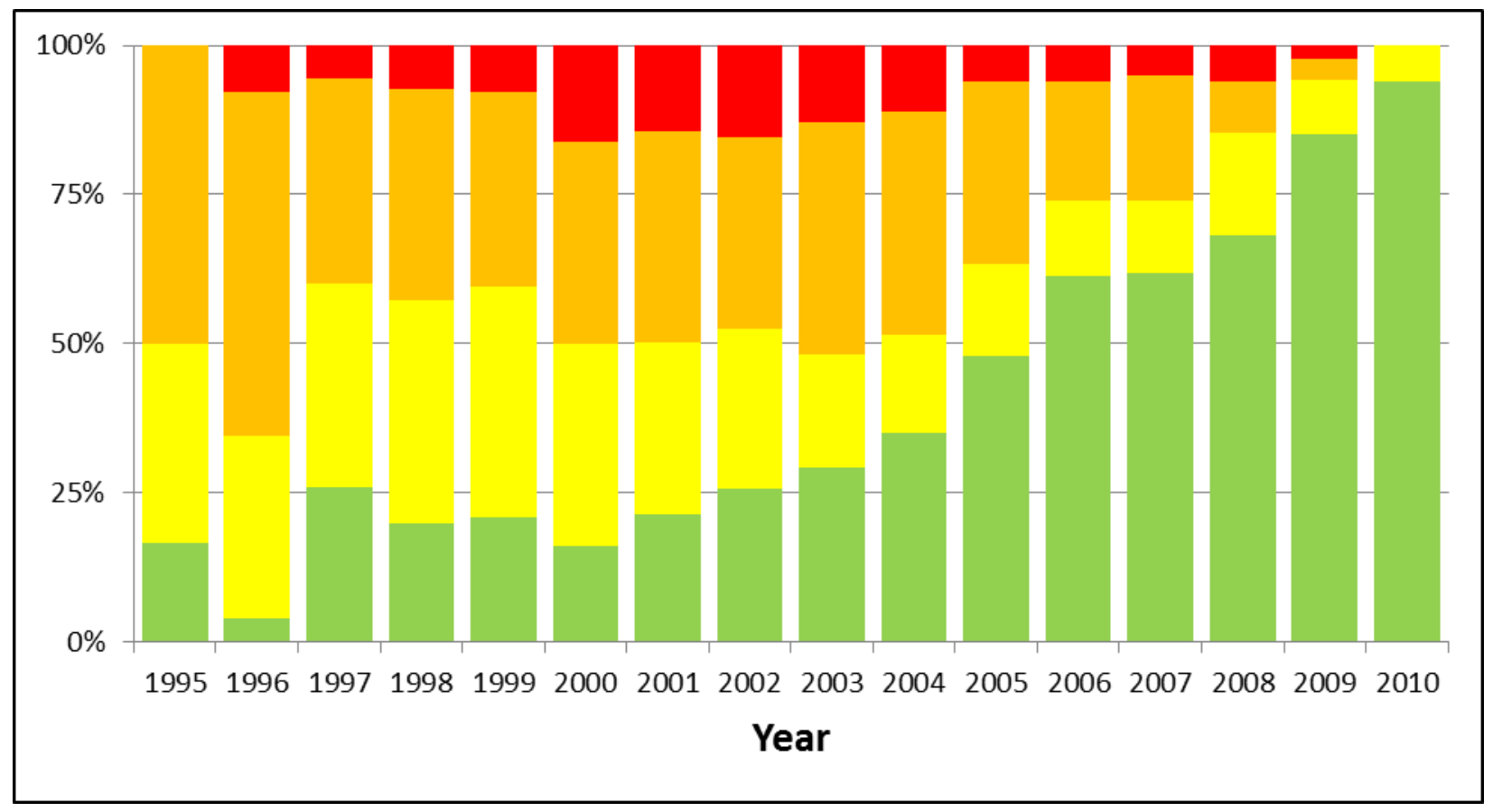

Figure B-3 - Element 32 Normalized Condition State Trend - Improvement Noted

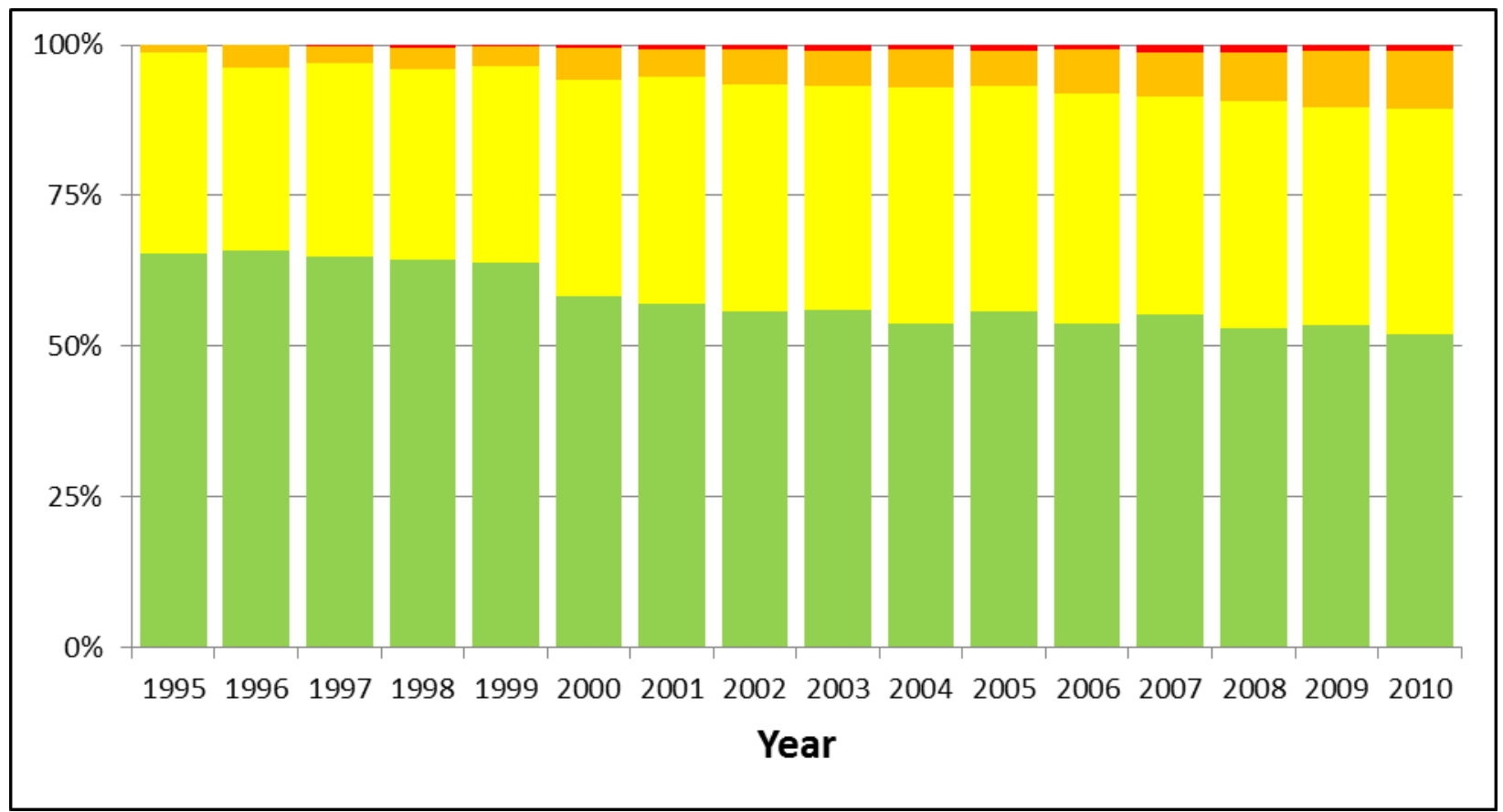

Figure B-4 - Element 32 Normalized Condition State Trend - Improvement Not Noted 


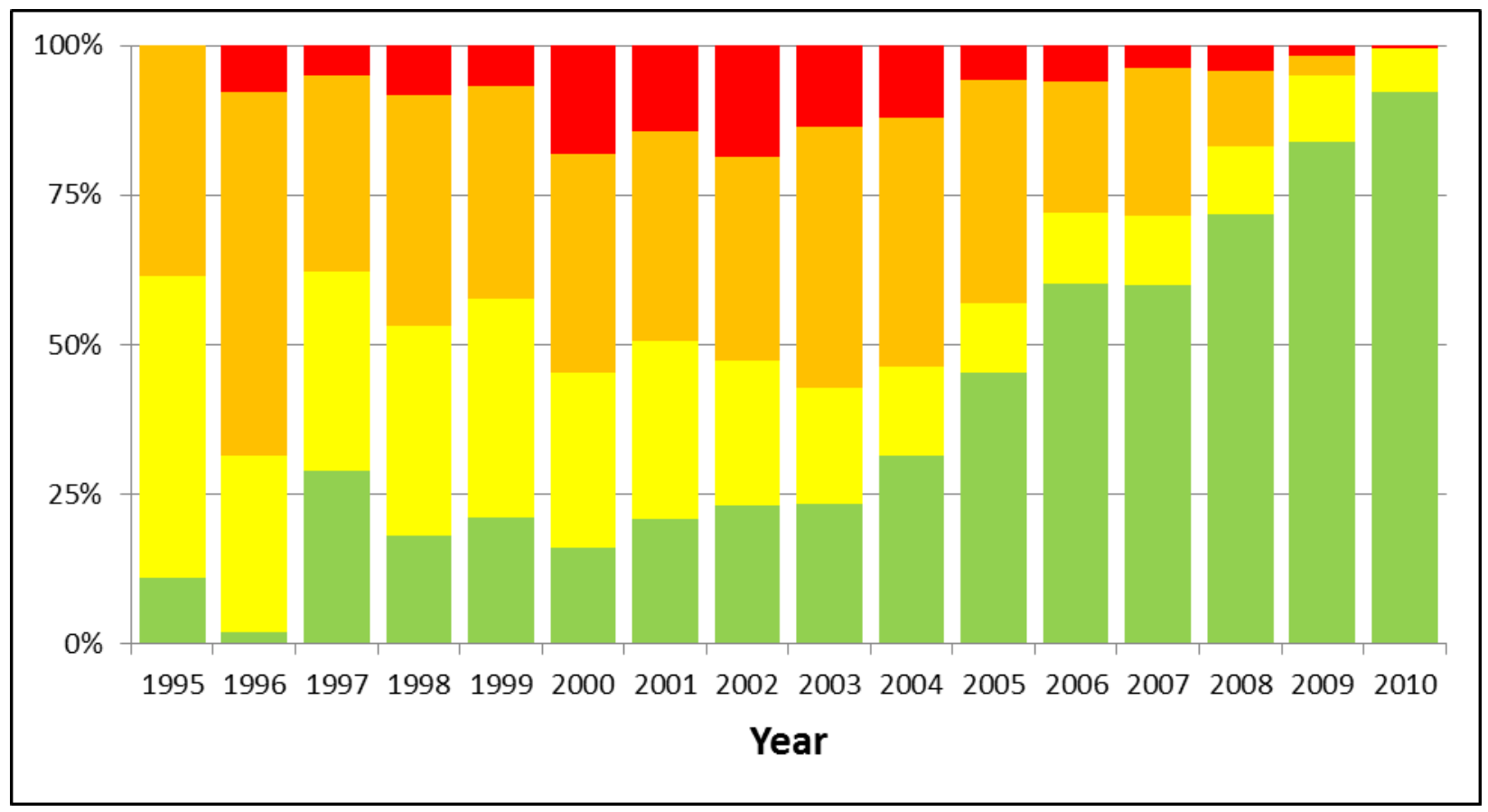

Figure B-5 - Element 32 Normalized Condition State Trend - Improvement Noted (Quantity Analysis)

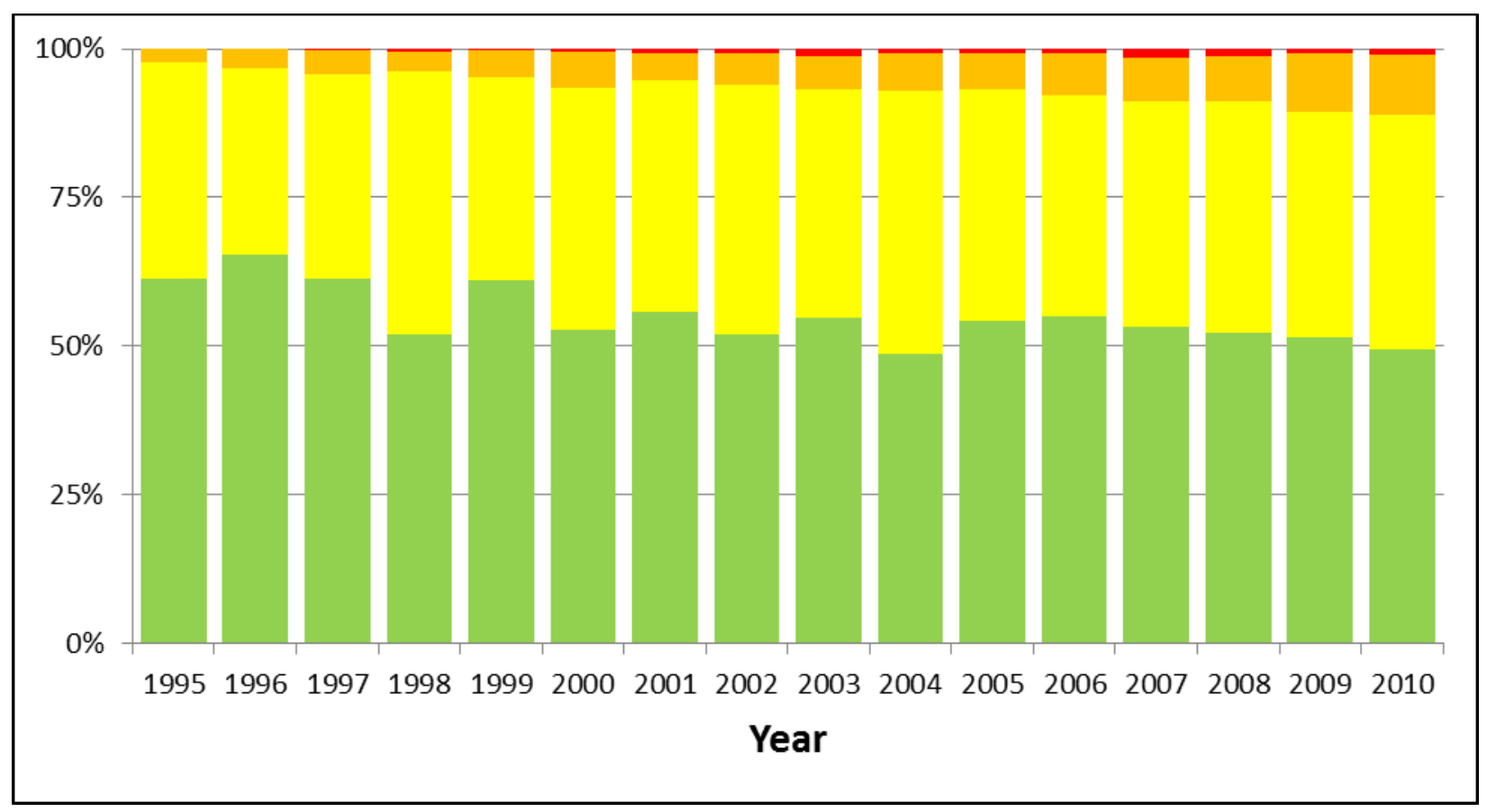

Figure B-6 - Element 32 Normalized Condition State Trend - Improvement Not Noted (Quantity Analysis) 


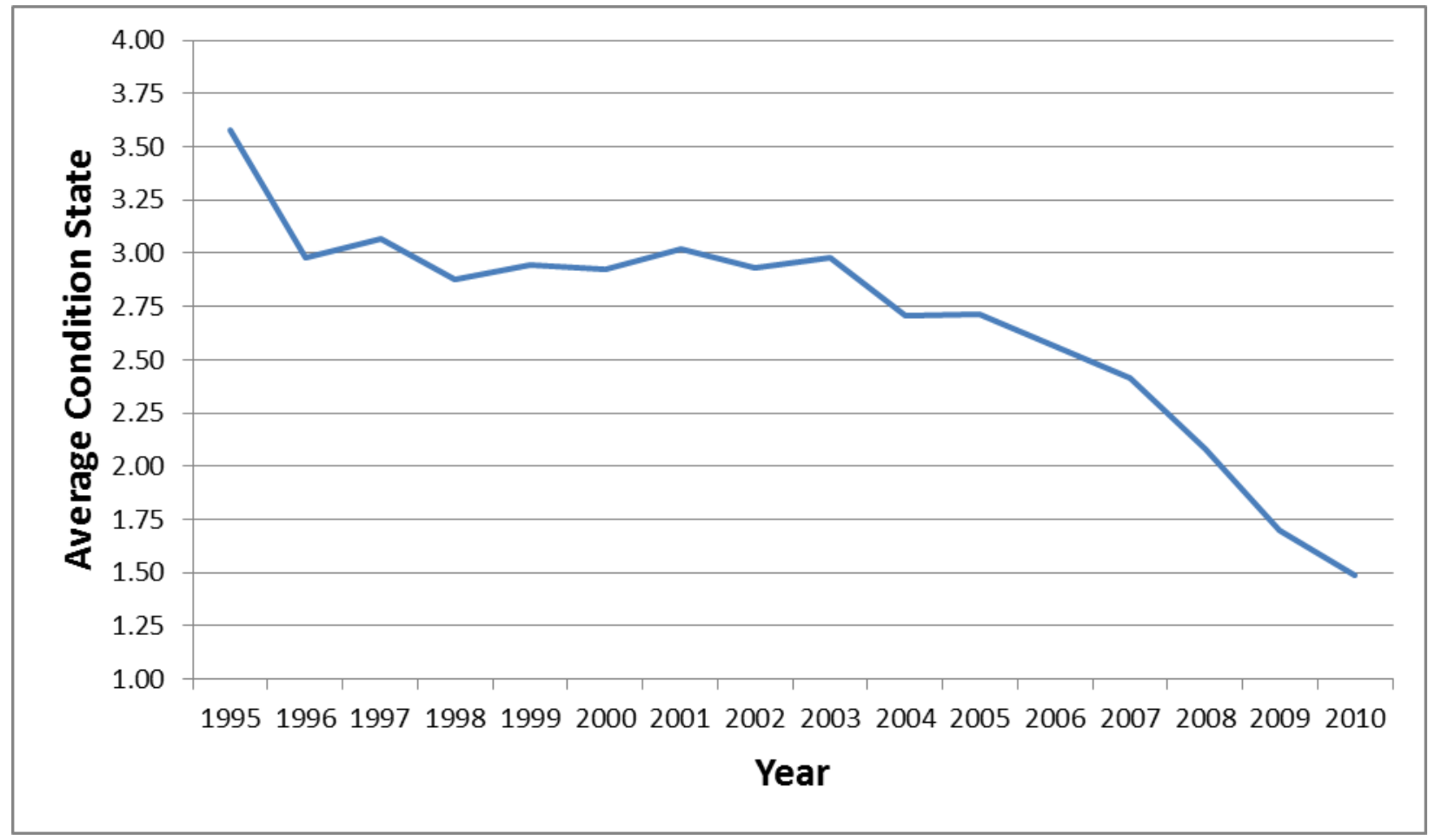

Figure B-7 - Element 108 (Steel Open Girder with Timber Deck - Coated and Uncoated) Average Condition State Trend - Improvement Noted

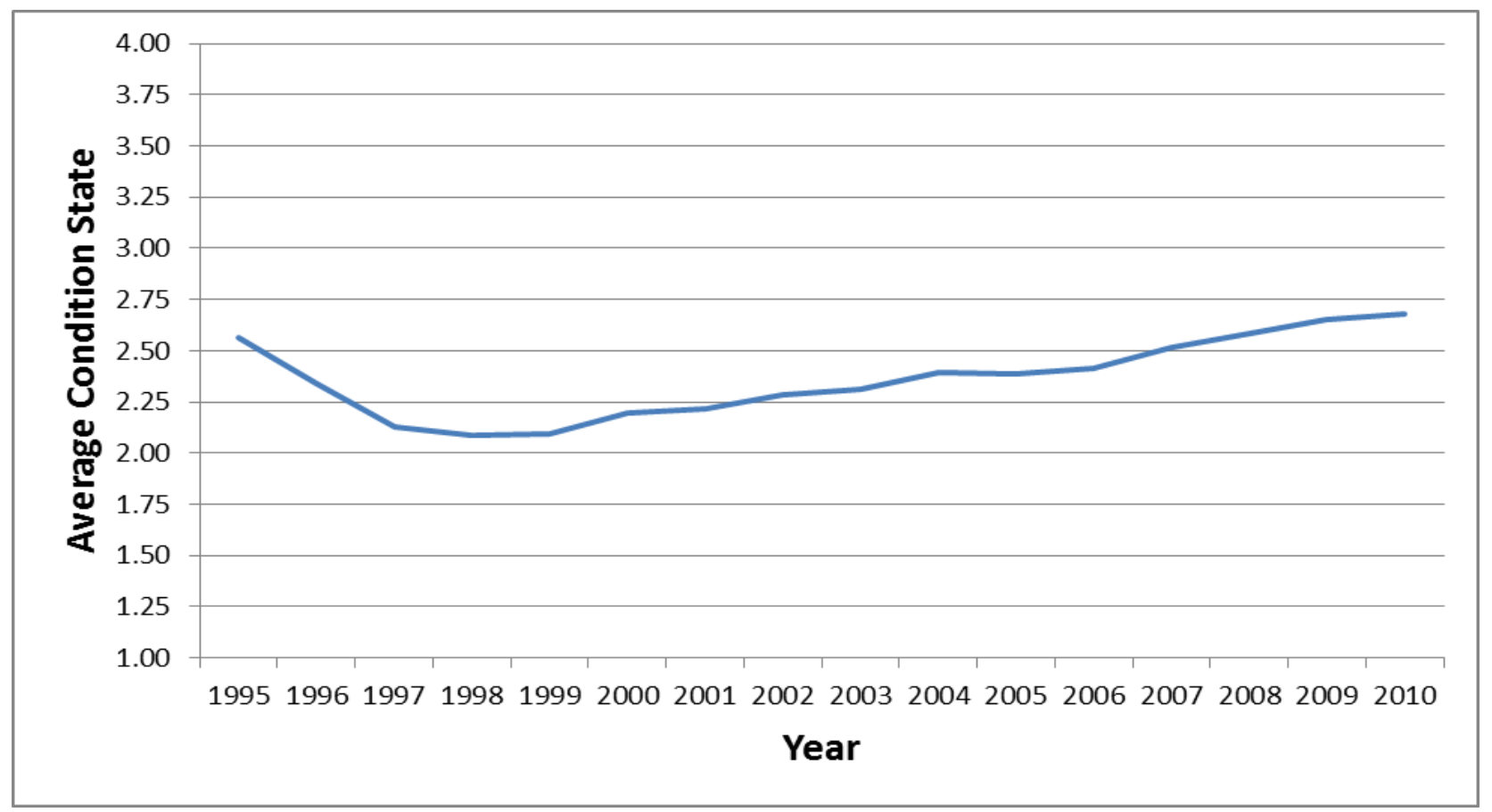

Figure B-8 - Element 108 Average Condition State Trend - Improvement Noted 


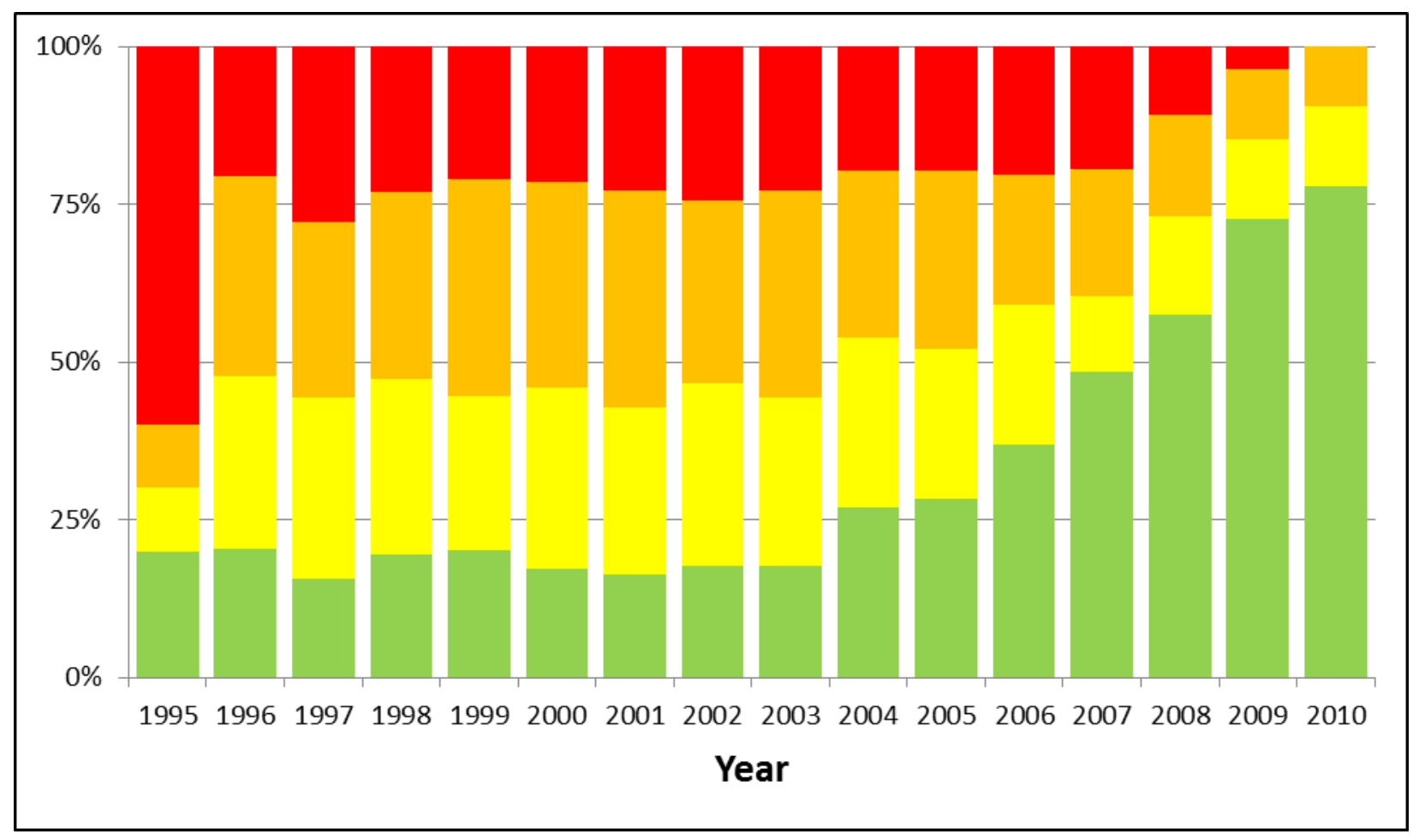

Figure B-9 - Element 108 Normalized Condition State Trend - Improvement Noted

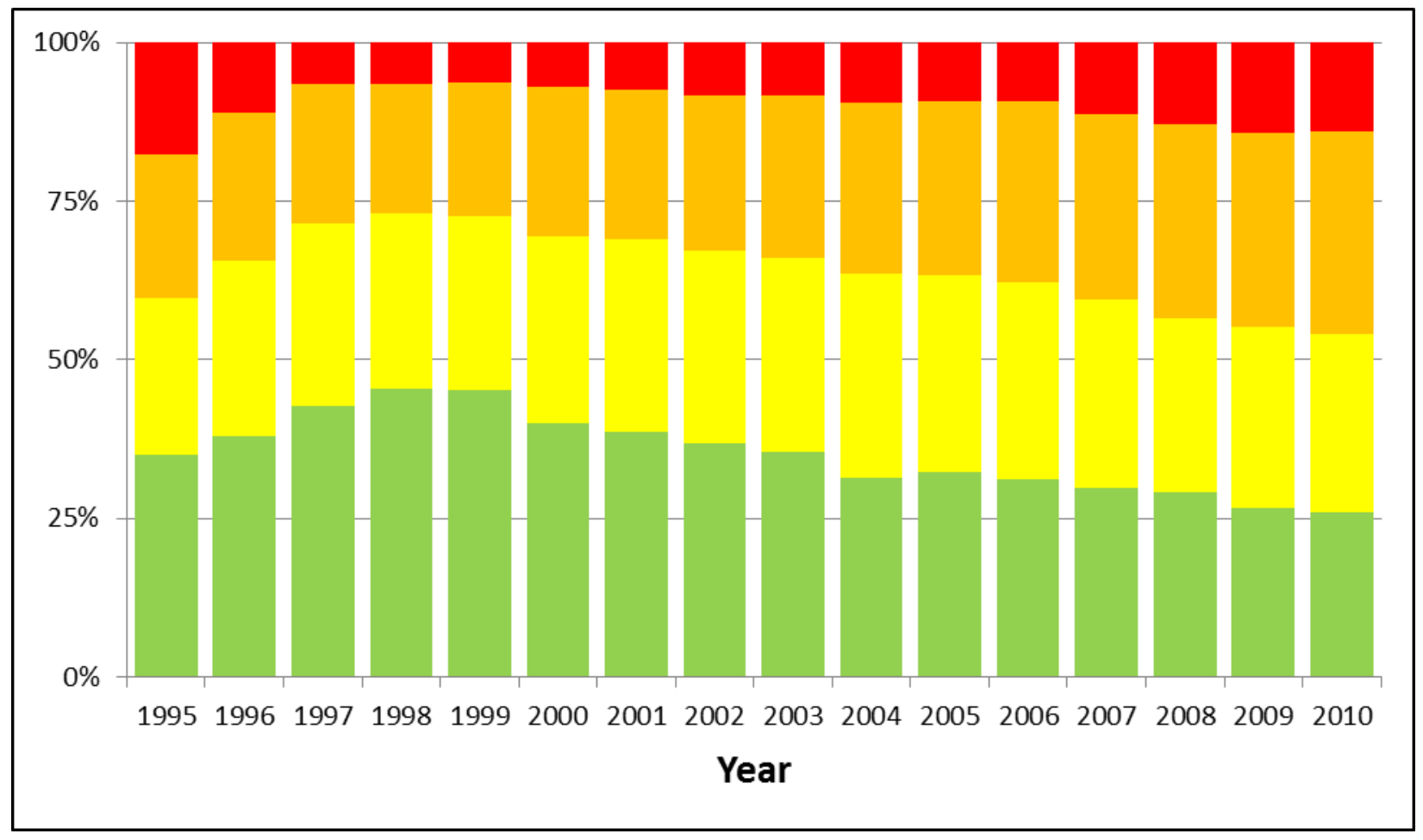

Figure B-10 - Element 108 Normalized Condition State Trend - Improvement Not Noted 


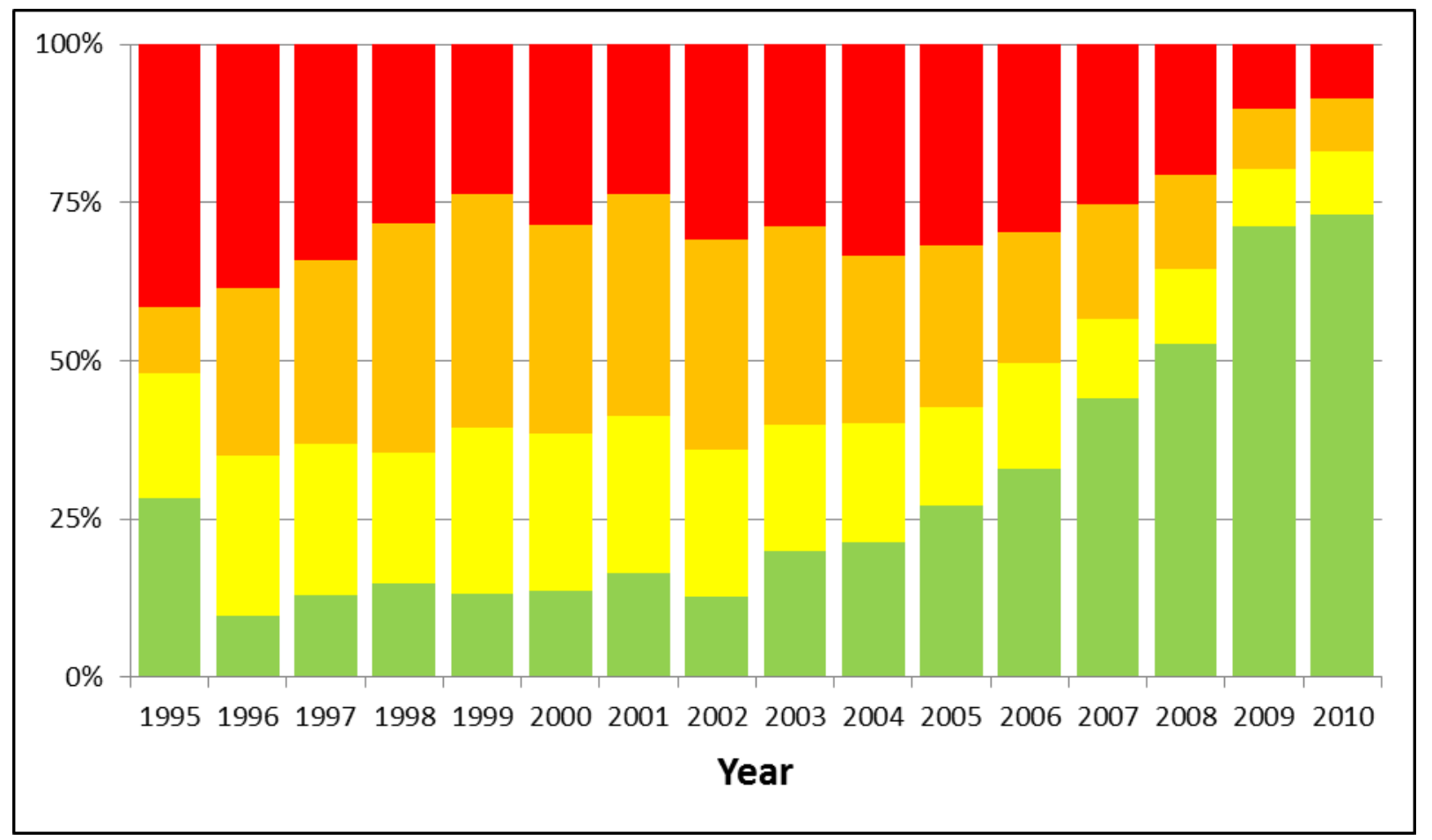

Figure B-11 - Element 108 Normalized Condition State Trend - Improvement Noted (Quantity Analysis)

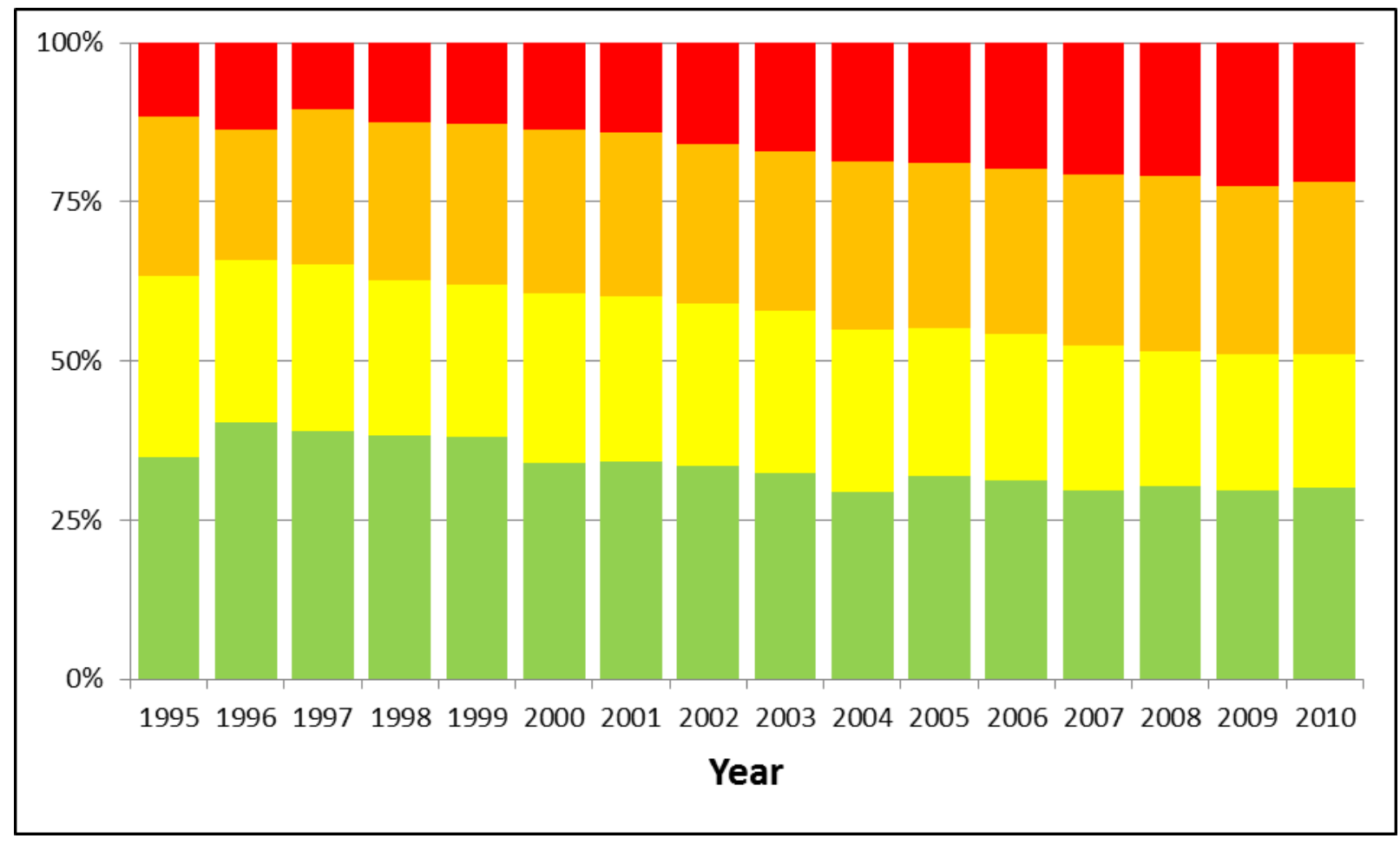

Figure B-12 - Element 108 Normalized Condition State Trend - Improvement Not Noted (Quantity Analysis) 


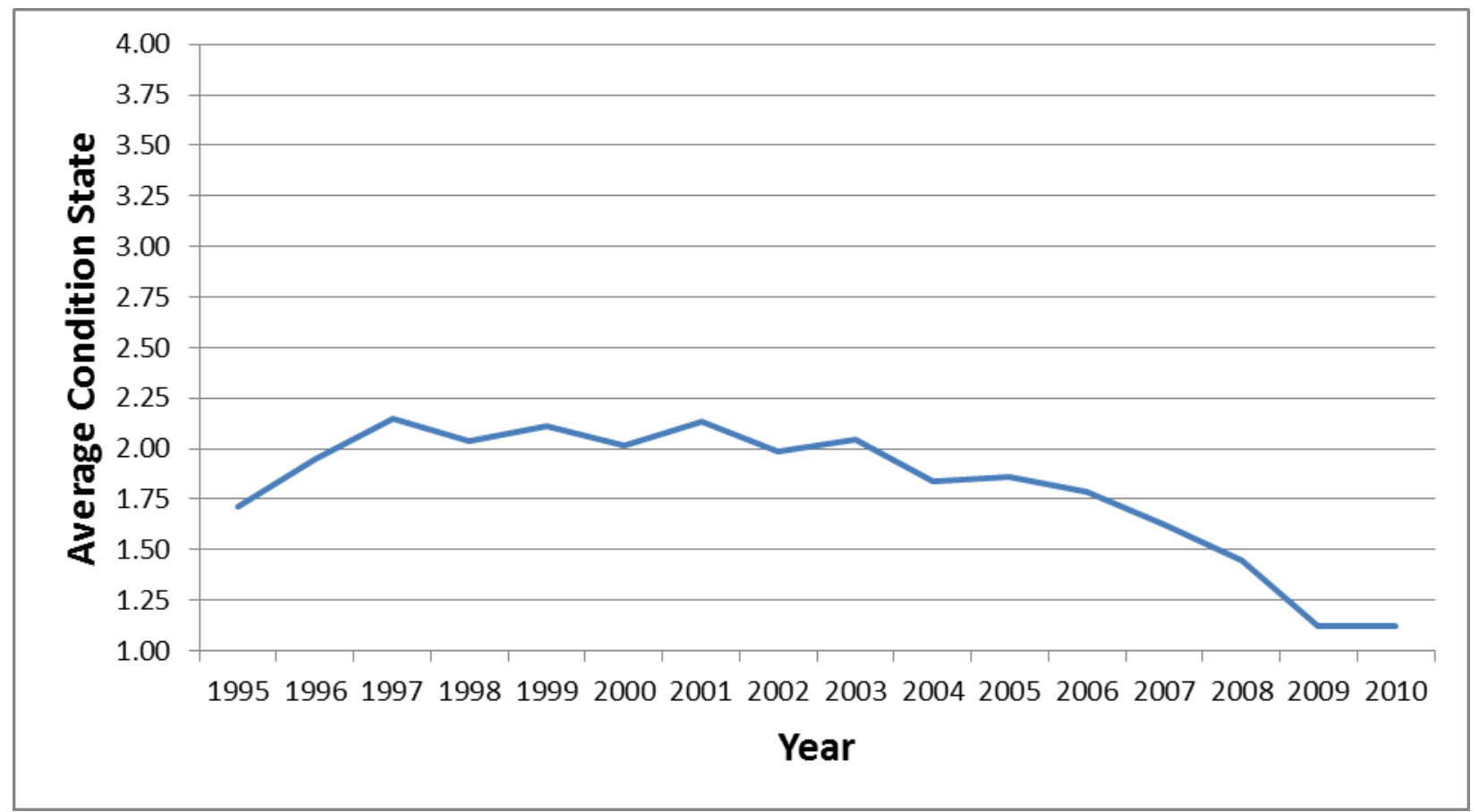

Figure B-13 - Element 302 (Compression Joint Seal) Average Condition State Trend - Improvement Noted

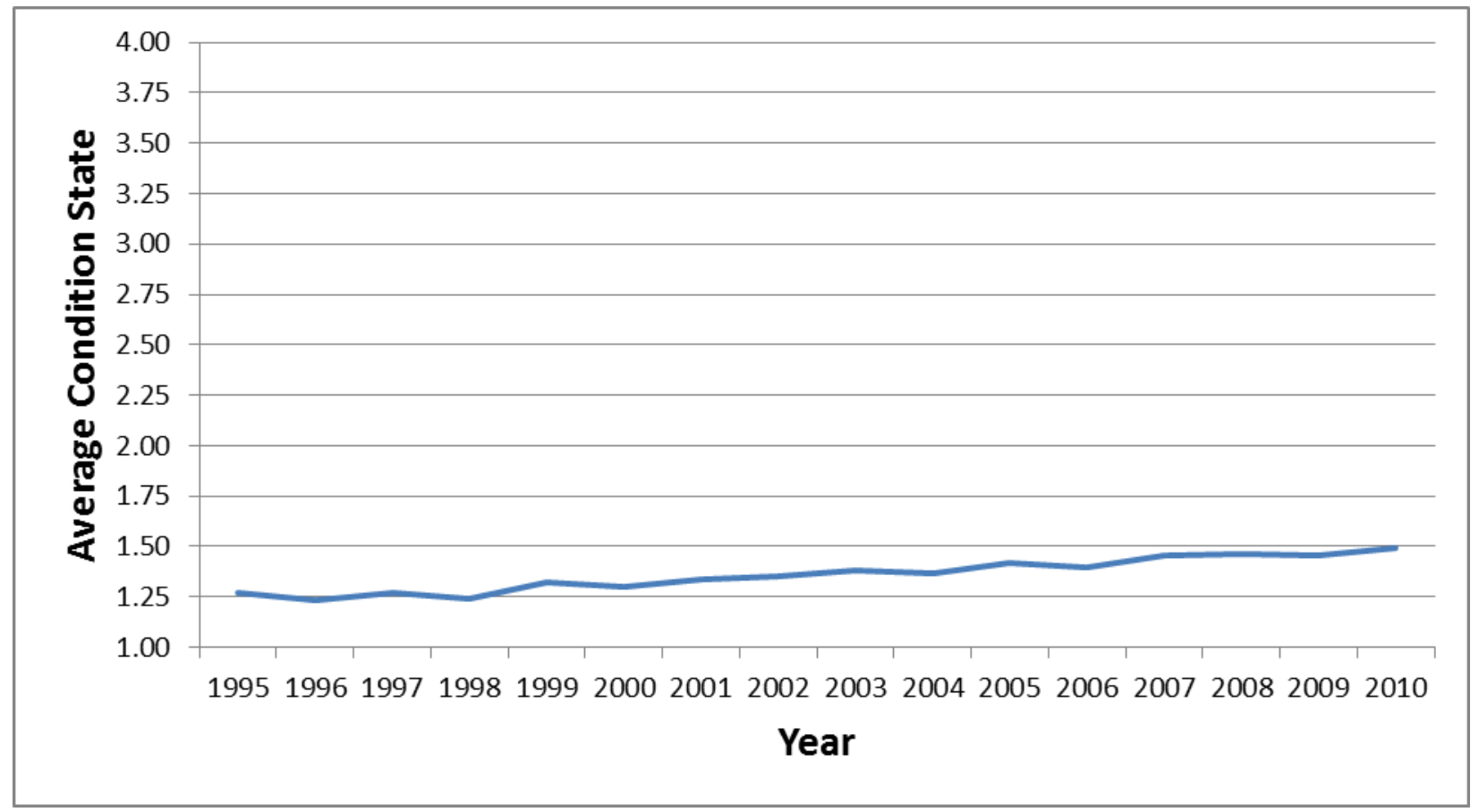

Figure B-14 - Element 302 Average Condition State Trend - Improvement Not Noted 


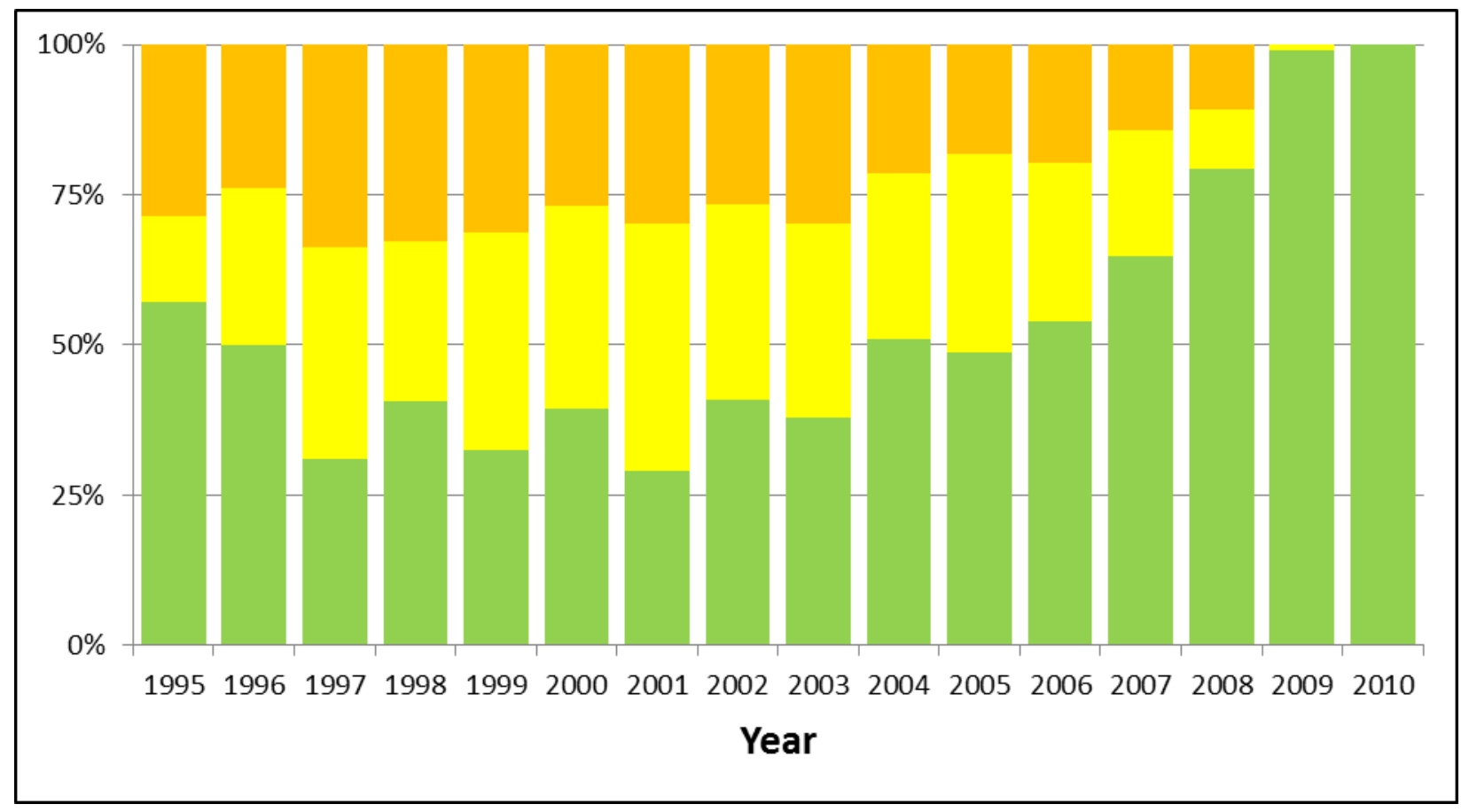

Figure B-15 - Element 302 Normalized Condition State Trend - Improvement Noted

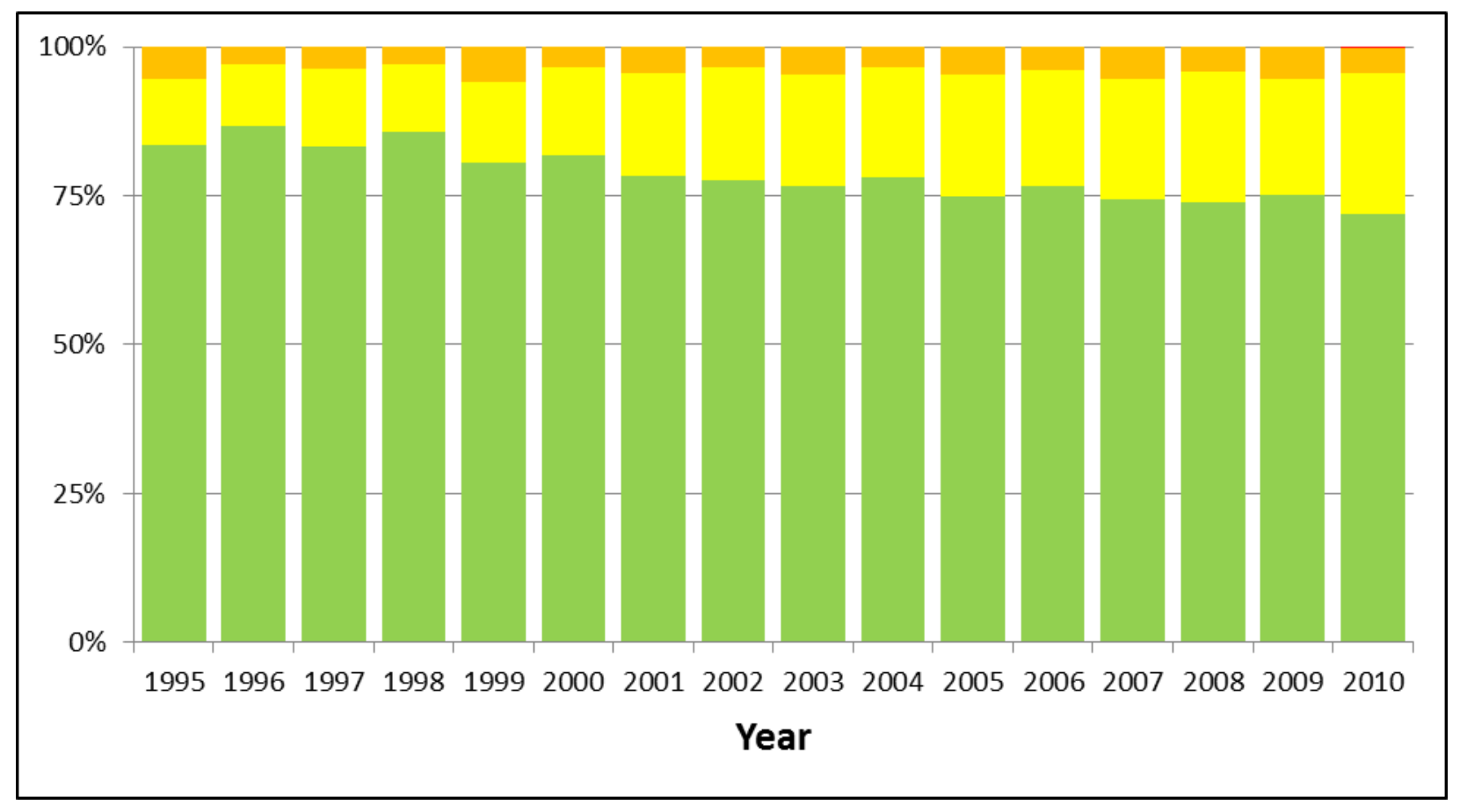

Figure B-16 - Element 302 Normalized Condition State Trend - Improvement Not Noted 


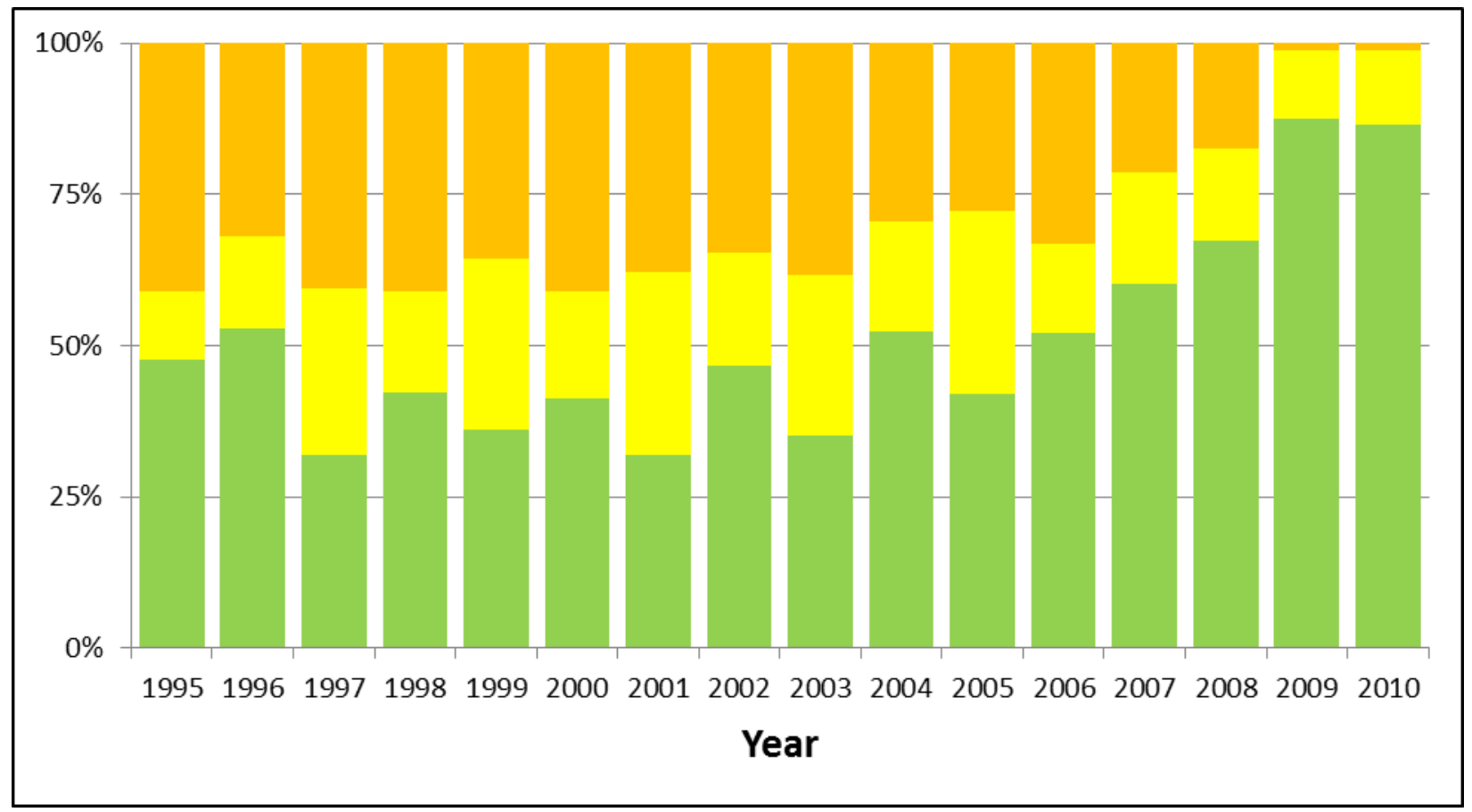

Figure B-17 - Element 302 Normalized Condition State Trend - Improvement Noted (Quantity Analysis)

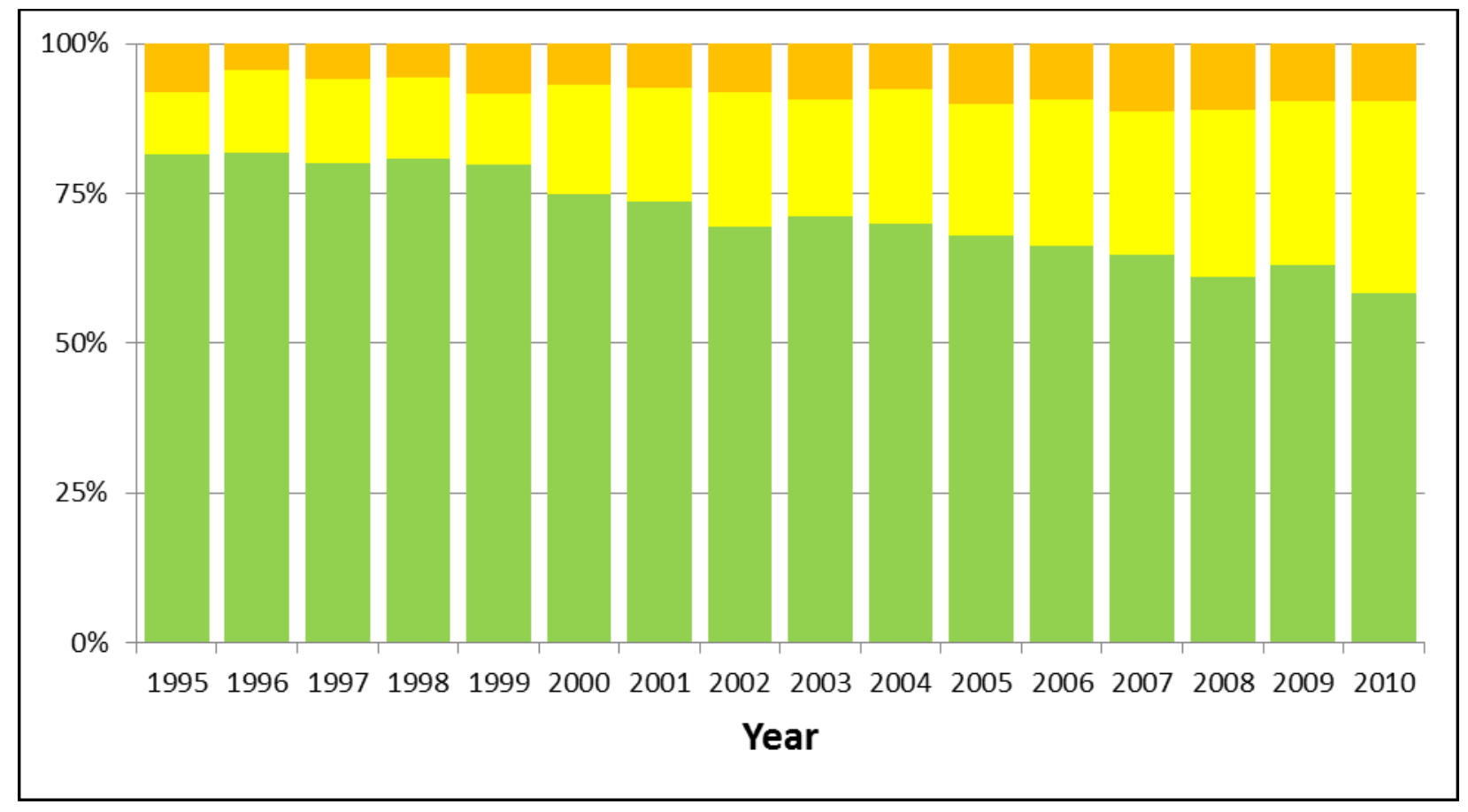

Figure B-18 - Element 302 Normalized Condition State Trend - Improvement Not Noted (Quantity Analysis) 


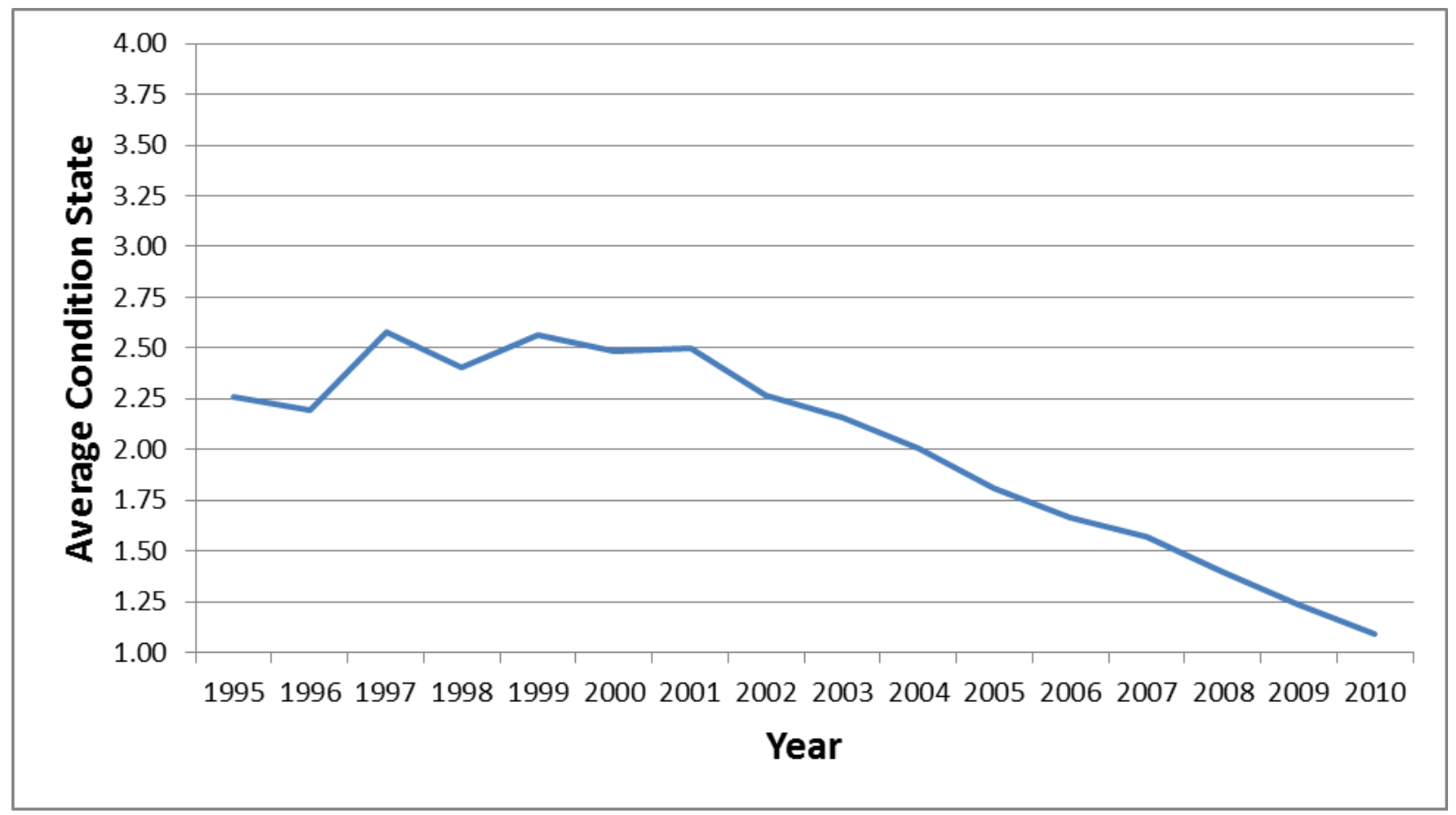

Figure B-19 - Element 334 (Metal Bridge Railing - Coating) Average Condition State Trend - Improvement Noted

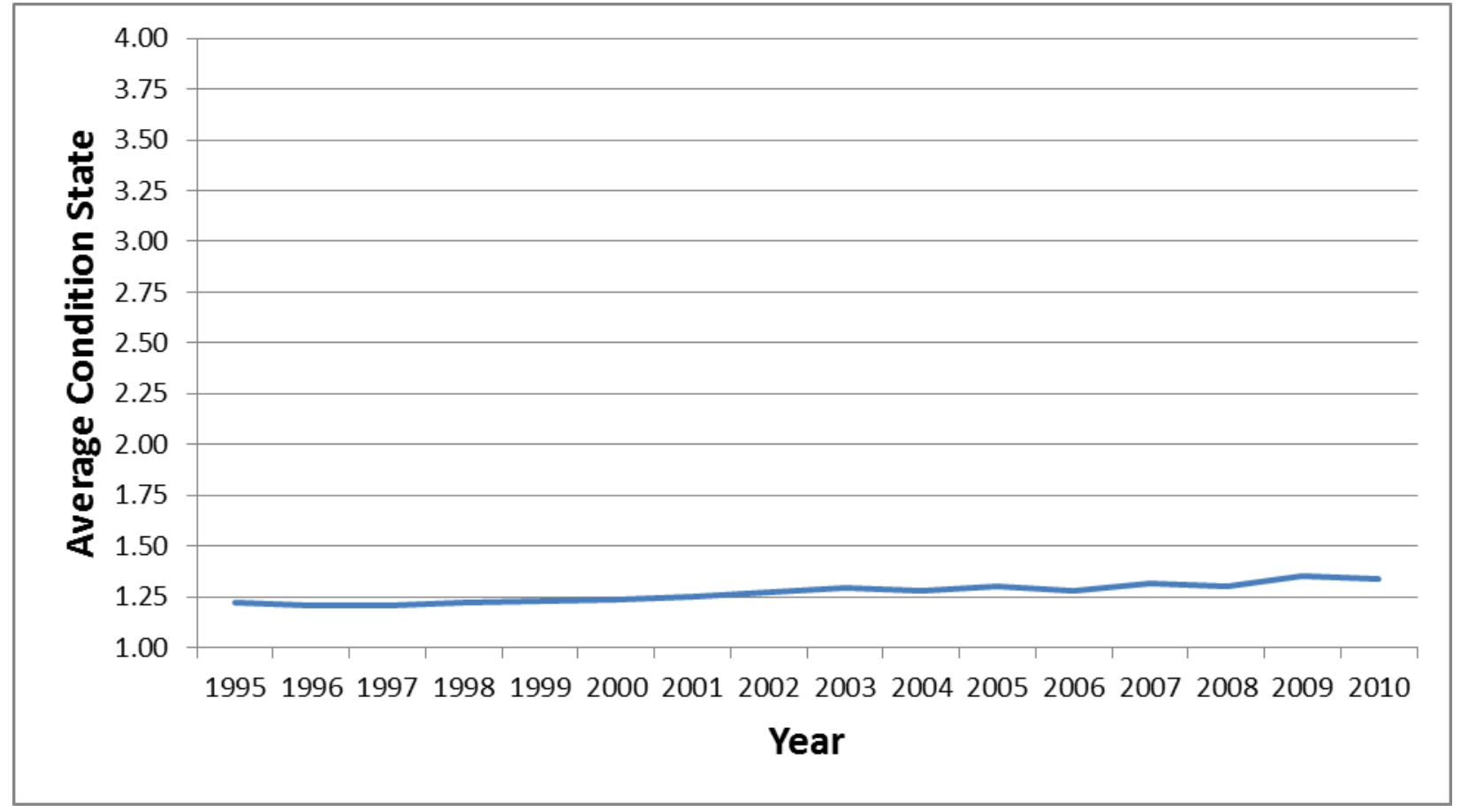

Figure B-20 - Element 334 Average Condition State Trend - Improvement Not Noted 


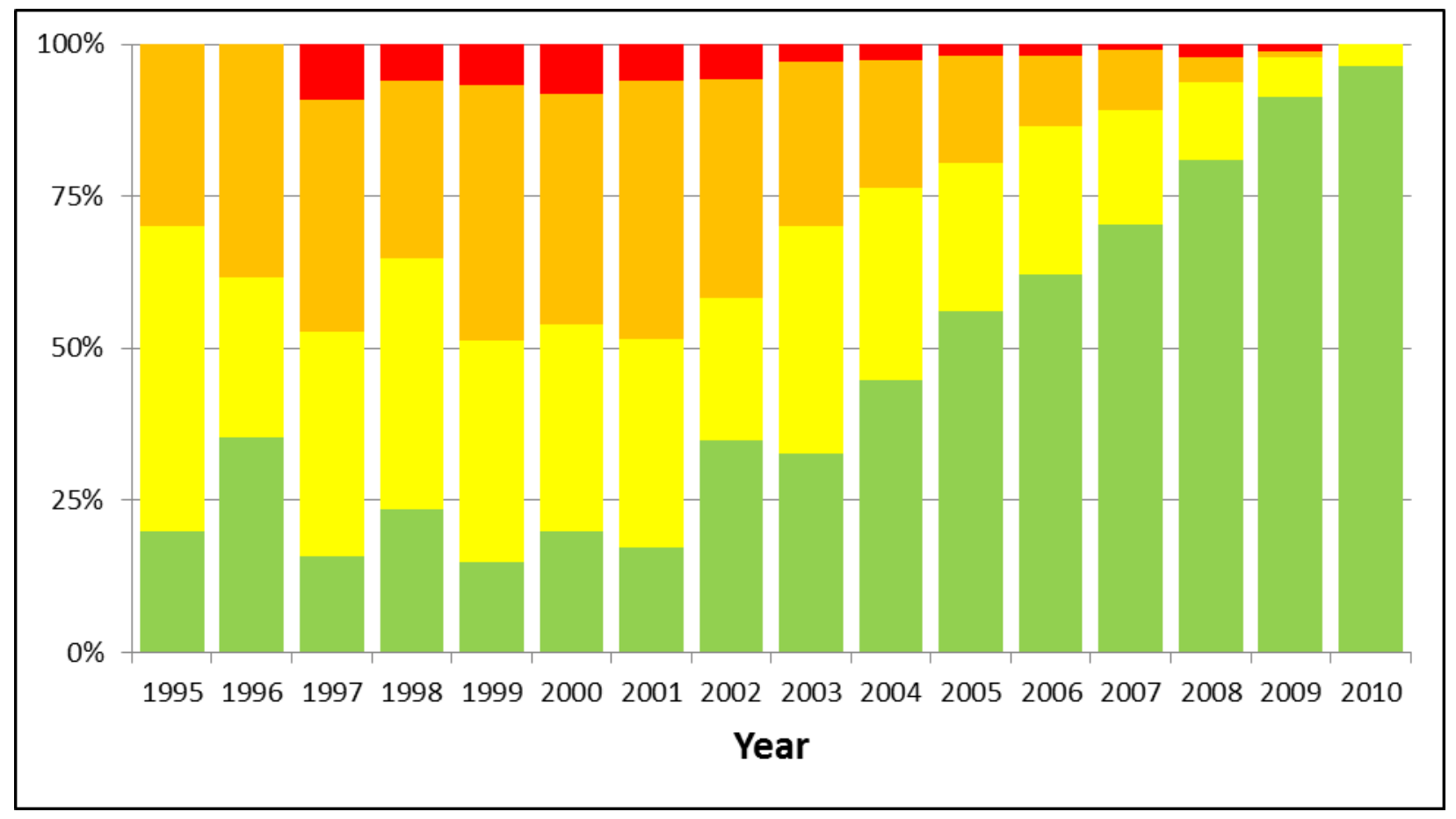

Figure B-21 - Element 334 Normalized Condition State Trend - Improvement Noted

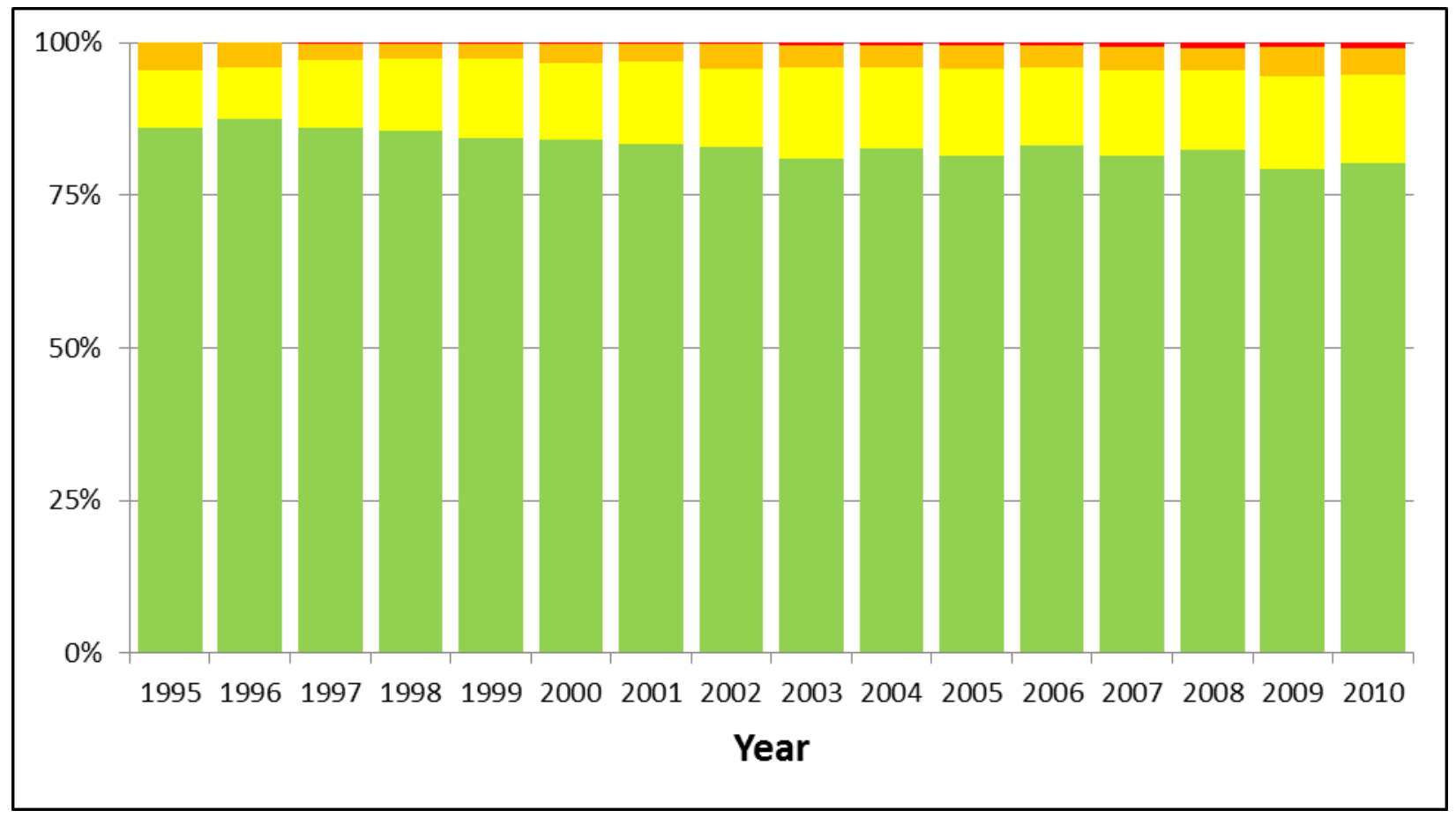

Figure B-22 - Element 334 Normalized Condition State Trend - Improvement Not Noted 


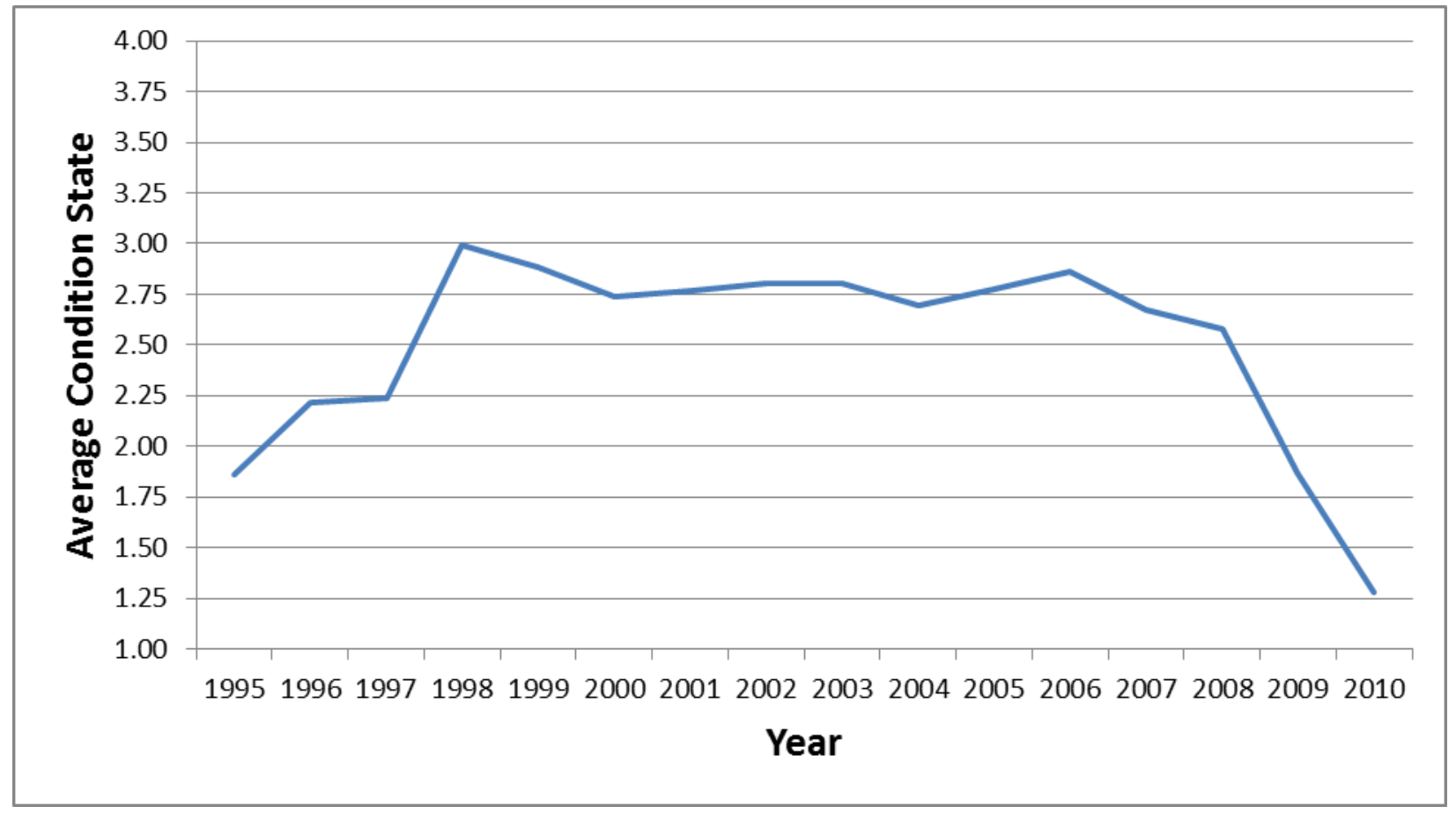

Figure B-23 - Element 240 (Metal Culvert) Average Condition State Trend - Improvement Noted

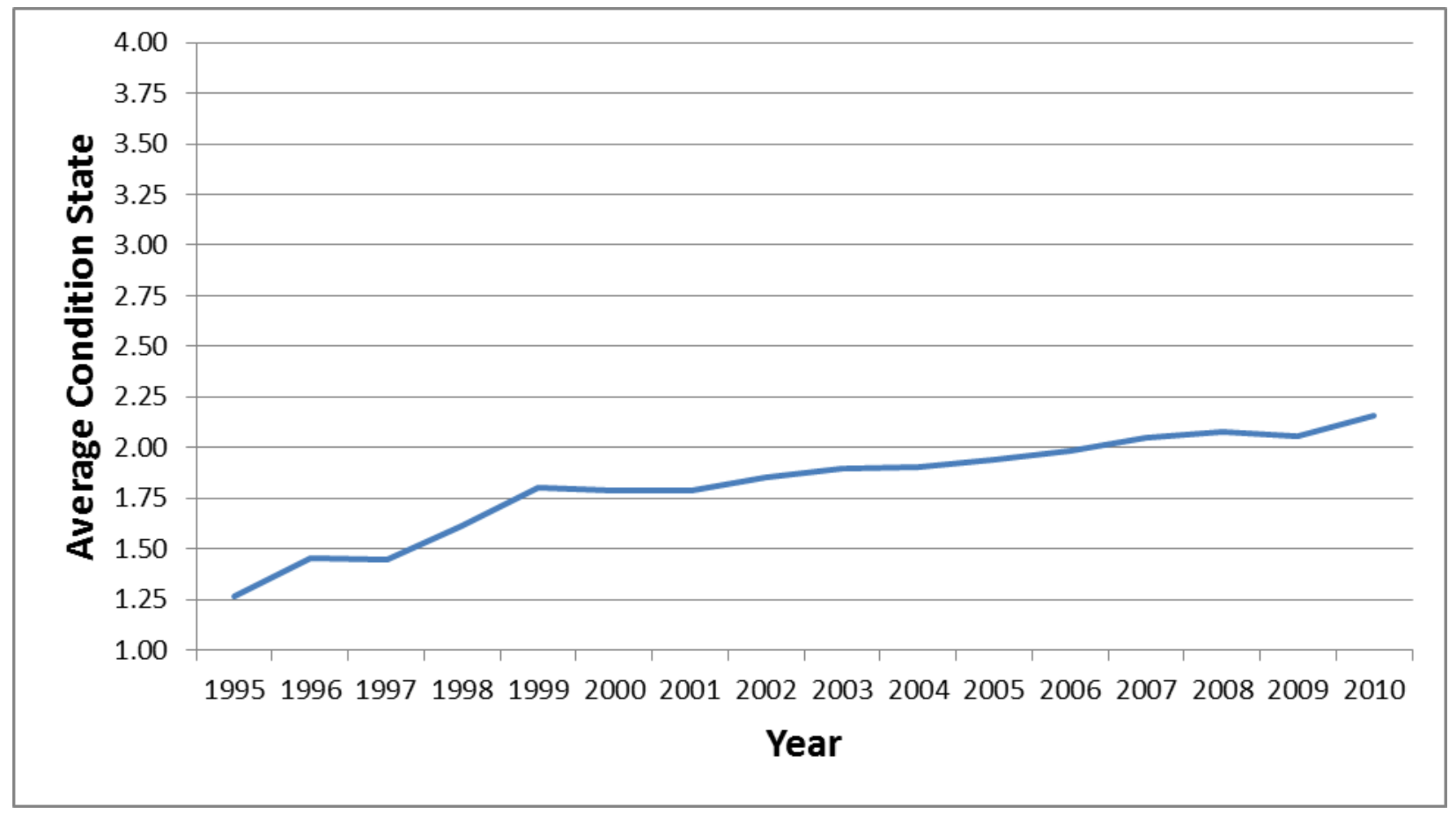

Figure B-24 - Element 240 Average Condition State Trend - Improvement Not Noted 


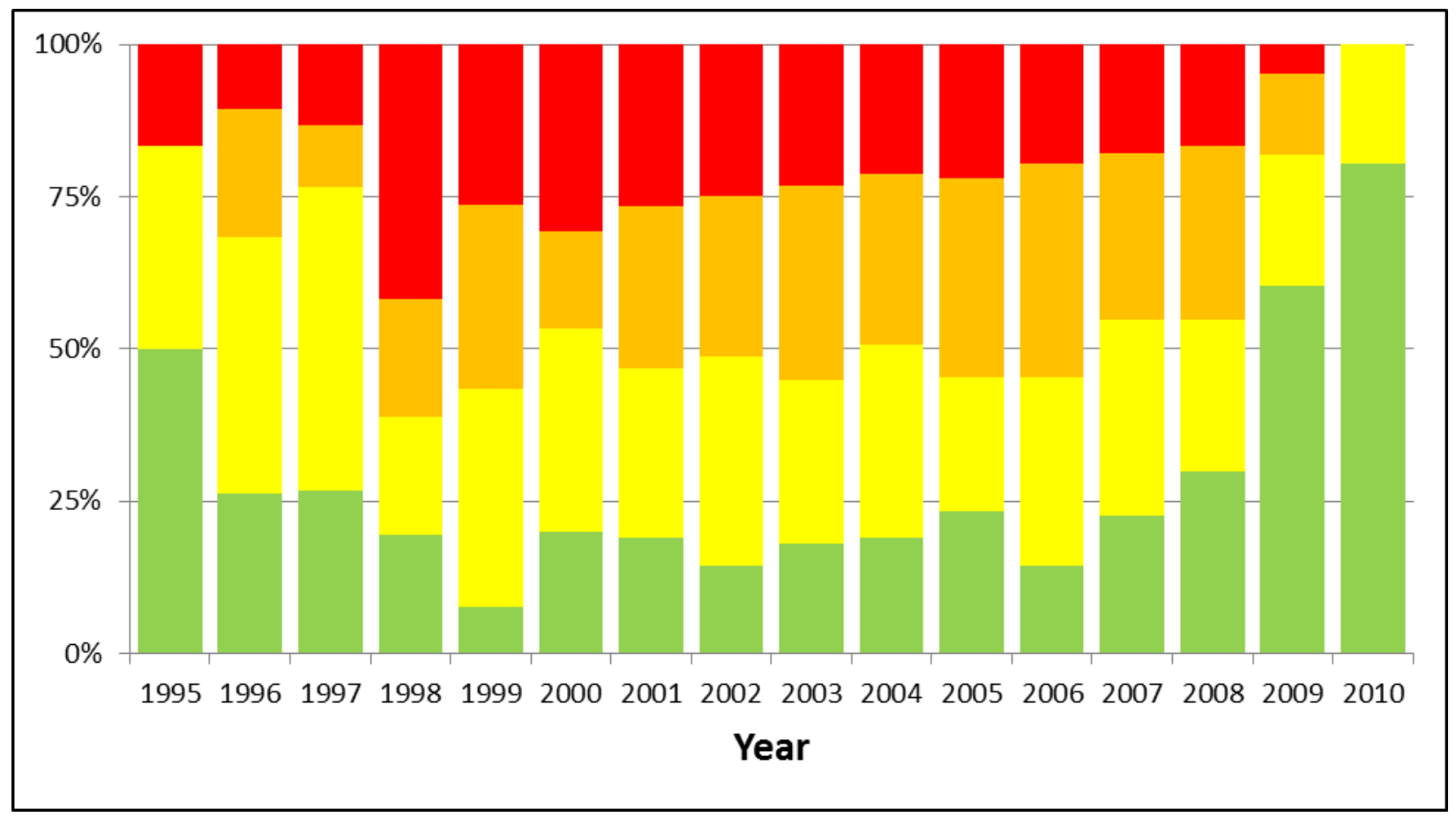

Figure B-25 - Element 240 Normalized Condition State Trend - Improvement Noted

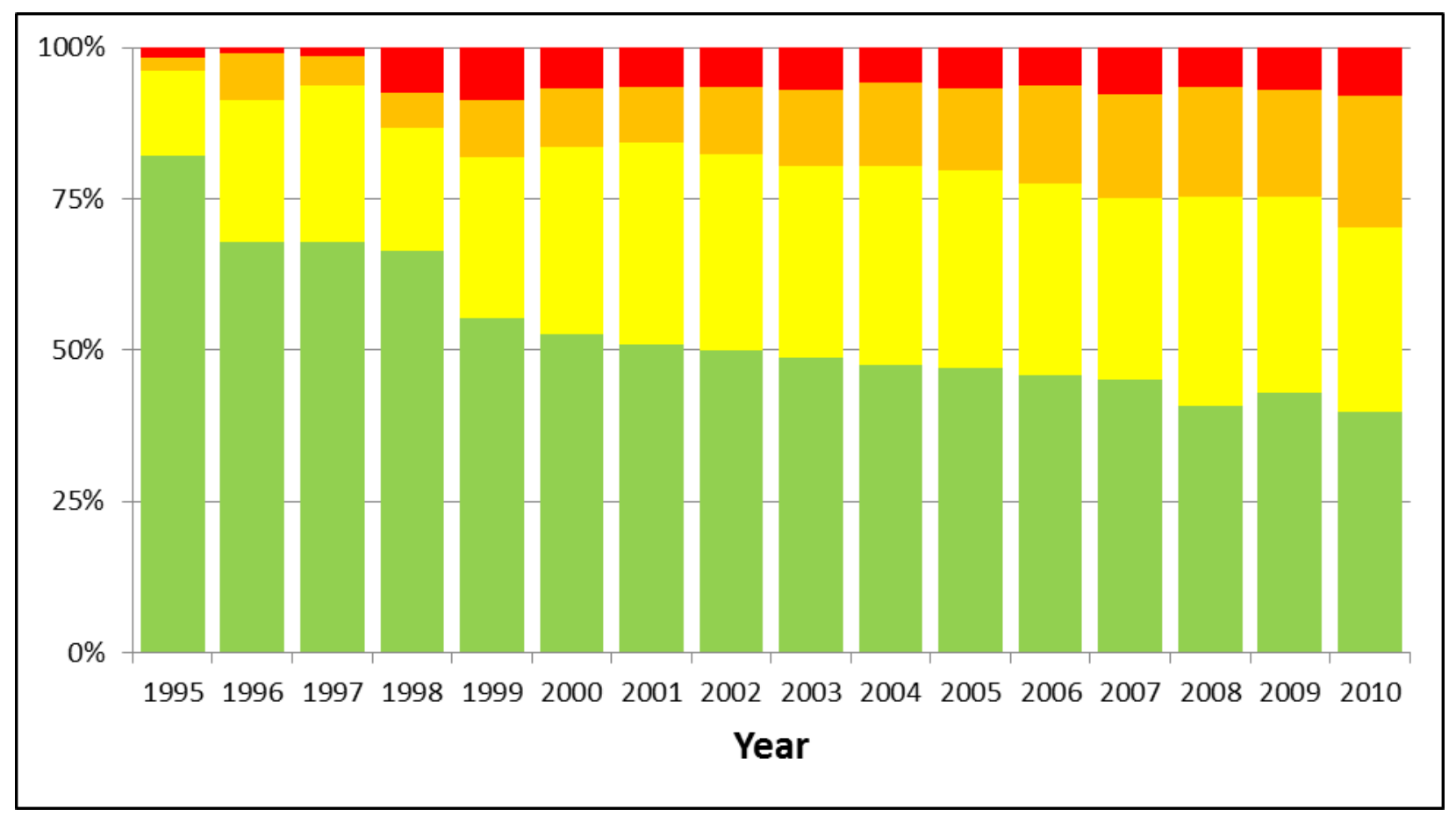

Figure B-26 - Element 240 Normalized Condition State Trend - Improvement Not Noted 


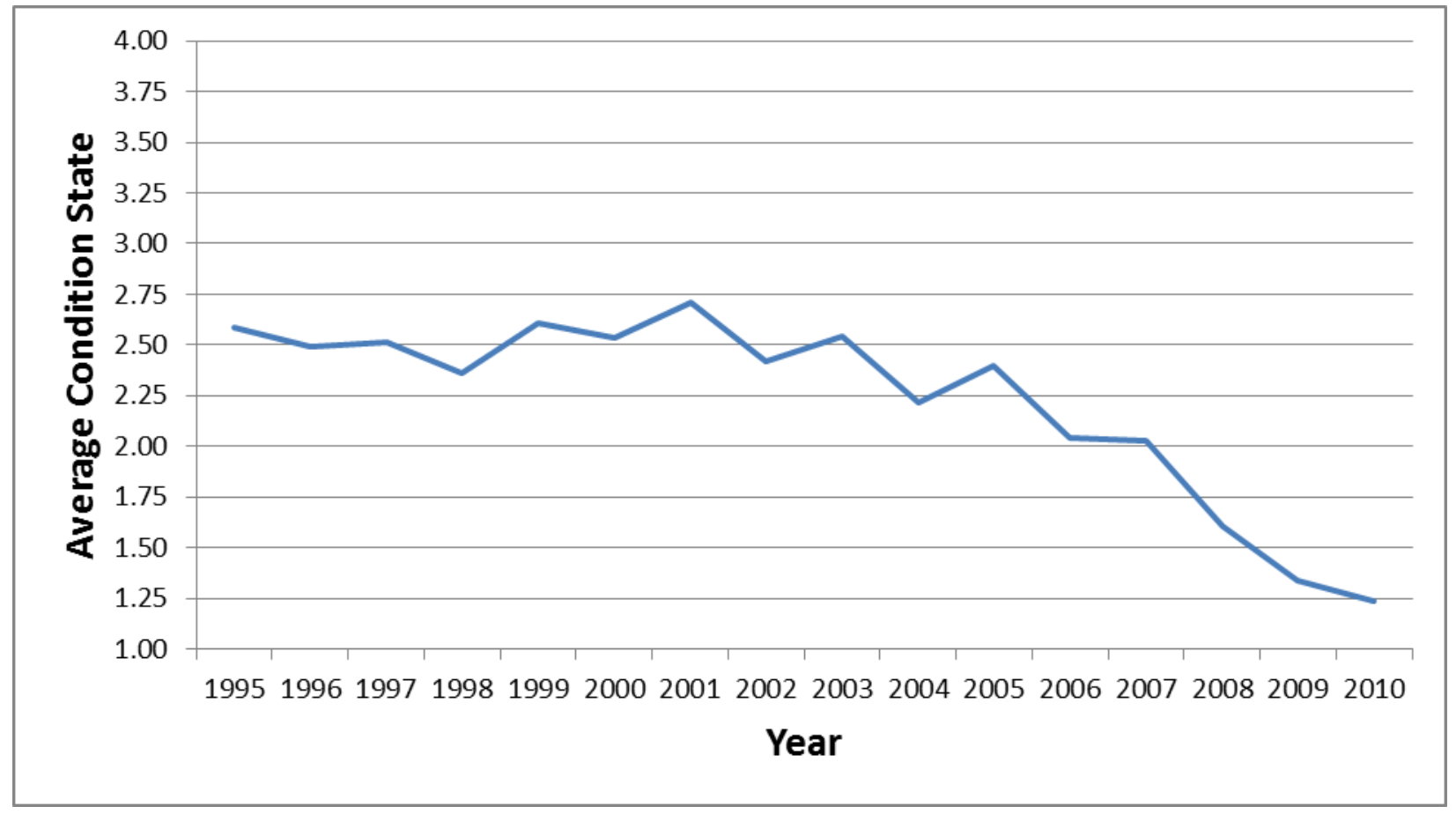

Figure B-27 - Element 107 (Steel Open Girder - Coated) Average Condition State Trend - Improvement Noted

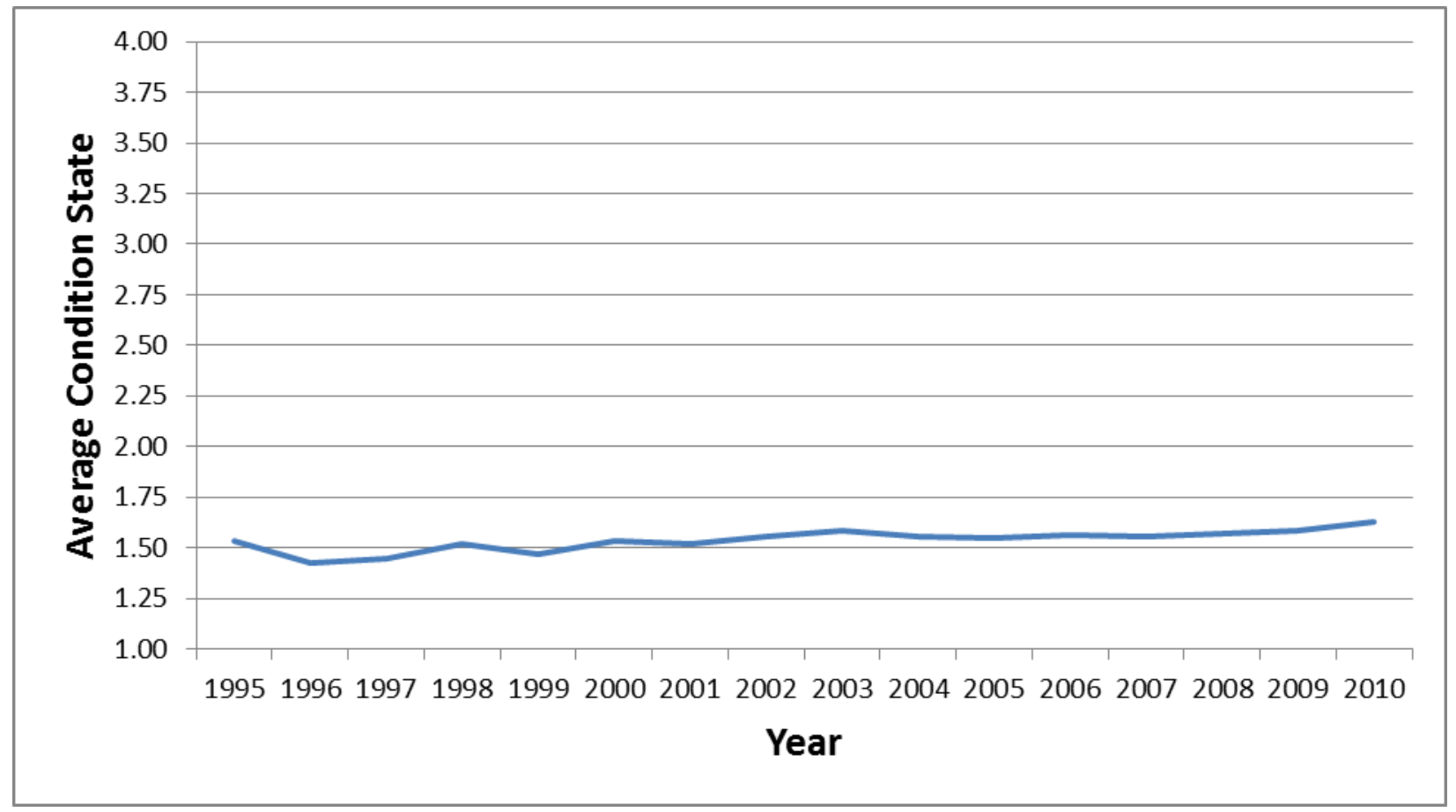

Figure B-28 - Element 107 Average Condition State Trend - Improvement Not Noted 


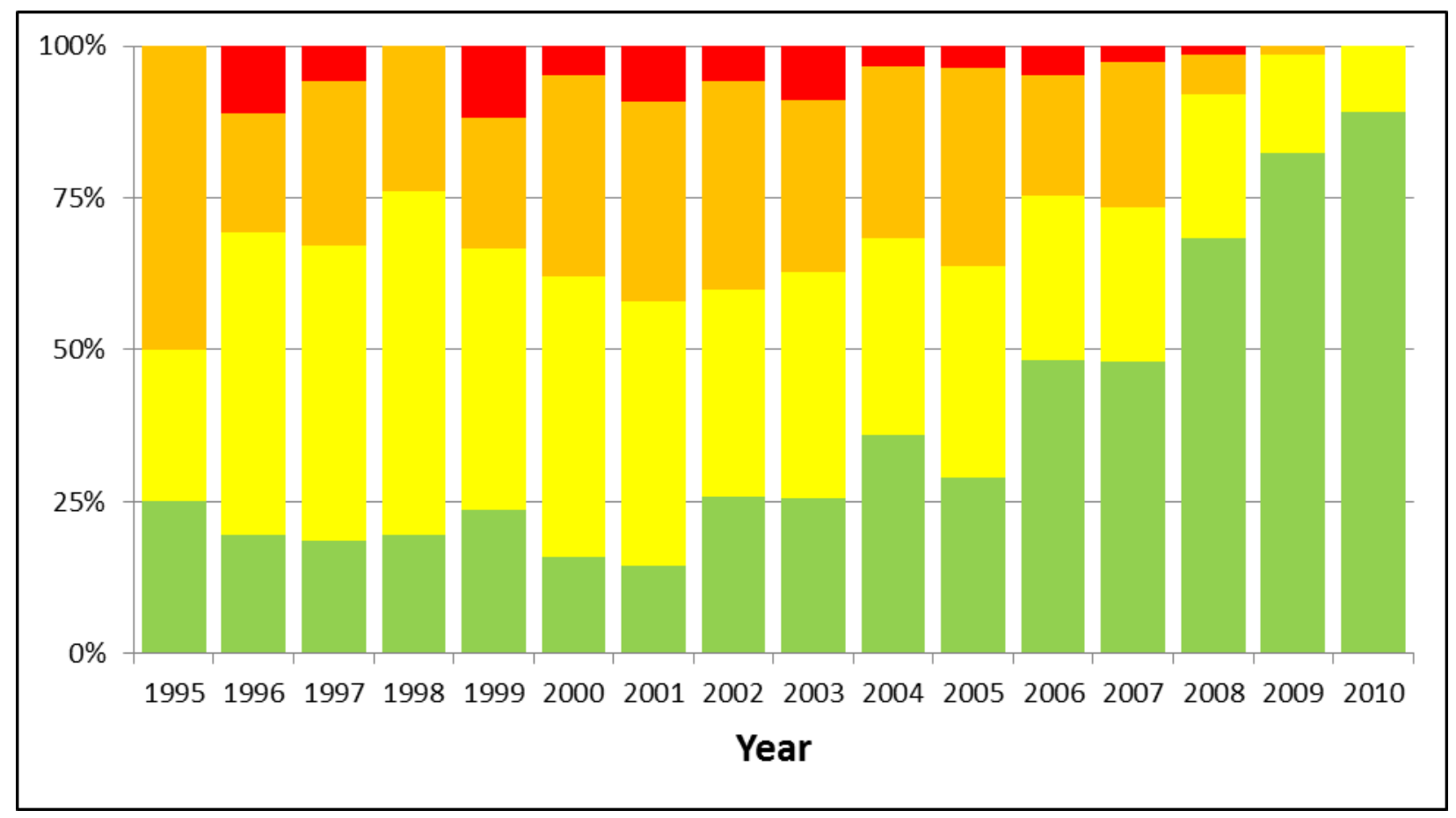

Figure B-29 - Element 107 Normalized Condition State Trend - Improvement Noted

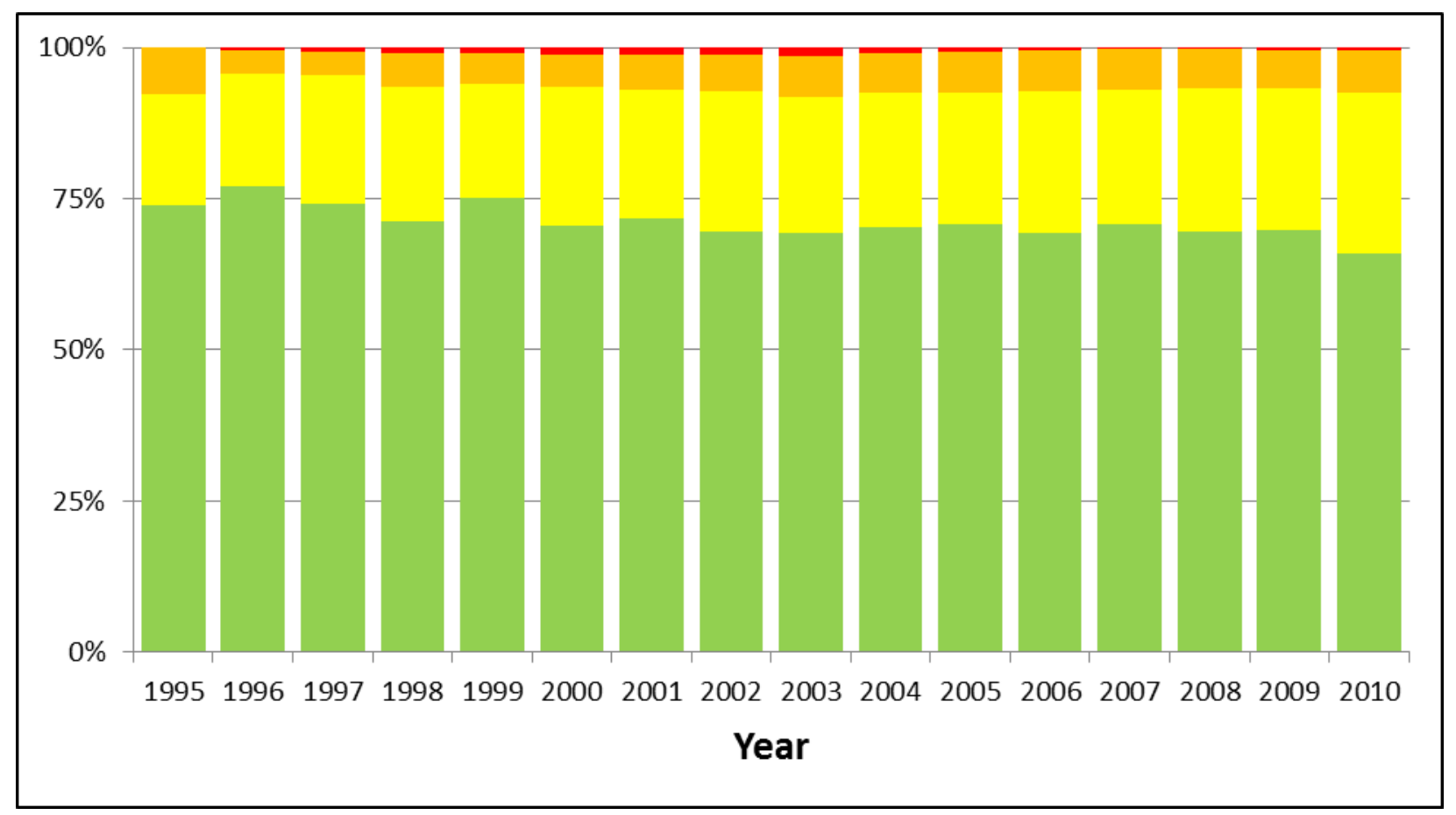

Figure B-30 - Element 107 Normalized Condition State Trend - Improvement Not Noted 


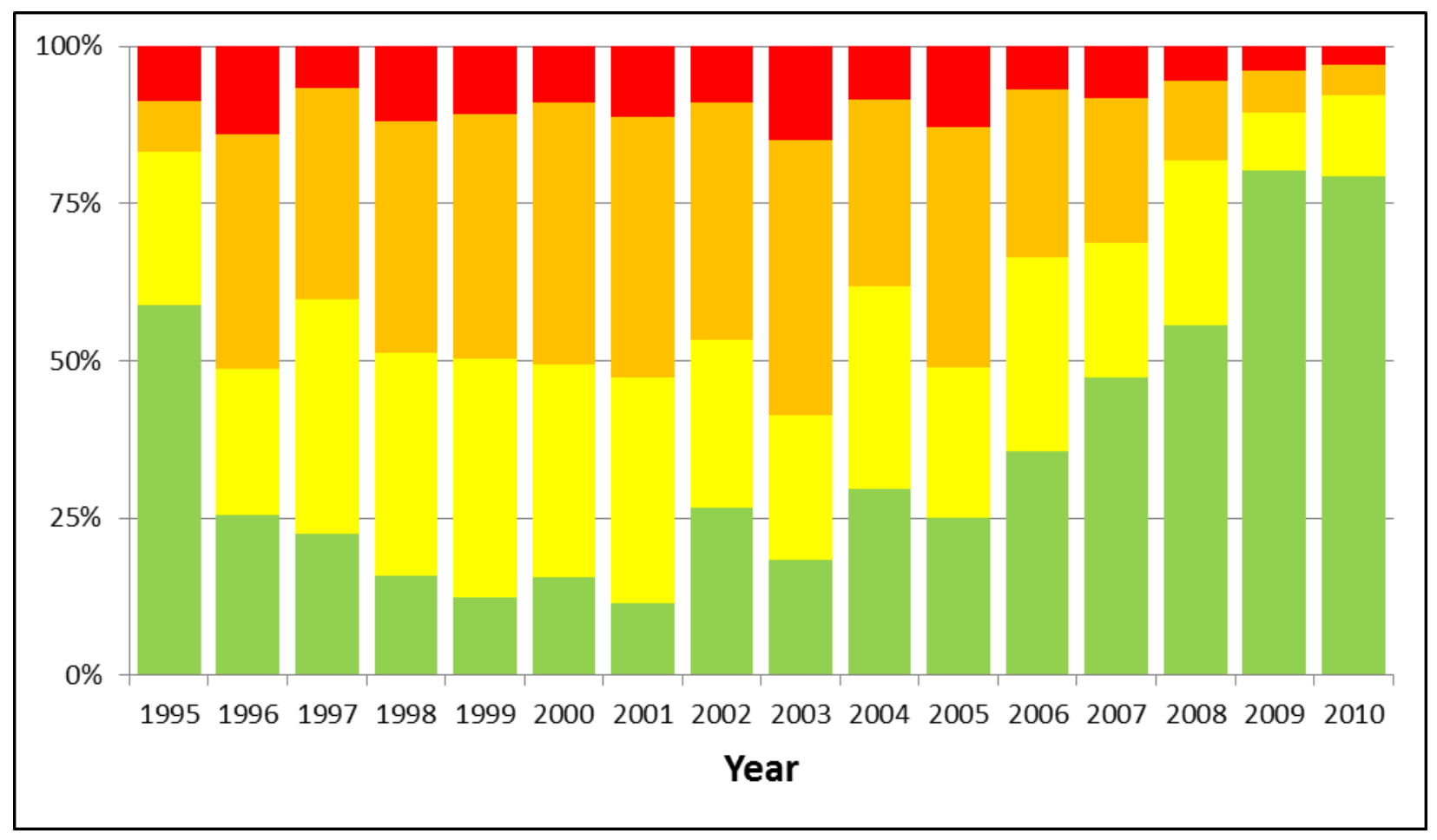

Figure B-31 - Element 107 Normalized Condition State Trend - Improvement Noted (Quantity Analysis)

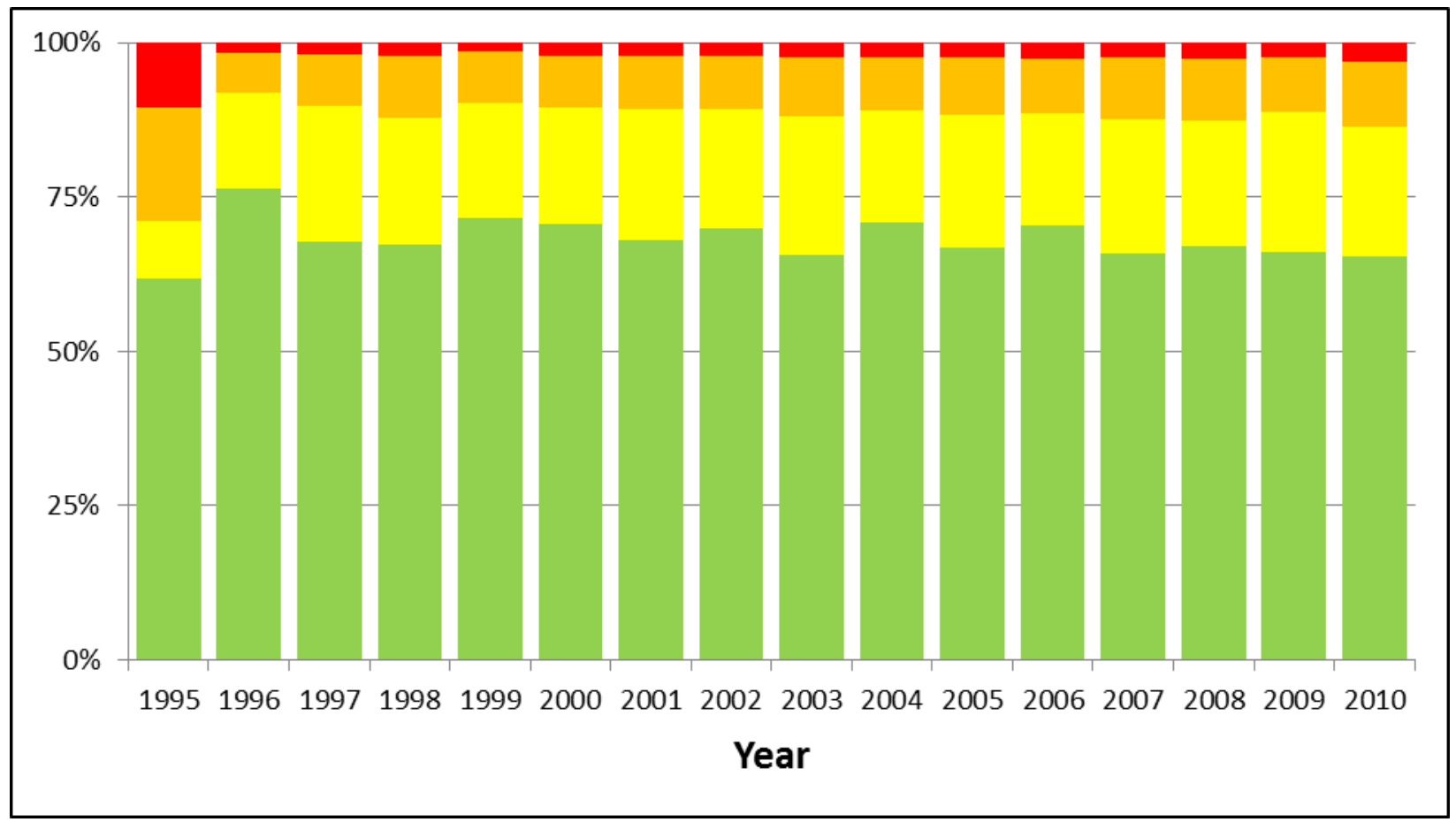

Figure B-32 - Element 107 Normalized Condition State Trend - Improvement Not Noted (Quantity Analysis) 


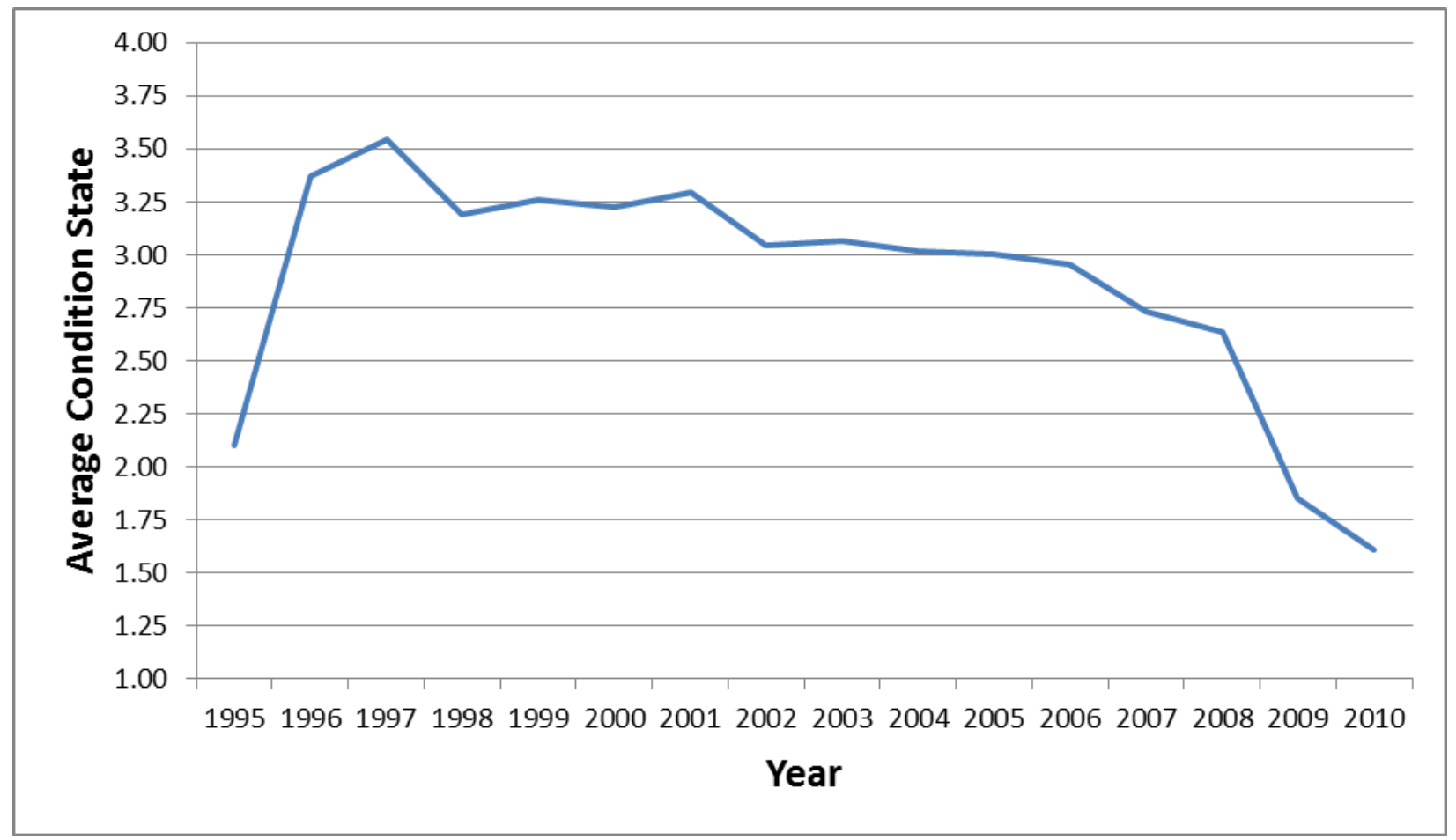

Figure B-33 - Smart Flag 359 (Soffit of Concrete) Average Condition State Trend - Improvement Noted

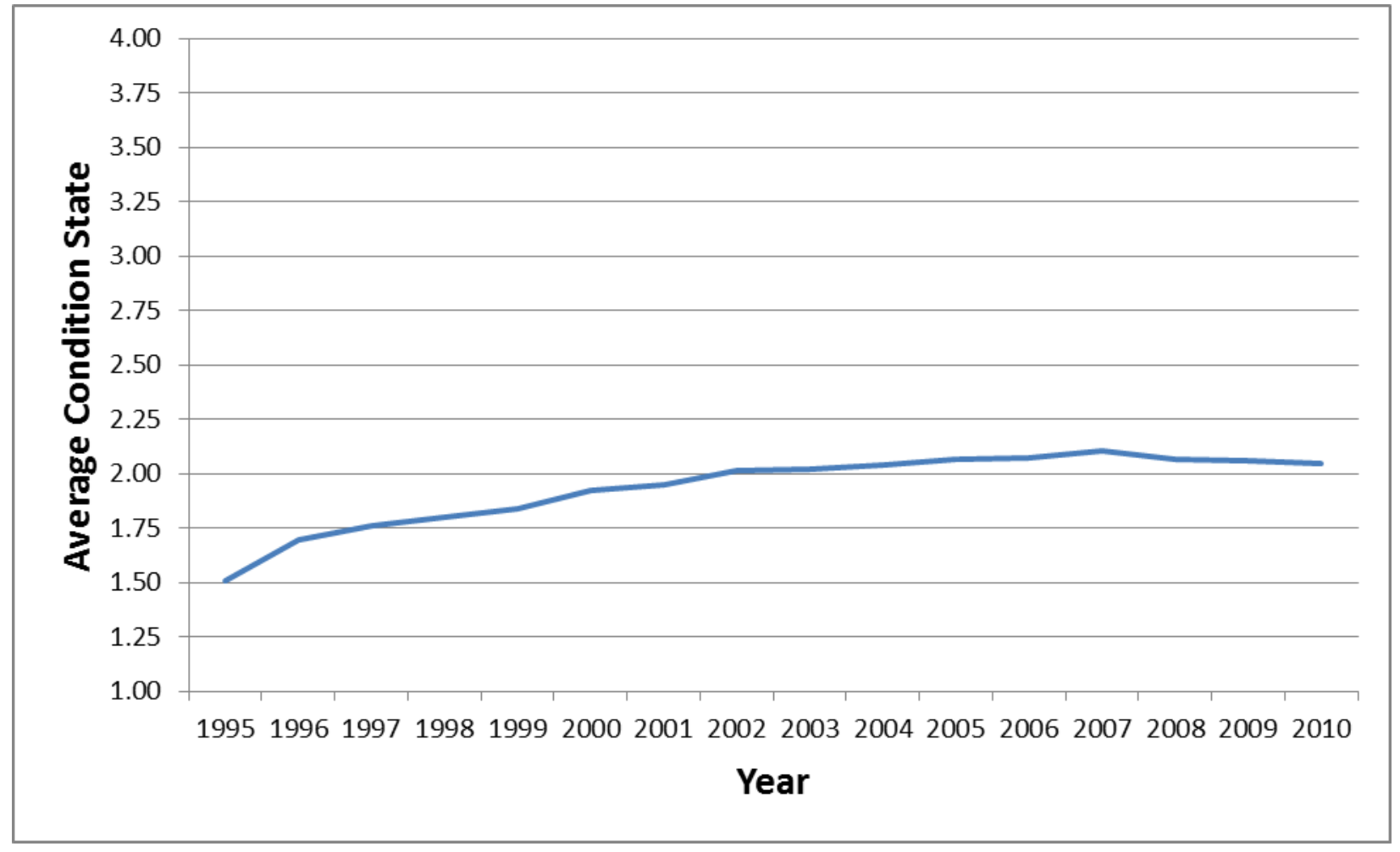

Figure B-34 - Smart Flag 359 Average Condition State Trend - Improvement Not Noted 


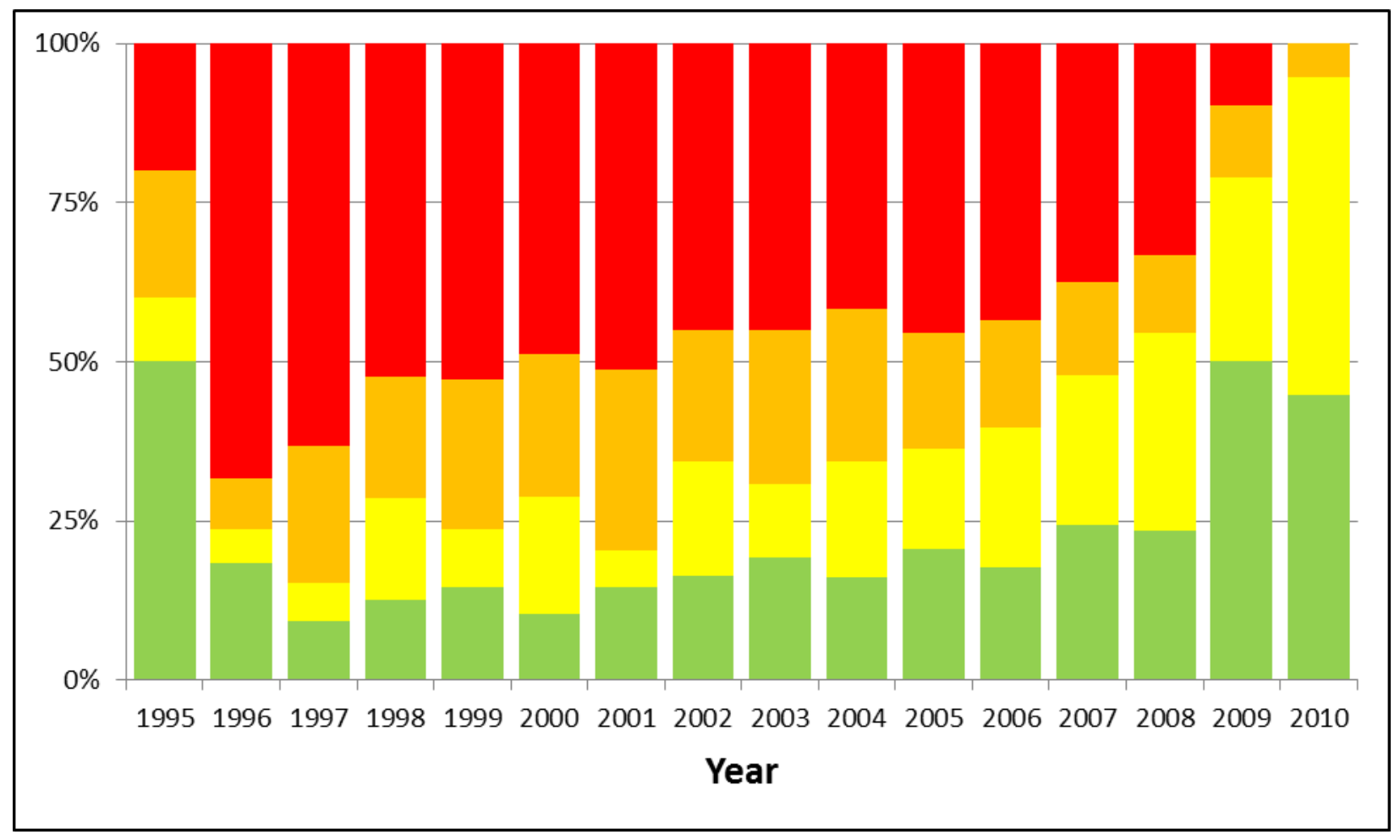

Figure B-35 - Smart Flag 359 Normalized Condition State Trend - Improvement Noted

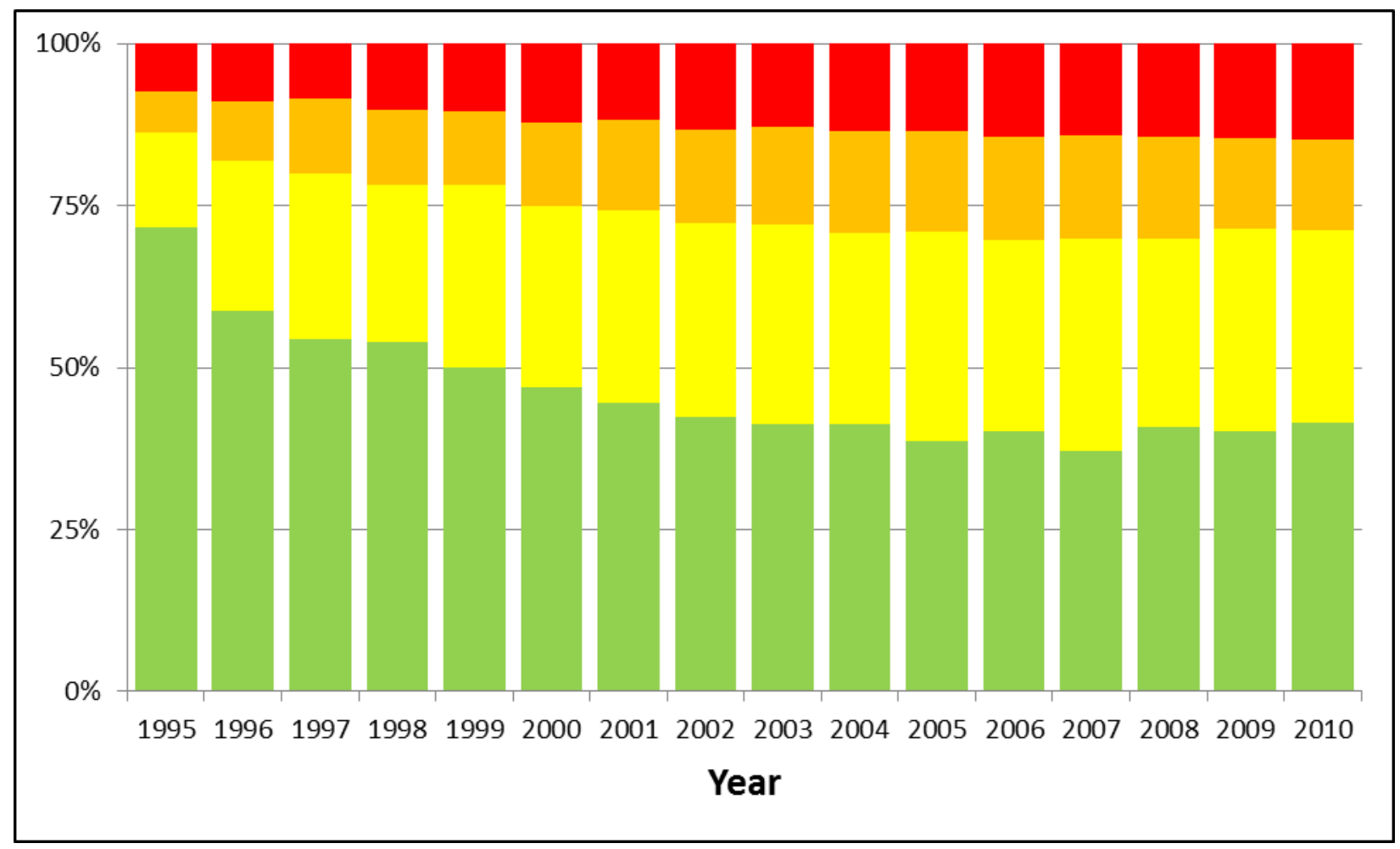

Figure B-36 - Smart Flag 359 Normalized Condition State Trend - Improvement Not Noted 


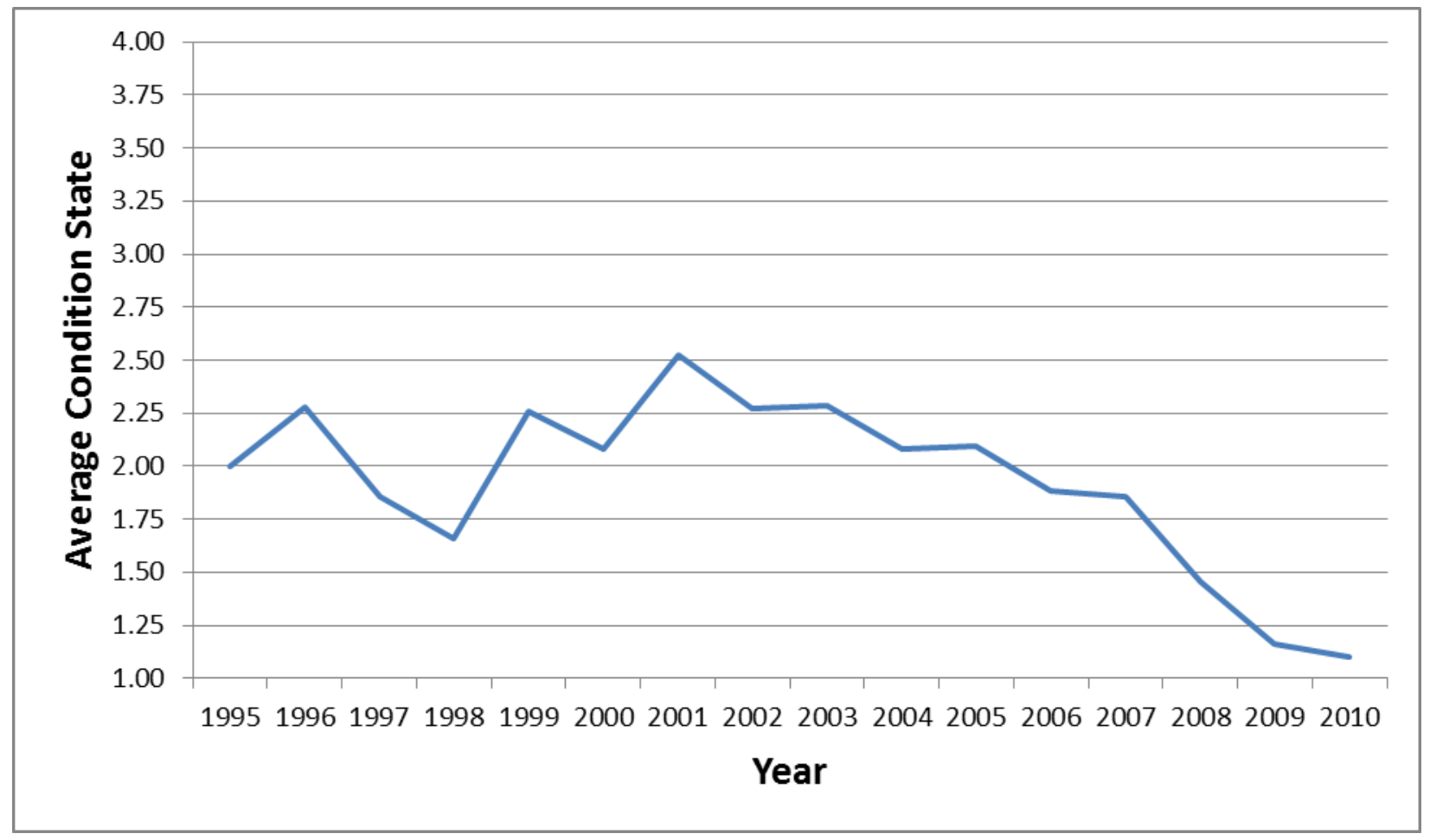

Figure B-37 - Smart Flag 702 (Drains) Average Condition State Trend - Improvement Noted

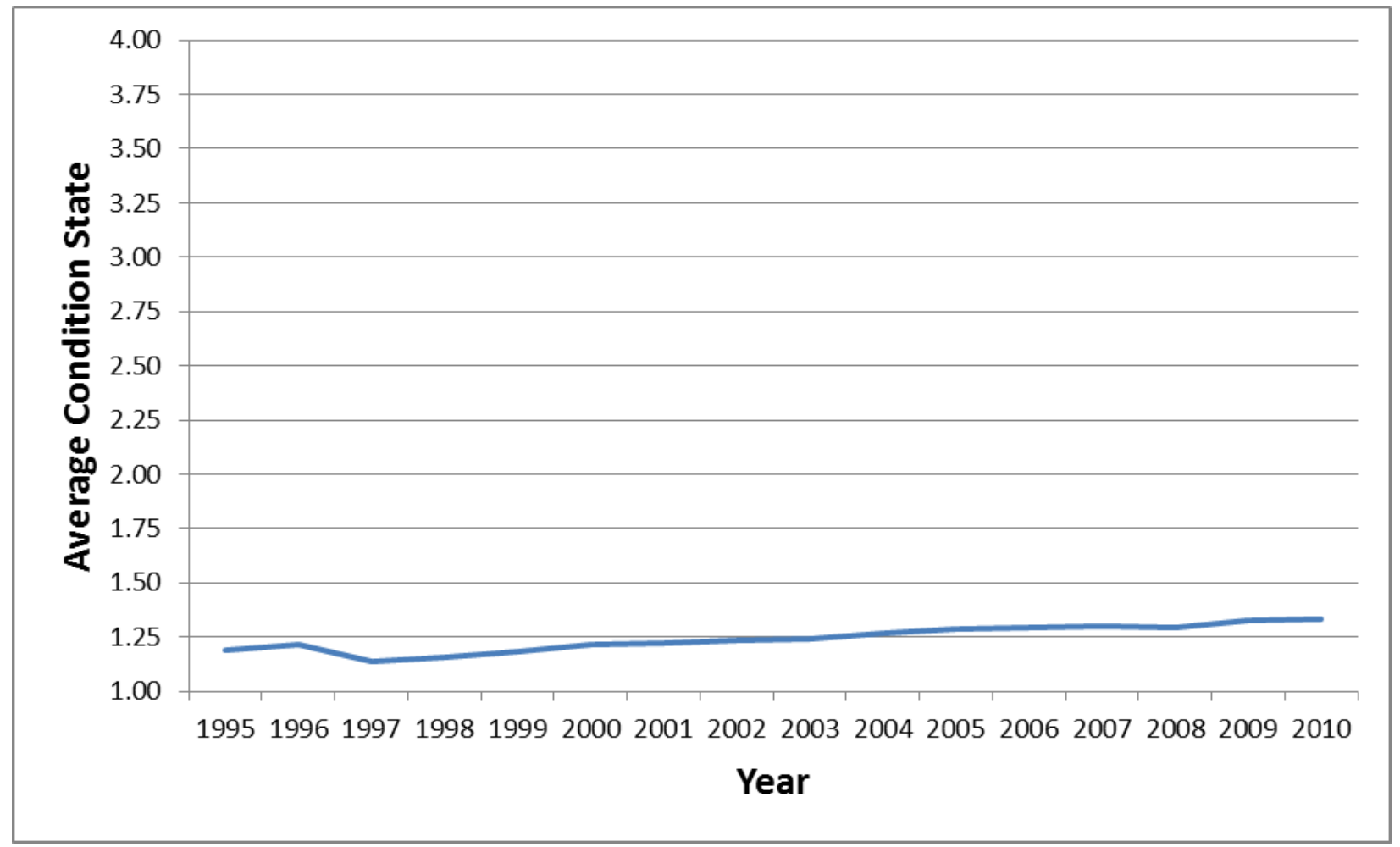

Figure B-38 - Smart Flag 702 Average Condition State Trend - Improvement Not Noted 


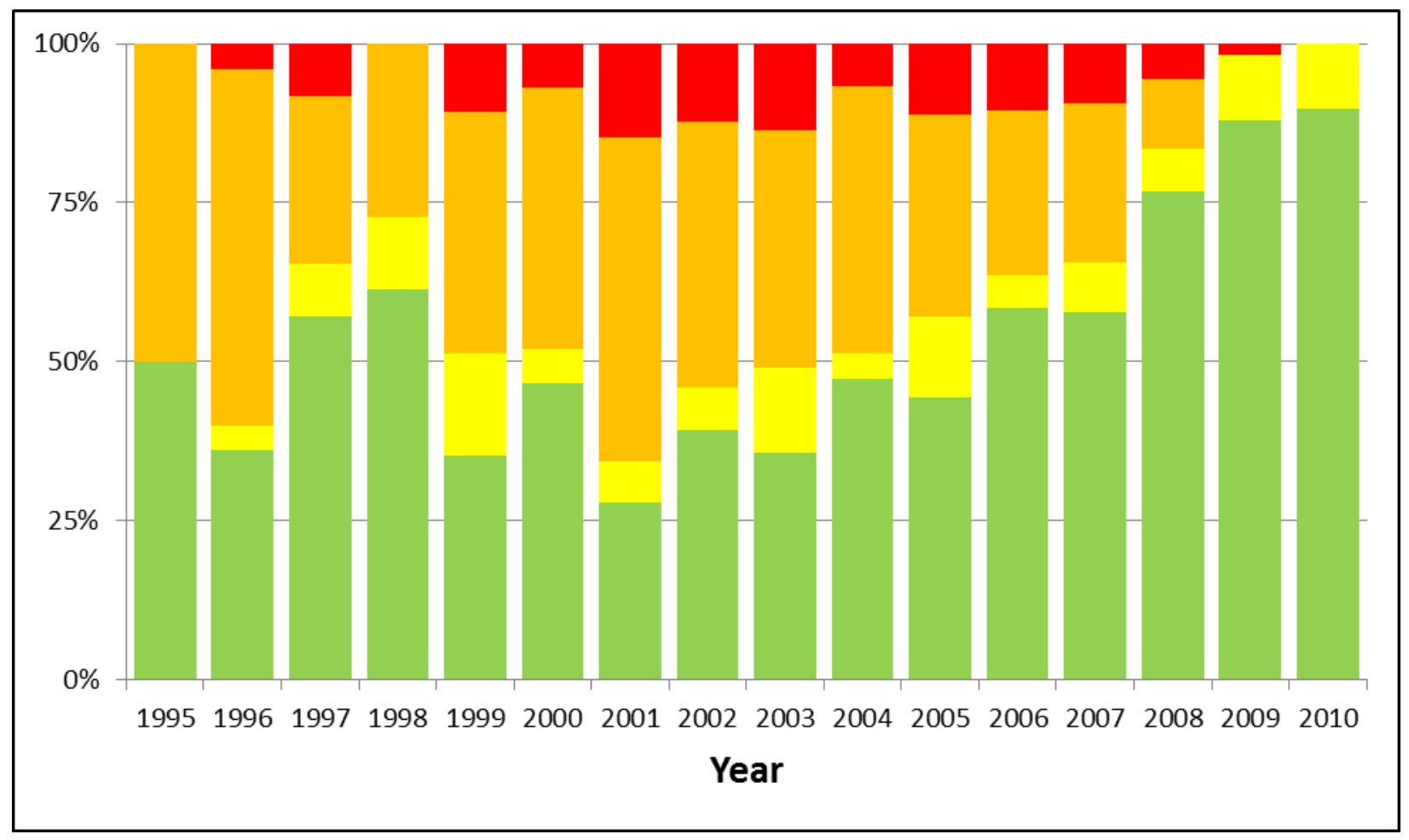

Figure B-39 - Smart Flag 702 Normalized Condition State Trend - Improvement Noted

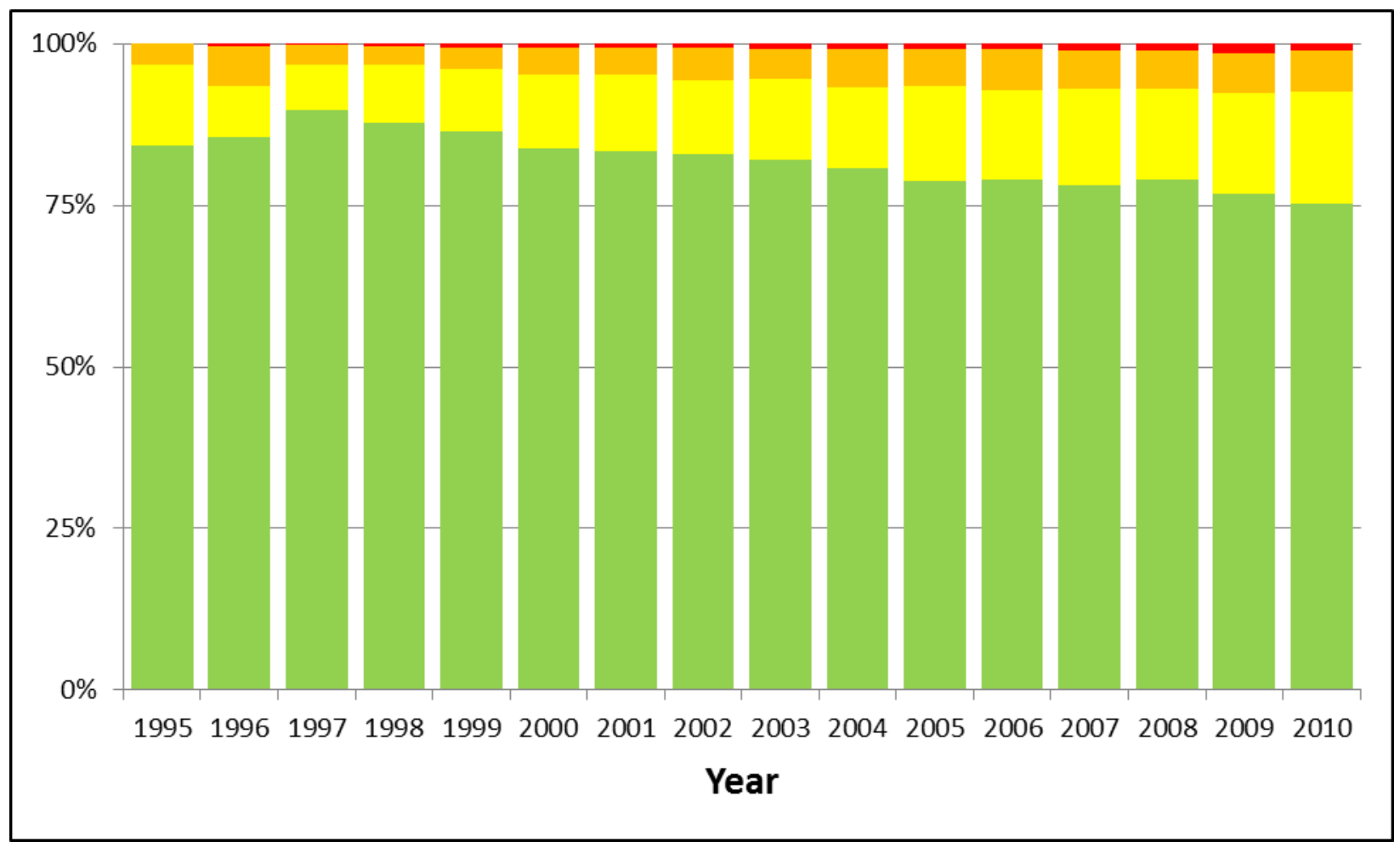

Figure B-40 - Smart Flag 702 Normalized Condition State Trend - Improvement Not Noted 


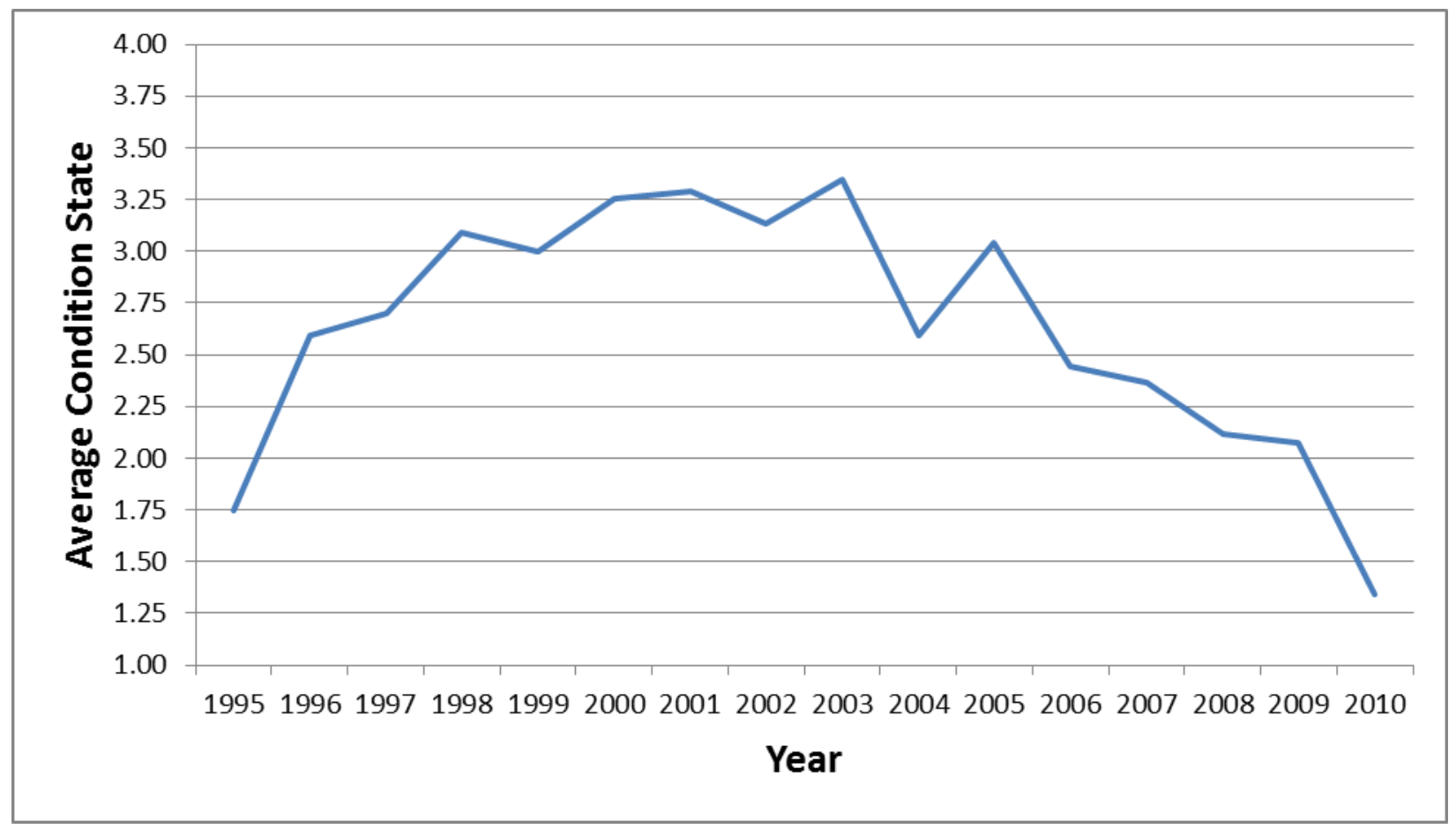

Figure B-41 - Smart Flag 706 (Soffit of Overhang of Concrete) Average Condition State Trend - Improvement Noted

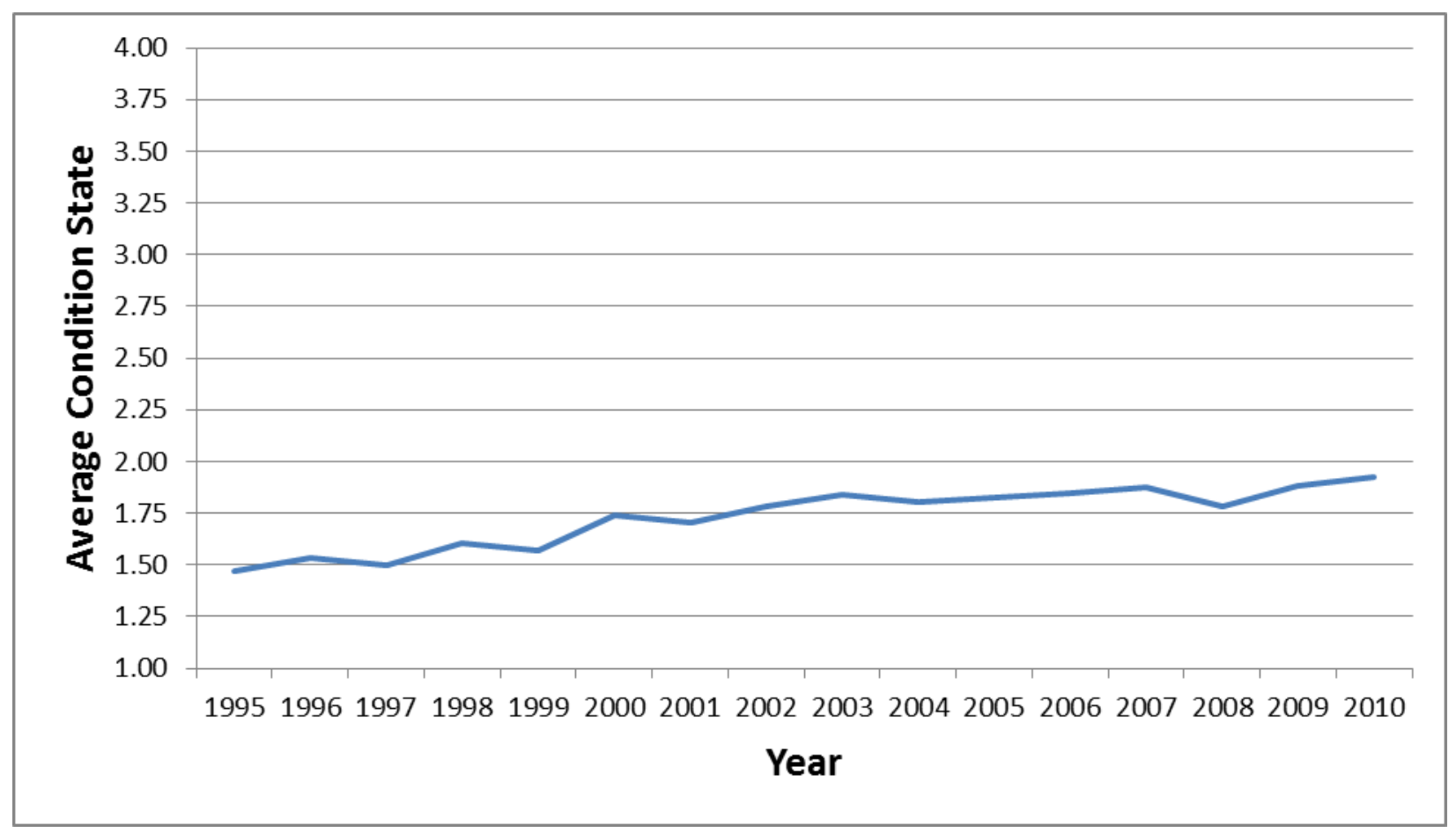

Figure B-42 - Smart Flag 706 () Average Condition State Trend - Improvement Not Noted 


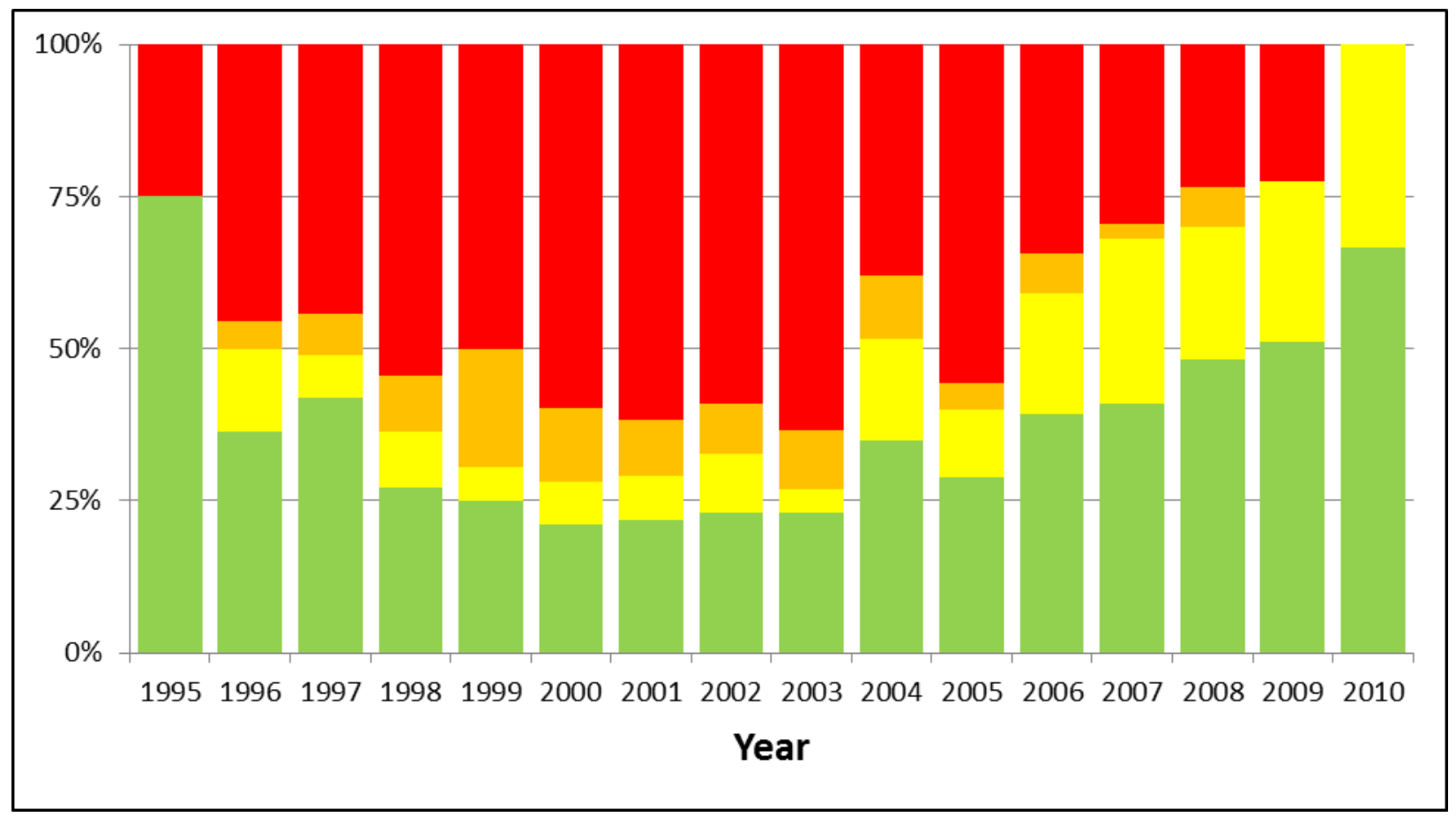

Figure B-43 - Smart Flag 706 Normalized Condition State Trend - Improvement Noted

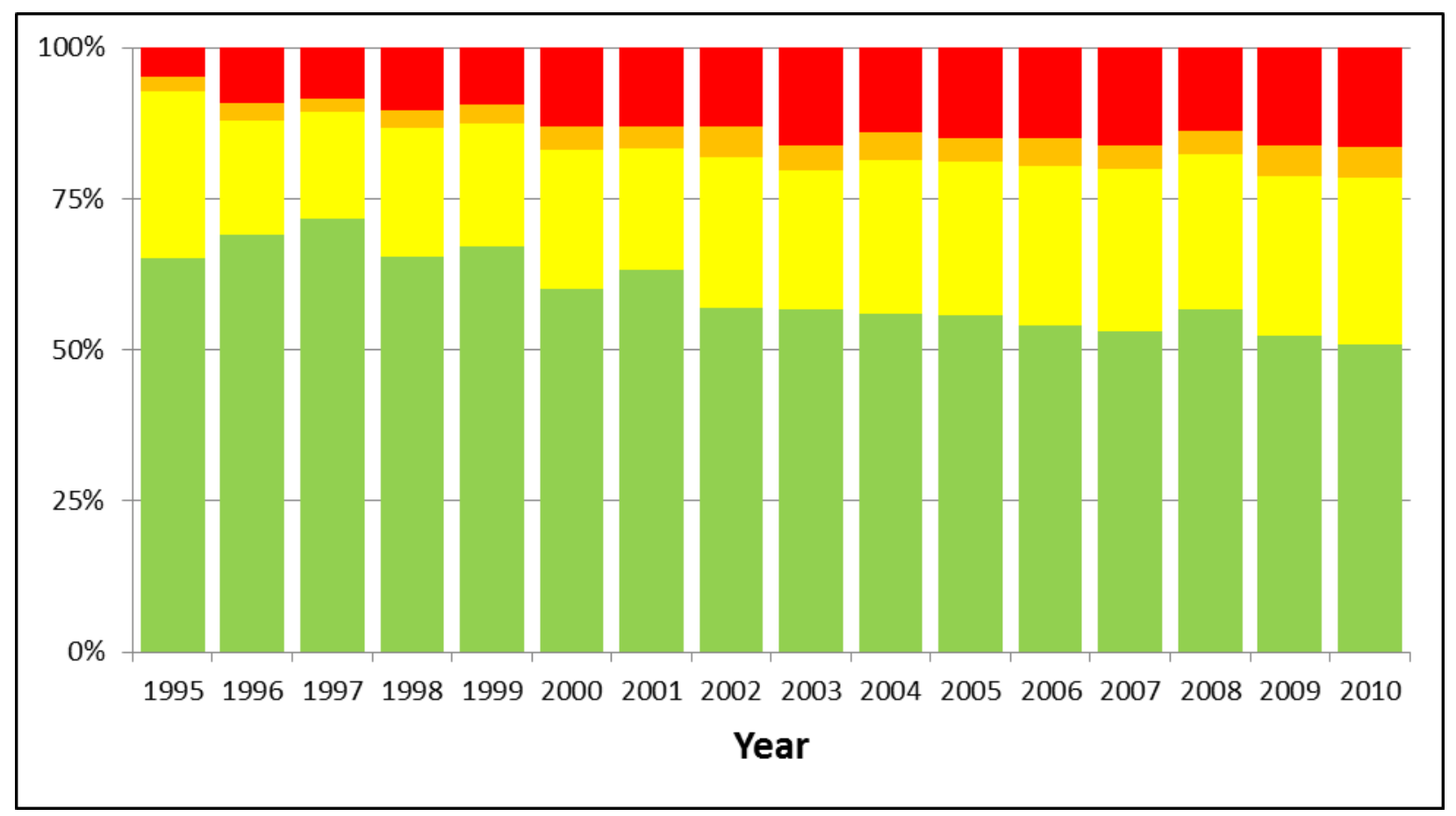

Figure B-44 - Smart Flag 706 Normalized Condition State Trend - Improvement Not Noted 


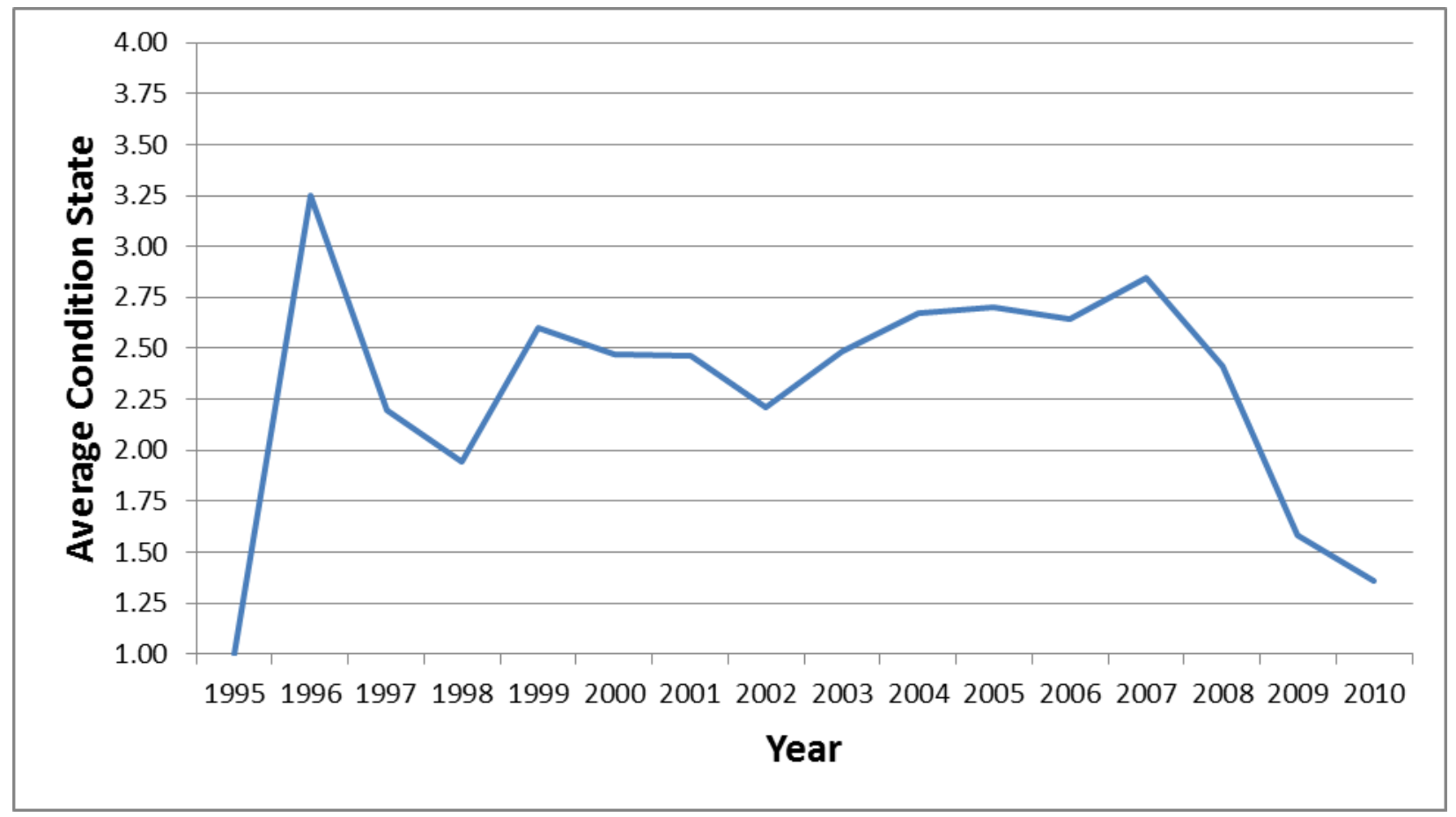

Figure B-45 - Smart Flag 363 (Section Loss) Average Condition State Trend - Improvement Noted

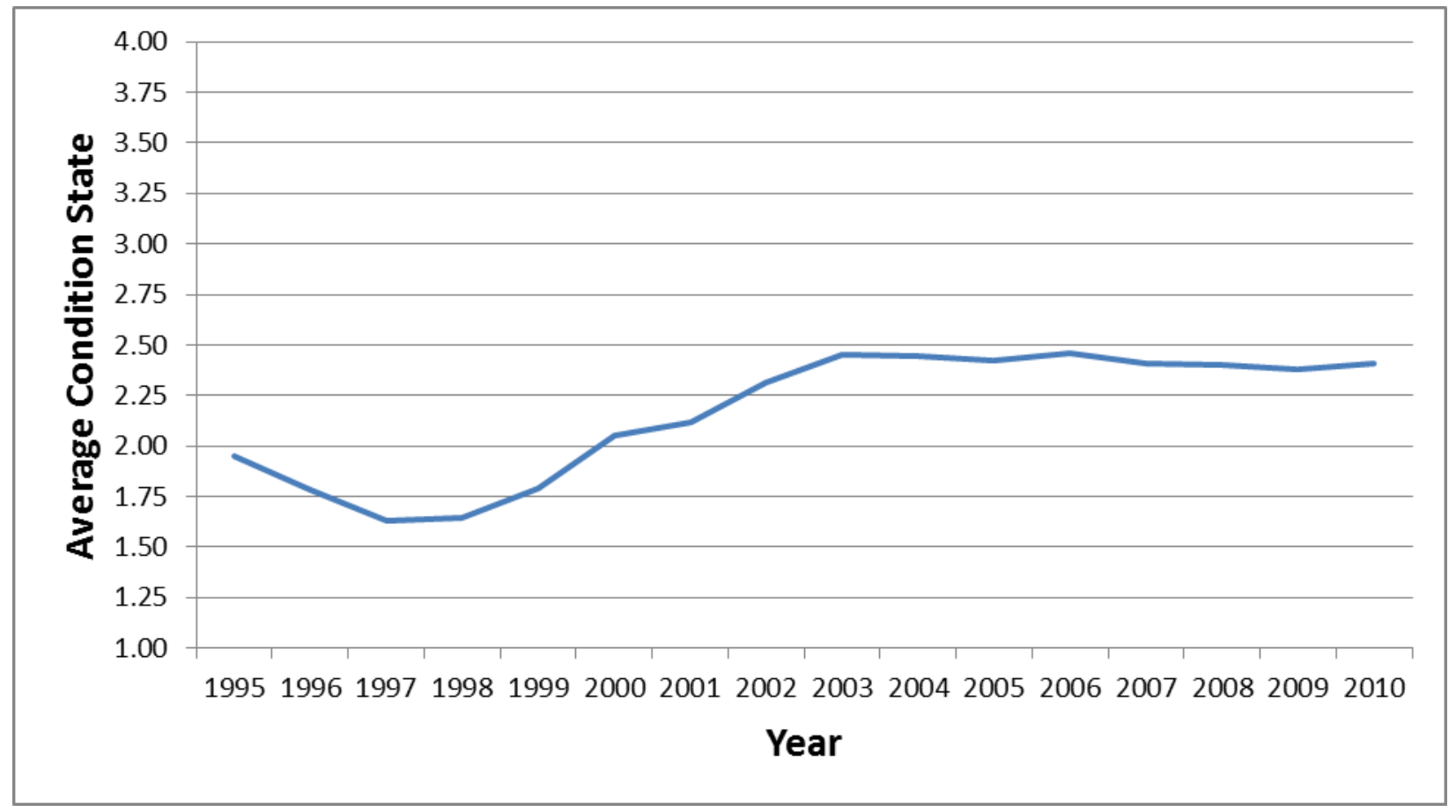

Figure B-46 - Smart Flag 363 Average Condition State Trend - Improvement Not Noted 


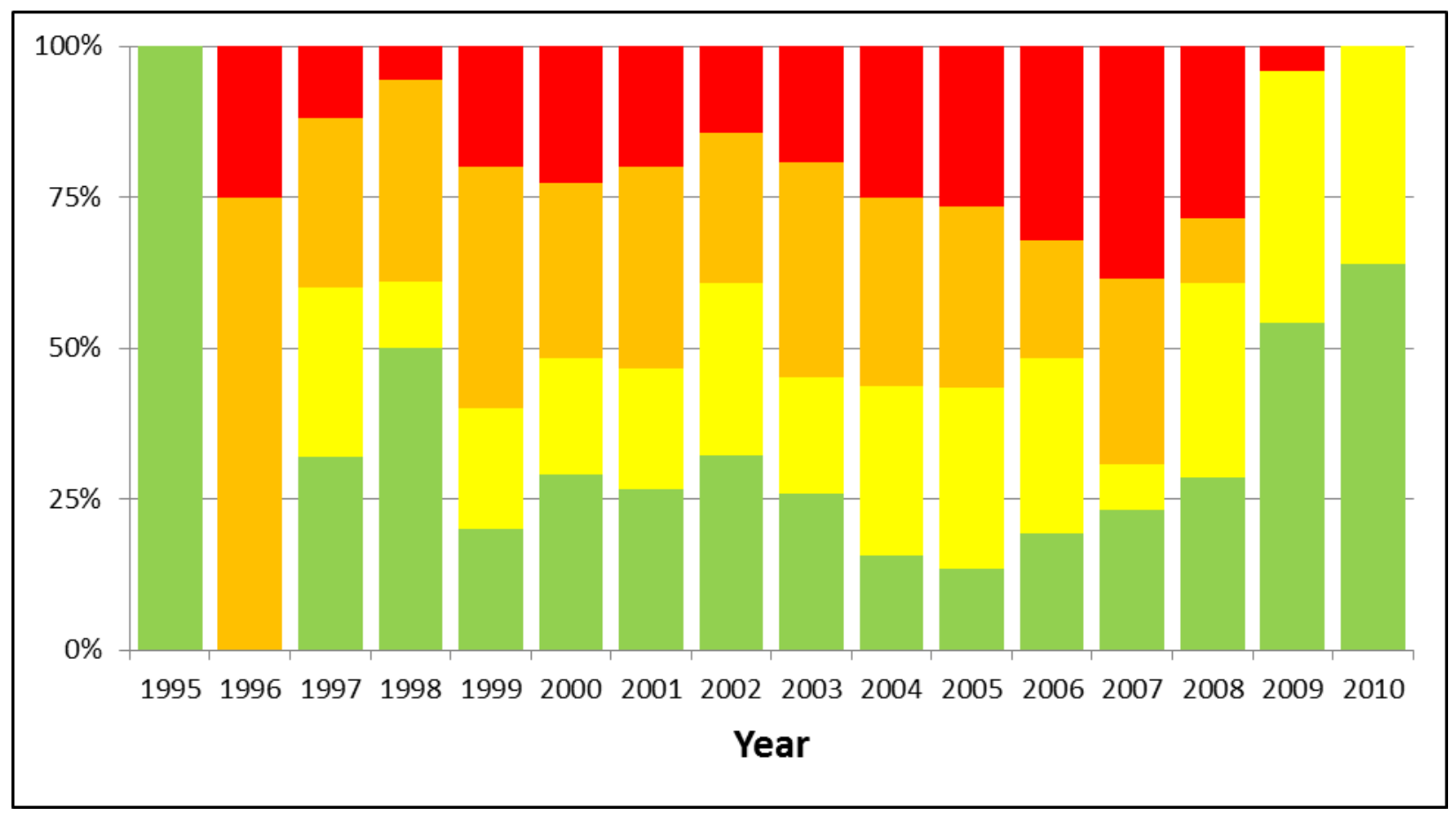

Figure B-47 - Smart Flag 363 Normalized Condition State Trend - Improvement Noted

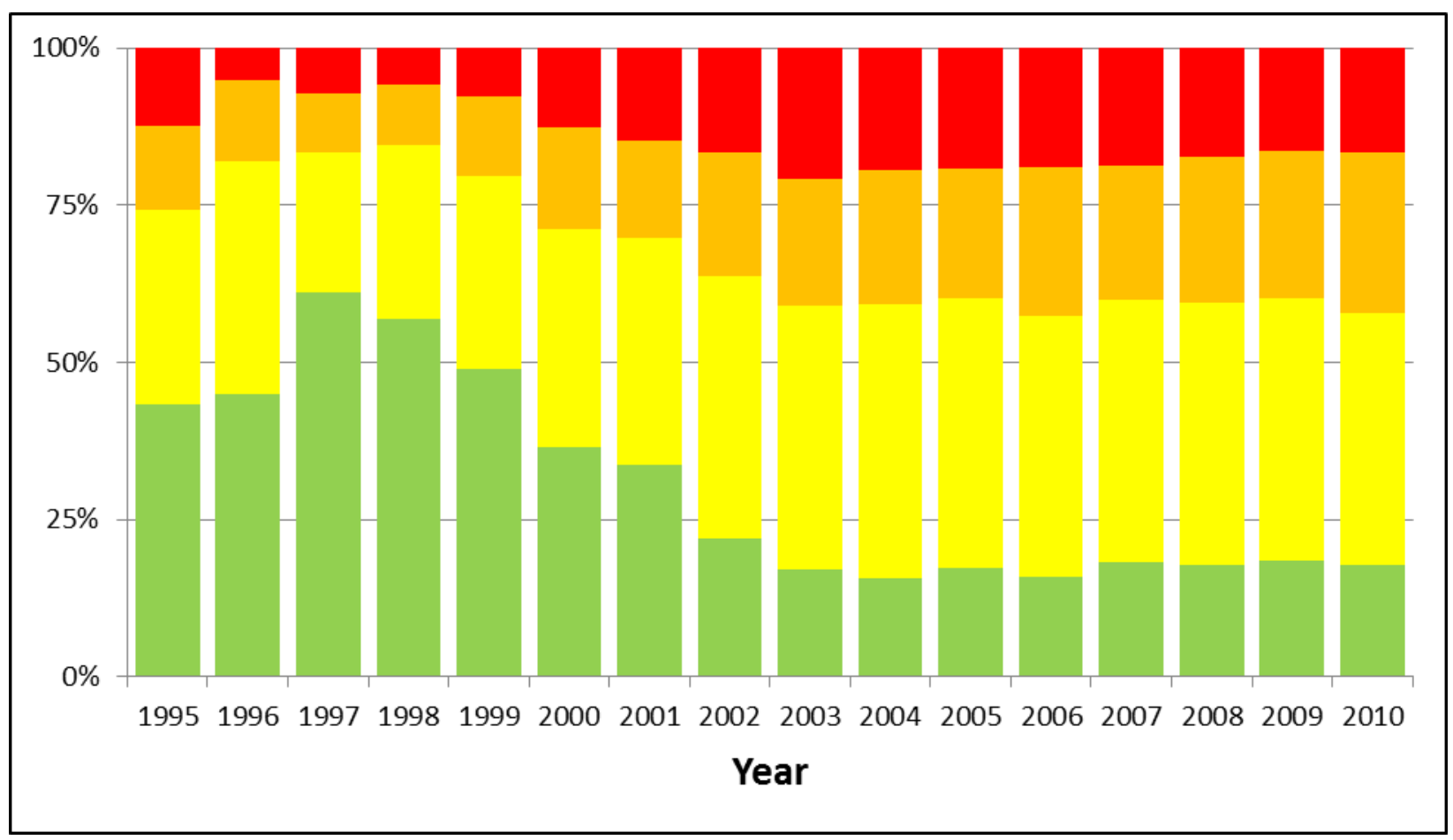

Figure B-48 - Smart Flag 363 Normalized Condition State Trend - Improvement Not Noted 


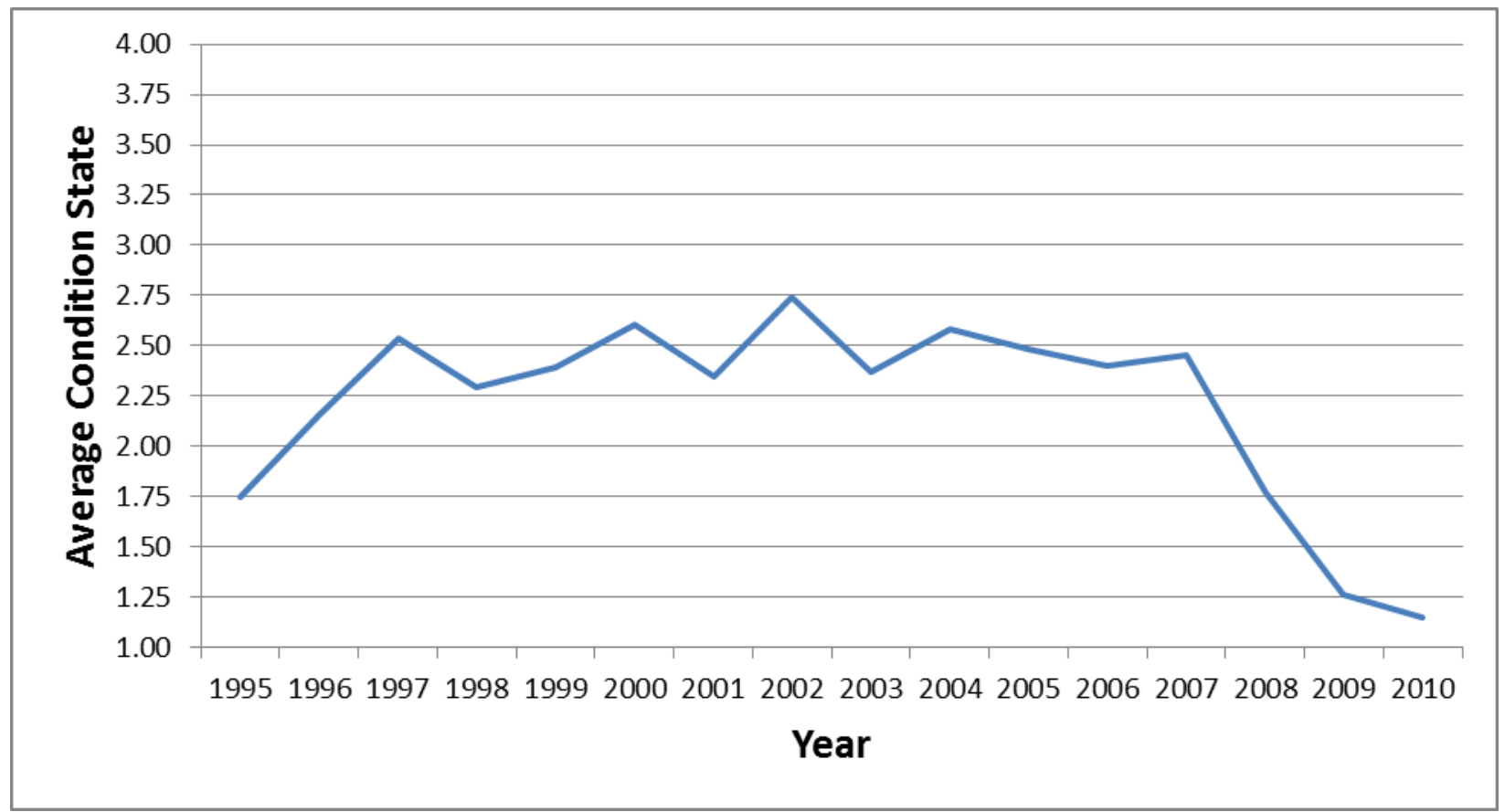

Figure B-49 - Smart Flag 358 (Deck Cracking) Average Condition State Trend - Improvement Noted

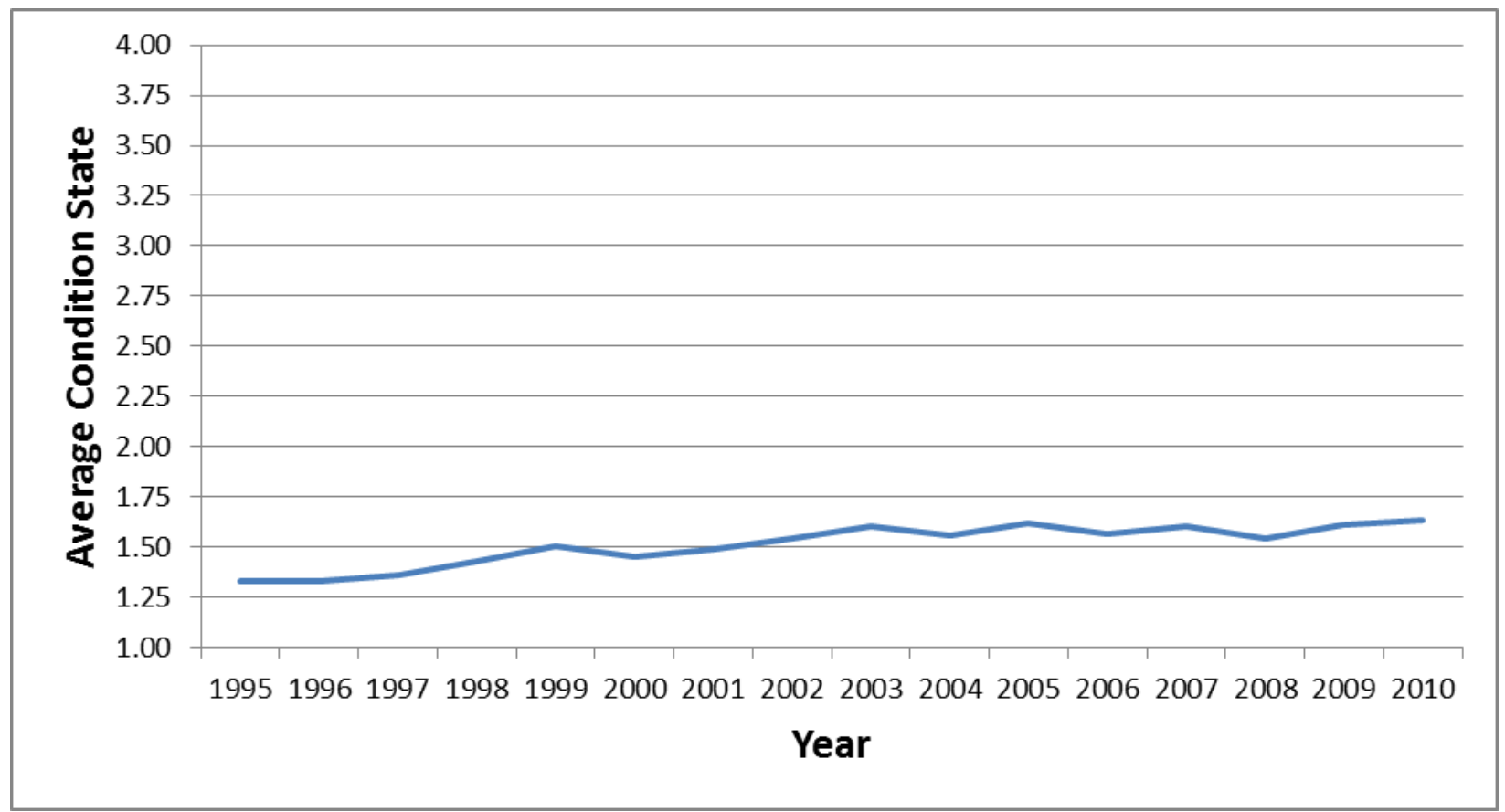

Figure B-50 - Smart Flag 358 Average Condition State Trend - Improvement Not Noted 


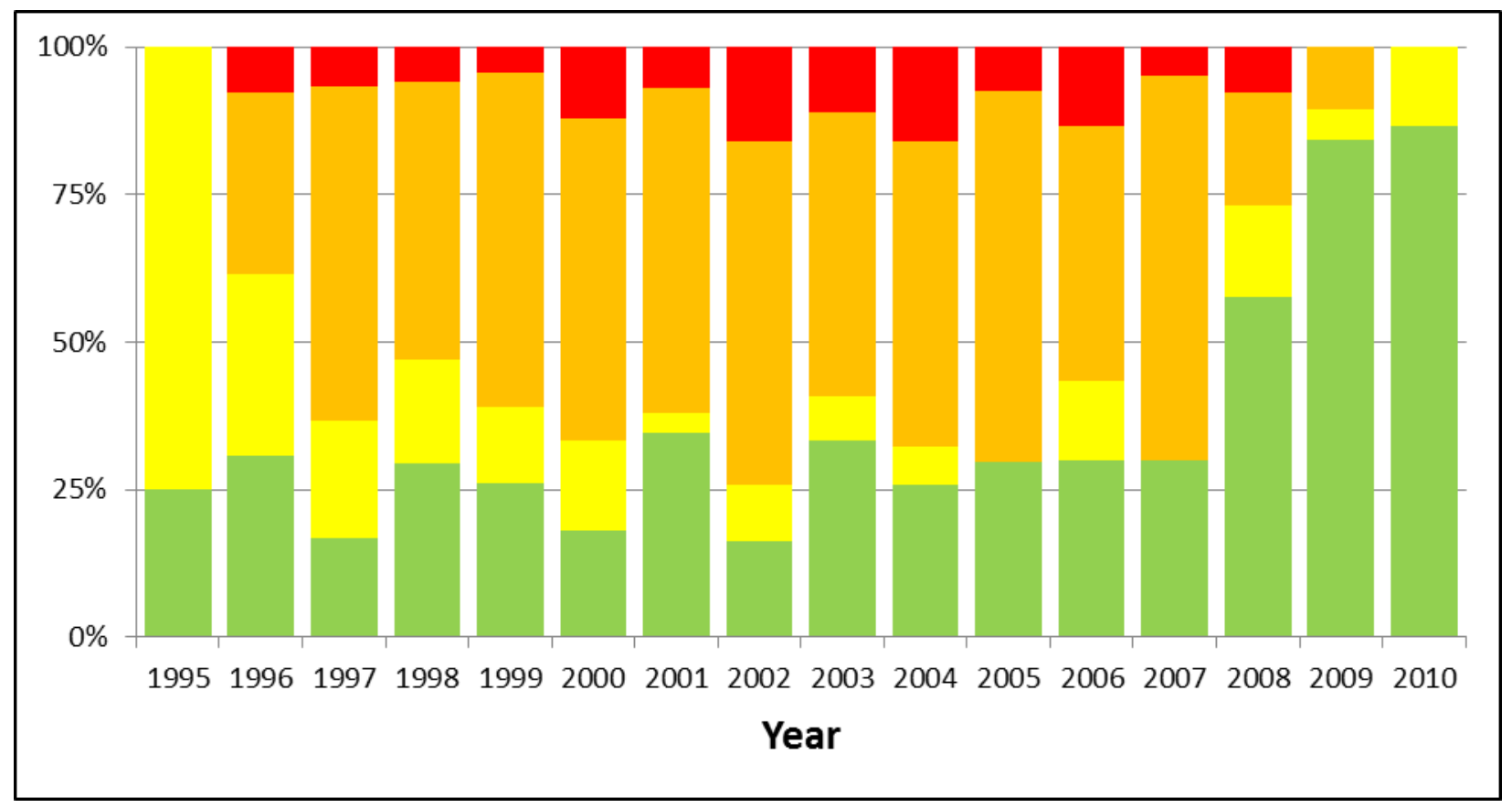

Figure B-51 - Smart Flag 358 Normalized Condition State Trend - Improvement Noted

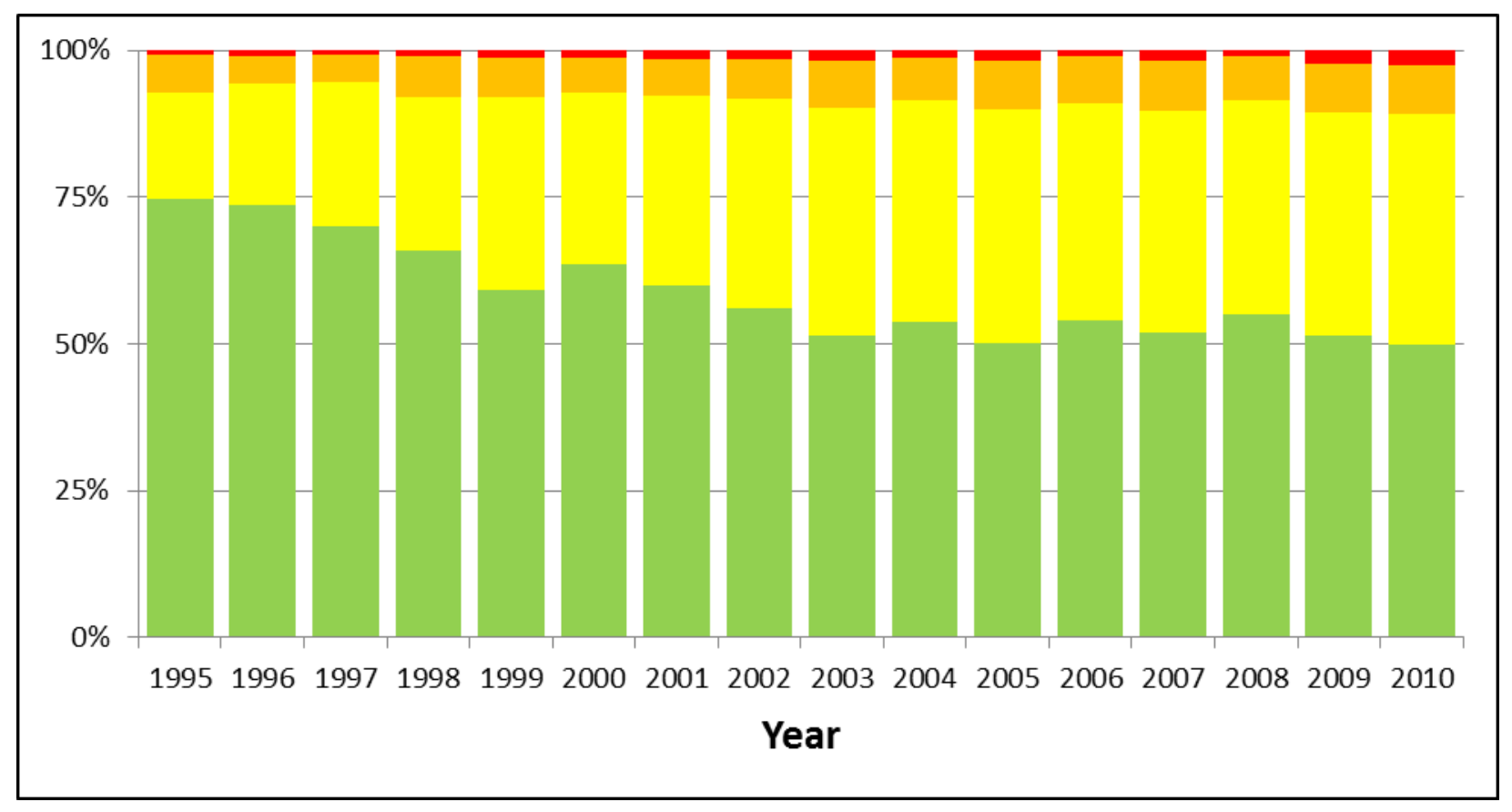

Figure B-52 - Smart Flag 358 Normalized Condition State Trend - Improvement Not Noted 


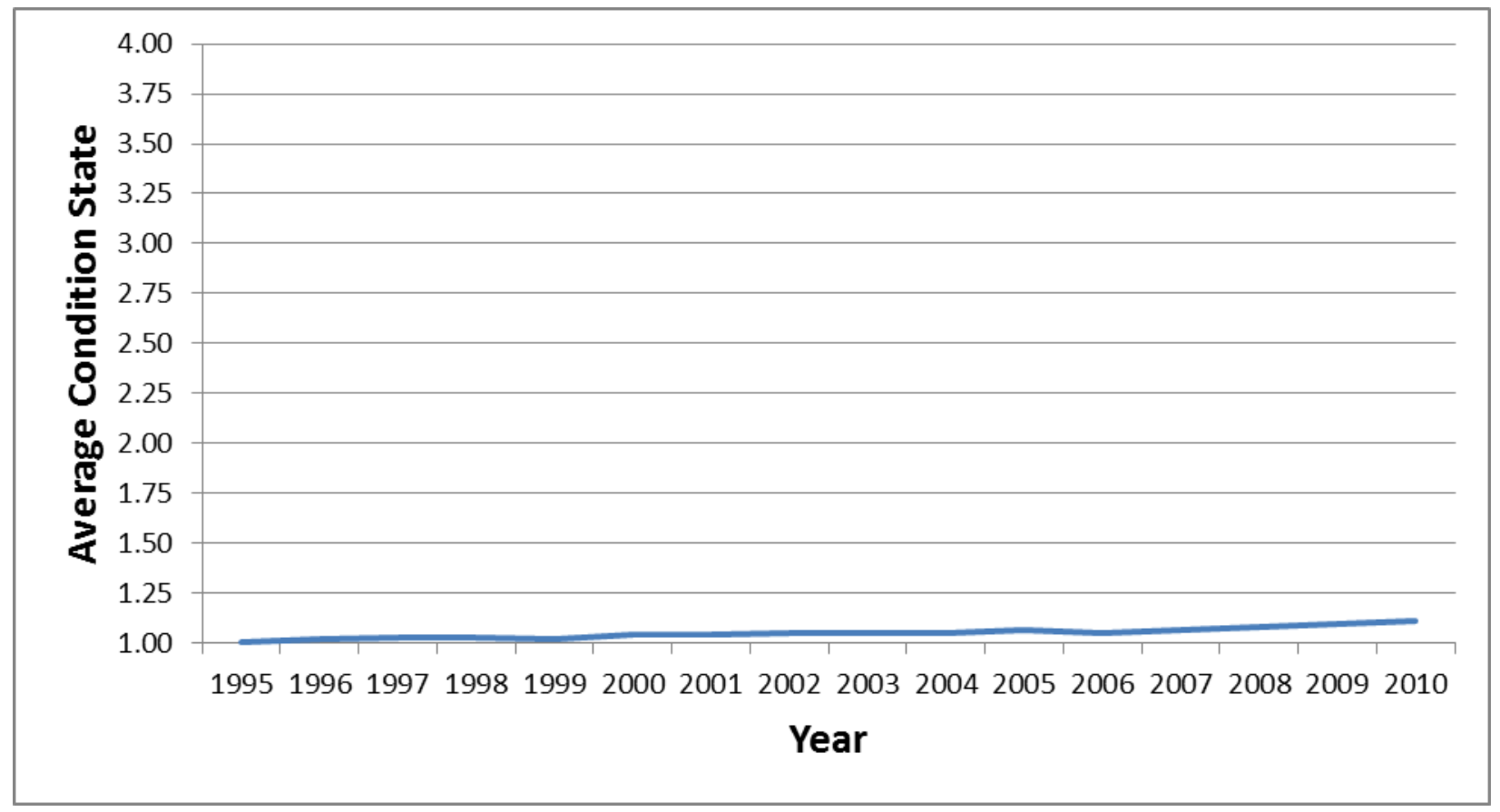

Figure B-53 - Smart Flag 704 (Roadway Over Culverts) Average Condition State Trend - Improvement Noted

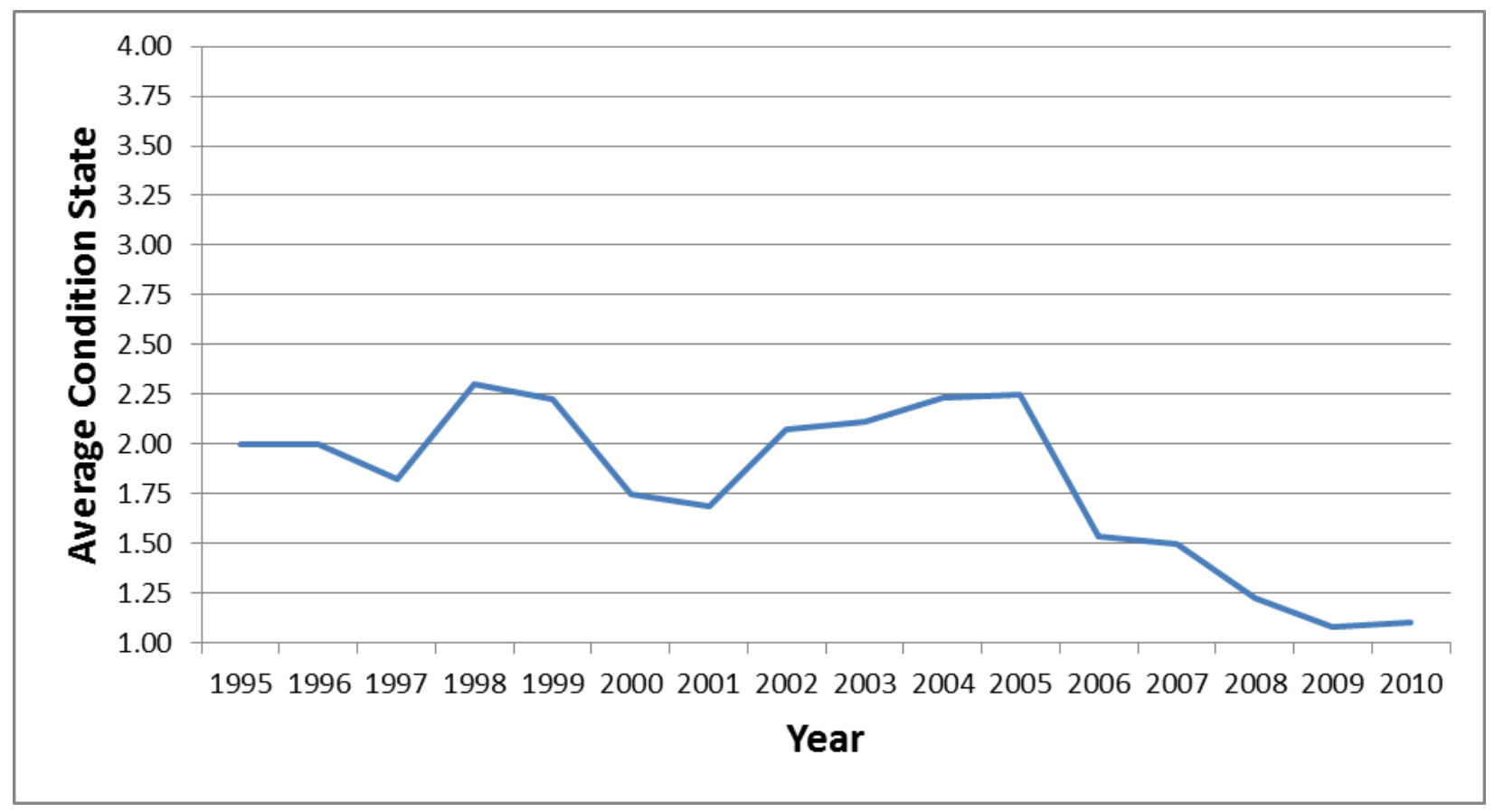

Figure B-54 - Smart Flag 704 Average Condition State Trend - Improvement Not Noted 


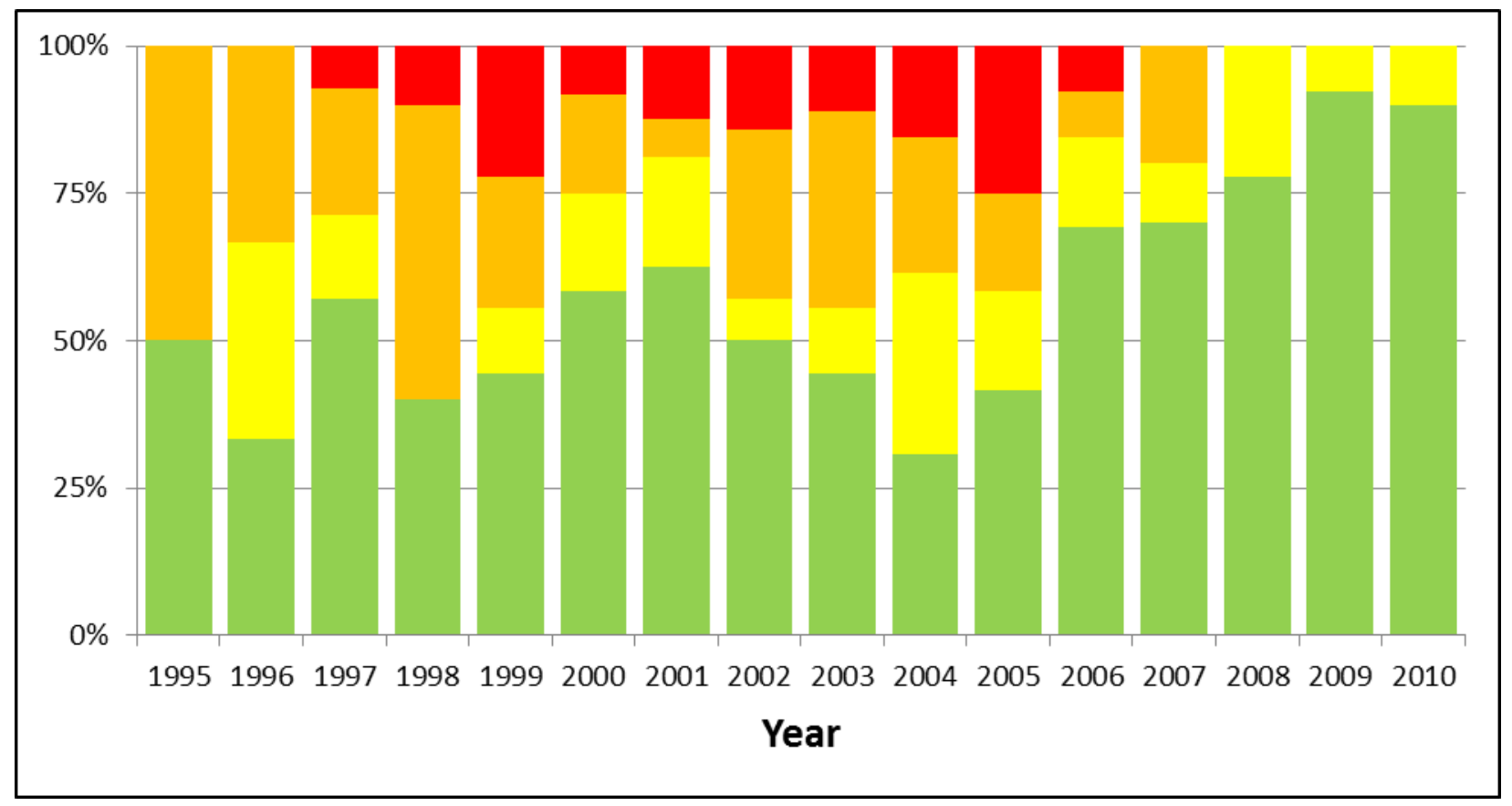

Figure B-55 - Smart Flag 704 Normalized Condition State Trend - Improvement Noted

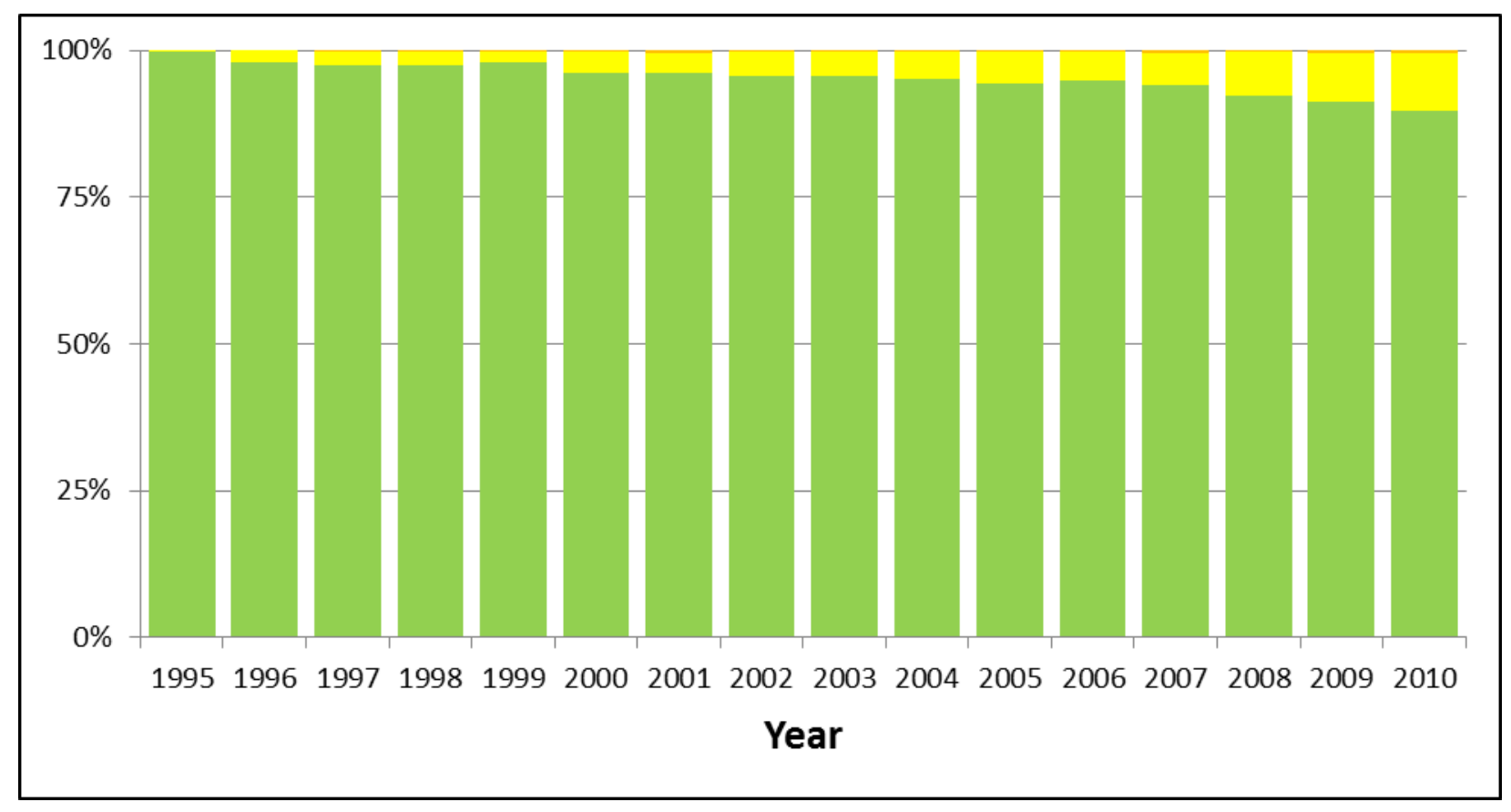

Figure B-56 - Smart Flag 704 Normalized Condition State Trend - Improvement Not Noted 


\begin{tabular}{|l|l|l|l|l|l|l|l|l|l|l|l|l|l|l|l|l|}
\hline Year & 1995 & 1996 & 1997 & 1998 & 1999 & 2000 & 2001 & 2002 & 2003 & 2004 & 2005 & 2006 & 2007 & 2008 & 2009 & 2010 \\
\hline Count & 0 & 0 & 1 & 1 & 14 & 14 & 13 & 20 & 12 & 16 & 32 & 19 & 14 & 17 & 14 & 15 \\
\hline Before & 0.00 & 0.00 & 3.00 & 4.00 & 3.25 & 3.14 & 3.19 & 3.35 & 3.25 & 3.34 & 3.18 & 3.16 & 3.43 & 3.29 & 3.36 & 3.40 \\
\hline After & 0.00 & 0.00 & 1.00 & 1.00 & 1.07 & 1.07 & 1.08 & 1.23 & 1.00 & 1.38 & 1.09 & 1.05 & 1.00 & 1.06 & 1.07 & 1.00 \\
\hline
\end{tabular}

Table B-1 - Statewide Condition State Improvements by Year for Element 32

\begin{tabular}{|l|l|l|l|l|l|l|l|l|l|l|l|l|l|l|l|l|}
\hline Year & 1995 & 1996 & 1997 & 1998 & 1999 & 2000 & 2001 & 2002 & 2003 & 2004 & 2005 & 2006 & 2007 & 2008 & 2009 & 2010 \\
\hline Count & 0 & 0 & 5 & 7 & 11 & 15 & 8 & 8 & 10 & 20 & 14 & 17 & 26 & 33 & 26 & 13 \\
\hline Before & 0.00 & 0.00 & 3.71 & 4.39 & 3.79 & 3.93 & 4.49 & 4.01 & 4.20 & 3.74 & 3.64 & 3.34 & 3.59 & 3.66 & 3.94 & 3.93 \\
\hline After & 0.00 & 0.00 & 1.45 & 1.93 & 1.49 & 1.75 & 1.41 & 1.20 & 1.42 & 1.77 & 1.71 & 1.28 & 1.25 & 1.69 & 1.67 & 1.75 \\
\hline
\end{tabular}

Table B-2 - Statewide Condition State Improvements by Year for Element 108

\begin{tabular}{|c|c|c|c|c|c|c|c|c|c|c|c|c|c|c|c|c|}
\hline Year & 1995 & 1996 & 1997 & 1998 & 1999 & 2000 & 2001 & 2002 & 2003 & 2004 & 2005 & 2006 & 2007 & 2008 & 2009 & 2010 \\
\hline Count & 0 & 0 & 0 & 1 & 7 & 8 & 7 & 22 & 17 & 16 & 16 & 11 & 24 & 27 & 36 & 15 \\
\hline Before & 0.00 & 0.00 & 0.00 & 3.00 & 2.75 & 2.94 & 2.82 & 2.71 & 2.81 & 2.81 & 2.86 & 2.76 & 2.67 & 2.55 & 2.63 & 2.84 \\
\hline After & 0.00 & 0.00 & 0.00 & 1.00 & 1.07 & 1.06 & 1.01 & 1.02 & 1.02 & 1.13 & 1.08 & 1.00 & 1.05 & 1.06 & 1.08 & 1.17 \\
\hline
\end{tabular}

Table B-3 - Statewide Condition State Improvements by Year for Element 302

\begin{tabular}{|l|l|l|l|l|l|l|l|l|l|l|l|l|l|l|l|l|}
\hline Year & 1995 & 1996 & 1997 & 1998 & 1999 & 2000 & 2001 & 2002 & 2003 & 2004 & 2005 & 2006 & 2007 & 2008 & 2009 & 2010 \\
\hline Count & 0 & 0 & 0 & 5 & 4 & 8 & 8 & 15 & 12 & 13 & 16 & 11 & 11 & 5 & 8 & 9 \\
\hline Before & 0.00 & 0.00 & 0.00 & 3.00 & 3.13 & 3.63 & 3.49 & 2.94 & 3.00 & 2.97 & 2.89 & 2.85 & 2.60 & 2.85 & 2.79 & 3.08 \\
\hline After & 0.00 & 0.00 & 0.00 & 1.33 & 1.00 & 1.62 & 1.19 & 1.12 & 1.11 & 1.21 & 1.20 & 1.12 & 1.00 & 1.00 & 1.15 & 1.09 \\
\hline
\end{tabular}

Table B-4 - Statewide Condition State Improvements by Year for Element 334 


\begin{tabular}{|l|l|l|l|l|l|l|l|l|l|l|l|l|l|l|l|l|}
\hline Year & 1995 & 1996 & 1997 & 1998 & 1999 & 2000 & 2001 & 2002 & 2003 & 2004 & 2005 & 2006 & 2007 & 2008 & 2009 & 2010 \\
\hline Count & 0 & 0 & 1 & 0 & 3 & 7 & 5 & 4 & 3 & 7 & 7 & 5 & 5 & 10 & 33 & 26 \\
\hline Before & 0.00 & 0.00 & 3.00 & 0.00 & 2.88 & 3.81 & 3.08 & 3.19 & 3.26 & 3.37 & 3.33 & 3.66 & 3.47 & 3.33 & 3.27 & 3.13 \\
\hline After & 0.00 & 0.00 & 1.00 & 0.00 & 1.50 & 1.32 & 1.00 & 1.38 & 1.67 & 1.30 & 1.41 & 1.27 & 1.56 & 1.69 & 1.49 & 1.27 \\
\hline
\end{tabular}

Table B-5 - Statewide Condition State Improvements by Year for Element 240

\begin{tabular}{|l|l|l|l|l|l|l|l|l|l|l|l|l|l|l|l|l|}
\hline Year & 1995 & 1996 & 1997 & 1998 & 1999 & 2000 & 2001 & 2002 & 2003 & 2004 & 2005 & 2006 & 2007 & 2008 & 2009 & 2010 \\
\hline Count & 0 & 0 & 1 & 2 & 5 & 5 & 3 & 15 & 9 & 7 & 6 & 8 & 20 & 17 & 20 & 12 \\
\hline Before & 0.00 & 0.00 & 3.00 & 3.43 & 3.20 & 2.86 & 2.49 & 2.78 & 3.23 & 3.60 & 2.59 & 2.80 & 3.05 & 3.10 & 2.88 & 2.42 \\
\hline After & 0.00 & 0.00 & 1.46 & 1.26 & 1.22 & 1.31 & 1.04 & 1.19 & 1.44 & 1.65 & 1.18 & 1.08 & 1.44 & 1.32 & 1.19 & 1.20 \\
\hline
\end{tabular}

Table B-6 - Statewide Condition State Improvements by Year for Element 107

\begin{tabular}{|c|c|c|c|c|c|c|c|c|c|c|c|c|c|c|c|c|}
\hline Year & 1995 & 1996 & 1997 & 1998 & 1999 & 2000 & 2001 & 2002 & 2003 & 2004 & 2005 & 2006 & 2007 & 2008 & 2009 & 2010 \\
\hline Count & 0 & 0 & 0 & 1 & 8 & 21 & 6 & 13 & 13 & 6 & 9 & 10 & 12 & 21 & 34 & 29 \\
\hline Before & 0.00 & 0.00 & 0.00 & 4.00 & 3.88 & 4.24 & 4.17 & 4.00 & 3.85 & 4.00 & 3.67 & 4.10 & 3.83 & 3.95 & 3.88 & 3.95 \\
\hline After & 0.00 & 0.00 & 0.00 & 2.00 & 1.25 & 1.79 & 1.50 & 1.23 & 1.31 & 1.67 & 1.33 & 1.63 & 1.71 & 1.74 & 1.50 & 1.67 \\
\hline
\end{tabular}

Table B-7 - Statewide Condition State Improvements by Year for Smart Flag 359

\begin{tabular}{|c|c|c|c|c|c|c|c|c|c|c|c|c|c|c|c|c|}
\hline Year & 1995 & 1996 & 1997 & 1998 & 1999 & 2000 & 2001 & 2002 & 2003 & 2004 & 2005 & 2006 & 2007 & 2008 & 2009 & 2010 \\
\hline Count & 0 & 0 & 0 & 3 & 2 & 4 & 3 & 8 & 7 & 13 & 4 & 11 & 9 & 17 & 14 & 9 \\
\hline Before & 0.00 & 0.00 & 0.00 & 3.33 & 3.00 & 3.00 & 3.00 & 3.00 & 3.14 & 3.23 & 3.00 & 3.00 & 3.33 & 3.24 & 3.29 & 3.33 \\
\hline After & 0.00 & 0.00 & 0.00 & 1.00 & 1.00 & 1.00 & 1.00 & 1.00 & 1.14 & 1.08 & 1.00 & 1.00 & 1.22 & 1.06 & 1.07 & 1.33 \\
\hline
\end{tabular}

Table B-8 - Statewide Condition State Improvements by Year for Smart Flag 702 


\begin{tabular}{|l|l|l|l|l|l|l|l|l|l|l|l|l|l|l|l|l|}
\hline Year & 1995 & 1996 & 1997 & 1998 & 1999 & 2000 & 2001 & 2002 & 2003 & 2004 & 2005 & 2006 & 2007 & 2008 & 2009 & 2010 \\
\hline Count & 0 & 0 & 0 & 0 & 0 & 6 & 6 & 6 & 5 & 14 & 4 & 4 & 12 & 12 & 8 & 19 \\
\hline Before & 0.00 & 0.00 & 0.00 & 0.00 & 0.00 & 3.83 & 3.83 & 4.33 & 3.80 & 4.00 & 3.50 & 4.00 & 4.00 & 4.25 & 4.00 & 4.32 \\
\hline After & 0.00 & 0.00 & 0.00 & 0.00 & 0.00 & 1.58 & 1.17 & 1.17 & 1.00 & 1.43 & 1.25 & 1.50 & 1.67 & 1.67 & 1.19 & 1.34 \\
\hline
\end{tabular}

Table B-9 - Statewide Condition State Improvements by Year for Smart Flag 706

\begin{tabular}{|l|l|l|l|l|l|l|l|l|l|l|l|l|l|l|l|l|}
\hline Year & 1995 & 1996 & 1997 & 1998 & 1999 & 2000 & 2001 & 2002 & 2003 & 2004 & 2005 & 2006 & 2007 & 2008 & 2009 & 2010 \\
\hline Count & 0 & 0 & 0 & 0 & 0 & 2 & 2 & 6 & 1 & 3 & 1 & 0 & 2 & 6 & 9 & 7 \\
\hline Before & 0.00 & 0.00 & 0.00 & 0.00 & 0.00 & 3.50 & 3.50 & 3.50 & 4.00 & 4.00 & 3.00 & 0.00 & 4.00 & 3.67 & 3.67 & 3.43 \\
\hline After & 0.00 & 0.00 & 0.00 & 0.00 & 0.00 & 1.50 & 1.00 & 1.33 & 1.00 & 2.00 & 1.00 & 0.00 & 1.50 & 1.75 & 1.56 & 1.29 \\
\hline
\end{tabular}

Table B-10 - Statewide Condition State Improvements by Year for Smart Flag 363

\begin{tabular}{|l|l|l|l|l|l|l|l|l|l|l|l|l|l|l|l|l|}
\hline Year & 1995 & 1996 & 1997 & 1998 & 1999 & 2000 & 2001 & 2002 & 2003 & 2004 & 2005 & 2006 & 2007 & 2008 & 2009 & 2010 \\
\hline Count & 0 & 0 & 0 & 1 & 5 & 2 & 3 & 3 & 1 & 2 & 0 & 3 & 2 & 8 & 9 & 11 \\
\hline Before & 0.00 & 0.00 & 0.00 & 4.00 & 3.20 & 3.00 & 3.00 & 3.00 & 3.00 & 3.00 & 0.00 & 3.33 & 3.00 & 3.25 & 3.11 & 3.36 \\
\hline After & 0.00 & 0.00 & 0.00 & 1.00 & 1.00 & 1.00 & 1.00 & 1.00 & 1.00 & 1.00 & 0.00 & 1.00 & 1.00 & 1.13 & 1.00 & 1.23 \\
\hline
\end{tabular}

Table B-11 - Statewide Condition State Improvements by Year for Smart Flag 358

\begin{tabular}{|l|l|l|l|l|l|l|l|l|l|l|l|l|l|l|l|l|}
\hline Year & 1995 & 1996 & 1997 & 1998 & 1999 & 2000 & 2001 & 2002 & 2003 & 2004 & 2005 & 2006 & 2007 & 2008 & 2009 & 2010 \\
\hline Count & 0 & 0 & 0 & 0 & 1 & 5 & 0 & 1 & 1 & 2 & 2 & 4 & 3 & 0 & 3 & 1 \\
\hline Before & 0.00 & 0.00 & 0.00 & 0.00 & 3.00 & 3.10 & 0.00 & 3.00 & 4.00 & 3.50 & 3.00 & 3.50 & 3.67 & 0.00 & 3.33 & 3.00 \\
\hline After & 0.00 & 0.00 & 0.00 & 0.00 & 1.00 & 1.20 & 0.00 & 1.00 & 2.00 & 1.50 & 1.00 & 1.00 & 1.00 & 0.00 & 1.00 & 1.00 \\
\hline
\end{tabular}

Table B-12 - Statewide Condition State Improvements by Year for Smart Flag 704 


\begin{tabular}{|c|c|c|c|c|c|c|c|c|c|c|c|c|c|c|c|c|}
\hline Age & 0 & 1 & 2 & 3 & 4 & 5 & 6 & 7 & 8 & 9 & 10 & 11 & 12 & 13 & 14 & 15 \\
\hline Total & 221.50 & 61.00 & 163.00 & 55.00 & 138.00 & 47.50 & 84.00 & 36.50 & 62.00 & 19.00 & 29.00 & 11.00 & 2.00 & 1.00 & 0.00 & 0.00 \\
\hline Count & 202 & 55 & 154 & 51 & 128 & 44 & 76 & 31 & 56 & 16 & 26 & 8 & 1 & 1 & 0 & 0 \\
\hline Average & 1.10 & 1.11 & 1.06 & 1.08 & 1.08 & 1.08 & 1.11 & 1.18 & 1.11 & 1.19 & 1.12 & 1.38 & 2.00 & 1.00 & & \\
\hline
\end{tabular}

Table B-13 - Statewide Condition State Trend by Age since Improvement for Element 32

\begin{tabular}{|c|c|c|c|c|c|c|c|c|c|c|c|c|c|c|c|c|}
\hline Age & 0 & 1 & 2 & 3 & 4 & 5 & 6 & 7 & 8 & 9 & 10 & 11 & 12 & 13 & 14 & 15 \\
\hline Total & 333.9 & 143.7 & 283.7 & 124.9 & 174.2 & 103.6 & 116.5 & 61.5 & 68.8 & 35.6 & 41.6 & 17.0 & 8.9 & 5.0 & 0.0 & 0.0 \\
\hline Count & 213 & 72 & 164 & 56 & 101 & 49 & 71 & 34 & 40 & 23 & 24 & 12 & 7 & 3 & 0 & 0 \\
\hline Average & 1.57 & 2.00 & 1.73 & 2.23 & 1.72 & 2.11 & 1.64 & 1.81 & 1.72 & 1.55 & 1.73 & 1.41 & 1.28 & 1.68 & & \\
\hline
\end{tabular}

Table B-14 - Statewide Condition State Trend by Age since Improvement for Element 108

\begin{tabular}{|c|c|c|c|c|c|c|c|c|c|c|c|c|c|c|c|c|}
\hline Age & 0 & 1 & 2 & 3 & 4 & 5 & 6 & 7 & 8 & 9 & 10 & 11 & 12 & 13 & 14 & 15 \\
\hline Total & 220.64 & 16.02 & 141.19 & 12.36 & 96.86 & 15.01 & 63.90 & 7.05 & 36.07 & 4.32 & 11.64 & 0.00 & 1.00 & 0.00 & 0.00 & 0.00 \\
\hline Count & 207 & 13 & 134 & 10 & 89 & 11 & 56 & 6 & 30 & 4 & 10 & 0 & 1 & 0 & 0 & 0 \\
\hline Average & 1.07 & 1.23 & 1.05 & 1.24 & 1.09 & 1.36 & 1.14 & 1.18 & 1.20 & 1.08 & 1.16 & & 1.00 & & & \\
\hline
\end{tabular}

Table B-15 - Statewide Condition State Trend by Age since Improvement for Element 302

\begin{tabular}{|c|c|c|c|c|c|c|c|c|c|c|c|c|c|c|c|c|}
\hline Age & 0 & 1 & 2 & 3 & 4 & 5 & 6 & 7 & 8 & 9 & 10 & 11 & 12 & 13 & 14 & 15 \\
\hline Total & 145.49 & 51.65 & 121.14 & 45.58 & 103.83 & 38.77 & 66.33 & 28.95 & 37.84 & 18.05 & 14.75 & 2.42 & 4.00 & 0.00 & 0.00 & 0.00 \\
\hline Count & 125 & 40 & 101 & 38 & 86 & 31 & 55 & 23 & 33 & 14 & 13 & 2 & 3 & 0 & 0 & 0 \\
\hline Average & 1.16 & 1.29 & 1.20 & 1.20 & 1.21 & 1.25 & 1.21 & 1.26 & 1.15 & 1.29 & 1.13 & & 1.33 & & & \\
\hline
\end{tabular}

Table B-16 - Statewide Condition State Trend by Age since Improvement for Element 334 


\begin{tabular}{|c|c|c|c|c|c|c|c|c|c|c|c|c|c|c|c|c|}
\hline Age & 0 & 1 & 2 & 3 & 4 & 5 & 6 & 7 & 8 & 9 & 10 & 11 & 12 & 13 & 14 & 15 \\
\hline Total & 162.22 & 19.40 & 48.80 & 10.79 & 48.88 & 11.43 & 25.18 & 5.88 & 19.94 & 4.88 & 6.06 & 0.00 & 1.00 & 0.00 & 0.00 & 0.00 \\
\hline Count & 116 & 12 & 35 & 6 & 37 & 6 & 16 & 3 & 16 & 3 & 5 & 0 & 1 & 0 & 0 & 0 \\
\hline Average & 1.40 & 1.62 & 1.39 & 1.80 & 1.32 & 1.90 & 1.57 & 1.96 & 1.25 & 1.63 & 1.21 & & 1.00 & & & \\
\hline
\end{tabular}

Table B-17 - Statewide Condition State Trend by Age since Improvement for Element 240

\begin{tabular}{|c|c|c|c|c|c|c|c|c|c|c|c|c|c|c|c|c|}
\hline Age & 0 & 1 & 2 & 3 & 4 & 5 & 6 & 7 & 8 & 9 & 10 & 11 & 12 & 13 & 14 & 15 \\
\hline Total & 167.44 & 26.29 & 112.14 & 21.77 & 70.79 & 12.22 & 49.63 & 11.21 & 31.55 & 5.19 & 12.42 & 2.55 & 3.27 & 0.00 & 0.00 & 0.00 \\
\hline Count & 130 & 16 & 85 & 11 & 55 & 8 & 36 & 8 & 22 & 5 & 8 & 2 & 3 & 0 & 0 & 0 \\
\hline Average & 1.29 & 1.64 & 1.32 & 1.98 & 1.29 & 1.53 & 1.38 & 1.40 & 1.43 & 1.04 & 1.55 & 1.27 & 1.09 & & & \\
\hline
\end{tabular}

Table B-18 - Statewide Condition State Trend by Age since Improvement for Element 107

\begin{tabular}{|c|c|c|c|c|c|c|c|c|c|c|c|c|c|c|c|c|}
\hline Age & 0 & 1 & 2 & 3 & 4 & 5 & 6 & 7 & 8 & 9 & 10 & 11 & 12 & 13 & 14 & 15 \\
\hline Total & 286.17 & 42.00 & 161.00 & 34.00 & 108.00 & 31.00 & 94.00 & 25.00 & 66.00 & 16.00 & 42.00 & 5.00 & 1.00 & 0.00 & 0.00 & 0.00 \\
\hline Count & 183 & 27 & 108 & 18 & 70 & 16 & 58 & 13 & 38 & 7 & 23 & 3 & 1 & 0 & 0 & 0 \\
\hline Average & 1.56 & 1.56 & 1.49 & 1.89 & 1.54 & 1.94 & 1.62 & 1.92 & 1.74 & 2.29 & 1.83 & 1.67 & 1.00 & & & \\
\hline
\end{tabular}

Table B-19 - Statewide Condition State Trend by Age since Improvement for Smart Flag 359

\begin{tabular}{|c|c|c|c|c|c|c|c|c|c|c|c|c|c|c|c|c|}
\hline Age & 0 & 1 & 2 & 3 & 4 & 5 & 6 & 7 & 8 & 9 & 10 & 11 & 12 & 13 & 14 & 15 \\
\hline Total & 113.00 & 25.00 & 77.00 & 17.00 & 50.00 & 12.00 & 33.00 & 6.00 & 17.00 & 4.00 & 7.00 & 0.00 & 3.00 & 0.00 & 0.00 & 0.00 \\
\hline Count & 104 & 23 & 70 & 16 & 49 & 12 & 33 & 6 & 17 & 4 & 7 & 0 & 3 & 0 & 0 & 0 \\
\hline Average & 1.09 & 1.09 & 1.10 & 1.06 & 1.02 & 1.00 & 1.00 & 1.00 & 1.00 & 1.00 & 1.00 & & 1.00 & & & \\
\hline
\end{tabular}

Table B-20 - Statewide Condition State Trend by Age since Improvement for Smart Flag 702 


\begin{tabular}{|c|c|c|c|c|c|c|c|c|c|c|c|c|c|c|c|c|}
\hline Age & 0 & 1 & 2 & 3 & 4 & 5 & 6 & 7 & 8 & 9 & 10 & 11 & 12 & 13 & 14 & 15 \\
\hline Total & 134.50 & 21.00 & 74.00 & 18.00 & 45.00 & 9.00 & 39.00 & 10.00 & 21.00 & 5.00 & 7.00 & 0.00 & 0.00 & 0.00 & 0.00 & 0.00 \\
\hline Count & 96 & 15 & 59 & 13 & 36 & 8 & 30 & 8 & 15 & 3 & 5 & 0 & 0 & 0 & 0 & 0 \\
\hline Average & 1.40 & 1.40 & 1.25 & 1.38 & 1.25 & 1.13 & 1.30 & 1.25 & 1.40 & 1.67 & 1.40 & & & & & \\
\hline
\end{tabular}

Table B-21 - Statewide Condition State Trend by Age since Improvement for Smart Flag 706

\begin{tabular}{|c|c|c|c|c|c|c|c|c|c|c|c|c|c|c|c|c|}
\hline Age & 0 & 1 & 2 & 3 & 4 & 5 & 6 & 7 & 8 & 9 & 10 & 11 & 12 & 13 & 14 & 15 \\
\hline Total & 57.50 & 25.00 & 26.00 & 14.00 & 13.00 & 4.00 & 9.00 & 3.00 & 6.00 & 2.00 & 2.00 & 0.00 & 0.00 & 0.00 & 0.00 & 0.00 \\
\hline Count & 39 & 17 & 18 & 7 & 9 & 3 & 6 & 2 & 5 & 1 & 1 & 0 & 0 & 0 & 0 & 0 \\
\hline Average & 1.47 & 1.47 & 1.44 & 2.00 & 1.44 & 1.33 & 1.50 & 1.50 & 1.20 & 2.00 & 2.00 & & & & & \\
\hline
\end{tabular}

Table B-22 - Statewide Condition State Trend by Age since Improvement for Smart Flag 363

\begin{tabular}{|c|c|c|c|c|c|c|c|c|c|c|c|c|c|c|c|c|}
\hline Age & 0 & 1 & 2 & 3 & 4 & 5 & 6 & 7 & 8 & 9 & 10 & 11 & 12 & 13 & 14 & 15 \\
\hline Total & 53.50 & 5.00 & 29.00 & 0.00 & 19.00 & 3.00 & 14.00 & 3.00 & 10.00 & 3.00 & 6.00 & 2.00 & 0.00 & 0.00 & 0.00 & 0.00 \\
\hline Count & 50 & 3 & 25 & 0 & 16 & 1 & 12 & 1 & 8 & 1 & 5 & 1 & 0 & 0 & 0 & 0 \\
\hline Average & 1.07 & 1.67 & 1.16 & & 1.19 & 3.00 & 1.17 & 3.00 & 1.25 & 3.00 & 1.20 & 2.00 & & & & \\
\hline
\end{tabular}

Table B-23 - Statewide Condition State Trend by Age since Improvement for Smart Flag 358

\begin{tabular}{|c|c|c|c|c|c|c|c|c|c|c|c|c|c|c|c|c|}
\hline Age & 0 & 1 & 2 & 3 & 4 & 5 & 6 & 7 & 8 & 9 & 10 & 11 & 12 & 13 & 14 & 15 \\
\hline Total & 26.00 & 9.00 & 11.00 & 9.00 & 12.00 & 5.00 & 4.00 & 0.00 & 4.00 & 0.00 & 3.00 & 0.00 & 0.00 & 0.00 & 0.00 & 0.00 \\
\hline Count & 23 & 8 & 10 & 6 & 11 & 3 & 4 & 0 & 4 & 0 & 3 & 0 & 0 & 0 & 0 & 0 \\
\hline Average & 1.13 & 1.13 & 1.10 & 1.50 & 1.09 & 1.67 & 1.00 & & 1.00 & & 1.00 & & & & & \\
\hline
\end{tabular}

Table B-24 - Statewide Condition State Trend by Age since Improvement for Smart Flag 704 


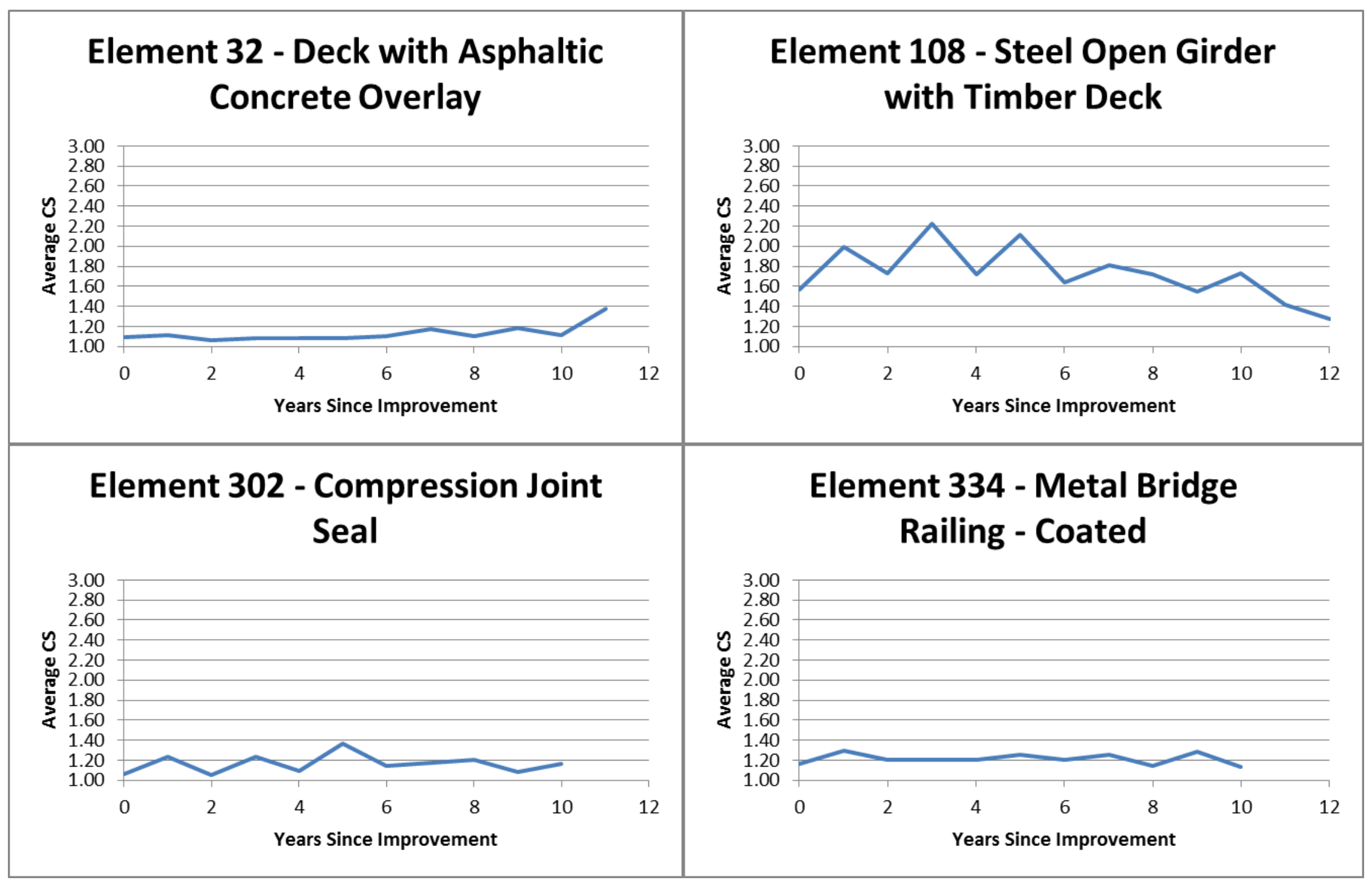

Figure B-57 - Statewide Condition State Trend Lines by Age since Improvement for Elements 32, 108, 302, and 334 


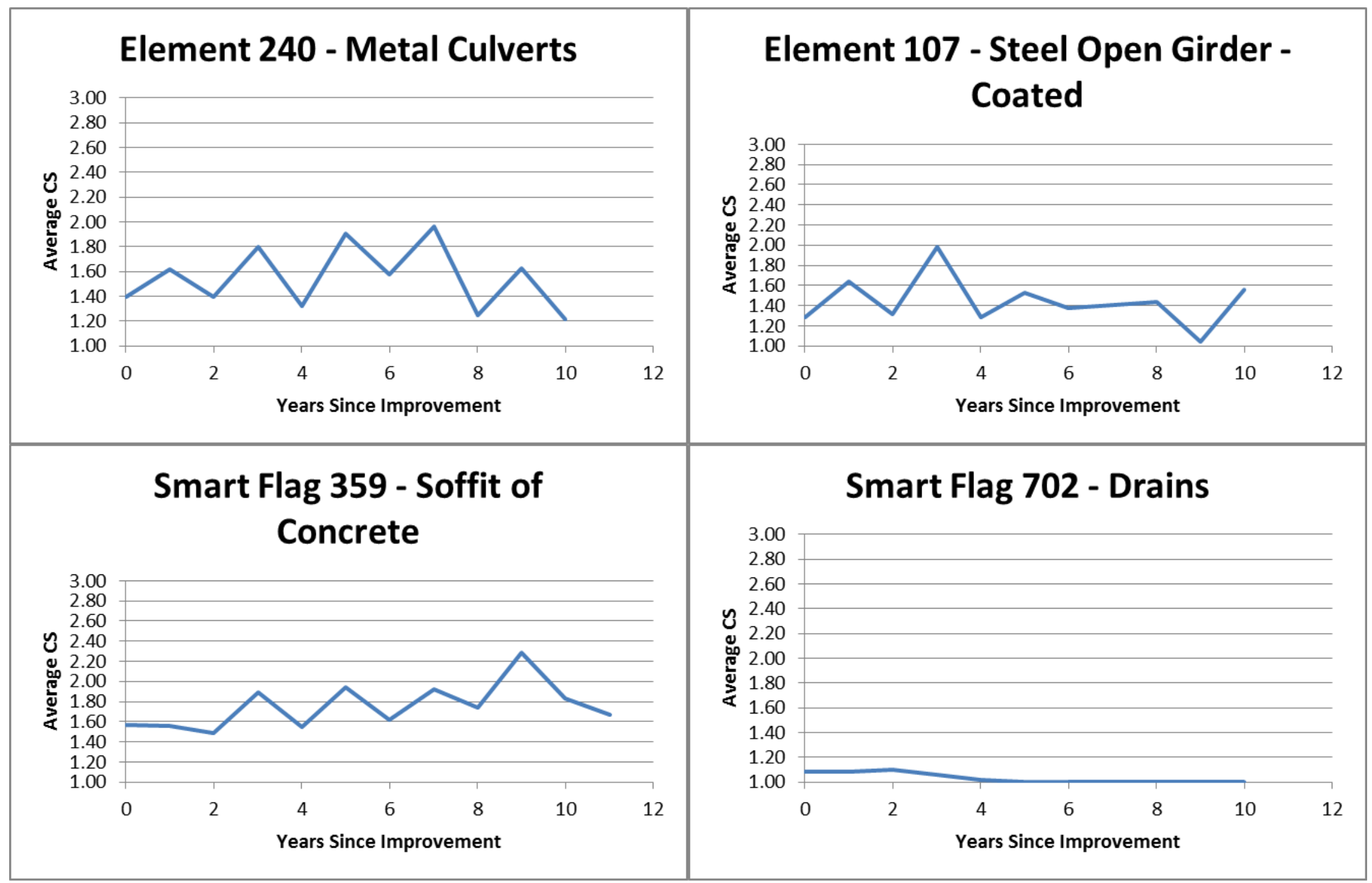

Figure B-58 - Statewide Condition State Trend Lines by Age since Improvement for Elements 240 and 107 and Smart Flags 359 and 702 


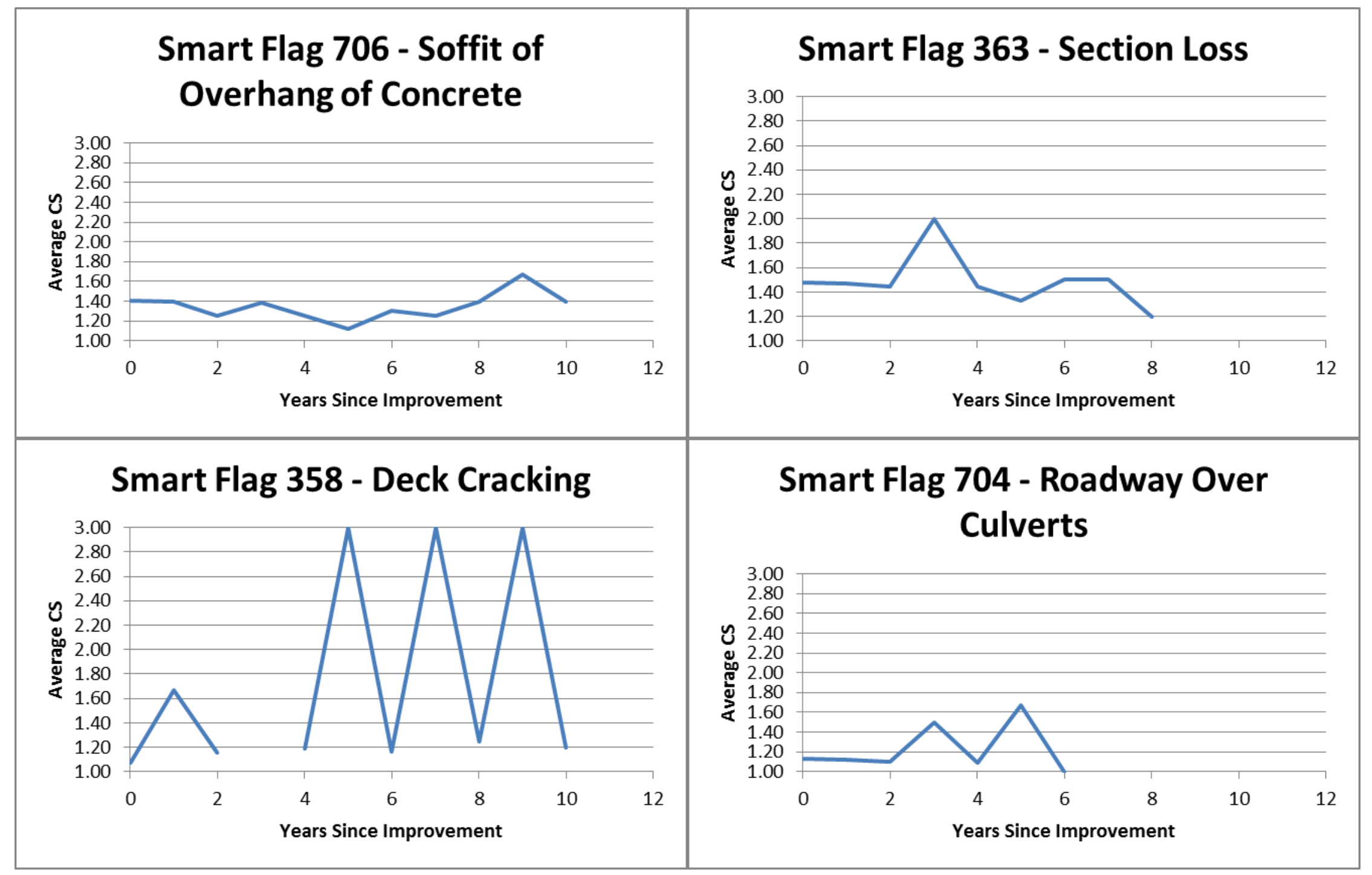

Figure B-59 - Statewide Condition State Trend Lines by Age since Improvement for Smart Flags 706, 363, 358, and 704 
APPENDIX C - DISTRICT VISIT RESULTS 


\begin{tabular}{|c|c|c|c|c|c|c|c|c|c|c|}
\hline Total & \multicolumn{10}{|c|}{ District } \\
\hline Element 107 & 1 & 2 & 3 & 4 & 5 & 6 & 7 & 8 & 9 & Total \\
\hline Count & 6 & 12 & 4 & 15 & 5 & 3 & 8 & 14 & 3 & 70 \\
\hline Avg. Quant. & 338.26 & 407.83 & 245.43 & 912.99 & 624.76 & 526.31 & 497.64 & 339.62 & 297.33 & 513.29 \\
\hline Tot. Quant. & 2029.56 & 4893.94 & 981.73 & 13694.82 & 3123.80 & 1578.94 & 3981.11 & 4754.63 & 892.00 & 35930.52 \\
\hline$C P$ & $50.0 \%$ & $66.7 \%$ & $0.0 \%$ & $40.0 \%$ & $20.0 \%$ & $66.7 \%$ & $75.0 \%$ & $71.4 \%$ & $0.0 \%$ & $51.4 \%$ \\
\hline$S R$ & $50.0 \%$ & $0.0 \%$ & $25.0 \%$ & $20.0 \%$ & $0.0 \%$ & $0.0 \%$ & $0.0 \%$ & $0.0 \%$ & $0.0 \%$ & $10.0 \%$ \\
\hline$R B$ & $0.0 \%$ & $0.0 \%$ & $0.0 \%$ & $6.7 \%$ & $0.0 \%$ & $0.0 \%$ & $0.0 \%$ & $0.0 \%$ & $0.0 \%$ & $1.4 \%$ \\
\hline$A Q$ & $0.0 \%$ & $0.0 \%$ & $0.0 \%$ & $0.0 \%$ & $0.0 \%$ & $0.0 \%$ & $0.0 \%$ & $0.0 \%$ & $0.0 \%$ & $0.0 \%$ \\
\hline$N M$ & $0.0 \%$ & $16.7 \%$ & $75.0 \%$ & $33.3 \%$ & $80.0 \%$ & $33.3 \%$ & $25.0 \%$ & $28.6 \%$ & $100.0 \%$ & $34.3 \%$ \\
\hline$T E$ & $0.0 \%$ & $16.7 \%$ & $0.0 \%$ & $0.0 \%$ & $0.0 \%$ & $0.0 \%$ & $0.0 \%$ & $0.0 \%$ & $0.0 \%$ & $2.9 \%$ \\
\hline$O T$ & $0.0 \%$ & $0.0 \%$ & $0.0 \%$ & $0.0 \%$ & $0.0 \%$ & $0.0 \%$ & $0.0 \%$ & $0.0 \%$ & $0.0 \%$ & $0.0 \%$ \\
\hline
\end{tabular}

Table C-1 - District Visit Maintenance Summary - Element 107 - All Road Classifications

\begin{tabular}{|c|c|c|c|c|c|c|c|c|c|c|}
\hline Interstate & \multicolumn{10}{|c|}{ District } \\
\hline Element 107 & 1 & 2 & 3 & 4 & 5 & 6 & 7 & 8 & 9 & Total \\
\hline Count & 1 & 3 & 0 & 4 & 3 & 0 & 5 & 7 & 0 & 23 \\
\hline Avg. Quant. & 506.94 & 443.98 & N/A & 974.14 & 305.93 & N/A & 483.86 & 291.27 & N/A & 483.10 \\
\hline Tot. Quant. & 506.94 & 1331.93 & 0.00 & 3896.55 & 917.80 & 0.00 & 2419.31 & 2038.89 & 0.00 & 11111.41 \\
\hline$C P$ & $100.0 \%$ & $100.0 \%$ & N/A & $25.0 \%$ & $0.0 \%$ & N/A & $80.0 \%$ & $57.1 \%$ & N/A & $56.5 \%$ \\
\hline$S R$ & $0.0 \%$ & $0.0 \%$ & N/A & $50.0 \%$ & $0.0 \%$ & N/A & $0.0 \%$ & $0.0 \%$ & N/A & $8.7 \%$ \\
\hline$R B$ & $0.0 \%$ & $0.0 \%$ & N/A & $0.0 \%$ & $0.0 \%$ & N/A & $0.0 \%$ & $0.0 \%$ & N/A & $0.0 \%$ \\
\hline$A Q$ & $0.0 \%$ & $0.0 \%$ & N/A & $0.0 \%$ & $0.0 \%$ & N/A & $0.0 \%$ & $0.0 \%$ & N/A & $0.0 \%$ \\
\hline$N M$ & $0.0 \%$ & $0.0 \%$ & N/A & $25.0 \%$ & $100.0 \%$ & N/A & $20.0 \%$ & $42.9 \%$ & N/A & $34.8 \%$ \\
\hline$T E$ & $0.0 \%$ & $0.0 \%$ & N/A & $0.0 \%$ & $0.0 \%$ & N/A & $0.0 \%$ & $0.0 \%$ & N/A & $0.0 \%$ \\
\hline$O T$ & $0.0 \%$ & $0.0 \%$ & N/A & $0.0 \%$ & $0.0 \%$ & N/A & $0.0 \%$ & $0.0 \%$ & N/A & $0.0 \%$ \\
\hline
\end{tabular}

Table C-2 - District Visit Maintenance Summary - Element 107 - Interstate Roads 


\begin{tabular}{|c|c|c|c|c|c|c|c|c|c|c|}
\hline Primary & \multicolumn{10}{|c|}{ District } \\
\hline Element 107 & 1 & 2 & 3 & 4 & 5 & 6 & 7 & 8 & 9 & Total \\
\hline Count & 3 & 2 & 1 & 10 & 2 & 2 & 0 & 1 & 3 & 24 \\
\hline Avg. Quant. & 274.38 & 415.21 & 332.00 & 947.72 & 1103.00 & 633.04 & N/A & 495.95 & 297.33 & 680.12 \\
\hline Tot. Quant. & 823.14 & 830.42 & 332.00 & 9477.19 & 2206.00 & 1266.08 & 0.00 & 495.95 & 892.00 & 16322.77 \\
\hline$C P$ & $66.7 \%$ & $100.0 \%$ & $0.0 \%$ & $50.0 \%$ & $50.0 \%$ & $50.0 \%$ & N/A & $0.0 \%$ & $0.0 \%$ & $45.8 \%$ \\
\hline$S R$ & $33.3 \%$ & $0.0 \%$ & $0.0 \%$ & $10.0 \%$ & $0.0 \%$ & $0.0 \%$ & N/A & $0.0 \%$ & $0.0 \%$ & $8.3 \%$ \\
\hline$R B$ & $0.0 \%$ & $0.0 \%$ & $0.0 \%$ & $0.0 \%$ & $0.0 \%$ & $0.0 \%$ & N/A & $0.0 \%$ & $0.0 \%$ & $0.0 \%$ \\
\hline$A Q$ & $0.0 \%$ & $0.0 \%$ & $0.0 \%$ & $0.0 \%$ & $0.0 \%$ & $0.0 \%$ & N/A & $0.0 \%$ & $0.0 \%$ & $0.0 \%$ \\
\hline NM & $0.0 \%$ & $0.0 \%$ & $100.0 \%$ & $40.0 \%$ & $50.0 \%$ & $50.0 \%$ & N/A & $100.0 \%$ & $100.0 \%$ & $45.8 \%$ \\
\hline$T E$ & $0.0 \%$ & $0.0 \%$ & $0.0 \%$ & $0.0 \%$ & $0.0 \%$ & $0.0 \%$ & N/A & $0.0 \%$ & $0.0 \%$ & $0.0 \%$ \\
\hline$O T$ & $0.0 \%$ & $0.0 \%$ & $0.0 \%$ & $0.0 \%$ & $0.0 \%$ & $0.0 \%$ & N/A & $0.0 \%$ & $0.0 \%$ & $0.0 \%$ \\
\hline
\end{tabular}

Table C-3 - District Visit Maintenance Summary - Element 107 - Primary Roads

\begin{tabular}{|c|c|c|c|c|c|c|c|c|c|c|}
\hline Secondary & \multicolumn{10}{|c|}{ District } \\
\hline Element 107 & 1 & 2 & 3 & 4 & 5 & 6 & 7 & 8 & 9 & Total \\
\hline Count & 2 & 7 & 3 & 1 & 0 & 1 & 3 & 6 & 0 & 23 \\
\hline Avg. Quant. & 349.74 & 390.23 & 216.58 & 321.09 & $\mathrm{~N} / \mathrm{A}$ & 312.86 & 520.60 & 369.96 & $\mathrm{~N} / \mathrm{A}$ & 369.41 \\
\hline Tot. Quant. & 699.48 & 2731.60 & 649.73 & 321.09 & 0.00 & 312.86 & 1561.80 & 2219.79 & 0.00 & 8496.34 \\
\hline$C P$ & $0.0 \%$ & $42.9 \%$ & $0.0 \%$ & $0.0 \%$ & $\mathrm{~N} / \mathrm{A}$ & $100.0 \%$ & $66.7 \%$ & $100.0 \%$ & $\mathrm{~N} / \mathrm{A}$ & $52.2 \%$ \\
\hline$S R$ & $100.0 \%$ & $0.0 \%$ & $33.3 \%$ & $0.0 \%$ & $\mathrm{~N} / \mathrm{A}$ & $0.0 \%$ & $0.0 \%$ & $0.0 \%$ & $\mathrm{~N} / \mathrm{A}$ & $13.0 \%$ \\
\hline$R B$ & $0.0 \%$ & $0.0 \%$ & $0.0 \%$ & $100.0 \%$ & $\mathrm{~N} / \mathrm{A}$ & $0.0 \%$ & $0.0 \%$ & $0.0 \%$ & $\mathrm{~N} / \mathrm{A}$ & $4.3 \%$ \\
\hline$A Q$ & $0.0 \%$ & $0.0 \%$ & $0.0 \%$ & $0.0 \%$ & $\mathrm{~N} / \mathrm{A}$ & $0.0 \%$ & $0.0 \%$ & $0.0 \%$ & $\mathrm{~N} / \mathrm{A}$ & $0.0 \%$ \\
\hline$N M$ & $0.0 \%$ & $28.6 \%$ & $66.7 \%$ & $0.0 \%$ & $\mathrm{~N} / \mathrm{A}$ & $0.0 \%$ & $33.3 \%$ & $0.0 \%$ & $\mathrm{~N} / \mathrm{A}$ & $21.7 \%$ \\
\hline$T E$ & $0.0 \%$ & $28.6 \%$ & $0.0 \%$ & $0.0 \%$ & $\mathrm{~N} / \mathrm{A}$ & $0.0 \%$ & $0.0 \%$ & $0.0 \%$ & $\mathrm{~N} / \mathrm{A}$ & $8.7 \%$ \\
\hline$O T$ & $0.0 \%$ & $0.0 \%$ & $0.0 \%$ & $0.0 \%$ & $\mathrm{~N} / \mathrm{A}$ & $0.0 \%$ & $0.0 \%$ & $0.0 \%$ & $\mathrm{~N} / \mathrm{A}$ & $0.0 \%$ \\
\hline
\end{tabular}

Table C-4 - District Visit Maintenance Summary - Element 107 - Secondary Roads 


\begin{tabular}{|c|c|c|c|c|c|c|c|c|c|c|}
\hline Total & \multicolumn{10}{|c|}{ District } \\
\hline Element 108 & 1 & 2 & 3 & 4 & 5 & 6 & 7 & 8 & 9 & Total \\
\hline Count & 10 & 13 & 17 & 11 & 10 & 3 & 6 & 14 & 11 & 95 \\
\hline Avg. Quant. & 93.91 & 136.66 & 111.17 & 188.70 & 117.87 & 63.67 & 126.70 & 104.50 & 64.50 & 115.62 \\
\hline Tot. Quant. & 939.06 & 1776.58 & 1889.85 & 2075.74 & 1178.72 & 191.00 & 760.21 & 1463.07 & 709.49 & 10983.72 \\
\hline$C P$ & $0.0 \%$ & $0.0 \%$ & $0.0 \%$ & $9.1 \%$ & $0.0 \%$ & $0.0 \%$ & $33.3 \%$ & $42.9 \%$ & $9.1 \%$ & $10.5 \%$ \\
\hline$S R$ & $60.0 \%$ & $84.6 \%$ & $70.6 \%$ & $36.4 \%$ & $50.0 \%$ & $0.0 \%$ & $50.0 \%$ & $42.9 \%$ & $18.2 \%$ & $51.6 \%$ \\
\hline$R B$ & $0.0 \%$ & $0.0 \%$ & $0.0 \%$ & $18.2 \%$ & $10.0 \%$ & $0.0 \%$ & $0.0 \%$ & $0.0 \%$ & $9.1 \%$ & $4.2 \%$ \\
\hline$A Q$ & $0.0 \%$ & $0.0 \%$ & $0.0 \%$ & $0.0 \%$ & $0.0 \%$ & $0.0 \%$ & $0.0 \%$ & $7.1 \%$ & $9.1 \%$ & $2.1 \%$ \\
\hline$N M$ & $20.0 \%$ & $15.4 \%$ & $23.5 \%$ & $27.3 \%$ & $40.0 \%$ & $33.3 \%$ & $16.7 \%$ & $7.1 \%$ & $45.5 \%$ & $24.2 \%$ \\
\hline$T E$ & $20.0 \%$ & $0.0 \%$ & $5.9 \%$ & $9.1 \%$ & $0.0 \%$ & $66.7 \%$ & $0.0 \%$ & $0.0 \%$ & $9.1 \%$ & $7.4 \%$ \\
\hline$O T$ & $0.0 \%$ & $0.0 \%$ & $0.0 \%$ & $0.0 \%$ & $0.0 \%$ & $0.0 \%$ & $0.0 \%$ & $0.0 \%$ & $0.0 \%$ & $0.0 \%$ \\
\hline
\end{tabular}

Table C-5 - District Visit Maintenance Summary - Element 108 - All Road Classifications

\begin{tabular}{|c|c|c|c|c|c|c|c|c|c|c|}
\hline Interstate & \multicolumn{10}{|c|}{ District } \\
\hline Element 108 & 1 & 2 & 3 & 4 & 5 & 6 & 7 & 8 & 9 & Total \\
\hline Count & 0 & 0 & 0 & 0 & 0 & 0 & 0 & 0 & 0 & 0 \\
\hline Avg. Quant. & N/A & N/A & N/A & N/A & N/A & N/A & N/A & N/A & N/A & N/A \\
\hline Tot. Quant. & 0.00 & 0.00 & 0.00 & 0.00 & 0.00 & 0.00 & 0.00 & 0.00 & 0.00 & 0.00 \\
\hline$C P$ & N/A & N/A & N/A & N/A & N/A & N/A & N/A & N/A & N/A & N/A \\
\hline$S R$ & N/A & N/A & N/A & N/A & N/A & N/A & N/A & N/A & N/A & N/A \\
\hline$R B$ & N/A & N/A & N/A & N/A & N/A & N/A & N/A & N/A & N/A & N/A \\
\hline$A Q$ & N/A & N/A & N/A & N/A & N/A & N/A & N/A & N/A & N/A & N/A \\
\hline NM & N/A & N/A & N/A & N/A & N/A & N/A & N/A & N/A & N/A & N/A \\
\hline$T E$ & N/A & N/A & N/A & N/A & N/A & N/A & N/A & N/A & N/A & N/A \\
\hline$O T$ & N/A & N/A & N/A & N/A & N/A & N/A & N/A & N/A & N/A & N/A \\
\hline
\end{tabular}

Table C-6 - District Visit Maintenance Summary - Element 108 - Interstate Roads 


\begin{tabular}{|c|c|c|c|c|c|c|c|c|c|c|}
\hline Primary & \multicolumn{10}{|c|}{ District } \\
\hline Element 108 & 1 & 2 & 3 & 4 & 5 & 6 & 7 & 8 & 9 & Total \\
\hline Count & 0 & 0 & 0 & 0 & 0 & 0 & 0 & 0 & 1 & 1 \\
\hline Avg. Quant. & N/A & N/A & N/A & N/A & N/A & N/A & N/A & N/A & 0.00 & 0.00 \\
\hline Tot. Quant. & 0.00 & 0.00 & 0.00 & 0.00 & 0.00 & 0.00 & 0.00 & 0.00 & 0.00 & 0.00 \\
\hline$C P$ & N/A & N/A & N/A & N/A & N/A & N/A & N/A & N/A & $0.0 \%$ & $0.0 \%$ \\
\hline$S R$ & N/A & N/A & N/A & N/A & N/A & N/A & N/A & N/A & $0.0 \%$ & $0.0 \%$ \\
\hline$R B$ & N/A & N/A & N/A & N/A & N/A & N/A & N/A & N/A & $100.0 \%$ & $100.0 \%$ \\
\hline$A Q$ & N/A & N/A & N/A & N/A & N/A & N/A & N/A & N/A & $0.0 \%$ & $0.0 \%$ \\
\hline NM & N/A & N/A & N/A & N/A & N/A & N/A & N/A & N/A & $0.0 \%$ & $0.0 \%$ \\
\hline$T E$ & N/A & N/A & N/A & N/A & N/A & N/A & N/A & N/A & $0.0 \%$ & $0.0 \%$ \\
\hline$O T$ & N/A & N/A & N/A & N/A & N/A & N/A & N/A & N/A & $0.0 \%$ & $0.0 \%$ \\
\hline
\end{tabular}

Table C-7 - District Visit Maintenance Summary - Element 108 - Primary Roads

\begin{tabular}{|c|c|c|c|c|c|c|c|c|c|c|}
\hline Secondary & \multicolumn{10}{|c|}{ District } \\
\hline Element 108 & 1 & 2 & 3 & 4 & 5 & 6 & 7 & 8 & 9 & Total \\
\hline Count & 10 & 13 & 17 & 11 & 10 & 3 & 6 & 14 & 11 & 95 \\
\hline Avg. Quant. & 93.91 & 136.66 & 111.17 & 188.70 & 117.87 & 63.67 & 126.70 & 104.50 & 57.35 & 114.79 \\
\hline Tot. Quant. & 939.06 & 1776.58 & 1889.85 & 2075.74 & 1178.72 & 191.00 & 760.21 & 1463.07 & 630.86 & 10905.09 \\
\hline$C P$ & $0.0 \%$ & $0.0 \%$ & $0.0 \%$ & $9.1 \%$ & $0.0 \%$ & $0.0 \%$ & $33.3 \%$ & $42.9 \%$ & $9.1 \%$ & $10.5 \%$ \\
\hline$S R$ & $60.0 \%$ & $84.6 \%$ & $70.6 \%$ & $36.4 \%$ & $50.0 \%$ & $0.0 \%$ & $50.0 \%$ & $42.9 \%$ & $18.2 \%$ & $51.6 \%$ \\
\hline$R B$ & $0.0 \%$ & $0.0 \%$ & $0.0 \%$ & $18.2 \%$ & $10.0 \%$ & $0.0 \%$ & $0.0 \%$ & $0.0 \%$ & $0.0 \%$ & $3.2 \%$ \\
\hline$A Q$ & $0.0 \%$ & $0.0 \%$ & $0.0 \%$ & $0.0 \%$ & $0.0 \%$ & $0.0 \%$ & $0.0 \%$ & $7.1 \%$ & $9.1 \%$ & $2.1 \%$ \\
\hline NM & $20.0 \%$ & $15.4 \%$ & $23.5 \%$ & $27.3 \%$ & $40.0 \%$ & $33.3 \%$ & $16.7 \%$ & $7.1 \%$ & $45.5 \%$ & $24.2 \%$ \\
\hline$T E$ & $20.0 \%$ & $0.0 \%$ & $5.9 \%$ & $9.1 \%$ & $0.0 \%$ & $66.7 \%$ & $0.0 \%$ & $0.0 \%$ & $9.1 \%$ & $7.4 \%$ \\
\hline$O T$ & $0.0 \%$ & $0.0 \%$ & $0.0 \%$ & $0.0 \%$ & $0.0 \%$ & $0.0 \%$ & $0.0 \%$ & $0.0 \%$ & $0.0 \%$ & $0.0 \%$ \\
\hline
\end{tabular}

Table C-8 - District Visit Maintenance Summary - Element 108 - Secondary Roads 


\begin{tabular}{|c|c|c|c|c|c|c|c|c|c|c|}
\hline Total & \multicolumn{10}{|c|}{ District } \\
\hline Element 301 & 1 & 2 & 3 & 4 & 5 & 6 & 7 & 8 & 9 & Total \\
\hline Count & 10 & 12 & 3 & 12 & 14 & 23 & 11 & 6 & 11 & 102 \\
\hline Avg. Quant. & 31.25 & 28.93 & 51.73 & 61.64 & 44.89 & 60.87 & 43.10 & 17.73 & 51.52 & 46.37 \\
\hline Tot. Quant. & 312.51 & 347.14 & 155.19 & 739.67 & 628.46 & 1400.08 & 474.12 & 106.35 & 566.69 & 4730.22 \\
\hline DJ & $70.0 \%$ & $41.7 \%$ & $66.7 \%$ & $33.3 \%$ & $42.9 \%$ & $60.9 \%$ & $72.7 \%$ & $50.0 \%$ & $27.3 \%$ & $51.0 \%$ \\
\hline AQ & $0.0 \%$ & $0.0 \%$ & $0.0 \%$ & $0.0 \%$ & $0.0 \%$ & $0.0 \%$ & $0.0 \%$ & $0.0 \%$ & $0.0 \%$ & $0.0 \%$ \\
\hline$S R$ & $0.0 \%$ & $0.0 \%$ & $0.0 \%$ & $25.0 \%$ & $7.1 \%$ & $0.0 \%$ & $0.0 \%$ & $0.0 \%$ & $0.0 \%$ & $3.9 \%$ \\
\hline$R R$ & $0.0 \%$ & $0.0 \%$ & $0.0 \%$ & $0.0 \%$ & $0.0 \%$ & $4.3 \%$ & $0.0 \%$ & $0.0 \%$ & $0.0 \%$ & $1.0 \%$ \\
\hline NM & $20.0 \%$ & $50.0 \%$ & $33.3 \%$ & $41.7 \%$ & $50.0 \%$ & $30.4 \%$ & $27.3 \%$ & $50.0 \%$ & $54.5 \%$ & $39.2 \%$ \\
\hline$T E$ & $10.0 \%$ & $8.3 \%$ & $0.0 \%$ & $0.0 \%$ & $0.0 \%$ & $4.3 \%$ & $0.0 \%$ & $0.0 \%$ & $9.1 \%$ & $3.9 \%$ \\
\hline OT & $0.0 \%$ & $0.0 \%$ & $0.0 \%$ & $0.0 \%$ & $0.0 \%$ & $0.0 \%$ & $0.0 \%$ & $0.0 \%$ & $9.1 \%$ & $1.0 \%$ \\
\hline
\end{tabular}

Table C-9 - District Visit Maintenance Summary - Element 301 - All Road Classifications

\begin{tabular}{|c|c|c|c|c|c|c|c|c|c|c|}
\hline Interstate & \multicolumn{10}{|c|}{ District } \\
\hline Element 301 & 1 & 2 & 3 & 4 & 5 & 6 & 7 & 8 & 9 & Total \\
\hline Count & 2 & 0 & 0 & 3 & 3 & 3 & 0 & 0 & 1 & 12 \\
\hline Avg. Quant. & 37.89 & N/A & N/A & 58.53 & 31.36 & 98.09 & N/A & N/A & 119.93 & 63.30 \\
\hline Tot. Quant. & 75.78 & 0.00 & 0.00 & 175.60 & 94.08 & 294.26 & 0.00 & 0.00 & 119.93 & 759.64 \\
\hline$D J$ & $100.0 \%$ & N/A & N/A & $33.3 \%$ & $33.3 \%$ & $33.3 \%$ & N/A & N/A & $0.0 \%$ & $41.7 \%$ \\
\hline$A Q$ & $0.0 \%$ & N/A & N/A & $0.0 \%$ & $0.0 \%$ & $0.0 \%$ & N/A & N/A & $0.0 \%$ & $0.0 \%$ \\
\hline$S R$ & $0.0 \%$ & N/A & N/A & $0.0 \%$ & $0.0 \%$ & $0.0 \%$ & N/A & N/A & $0.0 \%$ & $0.0 \%$ \\
\hline$R R$ & $0.0 \%$ & N/A & N/A & $0.0 \%$ & $0.0 \%$ & $33.3 \%$ & N/A & N/A & $0.0 \%$ & $8.3 \%$ \\
\hline NM & $0.0 \%$ & N/A & N/A & $66.7 \%$ & $66.7 \%$ & $33.3 \%$ & N/A & N/A & $100.0 \%$ & $50.0 \%$ \\
\hline$T E$ & $0.0 \%$ & N/A & N/A & $0.0 \%$ & $0.0 \%$ & $0.0 \%$ & N/A & N/A & $0.0 \%$ & $0.0 \%$ \\
\hline$O T$ & $0.0 \%$ & N/A & N/A & $0.0 \%$ & $0.0 \%$ & $0.0 \%$ & N/A & N/A & $0.0 \%$ & $0.0 \%$ \\
\hline
\end{tabular}

Table C-10 - District Visit Maintenance Summary - Element 301 - Interstate Roads 


\begin{tabular}{|c|c|c|c|c|c|c|c|c|c|c|}
\hline Primary & \multicolumn{10}{|c|}{ District } \\
\hline Element 301 & 1 & 2 & 3 & 4 & 5 & 6 & 7 & 8 & 9 & Total \\
\hline Count & 0 & 0 & 1 & 6 & 4 & 5 & 1 & 1 & 8 & 26 \\
\hline Avg. Quant. & N/A & N/A & 0.00 & 29.27 & 23.52 & 58.85 & 0.00 & 0.00 & 14.99 & 29.22 \\
\hline Tot. Quant. & 75.78 & 0.00 & 0.00 & 175.60 & 94.08 & 294.26 & 0.00 & 0.00 & 119.93 & 759.64 \\
\hline$D J$ & N/A & N/A & $100.0 \%$ & $33.3 \%$ & $50.0 \%$ & $20.0 \%$ & $0.0 \%$ & $0.0 \%$ & $37.5 \%$ & $34.6 \%$ \\
\hline$A Q$ & N/A & N/A & $0.0 \%$ & $0.0 \%$ & $0.0 \%$ & $0.0 \%$ & $0.0 \%$ & $0.0 \%$ & $0.0 \%$ & $0.0 \%$ \\
\hline$S R$ & N/A & N/A & $0.0 \%$ & $33.3 \%$ & $25.0 \%$ & $0.0 \%$ & $0.0 \%$ & $0.0 \%$ & $0.0 \%$ & $11.5 \%$ \\
\hline$R R$ & N/A & N/A & $0.0 \%$ & $0.0 \%$ & $0.0 \%$ & $0.0 \%$ & $0.0 \%$ & $0.0 \%$ & $0.0 \%$ & $0.0 \%$ \\
\hline NM & N/A & N/A & $0.0 \%$ & $33.3 \%$ & $25.0 \%$ & $80.0 \%$ & $100.0 \%$ & $100.0 \%$ & $50.0 \%$ & $50.0 \%$ \\
\hline$T E$ & N/A & N/A & $0.0 \%$ & $0.0 \%$ & $0.0 \%$ & $0.0 \%$ & $0.0 \%$ & $0.0 \%$ & $0.0 \%$ & $0.0 \%$ \\
\hline$O T$ & N/A & N/A & $0.0 \%$ & $0.0 \%$ & $0.0 \%$ & $0.0 \%$ & $0.0 \%$ & $0.0 \%$ & $12.5 \%$ & $3.8 \%$ \\
\hline
\end{tabular}

Table C-11 - District Visit Maintenance Summary - Element 301 - Primary Roads

\begin{tabular}{|c|c|c|c|c|c|c|c|c|c|c|}
\hline Secondary & \multicolumn{10}{|c|}{ District } \\
\hline Element 301 & 1 & 2 & 3 & 4 & 5 & 6 & 7 & 8 & 9 & Total \\
\hline Count & 8 & 12 & 2 & 3 & 7 & 15 & 10 & 5 & 2 & 64 \\
\hline Avg. Quant. & 9.47 & 0.00 & 0.00 & 58.53 & 13.44 & 19.62 & 0.00 & 0.00 & 59.96 & 11.87 \\
\hline Tot. Quant. & 75.78 & 0.00 & 0.00 & 175.60 & 94.08 & 294.26 & 0.00 & 0.00 & 119.93 & 759.64 \\
\hline$D J$ & $62.5 \%$ & $41.7 \%$ & $50.0 \%$ & $33.3 \%$ & $42.9 \%$ & $80.0 \%$ & $80.0 \%$ & $60.0 \%$ & $0.0 \%$ & $59.4 \%$ \\
\hline$A Q$ & $0.0 \%$ & $0.0 \%$ & $0.0 \%$ & $0.0 \%$ & $0.0 \%$ & $0.0 \%$ & $0.0 \%$ & $0.0 \%$ & $0.0 \%$ & $0.0 \%$ \\
\hline$S R$ & $0.0 \%$ & $0.0 \%$ & $0.0 \%$ & $33.3 \%$ & $0.0 \%$ & $0.0 \%$ & $0.0 \%$ & $0.0 \%$ & $0.0 \%$ & $1.6 \%$ \\
\hline$R R$ & $0.0 \%$ & $0.0 \%$ & $0.0 \%$ & $0.0 \%$ & $0.0 \%$ & $0.0 \%$ & $0.0 \%$ & $0.0 \%$ & $0.0 \%$ & $0.0 \%$ \\
\hline$N M$ & $25.0 \%$ & $50.0 \%$ & $50.0 \%$ & $33.3 \%$ & $57.1 \%$ & $13.3 \%$ & $20.0 \%$ & $40.0 \%$ & $50.0 \%$ & $32.8 \%$ \\
\hline$T E$ & $12.5 \%$ & $8.3 \%$ & $0.0 \%$ & $0.0 \%$ & $0.0 \%$ & $6.7 \%$ & $0.0 \%$ & $0.0 \%$ & $50.0 \%$ & $6.3 \%$ \\
\hline$O T$ & $0.0 \%$ & $0.0 \%$ & $0.0 \%$ & $0.0 \%$ & $0.0 \%$ & $0.0 \%$ & $0.0 \%$ & $0.0 \%$ & $0.0 \%$ & $0.0 \%$ \\
\hline
\end{tabular}

Table C-12 - District Visit Maintenance Summary - Element 301 - Secondary Roads 


\begin{tabular}{|c|c|c|c|c|c|c|c|c|c|c|}
\hline Total & \multicolumn{10}{|c|}{ District } \\
\hline Element 302 & 1 & 2 & 3 & 4 & 5 & 6 & 7 & 8 & 9 & Total \\
\hline Count & 9 & 12 & 14 & 9 & 15 & 18 & 8 & 18 & 13 & 116 \\
\hline Avg. Quant. & 74.18 & 47.95 & 57.19 & 39.79 & 64.54 & 45.63 & 60.83 & 48.44 & 72.85 & 56.01 \\
\hline Tot. Quant. & 667.65 & 575.43 & 800.68 & 358.07 & 968.15 & 821.29 & 486.60 & 871.83 & 947.08 & 6496.79 \\
\hline DJ & $44.4 \%$ & $75.0 \%$ & $92.9 \%$ & $11.1 \%$ & $53.3 \%$ & $27.8 \%$ & $50.0 \%$ & $77.8 \%$ & $15.4 \%$ & $51.7 \%$ \\
\hline AQ & $0.0 \%$ & $0.0 \%$ & $0.0 \%$ & $0.0 \%$ & $0.0 \%$ & $0.0 \%$ & $0.0 \%$ & $5.6 \%$ & $0.0 \%$ & $0.9 \%$ \\
\hline$S R$ & $11.1 \%$ & $0.0 \%$ & $0.0 \%$ & $22.2 \%$ & $0.0 \%$ & $0.0 \%$ & $0.0 \%$ & $0.0 \%$ & $0.0 \%$ & $2.6 \%$ \\
\hline$R R$ & $11.1 \%$ & $0.0 \%$ & $0.0 \%$ & $0.0 \%$ & $0.0 \%$ & $5.6 \%$ & $0.0 \%$ & $5.6 \%$ & $0.0 \%$ & $2.6 \%$ \\
\hline NM & $33.3 \%$ & $25.0 \%$ & $7.1 \%$ & $66.7 \%$ & $46.7 \%$ & $66.7 \%$ & $25.0 \%$ & $11.1 \%$ & $76.9 \%$ & $39.7 \%$ \\
\hline$T E$ & $0.0 \%$ & $0.0 \%$ & $0.0 \%$ & $0.0 \%$ & $0.0 \%$ & $0.0 \%$ & $12.5 \%$ & $0.0 \%$ & $7.7 \%$ & $1.7 \%$ \\
\hline$O T$ & $0.0 \%$ & $0.0 \%$ & $0.0 \%$ & $0.0 \%$ & $0.0 \%$ & $0.0 \%$ & $12.5 \%$ & $0.0 \%$ & $0.0 \%$ & $0.9 \%$ \\
\hline
\end{tabular}

Table C-13 - District Visit Maintenance Summary - Element 302 - All Road Classifications

\begin{tabular}{|c|c|c|c|c|c|c|c|c|c|c|}
\hline Interstate & \multicolumn{10}{|c|}{ District } \\
\hline Element 302 & 1 & 2 & 3 & 4 & 5 & 6 & 7 & 8 & 9 & Total \\
\hline Count & 1 & 1 & 0 & 5 & 9 & 2 & 2 & 10 & 4 & 34 \\
\hline Avg. Quant. & 66.00 & 73.07 & $\mathrm{~N} / \mathrm{A}$ & 39.20 & 75.28 & 63.10 & 69.24 & 49.75 & 109.79 & 65.11 \\
\hline Tot. Quant. & 66.00 & 73.07 & 0.00 & 195.98 & 677.51 & 126.20 & 138.48 & 497.48 & 439.17 & 2213.89 \\
\hline$D J$ & $100.0 \%$ & $0.0 \%$ & $\mathrm{~N} / \mathrm{A}$ & $20.0 \%$ & $33.3 \%$ & $50.0 \%$ & $50.0 \%$ & $80.0 \%$ & $25.0 \%$ & $47.1 \%$ \\
\hline AQ & $0.0 \%$ & $0.0 \%$ & $\mathrm{~N} / \mathrm{A}$ & $0.0 \%$ & $0.0 \%$ & $0.0 \%$ & $0.0 \%$ & $0.0 \%$ & $0.0 \%$ & $0.0 \%$ \\
\hline$S R$ & $0.0 \%$ & $0.0 \%$ & $\mathrm{~N} / \mathrm{A}$ & $40.0 \%$ & $0.0 \%$ & $0.0 \%$ & $0.0 \%$ & $0.0 \%$ & $0.0 \%$ & $5.9 \%$ \\
\hline$R R$ & $0.0 \%$ & $0.0 \%$ & $\mathrm{~N} / \mathrm{A}$ & $0.0 \%$ & $0.0 \%$ & $0.0 \%$ & $0.0 \%$ & $0.0 \%$ & $0.0 \%$ & $0.0 \%$ \\
\hline NM & $0.0 \%$ & $100.0 \%$ & $\mathrm{~N} / \mathrm{A}$ & $40.0 \%$ & $66.7 \%$ & $50.0 \%$ & $0.0 \%$ & $20.0 \%$ & $75.0 \%$ & $44.1 \%$ \\
\hline$T E$ & $0.0 \%$ & $0.0 \%$ & $\mathrm{~N} / \mathrm{A}$ & $0.0 \%$ & $0.0 \%$ & $0.0 \%$ & $0.0 \%$ & $0.0 \%$ & $0.0 \%$ & $0.0 \%$ \\
\hline$O T$ & $0.0 \%$ & $0.0 \%$ & $\mathrm{~N} / \mathrm{A}$ & $0.0 \%$ & $0.0 \%$ & $0.0 \%$ & $50.0 \%$ & $0.0 \%$ & $0.0 \%$ & $2.9 \%$ \\
\hline
\end{tabular}

Table C-14 - District Visit Maintenance Summary - Element 302 - Interstate Roads 


\begin{tabular}{|c|c|c|c|c|c|c|c|c|c|c|}
\hline Primary & \multicolumn{10}{|c|}{ District } \\
\hline Element 302 & 1 & 2 & 3 & 4 & 5 & 6 & 7 & 8 & 9 & Total \\
\hline Count & 4 & 5 & 6 & 2 & 3 & 12 & 5 & 0 & 6 & 43 \\
\hline Avg. Quant. & 95.94 & 48.20 & 89.84 & 52.99 & 57.03 & 43.02 & 62.25 & N/A & 38.43 & 58.12 \\
\hline Tot. Quant. & 383.75 & 241.01 & 539.06 & 105.98 & 171.08 & 516.30 & 311.23 & 0.00 & 230.57 & 2498.97 \\
\hline$D J$ & $25.0 \%$ & $100.0 \%$ & $83.3 \%$ & $0.0 \%$ & $66.7 \%$ & $33.3 \%$ & $60.0 \%$ & N/A & $16.7 \%$ & $48.8 \%$ \\
\hline$A Q$ & $0.0 \%$ & $0.0 \%$ & $0.0 \%$ & $0.0 \%$ & $0.0 \%$ & $0.0 \%$ & $0.0 \%$ & N/A & $0.0 \%$ & $0.0 \%$ \\
\hline$S R$ & $25.0 \%$ & $0.0 \%$ & $0.0 \%$ & $0.0 \%$ & $0.0 \%$ & $0.0 \%$ & $0.0 \%$ & N/A & $0.0 \%$ & $2.3 \%$ \\
\hline$R R$ & $0.0 \%$ & $0.0 \%$ & $0.0 \%$ & $0.0 \%$ & $0.0 \%$ & $0.0 \%$ & $0.0 \%$ & N/A & $0.0 \%$ & $0.0 \%$ \\
\hline NM & $50.0 \%$ & $0.0 \%$ & $16.7 \%$ & $100.0 \%$ & $33.3 \%$ & $66.7 \%$ & $40.0 \%$ & N/A & $83.3 \%$ & $48.8 \%$ \\
\hline$T E$ & $0.0 \%$ & $0.0 \%$ & $0.0 \%$ & $0.0 \%$ & $0.0 \%$ & $0.0 \%$ & $0.0 \%$ & N/A & $0.0 \%$ & $0.0 \%$ \\
\hline$O T$ & $0.0 \%$ & $0.0 \%$ & $0.0 \%$ & $0.0 \%$ & $0.0 \%$ & $0.0 \%$ & $0.0 \%$ & N/A & $0.0 \%$ & $0.0 \%$ \\
\hline
\end{tabular}

Table C-15 - District Visit Maintenance Summary - Element 302 - Primary Roads

\begin{tabular}{|c|c|c|c|c|c|c|c|c|c|c|}
\hline Secondary & \multicolumn{10}{|c|}{ District } \\
\hline Element 302 & 1 & 2 & 3 & 4 & 5 & 6 & 7 & 8 & 9 & Total \\
\hline Count & 4 & 6 & 8 & 2 & 3 & 4 & 1 & 8 & 3 & 39 \\
\hline Avg. Quant. & 54.48 & 43.56 & 32.70 & 28.06 & 39.85 & 44.70 & 36.89 & 46.79 & 92.45 & 45.74 \\
\hline Tot. Quant. & 217.90 & 261.35 & 261.61 & 56.11 & 119.56 & 178.79 & 36.89 & 374.36 & 277.35 & 1783.93 \\
\hline$D J$ & $50.0 \%$ & $66.7 \%$ & $100.0 \%$ & $0.0 \%$ & $100.0 \%$ & $0.0 \%$ & $0.0 \%$ & $75.0 \%$ & $0.0 \%$ & $59.0 \%$ \\
\hline$A Q$ & $0.0 \%$ & $0.0 \%$ & $0.0 \%$ & $0.0 \%$ & $0.0 \%$ & $0.0 \%$ & $0.0 \%$ & $12.5 \%$ & $0.0 \%$ & $2.6 \%$ \\
\hline$S R$ & $0.0 \%$ & $0.0 \%$ & $0.0 \%$ & $0.0 \%$ & $0.0 \%$ & $0.0 \%$ & $0.0 \%$ & $0.0 \%$ & $0.0 \%$ & $0.0 \%$ \\
\hline$R R$ & $25.0 \%$ & $0.0 \%$ & $0.0 \%$ & $0.0 \%$ & $0.0 \%$ & $25.0 \%$ & $0.0 \%$ & $12.5 \%$ & $0.0 \%$ & $7.7 \%$ \\
\hline$N M$ & $25.0 \%$ & $33.3 \%$ & $0.0 \%$ & $100.0 \%$ & $0.0 \%$ & $75.0 \%$ & $0.0 \%$ & $0.0 \%$ & $66.7 \%$ & $25.6 \%$ \\
\hline$T E$ & $0.0 \%$ & $0.0 \%$ & $0.0 \%$ & $0.0 \%$ & $0.0 \%$ & $0.0 \%$ & $100.0 \%$ & $0.0 \%$ & $33.3 \%$ & $5.1 \%$ \\
\hline$O T$ & $0.0 \%$ & $0.0 \%$ & $0.0 \%$ & $0.0 \%$ & $0.0 \%$ & $0.0 \%$ & $0.0 \%$ & $0.0 \%$ & $0.0 \%$ & $0.0 \%$ \\
\hline
\end{tabular}

Table C-16 - District Visit Maintenance Summary - Element 302 - Secondary Roads 


\begin{tabular}{|c|c|c|c|c|c|c|c|c|c|c|}
\hline Total & \multicolumn{10}{|c|}{ District } \\
\hline Element 311 & 1 & 2 & 3 & 4 & 5 & 6 & 7 & 8 & 9 & Total \\
\hline Count & 7 & 5 & 1 & 9 & 0 & 1 & 0 & 2 & 2 & 27 \\
\hline Avg. Quant. & 24.54 & 15.20 & 15.00 & 19.32 & N/A & 70.00 & N/A & 70.00 & 15.40 & 25.09 \\
\hline Tot. Quant. & 171.79 & 76.00 & 15.00 & 173.84 & 0.00 & 70.00 & 0.00 & 140.00 & 30.80 & 677.43 \\
\hline$B R$ & $14.3 \%$ & $0.0 \%$ & $100.0 \%$ & $11.1 \%$ & N/A & $0.0 \%$ & N/A & $50.0 \%$ & $50.0 \%$ & $18.5 \%$ \\
\hline$B P$ & $0.0 \%$ & $40.0 \%$ & $0.0 \%$ & $22.2 \%$ & N/A & $0.0 \%$ & N/A & $50.0 \%$ & $0.0 \%$ & $18.5 \%$ \\
\hline$S R$ & $71.4 \%$ & $0.0 \%$ & $0.0 \%$ & $11.1 \%$ & N/A & $0.0 \%$ & N/A & $0.0 \%$ & $0.0 \%$ & $22.2 \%$ \\
\hline$A Q$ & $0.0 \%$ & $0.0 \%$ & $0.0 \%$ & $0.0 \%$ & N/A & $0.0 \%$ & N/A & $0.0 \%$ & $0.0 \%$ & $0.0 \%$ \\
\hline NM & $0.0 \%$ & $40.0 \%$ & $0.0 \%$ & $55.6 \%$ & N/A & $100.0 \%$ & N/A & $0.0 \%$ & $50.0 \%$ & $33.3 \%$ \\
\hline$T E$ & $0.0 \%$ & $0.0 \%$ & $0.0 \%$ & $0.0 \%$ & N/A & $0.0 \%$ & N/A & $0.0 \%$ & $0.0 \%$ & $0.0 \%$ \\
\hline$O T$ & $14.3 \%$ & $20.0 \%$ & $0.0 \%$ & $0.0 \%$ & N/A & $0.0 \%$ & N/A & $0.0 \%$ & $0.0 \%$ & $7.4 \%$ \\
\hline
\end{tabular}

Table C-17 - District Visit Maintenance Summary - Element 311 - All Road Classifications

\begin{tabular}{|c|c|c|c|c|c|c|c|c|c|c|c|}
\hline Interstate & \multicolumn{10}{|c|}{ District } \\
\hline Element 311 & 1 & 2 & 3 & 4 & 5 & 6 & 7 & 8 & 9 & Total \\
\hline Count & 0 & 2 & 0 & 4 & 0 & 1 & 0 & 2 & 0 & 9 \\
\hline Avg. Quant. & N/A & 14.50 & N/A & 23.03 & N/A & 70.00 & N/A & 70.00 & N/A & 36.79 \\
\hline Tot. Quant. & 0.00 & 29.00 & 0.00 & 92.11 & 0.00 & 70.00 & 0.00 & 140.00 & 0.00 & 331.11 \\
\hline$B R$ & N/A & $0.0 \%$ & N/A & $0.0 \%$ & N/A & $0.0 \%$ & N/A & $50.0 \%$ & N/A & $11.1 \%$ \\
\hline$B P$ & N/A & $0.0 \%$ & N/A & $25.0 \%$ & N/A & $0.0 \%$ & N/A & $50.0 \%$ & N/A & $22.2 \%$ \\
\hline$S R$ & N/A & $0.0 \%$ & N/A & $25.0 \%$ & N/A & $0.0 \%$ & N/A & $0.0 \%$ & N/A & $11.1 \%$ \\
\hline$A Q$ & N/A & $0.0 \%$ & N/A & $0.0 \%$ & N/A & $0.0 \%$ & N/A & $0.0 \%$ & N/A & $0.0 \%$ \\
\hline NM & N/A & $50.0 \%$ & N/A & $50.0 \%$ & N/A & $100.0 \%$ & N/A & $0.0 \%$ & N/A & $44.4 \%$ \\
\hline$T E$ & N/A & $0.0 \%$ & N/A & $0.0 \%$ & N/A & $0.0 \%$ & N/A & $0.0 \%$ & N/A & $0.0 \%$ \\
\hline$O T$ & N/A & $50.0 \%$ & N/A & $0.0 \%$ & N/A & $0.0 \%$ & N/A & $0.0 \%$ & N/A & $11.1 \%$ \\
\hline
\end{tabular}

Table C-18 - District Visit Maintenance Summary - Element 311 - Interstate Roads 


\begin{tabular}{|c|c|c|c|c|c|c|c|c|c|c|}
\hline Primary & \multicolumn{10}{|c|}{ District } \\
\hline Element 311 & 1 & 2 & 3 & 4 & 5 & 6 & 7 & 8 & 9 & Total \\
\hline Count & 5 & 1 & 1 & 4 & 0 & 0 & 0 & 0 & 1 & 12 \\
\hline Avg. Quant. & 25.49 & 15.00 & 15.00 & 16.43 & N/A & N/A & N/A & N/A & 28.80 & 21.00 \\
\hline Tot. Quant. & 127.46 & 15.00 & 15.00 & 65.73 & 0.00 & 0.00 & 0.00 & 0.00 & 28.80 & 251.99 \\
\hline$B R$ & $20.0 \%$ & $0.0 \%$ & $100.0 \%$ & $25.0 \%$ & N/A & N/A & N/A & N/A & $0.0 \%$ & $25.0 \%$ \\
\hline$B P$ & $0.0 \%$ & $100.0 \%$ & $0.0 \%$ & $25.0 \%$ & N/A & N/A & N/A & N/A & $0.0 \%$ & $16.7 \%$ \\
\hline$S R$ & $60.0 \%$ & $0.0 \%$ & $0.0 \%$ & $0.0 \%$ & N/A & N/A & N/A & N/A & $0.0 \%$ & $25.0 \%$ \\
\hline$A Q$ & $0.0 \%$ & $0.0 \%$ & $0.0 \%$ & $0.0 \%$ & N/A & N/A & N/A & N/A & $0.0 \%$ & $0.0 \%$ \\
\hline NM & $0.0 \%$ & $0.0 \%$ & $0.0 \%$ & $50.0 \%$ & N/A & N/A & N/A & N/A & $100.0 \%$ & $25.0 \%$ \\
\hline$T E$ & $0.0 \%$ & $0.0 \%$ & $0.0 \%$ & $0.0 \%$ & N/A & N/A & N/A & N/A & $0.0 \%$ & $0.0 \%$ \\
\hline OT & $20.0 \%$ & $0.0 \%$ & $0.0 \%$ & $0.0 \%$ & N/A & N/A & N/A & N/A & $0.0 \%$ & $8.3 \%$ \\
\hline
\end{tabular}

Table C-19 - District Visit Maintenance Summary - Element 311 - Primary Roads

\begin{tabular}{|c|c|c|c|c|c|c|c|c|c|c|}
\hline Secondary & \multicolumn{10}{|c|}{ District } \\
\hline Element 311 & 1 & 2 & 3 & 4 & 5 & 6 & 7 & 8 & \multicolumn{1}{c|}{9} & Total \\
\hline Count & 2 & 2 & 0 & 1 & 0 & 0 & 0 & 0 & 1 & 6 \\
\hline Avg. Quant. & 22.17 & 16.00 & N/A & 16.00 & N/A & N/A & N/A & N/A & 2.00 & 15.72 \\
\hline Tot. Quant. & 44.33 & 32.00 & 0.00 & 16.00 & 0.00 & 0.00 & 0.00 & 0.00 & 2.00 & 94.33 \\
\hline$B R$ & $0.0 \%$ & $0.0 \%$ & N/A & $0.0 \%$ & N/A & N/A & N/A & N/A & $100.0 \%$ & $16.7 \%$ \\
\hline$B P$ & $0.0 \%$ & $50.0 \%$ & N/A & $0.0 \%$ & N/A & N/A & N/A & N/A & $0.0 \%$ & $16.7 \%$ \\
\hline$S R$ & $100.0 \%$ & $0.0 \%$ & N/A & $0.0 \%$ & N/A & N/A & N/A & N/A & $0.0 \%$ & $33.3 \%$ \\
\hline$A Q$ & $0.0 \%$ & $0.0 \%$ & N/A & $0.0 \%$ & N/A & N/A & N/A & N/A & $0.0 \%$ & $0.0 \%$ \\
\hline NM & $0.0 \%$ & $50.0 \%$ & N/A & $100.0 \%$ & N/A & N/A & N/A & N/A & $0.0 \%$ & $33.3 \%$ \\
\hline$T E$ & $0.0 \%$ & $0.0 \%$ & N/A & $0.0 \%$ & N/A & N/A & N/A & N/A & $0.0 \%$ & $0.0 \%$ \\
\hline$O T$ & $0.0 \%$ & $0.0 \%$ & N/A & $0.0 \%$ & N/A & N/A & N/A & N/A & $0.0 \%$ & $0.0 \%$ \\
\hline
\end{tabular}

Table C-20 - District Visit Maintenance Summary - Element 311 - Secondary Roads 


\begin{tabular}{|c|c|c|c|c|c|c|c|c|c|c|}
\hline Total & \multicolumn{10}{|c|}{ District } \\
\hline Element 313 & 1 & 2 & 3 & 4 & 5 & 6 & 7 & 8 & 9 & Total \\
\hline Count & 5 & 1 & 1 & 9 & 0 & 0 & 0 & 1 & 2 & 19 \\
\hline Avg. Quant. & 18.94 & 8.00 & 24.00 & 20.64 & N/A & N/A & N/A & 18.00 & 21.00 & 19.60 \\
\hline Tot. Quant. & 94.70 & 8.00 & 24.00 & 185.78 & 0.00 & 0.00 & 0.00 & 18.00 & 42.00 & 372.48 \\
\hline$B R$ & $20.0 \%$ & $0.0 \%$ & $100.0 \%$ & $11.1 \%$ & N/A & N/A & N/A & $0.0 \%$ & $0.0 \%$ & $15.8 \%$ \\
\hline$B P$ & $0.0 \%$ & $0.0 \%$ & $0.0 \%$ & $33.3 \%$ & N/A & N/A & N/A & $100.0 \%$ & $50.0 \%$ & $26.3 \%$ \\
\hline$S R$ & $40.0 \%$ & $0.0 \%$ & $0.0 \%$ & $11.1 \%$ & N/A & N/A & N/A & $0.0 \%$ & $0.0 \%$ & $15.8 \%$ \\
\hline$A Q$ & $0.0 \%$ & $0.0 \%$ & $0.0 \%$ & $0.0 \%$ & N/A & N/A & N/A & $0.0 \%$ & $0.0 \%$ & $0.0 \%$ \\
\hline NM & $40.0 \%$ & $100.0 \%$ & $0.0 \%$ & $44.4 \%$ & N/A & N/A & N/A & $0.0 \%$ & $50.0 \%$ & $42.1 \%$ \\
\hline$T E$ & $0.0 \%$ & $0.0 \%$ & $0.0 \%$ & $0.0 \%$ & N/A & N/A & N/A & $0.0 \%$ & $0.0 \%$ & $0.0 \%$ \\
\hline$O T$ & $0.0 \%$ & $0.0 \%$ & $0.0 \%$ & $0.0 \%$ & N/A & N/A & N/A & $0.0 \%$ & $0.0 \%$ & $0.0 \%$ \\
\hline
\end{tabular}

Table C-21 - District Visit Maintenance Summary - Element 313 - All Road Classifications

\begin{tabular}{|c|c|c|c|c|c|c|c|c|c|c|}
\hline \multirow{2}{*}{$\frac{\text { Interstate }}{\text { Element } \mathbf{3 1 3}}$} & \multicolumn{10}{|c|}{ District } \\
\hline & 1 & 2 & 3 & 4 & 5 & 6 & 7 & 8 & 9 & Total \\
\hline Count & 0 & 0 & 0 & 2 & 0 & 0 & 0 & 1 & 2 & 5 \\
\hline Avg. Quant. & $\mathrm{N} / \mathrm{A}$ & $\mathrm{N} / \mathrm{A}$ & $\mathrm{N} / \mathrm{A}$ & 29.56 & $\mathrm{~N} / \mathrm{A}$ & $\mathrm{N} / \mathrm{A}$ & $\mathrm{N} / \mathrm{A}$ & 18.00 & 21.00 & 23.82 \\
\hline Tot. Quant. & 0.00 & 0.00 & 0.00 & 59.11 & 0.00 & 0.00 & 0.00 & 18.00 & 42.00 & 119.11 \\
\hline$B R$ & $\mathrm{~N} / \mathrm{A}$ & $\mathrm{N} / \mathrm{A}$ & $\mathrm{N} / \mathrm{A}$ & $0.0 \%$ & $\mathrm{~N} / \mathrm{A}$ & $\mathrm{N} / \mathrm{A}$ & $\mathrm{N} / \mathrm{A}$ & $0.0 \%$ & $0.0 \%$ & $0.0 \%$ \\
\hline$B P$ & $\mathrm{~N} / \mathrm{A}$ & $\mathrm{N} / \mathrm{A}$ & $\mathrm{N} / \mathrm{A}$ & $50.0 \%$ & N/A & $\mathrm{N} / \mathrm{A}$ & $\mathrm{N} / \mathrm{A}$ & $100.0 \%$ & $50.0 \%$ & $60.0 \%$ \\
\hline$S R$ & $\mathrm{~N} / \mathrm{A}$ & $\mathrm{N} / \mathrm{A}$ & $\mathrm{N} / \mathrm{A}$ & $0.0 \%$ & $\mathrm{~N} / \mathrm{A}$ & $\mathrm{N} / \mathrm{A}$ & $\mathrm{N} / \mathrm{A}$ & $0.0 \%$ & $0.0 \%$ & $0.0 \%$ \\
\hline$A Q$ & $\mathrm{~N} / \mathrm{A}$ & $\mathrm{N} / \mathrm{A}$ & $\mathrm{N} / \mathrm{A}$ & $0.0 \%$ & $\mathrm{~N} / \mathrm{A}$ & $\mathrm{N} / \mathrm{A}$ & $\mathrm{N} / \mathrm{A}$ & $0.0 \%$ & $0.0 \%$ & $0.0 \%$ \\
\hline$N M$ & $\mathrm{~N} / \mathrm{A}$ & $\mathrm{N} / \mathrm{A}$ & $\mathrm{N} / \mathrm{A}$ & $50.0 \%$ & N/A & $\mathrm{N} / \mathrm{A}$ & $\mathrm{N} / \mathrm{A}$ & $0.0 \%$ & $50.0 \%$ & $40.0 \%$ \\
\hline$T E$ & $\mathrm{~N} / \mathrm{A}$ & $\mathrm{N} / \mathrm{A}$ & $\mathrm{N} / \mathrm{A}$ & $0.0 \%$ & $\mathrm{~N} / \mathrm{A}$ & $\mathrm{N} / \mathrm{A}$ & $\mathrm{N} / \mathrm{A}$ & $0.0 \%$ & $0.0 \%$ & $0.0 \%$ \\
\hline$O T$ & $\mathrm{~N} / \mathrm{A}$ & $\mathrm{N} / \mathrm{A}$ & $\mathrm{N} / \mathrm{A}$ & $0.0 \%$ & $\mathrm{~N} / \mathrm{A}$ & $\mathrm{N} / \mathrm{A}$ & $\mathrm{N} / \mathrm{A}$ & $0.0 \%$ & $0.0 \%$ & $0.0 \%$ \\
\hline
\end{tabular}

Table C-22 - District Visit Maintenance Summary - Element 313 - Interstate Roads 


\begin{tabular}{|c|c|c|c|c|c|c|c|c|c|c|}
\hline Primary & \multicolumn{10}{|c|}{ District } \\
\hline Element 313 & 1 & 2 & 3 & 4 & 5 & 6 & 7 & 8 & 9 & Total \\
\hline Count & 4 & 0 & 0 & 4 & 0 & 0 & 0 & 0 & 0 & 8 \\
\hline Avg. Quant. & 19.93 & N/A & N/A & 20.75 & N/A & N/A & N/A & N/A & N/A & 20.34 \\
\hline Tot. Quant. & 79.70 & 0.00 & 0.00 & 83.00 & 0.00 & 0.00 & 0.00 & 0.00 & 0.00 & 162.70 \\
\hline$B R$ & $25.0 \%$ & N/A & N/A & $0.0 \%$ & N/A & N/A & N/A & N/A & N/A & $12.5 \%$ \\
\hline$B P$ & $0.0 \%$ & N/A & N/A & $50.0 \%$ & N/A & N/A & N/A & N/A & N/A & $25.0 \%$ \\
\hline$S R$ & $50.0 \%$ & N/A & N/A & $0.0 \%$ & N/A & N/A & N/A & N/A & N/A & $25.0 \%$ \\
\hline$A Q$ & $0.0 \%$ & N/A & N/A & $0.0 \%$ & N/A & N/A & N/A & N/A & N/A & $0.0 \%$ \\
\hline NM & $25.0 \%$ & N/A & N/A & $50.0 \%$ & N/A & N/A & N/A & N/A & N/A & $37.5 \%$ \\
\hline$T E$ & $0.0 \%$ & N/A & N/A & $0.0 \%$ & N/A & N/A & N/A & N/A & N/A & $0.0 \%$ \\
\hline$O T$ & $0.0 \%$ & N/A & N/A & $0.0 \%$ & N/A & N/A & N/A & N/A & N/A & $0.0 \%$ \\
\hline
\end{tabular}

Table C-23 - District Visit Maintenance Summary - Element 313 - Primary Roads

\begin{tabular}{|c|c|c|c|c|c|c|c|c|c|c|}
\hline Secondary & \multicolumn{10}{|c|}{ District } \\
\hline Element 313 & 1 & 2 & 3 & 4 & 5 & 6 & 7 & 8 & 9 & Total \\
\hline Count & 1 & 1 & 1 & 3 & 0 & 0 & 0 & 0 & 0 & 6 \\
\hline Avg. Quant. & 15.00 & 8.00 & 24.00 & 14.56 & N/A & N/A & N/A & N/A & N/A & 15.11 \\
\hline Tot. Quant. & 15.00 & 8.00 & 24.00 & 43.67 & 0.00 & 0.00 & 0.00 & 0.00 & 0.00 & 90.67 \\
\hline$B R$ & $0.0 \%$ & $0.0 \%$ & $100.0 \%$ & $33.3 \%$ & N/A & N/A & N/A & N/A & N/A & $33.3 \%$ \\
\hline$B P$ & $0.0 \%$ & $0.0 \%$ & $0.0 \%$ & $0.0 \%$ & N/A & N/A & N/A & N/A & N/A & $0.0 \%$ \\
\hline$S R$ & $0.0 \%$ & $0.0 \%$ & $0.0 \%$ & $33.3 \%$ & N/A & N/A & N/A & N/A & N/A & $16.7 \%$ \\
\hline$A Q$ & $0.0 \%$ & $0.0 \%$ & $0.0 \%$ & $0.0 \%$ & N/A & N/A & N/A & N/A & N/A & $0.0 \%$ \\
\hline NM & $100.0 \%$ & $100.0 \%$ & $0.0 \%$ & $33.3 \%$ & N/A & N/A & N/A & N/A & N/A & $50.0 \%$ \\
\hline$T E$ & $0.0 \%$ & $0.0 \%$ & $0.0 \%$ & $0.0 \%$ & N/A & N/A & N/A & N/A & N/A & $0.0 \%$ \\
\hline$O T$ & $0.0 \%$ & $0.0 \%$ & $0.0 \%$ & $0.0 \%$ & N/A & N/A & N/A & N/A & N/A & $0.0 \%$ \\
\hline
\end{tabular}

Table C-24 - District Visit Maintenance Summary - Element 313 - Secondary Roads 


\begin{tabular}{|c|c|}
\hline Code & Corresponding Maintenance Action \\
\hline CP & Cleaned / Painted Structural Steel \\
\hline SR & Superstructure Replaced \\
\hline RB & Replaced Some Beams / Girders \\
\hline DJ & Deck Joints Resealed \\
\hline BR & Bearings Replaced \\
\hline BP & Bearings Painted \\
\hline AQ & Added Quantity (in CS 1) \\
\hline RR & Road Repaved \\
\hline NM & No Maintenance Noted \\
\hline TE & Typographical Error \\
\hline ND & No Data \\
\hline OT & Other \\
\hline
\end{tabular}

Table C-25 - Maintenance Action Codes and Descriptions

\begin{tabular}{|c|c|c|}
\hline Code & Corresponding Road Description & Road Classification \\
\hline 1 & Rural - Principal Arterial - Interstate & Interstate \\
\hline 2 & Rural - Principal Arterial - Other & Primary \\
\hline 6 & Rural - Minor Arterial & Primary \\
\hline 7 & Rural - Major Collector & Secondary \\
\hline 8 & Rural - Minor Collector & Secondary \\
\hline 9 & Rural - Local & Secondary \\
\hline 11 & Urban - Principal Arterial - Interstate & Interstate \\
\hline 12 & Urban - Principal Arterial - Freeway / Expressway & Primary \\
\hline 14 & Urban - Other Principal Arterial & Primary \\
\hline 16 & Urban - Minor Arterial & Primary \\
\hline 17 & Urban - Collector & Secondary \\
\hline 19 & Urban - Local & Secondary \\
\hline
\end{tabular}

Table C-26 - Road Type Codes, Descriptions, and Classifications 
APPENDIX D - FULL LLS AND MARKOV CHAIN RESULTS 


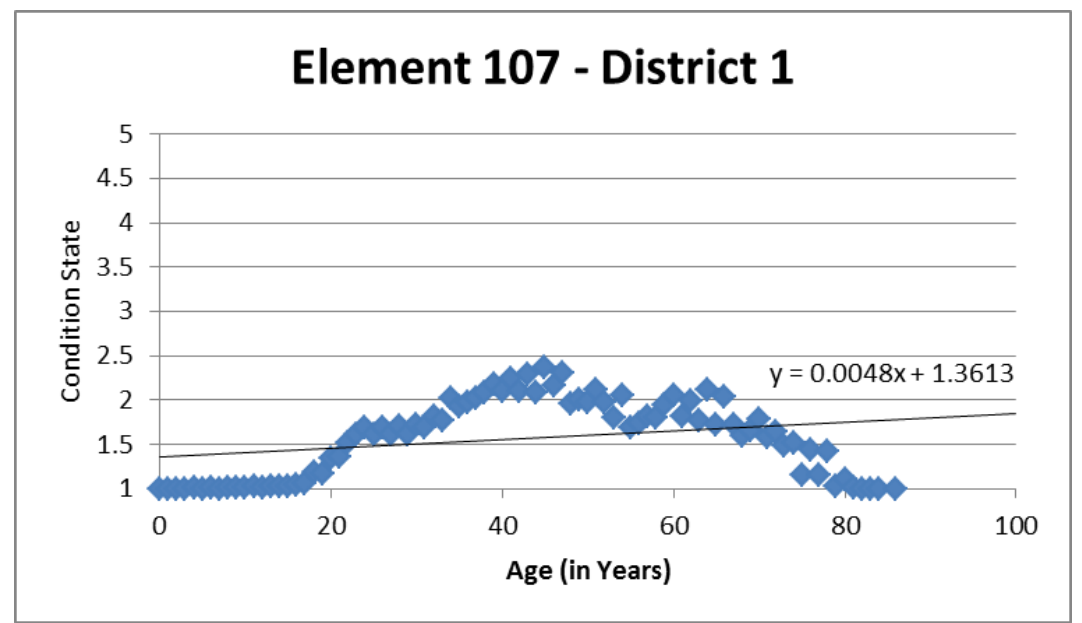

Figure D-1 - Condition State History and Linear Trendline by Age - Element 107 - District 1

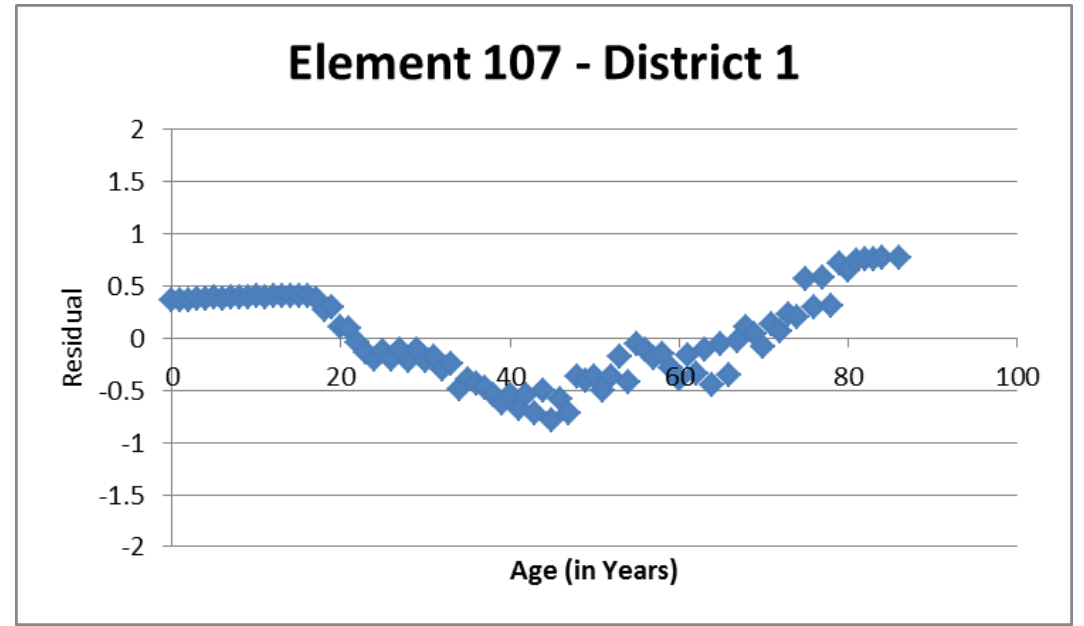

Figure D-2 - Condition States Residuals (Prediction Minus Actual) by Age - Element 107 - District 1

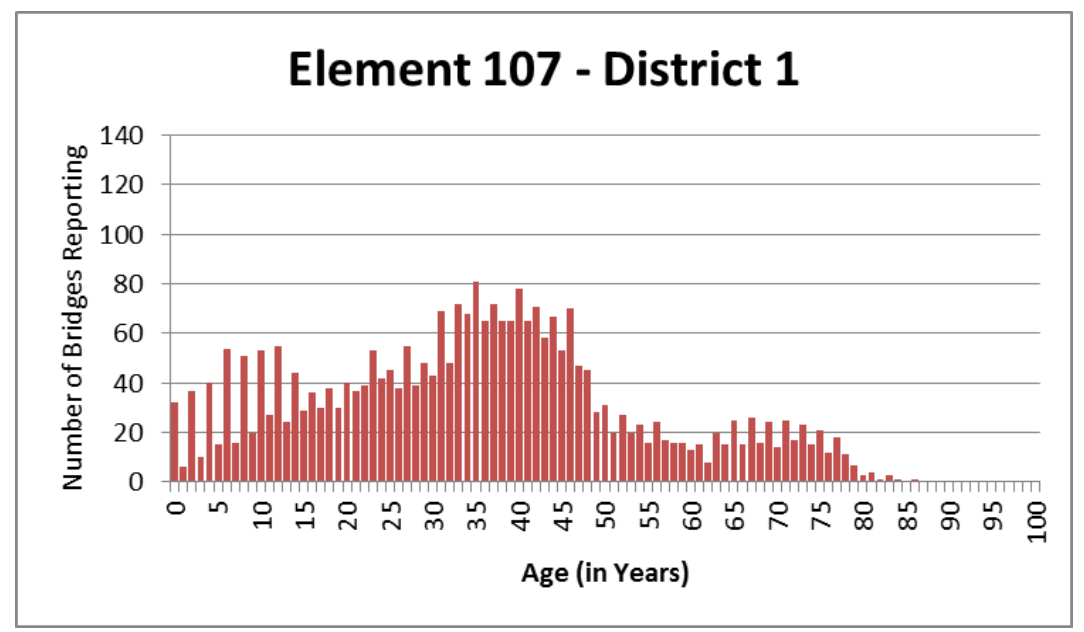

Figure D-3 - Number of Bridges Reporting by Age - Element 107 - District 1 


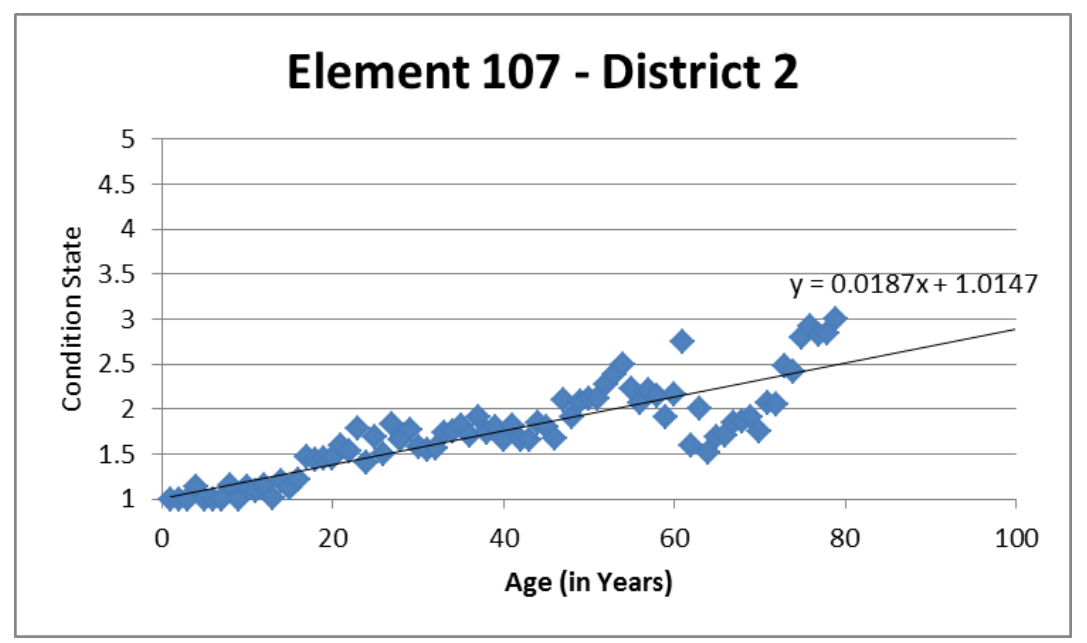

Figure D-4 - Condition State History and Linear Trendline by Age - Element 107 - District 2

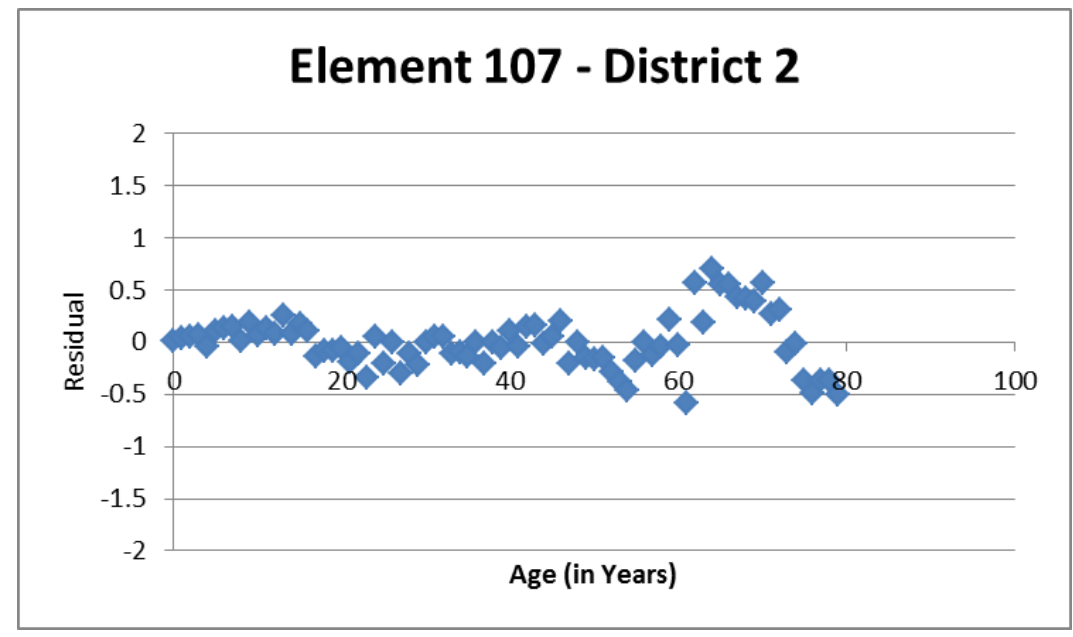

Figure D-5 - Condition States Residuals (Prediction Minus Actual) by Age - Element 107 - District 2

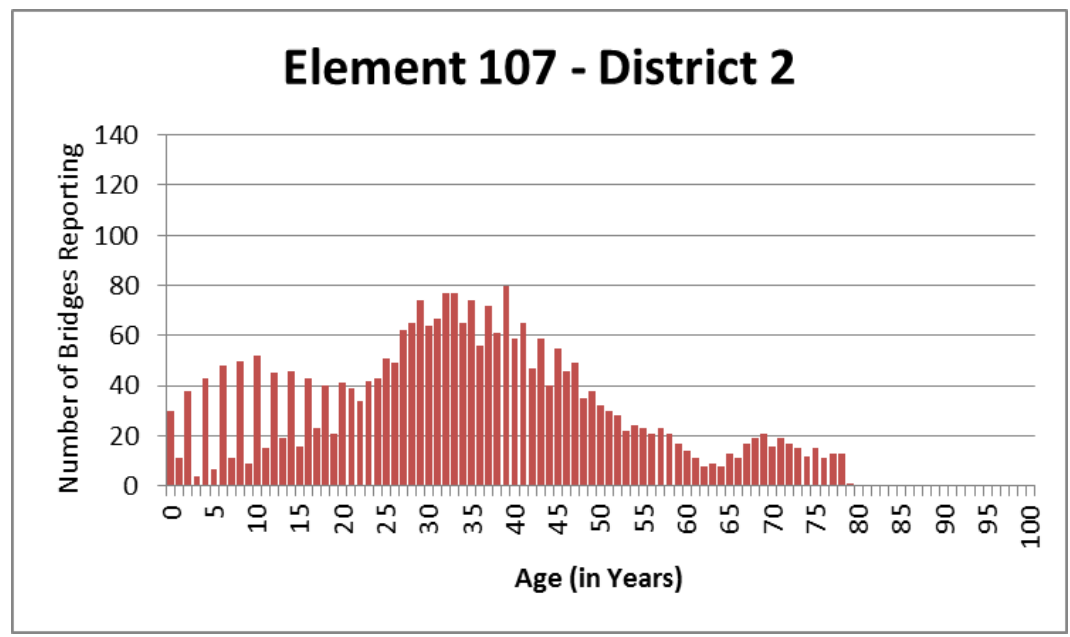

Figure D-6 - Number of Bridges Reporting by Age - Element 107 - District 2 


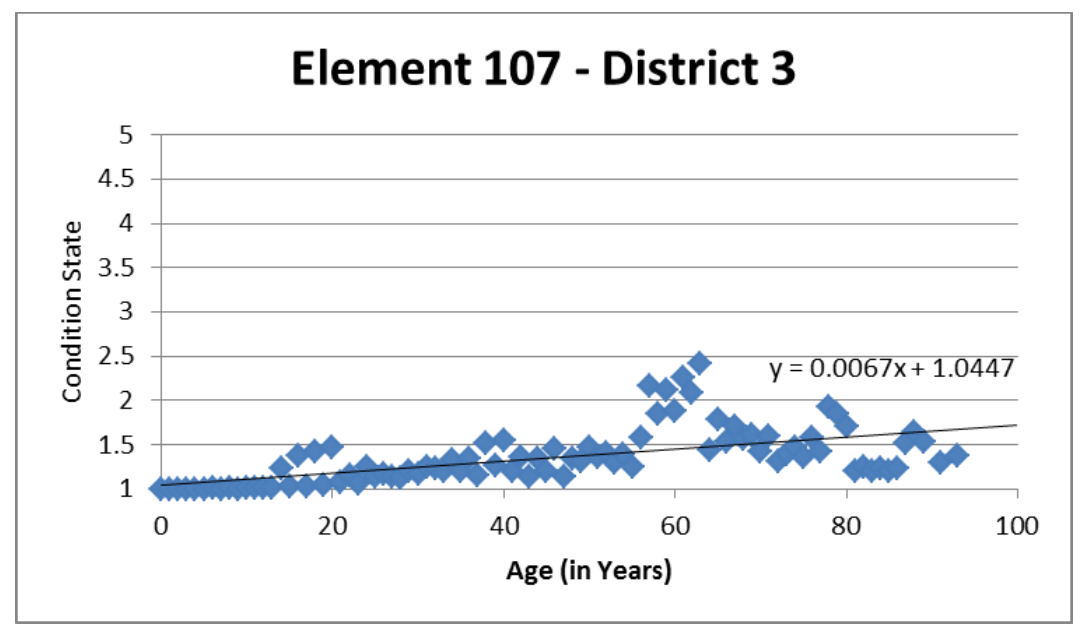

Figure D-7 - Condition State History and Linear Trendline by Age - Element 107 - District 3

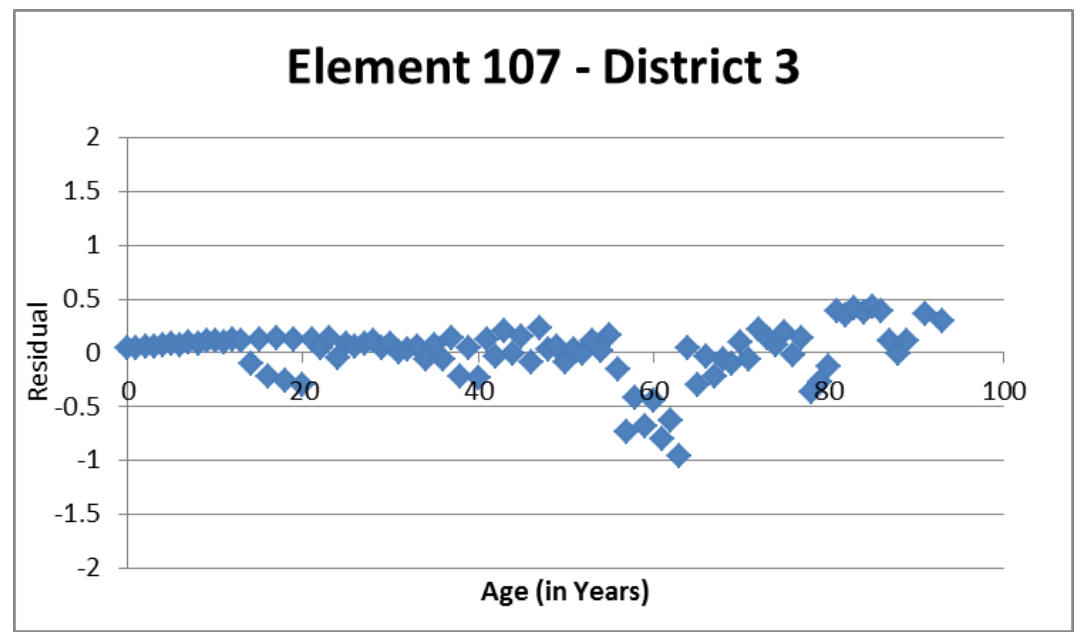

Figure D-8 - Condition States Residuals (Prediction Minus Actual) by Age - Element 107 - District 3

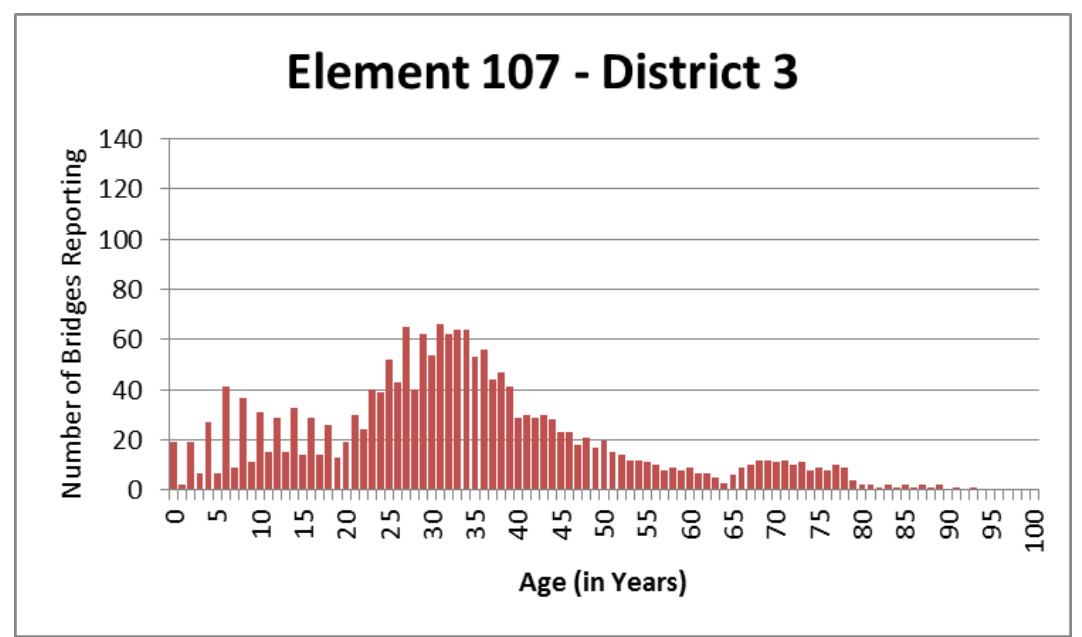

Figure D-9 - Number of Bridges Reporting by Age - Element 107 - District 3 


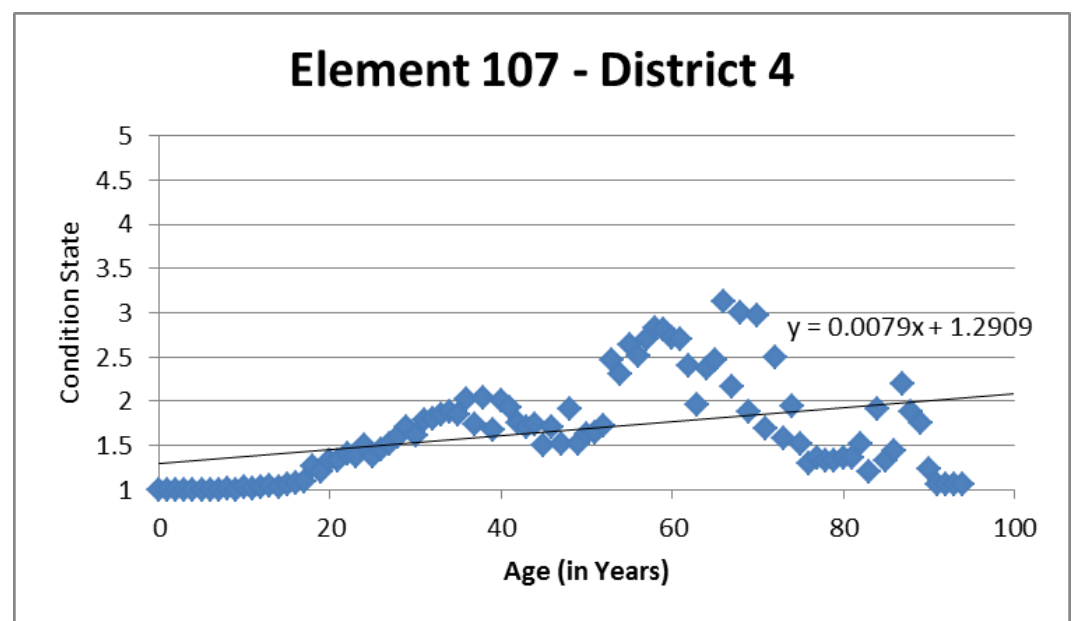

Figure D-10 - Condition State History and Linear Trendline by Age - Element 107 - District 4

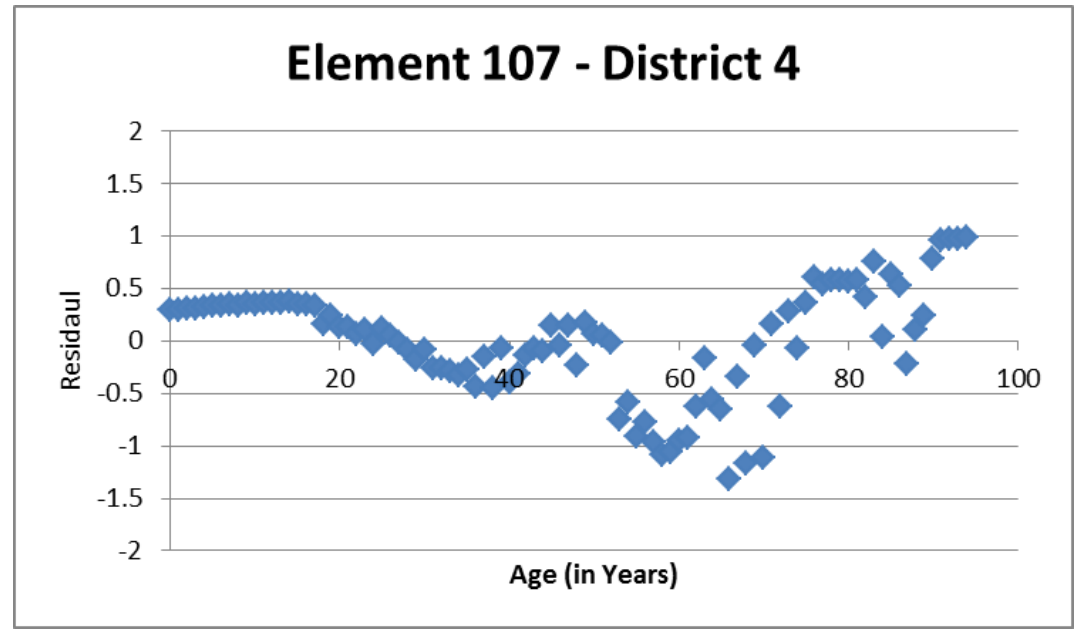

Figure D-11 - Condition States Residuals (Prediction Minus Actual) by Age - Element 107 - District 4

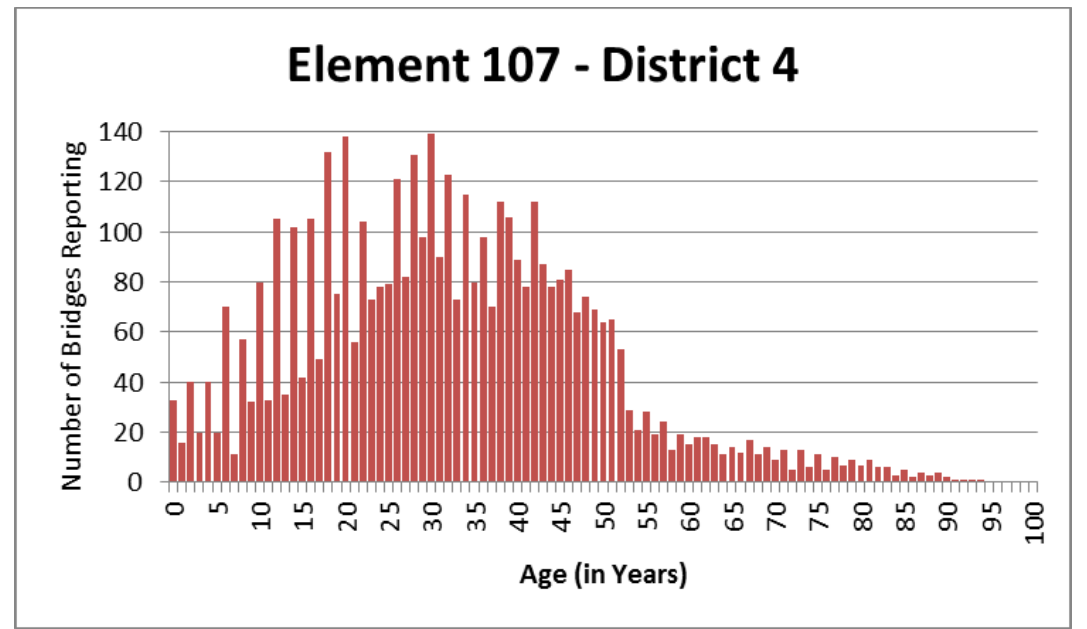

Figure D-12 - Number of Bridges Reporting by Age - Element 107 - District 4 


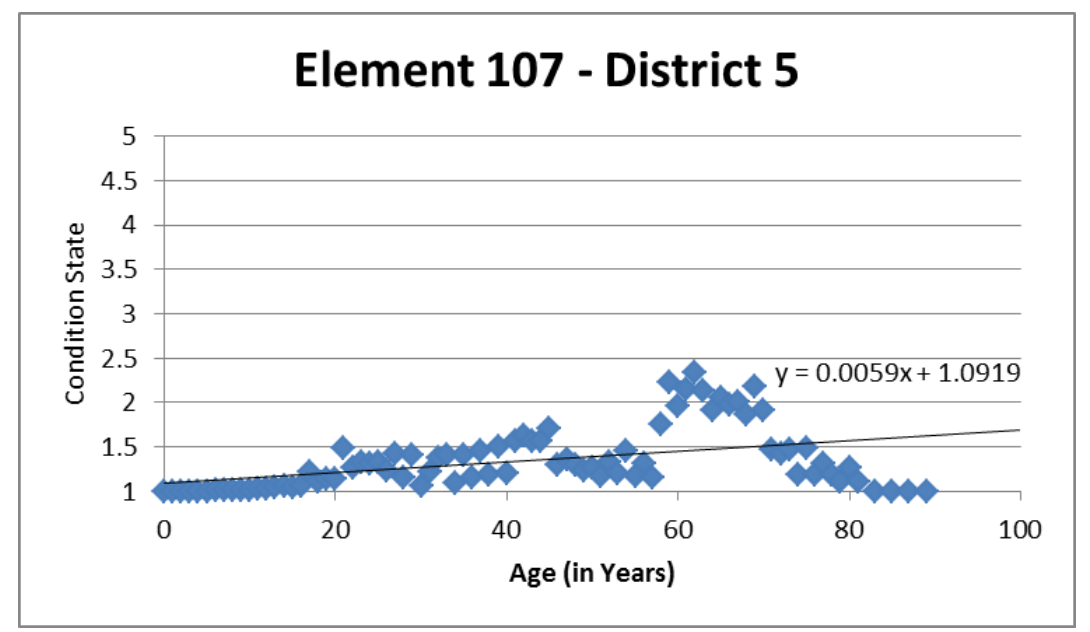

Figure D-13 - Condition State History and Linear Trendline by Age - Element 107 - District 5

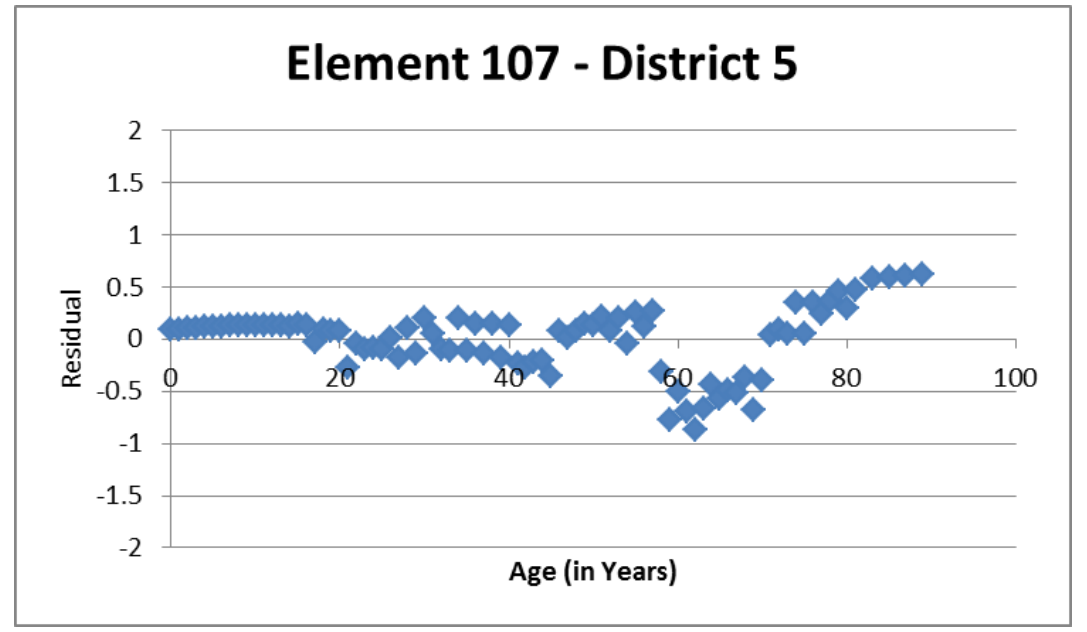

Figure D-14 - Condition States Residuals (Prediction Minus Actual) by Age - Element 107 - District 5

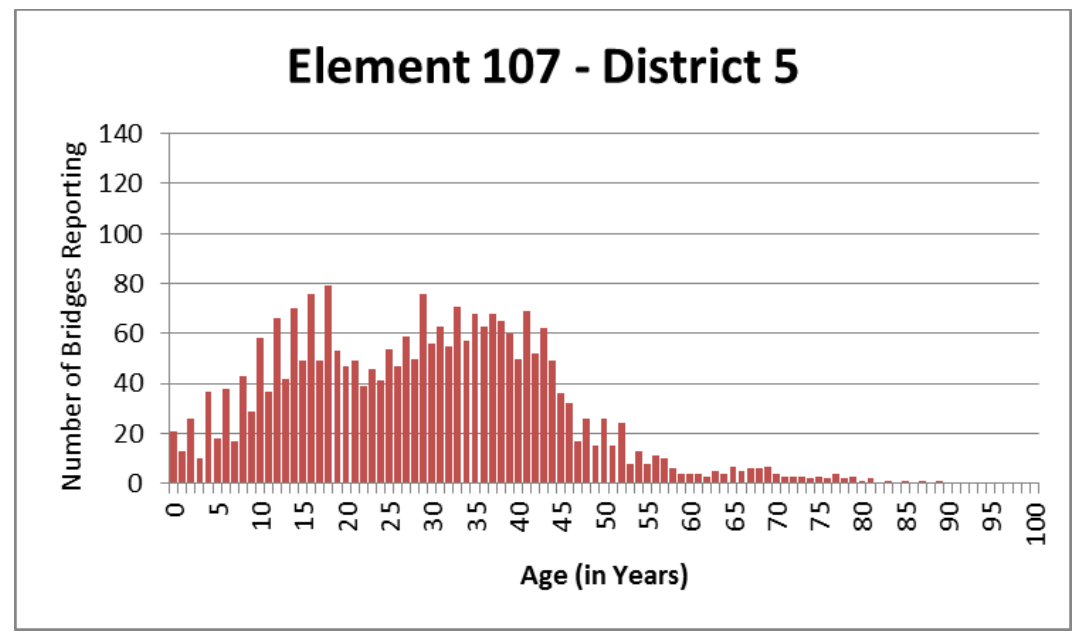

Figure D-15 - Number of Bridges Reporting by Age - Element 107 - District 5 


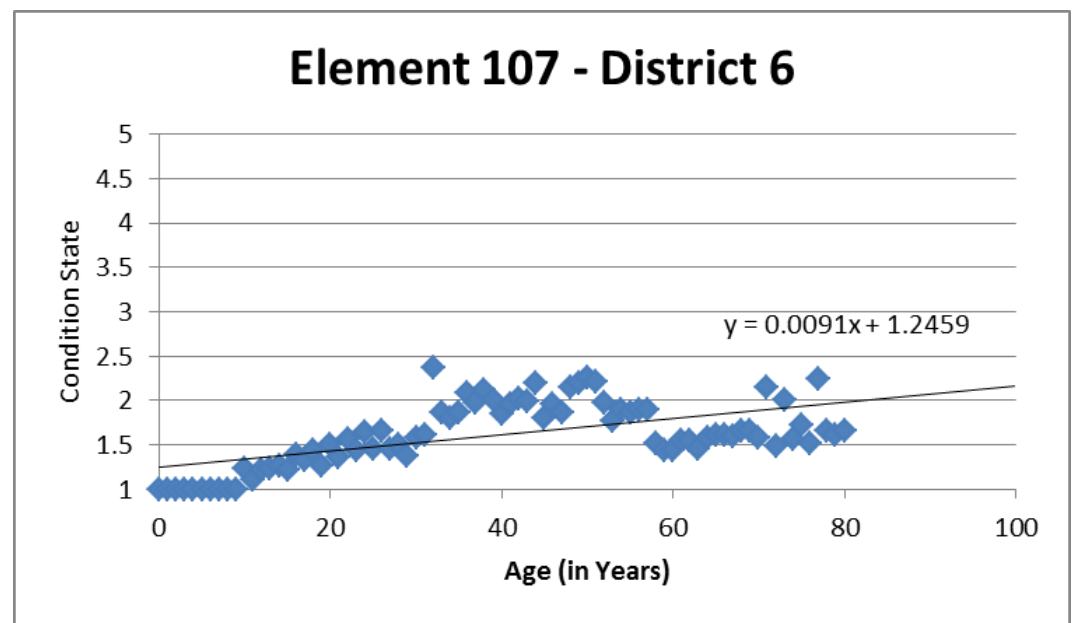

Figure D-16 - Condition State History and Linear Trendline by Age - Element 107 - District 6

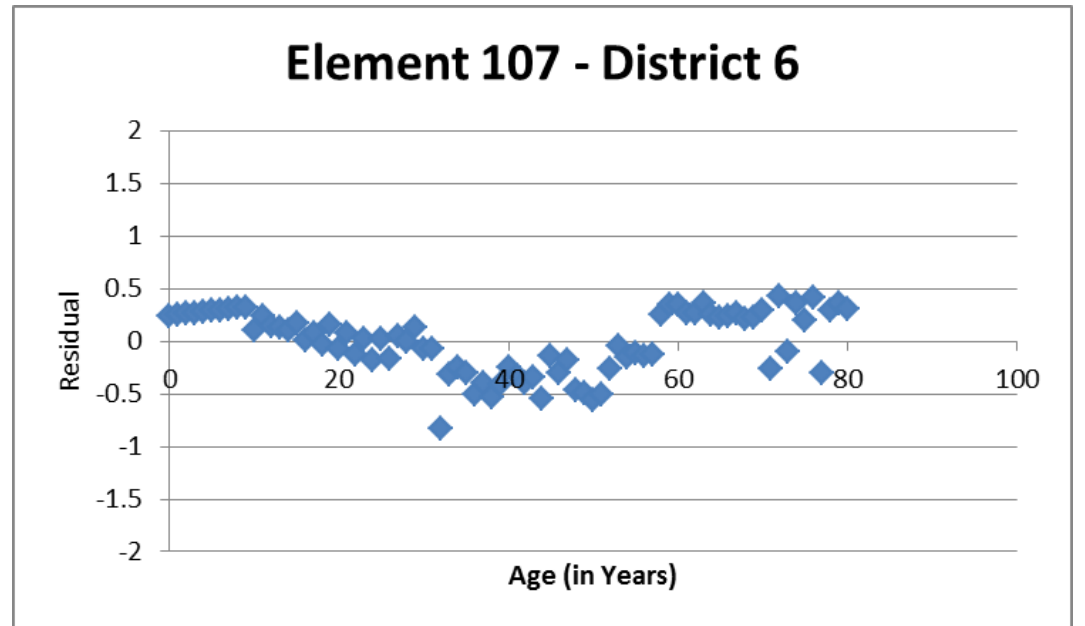

Figure D-17 - Condition States Residuals (Prediction Minus Actual) by Age - Element 107 - District 6

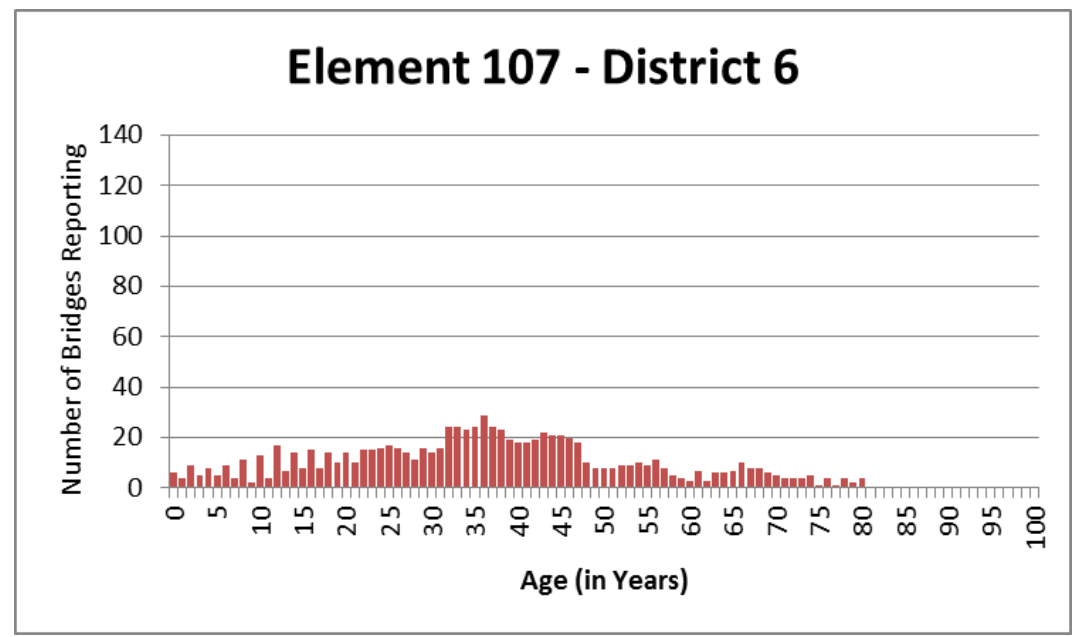

Figure D-18 - Number of Bridges Reporting by Age - Element 107 - District 6 


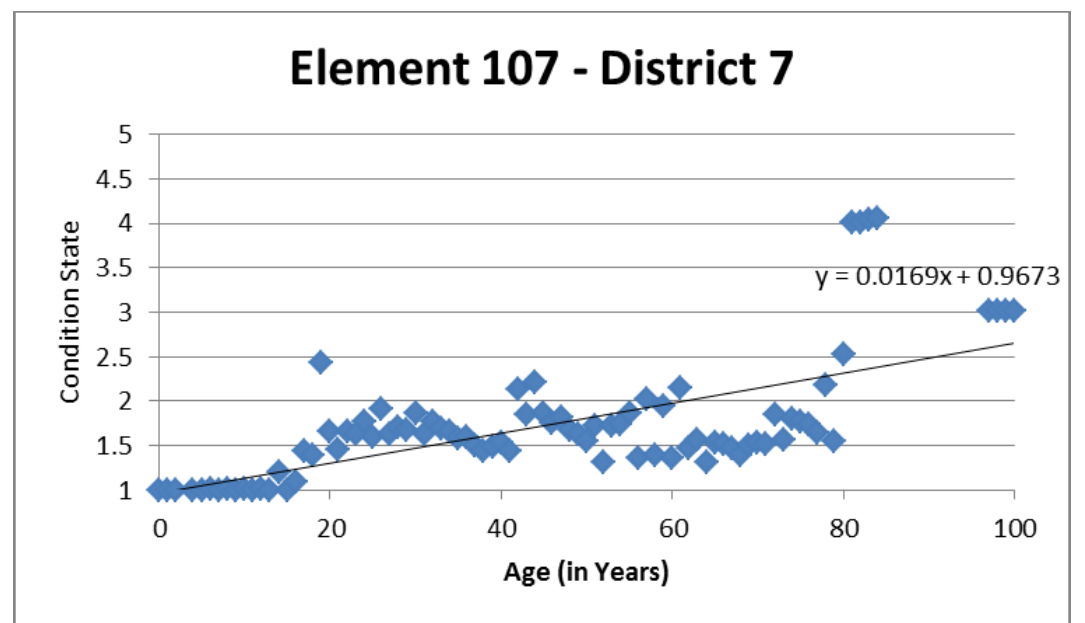

Figure D-19 - Condition State History and Linear Trendline by Age - Element 107 - District 7

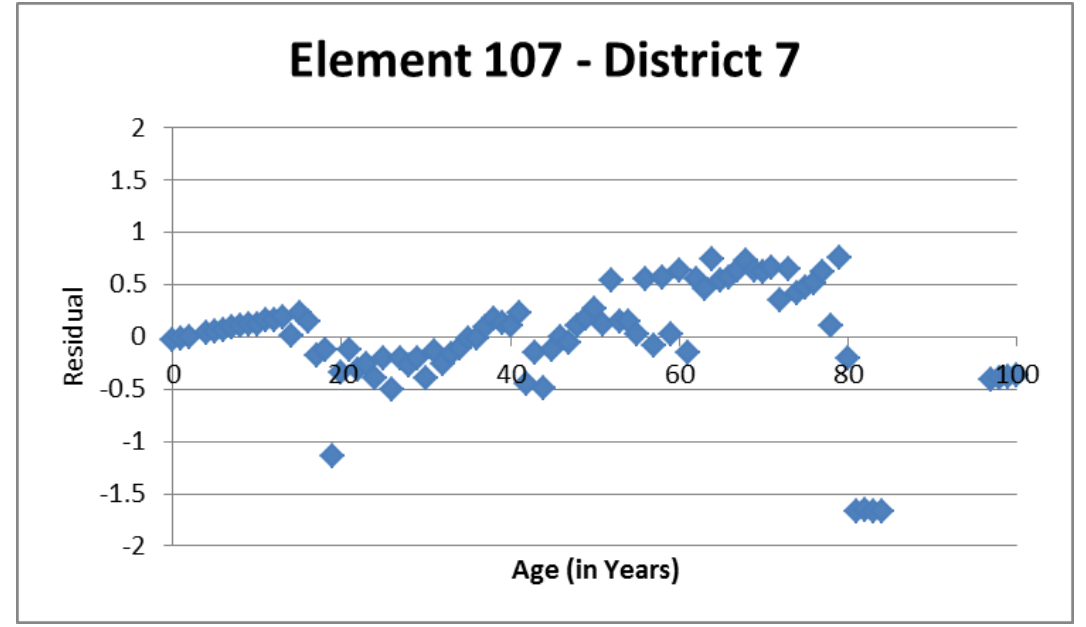

Figure D-20 - Condition States Residuals (Prediction Minus Actual) by Age - Element 107 - District 7

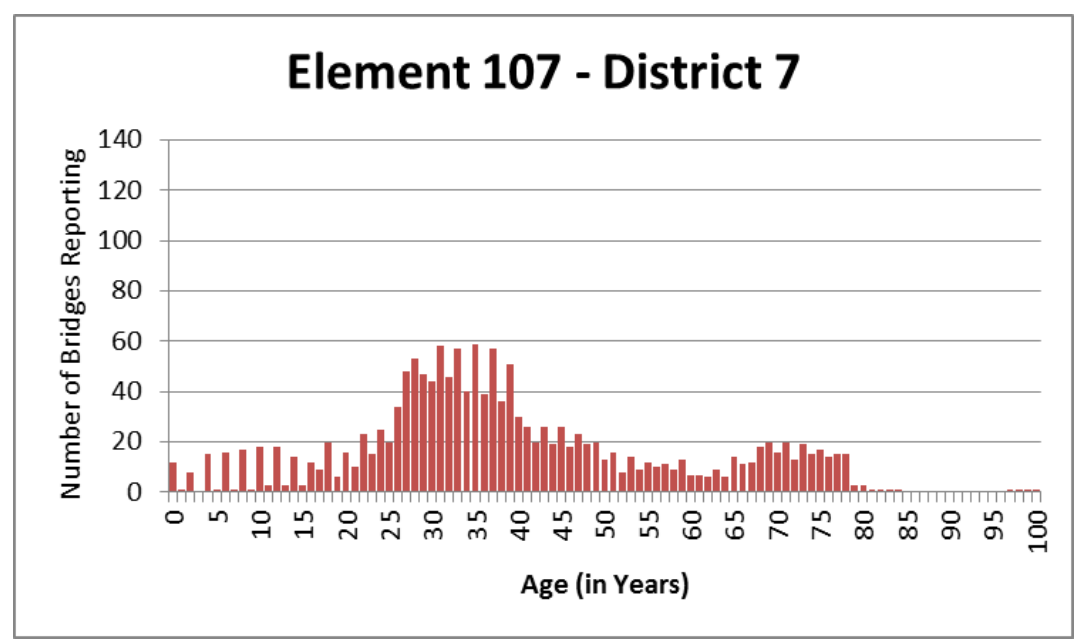

Figure D-21 - Number of Bridges Reporting by Age - Element 107 - District 7 


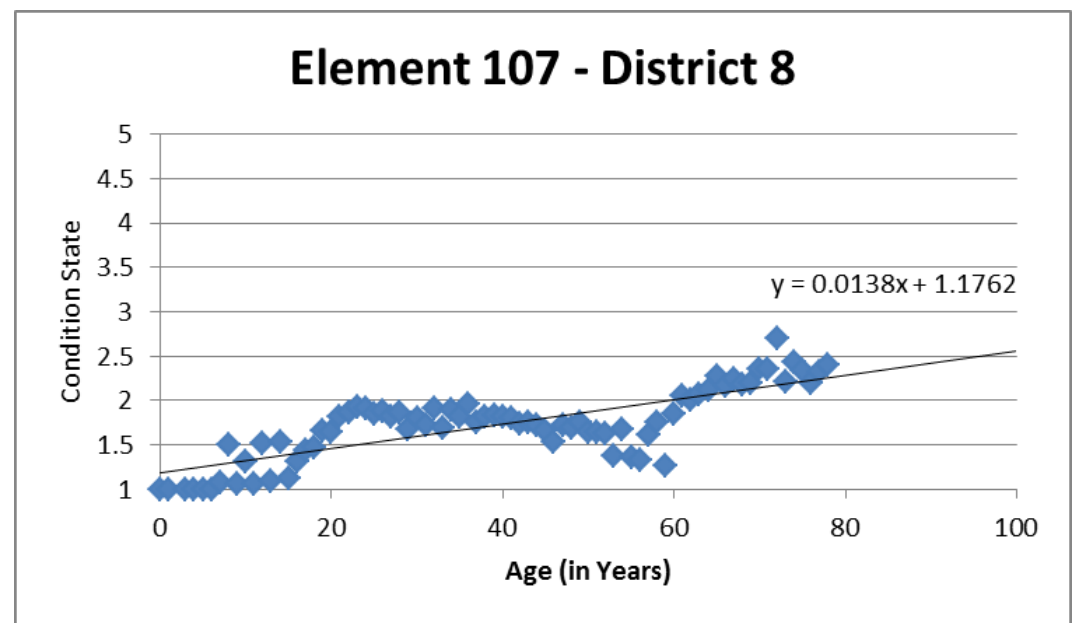

Figure D-22 - Condition State History and Linear Trendline by Age - Element 107 - District 8

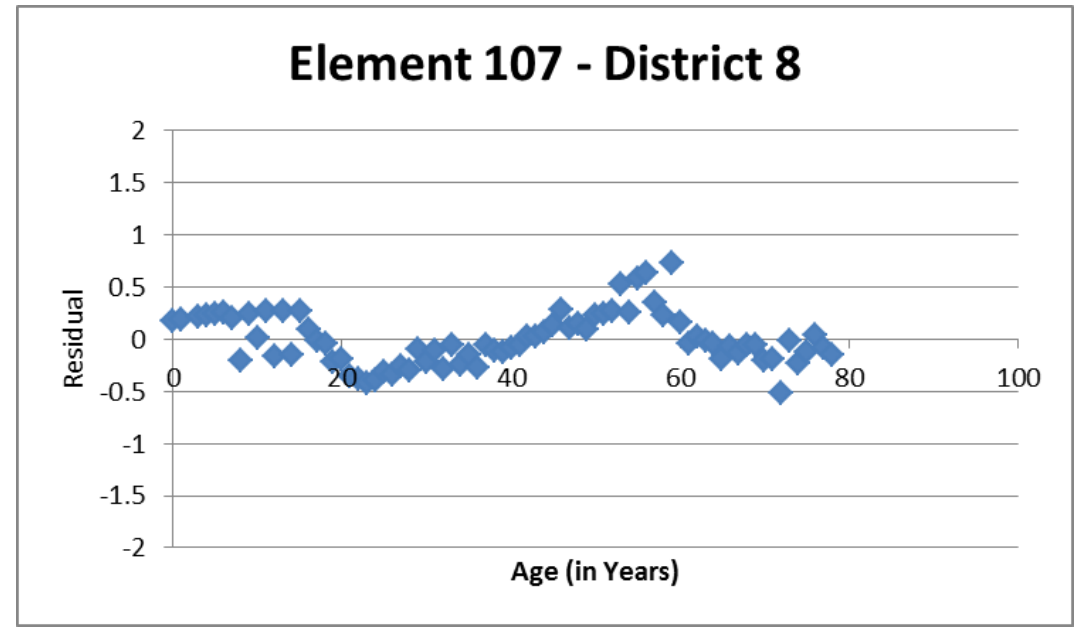

Figure D-23 - Condition States Residuals (Prediction Minus Actual) by Age - Element 107 - District 8

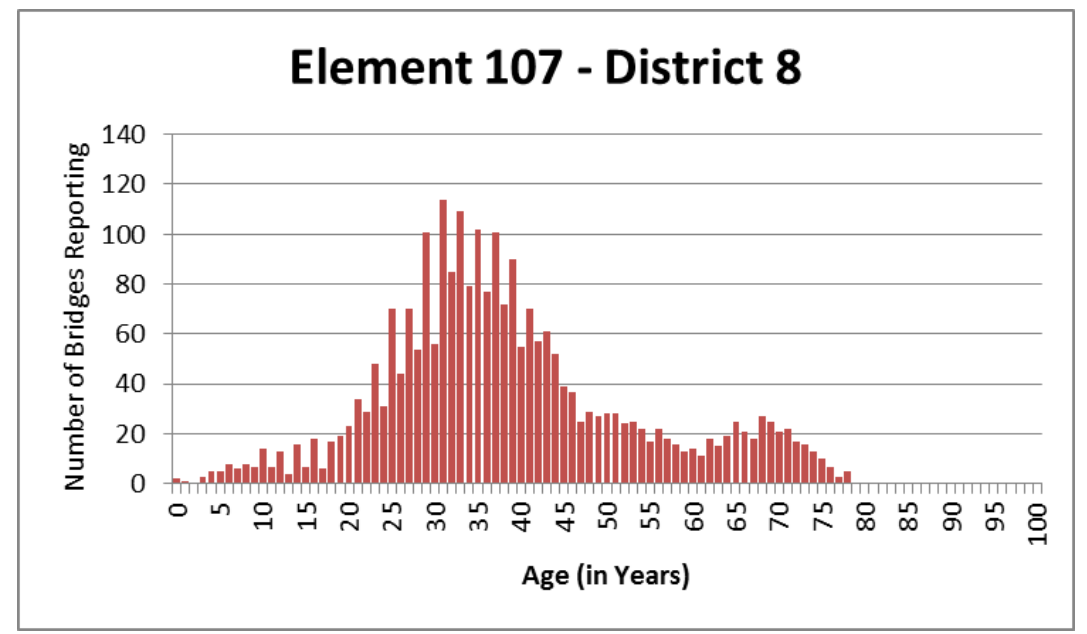

Figure D-24 - Number of Bridges Reporting by Age - Element 107 - District 8 


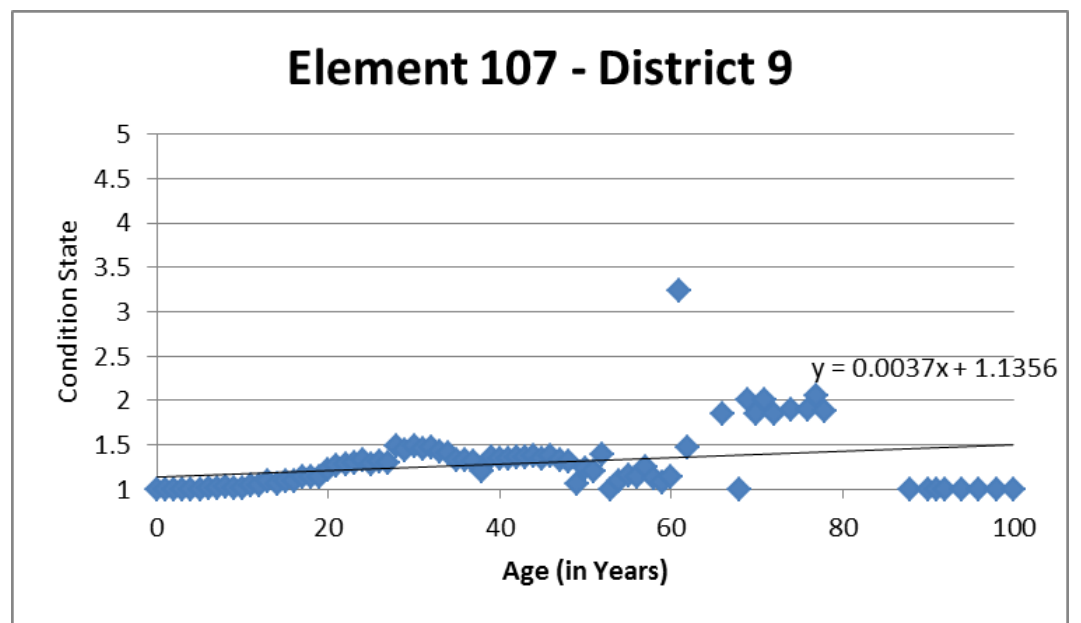

Figure D-25 - Condition State History and Linear Trendline by Age - Element 107 - District 9

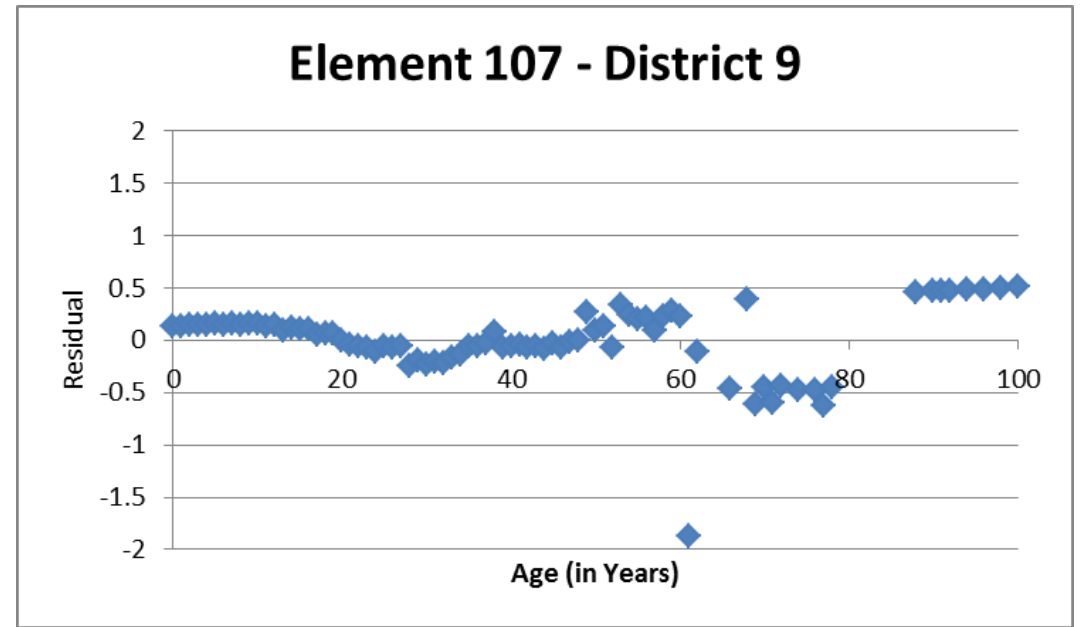

Figure D-26 - Condition States Residuals (Prediction Minus Actual) by Age - Element 107 - District 9

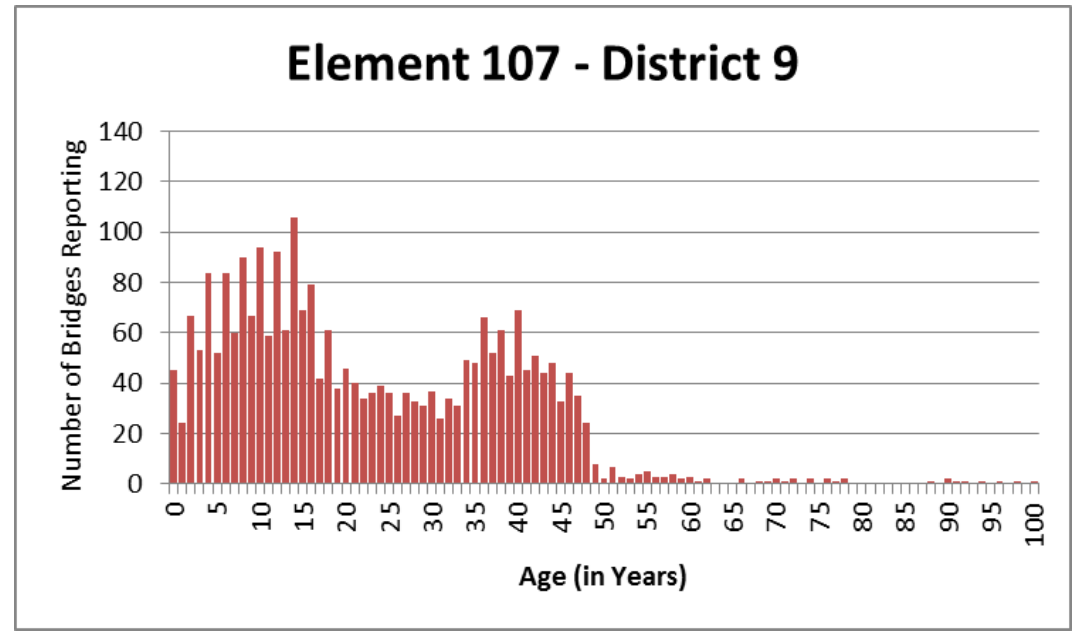

Figure D-27 - Number of Bridges Reporting by Age - Element 107 - District 9 


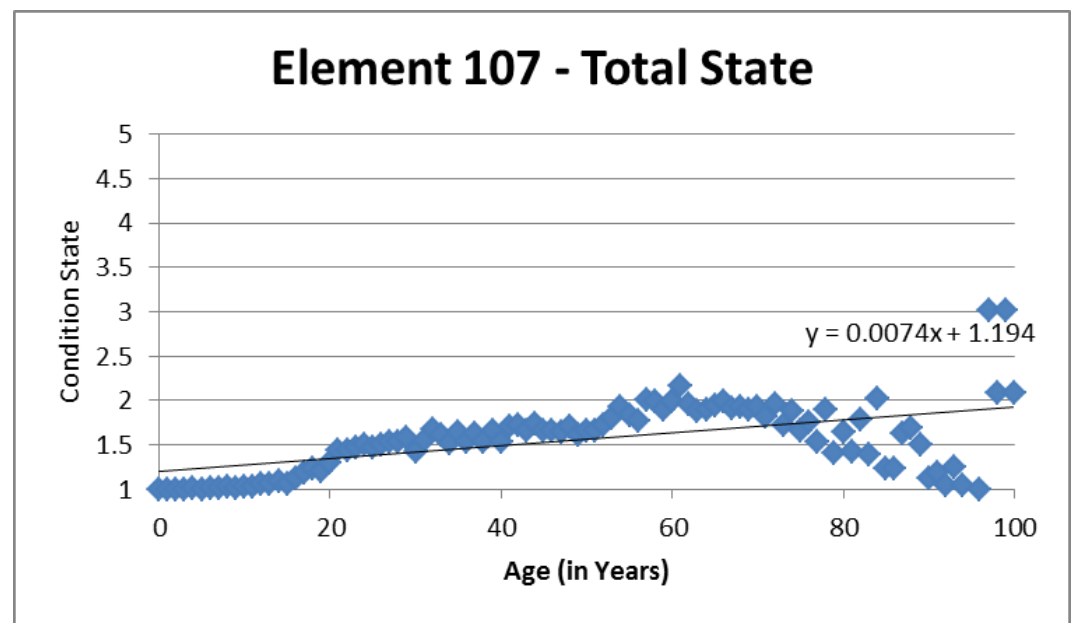

Figure D-28 - Condition State History and Linear Trendline by Age - Element 107 - Total State

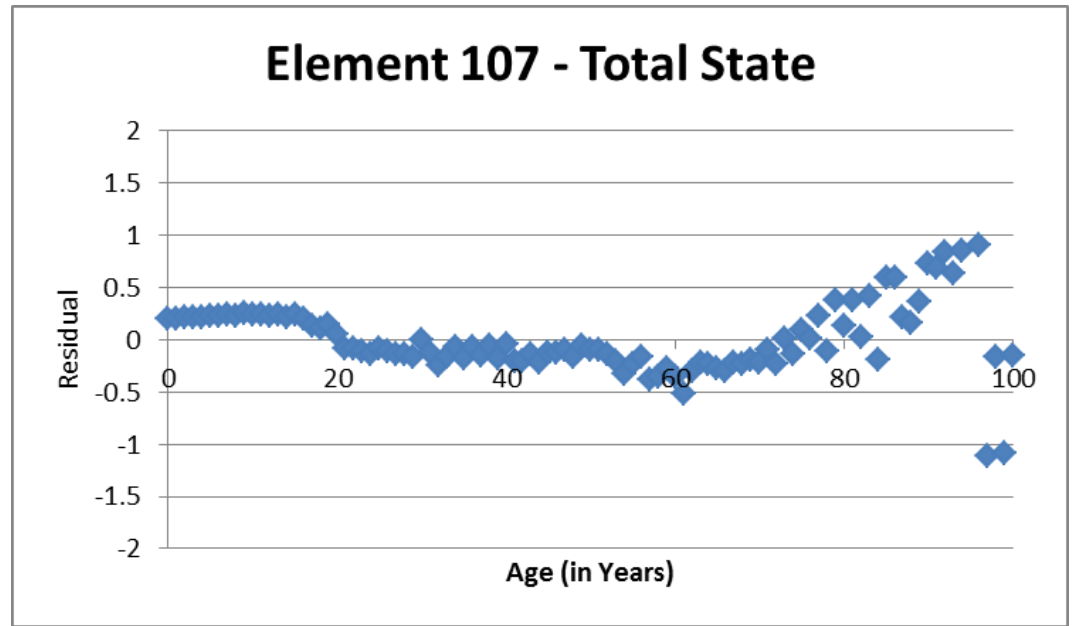

Figure D-29 - Condition States Residuals (Prediction Minus Actual) by Age - Element 107 - Total State

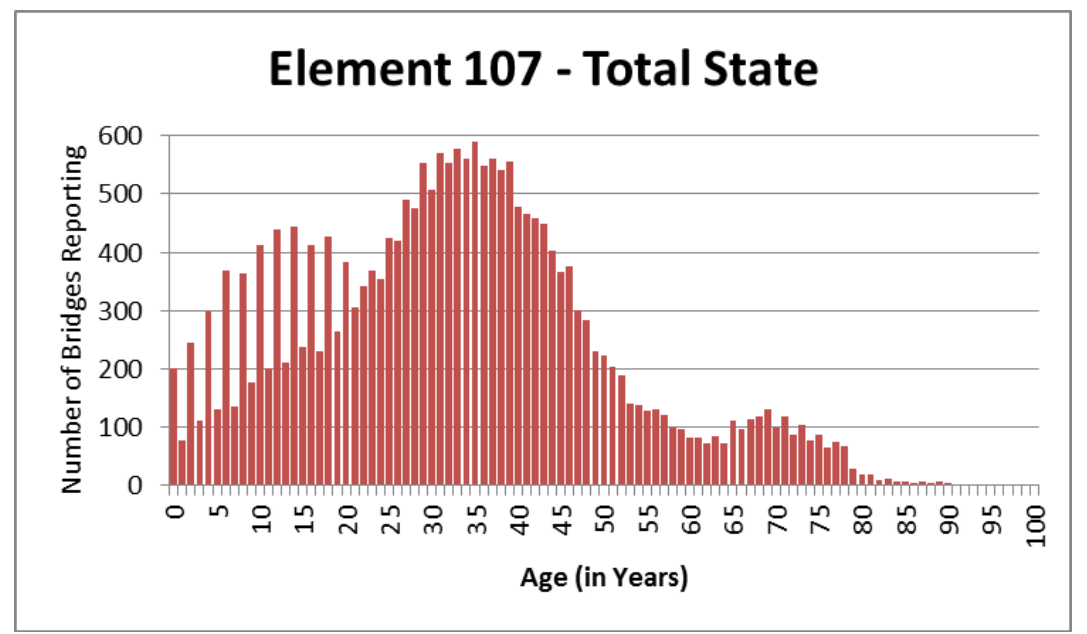

Figure D-30 - Number of Bridges Reporting by Age - Element 107 - Total State 


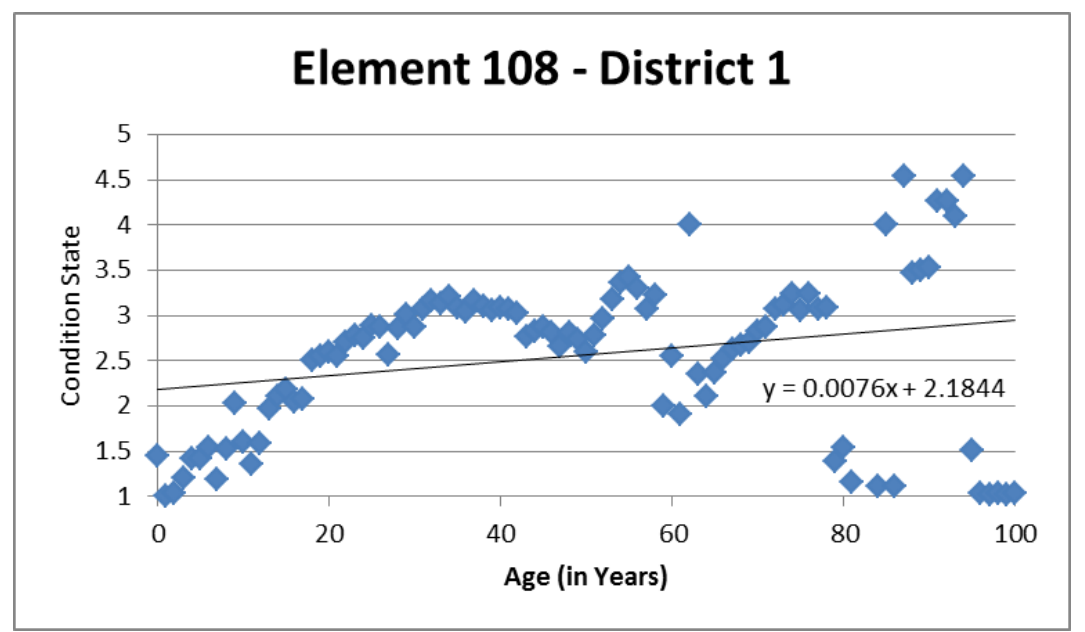

Figure D-31 - Condition State History and Linear Trendline by Age - Element 108 - District 1

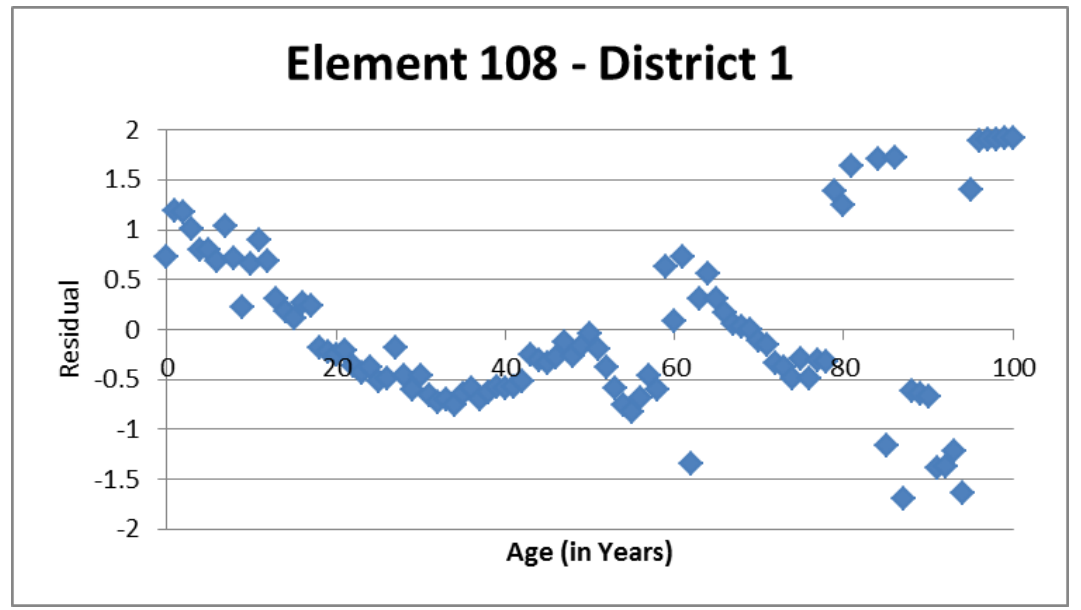

Figure D-32 - Condition States Residuals (Prediction Minus Actual) by Age - Element 108 - District 1

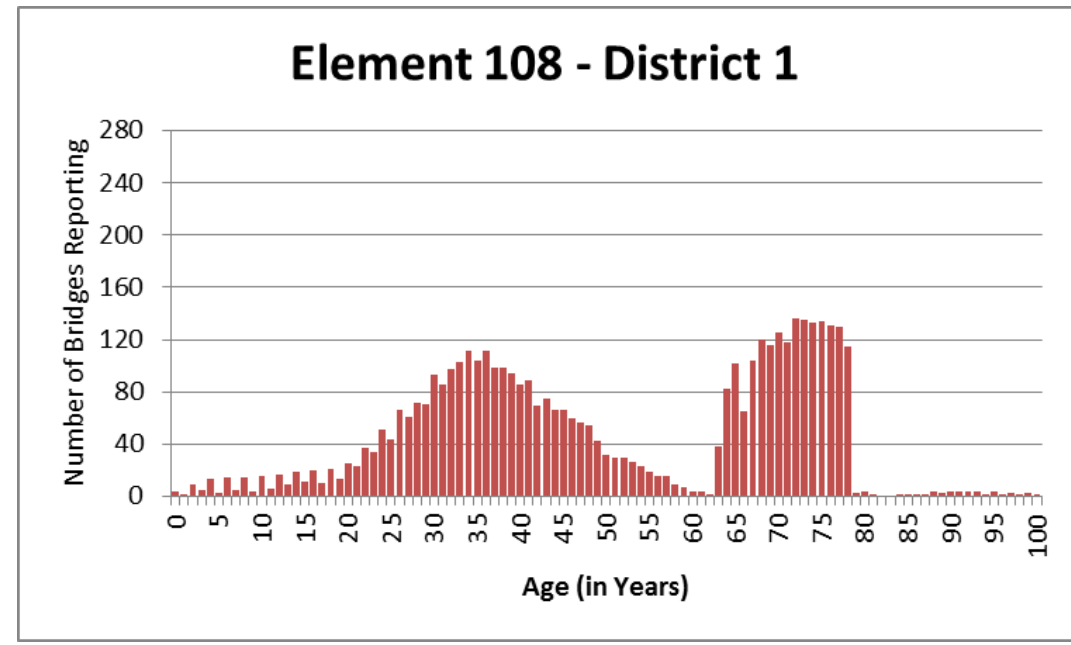

Figure D-33 - Number of Bridges Reporting by Age - Element 108 - District 1 


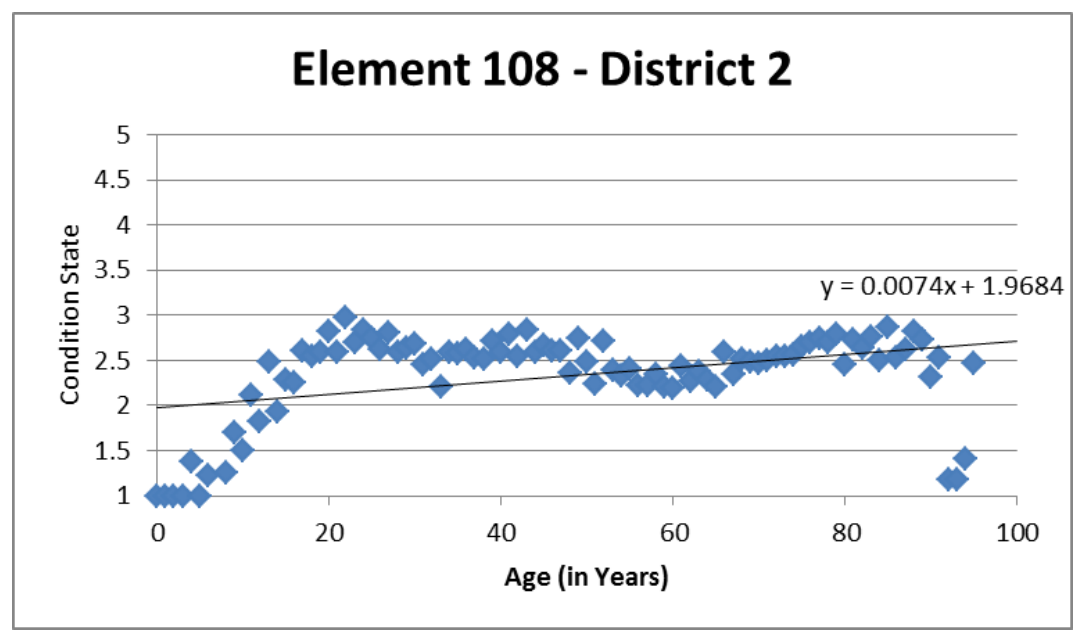

Figure D-34 - Condition State History and Linear Trendline by Age - Element 108 - District 2

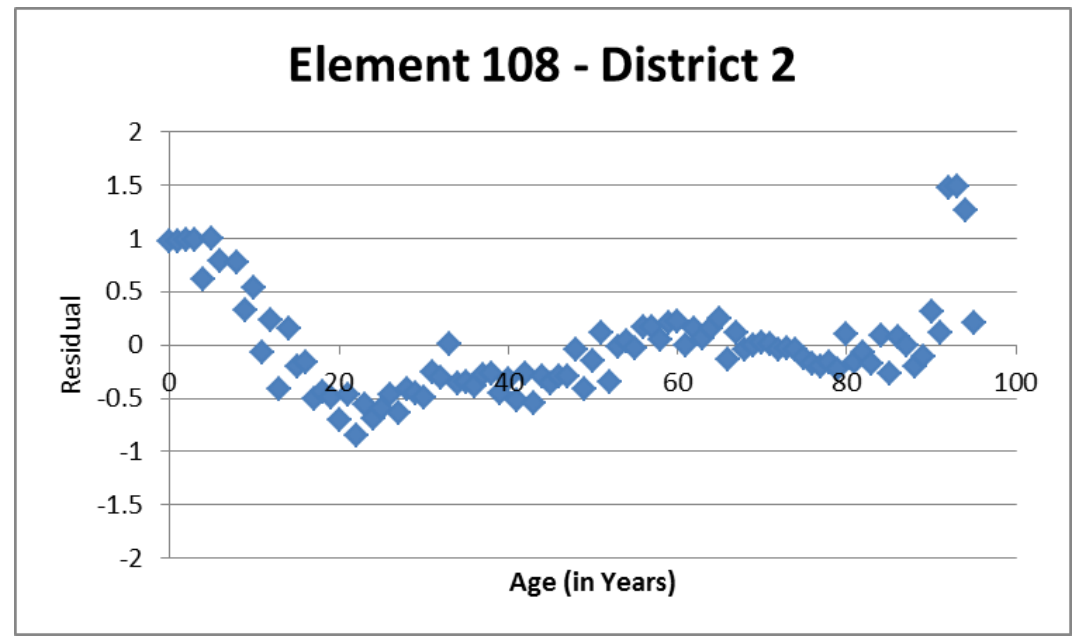

Figure D-35 - Condition States Residuals (Prediction Minus Actual) by Age - Element 108 - District 2

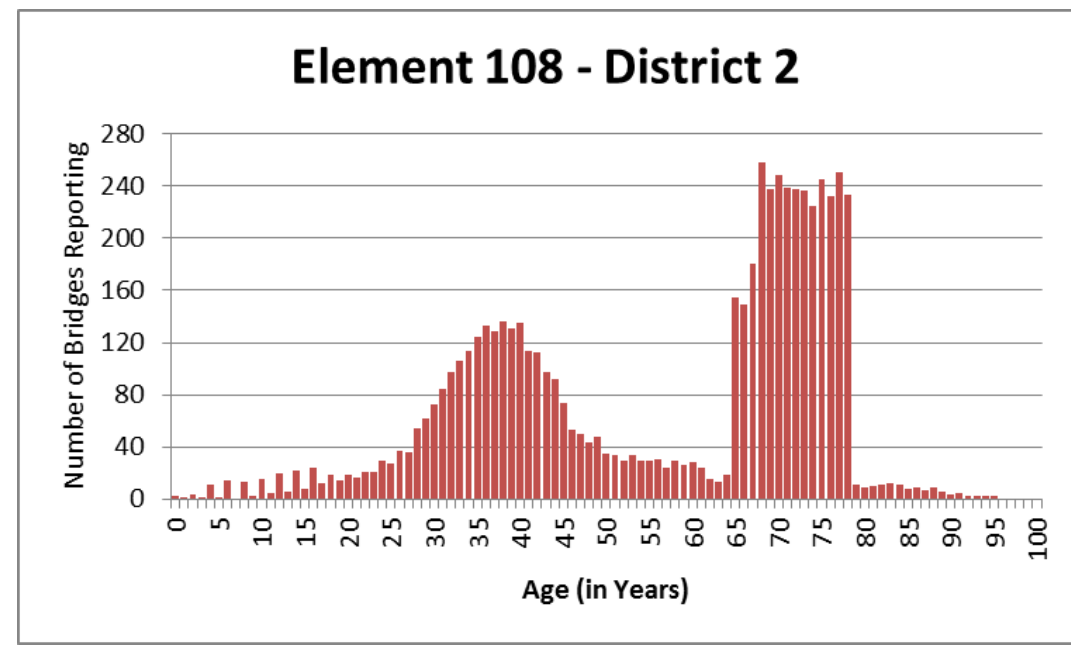

Figure D-36 - Number of Bridges Reporting by Age - Element 108 - District 2 


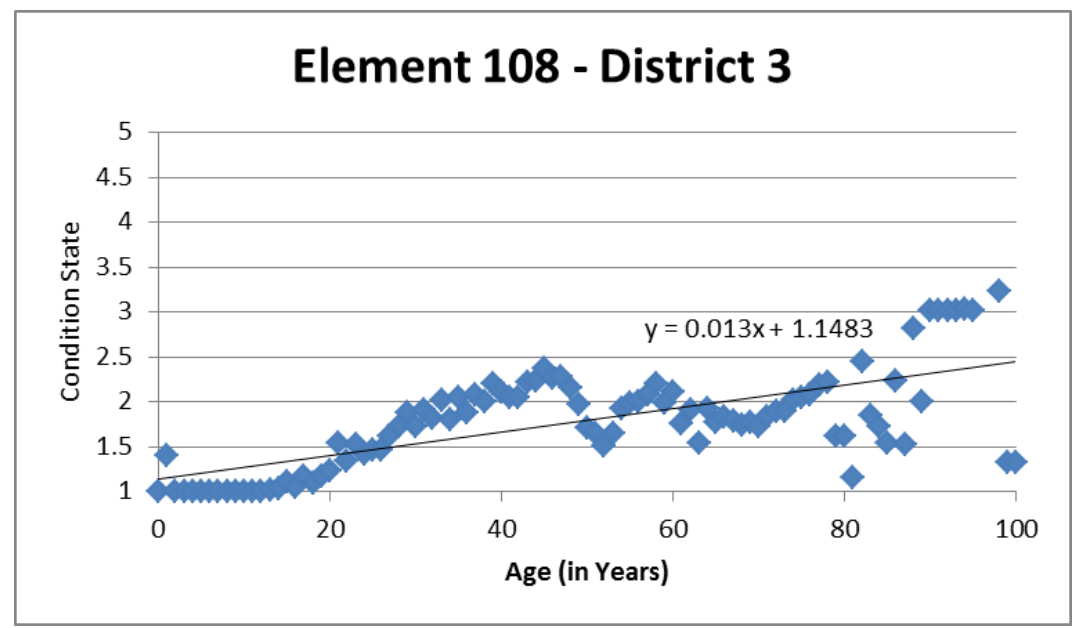

Figure D-37 - Condition State History and Linear Trendline by Age - Element 108 - District 3

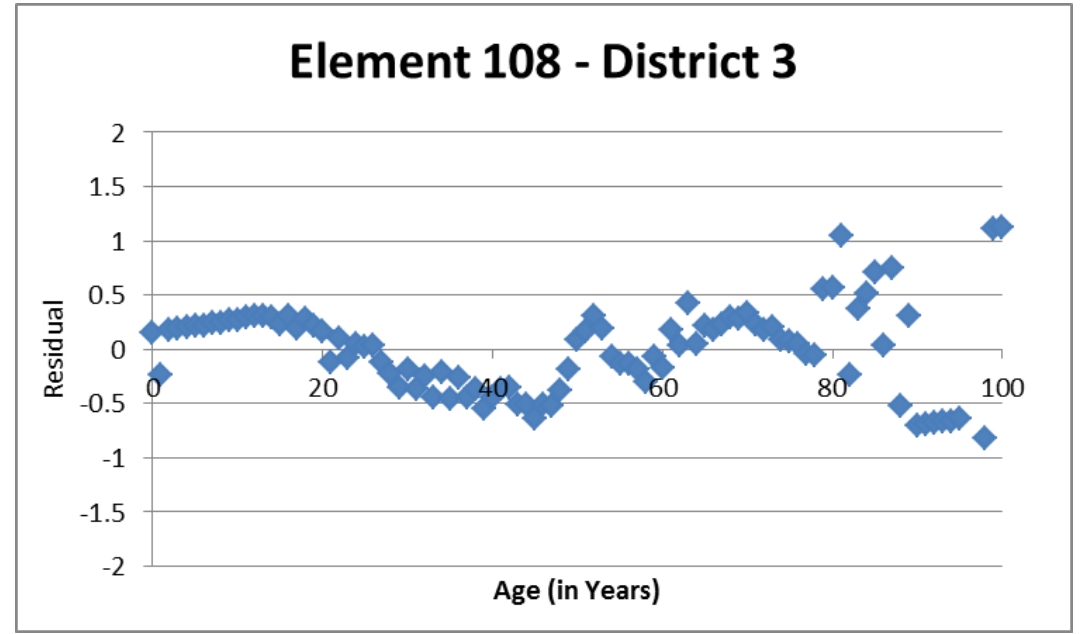

Figure D-38 - Condition States Residuals (Prediction Minus Actual) by Age - Element 108 - District 3

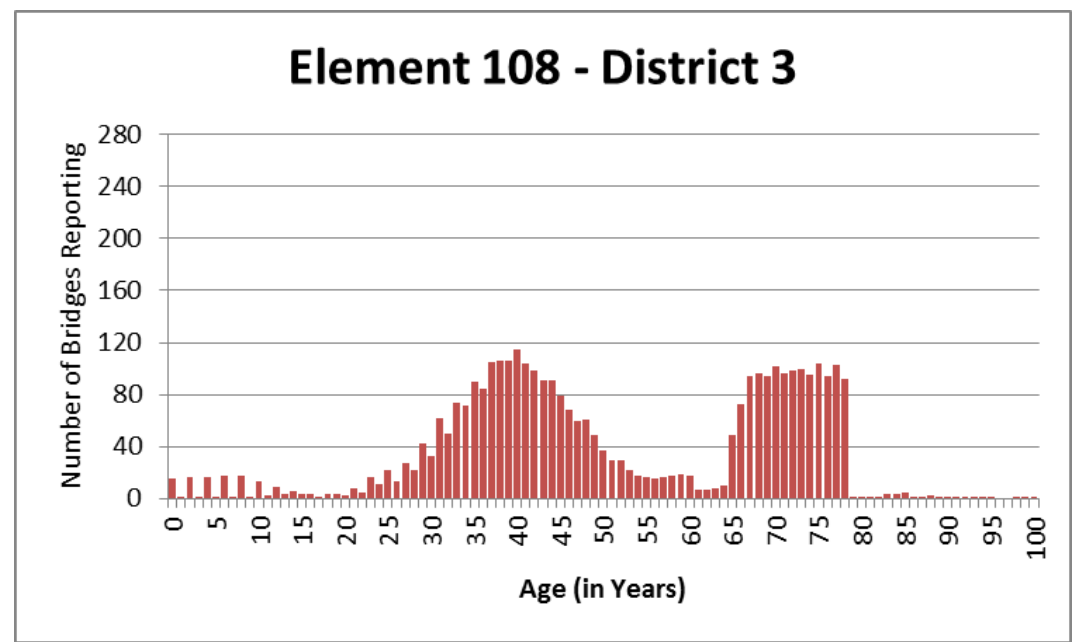

Figure D-39 - Number of Bridges Reporting by Age - Element 108 - District 3 


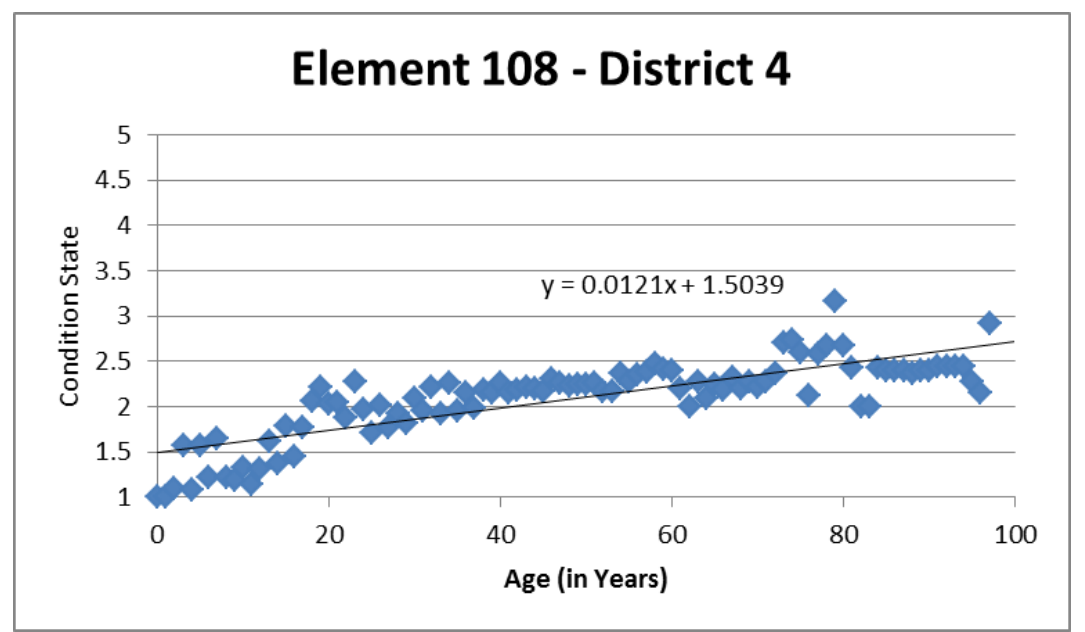

Figure D-40 - Condition State History and Linear Trendline by Age - Element 108 - District 4

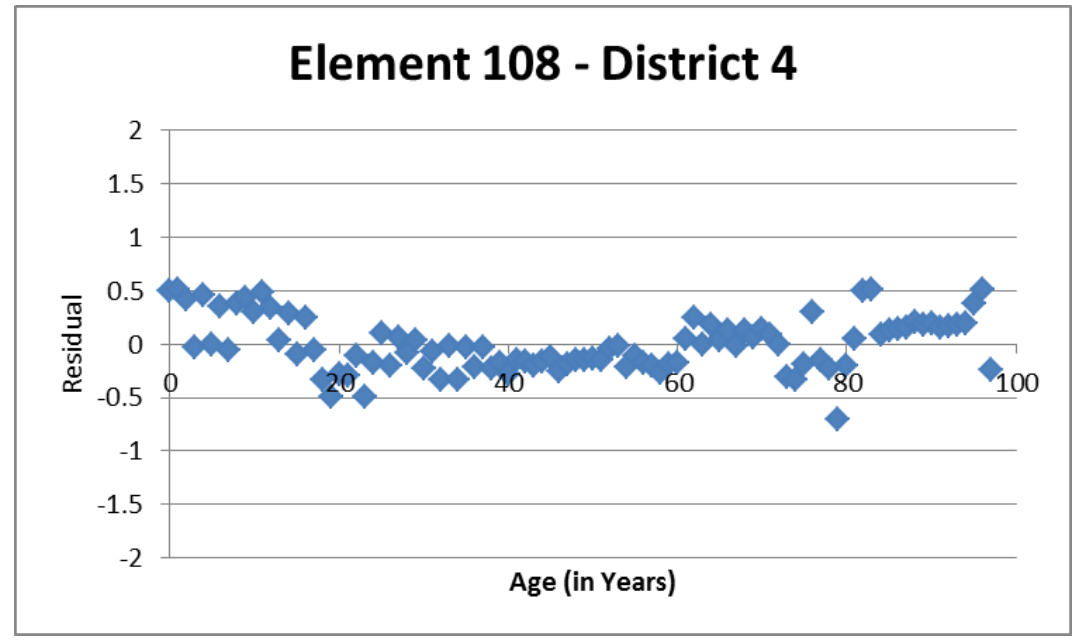

Figure D-41 - Condition States Residuals (Prediction Minus Actual) by Age - Element 108 - District 4

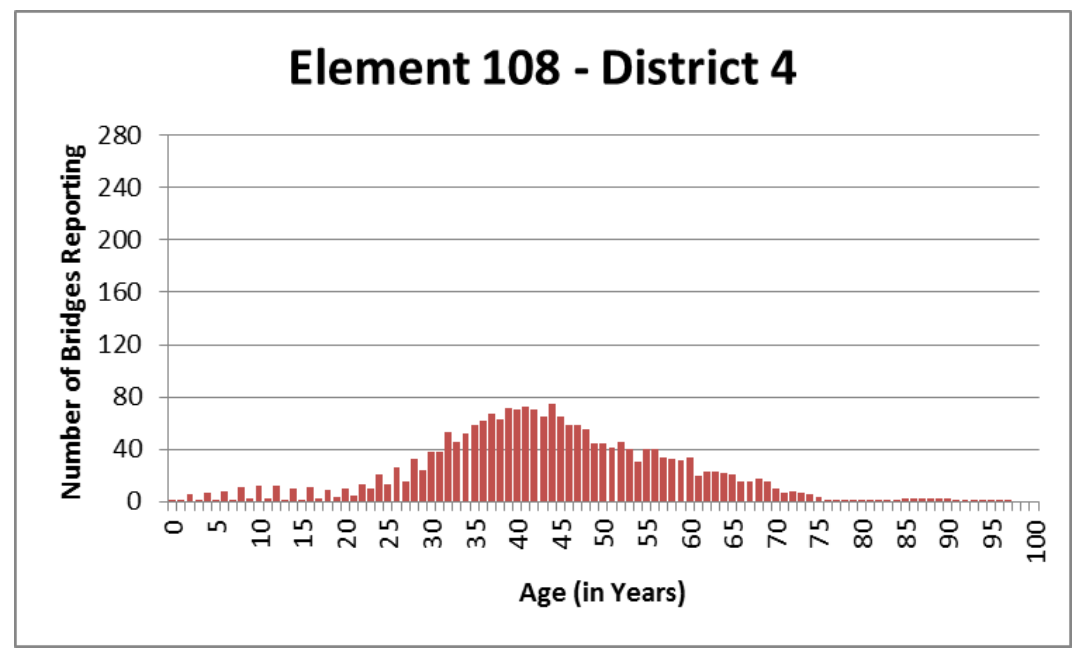

Figure D-42 - Number of Bridges Reporting by Age - Element 108 - District 4 


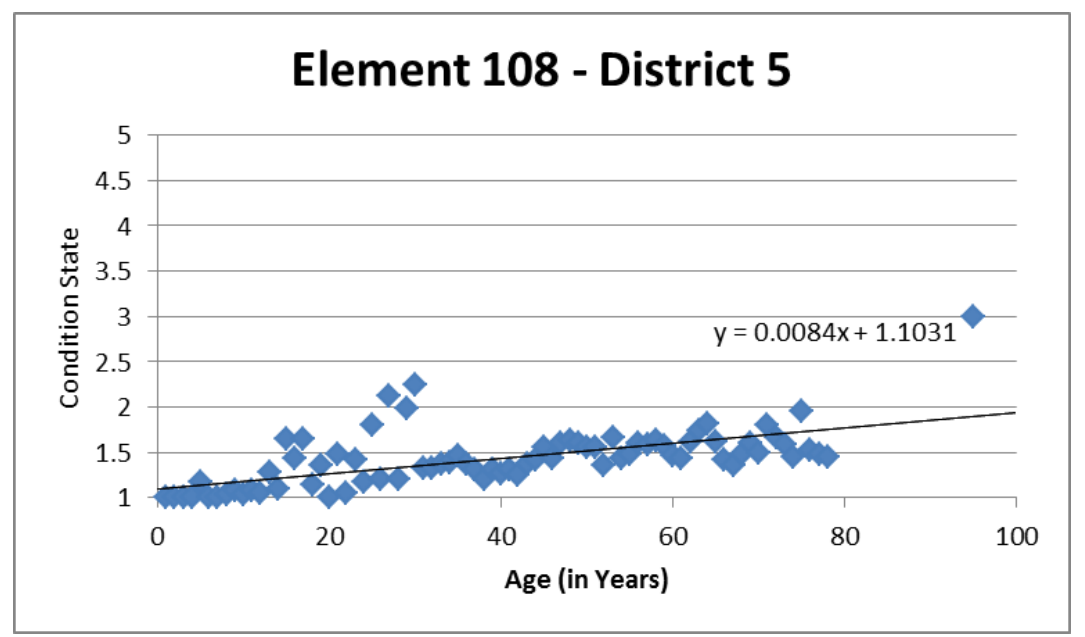

Figure D-43 - Condition State History and Linear Trendline by Age - Element 108 - District 5

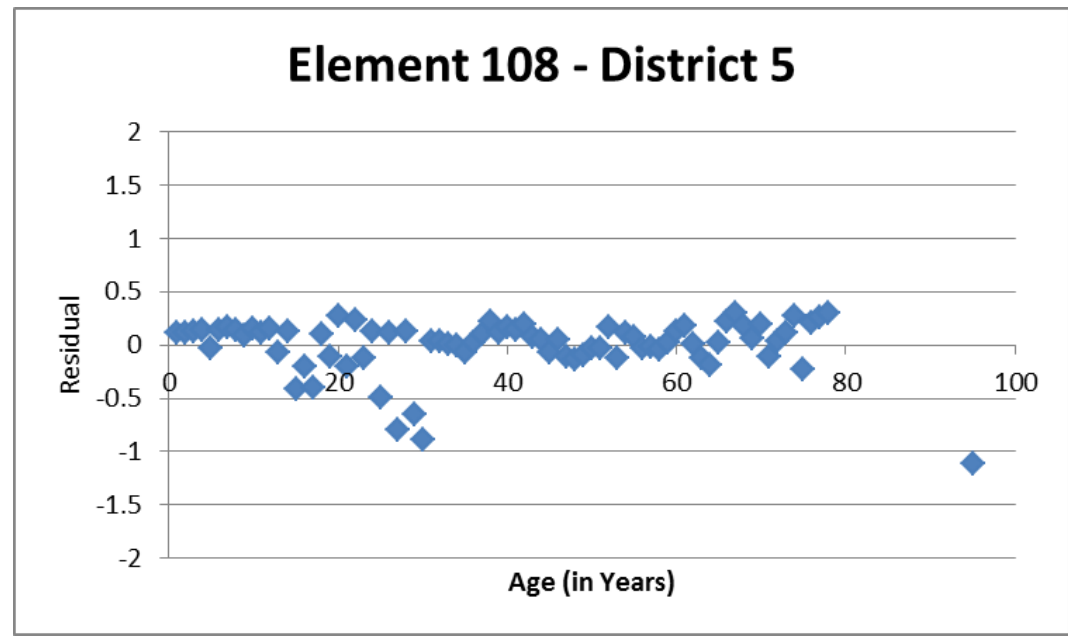

Figure D-44 - Condition States Residuals (Prediction Minus Actual) by Age - Element 108 - District 5

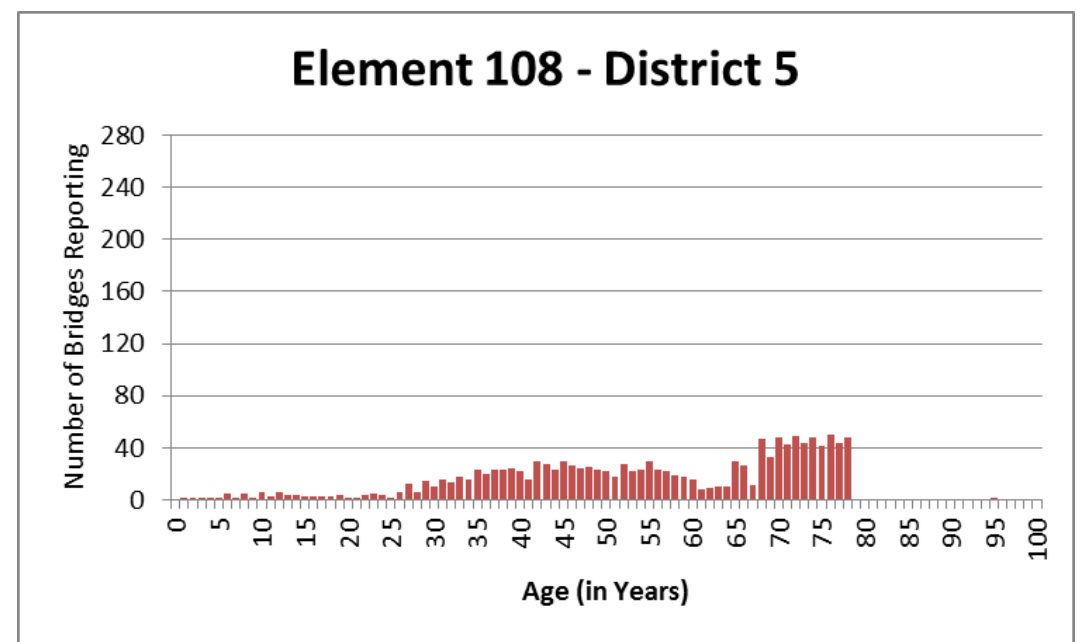

Figure D-45 - Number of Bridges Reporting by Age - Element 108 - District 5 


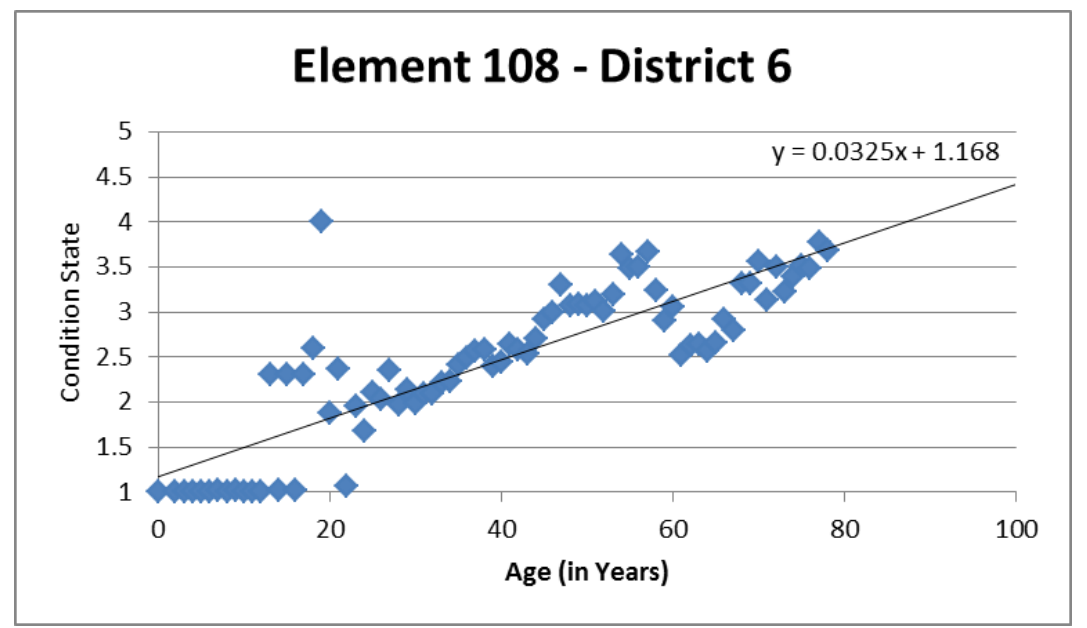

Figure D-46 - Condition State History and Linear Trendline by Age - Element 108 - District 6

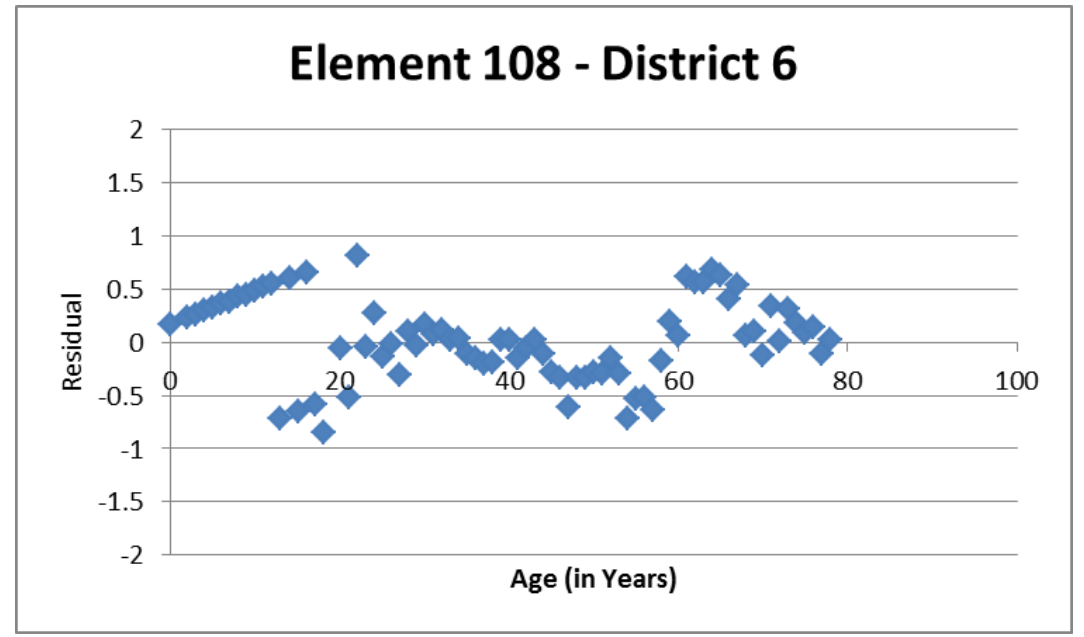

Figure D-47 - Condition States Residuals (Prediction Minus Actual) by Age - Element 108 - District 6

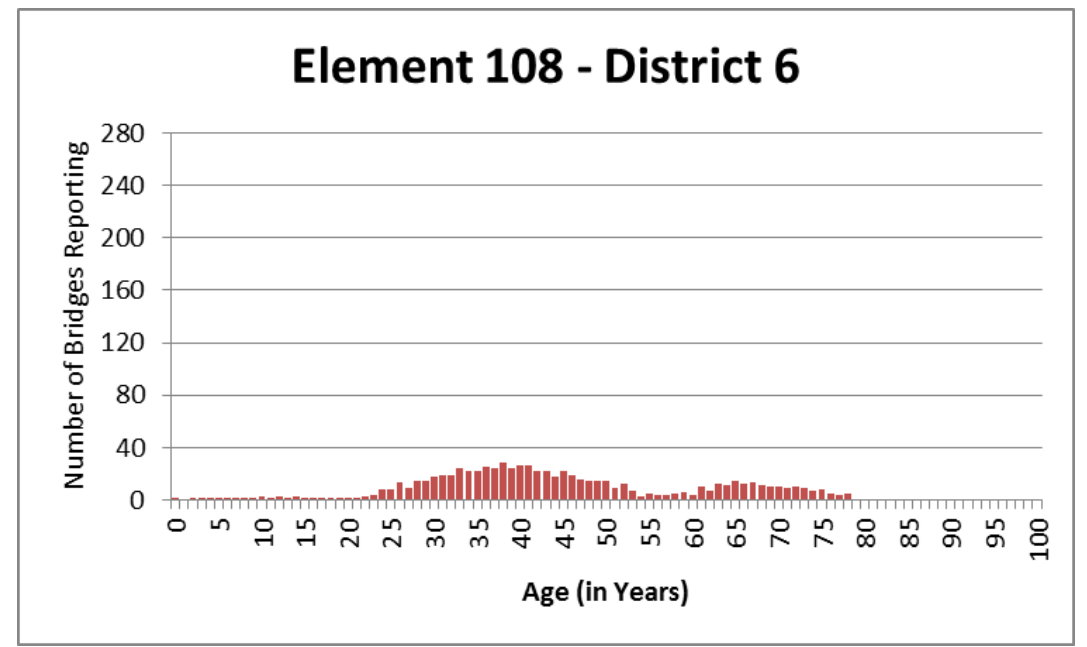

Figure D-48 - Number of Bridges Reporting by Age - Element 108 - District 6 


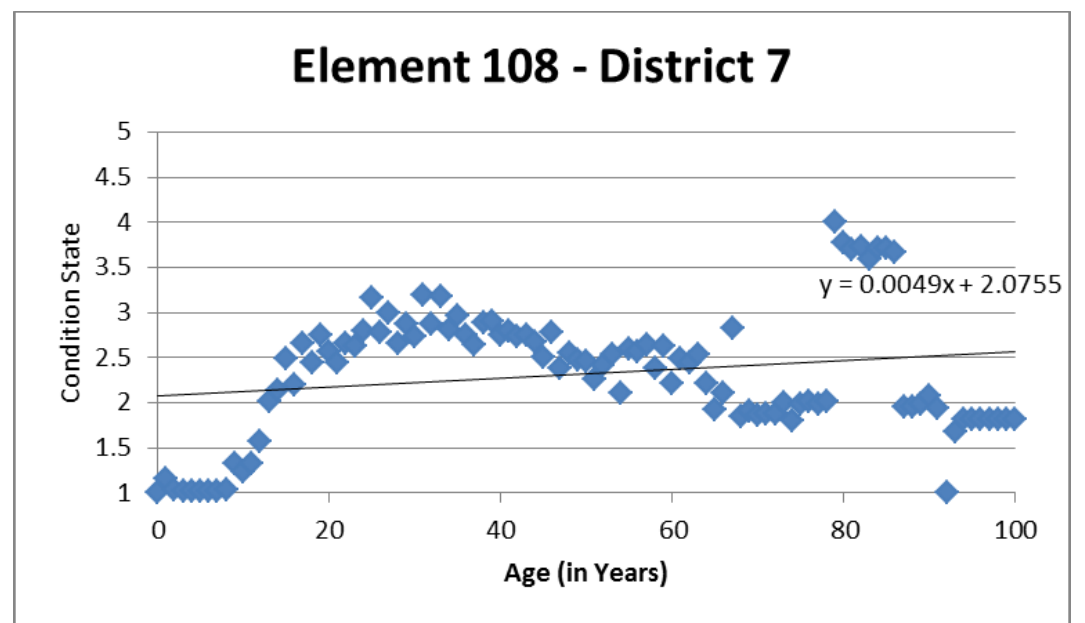

Figure D-49 - Condition State History and Linear Trendline by Age - Element 108 - District 7

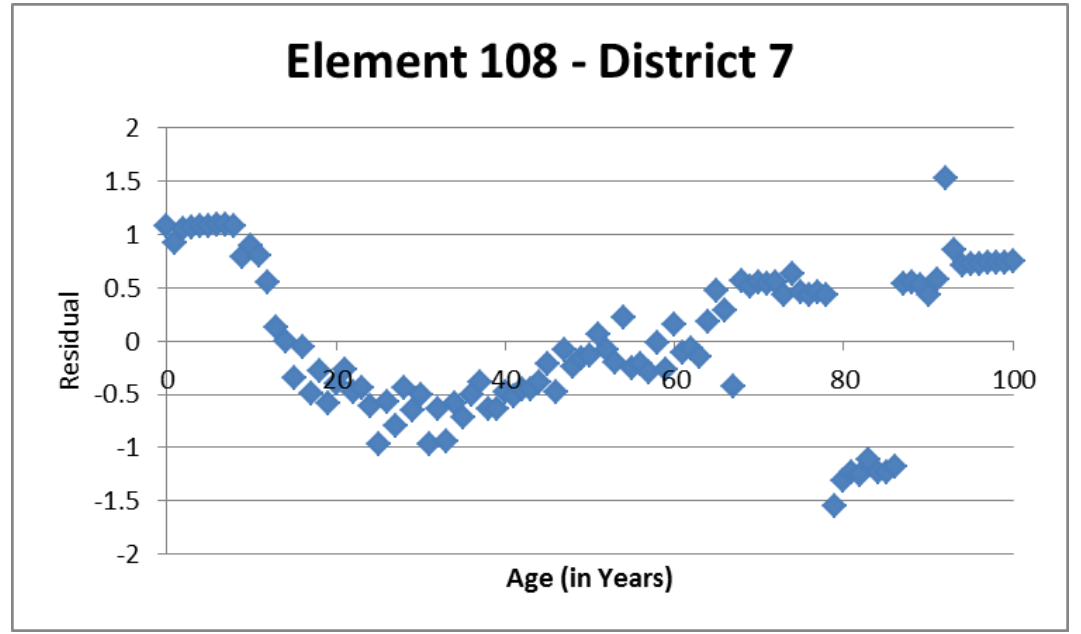

Figure D-50 - Condition States Residuals (Prediction Minus Actual) by Age - Element 108 - District 7

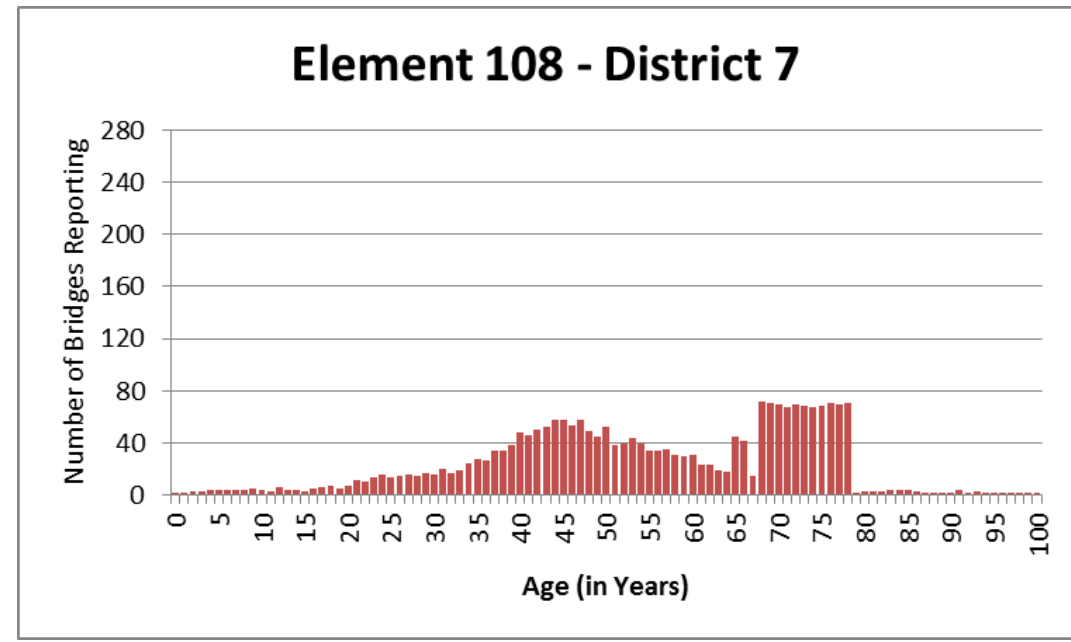

Figure D-51 - Number of Bridges Reporting by Age - Element 108 - District 7 


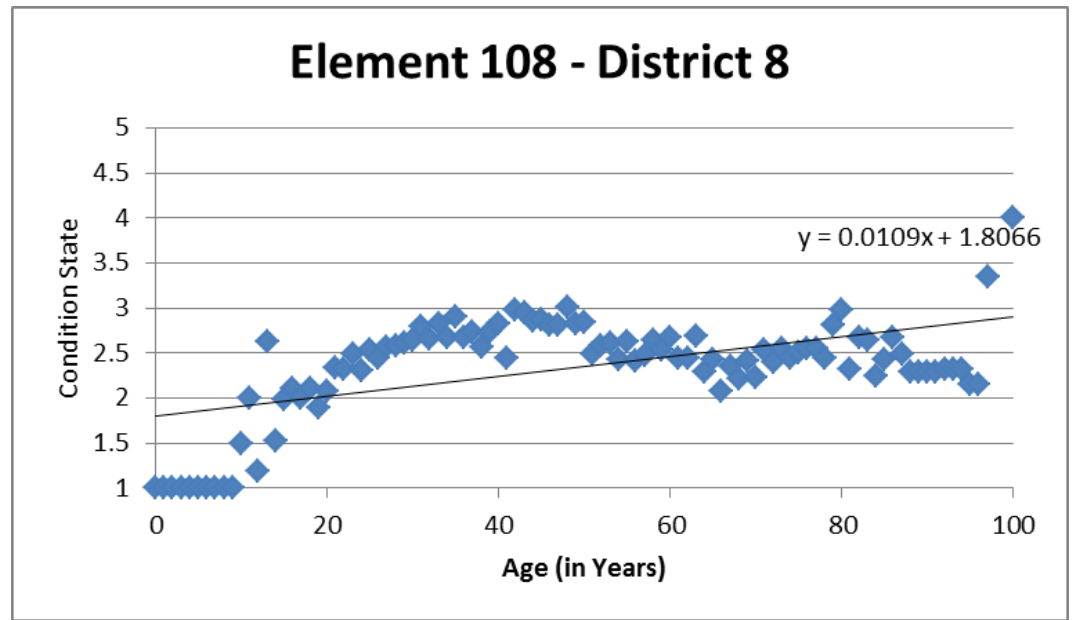

Figure D-52 - Condition State History and Linear Trendline by Age - Element 108 - District 8

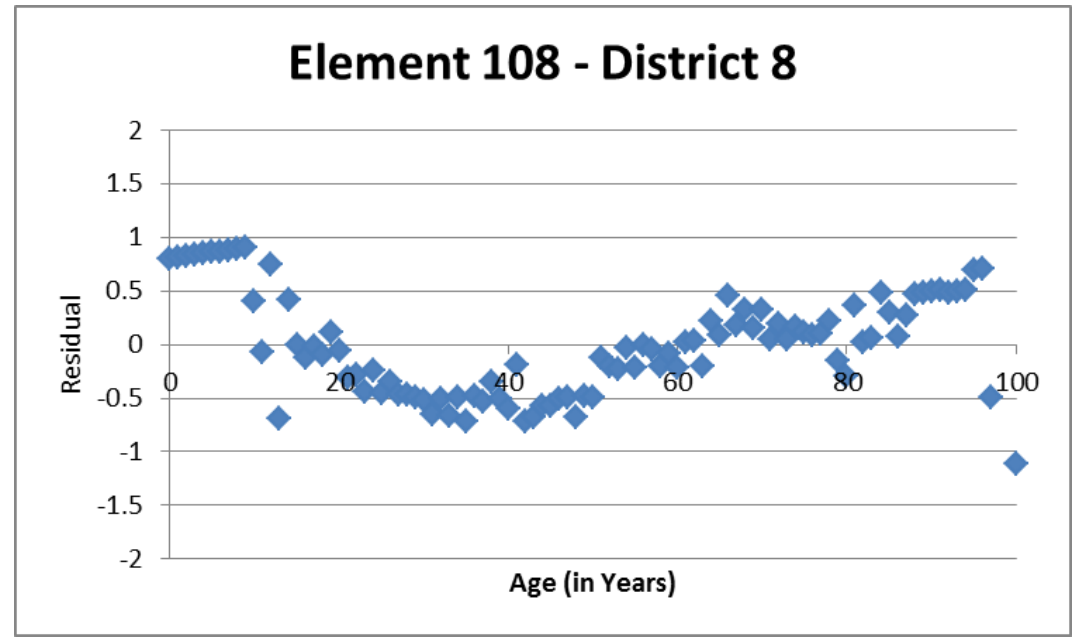

Figure D-53 - Condition States Residuals (Prediction Minus Actual) by Age - Element 108 - District 8

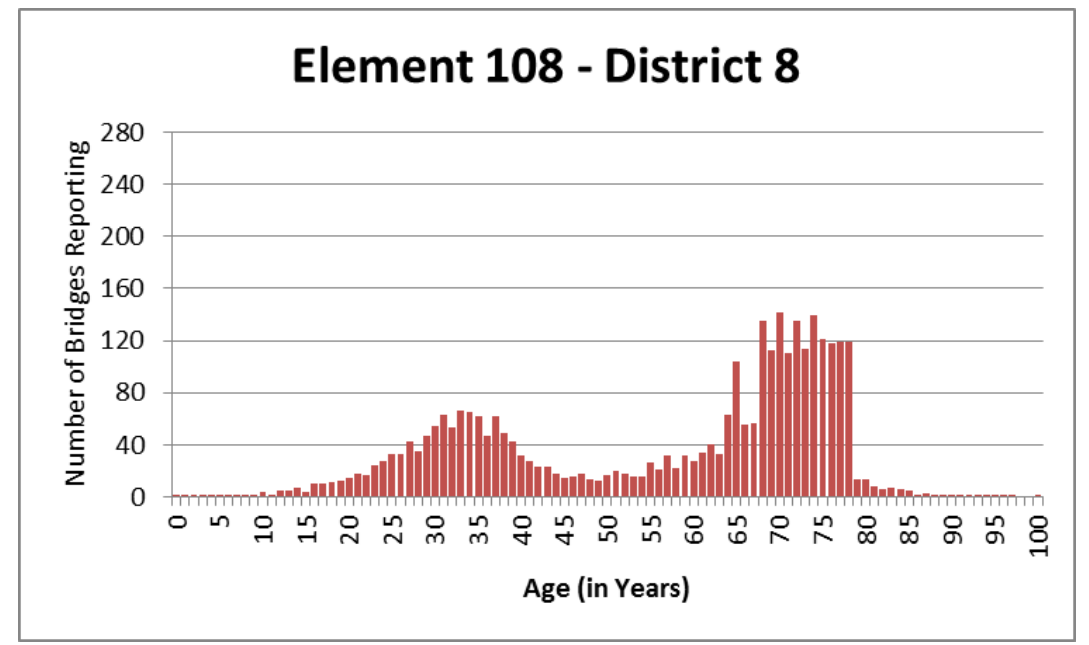

Figure D-54 - Number of Bridges Reporting by Age - Element 108 - District 8 


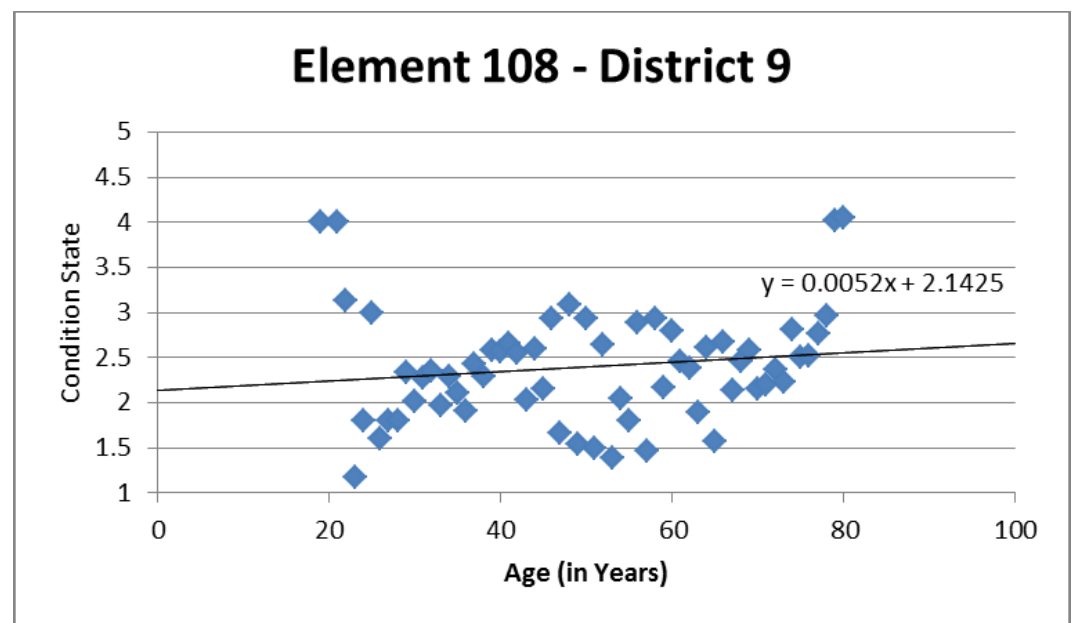

Figure D-55 - Condition State History and Linear Trendline by Age - Element 108 - District 9

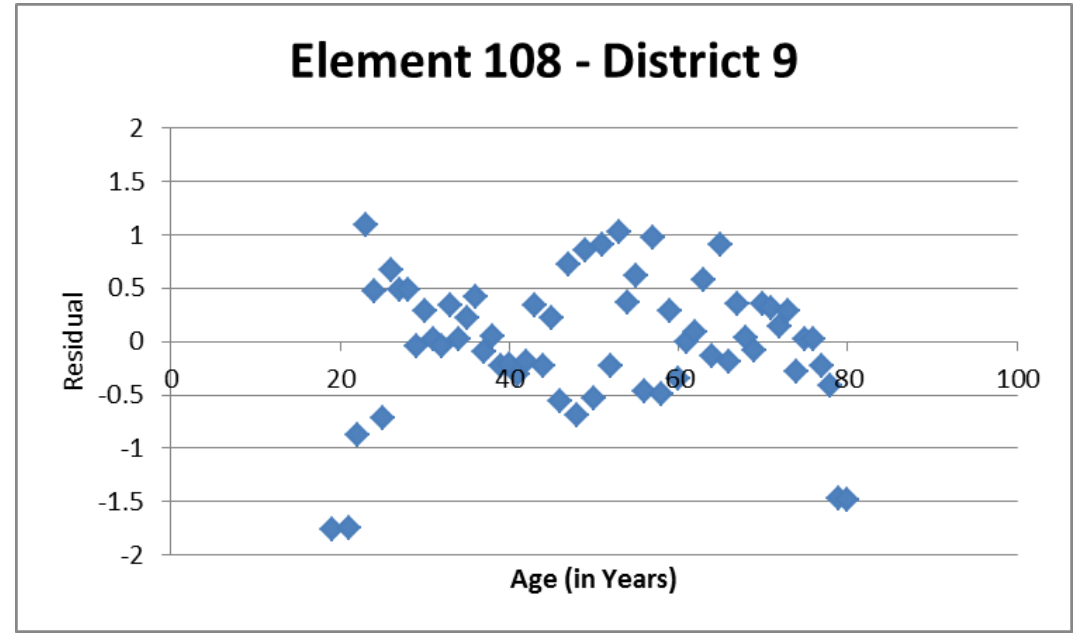

Figure D-56 - Condition States Residuals (Prediction Minus Actual) by Age - Element 108 - District 9

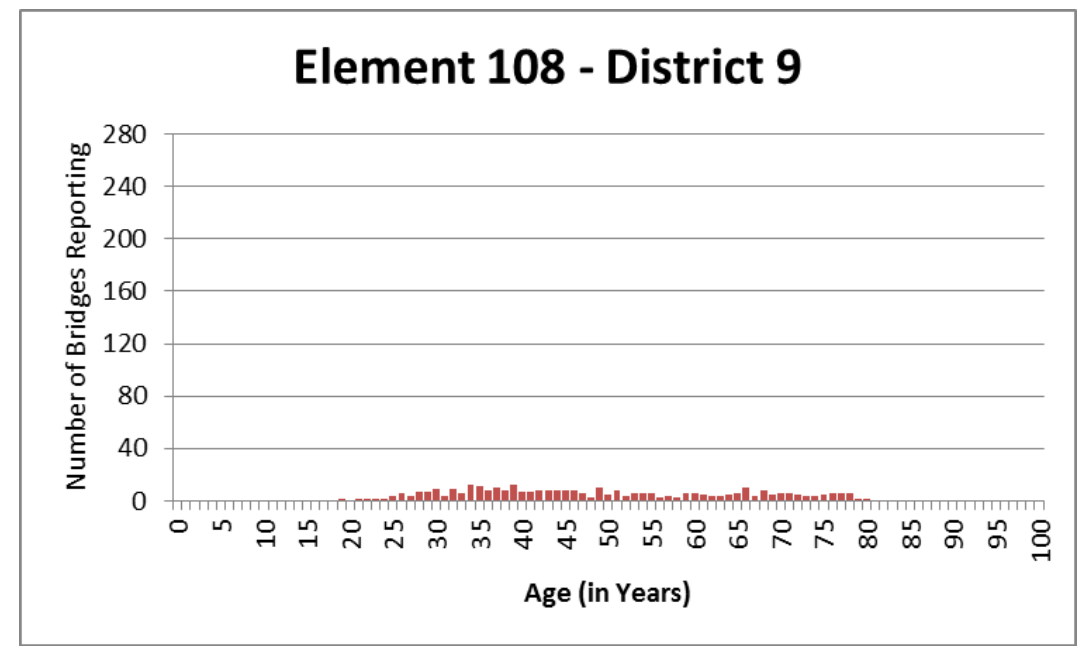

Figure D-57 - Number of Bridges Reporting by Age - Element 108 - District 9 


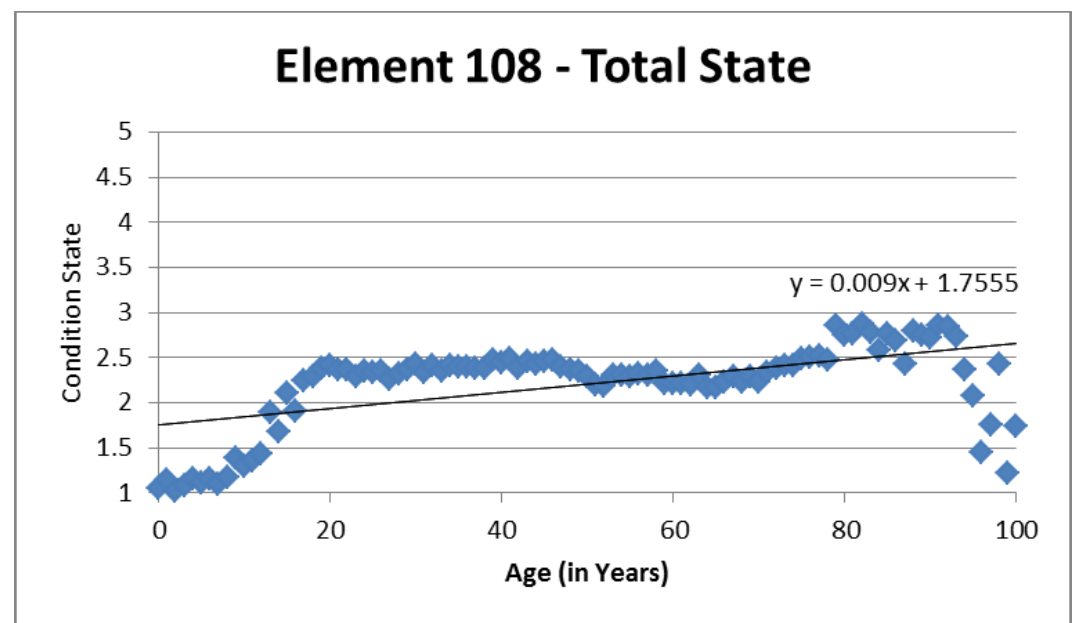

Figure D-58 - Condition State History and Linear Trendline by Age - Element 108 - Total State

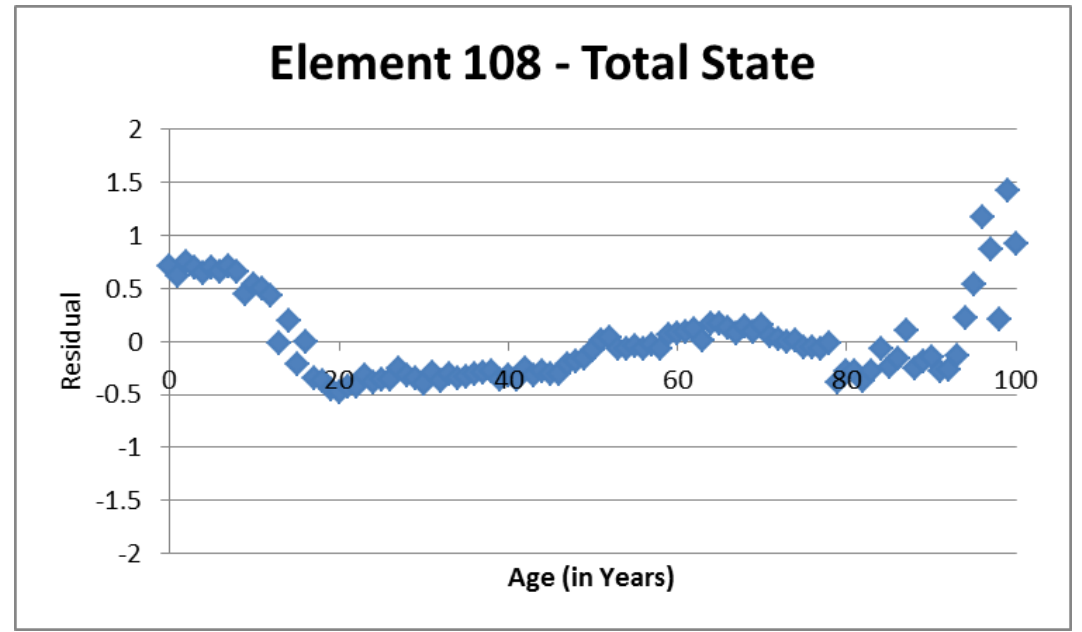

Figure D-59 - Condition States Residuals (Prediction Minus Actual) by Age - Element 108 - Total State

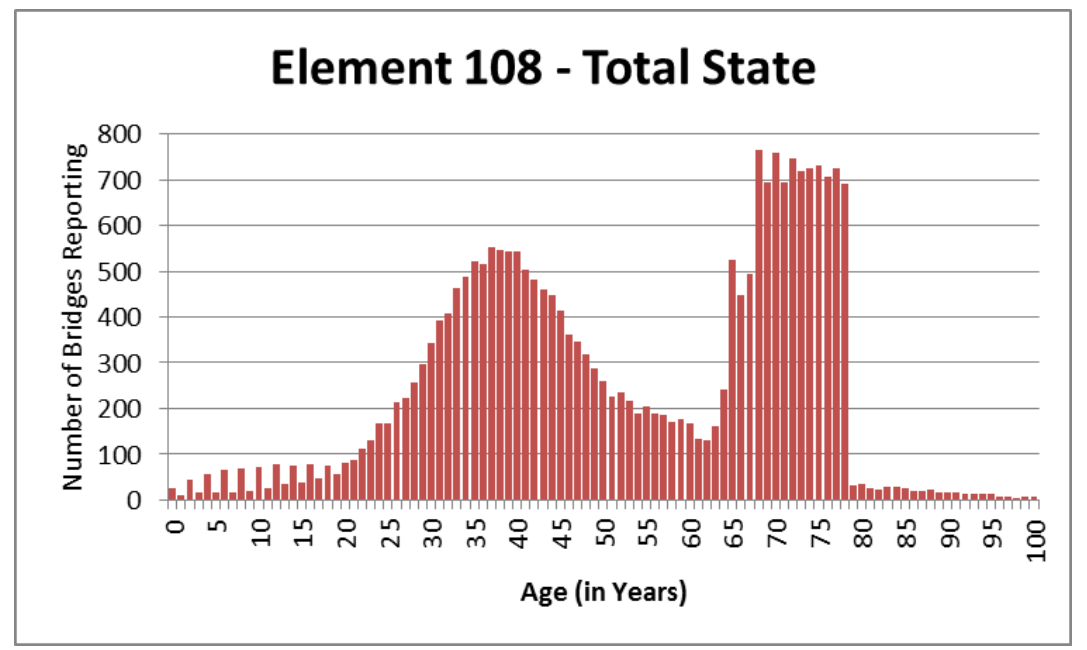

Figure D-60 - Number of Bridges Reporting by Age - Element 108 - Total State 


\begin{tabular}{|c|c|c|c|c|c|}
\hline D1 & 1 & 2 & 3 & 4 & 5 \\
\hline 1 & 0.990 & 0.010 & 0.000 & 0.000 & 0.000 \\
\hline 2 & 0.000 & 0.972 & 0.028 & 0.000 & 0.000 \\
\hline 3 & 0.000 & 0.000 & 0.976 & 0.024 & 0.000 \\
\hline 4 & 0.000 & 0.000 & 0.000 & 0.961 & 0.039 \\
\hline 5 & 0.000 & 0.000 & 0.000 & 0.000 & 1.000 \\
\hline
\end{tabular}

\begin{tabular}{|c|c|c|c|c|c|}
\hline D2 & 1 & 2 & 3 & 4 & 5 \\
\hline 1 & 0.982 & 0.018 & 0.000 & 0.000 & 0.000 \\
\hline 2 & 0.000 & 0.975 & 0.025 & 0.000 & 0.000 \\
\hline 3 & 0.000 & 0.000 & 0.993 & 0.007 & 0.000 \\
\hline 4 & 0.000 & 0.000 & 0.000 & 0.993 & 0.007 \\
\hline 5 & 0.000 & 0.000 & 0.000 & 0.000 & 1.000 \\
\hline
\end{tabular}

Table D-1 - Markov Transition Probability Matrices - Element 107 - Districts 1 and 2

\begin{tabular}{|c|c|c|c|c|c|}
\hline D3 & 1 & 2 & 3 & 4 & 5 \\
\hline 1 & 0.994 & 0.006 & 0.000 & 0.000 & 0.000 \\
\hline 2 & 0.000 & 0.977 & 0.023 & 0.000 & 0.000 \\
\hline 3 & 0.000 & 0.000 & 0.979 & 0.021 & 0.000 \\
\hline 4 & 0.000 & 0.000 & 0.000 & 0.992 & 0.008 \\
\hline 5 & 0.000 & 0.000 & 0.000 & 0.000 & 1.000 \\
\hline
\end{tabular}

\begin{tabular}{|c|c|c|c|c|c|}
\hline D4 & 1 & 2 & 3 & 4 & 5 \\
\hline 1 & 0.987 & 0.013 & 0.000 & 0.000 & 0.000 \\
\hline 2 & 0.000 & 0.972 & 0.028 & 0.000 & 0.000 \\
\hline 3 & 0.000 & 0.000 & 0.977 & 0.023 & 0.000 \\
\hline 4 & 0.000 & 0.000 & 0.000 & 0.985 & 0.015 \\
\hline 5 & 0.000 & 0.000 & 0.000 & 0.000 & 1.000 \\
\hline
\end{tabular}

Table D-2 - Markov Transition Probability Matrices - Element 107 - Districts 3 and 4

\begin{tabular}{|c|c|c|c|c|c|}
\hline D5 & 1 & 2 & 3 & 4 & 5 \\
\hline 1 & 0.994 & 0.006 & 0.000 & 0.000 & 0.000 \\
\hline 2 & 0.000 & 0.976 & 0.024 & 0.000 & 0.000 \\
\hline 3 & 0.000 & 0.000 & 0.970 & 0.030 & 0.000 \\
\hline 4 & 0.000 & 0.000 & 0.000 & 0.990 & 0.010 \\
\hline 5 & 0.000 & 0.000 & 0.000 & 0.000 & 1.000 \\
\hline
\end{tabular}

\begin{tabular}{|c|c|c|c|c|c|}
\hline D6 & 1 & 2 & 3 & 4 & 5 \\
\hline 1 & 0.987 & 0.013 & 0.000 & 0.000 & 0.000 \\
\hline 2 & 0.000 & 0.973 & 0.027 & 0.000 & 0.000 \\
\hline 3 & 0.000 & 0.000 & 0.982 & 0.018 & 0.000 \\
\hline 4 & 0.000 & 0.000 & 0.000 & 0.982 & 0.018 \\
\hline 5 & 0.000 & 0.000 & 0.000 & 0.000 & 1.000 \\
\hline
\end{tabular}

Table D-3 - Markov Transition Probability Matrices - Element 107 - Districts 5 and 6

\begin{tabular}{|c|c|c|c|c|c|}
\hline D7 & 1 & 2 & 3 & 4 & 5 \\
\hline 1 & 0.986 & 0.014 & 0.000 & 0.000 & 0.000 \\
\hline 2 & 0.000 & 0.978 & 0.022 & 0.000 & 0.000 \\
\hline 3 & 0.000 & 0.000 & 0.961 & 0.039 & 0.000 \\
\hline 4 & 0.000 & 0.000 & 0.000 & 1.000 & 0.000 \\
\hline 5 & 0.000 & 0.000 & 0.000 & 0.000 & 1.000 \\
\hline
\end{tabular}

\begin{tabular}{|c|c|c|c|c|c|}
\hline D8 & 1 & 2 & 3 & 4 & 5 \\
\hline 1 & 0.976 & 0.024 & 0.000 & 0.000 & 0.000 \\
\hline 2 & 0.000 & 0.987 & 0.013 & 0.000 & 0.000 \\
\hline 3 & 0.000 & 0.000 & 0.989 & 0.011 & 0.000 \\
\hline 4 & 0.000 & 0.000 & 0.000 & 0.982 & 0.018 \\
\hline 5 & 0.000 & 0.000 & 0.000 & 0.000 & 1.000 \\
\hline
\end{tabular}

Table D-4 - Markov Transition Probability Matrices - Element 107 - District 7 and 8 


\begin{tabular}{|c|c|c|c|c|c|}
\hline D9 & 1 & 2 & 3 & 4 & 5 \\
\hline 1 & 0.992 & 0.008 & 0.000 & 0.000 & 0.000 \\
\hline 2 & 0.000 & 0.996 & 0.004 & 0.000 & 0.000 \\
\hline 3 & 0.000 & 0.000 & 0.987 & 0.013 & 0.000 \\
\hline 4 & 0.000 & 0.000 & 0.000 & 0.997 & 0.003 \\
\hline 5 & 0.000 & 0.000 & 0.000 & 0.000 & 1.000 \\
\hline
\end{tabular}

\begin{tabular}{|c|c|c|c|c|c|}
\hline Total & 1 & 2 & 3 & 4 & 5 \\
\hline 1 & 0.990 & 0.010 & 0.000 & 0.000 & 0.000 \\
\hline 2 & 0.000 & 0.980 & 0.020 & 0.000 & 0.000 \\
\hline 3 & 0.000 & 0.000 & 0.977 & 0.023 & 0.000 \\
\hline 4 & 0.000 & 0.000 & 0.000 & 0.986 & 0.014 \\
\hline 5 & 0.000 & 0.000 & 0.000 & 0.000 & 1.000 \\
\hline
\end{tabular}

Table D-5 - Markov Transition Probability Matrices - Element 107 - District 9 and Total State

\begin{tabular}{|c|c|c|c|c|c|}
\hline D1 & 1 & 2 & 3 & 4 & 5 \\
\hline 1 & 0.970 & 0.030 & 0.000 & 0.000 & 0.000 \\
\hline 2 & 0.000 & 0.953 & 0.047 & 0.000 & 0.000 \\
\hline 3 & 0.000 & 0.000 & 0.960 & 0.040 & 0.000 \\
\hline 4 & 0.000 & 0.000 & 0.000 & 0.967 & 0.033 \\
\hline 5 & 0.000 & 0.000 & 0.000 & 0.000 & 1.000 \\
\hline
\end{tabular}

\begin{tabular}{|c|c|c|c|c|c|}
\hline $\mathrm{D} 2$ & 1 & 2 & 3 & 4 & 5 \\
\hline 1 & 0.973 & 0.027 & 0.000 & 0.000 & 0.000 \\
\hline 2 & 0.000 & 0.919 & 0.081 & 0.000 & 0.000 \\
\hline 3 & 0.000 & 0.000 & 0.984 & 0.016 & 0.000 \\
\hline 4 & 0.000 & 0.000 & 0.000 & 0.983 & 0.017 \\
\hline 5 & 0.000 & 0.000 & 0.000 & 0.000 & 1.000 \\
\hline
\end{tabular}

Table D-6 - Markov Transition Probability Matrices - Element 108 - Districts 1 and 2

\begin{tabular}{|c|c|c|c|c|c|}
\hline D3 & 1 & 2 & 3 & 4 & 5 \\
\hline 1 & 0.986 & 0.014 & 0.000 & 0.000 & 0.000 \\
\hline 2 & 0.000 & 0.977 & 0.023 & 0.000 & 0.000 \\
\hline 3 & 0.000 & 0.000 & 0.990 & 0.010 & 0.000 \\
\hline 4 & 0.000 & 0.000 & 0.000 & 0.987 & 0.013 \\
\hline 5 & 0.000 & 0.000 & 0.000 & 0.000 & 1.000 \\
\hline
\end{tabular}

\begin{tabular}{|c|c|c|c|c|c|}
\hline $\mathrm{D} 4$ & 1 & 2 & 3 & 4 & 5 \\
\hline 1 & 0.965 & 0.035 & 0.000 & 0.000 & 0.000 \\
\hline 2 & 0.000 & 0.986 & 0.014 & 0.000 & 0.000 \\
\hline 3 & 0.000 & 0.000 & 0.989 & 0.011 & 0.000 \\
\hline 4 & 0.000 & 0.000 & 0.000 & 0.989 & 0.011 \\
\hline 5 & 0.000 & 0.000 & 0.000 & 0.000 & 1.000 \\
\hline
\end{tabular}

Table D-7 - Markov Transition Probability Matrices - Element 108 - Districts 3 and 4

\begin{tabular}{|c|c|c|c|c|c|}
\hline D5 & 1 & 2 & 3 & 4 & 5 \\
\hline 1 & 0.991 & 0.009 & 0.000 & 0.000 & 0.000 \\
\hline 2 & 0.000 & 0.987 & 0.013 & 0.000 & 0.000 \\
\hline 3 & 0.000 & 0.000 & 0.976 & 0.024 & 0.000 \\
\hline 4 & 0.000 & 0.000 & 0.000 & 0.991 & 0.009 \\
\hline 5 & 0.000 & 0.000 & 0.000 & 0.000 & 1.000 \\
\hline
\end{tabular}

\begin{tabular}{|c|c|c|c|c|c|}
\hline D6 & 1 & 2 & 3 & 4 & 5 \\
\hline 1 & 0.968 & 0.032 & 0.000 & 0.000 & 0.000 \\
\hline 2 & 0.000 & 0.948 & 0.052 & 0.000 & 0.000 \\
\hline 3 & 0.000 & 0.000 & 0.955 & 0.045 & 0.000 \\
\hline 4 & 0.000 & 0.000 & 0.000 & 0.995 & 0.005 \\
\hline 5 & 0.000 & 0.000 & 0.000 & 0.000 & 1.000 \\
\hline
\end{tabular}

Table D-8 - Markov Transition Probability Matrices - Element 108 - Districts 5 and 6 


\begin{tabular}{|c|c|c|c|c|c|}
\hline D7 & 1 & 2 & 3 & 4 & 5 \\
\hline 1 & 0.971 & 0.029 & 0.000 & 0.000 & 0.000 \\
\hline 2 & 0.000 & 0.935 & 0.065 & 0.000 & 0.000 \\
\hline 3 & 0.000 & 0.000 & 0.971 & 0.029 & 0.000 \\
\hline 4 & 0.000 & 0.000 & 0.000 & 0.990 & 0.010 \\
\hline 5 & 0.000 & 0.000 & 0.000 & 0.000 & 1.000 \\
\hline
\end{tabular}

\begin{tabular}{|c|c|c|c|c|c|}
\hline D8 & 1 & 2 & 3 & 4 & 5 \\
\hline 1 & 0.956 & 0.044 & 0.000 & 0.000 & 0.000 \\
\hline 2 & 0.000 & 0.973 & 0.027 & 0.000 & 0.000 \\
\hline 3 & 0.000 & 0.000 & 0.986 & 0.014 & 0.000 \\
\hline 4 & 0.000 & 0.000 & 0.000 & 0.982 & 0.018 \\
\hline 5 & 0.000 & 0.000 & 0.000 & 0.000 & 1.000 \\
\hline
\end{tabular}

Table D-9 - Markov Transition Probability Matrices - Element 108 - Districts 7 and 8

\begin{tabular}{|c|c|c|c|c|c|}
\hline D9 & 1 & 2 & 3 & 4 & 5 \\
\hline 1 & 0.976 & 0.024 & 0.000 & 0.000 & 0.000 \\
\hline 2 & 0.000 & 0.964 & 0.036 & 0.000 & 0.000 \\
\hline 3 & 0.000 & 0.000 & 0.971 & 0.029 & 0.000 \\
\hline 4 & 0.000 & 0.000 & 0.000 & 0.989 & 0.011 \\
\hline 5 & 0.000 & 0.000 & 0.000 & 0.000 & 1.000 \\
\hline
\end{tabular}

\begin{tabular}{|c|c|c|c|c|c|}
\hline Total & 1 & 2 & 3 & 4 & 5 \\
\hline 1 & 0.975 & 0.025 & 0.000 & 0.000 & 0.000 \\
\hline 2 & 0.000 & 0.964 & 0.036 & 0.000 & 0.000 \\
\hline 3 & 0.000 & 0.000 & 0.980 & 0.020 & 0.000 \\
\hline 4 & 0.000 & 0.000 & 0.000 & 0.983 & 0.017 \\
\hline 5 & 0.000 & 0.000 & 0.000 & 0.000 & 1.000 \\
\hline
\end{tabular}

Table D-10 - Markov Transition Probability Matrices - Element 108 - District 9 and Total State

\begin{tabular}{|c|c|c|c|c|c|}
\hline D1 & 1 & 2 & 3 & 4 & 5 \\
\hline 1 & 0.993 & 0.007 & 0.000 & 0.000 & 0.000 \\
\hline 2 & 0.000 & 0.994 & 0.006 & 0.000 & 0.000 \\
\hline 3 & 0.000 & 0.000 & 1.000 & 0.000 & 0.000 \\
\hline 4 & 0.000 & 0.000 & 0.000 & 0.000 & 1.000 \\
\hline 5 & 0.000 & 0.000 & 0.000 & 0.000 & 0.000 \\
\hline
\end{tabular}

\begin{tabular}{|c|c|c|c|c|c|}
\hline $\mathrm{D} 2$ & 1 & 2 & 3 & 4 & 5 \\
\hline 1 & 0.987 & 0.013 & 0.000 & 0.000 & 0.000 \\
\hline 2 & 0.000 & 0.996 & 0.004 & 0.000 & 0.000 \\
\hline 3 & 0.000 & 0.000 & 1.000 & 0.000 & 0.000 \\
\hline 4 & 0.000 & 0.000 & 0.000 & 0.000 & 1.000 \\
\hline 5 & 0.000 & 0.000 & 0.000 & 0.000 & 0.000 \\
\hline
\end{tabular}

Table D-11 - Markov Transition Probability Matrices - Element 302 - Districts 1 and 2

\begin{tabular}{|c|c|c|c|c|c|}
\hline D3 & 1 & 2 & 3 & 4 & 5 \\
\hline 1 & 0.988 & 0.012 & 0.000 & 0.000 & 0.000 \\
\hline 2 & 0.000 & 0.975 & 0.025 & 0.000 & 0.000 \\
\hline 3 & 0.000 & 0.000 & 1.000 & 0.000 & 0.000 \\
\hline 4 & 0.000 & 0.000 & 0.000 & 0.000 & 1.000 \\
\hline 5 & 0.000 & 0.000 & 0.000 & 0.000 & 0.000 \\
\hline
\end{tabular}

\begin{tabular}{|c|c|c|c|c|c|}
\hline $\mathrm{D} 4$ & 1 & 2 & 3 & 4 & 5 \\
\hline 1 & 0.994 & 0.006 & 0.000 & 0.000 & 0.000 \\
\hline 2 & 0.000 & 0.980 & 0.020 & 0.000 & 0.000 \\
\hline 3 & 0.000 & 0.000 & 1.000 & 0.000 & 0.000 \\
\hline 4 & 0.000 & 0.000 & 0.000 & 0.000 & 1.000 \\
\hline 5 & 0.000 & 0.000 & 0.000 & 0.000 & 0.000 \\
\hline
\end{tabular}

Table D-12 - Markov Transition Probability Matrices - Element 302 - Districts 3 and 4 


\begin{tabular}{|c|c|c|c|c|c|}
\hline D5 & 1 & 2 & 3 & 4 & 5 \\
\hline 1 & 0.994 & 0.006 & 0.000 & 0.000 & 0.000 \\
\hline 2 & 0.000 & 0.976 & 0.024 & 0.000 & 0.000 \\
\hline 3 & 0.000 & 0.000 & 1.000 & 0.000 & 0.000 \\
\hline 4 & 0.000 & 0.000 & 0.000 & 0.000 & 1.000 \\
\hline 5 & 0.000 & 0.000 & 0.000 & 0.000 & 0.000 \\
\hline
\end{tabular}

\begin{tabular}{|c|c|c|c|c|c|}
\hline D6 & 1 & 2 & 3 & 4 & 5 \\
\hline 1 & 0.982 & 0.018 & 0.000 & 0.000 & 0.000 \\
\hline 2 & 0.000 & 0.951 & 0.049 & 0.000 & 0.000 \\
\hline 3 & 0.000 & 0.000 & 1.000 & 0.000 & 0.000 \\
\hline 4 & 0.000 & 0.000 & 0.000 & 0.000 & 1.000 \\
\hline 5 & 0.000 & 0.000 & 0.000 & 0.000 & 0.000 \\
\hline
\end{tabular}

Table D-13 - Markov Transition Probability Matrices - Element 302 - Districts 5 and 6

\begin{tabular}{|c|c|c|c|c|c|}
\hline D7 & 1 & 2 & 3 & 4 & 5 \\
\hline 1 & 0.988 & 0.012 & 0.000 & 0.000 & 0.000 \\
\hline 2 & 0.000 & 0.997 & 0.003 & 0.000 & 0.000 \\
\hline 3 & 0.000 & 0.000 & 1.000 & 0.000 & 0.000 \\
\hline 4 & 0.000 & 0.000 & 0.000 & 0.000 & 1.000 \\
\hline 5 & 0.000 & 0.000 & 0.000 & 0.000 & 0.000 \\
\hline
\end{tabular}

\begin{tabular}{|c|c|c|c|c|c|}
\hline D8 & 1 & 2 & 3 & 4 & 5 \\
\hline 1 & 0.992 & 0.008 & 0.000 & 0.000 & 0.000 \\
\hline 2 & 0.000 & 0.996 & 0.004 & 0.000 & 0.000 \\
\hline 3 & 0.000 & 0.000 & 1.000 & 0.000 & 0.000 \\
\hline 4 & 0.000 & 0.000 & 0.000 & 0.000 & 1.000 \\
\hline 5 & 0.000 & 0.000 & 0.000 & 0.000 & 0.000 \\
\hline
\end{tabular}

Table D-14 - Markov Transition Probability Matrices - Element 302 - Districts 7 and 8

\begin{tabular}{|c|c|c|c|c|c|}
\hline D9 & 1 & 2 & 3 & 4 & 5 \\
\hline 1 & 0.994 & 0.006 & 0.000 & 0.000 & 0.000 \\
\hline 2 & 0.000 & 0.985 & 0.015 & 0.000 & 0.000 \\
\hline 3 & 0.000 & 0.000 & 1.000 & 0.000 & 0.000 \\
\hline 4 & 0.000 & 0.000 & 0.000 & 0.000 & 1.000 \\
\hline 5 & 0.000 & 0.000 & 0.000 & 0.000 & 0.000 \\
\hline
\end{tabular}

\begin{tabular}{|c|c|c|c|c|c|}
\hline Total & 1 & 2 & 3 & 4 & 5 \\
\hline 1 & 0.990 & 0.010 & 0.000 & 0.000 & 0.000 \\
\hline 2 & 0.000 & 0.986 & 0.014 & 0.000 & 0.000 \\
\hline 3 & 0.000 & 0.000 & 1.000 & 0.000 & 0.000 \\
\hline 4 & 0.000 & 0.000 & 0.000 & 0.000 & 1.000 \\
\hline 5 & 0.000 & 0.000 & 0.000 & 0.000 & 0.000 \\
\hline
\end{tabular}

Table D-15 - Markov Transition Probability Matrices - Element 302 - District 9 and Total State

\begin{tabular}{|c|c|c|c|c|c|}
\hline D1 & 1 & 2 & 3 & 4 & 5 \\
\hline 1 & 0.988 & 0.012 & 0.000 & 0.000 & 0.000 \\
\hline 2 & 0.000 & 0.988 & 0.012 & 0.000 & 0.000 \\
\hline 3 & 0.000 & 0.000 & 0.987 & 0.013 & 0.000 \\
\hline 4 & 0.000 & 0.000 & 0.000 & 1.000 & 0.000 \\
\hline 5 & 0.000 & 0.000 & 0.000 & 0.000 & 0.000 \\
\hline
\end{tabular}

\begin{tabular}{|c|c|c|c|c|c|}
\hline D2 & 1 & 2 & 3 & 4 & 5 \\
\hline 1 & 0.992 & 0.008 & 0.000 & 0.000 & 0.000 \\
\hline 2 & 0.000 & 0.992 & 0.008 & 0.000 & 0.000 \\
\hline 3 & 0.000 & 0.000 & 0.989 & 0.011 & 0.000 \\
\hline 4 & 0.000 & 0.000 & 0.000 & 1.000 & 0.000 \\
\hline 5 & 0.000 & 0.000 & 0.000 & 0.000 & 0.000 \\
\hline
\end{tabular}

Table D-16 - Markov Transition Probability Matrices - Element 32 - Districts 1 and 2 


\begin{tabular}{|c|c|c|c|c|c|}
\hline D3 & 1 & 2 & 3 & 4 & 5 \\
\hline 1 & 0.982 & 0.018 & 0.000 & 0.000 & 0.000 \\
\hline 2 & 0.000 & 0.995 & 0.005 & 0.000 & 0.000 \\
\hline 3 & 0.000 & 0.000 & 0.988 & 0.012 & 0.000 \\
\hline 4 & 0.000 & 0.000 & 0.000 & 1.000 & 0.000 \\
\hline 5 & 0.000 & 0.000 & 0.000 & 0.000 & 0.000 \\
\hline
\end{tabular}

\begin{tabular}{|c|c|c|c|c|c|}
\hline D5 & 1 & 2 & 3 & 4 & 5 \\
\hline 1 & 0.989 & 0.011 & 0.000 & 0.000 & 0.000 \\
\hline 2 & 0.000 & 0.996 & 0.004 & 0.000 & 0.000 \\
\hline 3 & 0.000 & 0.000 & 0.975 & 0.025 & 0.000 \\
\hline 4 & 0.000 & 0.000 & 0.000 & 1.000 & 0.000 \\
\hline 5 & 0.000 & 0.000 & 0.000 & 0.000 & 0.000 \\
\hline
\end{tabular}

Table D-17 - Markov Transition Probability Matrices - Element 32 - Districts 3 and 5

\begin{tabular}{|c|c|c|c|c|c|}
\hline D7 & 1 & 2 & 3 & 4 & 5 \\
\hline 1 & 0.982 & 0.018 & 0.000 & 0.000 & 0.000 \\
\hline 2 & 0.000 & 0.994 & 0.006 & 0.000 & 0.000 \\
\hline 3 & 0.000 & 0.000 & 0.973 & 0.027 & 0.000 \\
\hline 4 & 0.000 & 0.000 & 0.000 & 1.000 & 0.000 \\
\hline 5 & 0.000 & 0.000 & 0.000 & 0.000 & 0.000 \\
\hline
\end{tabular}

\begin{tabular}{|c|c|c|c|c|c|}
\hline D8 & 1 & 2 & 3 & 4 & 5 \\
\hline 1 & 0.982 & 0.018 & 0.000 & 0.000 & 0.000 \\
\hline 2 & 0.000 & 0.992 & 0.008 & 0.000 & 0.000 \\
\hline 3 & 0.000 & 0.000 & 0.990 & 0.010 & 0.000 \\
\hline 4 & 0.000 & 0.000 & 0.000 & 1.000 & 0.000 \\
\hline 5 & 0.000 & 0.000 & 0.000 & 0.000 & 0.000 \\
\hline
\end{tabular}

Table D-18 - Markov Transition Probability Matrices - Element 32 - Districts 7 and 8

\begin{tabular}{|c|c|c|c|c|c|}
\hline Total & 1 & 2 & 3 & 4 & 5 \\
\hline 1 & 0.986 & 0.014 & 0.000 & 0.000 & 0.000 \\
\hline 2 & 0.000 & 0.992 & 0.008 & 0.000 & 0.000 \\
\hline 3 & 0.000 & 0.000 & 0.987 & 0.013 & 0.000 \\
\hline 4 & 0.000 & 0.000 & 0.000 & 1.000 & 0.000 \\
\hline 5 & 0.000 & 0.000 & 0.000 & 0.000 & 0.000 \\
\hline
\end{tabular}

Table D-19 - Markov Transition Probability Matrix - Element 32 - Total State 


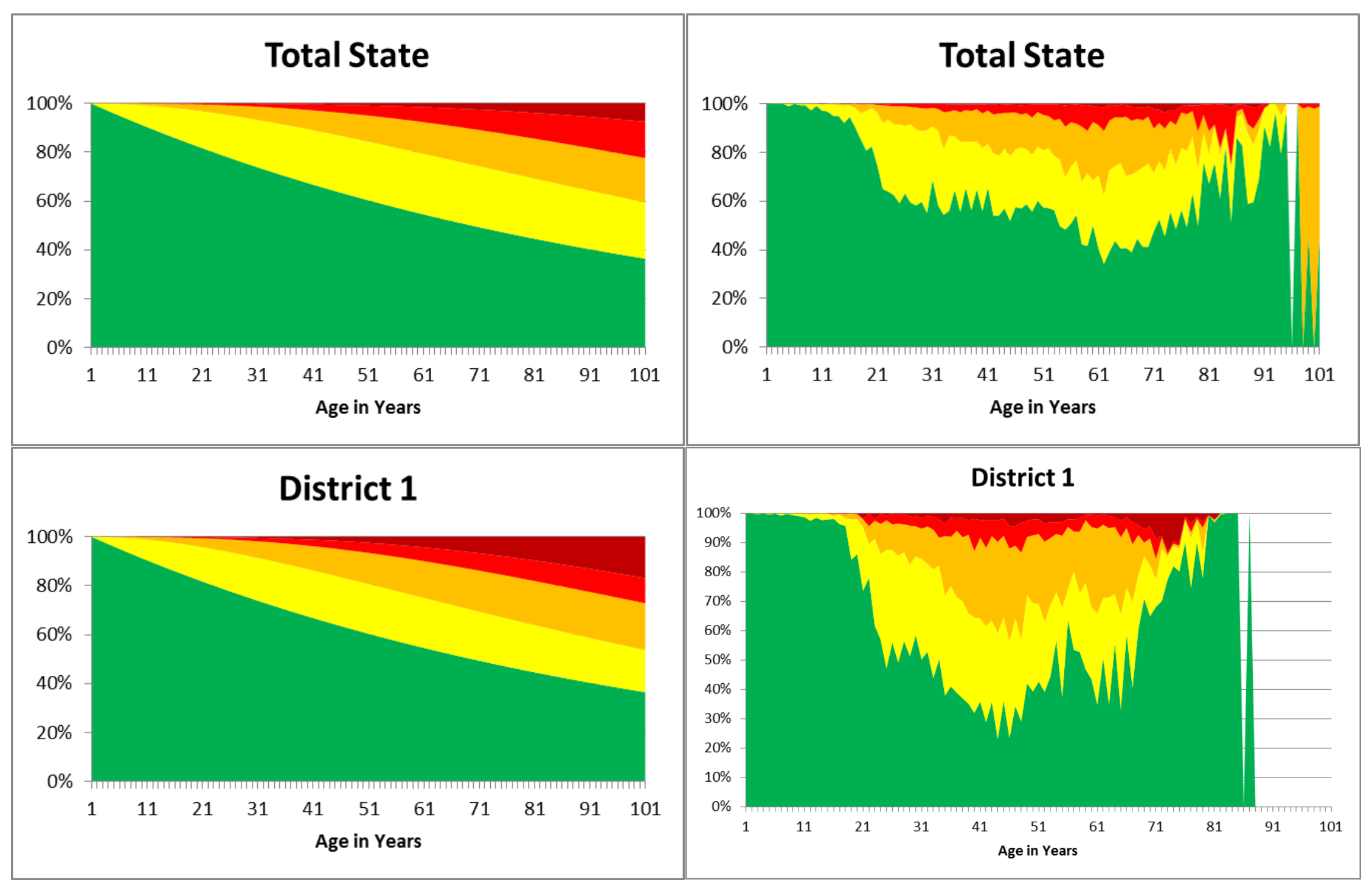

Figure D-61 - Markov Chain Prediction vs. Actual Graphs - Element 107 - Total State and District 1 


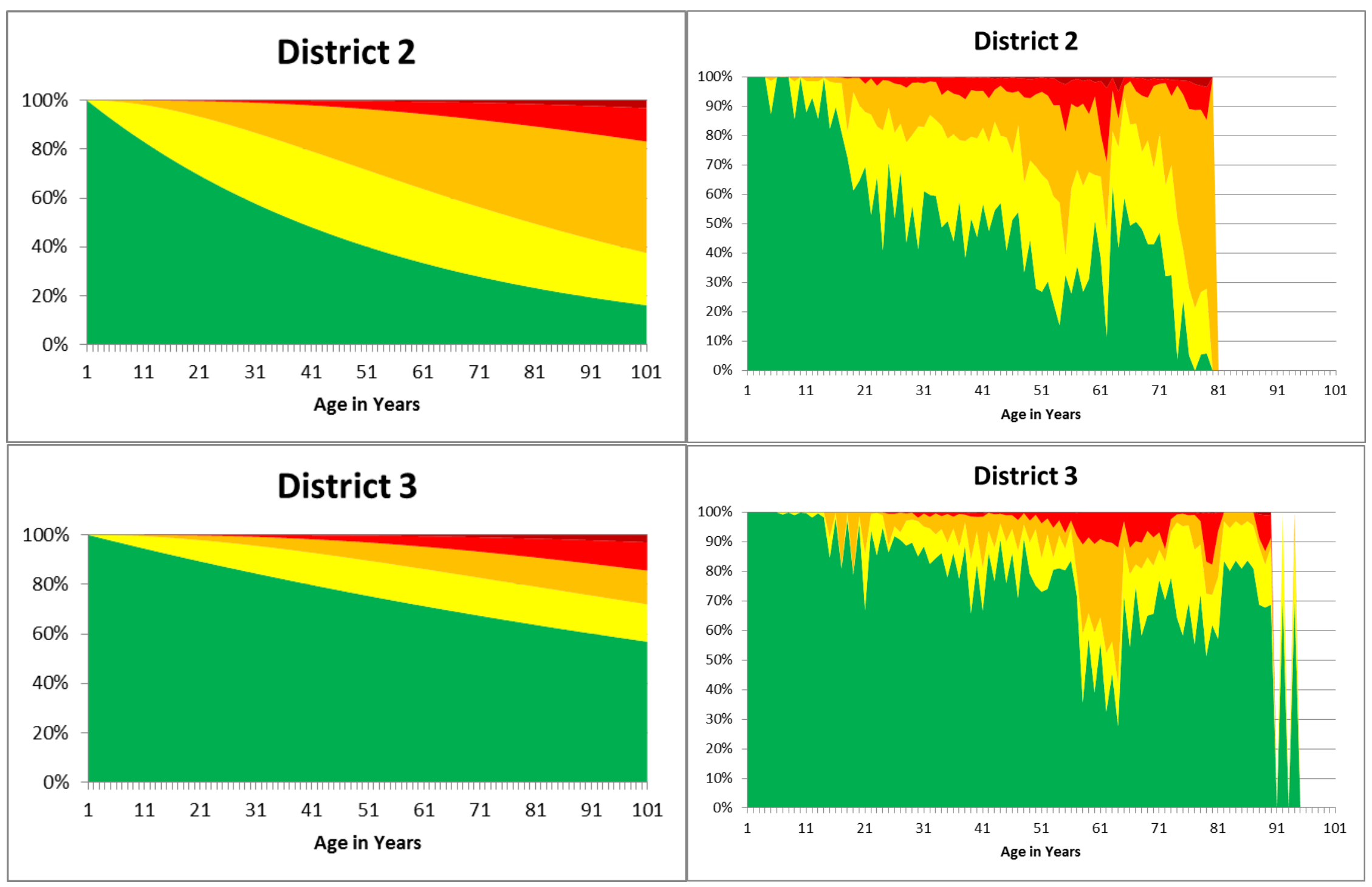

Figure D-62 - Markov Chain Prediction vs. Actual Graphs - Element 107 - Districts 2 and 3 


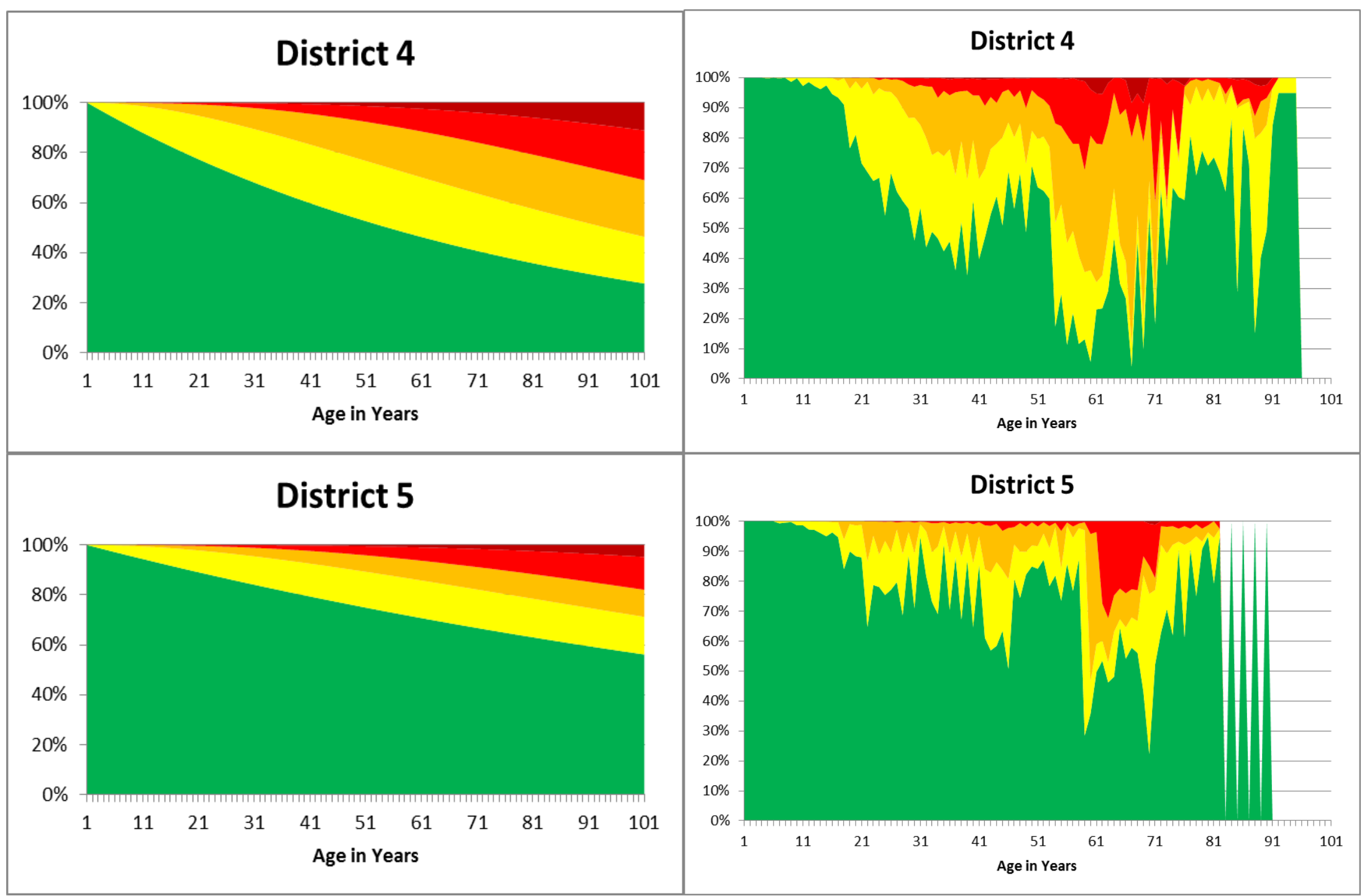

Figure D-63 - Markov Chain Prediction vs. Actual Graphs - Element 107 - Districts 4 and 5 


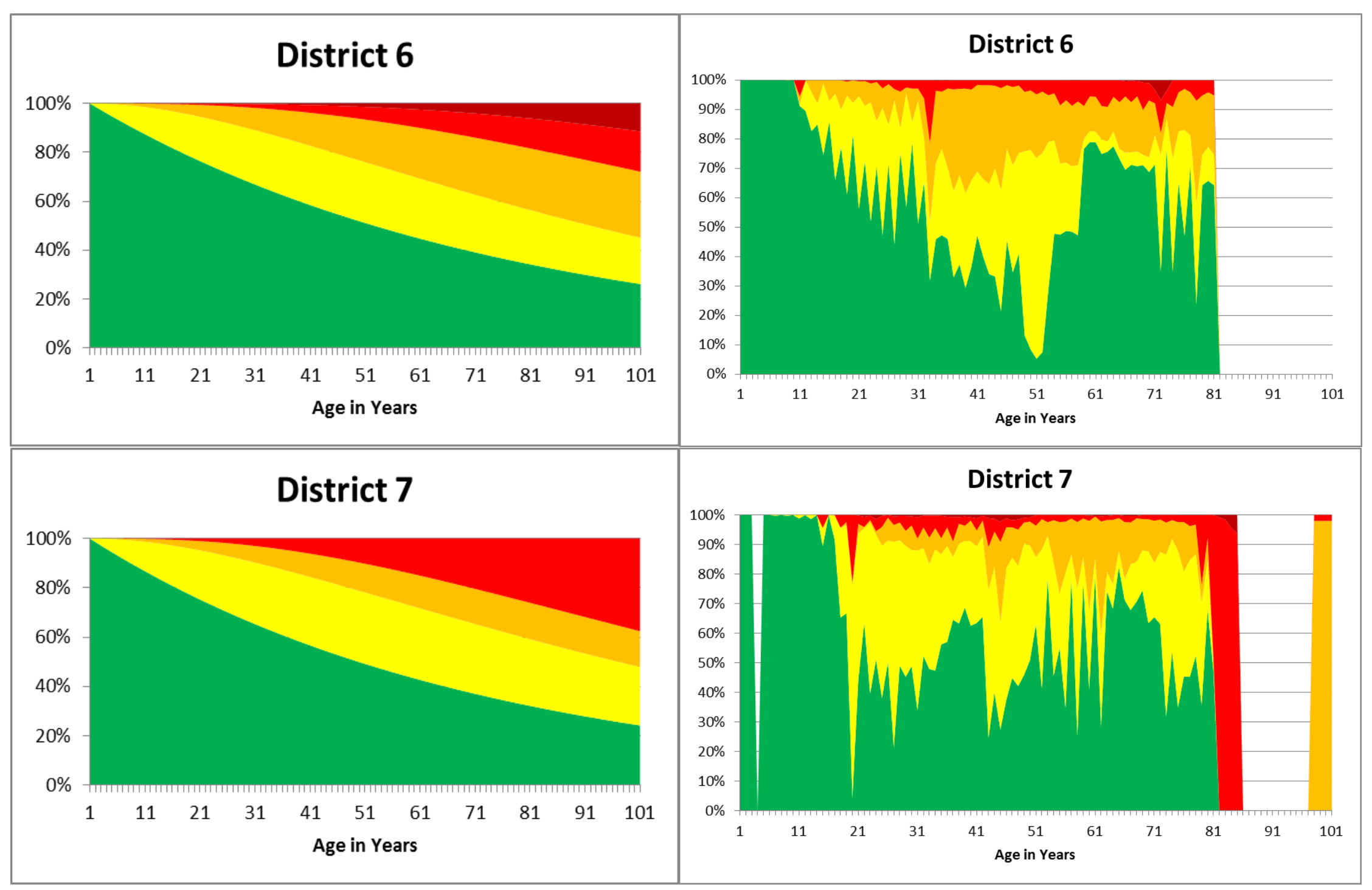

Figure D-64 - Markov Chain Prediction vs. Actual Graphs - Element 107 - Districts 6 and 7 


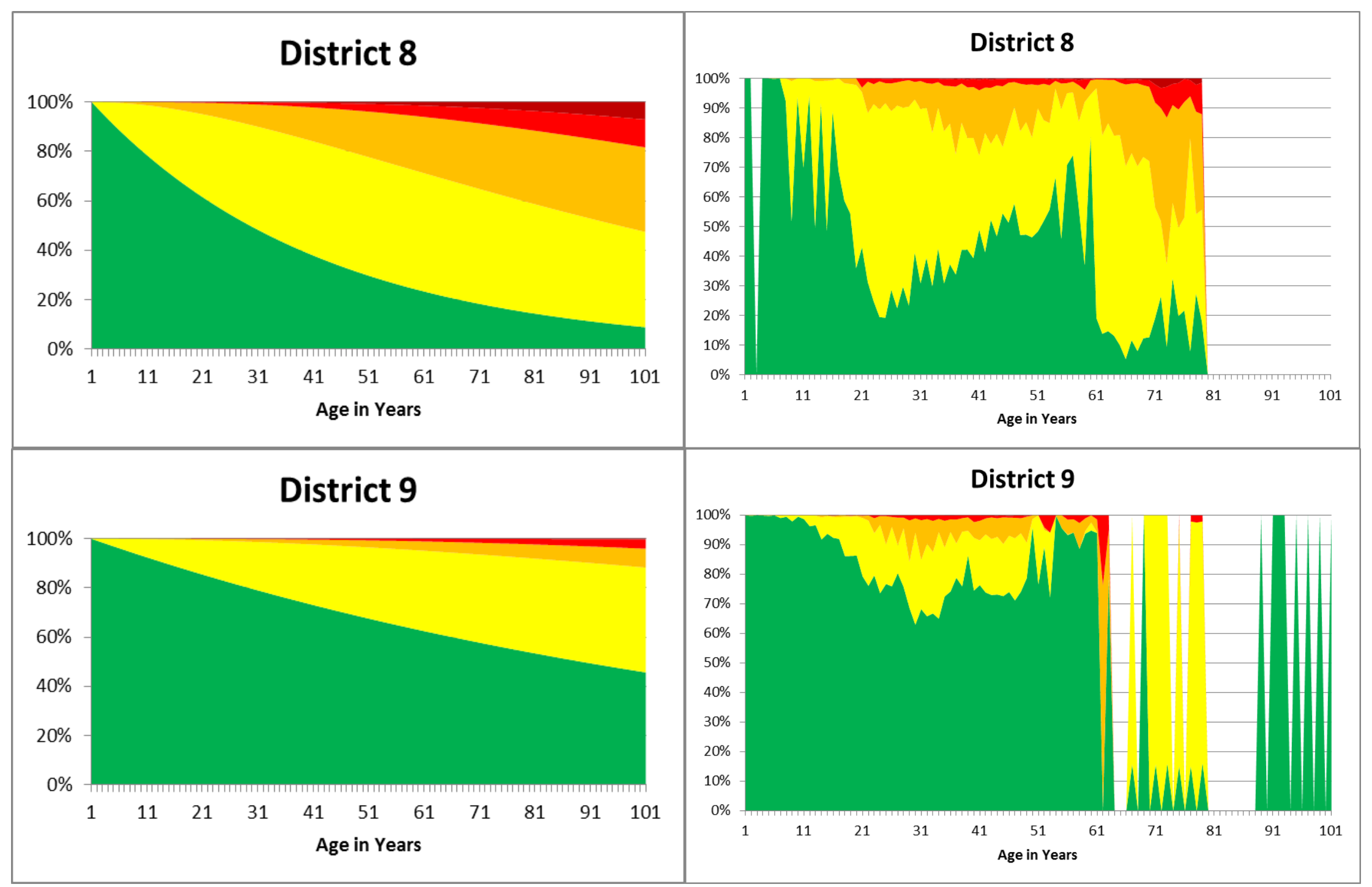

Figure D-65 - Markov Chain Prediction vs. Actual Graphs - Element 107 - Districts 8 and 9 


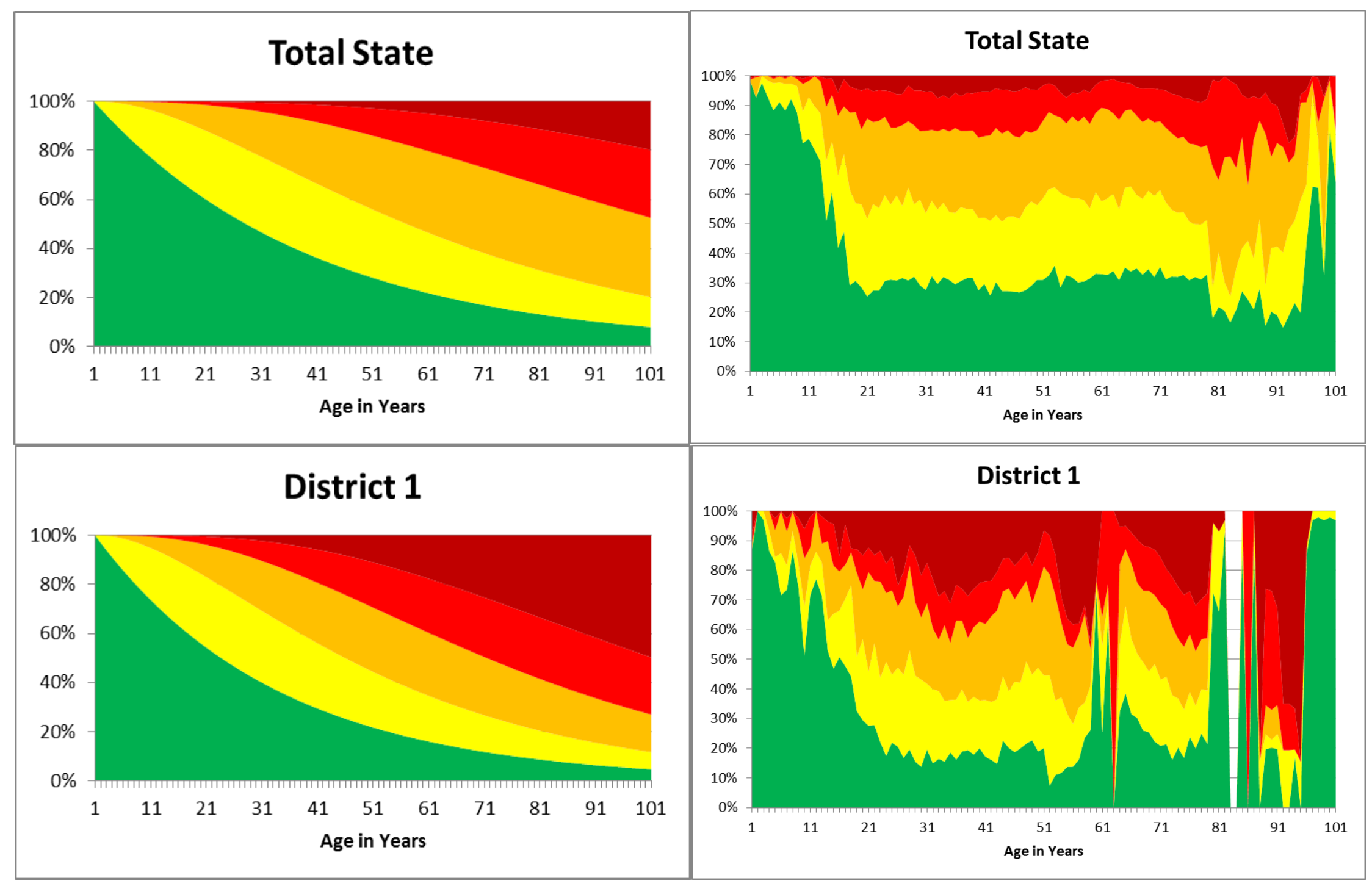

Figure D-66 - Markov Chain Prediction vs. Actual Graphs - Element 108 - Total State and District 1 


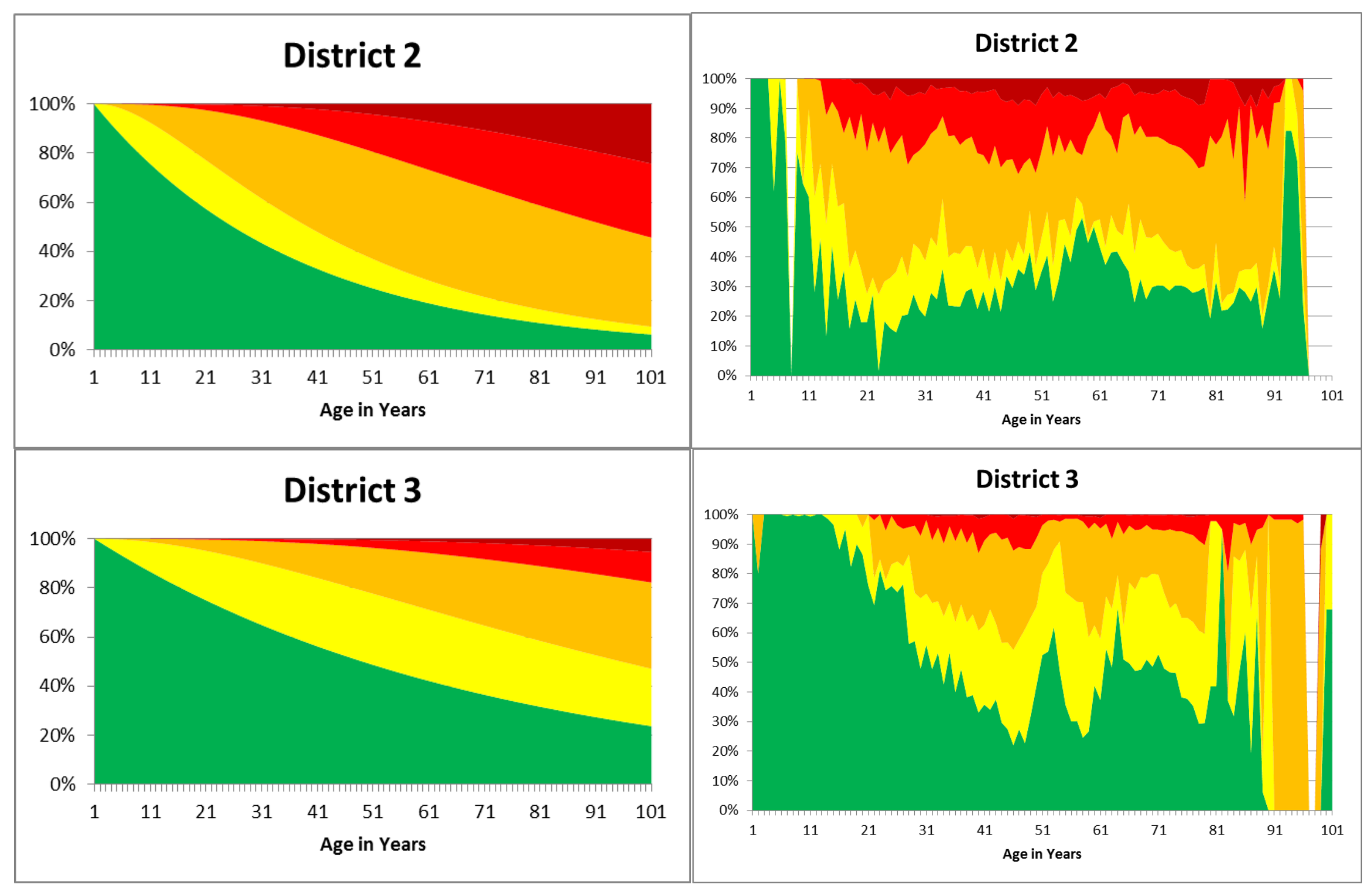

Figure D-67 - Markov Chain Prediction vs. Actual Graphs - Element 108 - Districts 2 and 3 


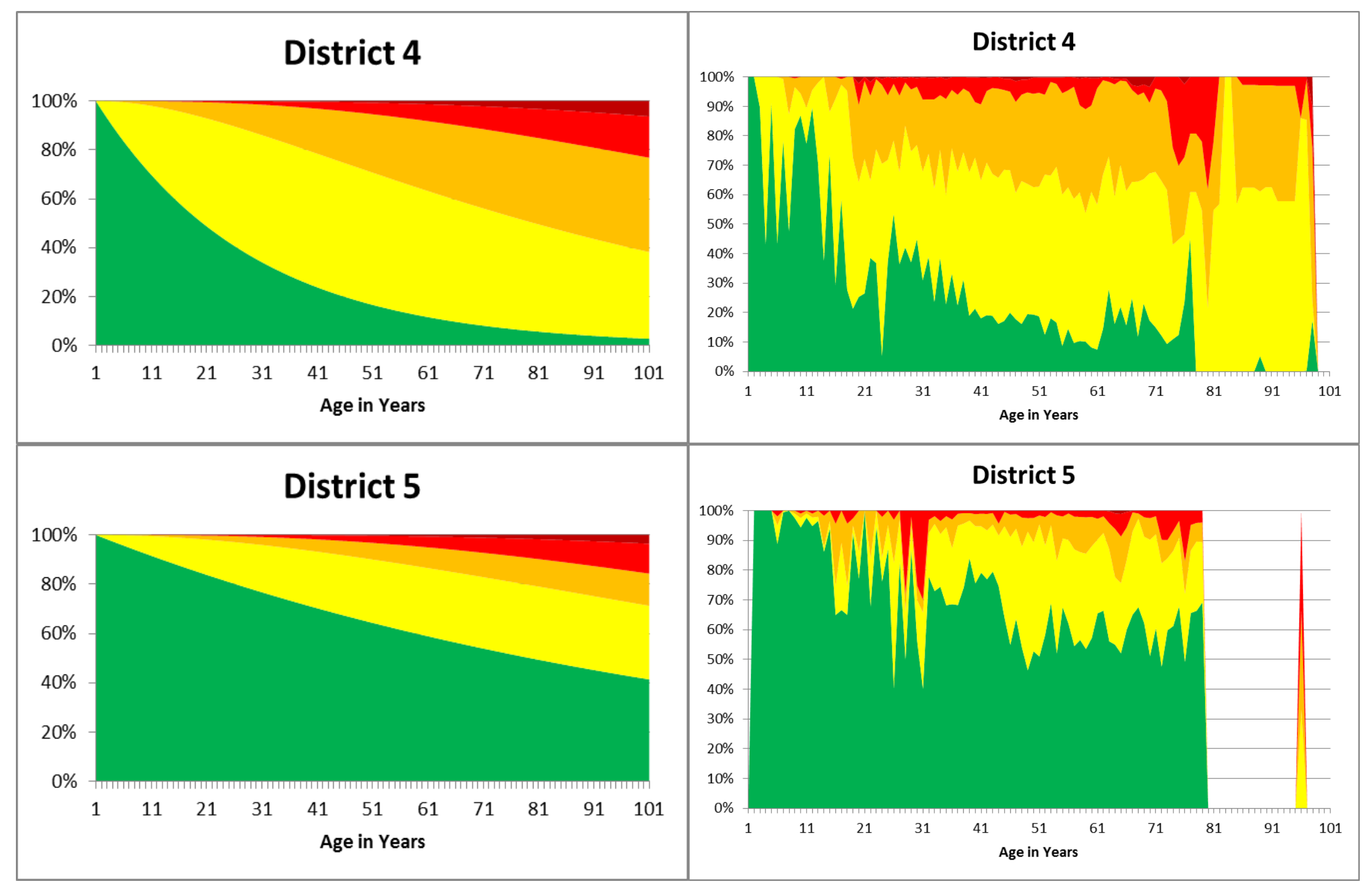

Figure D-68 - Markov Chain Prediction vs. Actual Graphs - Element 108 - Districts 4 and 5 


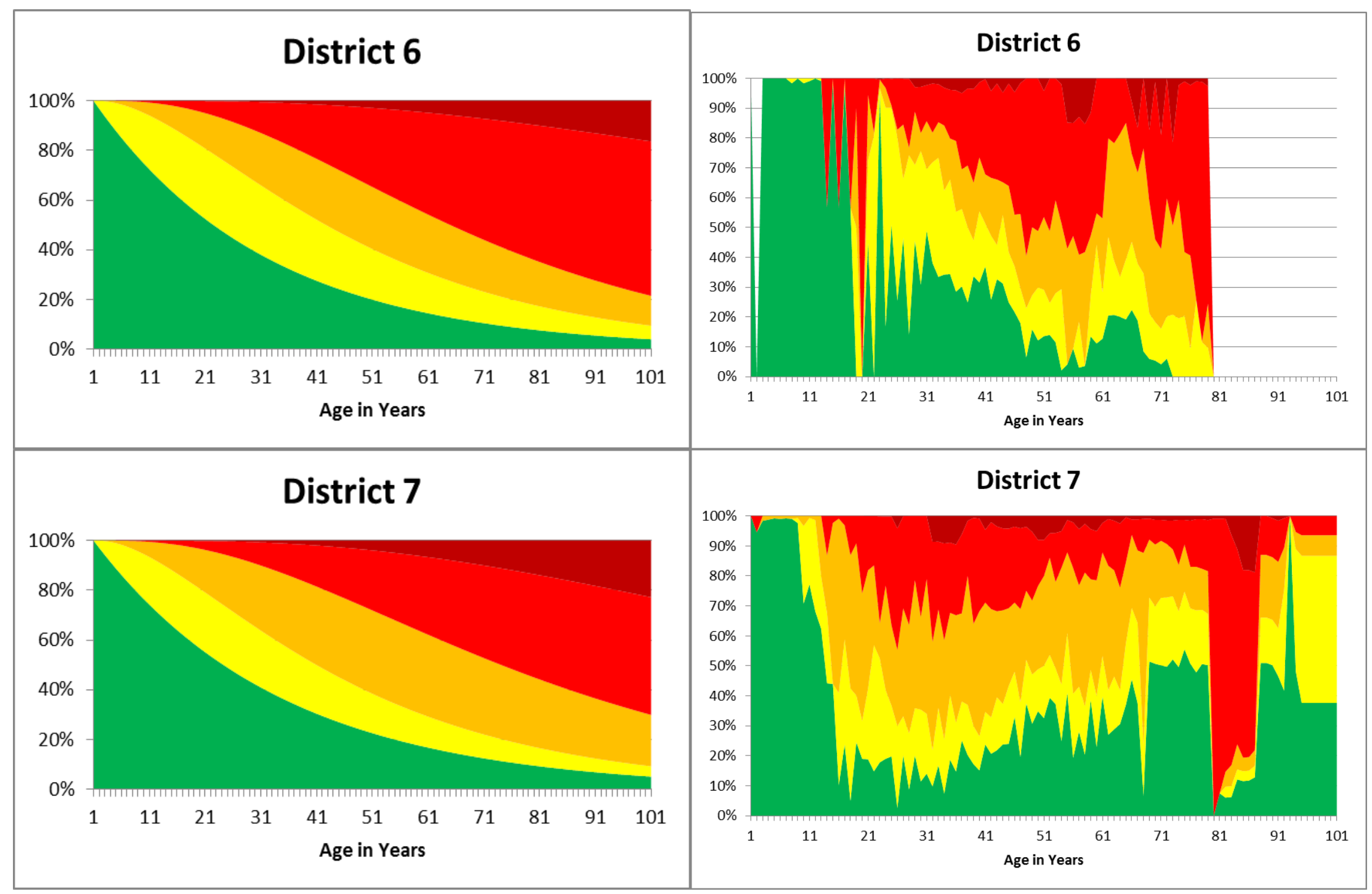

Figure D-69 - Markov Chain Prediction vs. Actual Graphs - Element 108 - Districts 6 and 7 


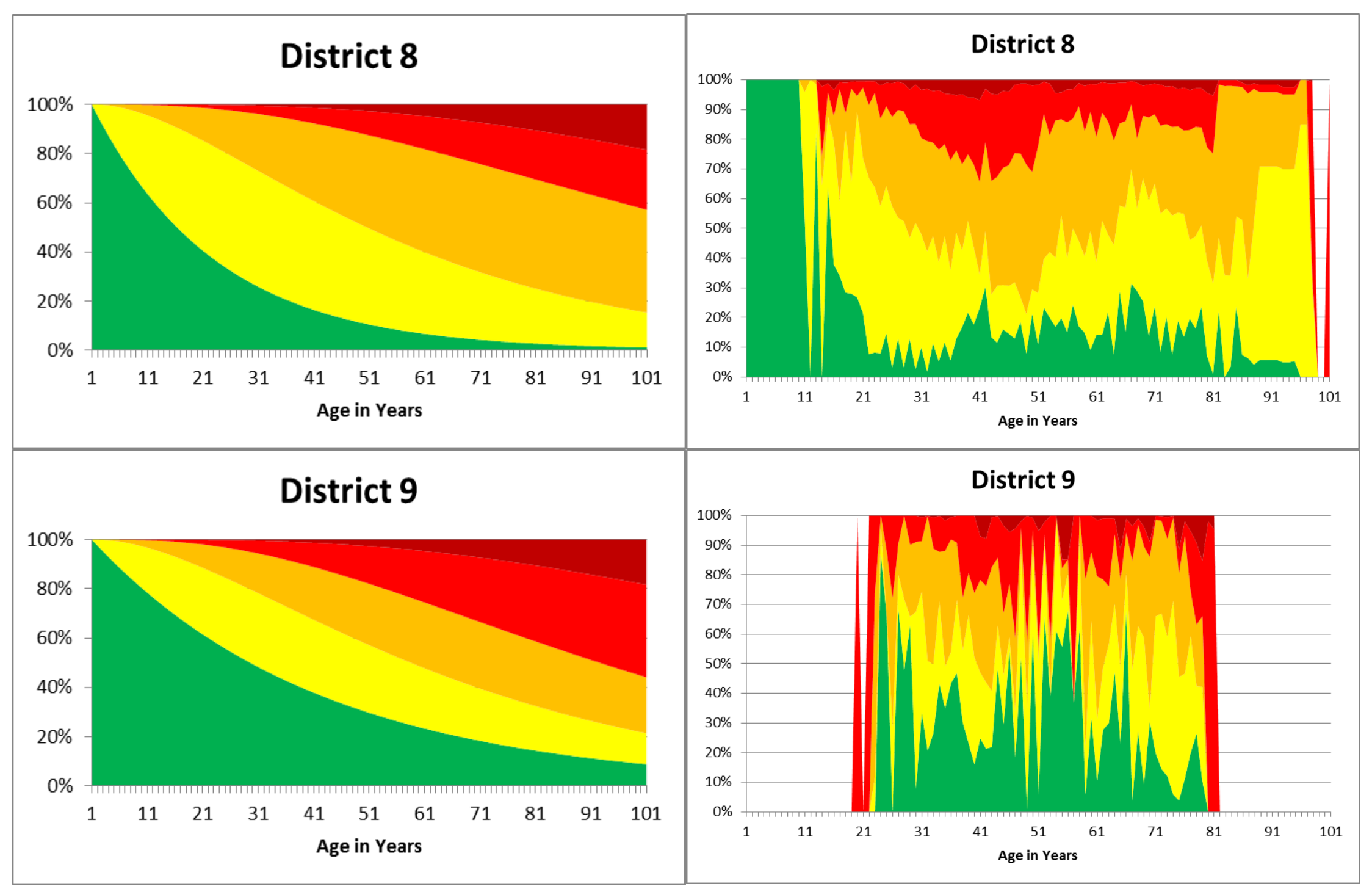

Figure D-70 - Markov Chain Prediction vs. Actual Graphs - Element 108 - Districts 8 and 9 


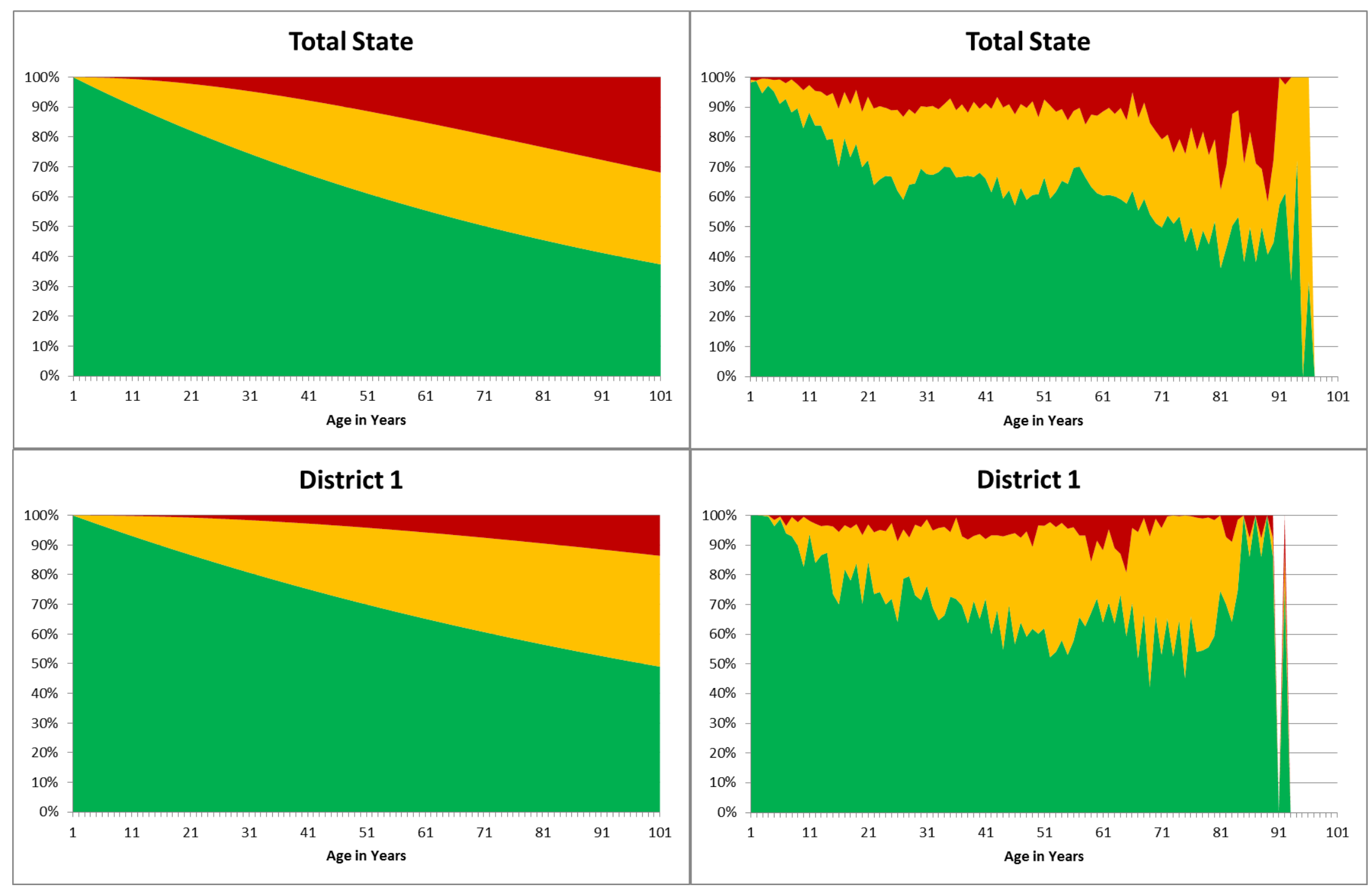

Figure D-71 - Markov Chain Prediction vs. Actual Graphs - Element 302 - Total State and District 1 


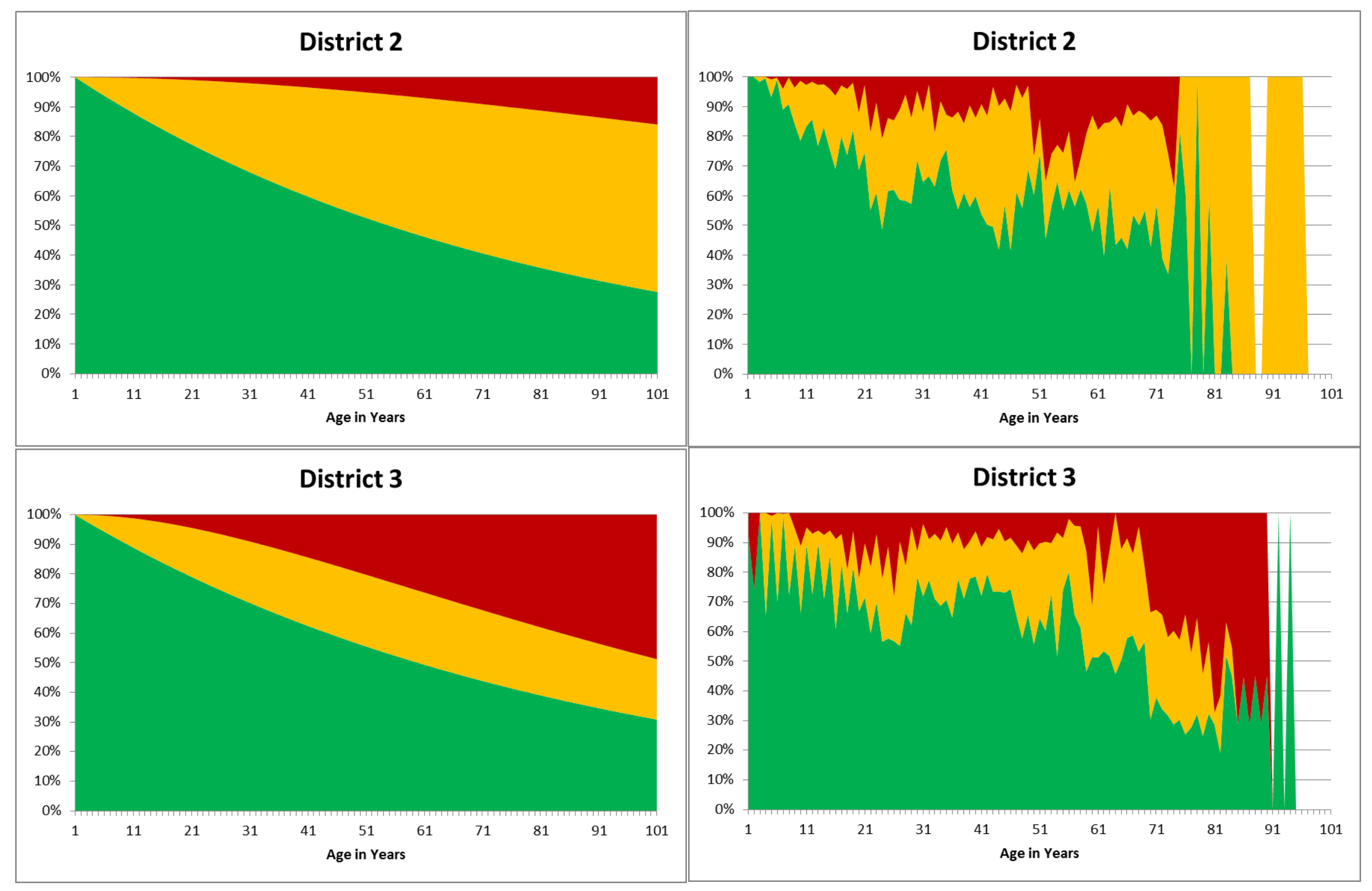

Figure D-72 - Markov Chain Prediction vs. Actual Graphs - Element 302 - Districts 2 and 3 


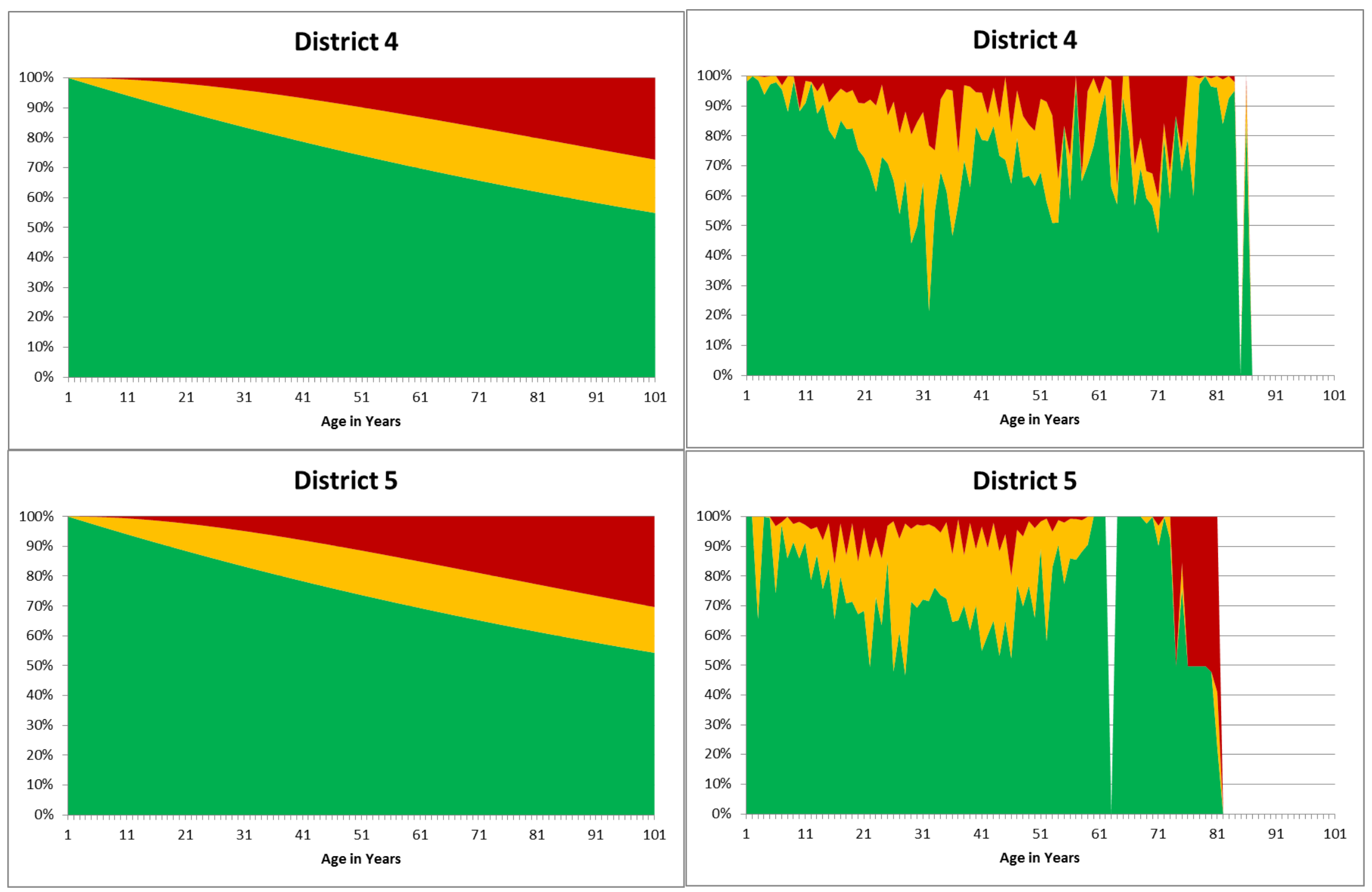

Figure D-73 - Markov Chain Prediction vs. Actual Graphs - Element 302 - Districts 4 and 5 


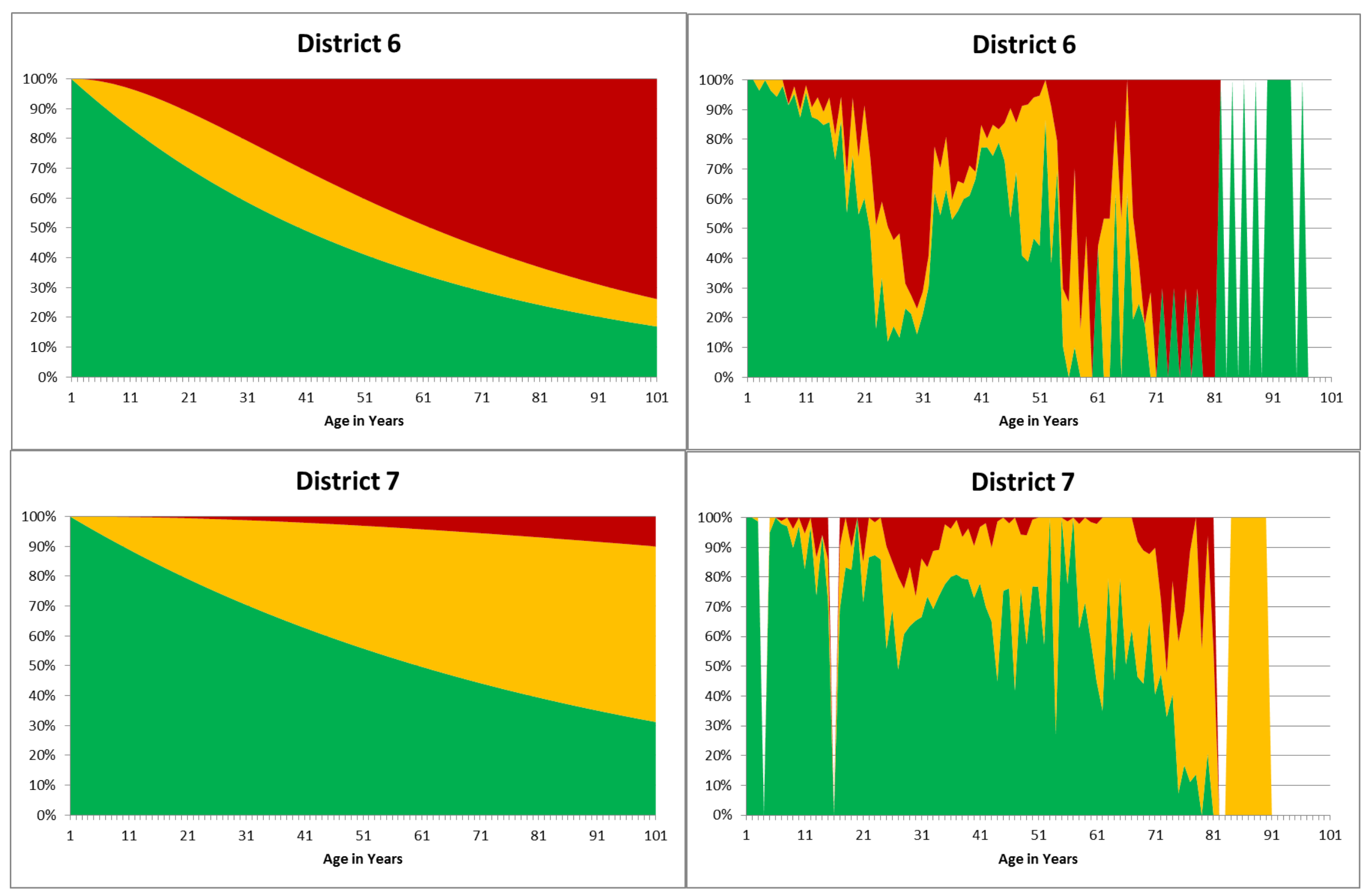

Figure D-74 - Markov Chain Prediction vs. Actual Graphs - Element 302 - Districts 6 and 7 


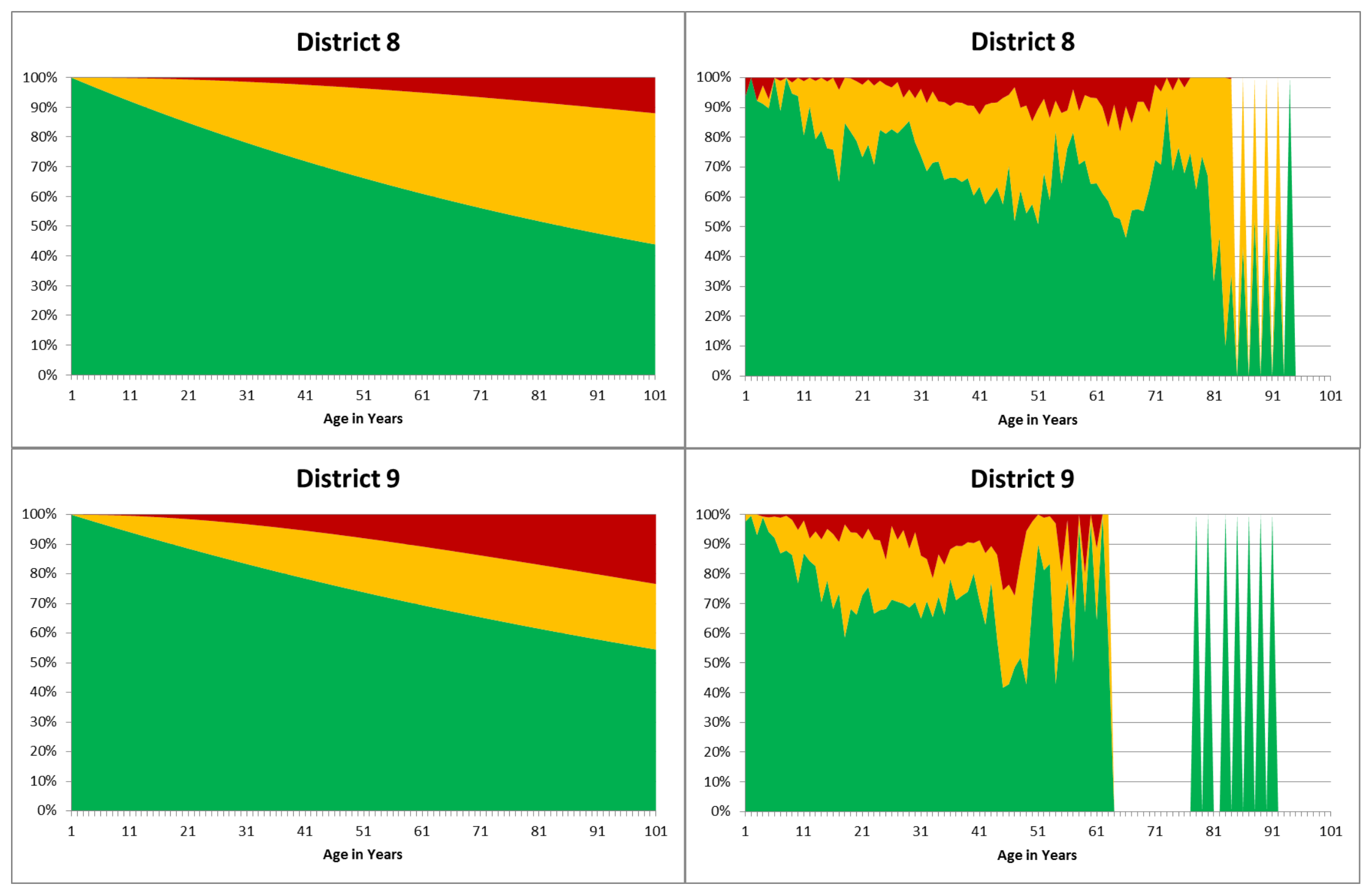

Figure D-75 - Markov Chain Prediction vs. Actual Graphs - Element 302 - Districts 8 and 9 


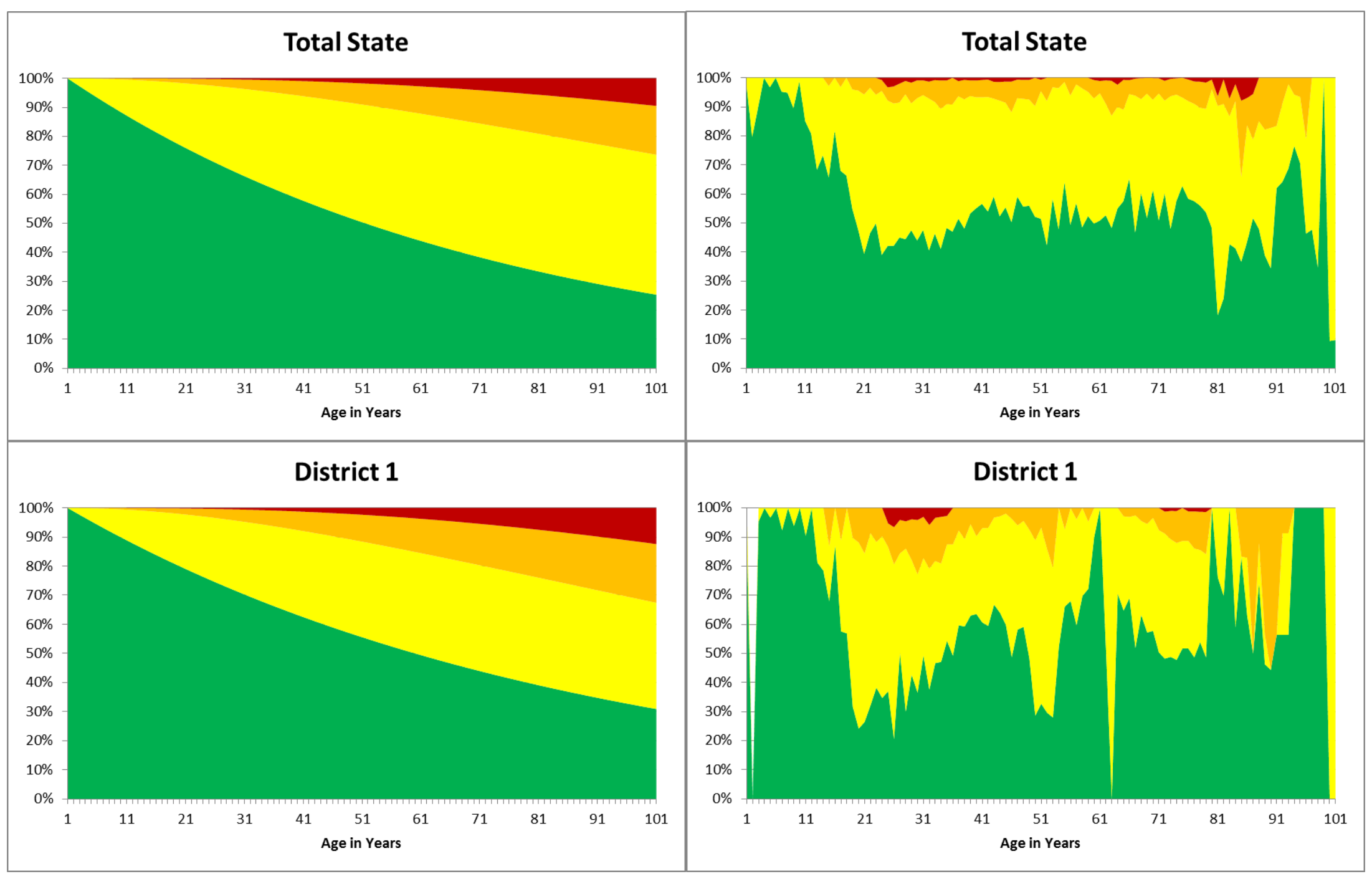

Figure D-76 - Markov Chain Prediction vs. Actual Graphs - Element 32 - Total State and District 1 


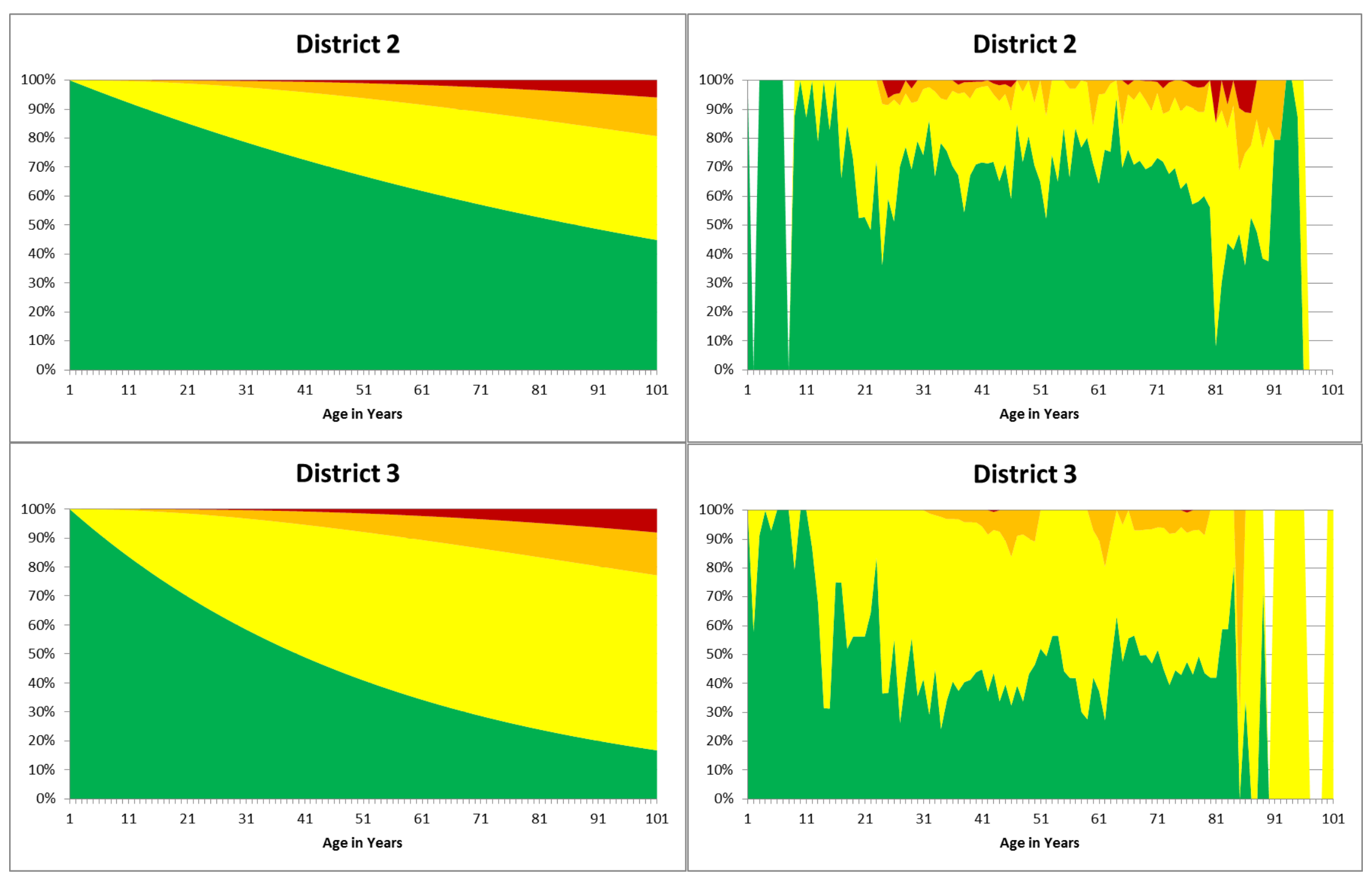

Figure D-77 - Markov Chain Prediction vs. Actual Graphs - Element 32 - Districts 2 and 3 


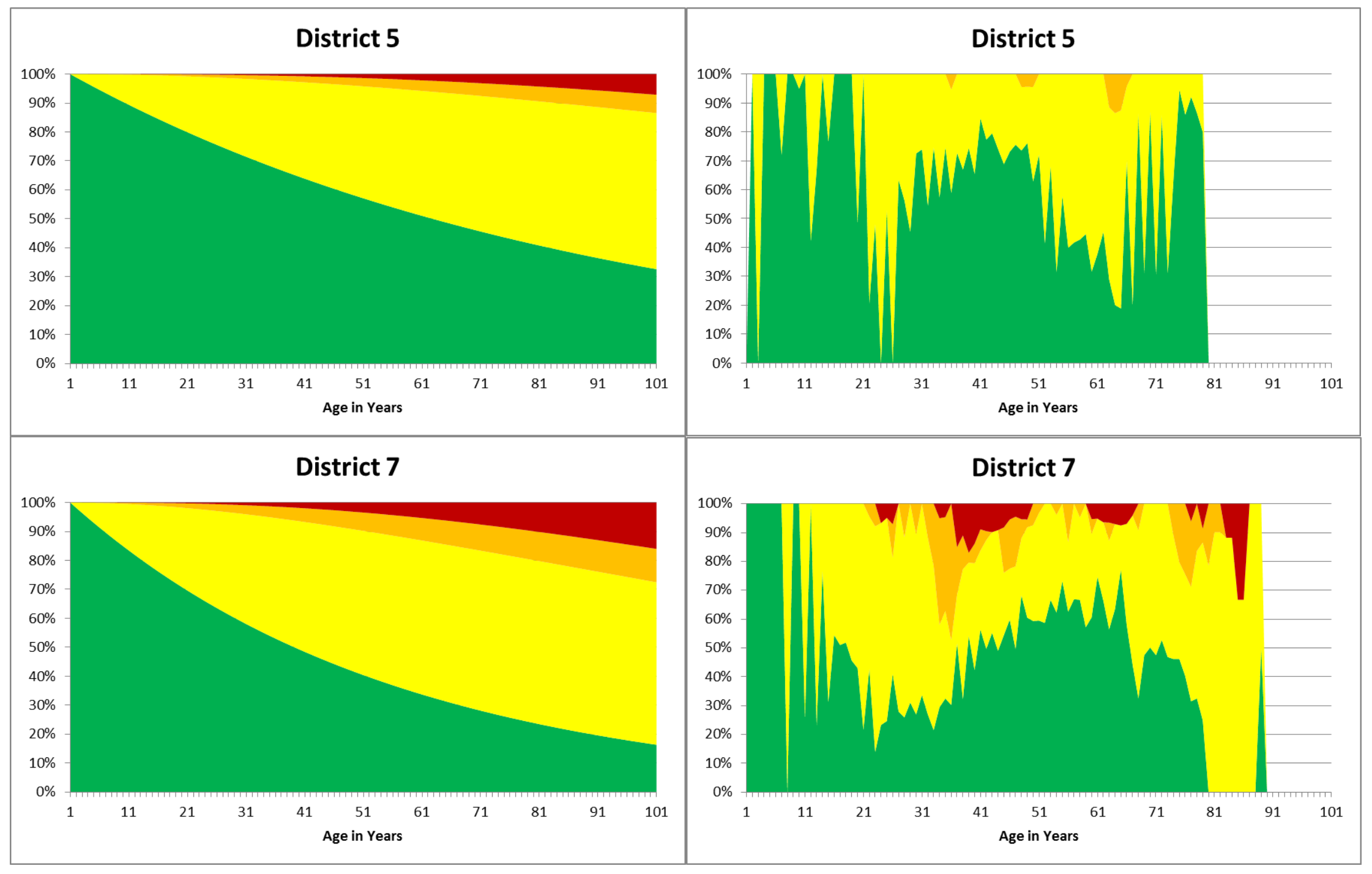

Figure D-78 - Markov Chain Prediction vs. Actual Graphs - Element 32 - Districts 5 and 7 


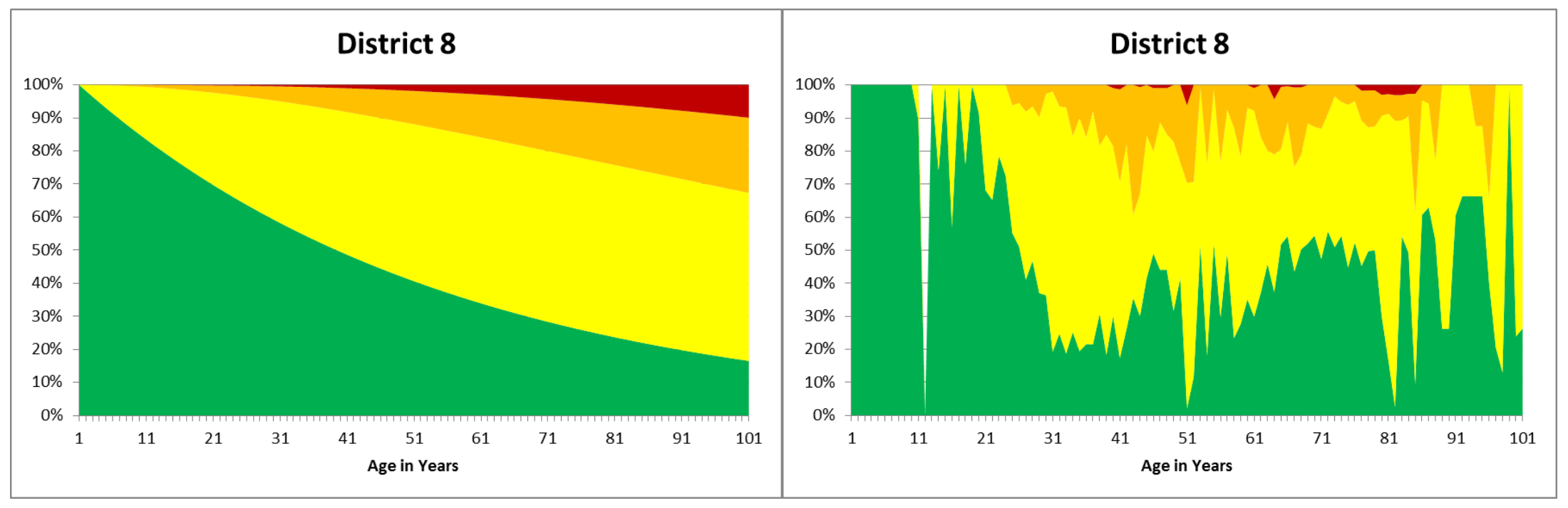

Figure D-79 - Markov Chain Prediction vs. Actual Graphs - Element 32 - District 8 